\title{
The 45th Annual Meeting of the European Society for Blood and Marrow Transplantation: Physicians - Oral Session
}

○ Springer Nature Limited 2019

\section{4-27 March 2019 Frankfurt, Germany}

Modified and published with permission from https://www.ebmt.org/annual-meeting

Sponsorship Statement: Publication of this supplement is sponsored by the European Society for Blood and Marrow Transplantation. All content was reviewed and approved by the EBMT Committee, which held full responsibility for the abstract selections.

Presenting author names are bold type in the contributor lists.

\section{Acute leukaemia}

O010

The impact of the starting day of graft-versus-host disease prophylaxis on haploidentical transplantation with post-transplant cyclophosphamide: A retrospective study on behalf of acute leukaemia working partyEBMT

Annalisa Ruggeri', Myriam Labopin ${ }^{2}$, Johanna Tischer ${ }^{3}$, Jean - Luis Diez Martin ${ }^{4}$, Benedetto Bruno ${ }^{5}$, Simona Sica $^{6}$, Luca Castagna ${ }^{7}$, Boris Afanasyev ${ }^{8}$, Antonin Vitek ${ }^{9}$, Montserrat Rovira $^{10}$, Arnon Nagler ${ }^{11}$, Mohamad Mohty ${ }^{2}$

${ }^{1}$ Ospedale Pediatrico Bambin Gesú, Roma, Italy, ${ }^{2}$ Saint Antoine Hospital, Paris, France, ${ }^{3}$ BMT Unit, Muenchen, Germany, ${ }^{4}$ BMT Unit, Madrid, Spain, ${ }^{5}$ Le Molinette, Torino, Italy, ${ }^{6}$ Universita Cattolica, Roma, Italy, ${ }^{7}$ Humanitas Clinical and Research Center Rozzano, Milano, Italy, ${ }^{8}$ BMT Unit, St Petersburg, Russian Federation, ${ }^{9}$ BMT Unit, Prague, Czech Republic, ${ }^{10}$ BMT Unit, Barcelona, Spain, ${ }^{11}$ Sheba Medical Center, Tel Aviv, Israel

Background: Post-transplant-Cyclophosphamide (PT-Cy) is being used successfully in the setting of unmanipulated haploidentical-transplant (haplo) as graft-versus-host-disease(GVHD) prophylaxis. However, the combined immunosuppressive compounds (IST) used as GVHDprophylaxis and the post-transplant initiation day varies and is not fully standardized.
Methods: Study aim was to evaluate the schedule of IST given in combination with PT-Cy as GVHD-prophylaxis post-haplo for acute leukemia (AL) and reported to the ALWP/EBMT registry. Patients were divided into 3 groups: received cyclosporine A-mycofenolate-mofetil(CSA$+\mathrm{MMF}$ ) initiated at day +1 (group-1, $\mathrm{n}=124$ ) or CSA + MMF both started at day +5 (group- $2, n=170$ ) and tacrolimus + MMF from day +5 (group-3, $n=215$ ). Transplants were performed from 2006-2017 and median follow up is 21 months (range 11-36). PT-CY was given on Day +3 and Day +5 in group -1 and on day +3 and day +4 in group-2 and 3.

Results: Acute myeloid leukemia (AML) was the most common indication for haplo (76\%) and approximately $45 \%$ of patients were transplanted in CR1. There were some differences among groups: patients in group-1 were younger (median age 46 years, $\mathrm{p}<0.02$ ) were transplanted in more recent year $(2015, \mathrm{p}<0.001)$, received more frequently a regimen based on TBF (thiotepa, fludarabine and busulfan) $(83 \%, \mathrm{p}<0.001)$ and bone marrow $(\mathrm{BM})$ as source of stem cells $(77 \%, \mathrm{p}<0.001)$, with no ATG $(100 \%$, $\mathrm{p}<0.001$ ). Probability of OS at 2 years was $59 \%, 48 \%$ and $44 \%$, for the 3 groups, respectively, $\mathrm{p}=0.15$. Probability of LFS and GRFS at 2 years were $52 \%$ and $46 \%, 43 \%$ and $36 \%, 39 \%$ and $33 \%$, for the 3 groups, respectively, (LFS $\mathrm{p}=0.05$, GRFS $\mathrm{p}=0.01$. Overall the cumulative incidence (CI) of grade II-IV acute GVHD was $18 \%, 39 \%$ and $25 \%$, for the 3 groups, respectively, $\mathrm{p}<0.001$, and the CI of chronic GVHD was $23 \%, 21 \%$ and $25 \%$; $\mathrm{p}=0.28$. The CI of relapse at 2 years was $26 \%, 37 \%$ and $35 \%(\mathrm{p}=0.01)$ and 
non-relapse mortality (NRM) was 21\%, 20\% and $26 \%$ $(\mathrm{p}=0.34)$ for group $-1,2$ and 3 , respectively. Disease recurrence, infections and GVHD were the most common causes of death in the whole cohort.In multivariate analysis, the early start of MMF at day +1 in addition to PT-CY was associated with reduced risk of chronic GVHD (HR 0.48, $\mathrm{p}=0.03$ ) and improved GRFS (HR 0.63, $\mathrm{p}=0.02$ ) while the effect on relapse was not confirmed. Stem cell source and type of conditioning regimen did not influenced the outcomes in multivariate analysis.The timing of immunosuppression did not influenced NRM and survival. Diagnosis of ALL (HR 1.64, $\mathrm{p}=0.04$ ), performance-status less than $90 \%$ (KPS90) (HR 1.72, $\mathrm{p}=0.001$ ), were associated with increased risk of NRM. Older age (HR 0.87, $p=0.02$ ), and active disease (HR 3.58, $\mathrm{p}<0.001)$ were associated with increased risk of relapse. KPS (OS, HR 0.59, p< 0.01; LFS, HR 0.62, p $<0.001$ ) and active disease (OS, HR 2.3, p< 0.01 ; LFS, HR 2.43, p< 0.001) were independently associated with reduced risk of OS and LFS.

Conclusions: The time of starting of immunosuppression drugs after haplo in combination with PT-Cy influences the results of unmanipulated haploidentical transplant. The early starting of CSA $+\mathrm{MMF}$ at day +1 decreases the risk of chronic GVHD, and improves GRFS.

Disclosure: No disclosure

\section{1}

Human acute myeloid leukemia stem cells selectively escape NKG2D-mediated NK cell control

Anna Paczulla ${ }^{1}$, Kathrin Rothfelder ${ }^{2,3}$, Simon Raffel ${ }^{4,5,6}$, Martina Konantz', Julia Steinbacher, ${ }^{2,3}$, Hui Wang ${ }^{1}$, Marcelle Christine Ndoh Mbarga ${ }^{I}$, Thorsten Schäfer ${ }^{I}$, Daniela Dörfel ${ }^{3}$, Mattia Falcone ${ }^{4}$, Claudia Tandler ${ }^{2,3}$, Jakob Passweg ${ }^{1}$, Pontus Lundberg ${ }^{1}$, Lothar Kanz ${ }^{3}$, Leticia Quintanilla-Martinez ${ }^{3}$, Alexander Steinle ${ }^{7}$, Andreas Trumpp $^{4,5}$, Helmut R. Salih ${ }^{2,3}$, Claudia Lengerke ${ }^{I}$

${ }^{1}$ University and University Hospital Basel, Basel, Switzerland, ${ }^{2}$ German Cancer Consortium (DKTK), Partner Site Tuebingen, Heidelberg, Germany, ${ }^{3}$ University of Tuebingen, Tuebingen, Germany, ${ }^{4}$ Deutsches Krebsforschungszentrum (DKFZ), Heidelberg, Germany, ${ }^{5}$ Heidelberg Institute for Stem Cell Technology and Experimental Medicine (HI-STEM gGmbH), Heidelberg, Germany, ${ }^{6}$ University of Heidelberg, Heidelberg, Germany, ${ }^{7}$ Goethe University Frankfurt, Frankfurt, Germany

Background: Patients with acute myeloid leukaemia (AML) often achieve remission but subsequently die of relapse driven by chemotherapy resistant leukemic stem cells (LSCs). Here we hypothesized that LSCs must also escape immunosurveillance to initiate and maintain cancer and investigate the interplay with $\mathrm{NKG} 2 \mathrm{D}$, a danger detector expressed by cytotoxic lymphocytes such as natural killer (NK) cells that recognizes stress-induced ligands (NKG2DL) of the MIC and ULBP protein families on AML cells.

Methods: 175 de novo AML were stained with antibodies against MICA, MICB and ULB2/5/6 or an NKG2D-Fe chimeric protein recognizing pan-NKG2DL. $\mathrm{NKG}_{2} \mathrm{DL}^{\text {pos }}$ and $\mathrm{NKG} 2 \mathrm{DL}^{\text {neg }}$ AML cells sorted from the same patient were analysed in colony forming assays, leukemogenesis assays in NSG mice, by RNAseq, gene expression arrays, qRT-PCR and targeted next generation sequencing. AML cells co-cultured or not with NK cells (control or anti-NKG2D pre-treated) were co-stained for additional stem/immunological markers. PARP1 expression was analysed by qRT-PCR and immunoblot, and binding to NKG2DL promoters by chromatin immunoprecipitation. PARP1 inhibition (PARPi) in AML cells was performed in vitro or in vivo using siRNAs or inhibitors (AG-14361, veliparib).

Results: Heterogeneous NKG2DL expression was detected among leukemic cells of the same patient (Fig. 1a). Interestingly, when compared to $\mathrm{NKG}_{2} \mathrm{DL}^{\mathrm{pos}}$ subpopulations, NKG2DL ${ }^{\text {neg }}$ AML cells isolated from the same patient showed immature morphology, enhanced in vitro clonogenicity $(39 \pm 47$ colonies vs. $1 \pm 4, \mathrm{p}<0.001, \mathrm{n}=32$ AML patients) and selective abilities to initiate leukemia (NKG2DL $^{\text {neg }}, 64 / 70,91 \%$; NKG2DL ${ }^{\text {pos }}, 0 / 78,0 \%$; p $<$ 0.00001 , Fig. $1 \mathrm{~b}, \mathrm{n}=19$ AML patients) and survive chemotherapy in NSG mice devoid of functional NK cells. In mice, NKG2DL ${ }^{\text {neg }}$ AML cells generated both NKG2DL ${ }^{\text {pos }}$ and NKG2DL ${ }^{\text {neg }}$ progeny of which again only latter was able to induce leukemia in re-transplant assays. Similar leukemia-specific mutations were detected in NKG2DL ${ }^{\text {neg }}$ compared to NKG2DL ${ }^{\text {pos }}$ AML cells from the same AML but published LSC (Fig. 1c), HSC and 17genes stemness score signatures were specifically enriched in NKG2DL ${ }^{\text {neg }}$ subfractions. NKG2DL ${ }^{\text {neg }}$ cells enriched for LSCs defined by alternative markers $\left(\mathrm{CD} 34^{+}, \mathrm{CD} 38^{-}\right.$, GPR56 ${ }^{+}$) but could identify cells with functional and molecular LSC activity also in CD34 non-expressing AML $(\mathrm{n}=11$ analyzed patients). NKG2DL expression was repressed by PARP1 recruitment at NKG2DL promoters. PARP1 inhibition (PARPi) induced NKG2DL surface expression in LSCs and co-treatment with PARPi and NK cells (but not with either alone) suppressed leukemogenesis in patient derived xenograft (PDX) models (Fig. 2c) cotransplanted with NK cells. Low NKG2DL surface or high PARP1 mRNA expression associated with poor outcome in AML patients. Furthermore, NKG2DL ${ }^{\text {neg }}$ and $\mathrm{CD} 34^{+}$LSCs showed reduced expression of other immune stimulatory molecules (e.g. CD112, CD155, CD80, CD86) and different 
expression of immune or inflammatory response gene signatures (GSEA).

Conclusions: These data indicate that LSCs escape NK cell recognition by selectively suppressing the surface expression of NKG2DL and other immunostimulatory molecules. Absence of NKG2DL can identify LSCs across genetic AML subtypes (including CD34 negative AMLs). This LSC specific mechanism of immune evasion could be overcome by treatment with PARP1 inhibitors, which in conjunction with functional NK cells holds promise to eradicate LSCs and promote immune-mediated cure of AML.

Disclosure: C.L.: Sanofi, Novartis, Otsuka (consultancy); Roche (research funding)

\section{2}

Abstract already published.

\section{3}

Second and third generation tyrosin kinase inhibitors in persisting minimal residual disease, molecular and hematological relapse after allogeneic stem cell transplantation for philadelphia chromosome positive adult all

Klaus Hirschbühl, Myriam Labopin ${ }^{2}$, Christoph Schmid $^{1}$, Mohamed Houhou ${ }^{2}$, Nathalie Fegueux ${ }^{3}$, Hélène Labussière-Wallet ${ }^{4}$, Bruno Lioure ${ }^{5}$, Dietrich Beelen ${ }^{6}$, Jan Cornelissen $^{7}$, Gerald Wulf ${ }^{8}$, Pavel Jindra ${ }^{9}$, Herve Tilly ${ }^{10}$, Jakob Passweg ${ }^{11}$, Riita Niittyvuopio ${ }^{12}$, Gesine Bug ${ }^{13}$, Arnon Nagler $^{14}$, Sebastian Giebel ${ }^{15}$, Mohamad Mohty ${ }^{2}$

${ }^{1}$ Klinikum Augsburg, Augsburg, Germany, ${ }^{2}$ Hôpital Saint Antoine, ALWP Office (EBMT), Paris, France, ${ }^{3} \mathrm{CHU}$ Lapeyronie, Montpellier, France, ${ }^{4}$ Centre Hospitalier Lyon Sud, France, ${ }^{5}$ Nouvel Hopital Civil, Strasbourg, France, ${ }^{6}$ University Hospital Essen, Essen, Germany, ${ }^{7}$ Erasmus MC Cancer Institute University Medical Center Rotterdam, Rotterdam, Netherlands, ${ }^{8}$ Universitätsklinikum Göttingen, Göttingen, Germany, ${ }^{9}$ Charles University Hospital, Pilsen, Czech Republic, ${ }^{10}$ Centre Henri Becquerel, Rouen, France, ${ }^{11}$ University Hospital, Basel, Switzerland, ${ }^{12} \mathrm{HUCH}$ Comprehensive Cancer Center, Helsinki, Finland, ${ }^{13}$ Universitätsklinikum Frankfurt, Frankfurt, Germany, ${ }^{14}$ TelHashomer and Tel-Aviv University, Chaim Sheba Medical Center, Tel Aviv, Israel, ${ }^{15}$ Maria Sklodowska-Curie Memorial Cancer Center and Institute of Oncology, Gliwice, Poland

Background: In contrast to imatinib, data on the use of $2^{\text {nd }}$ and $3^{\text {rd }}$ generation tyrosin kinase inhibitors (TKI) in the treatment of minimal residual disease (MRD), molecular and hematological relapse (MR/HR) after allogeneic stem cell transplantation (SCT) in Philadelphia chromosome positive $(\mathrm{Ph}+)$ acute lymphoblastic leukemia (ALL) are scarce.

Methods: We performed a retrospective, EBMT registry based analysis, including patients with documented use of $2^{\text {nd }}$ or $3^{\text {rd }}$ generation TKI given for persisting MRD, MR or HR after alloSCT in 2006-2016. Choice of TKI, efficacy, and toxicity of TKI and patient outcome were analysed.

Results: 140 patients (female 58, male 82) were identified, out of which 136 had also received a TKI (78\% imatinib) before alloSCT. Median age at transplant was 48 years (18-66), $81 \%$ were transplanted in first complete remission (CR1), $51 \%$ of the patients were in molecular CR. Conditioning was myeloablative in $71 \%$ and reduced in $29 \%$, donors were matched siblings (48\%), unrelated (41\%), haploidentical $(6 \%)$ and cord blood (5\%). After alloSCT, 111 patients developed HR, 23 MR and 6 had persisting MRD. For treatment, patients received dasatinib $(\mathrm{n}=104)$, nilotinib $(\mathrm{n}=18)$ and ponatinib $(\mathrm{n}=18)$. Median interval between diagnosis of persisting MRD or MR/HR and first application of a TKI was 10 days, median duration of TKI treatment was 154 days (range 4 2193). Fifty-eight patients were treated with TKI only (dasatinib, $n=50$, nilotinib, $n=3$, ponatinib, $n=5$ ), while 82 received additional treatment such as DLI, chemotherapy, or second alloSCT. Main toxicities of dasatinib were effusion, edema, or other pulmonary complaints (10 $15 \%$ of patients) and infections (13\%). No particular side effects were reported for nilotinib and ponatinib (no vascular events). Dose reduction of TKI was required in $32 \%$. Response rates were $71 \%$ (entire cohort) and $72 \%$ (patients receiving TKI only). For the entire cohort, 2- and 5 -year overall survival (OS) from first application of TKI was $49 \%$ and $33 \%$. Two-year OS was comparable in patients treated for persisting MRD/MR and for HR (48\% and 50\%). Among patients treated with TKI only, 2/5-year OS was $38 \% / 33 \%$. Rate of cGvHD was $11 \%$ for the whole population and $14 \%$ for the TKI alone cohort.

Conclusions: The use of $2^{\text {nd }}$ and $3^{\text {rd }}$ generation TKI, given alone or in combination with other therapies for treating persisting MRD, MR or HR after alloSCT in $\mathrm{Ph}+$ ALL was not associated with increased toxicities. Dasatinib was the most frequently used drug. Outcome compared favorably with published results on relapse after alloSCT in $\mathrm{Ph}$ negative ALL, suggesting that treatment with TKI could improve survival after post-transplant relapse, even when given as single therapy. Type of relapse did not influence response rates and outcome.

Disclosure: Nothing to declare

\section{4}

Multi-state modelling of the interplay between remission-induction chemotherapy and consolidation with alloSCT in newly diagnosed AML patients 
Katerina Bakunina ${ }^{1}$, Liesbeth C. de Wreede ${ }^{2}$, Jurjen Versluis $^{1}$, Markus G. Manz ${ }^{3}$, Dimitri A. Breems ${ }^{4}$, Bjorn T. Gjertsen $^{5}$, Yvette van Norden ${ }^{1}$, Jacqueline Cloos ${ }^{6}$, Jakob Passweg $^{7}$, Thomas Pabst ${ }^{8}$, Gert J. Ossenkoppele ${ }^{6}$, Bob Löwenberg ${ }^{1}$, Hein Putter ${ }^{2}$, Jan J. Conelissen ${ }^{1}$

${ }^{1}$ Erasmus University Medical Center, Rotterdam, Netherlands, ${ }^{2}$ Leiden University Medical Center, Leiden, Netherlands, ${ }^{3}$ University Hospital, Zurich, Switzerland, ${ }^{4}$ Ziekenhuis Netwerk Antwerpen, Antwerpen, Belgium, ${ }^{5}$ Haukeland University Hospital, Bergen, Norway, ${ }^{6}$ Amsterdam University Medical Center, Amsterdam, Netherlands, ${ }^{7}$ University Hospital Basel, Basel, Switzerland, ${ }^{8}$ University Hospital/Inselspital, Bern, Switzerland

Background: Many randomized phase III clinical trials for newly diagnosed patients with AML are designed to contrast the efficacy of a standard remission-induction chemotherapy with the addition of a new promising chemotherapeutic agent. After achieving CR, patients are consolidated with chemotherapy, autoSCT or alloSCT, according to estimated risk of relapse and NRM, and timing of CR. A remission-induction chemotherapy leading to higher or earlier CR rates thus would influence the accessibility of consolidation treatments, and the successive clinical outcomes. Nontrivial statistical methods like multistate models help to disentangle the direct effects of remission-induction chemotherapy and the indirect effects through consolidation therapy. They are of special relevance for modelling the impact of alloSCT considering the significance of its influence on subsequent outcomes.

Methods: Between August 2010 and September 2013, 795 eligible patients (708 AML, 87 MDS with excess blasts) aged 18-65 years have been randomized in the HOVON-102 / SAKK-30/09 prospective controlled clinical trial to receive standard induction chemotherapy with or without clofarabine $\left(10 \mathrm{mg} / \mathrm{m}^{2}\right.$ for 5 days) Consolidation therapy options included alloSCT, depending on the patients' prognostic risk score as described in the study protocol and the achievement of CR. A multi-state model was designed to evaluate the effect of the treatment arm on relapse and death, where distinction is made between events happening before and after consolidation with alloSCT.

Results: The study population and primary results have previously been reported (Blood 2017;129(12):1636-1645); after a median follow-up of 36 months no significant difference in CR rates, EFS and OS between the treatment arms has been found. Among the 402 patients randomized to the control arm, $184(46 \%)$ were consolidated with alloSCT, compared to $176(45 \%)$ of the 393 patients randomized to receive clofarabine. Using a multi-state model which distinguishes between relapse before and after alloSCT, we further investigated the previously published reduced rate of relapse in the clofarabine arm, discovering a significant difference between the treatment arms in the hazard of relapse only after alloSCT (HR $0.65,95 \% \mathrm{CI}$ $0.46-0.94$, p-value $=0.02)$, and not before alloSCT $(\mathrm{HR}$ $0.81,95 \%$ CI $0.58-1.13$, p-value $=0.20)$. In addition, we found increased NRM in the clofarabine arm before alloSCT (HR 1.95, 95\% CI 1.15-3.31, p-value $=0.01$ ), and not after alloSCT (HR 0.80, 95\% CI 0.50-1.28, p-value $=0.34)$. These effects are statistically significantly different (interaction test HR $0.41,95 \%$ CI $0.20-0.83$, p-value = 0.01 ). At two years after registration, $20.4 \%$ (95\% CI 16.6$24.1)$ of the patients in the control arm, and $19.9 \%$ (16.123.6) in the clofarabine arm were alive relapse-free in CR without alloSCT, while $20.9 \%(16.7-25.1)$ and $26.8 \%$ (22.3-31.4) were alive relapse-free after alloSCT.

Conclusions: Presented results suggest that the rate of relapse after alloSCT is lower among patients whose induction therapy includes clofarabine. These results could possibly be explained by higher rate of MRD negativity achieved in the clofarabine arm before proceeding to alloSCT. We also observed a higher NRM rate in CR before alloSCT in the clofarabine arm, indicating that the favorable effect of clofarabine on relapse may be compromised by toxicity.

Clinical Trial Registry: HOVON 102 study is registered at Netherlands Trial Registry \#NTR2187

Disclosure: Nothing to declare

\section{O015}

Haploidentical transplant with post-transplant cyclophosphamide for $\mathbf{T}$-cell acute lymphoblastic leukemia: Outcome strongly correlates with disease status; a report from the EBMT acute leukemia working party

Ali Bazarbachi, Myriam Labopin ${ }^{2}$, Emanuele Angelucci $^{3}$, Zafer Gülbas ${ }^{4}$, Hakan Ozdogu ${ }^{5}$, Mutlu Arat ${ }^{6}$, Luca De Rosa ${ }^{7}$, Corrado Tarella ${ }^{8}$, Pietro Pioltelli, Rovira Montserrat $^{10}$, Massimo Martino ${ }^{11}$, Fabio Ciceri ${ }^{12}$, Yener Koc $^{13}$, Gerard Socie $e^{14}$, Didier Blaise ${ }^{15}$, Concepcion Herrera Arroyo $^{16}$, Yves Chalandon ${ }^{17}$, Paolo Bernasconi ${ }^{18}$, Giuseppe Marotta ${ }^{19}$, Luca Castagna ${ }^{20}$, Andrew McDonald $^{21}$, Guiseppe Visani ${ }^{22}$, Giorgina Specchia ${ }^{23}$, Antonin Vitek $^{24}$, Bruno Lioure ${ }^{25}$, Boris Afanasyev ${ }^{26}$, Wolf Rösler ${ }^{27}$, J.L. Diez-Martin ${ }^{28}$, Arnon Nagler ${ }^{29}$, Eolia Brissot ${ }^{30}$, Sebastian Giebel ${ }^{31}$, Mohamad Mohty ${ }^{30}$

${ }^{1}$ American University of Beirut Medical Center, Beirut, Lebanon, ${ }^{2}$ EBMT Paris Study Office/CEREST-TC, Hôpital Saint Antoine, Paris, France, ${ }^{3}$ Hematology and Transplant Center, IRCCS Ospedale Policlinico San Martino, Genova, Italy, ${ }^{4}$ Anadolu Medical Center Hospital, Kocaeli, Turkey, ${ }^{5}$ Baskent University Hospital, Adana, Turkey, ${ }^{6}$ Florence Nightingale Sisli Hospital, Istanbul, Turkey, ${ }^{7}$ Ospedale S. 
Camillo-Forlanini, Rome, Italy, ${ }^{8}$ European Institute of Oncology, Milano, Italy, ${ }^{9}$ Ospedale San Gerardo, Monza, Italy, ${ }^{10}$ Hospital Clinic, Barcelona, Spain, ${ }^{11}$ Grande Ospedale Metropolitano Bianchi Melacrino Morelli - Centro Unico Trapianti A. Neri, Bianchi-Melacrino-Morelli, Reggio Calabria, Italy, ${ }^{12}$ Ospedale San Raffaele s.r.l., Milano, Italy, ${ }^{13}$ Medical Park Hospitals, Antalya, Turkey, ${ }^{14}$ Hopital St. Louis, Paris, France, ${ }^{15}$ Aix Marseille University, Marseille, France, ${ }^{16}$ Hosp. Reina Sofia, Córdoba Hospital, Cordoba, Spain, ${ }^{17}$ Hôpitaux Universitaires de Genève and Faculty of Medicine, University of Geneva, Geneva, Switzerland, ${ }^{18}$ Fondazione IRCCS Policlinico San Matteo, Pavia, Italy, ${ }^{19}$ Azienda Ospedaliera Universitaria Senese, Policlinico S.Maria alle Scotte, Siena, Italy, ${ }^{20}$ Istituto Clinico Humanitas, Milano, Italy, ${ }^{21}$ Pretoria East Hospital, Pretoria, South Africa, ${ }^{22}$ Pesaro Hospital Hematology \& Transplant Centre, Pesaro, Italy, ${ }^{23}$ U.O. Ematologia con Trapianto Azienda Ospedaliero Universitaria Policlinico Bari, Bari, Italy, ${ }^{24}$ Institute of Hematology and Blood Transfusion, Prague, Czech Republic, ${ }^{25}$ Nouvel Hopital Civil, Strasbourg, France, ${ }^{26}$ First State Pavlov Medical University of St. Petersburg, Raisa Gorbacheva Memorial Research Institute for Paediatric Oncology, Hematology, and Transplantation, St Petersburg, Russian Federation, ${ }^{27}$ University Hospital Erlangen, Erlangen, Germany, ${ }^{28}$ Hospital Gregorio Marañón, Madrid, Spain, ${ }^{29}$ Chaim Sheba Medical Center, Tel Hashomer, Israel, ${ }^{30}$ Hopital St. Antoine, Paris, France, ${ }^{31}$ Maria SklodowskaCurie Institute-Oncology Centre, Gliwice, Poland

Background: T-ALL represents 20 to $25 \%$ of ALL cases. Allogeneic stem cell transplantation (HCT) is recommended in second complete remission (CR2) or beyond, as well as in CR1 with high-risk features. For patients with no HLA identical donor, haploidentical transplant (haplo-HCT) is becoming the leading source of donors, particularly following the introduction of post-transplant cyclophosphamide (ptCy). However, data is scarce on haplo-HCT for T-ALL and on predictive factors for transplant outcomes in that setting, particularly that total body irradiation (TBI)based myeloablative conditioning (MAC) are less frequently used. The purpose of this study was to assess the influence of patient, disease and transplant characteristics on outcomes of haplo-HCT with ptCy for T-ALL.

Methods: We identified 122 adults (20\% females; median age 31 years; range 18-68) with T-ALL who received a haplo-HCT with ptCy between 2010 and 2017 at EBMT centers. Patients who received in vivo $\mathrm{T}$ cell depletion were excluded. Median white blood cells count at diagnosis was 33,5 G/L [Interquartile range; IQR: 8-82]. Karyotype was normal in 37 patients, abnormal in 21 and missing in 64 . More patients $(43 \%)$ were transplanted in first complete remission (CR1), 32\% in CR2 or beyond and
$25 \%$ with active disease. Ten patients had a previous autologous HCT. Karnovsky score was $<90 \%$ for 28 patients. Conditioning was TBI-based MAC in $28 \%$ of patients, chemotherapy-based MAC in 53\% (predominantly thiotepa/busulfan/fludarabine in $40 \%$ ), and reduced intensity (RIC) in $19 \%$. Patients received bone marrow (52\%) or peripheral blood stem cells (48\%), predominantly from male donors $(57 \%)$. Median donor age was 42 years (range 17-72). Most patients (76\%) and donors (77\%) were CMV positive. Thirty five percent of patients were males with a female donor. Median follow-up of alive patients was 23 months (IQR 12-38).

Results: 95\% of patients engrafted. Day 100 acute GVHD grade II-IV and grade III-IV were encountered in $22.5 \%$ and $13.5 \%$ of patients, respectively, whereas the 2year cumulative incidence of chronic and extensive chronic GVHD were $25.5 \%$ and $9.5 \%$, respectively. The 2-year relapse incidence and non-relapse mortality were $45 \%$ and $21 \%$, respectively. The 2-year leukemia free survival (LFS), overall survival (OS) and GVHD relapse free survival (GRFS) were $34 \%, 42 \%$ and $27 \%$, respectively. The 2-year LFS and OS were highly influenced by the disease status at transplant, being $49 \%$ and 55\% respectively for CR1, 34\% and $50 \%$ respectively for CR2, $8 \%$ and 12 respectively for patients with active disease ( $\mathrm{p}<0.0001$ for both). In multivariate analysis, only disease status affected LFS and OS. Transplantation in CR2 negatively affected LFS (HR $2.09 ; \mathrm{p}=0.02$ ), whereas active disease at haplo-HCT negatively affected LFS (HR 3.88; $<<10^{-4}$ ) and OS (HR $4.3 ; \mathrm{p}<10^{-4}$ ). When multivariate analysis was restricted to patients transplanted in $\mathrm{CR}$, only transplantation beyond CR1 affected LFS (HR 1.91; $p=0.045$ ), but not OS.

Conclusions: Haplo-HCT with ptCy produced encouraging results in this challenging disease, particularly when performed in CR. With the limitation of the small sample size, results were not affected by the type of conditioning, questioning the need for MAC TBI in that setting.

Disclosure: nothing to declare, no funding

\section{6}

Favourable outcome after allogeneic hematopoietic cell transplantation in first complete remission for AML with MLL (KMT2A) partial tandem duplication: An analysis by the ALWP

Marta Pratcoronal, Christophe Peczynski', Myriam Labopin $^{2}$, Dietrich Beelen ${ }^{3}$, Christoph Schmid ${ }^{4}$, Jean Henry Bouhris ${ }^{5}$, Xavier Poiré ${ }^{6}$, Arnold Ganser ${ }^{7}$, Pavel Jindra $^{8}$, Jorge Sierra ${ }^{1}$, Johanna Tischer ${ }^{9}$, Mar Tormo $^{10}$, Johan Maertens ${ }^{11}$, Marina Díaz-Beyál' ${ }^{12}$, Jürgen Finke ${ }^{13}$, Audrey Mailhol', Mohamad Mohty', Jordi Esteve ${ }^{12}$, Arnon Nagler ${ }^{2,14}$ 
${ }^{1}$ Hospital de la Santa Creu i Sant Pau, Barcelona, Spain, ${ }^{2}$ EBMT ALWP Office, Saint Antoine Hospital, Paris, France, ${ }^{3}$ University Hospital, Essen, Germany, ${ }^{4}$ University Hospital, Augsburg, Germany, ${ }^{5}$ Gustave Roussy, Institut de Cancérologie, Villejuif, France, ${ }^{6}$ Cliniques Universitaires St. Luc, Brussels, Belgium, ${ }^{7}$ Hannover Medical School, Hannover, Germany, ${ }^{8}$ Charles University Hospital Pilsen, Pilsen, Czech Republic, ${ }^{9}$ Klinikum Grosshadern, Munich, Germany, ${ }^{10}$ Hospital Clínico Universitario, Valencia, Spain, ${ }^{11}$ University Hospital Gasthuisberg, Leuven, Belgium,

${ }^{12}$ Hospital Clínic de Barcelona, Institut d'Investigacions Biomèdiques August Pi i Sunyer (IDIBAPS), Josep Carreras Leukemia Research Institute (IJC), Barcelona, Spain, ${ }^{13}$ University of Freiburg, Freiburg, Germany, ${ }^{14}$ Chaim Sheba Medical Center, Tel Hashomer, Israel

Background: Partial tandem duplication of $M L L$ ( $M L L-$ PTD) is an infrequent mutation in AML which produces a number gain of $5^{\prime}$ acetyltransferase domains of KMT2A protein as a result of a repeated exon 3-9/10 gene sequence. $M L L$-PTD leads to a disturbed histone acetylation and upregulation of determined $H O X$ genes. MLL-PTD AML defines a specific AML entity, distinguishable from cases of AML with $M L L$ rearrangement, with a characteristic pattern of co-mutations, including a high association with FLT3ITD (Q-Y Sun et al., Leukemia 2017). Prognosis of $M L L-$ PTD-AML is remarkably poor, with initial chemoresistance and high relapse rate; as a consequence, allogeneic hematopoietic cell transplantation (alloHCT) in early phase is recommended to overcome its high-risk prognostic impact (JP Patel et al., NEJM 2012; V Grossmann et al, Blood 2012). Nonetheless, studies focusing on transplant outcome have not been previously addressed.

Methods: For this purpose, we analysed the outcome of $M L L$-PTD AML adult patients reported to EBMT who had received an alloHCT from matched related or unrelated donors in CR1 during the period 2000-2016. Molecular screening of $M L L$-PTD was performed locally, but the presence of this mutation was verified specifically by a focused questionnaire among participating centres.

Results: Overall, we identified 58 patients fulfilling inclusion criteria (median age, 52.8 years; 29/58 female patients). Most patients harboured an intermediate risk cytogenetics (50/56; 89\% of available) and 14 (14/54, 26\% of available) patients presented with concomitant FLT3ITD. Donor was a matched sibling in 27 transplants (47\%) and an unrelated donor in the remaining cases. Conditioning was myeloablative (MAC) in 35 procedures $(61 \%)$ and reduced intensity in $22(40 \%)$. In vivo T-cell depletion was used in $36(64 \%)$ of transplants. At two years, cumulative incidence of relapse (CIR) was 33\% (95\% CI: 21-45), and non-relapse mortality (NRM) $16 \%$ (8-26); 100-day acute GvHD of grade III-IV was 7\% (2-16) and 2-yr chronic
GvHD was 38\% (extensive, 13\%, 6-23). Two-year overall survival (OS) was 56\% (45-71), with leukemia-free survival (LFS) and graft- and relapse-free survival (GRFS) of 51\% (40-65) and 39\% (27-58), respectively (Figure). Multivariate analysis did not identify any prognostic factor for LFS; notably, presence of FLT3-ITD, conditioning regimen intensity or donor type did not influence outcome.

Conclusions: These results support the potential clinical benefit of alloHCT performed in CR1 in patients with $M L L$ PTD AML, with a decreased relapse risk compared to previously reported series, suggesting the existence of a tangible graft-versus-leukemia effect $(\mathrm{GvL})$ in this setting.

Disclosure: Nothing to declare

\section{7}

Safety and efficacy of reduced intensity conditioning regimen combined with anti-thymocyte globulin and post-transplantation cyclophosphamide as graft versus host disease prophylaxis for acute myeloid leukemia

Maria Queralt Salas ${ }^{1}$, Shruti Prem ${ }^{1}$, Eshetu G. Atenafu, Arjun Datt Law ${ }^{I}$, Lam Wilson ${ }^{1}$, Fotios V Michelis, Santhosh Thyagu, Dennis (Dong Hwan) Kim ${ }^{1}$, Jeffrey Howard Lipton ${ }^{1}$, Rajat Kumar ${ }^{1}$, Auro Viswabandya ${ }^{I}$

${ }^{1}$ Princess Margaret Cancer Centre, Toronto, Canada,

Background: We aimed to evaluate the safety and efficacy of the use of reduced intensity conditioning regimen (RIC) combined with anti-thymocyte globulin (ATG) and posttransplant cyclophosphamide (PTCy) for graft versus host disease (GVHD) prophylaxis in patients diagnosed with acute myeloid leukemia (AML).

Methods: One hundred four adult patients were included. All patients received the same RIC regimen including fludarabine $(30 \mathrm{mg} / \mathrm{m} 2 /$ day day -5 to -2$)$, busulfan $(3.2 \mathrm{~kg} /$ $\mathrm{m} 2 /$ day day -3 and -2 ), and total body irradiation (200 cGy) (day -1) combined with rabbit-ATG $(4.5 \mathrm{mg} / \mathrm{kg}$ : day -3 to $-1)$, PTCy $(50 \mathrm{mg} / \mathrm{kg} /$ day: day $+3,+4)$, and cyclosporine. Unmanipulated peripheral blood stem cells were infused. Last follow-up was November 2018. Median follow-up was 19 months (range 5-35).

Results: Findings are summarized in Figure 1. One year overall survival (OS) progression-free survival (PFS) and non-relapse mortality (NRM) was $67.7 \%$ (95\% CI 58.676.8), $60.8 \%$ (95\% CI 51.1-70.5) and $16.4 \%$ (95\% CI 6.9$25.8)$ respectively. Main causes of death were relapse (18\%) and infection (13\%).

Three patients had residual disease prior-transplant and they had a significant worst OS $(p=0.000)$ and RFS $(\mathrm{p}=0.002)$. Patients who had Karnofsky performance status (KPS) $\leq 80 \%$ had a significantly worse OS $(\mathrm{p}=0.004)$ and PFS ( $p=0.014)$. The achievement of $\geq 95 \%$ chimerism of 
donor cells at day +60 was a significant predictor of better PFS $(p=0.004)$. Disease risk index, cytogenetic at diagnosis, and donor type did not have a significant impact in OS or PFS.

Conclusions: For patients diagnosed with AML, RIC combined with ATG and PTCy provides a route to potentially curative therapy with very low rate of acute and chronic GVHD and acceptable relapse rate. Donor type had not a significant impact in overall survival using the combination of ATG and PTCy.

Disclosure: Nothing to declare

\section{8}

Trends in the use of hematopoietic stem cell transplantation for adults with acute lymphoblastic leukemia: A report from the ALWP of the EBMT

Sebastian Giebel ${ }^{1}$, Ariane Boumendil' ${ }^{2}$,Myriam Labopin ${ }^{3}$, Anouchka Seesaghur, Frederic Baron ${ }^{5}$, Fabio Ciceri ${ }^{6}$, Jordi Esteve ${ }^{7}$, Norbert-Claude Gorin ${ }^{3}$, Bipin Savani, Christoph Schmid, Sally Wetten ${ }^{4}$, Mohamad Mohty, Arnon Nagler ${ }^{10}$

${ }^{1}$ Maria Sklodowska-Curie Institute - Oncology Center, Gliwice, Poland, ${ }^{2}$ Hospital Saint-Antoine, Paris, France, ${ }^{3}$ Hospital Saint-Antoine, Paris, France, ${ }^{4}$ Amgen Limited, London, United Kingdom, ${ }^{5}$ University of Liege, Liege, Belgium, ${ }^{6}$ IRCCS San Raffaele Scientific Institute, VitaSalute San Raffaele University, Milan, Italy, ${ }^{7}$ IDIBAPS, Hospital Clinic, Barcelona, Spain, ${ }^{8}$ Vanderbilt University, Nashville, TN, United States, ${ }^{9}$ Klinikum Augsburg, Ludwig-Maximilians-Universitaet, Munich, Munich, Germany,

${ }^{10}$ Chaim Sheba Medical Center, Tel Hashomer, Israel

Background: Indications for hematopoietic stem cell transplantation (HSCT) for adults with ALL evolve over time and vary among countries.

Methods: The goal of this study was to assess general trends in the number of various types of HSCTs performed between years 2001 and 2015 in Europe. Data reported to the EBMT registry were used for this analysis. In addition, we evaluated HSCT rates with respect to the incidence of ALL in selected European countries.

Results: Altogether, 15346 first allogeneic $(n=13460)$ or autologous $(n=1886)$ HSCTs were performed in the period 2001-2015. Comparing years 2013-2015 and 2001-2003, the number of alloHSCTs performed in first CR increased by $136 \%$, most prominently for transplantations from unrelated (272\%) and mismatched related donors (339\%). The number of HSCTs from matched sibling donors increased by $42 \%$, while the number of autoHSCTs decreased by $70 \%$.

The increase of the use of alloHSCT, irrespective of the disease stage, was stronger for $\mathrm{Ph}-$ pos $(166 \%)$ than $\mathrm{Ph}$ - neg ALL (38\%). Among patients aged $>55$ years, the number of alloHSCT increased by $559 \%$ while among younger adults (18-55 years), by 59\%. Between 2001 and 2003 , peripheral blood was used as source of stem cells in $61 \%$ cases of alloHSCT, compared to $84 \%$ between 2013 2015. The use of bone marrow decreased from $38 \%$ to $16 \%$, respectively. The proportion of alloHSCT with reduced-intensity conditioning (RIC) increased from $6 \%$ to $27 \%$. Among myeloablative transplantations, regimens based on total body irradiation were the preferable option (app. $80 \%$ over the whole study period). In contrast, among RIC regimens, the use of chemotherapy predominated (84\% between 2013-2015).

In most of analyzed individual countries, the estimated rates of alloHSCT (no. HSCT per 100 newly diagnosed ALL) for patients in CR1 increased over time. However, the values for a period 2013-2015 varied strongly, being highest in Finland (57.9), followed by the Netherlands (35.4) and Sweden (35.4) while lowest in Russia (2.6).

Conclusions: Results of our analysis indicate continued trend to increased use of alloHSCT for adults with ALL, which may be attributed to increasing availability of unrelated donors. However, it may also be speculated that the introduction of tyrosine kinase inhibitors allowed higher proportion of patients with Ph-pos ALL proceeding to transplantation. Finally, the implementation of RIC regimens contributed to wider use of alloHSCT among older adults. Limitations of the analysis include any assumptions made regarding ALL incidence for the specified time period and possible variation in reporting to the EBMT registry from different countries over time.

\begin{tabular}{llllll}
\hline & \multicolumn{7}{l}{ Year } \\
\cline { 2 - 6 } Type of HSCT & $2001-2003$ & $2004-2006$ & $2007-2009$ & $2010-2012$ & $2014-2015$ \\
\hline Total alloHSCT & 1006 & 1347 & 1816 & 2108 & 2374 \\
MSD-HSCT & 606 & 737 & 823 & 867 & 860 \\
URD-HSCT & 362 & 583 & 957 & 1169 & 1347 \\
MMRD-HSCT & 38 & 27 & 36 & 72 & 167 \\
AutoHSCT & 561 & 327 & 164 & 135 & 156 \\
$\begin{array}{l}\text { MSD, matched sibling donor; URD, unrelated donor; MMRD, mismatched } \\
\text { related donor }\end{array}$
\end{tabular}

[[O018 Table] 1. Numbers of HSCT procedures for ALL in first complete remission according to donor type]

Disclosure: SG has received honoraria for Amgen. AS and SW are employees of Amgen and own shares in Amgen. MM has received honoraria for speaking for Amgen and Pfizer.

\section{O019}

Outcome of allogeneic-HSCT in adult patients with PHpositive-all in the era of TKI: A retrospective analysis of 
the Italian blood and marrow transplantation society (GITMO)

Anna Candoni ${ }^{1}$, Renato Fanin ${ }^{1}$, Alessandro Rambaldi', Andrea Velardi ${ }^{3}$, William Arcese ${ }^{4}$, Fabio Ciceri ${ }^{5}$, Davide Lazzarotto $^{l}$, Federico Lussana ${ }^{6}$, Jacopo Olivieri ${ }^{1}$, Giovanni Grillo ${ }^{7}$, Matteo Parma ${ }^{8}$, Benedetto Bruno, ${ }^{90}$, Federica Sora ${ }^{11}$, Paolo Bernasconi ${ }^{12}$, Riccardo Saccardi $^{13}$, Roberto Foà ${ }^{14}$, Mariarosa Sessa ${ }^{15}$, Paola Bresciani $^{16}$, Fabio Giglio ${ }^{5}$, Raffaella Cerrettit, Alessandro Busca $^{10}$, Simona Sica ${ }^{11}$, Elisa Diral ${ }^{8}$, Anna Amelia Colombo $^{12}$, Stefano Tringali $^{17}$, Stella Santarone ${ }^{18}$, Giuseppe Irrera ${ }^{19}$, Stefano Mancini ${ }^{20}$, Francesco Zallio $^{21}$, Michele Malagola $^{22}$, Francesco Albano ${ }^{23}$, Angelo Michele Carella $^{24}$, Attilio Olivieri ${ }^{25}$, Cristina Tecchio ${ }^{26}$, Alida Dominietto $^{27}$, Adriana Vacca $^{28}$, Roberto Sorasio ${ }^{29}$, Enrico Orciuolo $^{30}$, Antonio Maria Risitano ${ }^{31}$, Agostino Cortelezzi $^{32}$, Sonia Mammoliti ${ }^{33}$, Elena Oldani ${ }^{34}$, Francesca Bonifazi, on behalf of GITMO. ${ }^{15}$

${ }^{1}$ University of Udine, Santa Maria Misericordia Hospital, Udine, Italy, ${ }^{2}$ Universita' degli Studi di Milano and Azienda Socio Sanitaria Territoriale Papa Giovanni XXIII, Bergamo, Bergamo, Italy, ${ }^{3}$ University of Perugia, Perugia, Italy, ${ }^{4}$ University 'Tor Vergata', Rome, Italy, ${ }^{5}$ University VitaSalute San Raffaele, San Raffaele Scientific Institute, Milano, Italy, ${ }^{6}$ Azienda Socio Sanitaria Territoriale Papa Giovanni XXIII, Bergamo, Bergamo, Italy, ${ }^{7}$ Ospedale Niguarda Ca' Granda, Milano, Italy, ${ }^{8}$ Ospedale San Gerardo, Clinica Ematologica e Centro Trapianto di Midollo, Monza, Italy, ${ }^{9}$ Università di Torino, Torino, Italy, ${ }^{10}$ Presidio Molinette - AOU Città della Salute e della Scienza, Trapianto Allogenico di Cellule Staminali, Torino, Italy, ${ }^{11}$ Fondazione Policlinico Universitario 'A.Gemelli', Istituto di Ematologia, Roma, Italy, ${ }^{12}$ Fondazione IRCCS Policlinico San Matteo, Centro Trapianti SC Ematologia, Pavia, Italy, ${ }^{13}$ Careggi University Hospital, Centro Trapianti di Midollo Osseo, Firenze, Italy, ${ }^{14}$ Sapienza University, Policlinico 'Umberto I', Cellular Biotechnologies and Hematology, Roma, Italy, ${ }^{15}$ University Hospital S. OrsolaMalpighi, Institute of Hematology 'L. and A. Seragnoli', Bologna, Italy, ${ }^{16}$ University of Modena and Reggio Emilia, Modena, Italy, ${ }^{17}$ AOR Villa Sofia-Cervello, UOS Trapianto di Midollo, Palermo, Italy, ${ }^{18}$ Ospedale Civile, Pescara, Italy, ${ }^{19}$ Grande Ospedale Metropolitano 'Bianchi-Melacrino-Morelli', Centro Unico Regionale Trapianto Cellule Staminali e Terapie Cellulari 'A. Neri', Reggio Calabria, Italy, ${ }^{20}$ Azienda Ospedaliera San Camillo Forlanini, Centro Trapianti di Cellule Staminali, Roma, Italy, ${ }^{21}$ Azienda Ospedaliera S. Antonio e Biagio, Unità Trapianti Midollo, Alessandria, Italy, ${ }^{22}$ University of Brescia, ASST-Spedali Civili, University of Brescia, Brescia, Italy, ${ }^{23}$ University of Bari, Bari, Italy, ${ }^{24}$ Fondazione IRCCS Casa Sollievo della
Sofferenza, UO Ematologia e Centro Trapianti TMO, San Giovanni Rotondo, Italy, ${ }^{25}$ University of Marche-Ospedali Riuniti, Ancona, Italy, ${ }^{26}$ University of Verona, Verona, Italy, ${ }^{27}$ IRCCS Ospedale Policlinico San Martino, U.O. Ematologia e Centro Trapianti, Genova, Italy, ${ }^{28}$ P.O. ' Roberto Binaghi', U.O. Ematologia-CTMO, Cagliari, Italy, ${ }^{29}$ Azienda Ospedaliera S Croce e Carle, Cuneo, Italy, ${ }^{30}$ Azienda Ospedaliero Universitaria Pisana, UO Ematologia, Pisa, Italy, ${ }^{31}$ Università di Napoli, Napoli, Italy, ${ }^{32}$ Università degli Studi e Ospedale Maggiore Policlinico di Milano, Milano, Italy, ${ }^{33}$ GITMO Clinical Trial CoOrdinator, Genova, Italy, ${ }^{34}$ Azienda Socio Sanitaria Territoriale Papa Giovanni XXIII, Bergamo, Italy

Background: We conducted a retrospective, nationwide analysis to describe the clinical outcome of adult patients with Philadelphia chromosome-positive acute lymphoblastic leukemia $(\mathrm{Ph}+\mathrm{ALL})$ undergoing allogeneic hematopoietic stem cell transplantation (HSCT) after being treated with a TKI based therapy.

Methods: A total of 441 patients were included in the study. The median age at HSCT was 44 (range: 18-70). All 441 patients $(100 \%)$ received TKI before HSCT (performed between 2005-2016). Of these patients, 404 (92\%) were in cytologic complete remission (CR) while $37(8 \%)$ had an active disease at the time of HSCT. Molecularly Measurable Residual Disease (MRD) was negative in 147 patients $(36 \%)$ at the time of HSCT. The donor was unrelated in $46 \%$ of cases. The prevalent source of stem cells was peripheral blood $(70 \%)$. The conditioning regimen was myeloablative in $82 \%$ of cases (TBI-based in 50\%) and included ATG in $51 \%$ of cases.

Results: With a median follow-up after HSCT of 39.4 months (range: 1-145), the probability of Overall Survival (OS) at 1,2 and 5 years was $69.6 \%, 61.1 \%$, and $50.3 \%$, respectively, with a median OS of 62 months. Progression Free Survival (PFS) at 1, 2 and 5 years was $60.2 \%, 52.1 \%$ and $43.7 \%$, respectively. OS and PFS were significantly better in patients with CR and MRD-negativity at the time of transplant compared to patients with $\mathrm{CR}$ but MRD-positive (50\% OS not reached vs. 36 months, $\mathrm{P}=0,015 ; 50 \%$ PFS not reached vs 26 months, $\mathrm{P}=0.003$ ). The cumulative incidence of relapse (CIR) at 5 years was significantly lower in patients with CR and MRD-negativity ( $19.5 \%$ vs. $35.4 \%, \mathrm{P}=0.001)$. The non relapse mortality (NRM) after 1, 2, and 5 years was $19.1 \%$ (95\%CI: 15.522.9), $20.7 \%$ (95\%CI: 17-24.7), and $24.1 \%$ (95\%CI: $20-$ 28.5), respectively. The subgroup of patients with MRDnegative both at HSCT and at 3rd month after HSCT had a better outcome (5 year OS 70\%). Conversely, the 37 patients who underwent HSCT with active $\mathrm{Ph}+\mathrm{ALL}$ had a median OS and PFS of 7 and 5 months, respectively. 
Conclusions: The median OS of all patients with $\mathrm{Ph}$ +ALL, treated with TKI based therapy and allografted in recent years at the GITMO Centers, is 62 months. The outcome of $\mathrm{Ph}+\mathrm{ALL}$ patients undergoing HSCT after TKI therapy has improved (with a 2 yrs NRM of 20,7\%), particularly for younger patients and those achieving a molecular remission before transplant (50\% OS and PFS not reached). HSCT remains a standard of care consolidation treatment for $\mathrm{Ph}+\mathrm{ALL}$ and only prospective randomized trials can suggest a survival benefit of non transplant based treatment strategies.

Clinical Trial Registry: Registered at Italian Clinical Trial Registry

Disclosure: Nothing to declare

\section{O020}

Haploidentical transplant in adult patients with acute lymphoblastic leukemia in Argentina: A comparison with matched related and unrelated donors

Ana Lisa Basquieral, Mariano Berro ${ }^{2}$, Sebastian Yantorno $^{3}$, Martin Castro ${ }^{4}$, Alejandro Requejo ${ }^{5}$, Miguel Sorrentino $^{6}$, Daniel Sutovsky ${ }^{7}$, Diego Giunta ${ }^{1}$, Silvina Palmer $^{8}$, Adriana Vitriu', Gonzalo Ferini, Georgina Bendek $^{10}$, Milagros Szelagowski ${ }^{3}$, Maria Leticia Rapan ${ }^{6}$, Nicolas Fernandez Escobar, Patricio Duarte ${ }^{11}$, Amalia Cerutti $^{12}$, Maximiliano Cattaneo ${ }^{13}$, Juliana Martinez, Rolon $^{14}$, Gregorio Jaimovich ${ }^{5}$, Javier Bordone, Vera Milovic $^{15}$, Gustavo Kusminsky ${ }^{2}$, Jorge Alberto Alberbide ${ }^{1}$

${ }^{1}$ Hospital Italiano de Buenos Aires, Ciudad Autonoma de Buenos Aires, Argentina, ${ }^{2}$ Hospital Universitario Austral, Pilar, Argentina, ${ }^{3}$ Hospital Italiano de La Plata, La Plata, Argentina, ${ }^{4}$ Sanatorio Anchorena, Ciudad Autonoma de Buenos Aires, Argentina, ${ }^{5}$ Fundación Favaloro, Ciudad Autonoma de Buenos Aires, Argentina, ${ }^{6}$ Sanatorio Sagrado Corazon, Ciudad Autonoma de Buenos Aires, Argentina, ${ }^{7}$ Hospital El Cruce, Florencio Varela, Argentina, ${ }^{8}$ Hospital Británico, Ciuidad Autonoma de Buenos Aires, Argentina, ${ }^{9}$ Instituto Alexander Fleming, Ciudad Autonoma de Buenos Aires, Argentina, ${ }^{10}$ Hospital Italiano de San Justo Agustin Rocca, San Justo, Argentina, ${ }^{11}$ Centro de Educación Médica e Investigactiones Clínicas Norberto Quirno (CEMIC), Ciudad Autonoma de Buenos Aires, Argentina, ${ }^{12}$ Centro de Hematología y Trasplante, Rosario, Argentina, ${ }^{13}$ Centro de Transplante Médula Osea de Rosario (CETRAMOR), Rosario, Argentina, ${ }^{14}$ FUNDALEU, Ciudad Autonoma de Buenos Aires, Argentina, ${ }^{15}$ Hospital Aleman, Ciudad Autonoma de Buenos Aires, Argentina

Background: Haploidentical (Haplo) donors have expanded patient transplant access. However, outcome of patients with acute lymphoblastic leukemia (ALL) undergoing allogeneic stem cell transplant (ASCT) with Haplo donors in Argentina has not been reported. We aimed to analyze the outcome of ASCT in patients with ALL, particularly results with Haplo donors.

Methods: We collected data from patients with an ALL diagnosis who underwent ASCT in first complete remission (CR1) and subsequent remissions (CR2+) in 15 centers in Argentina, affiliated to GATMO, between 2008 and 2017. Patients that underwent ASCT with matched donors (sibling and unrelated) and Haplo donors (with post-transplant cyclophosphamide) were included. Both donor categories were compared in terms of overall survival (OS), nonrelapsed mortality (NRM) and cumulative incidence of relapse (CIR). Graft versus host disease (GVHD) was also evaluated. Multivariate analysis was performed by Cox regression for OS and Fine-Gray for $\mathrm{CI}$ of competing events. A further propensity score (PS) adjustment was performed by donor group.

Results: In a 10-year period, 236 patients were included (mean age 31y; range 16-64; male 63.1\%); 188 (80\%) during last 5 years. ALL phenotype was B (79\%) and T (21\%). At diagnosis, 47/236 (20\%) had CNS involvement and 75/236 (32\%) were Philadelphia chromosome positive. ASCT was performed in CR1 $(\mathrm{n}=126 ; 53 \%)$ and in CR2+ $(\mathrm{n}=110 ; 47 \%)$ after a median time from ALL diagnosis to ASCT of 9 and 26 months, respectively. Comorbidity index (HCT-CI) was 0-1 in 199/236 (90\%). Donors were matched $(\mathrm{n}=175 ; 74 \% ; 146$ related and 29 unrelated) and Haplo $(\mathrm{n}=61 ; 26 \%)$. Conditioning regimen was myeloablative in 215/236 (91\%; 170 patients with total body irradiation), and this conditioning was more frequent in matched (95\%) than Haplo $(79 \%)(\mathrm{p}=0.001)$ donors. Two-years OS was $54 \%$ (95\%CI 46-60) for the entire population; 55\% (95CI 47-63) for matched donors and 49\% (95\% CI 34-62) for Haplo donors $(\mathrm{p}=0.350)$. In the multivariate analysis, pretransplant status (CR1 vs CR2+; HR 2.06, p< 0.001), CNS status at diagnosis (yes vs no; HR 1.70; $\mathrm{p}=0.019$ ) and unrelated donors (yes vs no; HR 1.77; $\mathrm{p}=0.036$ ) were independently associated with OS; donor category had not impact in the OS. By adjusting the PS term (ROC area 0.787), no difference was found by donor category. Twoyears NRM was $24 \%$ (95\%CI 18-31) for matched and $22 \%$ (95\%CI 12-33) for Haplo $(\mathrm{p}=0.999)$ donors; older donors $(\mathrm{p}=0.049)$ and unrelated donors $(\mathrm{p}=0.001)$ were associated with higher NRM. Two-years CIR was $25 \%$ (95\% CI 19-33) for matched and 38\% (95\% CI 24-51) for Haplo $(\mathrm{p}=0.137)$ donors; only male donors were associated with higher CIR $(p=0.031)$. CI of grade 3-4 acute GVHD was $20 \%$ vs $17 \%$ $(\mathrm{p}=0.784)$ and chronic GVHD was $35 \%$ vs $27 \%(\mathrm{p}=0.057)$ for matched and Haplo donors respectively. In both groups, matched and Haplo donors, the half of deaths were due to relapse. 
Conclusions: Patients with ALL undergoing ASCT were young with a low HCT-CI. In this setting, an Haplo donor represents an alternative widely available in the absence of a matched donor. Relapse remains a challenge for all donor categories.

Disclosure: Nothing to declare.

\section{1}

TIM-3-mediated immunosurveillance of acute lymphoblastic leukemia by $\mathrm{CD}^{+}$bone marrow $\mathrm{T}$ cells: Implications for relapse and graft-versus-leukemia effects

Franziska Blaeschke ${ }^{I}$, Semjon Willier ${ }^{I}$, Dana Stenger ${ }^{I}$, Mareike Lepenies ${ }^{1}$, Martin A. Horstmann ${ }^{2}$, Gabriele Escherich $^{2}$, Martin Zimmermann ${ }^{3}$, Francisca Rojas Ringeling $^{4}$, Theresa Kaeuferle ${ }^{1}$, Meino Rohlfs ${ }^{1}$, Irene Schmid ${ }^{1}$, Michael H. Albert', Vera Binder ${ }^{1}$, Stefan Canzar, Christoph Klein ${ }^{1}$, Tobias Feuchtinger ${ }^{1}$

${ }^{1}$ Dr. von Hauner University Children's Hospital, Ludwig Maximilian University Munich, Munich, Germany, ${ }^{2} \mathrm{Clinic}$ of Pediatric Hematology and Oncology, University Medical Center Hamburg-Eppendorf, Hamburg, Germany, ${ }^{3}$ Hannover Medical School, Hannover, Germany, ${ }^{4}$ Gene Center Munich, Ludwig Maximilian University Munich, Munich, Germany

Background: Relapse is the most important cause for treatment failure in pediatric B-precursor acute lymphoblastic leukemia (BCP-ALL) occurring in 10-20\% of patients. Mechanisms of ineffective graft-versus-leukemia (GvL) effects or T-cell responses against ALL remain to be investigated.

Methods: We analyzed parameters of immunosurveillance in bone marrow (BM) samples of 100 pediatric patients to identify potential mechanisms of T-cell suppression. Expression of co-stimulatory/ co-inhibitory molecules was analyzed to identify implications for GvL. Expression was correlated with clinical outcome (8 years mean followup). T-cell immunoglobulin and mucin-domain containing3 (TIM-3) overexpression and CRISPR/Cas9-mediated knockout $(\mathrm{KO})$ in primary $\mathrm{T}$ cells were performed to analyze its role for anti-leukemic T-cell functionality. To induce an interaction of $\mathrm{T}$ cells with leukemic blasts, antiCD19/-CD3 bispecific T-cell engager (BiTE) was added and T-cell activation/ proliferation were analyzed. Fold change (FC) was created by comparing levels of T-cell activation/ proliferation before vs. after co-culture. Transcriptome analysis of primary BM samples identified expression levels of known TIM-3 inducers.

Results: Flow cytometric analyses of 100 BCP-ALL samples showed increased TIM- 3 expression on $\mathrm{CD} 4^{+} \mathrm{BM}$
$\mathrm{T}$ cells at initial diagnosis in patients with relapse in the course of disease. Multivariate analysis confirmed 7-fold increased relapse risk in TIM-3 high $(\mathrm{n}=37)$ vs. TIM-3 low expressing $(n=37)$ patients. PD-1 expression on BM T cells alone had no impact on relapse-free survival (RFS), whereas patients with high percentage of TIM-3/PD-1 double positive $\mathrm{CD}^{+} \mathrm{BM}$ T cells showed significantly decreased RFS (87.8\% vs $41.7 \%)$. Co-culture experiments revealed that TIM-3 is induced in primary $\mathrm{T}$ cells by contact with leukemic cells (mean TIM-3 expression $51.1 \%$ vs. $29.7 \%$ on $\mathrm{T}$ cells with vs. without addition of leukemic cells, $\mathrm{n}=3$ ). Transcriptome analysis was performed to identify expression levels of known TIM-3 ligands/ inducers in BM samples with high vs. low TIM-3 expression. No significant differences in expression levels of High-Mobility-Group-Protein B1 (HMGB1), Carcinoembryonic antigen-related cell adhesion molecule 1 (CEACAM1) or Galectin 9 were observed. Known TIM-3 inducers IL-12, IL-15, IL-27, IL-7 or Transforming growth factor beta 1 (TGF- $\beta 1$ ) were not differentially expressed indicating that another mechanism must be responsible for TIM-3 overexpression. TIM-3 overexpression and CRISPR/ Cas9-mediated TIM-3 KO were performed to analyze functional relevance of TIM-3 expression in an in vitro leukemia model. $\mathrm{T}$ cells of healthy donors were co-cultured with leukemic cells and anti-CD19/-CD3 BiTE to induce anti-leukemic T-cell response. TIM-3 KO T cells showed significantly increased activation compared to wildtype $\mathrm{T}$ cells (FC of CD69 expression 5.0 vs. 3.2, $\mathrm{n}=3$ ). In contrast, proliferation of TIM-3 overexpressing T cells was significantly impaired (FC 1.6 vs. $2.3, \mathrm{n}=3$ ), whereas TIM$3 \mathrm{KO} \mathrm{T}$ cells showed higher proliferation levels compared to controls (FC 6.5 vs. $2.4, \mathrm{n}=3$ ).

Conclusions: TIM-3 expression on $\mathrm{CD}^{+}{ }^{+} \mathrm{BM}$ T cells is a strong predictor for pediatric BCP-ALL relapse and is induced by T-cell interaction with leukemic cells. TIM-3 expression decreases anti-leukemic T-cell activation and proliferation and thus constitutes a new mechanism of immune escape and potentially insufficient GvL effects in pediatric BCP-ALL. Targeting the TIM-3 axis can be of interest to improve future immunotherapy of advanced BCP-ALL.

Disclosure: Nothing to declare.

\section{O022}

Abstract already published.

\section{3}

Multiparameter flow cytometric minimal residual disease before myeloablative allogeneic hematopoietic cell transplantation in acute myeloid leukemia influences patients survival in first and second complete remission 
Anna Czyz ${ }^{1}$, Barbara Nasilowska-Adamska ${ }^{2}$, Agnieszka Piekarska ${ }^{3}$, Patrycja Mensah-Glanowska ${ }^{4}$, Anna LojkoDankowska $^{5}$, Malgorzata Sobczyk-Kruszelnicka ${ }^{6}$, Tomasz Czerw $^{6}$, Jolanta Wozniak ${ }^{2}$, Agnieszka Balana ${ }^{4}$, Jolanta Parulska ${ }^{5}$, Donata Szymczak', Tomasz Wrobel, Kazimierz Halaburda ${ }^{2}$, Maria Bieniaszewska ${ }^{3}$, Lidia Gil ${ }^{5}$, Sebastian Giebel ${ }^{6}$

${ }^{1}$ Wroclaw Medical University, Wroclaw, Poland, ${ }^{2}$ Institute of Hematology and Transfusion Medicine, Warszawa, Poland, ${ }^{3}$ Medical University of Gdansk, Gdansk, Poland, ${ }^{4}$ Collegium Medicum Jagiellonian University, Krakow, Poland, ${ }^{5}$ University of Medical Sciences, Poznan, Poland, ${ }^{6}$ Maria Sklodowska-Curie Institute - Oncology Center, Gliwice Branch, Gliwice, Poland

Background: The growing evidence from the literature strongly suggest that multiparameter flow cytometric (MFC) minimal residual disease (MRD) assessment in AML can be used to risk-stratify patients at the time of allogeneic hematopoietic stem cell transplantation (alloHCT). We sought to determine the significance of MFC-MRD status in patients with AML in first or second complete remission (CR) treated with myeloablative conditioning (MAC) alloHCT at six centers of the Polish Adult Leukemia Group (PALG).

Methods: MRD was assessed by 6-color (8-color since 2017) MFC performed on bone marrow aspirates obtained as routine assessment before alloHCT. All consecutive patients undergoing MAC alloHCT were included in the analysis if they underwent pre-HCT MFC-MRD analysis from May 2013 until January 2018. The abnormal population was quantified as a percentage of the total $\mathrm{CD} 45^{+}$white cell events. Residual disease at a $\geq 0.1 \%$ level was considered MRD-positive (MRD+).

Results: We identified 83 adult patients (median age 41 years, range 19-64) with AML undergoing alloHCT from either HLA-identical sibling $(\mathrm{n}=30)$ or unrelated donor $(\mathrm{n}=53)$, in CR1 $(\mathrm{n}=72)$ or CR2 $(\mathrm{n}=11)$, who were conditioned with i.v. busulfan given in myeloablative dose $(9,6-12,8 \mathrm{mg} / \mathrm{kg})$ in combination with Flu $(\mathrm{n}=46)$ or $\mathrm{Cy}$ $(n=37)$. GvHD prophylaxis consisted of calcineurin inhibitor combined with MTX plus ATG in alloHCT from unrelated donors. Positive MRD status before alloHCT was detected in 30/72 (42\%) pts in CR1 and 6/11 (55\%) pts in CR2. The MRD(-) and MRD(+) groups did not differ in terms of gender, age, ELN cytogenetic and molecular genetic risk, first and second $\mathrm{CR}$, conditioning regimen, HSC source, and type of donor. The 3-year overall survival (OS) for MRD(-) and MRD(+) patients were $77 \%$ and $49 \%$ (log-rank $\mathrm{p}=0.023$ ). The respective 3-year leukemia-free survival (LFS) were $61 \%$ and $37 \%$ (log-rank $p=0.012$ ). In univariate and multivariate Cox proportional hazard model the only significant adverse prognostic factors for LFS were $\mathrm{MRD}(+)(\mathrm{HR} 2.45,95 \% \mathrm{CI} 1.12-5.33 ; \mathrm{p}=0.025)$ and high ELN genetic risk (HR 3.70, 95\%CI 1.71-8.0; $\mathrm{p}=0.0009$ ). The same factors significantly influenced OS [HR 2.48, 95\%CI 1.06-5.83; $\mathrm{p}=0.036$ and HR 3.47, 95\% CI 1.49-8.05; $\mathrm{p}=0.004$ for $\mathrm{MRD}(+)$ and high ELN risk, respectively].

Conclusions: Our findings confirm that pre-transplant residual disease at $\mathrm{a} \geq 0.1 \%$ level assessed by MFC is independent risk factor for both LFS and OS in patients undergoing alloHCT. In addition, the results of our study show that MAC alloHSCT outcomes in patients with AML in first and second CR are significantly influenced by both MFC-MRD status and ELN cytogenetic and molecular genetic risk.

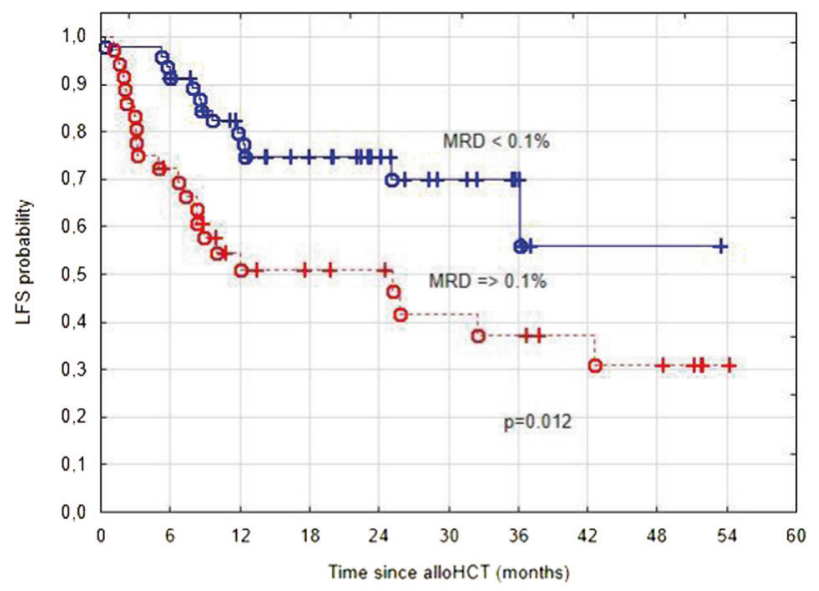

[[O023 Image] 1. Leukemia-free survival according to pre-transplant minimal residual disease level]

Disclosure: Nothing to declare

\section{O024}

Second allogeneic stem cell transplantation in acute lymphoblastic leukemia patients in second complete remission or relapse: A study on behalf of the ALWP / EBMT

Arnon Nagler ${ }^{1,2}$, Myriam Labopin ${ }^{3}$, Jürgen Finke ${ }^{4}$, Arne Brecht $^{5}$, Urs Schanz, ${ }^{6}$, Riitta Niittyvuopio ${ }^{7}$, Andreas Neubauer $^{8}$, Martin Bornhäuser', Stella Santarone ${ }^{10}$, Dietrich Beelen"11, Avichai Shimoni' ${ }^{12}$, Wolf Rösler ${ }^{13}$, Zinaida Peric ${ }^{14}$, Bipin N Savani ${ }^{15}$, Sebastian Giebel ${ }^{16}$, Mohamad Mohty ${ }^{3}$

${ }^{1}$ Chaim Sheba Medical Center, Tel Aviv University, Ramat Gan, Israel, ${ }^{2}$ EBMT ALWP Office, Saint Antoine Hospital, Paris, France, ${ }^{3}$ Saint Antoine Hospital, Paris, France, ${ }^{4}$ University of Freiburg, Freiburg, Germany, ${ }^{5}$ Deutsche Klinik fuer Diagnostik, KMT Zentrum, Wiesbaden, Germany, 
${ }^{6}$ University Hospital, Clinic of Hematology, Zurich, Swaziland, ${ }^{7} \mathrm{HUCH}$ Comprehensive Cancer Center, Helsinki, Finland, ${ }^{8}$ Philipps Universitaet Marburg, University Hospital Giessen and Marburg, Marburg, Germany, ${ }^{9}$ Universitaetsklinikum Dresden, Dresden, Germany, ${ }^{10}$ Ospedale Civile, Pescara, Italy, ${ }^{11}$ University Hospital, Essen, Germany, ${ }^{12}$ Chaim Sheba Medical Center, Tel Aviv University, Ramat Gan, Israel, ${ }^{13}$ University Hospital Erlangen, Erlangen, Germany, ${ }^{14}$ University of Zagreb, Zagreb, Croatia, ${ }^{15}$ Vanderbilt University Medical Center, Nashville, TN, United States, ${ }^{16}$ Maria Sklodowska-Curie Memorial Cancer and Institute of Oncology, Gliwice, Poland

Background: Second allogeneic transplantation (HSCT2) is a therapeutic option for patients (pts) relapsing (Rel) after first HSCT (HSCT1) however most of the available data is in acute myelogenous leukemia (AML) and there is very limited data on HSCT2 in patients (pts) with ALL. Therefore, the ALWP of the EBMT performed a large registry analysis to study the outcome of HSCT2 in pts with ALL.

Methods: We studied 245 pts receiving HSCT2 as a salvage treatment between the years (y) 2000-2017 for Rel following HSCT1 in CR1. Median follow-up of surviving pts was 58 months (IQR: 24-98).

Results: 142 pts (58\%) were males and median age at HSCT2 was 34.6 years (range: 18-74). Median time from HSCT1 to HSCT2 was 463 (63-5482) days (d) and from Rel to HSCT2 it was 114 (15-348) d. At the time of HSCT2 $157(64 \%)$ pts were in CR2 while $88(36 \%)$ had advanced disease.101 (41\%) pts received sibling donor (MSD) and $144(59 \%)$ unrelated donor (UD) HSCT2 (10/10-63; 9/1010 , missing HLA- 71). In $34 \%$ of pts with available data the HSCT2 was performed from the same donor. The majority of pts with available MRD data transplanted in CR2 (64/84 pts) were MRD negative pre-HSCT2. Karnofsky performance status was $\geq 90$ in $60 \%$ of pts. $93 \%$ were transplanted with PB graft. 204 (83\%) pts received chemotherapy based conditioning (reduced intensity 63\%, myeloablative 37\%) while it was TBI based in 41(17\%) pts. 109 (52\%) pts received in vivo T-cell depleted (TCD) grafts. $94 \%$ of the pts engrafted. Acute graft-versus-host disease (aGVHD) $\geq$ II and $\geq$ III-IV occurred in $33 \%$ and $17 \%$ of the pts. Incidence of $2 y$ total and extensive chronic GVHD was $38 \%$ and $19 \%$, respectively. Main causes of death were leukemia recurrence in 54\%, GVHD in $18 \%$ and infections in $19 \%$. At 2 and $5 \mathrm{y}$, the cumulative incidence of NRM, RI, LFS, OS and GRFS were $24 \%$ \& $26 \%: 56 \%$ \& $62 \%, 20 \% \& 12 \%$, $30 \% \& 14 \%$ and $12 \% \& 7 \%$, respectively. In MVA no factor predict NRM. In multivariate analysis, RI was independently associated with time from HSCT1 to Rel, aGVHD $\geq$ II after HSCT1 and in vivo TCD and LFS the prognostic factors were time from HSCT1 to Rel and KPS $\geq 90$ at HSCT2. Factors associated with OS were age (per 10 years), time from HSCT1 to Rel, RIC at HSCT1, KPS $\geq 90$ at HSCT2 and UD vs MSD. Longer time internal from Rel to HSCT2 and in vivo TCD was associated with inferior cGVHD. Lastly for GRFS the prognostic factors were time from HSCT1 to Rel, aGVHD $\geq$ II after HSCT1, RIC at HSCT2 and KPS $\geq 90$ at HSCT2.

Conclusions: Results of HSCT2 in ALL pts with Rel or CR2 are poor with only $14 \%$ OS and 7\% GRFS at 5 y with very high RI of $62 \%$. The prognostic factors are similar to those previously reported for HSCT2 in AML. The future goals are to prevent and treat relapsed ALL by MRD driven novel monoclonal antibodies and CAR-T cell therapy.

Disclosure: Nothing to declare.

\section{Aplastic anaemia}

\section{5}

Outcomes of allogeneic stem cell transplantation (hsct) for older patients ( $>\mathbf{5 0}$ years) with severe aplastic anaemia using alemtuzumab-based ('FCC') regimen: King's college hospital experience

Vipul Sheth ${ }^{1}$, Inam Shafqat, Austin Kulasekararaj, Shreyans Gandhi ${ }^{1}$, Hugues Delavallade ${ }^{I}$, Petra Muus ${ }^{1}$, Antonio Pagliuca ${ }^{I}$, Carmel Rice ${ }^{I}$, Varun Mehra ${ }^{I}$, Ghulam Mufti $^{I}$, Victoria Potter ${ }^{I}$, Judith Marsh ${ }^{1}$

${ }^{1}$ Kings College Hospital NHS Trust, London, United Kingdom

Background: Treatment of older patients with severe aplastic anaemia (SAA) is problematic with poor long-term survival after treatment with antithymocyte globulin (ATG) and/or ciclosporin (CSA). Use of fludarabine, low dose cyclophosphamide (CY) and ATG ('FCATG') conditioning suggests better outcomes among older patients transplanted from matched sibling donors compared to high dose CY/ ATG conditioning, but GVHD remains a serious concern. We have transplanted SAA patients aged $>50$ years, predominantly from unrelated donors, using alemtuzumabbased ('FCC') regimen.

Methods: From our FCC SAA database of 65 patients, 27 aged > 50 years were transplanted between 2007-18. Median age was 61 years (range 51-71); 12 aged 50-59 and 15 aged $\geq 60$ years. Donor was matched sibling (MSD) in $6(22 \%), 10 / 10$ matched unrelated (MUD) in 18 (66\%), 9/ 10 unrelated (MMUD) in $3(12 \%)$. Conditioning was fludarabine $30 \mathrm{mg} / \mathrm{m}^{2}$ x 4 , CY $300 \mathrm{mg} / \mathrm{m}^{2}$ x 4, Alemtuzumab $0.2 \mathrm{mg} / \mathrm{kg}$ daily from day -7 to -3 . Post graft immune suppression was CSA alone. 2Gy TBI was added to FCC for MMUD HSCT. All patients received peripheral blood (PBSC) as stem cell source. 10/27 (36\%) patients were 
HLA alloimmunised. PB telomere length (TL) by multiplex qPCR measured in 17 patients, was $<1^{\text {st }}$ centile in $3(17 \%)$, $<10^{\text {th }}$ centile in $2(11 \%)$ and normal in $12(70 \%)$ patients. First line HSCT was performed in 2/6 (38\%) MSD and 3/21 $(12 \%)$ among unrelated donors. HCT comorbidity index (HTC-CI) score was 0-1 in $10(37 \%)$; 2 in $7(25 \%)$ and $>2$ in $10(37 \%)$.

Results: Three patients had invasive fungal infection at time of HCT and died day +14 to 21 , and one patient died at 11 months from multiorgan failure with recurrent parainfluenza virus and CMV. Median CD3 chimerism was $71 \%$ (1-100), $77 \%(23-100)$ and $60 \%(22-98)$ at day +100 , Iyr and $3 y r$ post HSCT. One late graft failure at 6 months was associated with low CSA blood levels, and was followed by successful $2^{\text {nd }}$ transplanted with no GVHD. 5-year OS was 86\%, compared to $96 \%$ among 27 patients aged $<50$ years $(\mathrm{p}=0.14)$. OS was $89 \%$ and $82 \%$ for patients aged 50-59 and $\geq 60$ year, respectively, $\mathrm{p}=0.8$. OS for MSD, MUD and 9/10 MMUD was 100\%, 80\% and $100 \%$, respectively. HTC-IC score of $>2$ was associated with worse OS of $72 \%$ compared to $94 \%$ with score $<2$, $\mathrm{p}=0.13$. Outcomes were comparable irrespective of telomere length ( $84 \%$ vs $80 \%$ for normal vs short telomere, $\mathrm{p}=0.76$ ). Cumulative rates of acute and chronic GVHD were $5 \%$ and $12 \%$, respectively. All cases of acute GVHD were confined to skin and grade I/II only, and no cases of severe chronic GVHD. 17 (62\%) patients needed dose reduction of CSA with addition of mycophenolate due to renal dysfunction. Rates of CMV and EBV reactivation were $25 \%$ and $27 \%$ respectively, with no CMV or EBV disease.

Conclusions: FCC conditioning regimen enabled high survival and low risk of GVHD among older patients with HCT-CI score $<2$ and who did not have established invasive fungal disease at time of HSCT.

Clinical Trial Registry: Not applicable

Disclosure: No conflicts of interest to declare

\section{6}

Haploidentical transplantation with post-transplant cyclophosphamide (Haplo-PTCy) for 71 patients with acquired or inherited bone marrow failure syndromes (BMF): The experience from Curitiba, Brazil

Carmem Bonfim ${ }^{1,2,3}$, Samantha Nichele ${ }^{1,2}$, Lisandro Ribeiro $^{1,2}$, Gisele Loth ${ }^{1,3}$, Cilmara Kuwahara ${ }^{3}$, Ana Luiza Melo Rodrigues, ${ }^{3}$, Fernanda Moreira Lara Benini, Adriana Koliski, Daniela Marinhol, Rebeca Toassa Gomes Mousquer ${ }^{1}$, Suellen Renoste, ${ }^{2,3}$,Joanna Trennepohl $^{1}$, Adriana Mello Rodrigues ${ }^{I}$, Alberto Cardoso Martins Lima ${ }^{I}$, Daniela Pilonetto ${ }^{I}$, Noemi Farah Pereira ${ }^{l}$, Ricardo Pasquini ${ }^{1,2}$
${ }^{1}$ Federal University of Parana, Curitiba, Brazil, ${ }^{2}$ Hospital Nossa Senhora das Graças, Curitiba, Brazil, ${ }^{3}$ Hospital Infantil Pequeno Principe, Curitiba, Brazil

Background: Availability of unrelated donors as well as time to find a donor and the costs related to graft acquisition are important limitations in countries with ethnical minorities and fewer resources.

Methods: We describe the experience of 78 transplants in 71 patients(pts) with acquired or inherited BMF submitted to an Haplo-PTCy transplantation between 04.2008 and 08.2018. The median age was $9 y$ s, $70 \%$ were male and $94 \%$ were CMV positive. Haplo-PTCy was the $1^{\text {st }}$ transplant for 62 pts, second or third for 9pts.

Diagnosis: Fanconi Anemia (FA, $\mathrm{N}=48$ ), acquired severe aplastic anemia (aSAA, $\mathrm{N}=10)$, Telomere diseases $(\mathrm{N}=6)$; other inherited BMF $(\mathrm{N}=7)$. All pts had failed prior therapies and $96 \%$ had previous blood transfusions. The majority received a RIC regimen with low dose TBI $(\mathrm{N}=69$, $97 \%)$. Donors were father $(\mathrm{N}=28)$, mother $(\mathrm{N}=31)$, other relatives $(\mathrm{N}=12)$. Bone marrow was the stem cell source in 70pts. All pts received GVHD prophylaxis that included PTCy followed by cyclosporine and Mycophenolate mofetil. FA pts received a modified preparatory regimen and PTCy at a total dose of $50 \mathrm{mg} / \mathrm{kg}(\mathrm{n}=32)$ or $60 \mathrm{mg} / \mathrm{kg}$ $(\mathrm{n}=16)$ while other BMF received $100 \mathrm{mg} / \mathrm{kg}$

Results: FA pts: 14 pts did not receive ATG in the preparatory regimen and all engrafted, despite the presence of donor specific antibodies(DSA) in 2 pts. Three pts had AML and 2 are in remission 3 and 6ys after transplant. $7 \mathrm{pts}$ died due to GVHD $(\mathrm{N}=5)$; toxoplasmosis/CMV pneumonia $(\mathrm{N}=1)$ or relapse $(\mathrm{N}=1) .7 / 14$ pts are alive with a median follow-up(FU) of 6.7ys. In the subgroup of FA pts receiving $\mathrm{r}-\mathrm{ATG}(\mathrm{N}=34)$, 3pts presented primary or secondary graft failure(GF), none had DSA and all died despite a $2^{\text {nd }}$ HaploPTCy with different donors. 6 pts had advanced disease and 4 are in remission at the last FU. 26/34pts are alive at a median of 4 ys after transplant. Eight pts died due to GvHD $(\mathrm{N}=4)$; RSV pneumonitis $(\mathrm{N}=1)$ and $\mathrm{GF}(\mathrm{N}=3)$. All $10 \mathrm{pts}$ transplanted with aSAA are alive and fully engrafted at a median of 5.6ys after transplant and none developed GvHD, Nine out of 13pts transplanted for other inherited BMF are alive at a median of 3ys after transplant. GF occurred in 4 pts, all received a $2^{\text {nd }}$ Haplo-PTCy from different donors and 2 are alive and engrafted. 4 pts died due to GvHD $(\mathrm{N}=1), \mathrm{GF}(\mathrm{N}=2)$ and TMA $(\mathrm{N}=1)$. Altogether CMV reactivation occurred in 51pts (72\%), at a median of 31 days (range:15-90) and hemorrhagic cystitis in 31pts (44\%) at a median of 44 days (range:8-65). after transplant.

Conclusions: Haplo-PTCy for pts with acquired or inherited BMF should be offered for those who need an immediate transplant but lack a matched donor. $70 \%$ of pts are alive at a median FU of 3ys but GvHD is a major 
complication for pts with inherited BMF, especially FA. New approaches to GVHD prophylaxis and treatment are needed in order to improve quality of survival for these pts.

Disclosure: Nothing to declare

\section{O027}

Relationship between plasma rabbit anti-thymocyte globulin level and response to immunosuppressive therapy in patients with severe aplastic anemia: Results of a multicenter, prospective, randomized study

Atsushi Narita ${ }^{I}$, Hideki Muramatsu ${ }^{I}$, Yinyan $\mathrm{Xu}^{l}$, Eri Nishikawal, Nozomu Kawashimal, Yusuke Okuno ${ }^{1}$, Nobuhiro Nishiol, Asahito Hama ${ }^{1}$, Hirohito Yamazaki', Shinji Nakao $^{3}$, Seiji Kojima ${ }^{1}$, Yoshiyuki Takahashi ${ }^{1}$

\footnotetext{
${ }^{1}$ Nagoya University Graduate School of Medicine, Nagoya, Japan, ${ }^{2}$ Kanazawa University Hospital, Kanazawa, Japan, ${ }^{3}$ Kanazawa University, Kanazawa, Japan
}

Background: Patients with acquired aplastic anemia (AA) who do not have HLA-matched donors receive immunosuppressive therapy (IST) with anti-thymocyte globulin (ATG). Previous studies have suggested several variables that predict response to IST. However, no studies have investigated the plasma ATG level as a variable. In this study, we assessed the relationship between plasma rabbit ATG (r-ATG) level and response to IST in patients with severe AA.

Methods: Patients with severe AA who required initial IST were enrolled from May 2012 to October 2017. The IST regimen included r-ATG (thymoglobulin ${ }^{\circledR}$, Sanofi, Cambridge, 2.5 or $3.5 \mathrm{mg} / \mathrm{kg} / \mathrm{day}$ for 5 days) and cyclosporine A $(6 \mathrm{mg} / \mathrm{kg} / \mathrm{day}$ for minimum 6 months). Plasma r-ATG level was measured using a rabbit IgG ELISA kit on days 14 and 28. Response rate was defined as complete and partial responses at 6 months. Receiver operator characteristic curves were generated to discriminate between response and no response to IST.

Results: A total of 81 patients (aged 1.7-67.9 years) were randomized; 43 and 38 patients received 2.5 and $3.5 \mathrm{mg} / \mathrm{kg}$ of r-ATG, respectively. In the $2.5 \mathrm{mg}$ group, the response rate was $63 \%$, which was comparable with that in the 3.5 $\mathrm{mg}$ group $(58 \%)(\mathrm{P}=0.820)$. Plasma $\mathrm{r}-\mathrm{ATG}$ level greatly varied in both groups. Median r-ATG level on days 14 and 28 after IST was $15.2(0.0-97.7)$ and $1.8(0.0-74.9 \mu \mathrm{g} / \mathrm{mL})$, respectively, which was not significantly different between two dosages of ATG groups (day 14, $\mathrm{P}=0.498$; day 28, $\mathrm{P}=0.404$ ). According to the $\mathrm{r}-\mathrm{ATG}$ level, response rates were significantly higher in the group with higher r-ATG level than in that with lower ATG level (day 14, $88 \%$ vs. $52 \%$, respectively; $\mathrm{P}=0.006$ and day $28,79 \%$ vs. $46 \%$; $\mathrm{P}=0.005$ ) (Figure). Cut-off levels at days 14 and 28 were 21.6 and $4.8 \mu \mathrm{g} / \mathrm{mL}$, respectively. The vast majority (90\%) of patients with levels higher than cut-off levels on day 14 responded to IST. In multivariate analysis, higher ATG levels at day 28 were independent favorable predictors of response to IST at 6 months (OR $=0.29$; 95\% CI: 0.09 $0.93 ; \mathrm{P}=0.037$ ). There were no significant differences in the kinetics of lymphocyte subsets among patients treated with different dosages of ATG. However, higher ATG level was associated with lower $\mathrm{CD} 4+\mathrm{T}$ and regulatory $\mathrm{T}$ cell numbers for the entire 6-month period.

Conclusions: The present data indicate interindividual variability in plasma r-ATG level. Higher ATG level resulted in improved response to IST and correlated with prolonged immune reconstitution. Individualized dosing of ATG via a pharmacokinetic model may improve the response rate to IST and reduce the number of patients who require allogeneic stem cell transplantation following IST.

Clinical Trial Registry: www.umin.ac.jp UMIN000011134 Disclosure: This study was partly supported by Sanofi K.K.

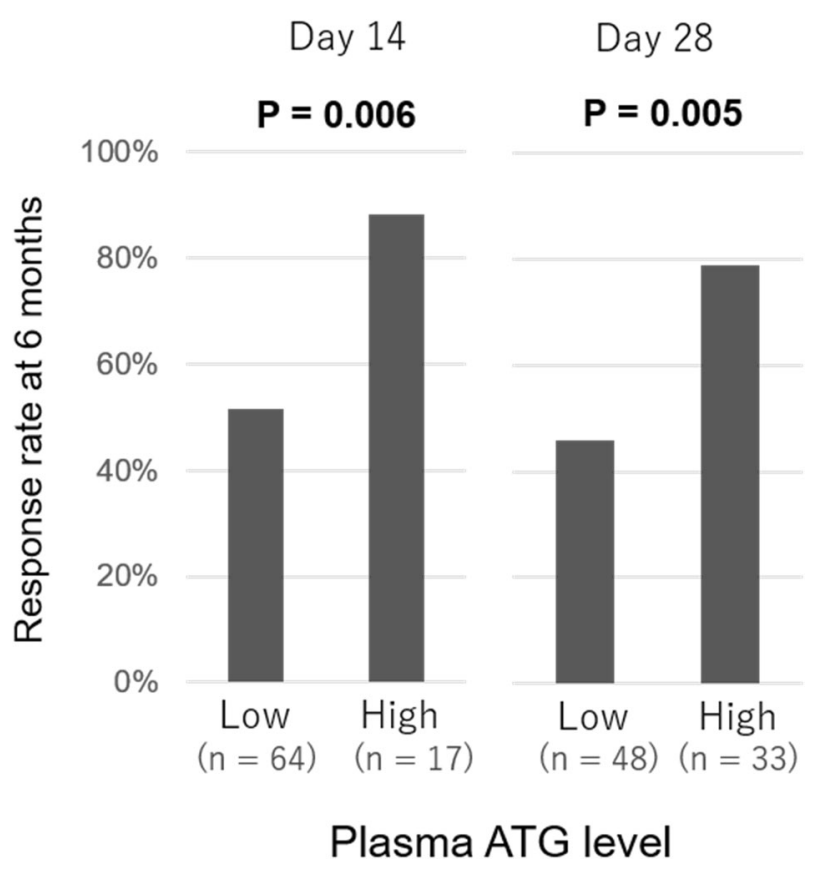

[[O027 Image] 1. Response rates of IST at 6 months according to plasma ATG levels]

O028

Abstract already published.

0029

Hematopoietic cell transplantation without radiation or dna alkylating agents in bone marrow failure with short telomeres 
Suneet Agarwal ${ }^{1,2}$, Kasiani Myers ${ }^{3}$, Joseph Antin ${ }^{4}$, Alison Bertuch $^{5}$, Lauri Burroughs ${ }^{6}$, Inga Hofmann ${ }^{7}$, Mark Juckett $^{8}$, Ghadir Sasa ${ }^{9}$, Sundin Mikael ${ }^{10}$, Farid Boulad ${ }^{11}$, Timothy Olson ${ }^{12}$, Michael Pulsipher ${ }^{13}$, Wendy London ${ }^{1,2}$, David Williams $^{1,2}$, Leslie Lehmann ${ }^{1,2}$

${ }^{1}$ Dana-Farber/Boston Children's Cancer \& Blood Disorders Center, Boston, MA, United States, ${ }^{2}$ Harvard Medical School, Boston, MA, United States, ${ }^{3}$ Cincinnati Children's Hospital Medical Center, Bone Marrow Transplantation and Immune Deficiency, Cincinnati, OH, United States, ${ }^{4}$ DanaFarber Cancer Institute, Hematologic Malignancies, Boston, MA, United States, ${ }^{5}$ Baylor College of Medicine, Pediatrics, Hematology/Oncology, Houston, TX, United States, ${ }^{6}$ Fred Hutchinson Cancer Research Center, Seattle, WA, United States, ${ }^{7}$ University of Wisconsin - Madison, Hematology/Oncology and Bone Marrow Transplantation, Madison, WI, United States, ${ }^{8}$ University of Wisconsin Madison, Carbone Cancer Center, Madison, WI, United States, ${ }^{9}$ Center for Cell and Gene Therapy, Houston, TX, United States, ${ }^{10}$ Karolinksa University, Pediatric Hematology, Immunology and HCT, Stockholm, Sweden, ${ }^{11}$ Memorial Sloan Kettering Cancer Center, Pediatrics, New York, NY, United States, ${ }^{12}$ Children's Hospital of Philadelphia, Pediatrics, Philadelphia, PA, United States, ${ }^{13}$ Children's Hospital Los Angeles, Los Angeles, CA, United States

Background: Radiation and DNA alkylating agents used in hematopoietic cell transplantation (HCT) can cause organ damage, malignancy and death. These risks are heightened in patients with genetic bone marrow failure (BMF) syndromes driven by defects in cellular proliferation or DNA repair, including dyskeratosis congenita (DC), which arises from impaired telomere maintenance. We hypothesized that proliferative defects in hematopoietic cells of patients with BMF and very short telomeres might permit myeloid engraftment following HCT without the need for radiation or DNA alkylating agents. We conducted a multi-center prospective trial (NCT01659606) evaluating engraftment after HCT without these agents.

Methods: We enrolled BMF patients with genetic validation of DC or lymphocyte telomere length $<1^{\text {st }}$ percentile by flow-FISH. We performed HCT using bone marrow allografts from related or unrelated donors matched at 7 or 8 of 8 HLA alleles after a preparative regimen consisting of only fludarabine and alemtuzumab. Graft versus host disease (GVHD) prophylaxis consisted of cyclosporine A and mycophenolate mofetil. The primary endpoint of the trial was donor myeloid engraftment, defined as an absolute neutrophil count $\geq 500$ cells $/ \mu \mathrm{L}$ by day +42 and donor myeloid chimerism $>50 \%$ by day +100 .

\section{A. Primary engraftment}

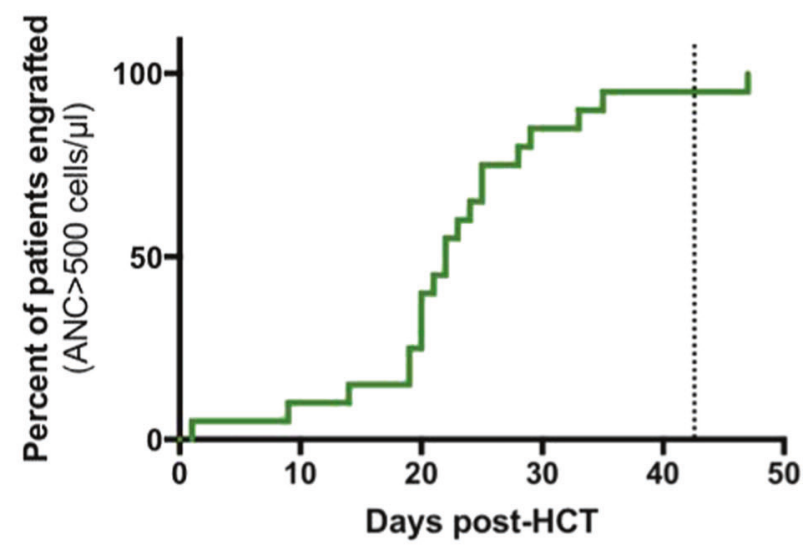

B. Survival

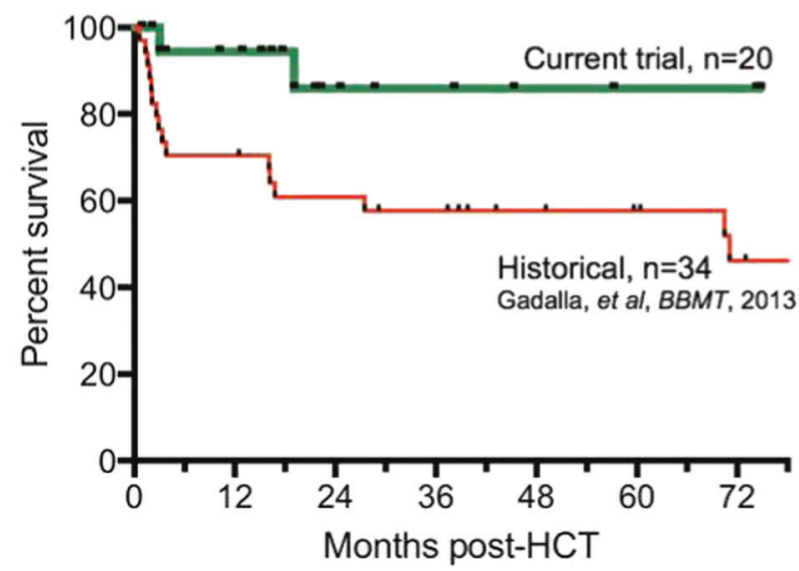

[[O029 Image] 1. Engraftment and Survival after Radiation- and Alkylator-Free HCT for BMF with Very Short Telomeres]

Results: Twenty patients (age 1.7-31.5 years old at HCT) received treatment between August 2012 - October 2018 at 7 institutions. Eighteen of the 20 patients received unrelated donor grafts (15 matched, 3 single-allele mismatched). Primary myeloid engraftment was achieved in 19 of 20 patients $(95 \%)$ at a median 22 days post-HCT (range 1-35 days). The single patient with primary graft failure had DC-related liver disease and hypersplenism; in this case, splenectomy at day +47 promptly revealed donor myeloid engraftment. Of the other 19 patients, 16 had sustained myeloid engraftment, with a median post-HCT follow-up of 21 months (range 1-74 months). Three patients had secondary graft failure. Two of these had early graft rejection and underwent successful repeat HCT using higher intensity regimens. The third patient maintained high donor chimerism after primary engraftment but developed severe neutropenia in the setting of multiple viral reactivations, and died of a fungal infection 90 days post-HCT. There was one 
other death, due to DC-related gastrointestinal complications 19 months post-HCT. None of the 16 patients who engrafted durably under the protocol regimen had acute GVHD. Four had chronic GVHD (3 limited, 1 extensive), treated successfully with limited courses of topical or oral steroids.

Conclusions: We conclude that this radiation- and alkylator-free HCT conditioning regimen is an effective strategy for BMF in patients with DC or very short lymphocyte telomeres. Eliminating DNA damaging agents may reduce HCT complications including GVHD and enable transplant in patients with high-risk comorbidities.

Clinical Trial Registry: NCT01659606

https://clinicaltrials.gov/ct2/show/NCT01659606

Disclosure: Nothing to declare

\section{O030}

Abstract already published.

\section{O031}

\section{HLA-Alloimmunization in acquired aplastic anemia}

Katja Julen ${ }^{1}$, Thomas Volken ${ }^{2}$, Andreas Holbro ${ }^{1,3}$, Laura Infanti ${ }^{1,3}$, Jörg Halter ${ }^{3}$, Stefan Schaub ${ }^{3}$, Tamara Diesch ${ }^{4}$, Jakob Passweg ${ }^{3}$, Andreas Buser ${ }^{1,3}$, Beatrice Drexler ${ }^{1,3}$

${ }^{1}$ Blood Transfusion Center, Swiss Red Cross, Basel, Switzerland, ${ }^{2}$ Zurich University of Applied Sciences, Winterthur, Switzerland, ${ }^{3}$ University Hospital, Basel, Switzerland, ${ }^{4}$ University Children's Hospital, Basel, Switzerland

Background: The standard treatment of acquired aplastic anemia (AA) is either intensive immunosuppressive therapy (IST) or allogeneic hematopoietic cell transplantation (HCT). As supportive measures, red blood cells and platelet transfusions are the mainstay of therapy and patients are often multitransfused, which in turn can lead to anti-human leukocyte (HLA) alloimmunization. In acquired AA the rate of HLA-alloimmunization has previously shown a higher frequency in patients with AA compared to hematological malignancies. However, these results date back before the general introduction of leukoreduction of blood products and photochemical pathogen reduction of platelet components, and are based on cell-based assays. In recent years, leukoreduction and pathogen reduction of blood products became standard in Switzerland and the solid-phase assay (Lumine ${ }^{\circledast}$ technology) is now widely available to test for HLA-antibodies, allowing a more extensive and detailed characterization of HLA-antibodies. With these techniques, less is known on the exact incidence of HLA-antibodies and their magnitude, associated cofactors and its impact on treatment outcomes in acquired AA.
Methods: We retrospectively investigated 54 AA patients treated with IST $(n=44)$ and/or HCT $(n=25)$ at the University Hospital of Basel and the University Children's Hospital of Basel (Switzerland) regarding HLA antibodies since the introduction of testing with the Luminex ${ }^{\circledR}$ at our center in 2008. At least one HLA antibody measurement before and/or after therapy was available per patient. All patients received leukoreduced blood products and as of 2011 platelets treated with INTERCEPT $^{\circledR}$ (UV+ amotosalen).

Results: Overall, HLA-antibodies were detected in 40 (74\%) patients with a higher rate of HLA alloimmunziation by severity of AA $(p<0.01)$. The median number of HLAantibodies in each patient before therapy (i.e. IST or HCT) was 3 (IQR 0-25). In patients undergoing HCT HLAantibodies were more frequent before treatment start as compared to patients with IST treatment (median 13 (IQR $0-45$ ) versus 2.5 (IQR 0-16.5), $\mathrm{p}<0.05$ ). Differences between treatments remained after adjusting for all covariates $(\mathrm{p}<0.01)$. There was no statistically significant difference regarding the HLA antibody mean fluorescence intensity (MFI) between the two treatment forms (overall mean MFI of $1580+/$ - 2075). The highest mean HLAantibody MFI before therapy was $4747(+/-7221)$ with a maximum of 24020 . Females showed a significantly higher number of HLA-antibodies $(\mathrm{p}<0.01)$ and also higher mean MFI ( $p<0.05)$. Furthermore, the number of pregnancies was associated with higher numbers of HLA-antibodies $(\mathrm{p}<$ 0.01 ), however the number of transfusions did not have significant impact on HLA-antibody number and MFI. Regarding outcome, there was no significant association between the number of HLA-antibodies and engraftment as well as bleeding events.

Conclusions: HLA-alloimmunization is still frequent in patients with acquired AA but today number of pregnancies and gender seem to be more important for development of HLA-alloimmunization than number of transfusions. Interestingly, patients treated by HCT show a higher rate of HLA-alloimmunization before treatment start in comparison to IST, thereby emphazing the importance of blood management and donor selection in HCT in acquired AA as HLA-antibodies can cause platelet refractoriness and can represent donor-specific antibodies in the setting of mismatched HCT (e.g. haploidentical).

Disclosure: Nothing to declare.

\section{Autoimmune diseases}

\section{2}

Health-related quality of life in systemic sclerosis before and after autologous hematopoietic stem cell transplant - a systematic review 
Mathieu Puyade ${ }^{1}$, Nancy Maltez ${ }^{2}$, Gregory Pugnet ${ }^{3}$, Zora Marjanovic $^{4}$, Anne Huyn ${ }^{5}$, Inés Colmegna ${ }^{6}$, Marie Hudson $^{2}$, Dominique Farge-Bancel ${ }^{4}$

${ }^{1} \mathrm{CHU}$ de Poitiers, Poitiers, France, ${ }^{2} \mathrm{Mc}$ Gill University/ Jewish General Hospital and Lady David Research Institute, Montreal, Canada, ${ }^{3} \mathrm{CHU}$ de Toulouse Service de Médecine Interne, Toulouse, France, ${ }^{4}$ APHP Hopital Saint Antoine, Paris, France, ${ }^{5}$ IUCT Oncopole, Toulouse, France, ${ }^{6} \mathrm{Mc}$ Gill University, Montreal, Canada,

Background: Autologous hematopoietic stem cell transplantation (AHSCT) for severe rapidly progressive Systemic Sclerosis (SSc) allows significant regression in skin and lung fibrosis and improvements in overall and event free survival up to 7 years after transplant. We undertook this study to synthesize the evidence on changes in healthrelated quality of life (HRQoL) associated with AHSCT for SSc.

Methods: Autologous hematopoietic stem cell transplantation (AHSCT) for severe rapidly progressive Systemic Sclerosis (SSc) allows significant regression in skin and lung fibrosis and improvements in overall and event free survival up to 7 years after transplant. We undertook this study to synthesize the evidence on changes in healthrelated quality of life (HRQoL) associated with AHSCT for SSc.

Results: The search returned 656 articles. Eight were selected: 3 uncontrolled phase I or II trials, 2 cohort studies and 3 RCT (ASSIST, ASTIS, SCOT). HRQoL data from 289 SSc patients treated with AHSCT and 125 with intravenous cyclophosphamide (CYC) as a comparator were extracted. HRQoL was assessed using the Health Assessment Questionnaire-Disability Index (HAQ-DI) $(\mathrm{n}=275$ patients), the Short-Form Health Survey (SF-36) $(n=249$ patients) and the EuroQol 5 Dimensions (EQ-5D) ( $\mathrm{n}=138$ patients). HRQoL was analyzed as a secondary outcome in all studies. Quality of the data was assessed as high.

HAQ-DI improved significantly more with AHSCT compared to CYC $(-0.58$ vs $-0.19, \mathrm{p}=0.02$ at 2 years in ASTIS; $53 \%$ vs $16 \%$ improved at 4.8 years in SCOT). Scores also improved pre-post AHSCT in the uncontrolled studies (ranging from -0.6 to -1.7 points at one year (all $\mathrm{p}<$ $0.05)$, and up to -1.5 points at 7.5 years $(\mathrm{p}<0.001)$ ). SF-36 Physical Component Summary scores were significantly better in subjects treated with AHSCT compared to CYC (between group differences ranging from 26 points at one year in ASSIST and 6.1 points at 2 years in ASTIS $(p=0.01) ; 56 \%$ vs $15 \%$ improved at 4.8 years in SCOT $(\mathrm{p}=0.02))$. Similar differences pre-post treatment scores were also reported in an uncontrolled study (increase of 20 points, $\mathrm{p}<0.0001$ ). In ASSIST, there was a trend for the SF-
36 Mental Component Summary score to improve in the AHSCT arm (46 vs 58, $\mathrm{p}=0.07$ ) and worsen in the CYC $\operatorname{arm}(56$ vs $42, p=0.04)$ at one year. There were no significant differences between the AHSCT and CYC arms in ASTIS and SCOT with 2.0 and 4.8 years of follow-up, respectively. Post-treatment scores improved significantly compared to pre-treatment in an uncontrolled study (from 51 to 64 points, $p=0.005$ ). ASTIS showed a significant difference in the index-based utility score of the EQ-5D $(0.29, p<0.001)$ and a non-significant difference in the general health visual analogue scale $(6.7, p=0.36)$ at two years, in favour of AHSCT compared to CYC.

Conclusions: Although there is heterogeneity in the reported data, AHSCT in SSc was consistently associated with marked and sustained improvement in HRQoL. This analysis provides additional compelling data for the role of AHSCT in SSc when assessing patient's point of view.

Clinical Trial Registry: NA

Disclosure: No conflict of interest

\section{$\mathbf{O 0 3 3}$}

Immune reconstitution following autologous haematopoietic stem cell transplantation for multiple sclerosis is driven by sustained thymic reactivation

Jennifer Massey ${ }^{1}$, Carole Ford $^{I}$, Melissa Khoo ${ }^{1}$, Remi Cheynier $^{2}$, Benedicte Charmantu ${ }^{2}$, John Zaunders ${ }^{3}$, Kevin Hendrawan $^{1}$, Ian Sutton ${ }^{1}$, Barbara Withers ${ }^{1}$, Sam Milliken $^{I}$, David Ma ${ }^{I}$, John Moore ${ }^{I}$

${ }^{1}$ St Vincents Hospital, Sydney, Australia, ${ }^{2}$ INSERM, Institut Cochin, Paris, France, ${ }^{3}$ Kirby Institute, Sydney, Australia

Background: Autologous haematopoietic stem cell transplantation (AHSCT) is a promising strategy for Multiple Sclerosis (MS) patients that do not respond to conventional treatments, but the mechanisms enabling clinical improvement of MS in transplant recipients are not fully understood. We hypothesised that AHSCT induces a rebooting of thymic function, resulting in the re-development of a functional, tolerant immune system. We aimed to examine cellsurface and DNA markers of recent thymic emigrants (RTE's) longitudinally in a cohort of MS patients postAHSCT in order to identify markers that correlate with a durable treatment response.

Methods: Peripheral blood mononuclear cells (PBMNCs) were collected from patients enrolled in the Phase II trial at St Vincent's Hospital Sydney for AHSCT in MS (Moore et al, JNNP in press). A multicolour flow cytometry panel to optimally identify RTE's was performed on 10 patient samples at $0,6,12,24$ and 36 month timepoints, allowing us to track changes in thymic output longitudinally 
following AHSCT. DNA markers of thymic function - Sj:b TREC ratio was performed in the same cohort of patients, on the same bio-banked sample to enhance the validity of observed changes. Statistical analysis was performed with GraphPad Prism.

Results: A sustained, significant increase in RTE's and Sj:b TREC was detected between pre-transplant and 24 month post-transplant specimens $(\mathrm{p}=0.024)$. In patients where similar analysis was able to be performed at 36 months a trend to increased markers of thymic output was observed. A correlation between RTE's and Sj:b TREC was observed $(r=0.70, p=0.003)$. Contrary to other publications in the field, TREC as a marker of thymic output did not appear to be lower when patients were analysed by age $(<30$ yrs vs. $>30$ yrs). Greater thymic output as determined by $\mathrm{Sj}: \mathrm{b}$ TREC was observed at all timepoints in patients who had evidence of sustained disease remission as opposed to patients who experienced disease relapse.

Conclusions: We have seen evidence that sustained thymic reactivation is a component of immune reconstitution following AHSCT for MS. Previous studies have only demonstrated thymic activity at 12 months but this study confirms that thymic activity remains prominent at 24 and even 36 months. This thymic regeneration may contribute to a durable clinical response in patients with MS post HSCT.

Clinical Trial Registry: Australia and New Zealand Clinical Trials Registry

ACTRN12613000339752

www.anzctr.org.au

Disclosure: Nothing to declare

$\mathrm{SJ}: \mathrm{B}(1+2)$ TREC AHSCT by age - mean and SEM

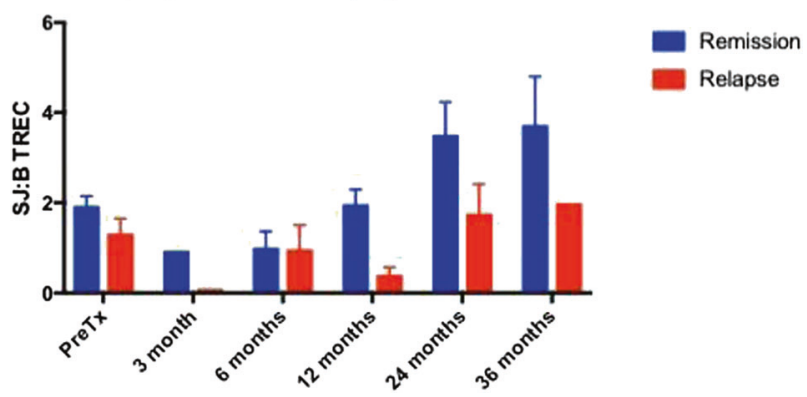

[[O033 Image] 1. Thymic output post HSCT for MS]

\section{4}

Allogeneic hsct for autoimmune diseases: A retrospective study from the ebmt autoimmune diseases, inborn errors and paediatric working parties

Raffaella Greco' $^{1}$, Myriam Labopin ${ }^{2}$, Manuela Badoglio', Paul Veys, ${ }^{3}$ Juliana Silva ${ }^{3}$, Mario Abinun ${ }^{4}$, Francesca

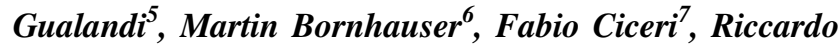
Saccardi $^{8}$, Arjan Lankester ${ }^{9}$, Tobias Alexander ${ }^{10}$, Andrew Gennery $^{11}$, Peter Bader ${ }^{12}$, Dominique Farge ${ }^{13}$, John Snowden $^{14}$

${ }^{1}$ IRCCS San Raffaele Scientific Institute, Milano, Italy, ${ }^{2}$ EBMT Paris Study Office / CEREST-TC, Saint Antoine Hospital - INSERM UMR 938 - Université Pierre et Marie Curie, Paris, France, ${ }^{3}$ Great Ormond Street Hospital, London, London, United Kingdom, ${ }^{4}$ Great North Children's Hospital, Newcastle University, Newcastle upon Tyne, United Kingdom, ${ }^{5}$ IRCCS AOU San Martino-IST, Genova, Italy, ${ }^{6}$ Carl Gustav Carus University Hospital, Dresden, Germany, ${ }^{7}$ Salute San Raffaele University, Milano, Italy, ${ }^{8}$ Careggi University Hospital, Florence, Italy, ${ }^{9}$ University Medical Centre, Leiden, Netherlands, ${ }^{10}$ Charité-University Medicine Berlin, Berlin, Germany, ${ }^{11}$ Newcastle University, Great North Childrens' Hospital, Newcastle-upon-Tyne, United Kingdom, ${ }^{132}$ University Hospital Frankfurt, Frankfurt, Germany, ${ }^{14}$ Saint Louis Hospital, Paris, France, ${ }^{15}$ Sheffield Teaching Hospitals NHS Foundation Trust, Sheffield, United Kingdom

Background: Allogeneic HSCT offers the potential replacement of an aberrant immune system. This retrospective study assessed long-term outcomes of this strategy in patients treated for severe autoimmune diseases (ADs), reported to the EBMT registry.

Methods: Among the total 126 patients who received allogeneic HSCT between 1997-2014, we received detailed questionnaires on long-term outcomes from 64 patients. The diagnosis of $\mathrm{AD}$ was hematological $(\mathrm{n}=21)$ and nonhematological $(n=43)$, among pediatric $(=45)$ and adult (=19) populations. The median age of patients at HSCT was 11.14 years (pediatric: median 8.07 years, range 1.2217.77); adult : median 31.30 years, range 21.43-51.57). All patients were refractory to previous immunosuppressive therapies (median of 4 lines of treatments, range 1-13), and eight of them received a previous autologous transplant.

The graft source was PBSCs in $38, \mathrm{BM}$ in 24 , and cord blood in 2 patients. Donors were as follows; $41 \%$ MRD, 42\% MUD, 9\% MMRD, 5\% syngeneic and 3\% cord blood. Conditioning was MAC in 35 and RIC in 29 patients. Serotherapy with ATG was given in 16 patients, while 30 patients received alemtuzumab. Post-transplant GvHD prophylaxis was cyclosporine-based for the majority of patients $(\mathrm{n}=54)$.

Results: Median follow-up was 67 months (range 7.9189 months). Toxicity profile was similar to allogeneic HSCT in other contexts. The incidence of grades II-IV acute GvHD was $16.4 \%$ (95\% CI: $8.4-26.8$ ) at 100-days; severe acute GvHD was reported in $6.5 \%$ of them. Cumulative incidence of chronic GvHD was 32.5\% (95\% CI: 20.8 - 
44.8) at 5-year; extensive manifestations were reported for $58 \%$ of chronic GvHD. Overall graft rejection rate was $4.9 \%$.

Seven secondary AD and one case of new malignancy (lymphoma) occurred. Viral reactivations were reported in a total of 33 patients, including CMV $(n=14), \operatorname{EBV}(n=10)$, Adenovirus $(\mathrm{n}=5)$, BK virus $(\mathrm{n}=3)$, HSV $(\mathrm{n}=2)$, HHV6 $(n=3)$ and VZV $(n=2)$. Seven cases of invasive fungal infection were reported (one aspergillosis and three candidiasis). Ten bacterial infections (only 4 patients developed infection from Gram-negative bacteria) and four pneumonia were observed.

At the last follow-up complete clinical response was obtained in $69.4 \%$ of patients, while partial remission was reported in $6.5 \%$. Relapse incidence (RI) was $21.9 \%$ (95\% CI: 11.8 - 33.9) at the last follow-up. Post-HSCT autoimmune disease specific treatment was required for 12 patients. In subgroup analysis among different diseases, the OS rates were similar between immune cytopenia and other ADs.

At 5 years, OS was $76 \%$ (95\% CI: 64.8 - 87.3), NRM was $13.3 \%$ (95\% CI: 6.1 - 23.2) and PFS was $64.8 \%$ (95\% CI: 52.1 - 77.6), with no differences between immune cytopenia (73.8\%) and other ADs (64.3\%).

By multivariate analysis, only one prognostic factor remained significantly associated with long-term outcomes: a more recent year of transplant (better OS, $\mathrm{p}=0.007$; lower chronic GvHD, $\mathrm{p}=0.047$ ).

Conclusions: This large retrospective survey of the EBMT registry confirms the potential of allogeneic HSCT to produce long-term disease remission in a large proportion of refractory ADs, with acceptable toxicities and NRM. Outcomes have improved in recent years.

Clinical Trial Registry: NA

Disclosure: Nothing to declare

\section{5}

Hematopoietic stem cell transplantation improves functional capacity and quality of life of systemic sclerosis patients

Karla Ribeiro Costa-Pereira ${ }^{1}$, Juliana Elias ${ }^{1}$, Daniela Moraes $^{1}$, Ana Luisa Guimaraes ${ }^{1}$, Vania Mara Silva ${ }^{1}$, Erika Arantes Oliveira Cardoso ${ }^{1}$, Marilia De Fatima Cirioli $^{I}$, Vanessa Leopoldo ${ }^{I}$, Andreia Ferreira Zombrilli, Thalita C M Costa ${ }^{1}$, Belinda Simoes ${ }^{I}$, Maria Carolina Oliveira ${ }^{1}$

${ }^{1}$ University of Sao Paulo, Ribeirao Preto, Brazil

Background: Systemic sclerosis ( $\mathrm{SSc}$ ) is a chronic autoimmune disease characterized by skin thickening and visceral involvement, leading to impairment of physical function, daily life activities and quality of life. Severe cases usually have poor prognosis, despite conventional immunosuppressive treatment. Autologous hematopoietic stem cell transplantation (AHSCT) has been investigated as treatment for patients with severe and rapidly progressive $\mathrm{SSc}$ and promotes reduction of skin thickening and at least stabilization of pulmonary involvement. There are no reports in the literature addressing the influence of AHSCT on the functional outcomes of SSc patients. The aim of this study was to evaluate the impact of AHSCT on skin involvement, pulmonary function, functional capacity and quality of life (QoL) of SSc patients, and to evaluate if pulmonary function measurements correlate with the other evaluated variables.

Methods: in this prospective longitudinal study, 27 SSc patients were evaluated before, and at six and twelve months after AHSCT. Assessments included modified Rodnan's skin score (mRSS), pulmonary function test (forced vital capacity - FVC and carbon monoxide diffusion capacity - DLCO), respiratory muscle strength test (maximal inspiratory pressure - MIP and maximal expiratory pressure - MEP), six-minute walk test (6MWT) and quality of life questionnaire (SF-36). Results underwent statistical analyses and significance levels were established at $\mathrm{p}<0.05$.

Results: Twenty-seven patients were evaluated before and at 6 months after transplant, 22 of which were additionally evaluated at 12 months. At 6 and 12 months after AHSCT, patients presented significant improvement of mRSS ( $p<0.01)$, MIP $(\mathrm{p}<0.01), \operatorname{MEP}(\mathrm{p}<0.01), 6$ MWT distance $(\mathrm{p}=0.02)$, and physical $(\mathrm{p}<0.01)$ and mental $(\mathrm{p}=0.02)$ components of the SF-36, when compared to pretransplant evaluations. No changes were observed in FVC after treatment. Despite a transient decline in DLCO at 6 months $(\mathrm{p}<0.01)$ after transplant, DLCO levels at 12 months were not different from baseline (pre-transplant). Significant correlations were observed between the 6MWT distance and physical component score of quality of life $(\mathrm{R}=0.62, \mathrm{p}<0.01)$. No significant correlation was observed between pulmonary function and the other evaluated variables (mRSS, respiratory muscle strength, physical capacity and quality of life).

Conclusions: AHSCT significantly improves the functional status of SSc patients in the first year of follow-up. Although the pulmonary function remained stable after AHSCT, there was significant increase in the physical capacity and quality of life of patients. These results can be interpreted as positive outcomes of AHSCT for SSc.

Disclosure: Nothing to declare.

\section{6}

Model of multidisciplinary and multicenter approach for HSCT for children with multiple sclerosis: Long- 
term follow-up, late effects and transfer to adult healthcare

Kirill Kirgizov ${ }^{1,2}$, Ekaterina Pristanskova ${ }^{1}$, Elvira Volkoval, Oksana Blagonravoval, Nataliya Sidorova', Veronika Konstantinova ${ }^{1}$, Elena Machneva ${ }^{1}$, Sergey Piliya ${ }^{1}$, Olga Filina ${ }^{1}$, Julia Nikolaeva ${ }^{1}$, Anastasiya Mezentseva ${ }^{1}$, Raisa Bembeeva ${ }^{1}$, Elena Skorobogatova ${ }^{1}$

${ }^{1}$ The Russian Children's Research Hospital, Moscow, Russian Federation, ${ }^{2}$ Dmitry Rogachev National Medical Research Center of Pediatric Hematology,

\section{Moscow, Russian Federation}

Background: HSCT for children with multiple sclerosis (MS) proved effectiveness and safety. It is required to improve the results by analysis of long-term follow-up and late effects. Several challenges identified in multidisciplinary collaboration for successful treatment as well as a problem of switching these patients to the adult healthcare. We aimed to create a model of organization of help for children with severe refractory multiple sclerosis based on multidisciplinary and multicenter approach.

Methods: Fifteen children with MS received autologous HSCT (aHSCT) from January 2010 to May 2018. Gender: females - 11, males - 4. Mean length of MS prior aHSCT was $22.7 \pm 8.4$ months and mean age of MS debut was 12.7 \pm 2.1 years old. All patients had severe refractory MS treated with corticosteroids, interferons, plasmapheresis and mitoxantron with negative results. These patients presented signs of neuroinflammation. Mean baseline EDSS before the start of mobilization was $4.8 \pm 1.4$. Procedures included mobilization with the help of cyclophosphamide and filgrastim and conditioning: Cyclophosphamide $200 \mathrm{mg} / \mathrm{kg}$ and hATG $160 \mathrm{mg} / \mathrm{kg}$. All patients received at least $2 \times 10^{\wedge} 6 / \mathrm{kg} \mathrm{CD} 34$ + hematopoietic stem cells (mean $4.3 \times 10^{\wedge} 6 \pm 2.6 \times 10^{\wedge} 6$ ). We analysed the incidence and nature of late effects in patients with at least one year of follow-up (based on the standard protocol for late effects). Fertility preservation proposed for patients. aHSCT as well as pre- and posttransplant care was done by multidisciplinary team involved both transplant and neurological team. Technology of transfer patients to adult center for post-transplant observation was identified.

Results: All patients survived. Mean time to engraftment was $11 \pm 1.6$ days. Eleven patients experienced culture negative fever, one patient - cystitis, and one patient had CMV reactivation within 100 days of the transplant. No patient experienced an EDSS increase post-HSCT above baseline, and all patients improved. Mean improvement of EDSS was $2.8 \pm 1.2$ during the first 60 days after aHSCT (fast recovery). In-time transplanted patients improved better. Improvement confirmed by immunological data (increasing of immune regulation index and T-regs in comparison with the baseline). Median follow-up period was 48 months (8-93 months). Four patients $(26.7 \%)$ experienced exacerbations (both neurological and MRI) in median of 2 years (1-3 years) after aHSCT. No onsets of secondary autoimmune disease and malignancies was seen. Cardiocascular late effects were seen in 6 patients and endocrine - in 3 patients (all females). All these late effects were successfully treated. Patients after the age of 18 years old were transferred to partnering adult center. This center uses the same protocol for HSCT (in adults) and posttransplant observation. Detailed scheme of transfer developed.

Conclusions: aHSCT for pediatric patients with severe refractory MS appears to be safe and effective method and in-time HSCT can significantly improve the outcomes. Most of the patients did not experienced exacerbations. Late effects found in patients were successfully treated. We provide a best care for these patients in both childhood and adulthood by transferring them to adult center. Thus, a unique multicenter and multidisciplinary model of care for children with severe refractory MS was found.

Disclosure: Nothing to declare

\section{O037}

Autologous non-myeloablative hematopoietic stem cell transplantation in patients with neuromyelitis optica spectrum disorder (NMOSD): An open-label pilot study

\section{Richard Burt ${ }^{1}$, Xiaoqiang Han ${ }^{1}$, Jiraporn Jitprapaikul-} $\operatorname{san}^{2}$, Sean Pittock ${ }^{2}$

${ }^{1}$ Northwestern University, Chicago, IL, United States, ${ }^{2}$ Mayo Clinic Rochester, Rochester, MN, United States

Background: Neuromyelitis optica spectrum disorder (NMOSD) is an inflammatory central nervous system disorder characterized, despite immunotherapy treatments, by life-long, severe, and disabling attacks of optic neuritis and myelitis. The aim is to determine if autologous nonmyeloablative hematopoietic stem cell transplantation could be an alternative treatment option.

Methods: Following stem cell mobilization with cyclophosphamide $\left(2 \mathrm{~g} / \mathrm{m}^{2}\right)$ and filgrastim, patients were treated with cyclophosphamide (200 mg/kg) divided as $50 \mathrm{mg} / \mathrm{kg}$ intravenously (IV) on day -5 to day -2 , rATG (thymoglobulin) given IV at $0.5 \mathrm{mg} / \mathrm{kg}$ on day $-5,1 \mathrm{mg} / \mathrm{kg}$ on day -4 , and $1.5 \mathrm{mg} / \mathrm{kg}$ on days $-3,-2$, and -1 (total dose $6 \mathrm{mg} / \mathrm{kg}$ ), and rituximab $500 \mathrm{mg}$ IV on days -6 and +1 . Unselected peripheral blood stem cells were infused on day 0. AQP4IgG antibody status was determined by CLIA validated ELISA or flow cytometry assays. Cell killing activity was measured using a flow cytometry based complement assay. 
Results: Twelve (eleven AQP4-IgG positive) patients were treated with a median follow-up of 54 months. Ten patients are more than five years post-transplant. At five years, $80 \%$ of patients were relapse-free off all immunosuppression $(p<0.001)$. At one and five years after HSCT, EDSS improved from a baseline mean of 4.3 to 2.8 $(\mathrm{P}<0.001)$ and $3.25(\mathrm{P}<0.001)$, respectively. NRS improved after HSCT from a baseline mean of 69.5 to 83.8 at one year $(\mathrm{p}<0.001)$ and 85.9 at five years $(\mathrm{P}<0.001)$. The SF-36 quality of life total score improved from mean 34.2 to 55.1 $(\mathrm{P}=0.03)$ and 62.1 $(\mathrm{P}=0.001)$. AQP4-IgG serostatus converted to negative in nine patients and complement activating and cell killing ability of patient serum was switched off. Two patients remained AQP4-IgG seropositive (with persistent cell killing ability) and relapsed within two years of HSCT $(\mathrm{P}<0.01)$

Conclusions: Prolonged drug-free remission with AQP4IgG seroconversion to negative following nonmyeloablative autologous HSCT warrants further investigation in larger randomized controlled trial.

Clinical Trial Registry: Identifier: NCT00787722

ClinicalTrials.gov

Disclosure: Nothing to declare

\section{O038}

\section{Abstract already published.}

\section{O039}

Autoimmune haemolytic anaemia after haematopoietic stem cell transplantation in children: A french multicenter study

Aurélie Guinot ${ }^{1}$, Marc Arca ${ }^{I}$, Nicole Raus ${ }^{2}$, Cecile Renard $^{3}$, Claire Galambrun ${ }^{4}$, Pierre-Simon Rohrlich ${ }^{5}$, Benedicte Bruno $^{6}$, Virginie Gandemer ${ }^{7}$, Cecile Pochon ${ }^{8}$, Jean-Hugues Dalle, Nathalie Aladjidi ${ }^{10}$, Catherine Paillard $^{1}$

${ }^{1}$ University Hospital of Strasbourg, Strasbourg, France, ${ }^{2}$ Société Francophone de Greffe de Moelle et de Thérapie Cellulaire (SFGM-TC), Lyon, France, ${ }^{3}$ Lyon University Hospital, Lyon, France, ${ }^{4}$ APHM, Timone Hospital, AixMarseille University, Marseille, France, ${ }^{5}$ Nice University Hospital, Nice, France, ${ }^{6}$ University of Lille Nord de France, Lille, France, ${ }^{7}$ University Hospital of Rennes, Rennes, France, ${ }^{8}$ Nancy University Hospital, Nancy, France, ${ }^{9}$ APHP, Robert Debré Hospital, Paris Diderot University, Paris, France, ${ }^{10}$ University Hospital of Bordeaux, Bordeaux, France

Background: Autoimmune cytopenias (AIC) are a rare but serious complication of haematopoietic stem cells transplantation (HSCT). The auto immune haemolytic anaemia
(AIHA) is the most frequent of these complications in paediatrics and is difficult to treat. So far, there has been no nationwide post transplantation AIHA study.

Methods: This observational, retrospective and multicentric study focused on French paediatric cases of AIHA after HSCT between January 2007 and January 2018.

Data was collected from national reference databases and direct interview of physicians.

The inclusion criteria were patients between 0 to 18 years old who developed an AIHA or an Evans syndrome after HSCT. Data concerning patient, primary diagnosis, HSCT procedure, pre-transplant viral status, blood group compatibility, characteristics of cytopenia, delay transplant-cytopenia, graft vs host reaction. Laboratory characteristics and therapies were analyzed as well as the response to first, second or third line treatments.

Results: 2856 paediatric HSCT were performed in 16 French paediatric centers between 2007 and 2018. Among them, 36 children developed an AIC: 30 AIHA, 1 pancytopenia and 5 Evans syndromes. The median age at HSCT was 4,9 years old (0,3-14,8). Average delay between transplantation and AIC was 192 days (20-600). The median follow-up after transplant was 35,8 months (5-94).

23 patients were transplanted for non-malignant disease (12 benign hemopathies, 11 immunodeficiencies) and 13 for a malignant one.

Stem cell source was bone marrow for $50 \%$ of patients, peripheral blood stem cells for $29 \%$ and cord blood for $12 \%$. The donor was unrelated for $65 \%$ of HSCT, matched related for $15 \%$ and $12 \%$ of procedures were haploidentical. $76 \%$ of patients received ALS as part of their conditioning regimen, it was myeloablative in $65 \%$ (TBI based for $6 \%$ ) and reduced intensity in $20 \%$.

Direct anti-globulin test (DAT) was positive to $\operatorname{IgG}+/$ C3d for $52 \%$ of patients, 16 patients had a blood group incompatibility ( 8 minor and 8 major).

$64 \%$ of patients had an acute $\mathrm{GvH}$ and chronic $\mathrm{GvH}$ on time of AIHA was find in $12 \%$ of patients.

For $70 \%$ of patients the first line treatment was a combination of steroids $(2 \mathrm{mg} / \mathrm{kg} /$ day $)$, intravenous immunoglobulin and rituximab $\left(375 \mathrm{mg} / \mathrm{m}^{2}\right.$ per week). For $41 \%$ of patients a second or third-line treatment was required (Imurel, Cellcept, Cyclophosphamide, Sirolimus, Campath, Bortezomib). 8 patients died (4 relapse, 2 infections and 1 severe anaemia, 1 multiple organ failure).

Conclusions: This is the first study describing precisely the AIHA post HSCT in pediatrics in France along with the various treatments used. It's a rare but severe complication with multiple risk factors associated with a high mortality rate and no standardized therapy at this time. Further studies would allow us to understand the disease better in order to prevent its occurrence and treat it more efficiently.

Disclosure: Nothing to declare 


\section{Cellular Therapy}

\section{0}

Updated analysis of CART19-BE-01 trial: An academic trial on the use of ARI-0001 cells (A3B1:CD8:4-1BB: CD3Z CAR19 cells) in patients with CD19+ relapsed / refractory malignancies

Valentín Ortiz-Maldonado', Anna Alonso-Saladrigues', Miguel Caballero Baños ${ }^{1}$, María Castella', Anna Boronat, Enric Garcia Rey, Tycho Baumann ${ }^{3}$, Montse Torrebadell ${ }^{2}$, Joan Cid ${ }^{1}$, Miquel Lozano ${ }^{1}$, Cristina Llanos $^{2}$, Berta Marzal', Alex Bataller ${ }^{1}$, Aina Oliver ${ }^{1}$, Andrea Rivero $^{1}$, Pedro Castro ${ }^{1}$, Yolanda Jordan', Jordi Esteve ${ }^{1}$, Josep María Canals ${ }^{4}$, Esteve Trias ${ }^{3}$, Jordi Yagüe ${ }^{1}$, Montserrat Rovira', Alvaro Urbano-Izpizúa', Manel Juan', Susana Rives', Julio Delgado ${ }^{1}$

${ }^{1}$ Hospital Clínic de Barcelona, Barcelona, Spain, ${ }^{2}$ Hospital Sant Joan de Déu, Barcelona, Spain, ${ }^{3}$ Banc de Sang i Teixits, Barcelona, Spain, ${ }^{4}$ University of Barcelona, Barcelona, Spain

Background: The prognosis of relapsed/refractory acute lymphoid leukemia (ALL), non Hodgkin lymphoma (NHL) or chronic lymphocytic leukemia (CLL) is very poor, particularly in patients relapsing after autologous (autoHCT) or allogeneic hematopoietic cell transplantation (alloHCT). In the last decade, several chimeric antigen receptor anti-CD19 (CAR19) constructs have been developed. Two of them (tisa-cel and axi-cel) are already approved by the FDA and EMA for ALL and NHL. However, the availability and affordability of these commercial CARTs remains a challenge in Europe.

Methods: The first academic pilot clinical trial (clinicaltrials.gov NCT03144583) using our fully academic (A3B1:CD8:4-1BB:CD3Z) CAR19 was approved by the Spanish Agency of Medicines on May/2017. Eligibility criteria included R/R ALL (adult and pediatric), NHL and CLL who had failed standard available therapy. The primary objective of the study was safety, and secondary objectives were response rate and its duration (progressionfree survival). Here we report an updated analysis of this trial.

Results: As of December/2018 we have included 35 patients in the study. Of these, we performed lymphoapheresis to 34 and we processed the cells of 33, although for the moment we have only infused to 25 of them. The diagnoses of these 25 patients were ALL in 20 (13 adults), NHL in 4 and CLL in 1. Median age was 23 (3-54) years and $40 \%$ were women. Of the 20 patients with ALL, 17 had relapsed after alloHCT, while 3/4 patients with NHL had relapsed after autoHCT. After conditioning with fludarabine $(90 \mathrm{mg} /$ $\mathrm{m}^{2}$ ) and cyclophosphamide $\left(900 \mathrm{mg} / \mathrm{m}^{2}\right)$, we infused $0.4-5$ $\mathrm{x} 10^{6}$ ARI-0001 cells $/ \mathrm{kg}$, first as a single infusion (first 19 patients, cohort 1), and then in 2-3 fractions (last 6 patients, cohort 2). We have observed $5(20 \%)$ cases of severe cytokine release syndrome (CRS) and the non-relapse mortality (NRM) was $12 \%(3 / 25)$. These deaths were due to CRS (2) and pseudomembranous colitis (1), and all happened in cohort 1 . We had no cases of severe neurotoxicity, and grade II neurotoxicity was only seen in 2 patients. Of the 14 patients with active ALL at inclusion, 11 had enough follow-up for efficacy analysis, and all of them achieved a CR ( 9 of them with negative MRD). With a median follow-up of 8.7 months (range, 1.3-14.8), relapses were observed on days $+223(\mathrm{CD} 19+),+105$ and +284 (CD19-), leading to a progression-free survival of $53 \%$ at 12 months. In patients with NHL/CLL we have documented $\mathrm{CR}$ in 2, one of them (NHL) relapsing at day +259 . One NHL patient has not been evaluated yet and the other 2 patients did not respond and have died due to their lymphoma. The CLL patient, refractory to 5 lines of treatment, achieved a CR, which is maintained at day +377 .

Conclusions: Treatment with ARI-0001 cells is effective with a response rate of $78.5 \%$ in ALL (PFS of $53 \%$ at 1y) and $50 \%$ in NHL/CLL. We also observed cases of severe CRS in $20 \%$ and a NRM of $12 \%$ of patients, though dose fractioning seems to improve safety profile. Still, longer median follow-up and further patient inclusions are needed.

Clinical Trial Registry: Phase I, CART19-BE-01 clinical trial (EudraCT: 2016-002972-29, NCT03144583) for relapsed/refractory CD19+ leukemia and lymphoma at the Hospital Clínic de Barcelona and Hospital Sant Joan de Déu, Barcelona (Spain).

Disclosure: Source of funding: Proyecto ARI, Fundació Gloria Soler, ISCIII, CatSalut.

\section{O041}

Abstract already published.

\section{2}

High expansion level and long term persistence of BCMA CAR-T cells contribute to potent anti-tumor activity against heavily treated multiple myeloma patients

Yongxian $\mathrm{Hu}^{1}$, Yanlei Zhang ${ }^{2}$, Guoqing Wei', Huijun $\mathbf{X u}^{1}$, Wenjun $\mathrm{Wu}^{1}$, Shaohui Liu ${ }^{2}$, Jiazhen Cui', Alex Hongsheng Chang', He Huang ${ }^{1}$

${ }^{1}$ Bone Marrow Transplantation Center, The First Affiliated Hospital, Zhejiang University, Hangzhou, China, ${ }^{2}$ Shanghai YaKe Biotechnology Ltd, Shanghai, China 
Background: BCMA CAR-T cells have demonstrated substantial preclinical and clinical activity for relapsed/ refractory multiple myeloma (RRMM) patients. In different clinical trials, the overall response rate (ORR) varied from $50 \%$ to $100 \%$. Complete remission (CR) rate varied from $7 \%$ to $60 \%$. Previous studies indicated higher CAR-T cell expansion in vivo achieved better remission. Here we developed a BCMA CAR-T cell product manufactured via lentiviral vector-mediated transduction of activated $\mathrm{T}$ cells to express a second-generation CAR with 4-1BB costimulatory domain.

Methods: Our trial (ChiCTR1800017404) was initiated to evaluate the safety and efficacy of autologous BCMA CAR-T treatment for RRMM. The enrolled RRMM patients either had received at least 3 prior treatment regimens, including a proteasome inhibitor, an immunomodulatory agent, anti-CD38 monoclonal antibody or were double or triple-refractory, and have over 30\% BCMA expression. Patients were subjected to a lymphodepleting regimen with Flu daily for 3 days $\left(30 \mathrm{mg} / \mathrm{m}^{2}\right.$, D4 to D2) and Cy (500mg/ $\mathrm{m}^{2}, \mathrm{D}-3$ to D-2) prior to the CAR-T cell infusion (D0) at a dose range of $1-5 \times 10^{6} \mathrm{CAR}+$ cells $/ \mathrm{kg}$.

Results: As of the data cut-off date (Nov 28th, 2018), 17 patients, median age 61.8 (49 to 75 ) years old, with a median of 4.9 (0.4 to 10.8) years since MM diagnosis, were infused with BCMA CAR-T cells. Patients had a median of 14 prior regimens (range 4 to 24) (Figure 1A). All the 17 patients were eligible for initial evaluation of early clinical response with a median observation period of 12 ( 0.9 to 40) weeks. ORR was $100 \%$. All the patients achieved MRD negative in bone marrow by flow cytometry within 1-2 weeks after CAR-T infusion. Partial response (1 PR, 5.9\%), very good PR (6 VGPR, 35.3\%), and complete response (10 $\mathrm{CR}, 58.9 \%$ ) within 12 weeks post CAR-T infusion were achieved. Durable responses from 4 weeks towards the data cut-off date were found in $15 / 17$ patients $(88.2 \%)$. The disease progressed in 1 patient from PR by week 5 (Figure 1B). One patient died of severe infection by Day 9. All patients had detectable CAR-T expansion by flow cytometry from Day 3 post CAR-T cell infusion. Expansion peaks were found between Day 6 to Day 17. The peak CAR-T cell expansion in $\mathrm{CD} 3+$ lymphocytes of peripheral blood (PB) varied from $35 \%$ to $95 \%$ with a median percentage of $83.5 \%$ (Figure 1C). The peak absolute number of CAR-T cells in PB varied from 530 to 23000 per $\mu \mathrm{L}$ with a median number of 10300 per $\mu \mathrm{L}$. Cytokine release syndrome (CRS) was reported in all the 17 patients, including 2 with Grade 1 , 4 with Grade 2 and 11 with Grade 3. CAR-T -related AEs were 9 pancytopenia (52.9\%), 14 fever $(82.3 \%), 8$ nausea (47.1\%), 1 heart failure (5.8\%). No patient died of CRS complication.

Conclusions: Our data showed BCMA CAR-T treatment is safe with prominent efficacy. We also observed high expansion level and long term persistence of BCMA CAR$\mathrm{T}$ cells contribute to potent anti-myeloma activity. These initial data provide strong evidence to support the further development of this anti-myeloma cellular immunotherapy.

Clinical Trial Registry: ChiCTR1800017404

Disclosure: Nothing to declare

\section{3}

Chimeric antigen receptor (CAR)-mediated BCL11B suppression in lymphoid progenitor cells propagates natural killer-like cell development

\section{Marcel Maluski, Arnab Ghosh ${ }^{2}$, Jessica Herbst ${ }^{3}$, Vanessa Scholl, Rolf Baumann, Robert Geffers, Johann Meyer ${ }^{6}$, Andreas Krueger ${ }^{7}$, Axel Schambach, Marcel van den Brink ${ }^{2}$, Martin Sauer ${ }^{3}$}

${ }^{1}$ Medizinische Hochschule Hannover, Pediatric Hematology Oncology, Hannover, Germany, ${ }^{2}$ Memorial Sloan Kettering Cancer Center, New York, NY, United States, ${ }^{3}$ Medizinische Hochschule Hannover, Pediatric Hematology and Oncology, Hannover, Germany, ${ }^{4}$ Clinic for Radiation Oncology, Hannover, Germany, ${ }^{5}$ Genome Analytics, Helmholtz Centre for Infection Research, Braunschweig, Germany, ${ }^{6}$ Medizinische Hochschule Hannover, Experimental Hematology, Hannover, Germany, ${ }^{7}$ Goethe University Frankfurt, Frankfurt, Germany

Background: The transcription factor B Cell CLL/Lymphoma 11B (Bcl11b) is indispensable for $\mathrm{T}$ lineage development of lymphoid progenitors. Pre-fabricated $\mathrm{T}$ cell products, allowing for a wider choice of effectively targetable antigens, being applicable to a wider range of patients, and minimizing the risk of long-term sequalae from on target/off tumor effects would be highly desirable. Here we hypothesized that antigen receptor engineering of hematopoietic stem cells would fundamentally impact lymphoid progenitor cell fate and as a consequence biological properties thereby allowing to generate an ubiquitously available lymphoid cell product for targeted immunotherapy across MHC barriers.

Methods: Murine hematopoietic stem cells were transduced with a broad panel of $1^{\text {st }}$ and $2^{\text {nd }}$ generation murine CARs containing different costimulatory domains and numbers of active immune receptor-based activation motifs (ITAMs) against CD19 in a lentiviral backbone and consecutively differentiated into lymphoid progenitors using the OP9-DL1 feeder layer system. Resulting products were comparatively assessed in vitro and in vivo (FACS, functional assays, microarray gene transcript analysis, western blotting, timely controlled transgene expression, leukemia challenge experiments, in vivo lymphoid depletion experiments) upon co-transplantation into a MHC- 
mismatched myeloablative transplantation model for relapsed leukemia.

Results: CAR expression on early ex vivo generated lymphoid progenitors suppresses Bcl11b (Figure 1B) and leads to decreased $\mathrm{T}$ cell-associated gene expression. Concomitantly, suppressed Bcl11b permits lymphoid progenitors to acquire NK cell-like properties (Figure 1A) and upon adoptive transfer into hematopoietic stem cell transplant recipients they differentiate into CARinduced killer cells (CARiK) that mediate potent antigendirected antileukemic activity across MHC barriers (Figure 1C,D). A CD28, but not 4-1BB, costimulatory domain and active ITAMs are critical for a functional CARiK phenotype.

Conclusions: These results give important insights into differentiation of lymphoid progenitors driven by synthetic CAR transgene-expression and inform the potential use of ex vivo generated CARiK as an "off-the-shelf" product for targeted immunotherapy.

Disclosure: M.v.d.B has IP Licensing with Seres Therapeutics and Juno Therapeutics. M.v.d.B. has also received honorariums from Flagship Ventures, Novartis, Evelo, Seres, Jazz Pharmaceuticals, Therakos, Amgen, Merck \& Co, Inc., Acute Leukemia Forum (ALF), and DKMS Medical Council (Board) and research support and has stock options with Seres Therapeutics. A.G. has received research support from Aprea therapeutics and Infinity therapeutics. All remaining authors have no conflict of interest.

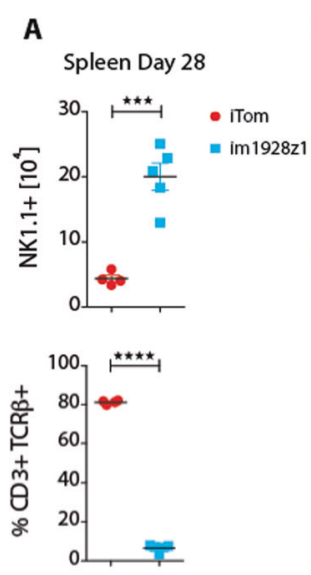

B
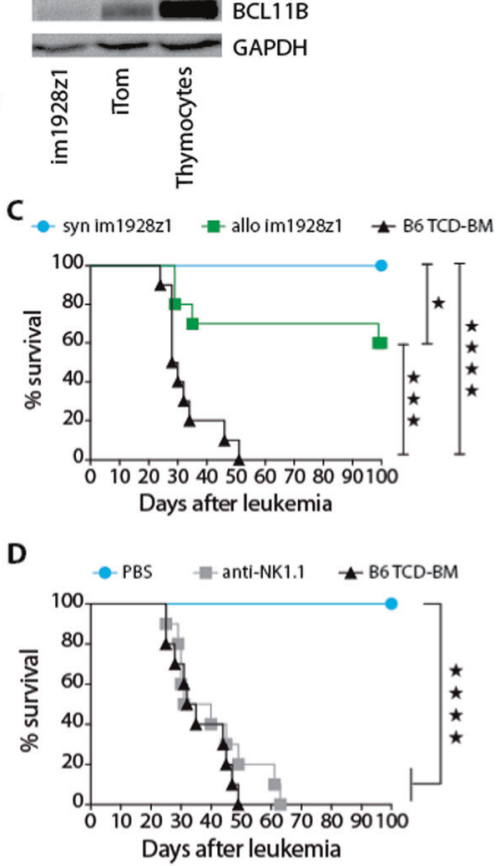

[[O043 Image] 1. Figure 1: CAR-induced killer (CARiK) cells provide strong anti-leukemia effects in vivo.]
(A) C57BL/6 (B6) recipients received $3 \times 10^{6}$ B6 TCDBM only or additionally with $8 \times 10^{6}$ syngeneic im1928z1lymphoid progenitors. Numbers of NK $1.1^{+} \mathrm{CARiK}$ cells and frequencies of $\mathrm{CD}^{+}{ }^{+} \mathrm{TCR} \beta^{+}$progeny within the Tom + gate in spleens on day 28 (im1928z1, n= 5; iTom, $n=4)$. Statistics was performed by using Students t-test (twotailed). Data represent means \pm s.e.m. (B) Western blot analysis for BCL11B in lysates from iTom lymphoid progenitors, im1928z1-lymphoid progenitors or B6 WT thymocytes. (C) Recipients co-transplanted with either syngeneic (syn) or MHC class I and II mismatched (allo) im1928z1-expressing lymphoid progenitors $(n=10$ mice, respectively) received $1.2 \times 10^{6}$ C1498-mCD19 leukemic cells on day 21 after transplantation and monitored for survival. (D) CAR-lymphoid progenitor-co-transplanted mice were treated with weekly i.p. injections of either an

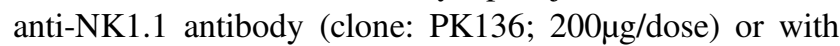
PBS for control ( $\mathrm{n}=10$ per group). All mice received $1.2 \mathrm{x}$ $10^{6}$ C1498-mCD19 cells on day 21 after transplantation and were monitored for survival. Survival curves were compared using Mantel-Cox (log-rank) test. Significant differences are indicated by $* \mathrm{P}<0.05, * * * \mathrm{P}<0.001$, **** $\mathrm{P}<$ 0.0001 .

\section{O044}

Characterization of an HLA-DPB1 specific T-cell receptor for adoptive immunotherapy

Sebastian Klobuch ${ }^{1}$, Elisabeth Neidlinger ${ }^{1}$, Carina Mirbeth $^{1}$, Wolfgang Herr ${ }^{1,2}$, Simone Thomas ${ }^{1,2}$

${ }^{1}$ University Hospital Regensburg, Regensburg, Germany, ${ }^{2}$ University of Regensburg, Regensburg Center for Interventional Immunology, Regensburg, Germany

Background: HLA-DPB1 mismatches occur in up to $80 \%$ of allogeneic hematopoietic stem cell transplantations from HLA 10/10 matched donors and were shown to be associated with a decreased risk of leukemia relapse. Therefore, targeting HLA-DPB1 mismatched antigens by donor T cells seems to be an attractive strategy to enhance graft-versusleukemia effect. We recently established a reliable method to generate and isolate HLA-DPB1 mismatch reactive $\mathrm{T}$ cells receptors (TCR). TCR-modified $\mathrm{T}$ cells showed leukemia eradication of primary human AML blasts in a xenogeneic NOD/SCID/IL2Rgc ${ }^{-/}$(NSG) mouse model. However, human fibroblasts used as surrogate cells for graft-versus-host $(\mathrm{GvH})$ reactivity were also recognized by HLA-DPB1-specific $\mathrm{T}$ cells upon IFN- $\gamma$ pretreatment, which up-regulates HLA-class II expression on these cells. In this project, we aim at the isolation of TCRs recognizing mismatched HLA-DPB1 only on hematopoietic cells, which might lower their risk for GvHD. 
Methods: Naive-enriched CD4 T cells were stimulated with autologous dendritic cells expressing allo-HLA-DPB1 alleles upon RNA transfection. To drive the outgrowth of CD4 T-cell populations expressing 'CD4-independent' TCR which allow the redirection of both CD4 and CD8 T cells, we blocked the CD4/HLA interactions by the addition of CD4 binding antibody. Allo-HLA-DPB1 reactive cultures were analyzed for their recognition of primary AML blasts in IFN- $\gamma$ ELISpot as well as ${ }^{51}$ Chromium-release assays. Highly reactive $\mathrm{CD} 4 \mathrm{~T}$ cell populations were further analyzed for their IFN- $\gamma$ secretion against nonhematopoietic cells. TCRs from most promising CD4 T cell clones with an HLA-DPB1 specific recognition of hematopoietic but not non-hematopoietic cells were isolated and further analyzed.

Results: Two CD4-independent $\mathrm{T}$ cell clones with reactivity to the HLA-DPB $1 * 03: 01$ mismatch allele specifically lysed HLA-DPB $1 * 03: 01^{+}$primary AML blasts. Most importantly, one of these $\mathrm{T}$ cell clones did not show IFN- $\gamma$ secretion upon co-culture with IFN- $\gamma$ pretreated primary fibroblasts. Therefore, we isolated this TCR and transferred it into CD4 and CD8 T cells from healthy donors. $\mathrm{TCR}_{\mathrm{DP} 03}$ re-directed $\mathrm{CD} 4$ and $\mathrm{CD} 8 \mathrm{~T}$ cells specifically recognized and lysed primary AML blasts from several HLA-DPB $1 * 03: 01^{+}$patients in vitro. Again, cells of non-hematopoietic origin (fibroblasts) were not recognized even after IFN- $\gamma$ pretreatment and HLA-DP upregulation.

To optimize TCR expression and activity, we exchanged the constant domains of the TCR by their murine counterparts. This modification not only led to a higher IFN- $\gamma$ production and lysis of AML blasts, but also induced recognition of IFN- $\gamma$ pretreated fibroblasts. However, tumor cell lines overexpressing HLA-DPB1*03:01 were also recognized by $\mathrm{CD} 4 \mathrm{~T}$ cells engineered with the wild-type or murinized $\mathrm{TCR}_{\mathrm{DP} 03}$, suggesting that recognition of hematopoietic and non-hematopoietic cells is rather triggered by the avidity between the $\mathrm{T}$ cell and the target cell than by the TCR target epitope.

Conclusions: In conclusion, our data suggest that alloHLA-DPB1 specific TCRs are powerful therapeutic off-theshelf reagents in allogeneic T-cell therapy of leukemia. The isolation of allo-HLA-DPB1 specific TCR without crossreactivity to non-hematopoietic cells might be one strategy to avoid HLA-DPB1 specific GvH reactivity upon inflammatory situations (e.g. viral infections). However, our data also indicate that finding of the most suitable TCR candidate is challenging.

Disclosure: Nothing to declare.

\section{5}

Abstract already published.

\section{6}

Infusion of memory $T$ cell (CD45RA-Depleted) DLI improves CMV-specific immune response early after ABT cell-depleted HSCT: First results of a prospective randomized trial

Zhanna Shekhovtsova ${ }^{1}$, Maria Dunaykina ${ }^{I}$, Larisa Shelikhova $^{I}$, Dmitry Balashov ${ }^{I}$, Elena Kurnikova ${ }^{1}$, Yakov Muzalevsky ${ }^{I}$, Alexey Kazachenok ${ }^{I}$, Elena Osipova ${ }^{I}$, Viktoria Kiseleval, Dmitry Pershin ${ }^{1}$, Daria Shasheleva ${ }^{1}$, Rimma Khismatullina ${ }^{l}$, Sergey Blagov ${ }^{1}$, Andrei Abrosimov $^{I}$, Irina Shipitsina ${ }^{I}$, Elena Gutovskaya ${ }^{I}$, Galina Novichkova $^{1}$, Alexei Maschan ${ }^{1}$, Michael Maschan ${ }^{I}$

${ }^{1}$ Dmitry Rogachev National Medical Research Center of Pediatric Hematology, Oncology and Immunology, Moscow, Russian Federation

Background: abT cell depletion effectively prevents severe GVHD in mismatched HSCT, but in a proportion of cases delayed immune recovery leads to increased infection risk and NRM. We've shown in a pilot study that infusion of low-dose memory $\mathrm{T}$ cells (CD45-RA depleted) is safe after engraftment among recipients of ab $\mathrm{T}$ cell-depleted grafts (PMID:29269793). We initiated a prospective trial to directly test the efficiency of this approach. We report here an interim result of a prospective, randomized, single-centre trial (NCT02942173).

Methods: A total of 100 paediatric patients with malignant disorders (ALL, $n=56$; AML, $n=30$; NHL, $n=8$; acute mixed lineage leukemia, $n=5$ and MPD, $n=1$ ) were enrolled between October 2016 and September 2018. Patients were randomly assigned to receive CD45RA-depleted DLIs (experimental arm), $\mathrm{n}=54$, or not (control arm), $\mathrm{n}=46$. Median age at HSCT was 8.9 years, m:f ratio - 42:58. The conditioning consisted of either treosulfan $(n=50)$ or TBI $(\mathrm{n}=50)$ in combination with fludarabine and thiotepa. GVHD prophylaxis included tocilizumab at $8 \mathrm{mg} / \mathrm{kg}$ at day 0 , abatacept at $10 \mathrm{mg} / \mathrm{kg}$ at day $0,+7,+14$ and +28 , and bortezomib at $1,3 \mathrm{mg} / \mathrm{m}^{2}$ at days $-5,-2,+2,+5$. Neither antithymocyte globulin nor calcineurin inhibitors were used. Donors were HLA-haploidentical $(n=94)$ or matched $(n=6)$. All donors and $76 \%$ of the recipients were CMV seropositive. PMBC grafts were split and TCR $\alpha \beta / C D 19$ depletion and CD45RA depletion were performed with CliniMACS Prodigy. The median dose of CD34+ cells was $10 \times 10^{6} / \mathrm{kg}$, $\alpha \beta T$ cells $-28 \times 10^{3} / \mathrm{kg}$. In the experimental arm memory DLIs were infused on day 0 at $25 \times 10^{3} / \mathrm{kg}$ and on days $+30,+60$, $+90,+120$ at $50 \times 10^{3} / \mathrm{kg}$. In the control arm 8 patients received DLI after engraftment to prevent relapse $(n=6)$ or treat infections $(n=2)$. Primary endpoints were the cumulative incidence (CI) of CMV viremia ( $>500$ copies $/ \mathrm{ml}$ ) by day +100 and the CI of acute GvHD grade $\geq$ II. 
Results: Median follow-up for survivors was 1 year $(0,2-$ 2). Engraftment of WBC and platelets was achieved in 99 pts, one patient died at day +8 . WBC and platelets engrafted at a median of 11 days and 14 days, respectively. The incidence of CMV viremia was $45 \%$ (36-56) overall, $41 \%(30-56)$ in the experimental arm vs. $50 \%(38-67)$ in the control arm, $\mathrm{p}=\mathrm{ns}$. The $\mathrm{CI}$ of $\mathrm{aGvHD} \geq$ grade II was $10 \%$ (6-18) overall, $10 \%$ (4-23) in the experimental arm vs. $9 \%$ (4-24) in the control arm, $\mathrm{p}=\mathrm{ns}$. Two patients died, one per treatment arm, resulting in 2\% (0-14) CI of TRM at 1 year among the whole cohort. Causes of death were preengraftment bloodstream infection and disseminated adenovirus infection. Patients randomized to experimental arm acquired anti-CMV reactivity significantly earlier, according to IFN-g ELISPOT assay on day +30 after HSCT $(\mathrm{p}=0,0001)$.

Conclusions: Co-infusion of donor-derived memory DLI with the $\alpha \beta \mathrm{T}$ cell-depleted graft is safe and improves recovery of virus-specific immune responses. Replacement of ATG with targeted blockade of CD28/CD80 costimulation and IL-6 receptor does not compromise engraftment and GVHD control, and is associated with low rate of non-relapse mortality.

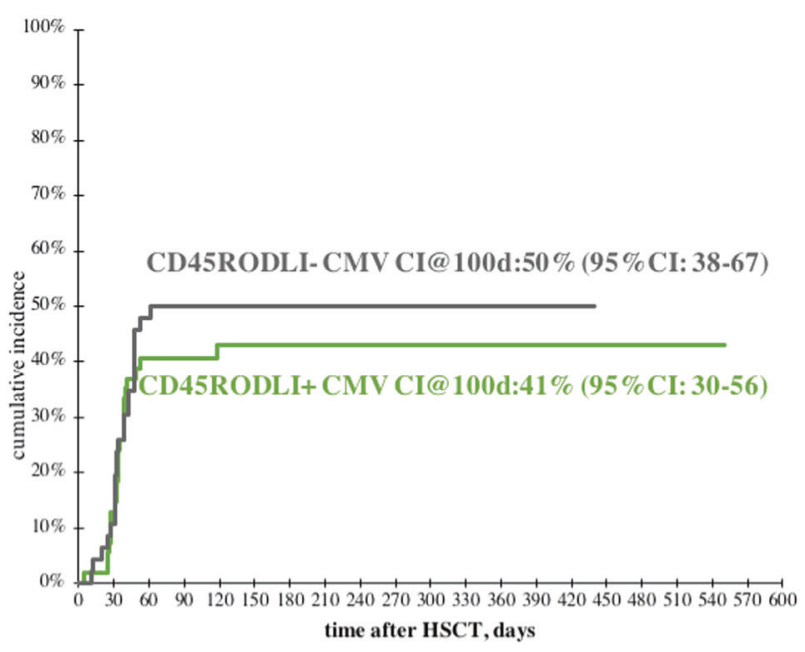

[[O046 Image] 1. Incidence of CMV viremia]

Disclosure: Nothing to declare

\section{O047}

conditioning prior to CD19-specific CAR (19-28Z) $T$ cells predicts response and survival in pediatric relapse/refractory $(\mathrm{R} / \mathrm{R}) \mathrm{B}-\mathrm{ALL}$

Kevin Curran', Steven Margossian', Nancy Kernan', Susan Prockop ${ }^{1}$, Farid Boulad ${ }^{1}$, Maria Cancio ${ }^{1}$, Victoria Szenes $^{1}$, Andrew Kung ${ }^{1}$, Jaap Jan Boelens ${ }^{1}$, Richard
O'Reilly', Isabelle Riviere $^{3}$, Michel Sadelain ${ }^{3}$, Renier Brentjens $^{3}$

${ }^{1}$ Memorial Sloan Kettering Cancer Center, Pediatrics, New York, NY, United States, ${ }^{2}$ Dana-Farber Cancer Institute, Boston, MA, United States, ${ }^{3}$ Memorial Sloan Kettering Cancer Center, New York, NY, United States

Background: CD19-specific CAR T cells have demonstrated clinical benefit in patients with $\mathrm{R} / \mathrm{R}$ B-cell acute lymphoblastic leukemia (B-ALL). Several factors have been associated with response including conditioning chemotherapy, CD4/8 ratio, and post infusion CAR $\mathrm{T}$ cell expansion.

Methods: We studied the feasibility of a multi-center trial of a MSK-developed CD19-specific CAR (19-28z) for the treatment of R/R B-ALL, the toxicity following infusion, and performed predictor analysis for optimal response. Pediatric and young adult patients with R/R B-ALL were eligible for infusion. Patients received a cyclophosphamidebased (Cy) conditioning of high dose (HD; 3g/m2) or low dose (LD; $1.5 \mathrm{~g} / \mathrm{m} 2)$ chemotherapy. Outcomes of interest were complete response (CR), overall survival (OS). Variables considered were conditioning regimen (HD-Cy vs LD-Cy), pre-treatment disease burden (MRD vs morphologic), complete remission (CR) status, absolute lymphocyte count (ALC) change, and in vivo CAR $\mathrm{T}$ cell expansion.

Results: 25 patients were included; 17 patients received HD-Cy and 8 received LD-Cy prior to CAR T cell infusion. Among evaluable patients $(n=24), C R$ or $C R$ with incomplete count recovery was demonstrated in $94 \%$ and $38 \%$ for HD-Cy vs LD-Cy cohorts respectively $(\mathrm{p}=0 \cdot 01)$. OS was superior in the HD-Cy cohort as compared to the LD-Cy cohort (median OS = not reached; NR) vs. 4.7 months ( $\mathrm{p}=0.004$; Figure 1). Lymphodepletion (Delta ALC: prior to and following Cy) was significantly higher in the HD-Cy cohort as compared to the LD-Cy cohort $(\mathrm{p}<$ 0.001; Figure 2). The in vivo CAR T cell expansion (peak CAR T cell vector copy number/ml) in peripheral blood was higher in the HD-Cy cohort as compared to the LD-Cy cohort $(p=0 \cdot 01$; Figure 2$)$. To less extent, disease burden prior to treatment with conditioning chemotherapy and CAR T cells impacted response. Disease response was 93\% $(13 / 14)$ in low disease burden group (MRD-cohort) compared to $50 \%(5 / 10)$ in the high disease burden group (morphologic cohort) $(\mathrm{p}=0 \cdot 05)$. OS was also superior in the low disease burden group (median OS $=\mathrm{NR}$ ) compared to high disease burden group (median $\mathrm{OS}=4.3$ months; $\mathrm{p}=0 \cdot 01$ ). Combined response for HD-Cy/MRD was $100 \%$ (12/12), HD-Cy/Morphologic 75\% (3/4), LD-Cy/MRD $50 \%$ (1/2), and LD-Cy/Morphologic 33\% (2/6). Grade III/ IV toxicity occurred in $32 \%(8 / 25)$ of patients including 
severe cytokine release syndrome (sCRS) in $16 \%$ of patients and severe CAR-associated neurotoxicity in $28 \%$ of patients.

Conclusions: In this preliminary analysis we demonstrate that dose intensity of conditioning chemotherapy positively correlated with response and overall survival for patients treated with CAR T cells and confirms, to a lesser extent, pre-treatment disease burden impacts both response and overall survival.

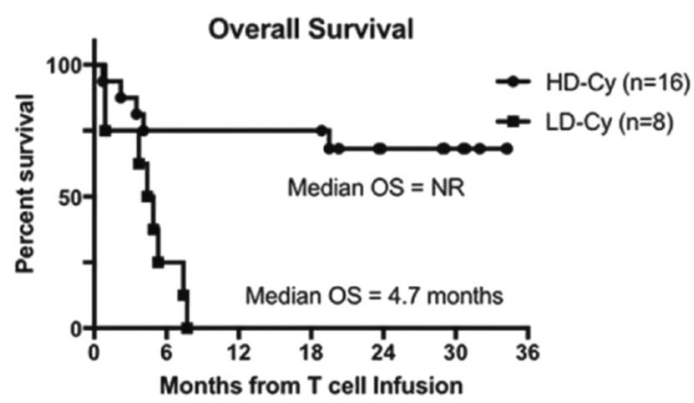

Figure 1: OS following CD19-specific CAR T cells based on dose intensity of conditioning chemotherapy. Eighty-three percent (15/18) of responding patients underwent consolidation with allo-HSCT.
A.

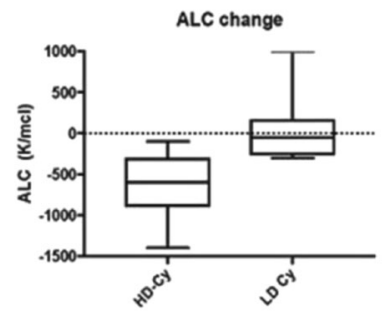

B.

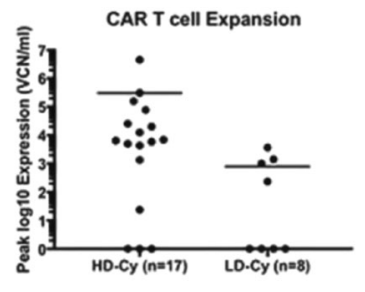

Figure 2: (A) ALC prior to and following cyclophosphamide (ALC change) was available for twenty-three $(n=23)$ patients and was significantly greater in the HD-Cy cohort ( $p<0.001$; Figure 2). (B) Differences in the in vivo CAR T cell expansion (peak CAR T cell vector copy number/ml) in peripheral blood was also significantly higher in the HD-Cy cohort $(p=0.01)$

\section{[[O047 Image] 1. Figure1_2]}

\section{Clinical Trial Registry: NCT01860937}

Disclosure: The authors acknowledge William Lawrence and Blanche Hughes Foundation provided funding for the conduct of this study with Juno Therapeutics providing funding for an extension cohort of patients. K.J.C. has received research support from Juno Therapeutics; has consulted, participated in advisory boards, or participated in educational seminars for Juno Therapeutics, and Novartis. R. J.B. M.S. I.R. are co-founder, stock holders, and consultants for Juno Therapeutics. C.S.S. has received research support from Juno Therapeutics; has consulted or participated in advisory boards for Juno Therapeutics, Kite and Novartis.

\section{8}

Early and late hematologic toxicities in children and adults treated with CD19-CAR T cells
Elad Jacoby ${ }^{1,2}$, Shalev Fried ${ }^{1,2}$, Abraham Avigdor ${ }^{1,2}$, Bella Bielorai $^{1,2}$, Amilia Meir ${ }^{1}$, Michal Besser ${ }^{1,2}$, Jacob Schachter $^{1,2}$, Avichai Shimoni ${ }^{1,2}$, Arnon Nagler ${ }^{1,2}$, Amos Toren $^{1,2}$

${ }^{1}$ Sheba Medical Center, Ramat Gan, Israel, ${ }^{2}$ Tel Aviv University, Tel Aviv, Israel

Background: Autologous T cells transduced with CD19directed chimeric antigen receptors show notable remission rate in advanced patients, leading to approval by the FDA and EMA for treatment of relapsed and refractory acute lymphoblastic leukemia (ALL) and non-Hodgkin lymphoma (NHL). The most common adverse events reported are cytokine-release syndrome (CRS) and neurotoxicity. Here we study and profile of hematologic toxicity of patients following locally produced CAR $\mathrm{T}$ cells.

Methods: We studied the first 38 patients treated on a phase 1b/2 clinical trial using CD19 CAR-T cells for B-cell malignancies, focusing on hematologic toxicities (neutropenia, thrombocytopenia and anemia), from the initial lymphodepleting regimen till progression or an additional treatment was administered. Cytokine levels were studied using Milliplex Map, human cytokine/chemokine panel II.

Results: Between July 2016 and March 2018, 38 patients were enrolled on the trial, and 35 patients who received CAR-T cells and survived more than 21 days were included in this analysis. Neutropenia, thrombocytopenia and anemia occurred frequently $(94 \%, 80 \%$ and $51 \%$, respectively) after CAR T cell infusion, and were associated with a prolonged or biphasic nature: In $93 \%$ of patients hematologic toxicity occurred or were ongoing after 21 days from cell infusion, and in 52\% (neutropenia) and 44\% (thrombocytopenia), two trough levels were noted, the second trough occurring after day +21 . Later events of cytopenia, following more than 42 days from CAR infusion and in absence of further therapy, occurred in 62\% (neutropenia), 44\% (thrombocytopenia) and $17 \%$ (anemia requiring PRBC transfusion) of patients.

We identified a strong correlation between the late hematologic toxicities (thrombocytopenia and neutropenia, $\mathrm{p}=0.018$, thrombocytopenia and anemia, $\mathrm{p}<0.0001$, anemia and neutropenia $\mathrm{p}=0.05$ ).

On univariate analysis, factors affecting late cytopenia were prior HSCT $(p=0.0015,0.0083$ and 0.02 for anemia, thrombocytopenia and neutropenia respectively) and higher CRS grade $\mathrm{p}=0.003,0.018$ and 0.04 for late anemia, thrombocytopenia and anemia respectively). Diagnosis (ALL vs NHL) or age were not correlated to the incidence of early or late post-CAR cytopenia. To further study potential causes of late events in patients who were in remission, and in absence of signs of hemophagocytosis, patients' serum was analyzed for chemokine panel at 
different time points. As expected, serum thrombopoietin levels were correlated with the platelet count. We observed that only in late events (more than 21 days from infusion) SDF-1 serum levels correlated to neutrophil count $\left(\mathrm{R}^{2}=0.3\right.$, $\mathrm{p}=0.04)$.

Conclusions: Cytopenia are common events following CD19 CAR T cell therapy, and may have a prolonged and even biphasic course. Patients at risk include those following a recent HSCT or with high grade CRS. Late neutropenia events which occurred later than expected recovery from conditioning chemotherapy and following resolution of CRS, were correlated with serum SDF1 levels, similar to prior observations with late onset neutropenia related to rituximab (Dunleavy, Blood 2005). As CAR T cell products are entering standard of care for relapsed patients, detection and proper management of hematologic toxicities is warranted.

Clinical Trial Registry: clinicaltrials.gov NCT02772198

Disclosure: Elad Jacoby - Advisory board, Novartis

\section{9}

Natural killer cells infusion as consolidative strategy in AML pediatric patients

Luisa Sisinni', José Luis Fuster², Antonia Pascual ${ }^{3}$, José Manuel Vagace ${ }^{4}$, María Vela ${ }^{1}$, Berta González', David Bueno $^{1}$, Yasmina Mozo ${ }^{1}$, Blanca Rosich ${ }^{1}$, Jaime Valentín', Mario Muñoz', Antonio Balas', Isabel Mirones ${ }^{I}$, Lucia Fernández, ${ }^{5}$ Antonio Pérez-Martínez ${ }^{1}$

${ }^{1}$ Hospital Universitario La Paz, Madrid, Spain, ${ }^{2}$ Hospital Universitario Virgen de la Arrixaca, Murcia, Spain, ${ }^{3} \mathrm{Hos}-$ pital Pediatrico, Malaga, Spain, ${ }^{4}$ Badajoz Hospital, Badajoz, Spain, ${ }^{5}$ Spanish National Cancer Research Centre (CNIO), Madrid, Spain

Background: Acute myeloblastic leukemia (AML) represents $40 \%$ of all leukemias of western countries, being the second malignant hemopathy in pediatric population. In the last decades, the survival rate has maintained around $60 \%$, being relapse the main problem. It has been highlighted the role of immune system for the control of AML and new therapeutic strategies have been developed. In this setting, the use of alloreactive Natural Killer (NK) cells could play a key role not only in the context of hematopoietic stem cell transplantation (HSCT) but also as adoptive immunotherapy in patients with minimal residual disease. In this project we proposed including the cellular therapy with haploidentical activated and expanded NK cells as adjuvant therapy in pediatric patients affected by AML in complete remission and without indication of HSCT.

Methods: It is a multicentre, open, prospective and no randomized phase II clinical trial, to evaluate the efficacy and safety of allogenic NK cells infusion from haploidentical donor after a lympho- ablative chemotherapy in pediatric patients affected by low and intermediate risk AML in first complete cytological remission. Patients were treated according to Spanish protocol (SEHOP 2002) including 2 inductions plus 2 consolidation cycles. After chemotherapy patients received the infusion of activated and expanded NK cells (NKAE) after a lympho-ablative treatment based on cyclophosphamide $60 \mathrm{mg} / \mathrm{Kg}$ and fludarabine $125 \mathrm{mg} / \mathrm{Kg}$. NK cells were obtained from 250 $\mathrm{ml}$ of peripheral blood from haploidentical donors selected based on alloreactivity of KIR inhibitors and KIR activators receptors. NK cells were activated and expanded for 3 weeks trough co-culture with the cellular line K562mbil15-41bbl suitable for clinical use in humans (Good Manufacturing Practices, GMP).

Results: At this time 14 products in 7 patients have been infused, with the following characteristics: NK cells (CD45 $\left.{ }^{+} \mathrm{CD}^{+}{ }^{+} \mathrm{CD}^{-}\right) 86.64 \pm 11.36 \%$; $\mathrm{T}$ lymphocytes $\left(\mathrm{CD} 45^{+}\right.$ $\mathrm{CD} 56^{-} \mathrm{CD}^{+}$) $2.94 \pm 3.55 \%$. One product was rejected for quality criteria. A mean of $51.14 \pm 53.99 \times 10^{6} \mathrm{NK}$ cells $/ \mathrm{kg}$ and $0.69 \pm 0.35 \times 10^{6} \mathrm{~T}$ cells $/ \mathrm{kg}$ were infused. Median age of the patients were 7 years (1-16). Treatment and infusions were well tolerated. The main adverse event was neutropenic fever in one patient. After a median follow up of 530 days (371-813) all the 7 patients are alive. Only one patient showed relapse at day +307 after NK cells infusion and was submitted to an HSCT from a matched unrelated donor.

Conclusions: The preliminary results of this trial suggest that NK cells infusion as consolidative strategy in pediatric patients with AML, is feasible and secure. The therapeutic effect should be confirmed in a larger cohort of patients.
Clinical
Trial
Registry:
Clinicaltrials.gov

(NCT02763475) and EudraCT (2015-001901-15).

Disclosure: Authors have no conflict of interest.

The trial was funded by "Fundación Mutua Madrileña" Grant

\section{0}

TCR $\alpha \beta / C D 19$ depleted and IL-15 stimulated donor cell infusions can exert anti-tumor effects after allosct without inducing GVHD in vitro and in vivo

Anne-Marie Lang ${ }^{1}$, Patrick Hundsdörfer ${ }^{2}$, Florian Heubach $^{1}$, Emmanuelle Moraes Ribeiro', Armin Rabsteyn ${ }^{I}$, Annette Künkele ${ }^{2}$, Patrick Schlegel, Christian Seitz ${ }^{1}$, Michaela Döring ${ }^{1}$, Michael Schumm ${ }^{1}$, Angelika Eggert ${ }^{2}$, Rupert Handgretinger ${ }^{1}$, Peter Lang ${ }^{1}$, Johannes Schulte ${ }^{2}$

${ }^{1}$ University Children's Hospital Tuebingen, Tuebingen, Germany, ${ }^{2}$ Charité University Medicine Berlin, Berlin, Germany 
Background: Natural killer (NK) cells and $\gamma \delta$ T-cells have been shown to play a significant role in GvL effects after allogeneic stem cell transplantation (SCT). Both cell types are not restricted by MHC molecules which makes them unlikely to elicit graft versus host disease (GvHD) even in mismatched SCT and therefore are suited also for cell-based posttransplant immunotherapy. Incubation with cytokines strongly enhances their spontaneous and antibody dependent cellular cytotoxicity (ADCC).

Methods: Thus, we investigated the efficacy of a combination of IL-15 stimulated NK- and $\gamma \delta$ T-cells from mismatched donors in vitro and based on urgent medical need in ultra high risk patients.

Results: In vitro data: Spontaneous cytotoxic activity and ADCC with TCR $\alpha \beta / C D 19$ depleted PBMCs from healthy donors against LS (neuroblastoma) and Nalm-6 (leukemia) target cells after overnight stimulation with IL-15 was significantly increased compared to non-stimulated cells (E: T ratio 20:1; LS cells: $43 \%$ and $61 \%$ vs $19 \%$; Nalm- 6 cells: $44 \%$ and $68 \%$ vs $26 \%$ ). The results for CD107a assays are in line with the cytotoxicity approaches. NK and $\gamma \delta \mathrm{T}$-cells showed a significantly stronger stimulation after IL-15 incubation $+/$ - ADCC, as measured by secreting cytokines (CD107a, INF $\gamma$, TNF $\alpha$ ).

Moreover, 7 patients at extremely high risk for relapse received NK-/ $\gamma \delta$ T-cell infusions after previous transplantation from haploidentical $(n=5)$ or matched donors $(n=2)$; Diagnoses: T-ALL, relapse after $1^{\text {st }} \mathrm{SCT} n=1$; B-lineageALL, CR after $2^{\text {nd }} \mathrm{SCT}, \mathrm{n}=3$; AML, relapse after $1^{\text {st }} \mathrm{SCT}$, $\mathrm{n}=1$; relapsed neuroblastoma, PR after $2^{\text {nd }} \mathrm{SCT}, \mathrm{n}=1$.

For that purpose magnetic microbeads and the CliniMACS $^{\oplus}$ device were used for TCR $\alpha \beta / C D 19$ depletion of leukapheresis products from the original donors. Afterwards, remaining cells (NK, $\gamma \delta \mathrm{T}$ and myeloic cells) were stimulated with IL-15 $(10 \mathrm{ng} / \mathrm{ml})$ overnight. Efficacy of the stimulation procedure was measured in 3 leukapheresis products: after incubation with target cells (LS) both, NKcells as well as $\gamma \delta$ T-cells showed a significant increase in CD107a secretion compared to non-stimulated effectors $(\mathrm{n}=4)$ from healthy donors (NK-cells: $75 \%$ vs $12 \% ; \gamma \delta \mathrm{T}-$ cells: $44 \%$ vs $2 \%$ ). A median number of $12.4 \times 10^{6}$ NK-cells and $1.66 \times 10^{6} \gamma \delta$ T-cells and $1.08 \times 10^{3}$ residual TCR $\alpha \beta$ cells/ $\mathrm{kg}$ were infused. Four patients had received therapeutic mAbs (anti-GD2, anti-CD19 or anti-CD38) within 48 hours after cell infusion to induce ADCC.

Side effects were treatable cytokine release syndrome in 3 patients; no GvHD occurred. Infused cells could be tracked by CD69 expression for up to 26 days in peripheral blood.

Outcome: 2 patients with T-ALL and neuroblastoma responded and achieved another CR, 2 patients maintained their previous $\mathrm{CR}$ for up to now 55/222 days; 3 patients progressed/relapsed after 26/69/89 days. Additional therapies were applied in $4 / 7$ patients. 4 patients are alive (median follow up: 248 days after infusion), 2/4 are disease free.

Conclusions: Infusion of a combination of NK/ $\gamma \delta \mathrm{T}$ cells can increase anti-tumor effects and ADCC with appropriate therapeutic mAbs without inducing GvHD even after mismatched SCT. Further investigations are needed to evaluate the role of this cellular therapy.

Disclosure: Nothing to declare

\section{1}

\section{Abstract already published.}

\section{2}

Identifying chimeric antigen receptor (CAR) centers and CAR activity in Europe, a survey on behalf of the cellular therapy \& immunobiology working-party (CTIWP) of EBMT

Alvaro Urbano ${ }^{1}$, Mickael Hudecek ${ }^{2}$, Francesco Dazzi, Rose Ellard ${ }^{4}$, Katharina Fleischhauer ${ }^{2}$, Ulrike Koeht, Jurgen Kuball, Franco Locatelli ${ }^{7}$, Florent Malard, Paolo Pedrazzoli, Luca Vago ${ }^{10}$, Lotte Wieten ${ }^{11}$, Chiara Bonini $^{10}$, Anja Van Biezen ${ }^{12}$, Steffie Vanderwerf ${ }^{12}$, Junfan Wang $^{12}$, Annalisa Ruggeri ${ }^{13}$, Christian Chabannon $^{14}$

${ }^{1}$ Hospital Clínic de Barcelona, Barcelona, Spain, ${ }^{2}$ BMT Unit, Essen, Germany, ${ }^{3}$ King's College Hospital NHS Foundation Trust, London, United Kingdom, ${ }^{4}$ BMT Unit, London, United Kingdom, ${ }^{5}$ BMT Unit, Hannover, Germany, ${ }^{6}$ BMT Unit, Utrecht, Netherlands, ${ }^{7}$ Bambino Gesù Children's Hospital IRCCS, Rome, Italy, ${ }^{8}$ Saint Antoine Hospital, Paris, France, ${ }^{9}$ San Matteo, Pavia, Italy, ${ }^{10}$ San Raffaele Scientific Institute, Milano, Italy, ${ }^{11}$ BMT Unit, Leiden, Netherlands, ${ }^{12}$ EBMT Data Office Leiden, Leiden, Netherlands, ${ }^{13}$ Bambino Gesù Children's Hospital IRCCS, Roma, Italy, ${ }^{14}$ Institut Paoli Calmettes, Marseille, France.

Background: The use of chimeric antigen receptor (CAR) $\mathrm{T}$ cells has shown outstanding efficacy in patients with relapsed/refractory B-cell lymphoid malignancies, as reported by many groups in United States (US) and China. CAR-T cell based activity in Europe is still at early stages. Few European academic and pharma-sponsored clinical trials are currently opened to inclusions, and access to the two EMA-approved autologous CAR-T products remains limited for the majority of European centers, since they must undergo a tedious and non-harmonized qualification process imposed by the manufacturer as part of the drug label. Improved awareness of CAR-T centers and ongoing CAR-T activities in Europe are essential to promote European networking activities, improve competitiveness of EU medical centers, and develop clinical trials, education, 
accreditation, and registration/long-term follow-up, thus fulfilling the needs of stakeholders.

Methods: A survey questionnaire was prepared by the committee members of the EBMT Cellular-Therapy\&-Immunobiology-Working-Party (CTIWP). Questions were formulated to identify CAR-T centers and CAR-T based activity in Europe, and to know the characteristics of centers and the organization for implementing a clinical CAR-T program. Using monkey survey, the survey was sent to 566 EBMT-centers.

Results: 123 centers replied to the survey. 340 patients (ALL 45\%, NHL 32\%, MM 10\%, CLL 5\%, AML 1.6\%, Solid tumors $7 \%$ ) have been treated so far in 31 EBMT centers from 12 countries. Most of these centers are JACIE-accredited (90\% for clinical activities; 93\% for cell collection activities). 54 additional centers reported plans to start CAR-T cell administration within the following 6-months. Most patients were treated in the setting of academic clinical trials $(58.6 \% 1)$ or pharma sponsored trials $(36 \% \mathrm{l})$. Few patients $(7 \%)$ received marketed CAR-T cells. PCR (36\%) and flow-cytometry (60\%) were used to monitoring the persistence of CAR-T cells after infusion. Cytokine levels were measured in $78 \%$ of the cases, and NGS was performed in $45 \%$ of the centers. Half of the centers reported to use "point of care" manufacturing. Patient management was mainly carried out by bone marrow transplant (BMT) physicians (85\%).

However, $77 \%$ of the centers reported to have also implemented a "CAR-T team", as a multidisciplinary team managed by a coordinator, with weekly clinical meetings. In more than $90 \%$ of the centers, there were specific rules for transferring patients to the intensive-care-unit. In contrast, only $40 \%$ of the centers had a nurse coordinator for management assistance of these patients. $77 \%$ of the centers plan to expand the capacity of their own department for CAR-T program.

Conclusions: This survey is the first attempt to gather information about CAR-T cell activity in Europe. This first results confirms active and growing involvement and high quality standards of European centers having CAR-T cell program.

The expanding use of these complex ATMP approaches could be facilitated through harmonization of the clinical practice, shared analysis of good quality data, and by a centralized European clinical trial office.

The cellular therapy CAR-T form is readily available to capture in more detail safety and efficacy of this intervention and will allow sharing in a transparent process registry data with stakeholders.

Disclosure: No disclosure

\section{3}

Coagulation disorders during CRS of a BCMA targeted CAR $T$ cell therapy in relapsed/refractory multiple myeloma: Periodic results of a phase I clinical trial
Mi Shao ${ }^{I}$, Yongxian $\mathrm{Hu}^{I}$, Huijun $\mathrm{Xu}^{\mathbb{I}}$, Yanlei Zhang ${ }^{I}$, Jiazhen Cui ${ }^{1}$, He Huang ${ }^{1}$

${ }^{1}$ The First Affiliated Hospital, Zhejiang University, Hangzhou, China

Background: Despite rapid advances in the treatment of multiple myeloma (MM), outcomes remain poor in relapse/ refractory patients. B-Cell Maturation Antigen (BCMA) chimeric antigen receptor (CAR) T cells, are showing promising responses rates in early trials. But there are still lots of uncertainties pending research. Herein, we conducted a clinical study aiming to determine the safety, efficacy and cytokinetic of CAR-BCMA T cells in patients with BCMApositive refractory or relapsed multiple myeloma (RRMM).

Methods: This is an open-label, phase 1 study (ChiCTR1800017404). Eligible patients had RRMM, confirmed as BCMA-positive by flow cytometry or pathological examination. Autologous T cells transduced with a BCMAtargeted chimeric antigen receptor (CAR-BCMA T cells) are used $\left(1 \times 10^{\wedge} 7 / \mathrm{kg} \mathrm{CAR}+\mathrm{T}\right.$ cells), following pre-infusion treatment-intravenous administration and lymphodepletion conditioning (fludarabine and cyclophosphamide). The primary outcome measure is incidence of adverse events (AEs) especially clinical events, including CRS, neurotoxicity and coagulopathy, et al. Additional outcome measures are duration and expansion of BCMA CAR T cells.

Results: 19 patients (median age of 61, range 41 to 71) with RRMM have received treatment. The median \%PC in BM at enrollment was $30.23 \%$ (range $2 \%$ to $72 \%$ ). $4 / 19$ had high tumor burden, defined as $\geq 50 \%$ bone marrow plasma cells. 3/19 had prior autologous stem cell transplant. As of data cut-off (Dec 1,2018), CRS grade 1-2 occurred in 8 patients, grade 3-4 occurred in 11. Surprisingly, not a few patients developed coagulation disfunction, manifesting elevated D-dimer, prolonged APTT, PT, TT, and decreased fibrinogen, with 0 case of organ bleeding. Laboratory values correlating with $\mathrm{CRS}$ reaching grade $3-4(\mathrm{~N}=11)$ compared to those with milder CRS $(\mathrm{N}=8)$ included peak ferritin (mean: 31095.23 vs $1665.53 \mathrm{ug} / \mathrm{L}, \mathrm{p}<0.005$ ), peak IL-6 (mean: 8001.07 vs $266.92 \mathrm{pg} / \mathrm{mL}, \mathrm{p}<0.005$ ), peak IL-10 (mean: 324.89 vs $43.81 \mathrm{pg} / \mathrm{mL}, \mathrm{p}<0.01)$, and peak IFN- $\gamma($ mean: 2936.46 vs $58.21 \mathrm{pg} / \mathrm{mL}, \mathrm{p}<0.05)$. No significant difference was seen in peak CRP, IL-2 and TNF- $\alpha$.

Patients with CRS 3-4 had a higher $\triangle$ APTT (mean: 38.86 vs 12.09 s, p< 0.01), $\triangle$ D-dimer (mean: 44509.18 vs $13347.75 \mathrm{ug} / \mathrm{L}, \mathrm{p}<0.05$ ) and $\triangle$ fibrinogen (mean: $-1.68 \mathrm{vs}$ $-0.34 \mathrm{~g} / \mathrm{L}, \mathrm{p}<0.05)$ compared to those with milder CRS. There was no significant difference in $\triangle P T$ and $\triangle T T$.The changes of D-dimer and APTT were in line with the indictors of CRS, such as CRP, IL-6 and IL-10 in some individual cases. The significant correlation between APTT and CRP $(\mathrm{p}<0.05)$ appeared on 17 patients $(89.5 \%)$, while 
the significant correlation between IL- 6 and APTT as well as between IL-6 and D-dimer only appeared on 7 patients $(36.84 \%)$.

Conclusions: Early safety and efficacy results of BCMA CAR $\mathrm{T}$ therapy in RRMM are encouraging. AEs of coagulation were observed. Changes of APTT, D-dimer and fibrinogen of patients with high CRS level were more significant than those with milder CRS. On the individual level, APTT correlated with CRP in the course of therapy. Given extensive cross-talk between inflammation and coagulation, coagulation-related indictors are more convenient for monitoring CRS. Also, our study suggests the importance in diagnosis and early management for coagulopathy to avoid CRS-related mortality.

Clinical Trial Registry: ChiCTR1800017404 http:// www.chictr.org.cn/abouten.aspx

Disclosure: Nothing to declare

\section{4}

Abstract already published.

\section{5}

Point-of-care production of CD19 CAR-T cells in an automated closed-system bioreactor: Report of first clinical experience

O Molostova ${ }^{1}$, Larisa Shelikhova ${ }^{1}$, Y Muzalevsky ${ }^{I}, A$ Kazachenok $^{1}$, D Pershin ${ }^{1}$, E Kurnikova ${ }^{1}$, M Dunaylkina ${ }^{1}$, Y Abugova', D Abramov', P Trakhtman', B Boro

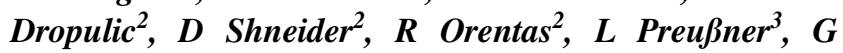
Rauser $^{3}$, R Alex $^{3}$, M Ess $^{3}$, N Miakova ${ }^{1}$, A Karachunskiy ${ }^{1}$, D Litvinov ${ }^{1}, G$ Novichkova $^{1}, A$ Maschan ${ }^{1}$, Michael Maschan ${ }^{1}$

${ }^{1}$ Dmitry Rogachev National Medical Research Center of Pediatric Hematology, Oncology and Immunology, Moscow, Russian Federation, ${ }^{2}$ Lentigen, A Miltenyi Biotec Company, Gaithersburg, MD, United States, ${ }^{3}$ Miltenyi Biotec GmbH, Bergisch Gladbach, Germany

Background: CD19 CAR-T cell products are approved as therapy for advanced B-cell malignancies. The predominant manufacturing model is a centralized industrial-type production process. An alternative approach to CAR-T cell production and delivery to the patient is via a point-of care manufacturing process.

Methods: Between February 2018 to November 2018 a total of 15 pts with relapsed/refractory B-cell malignancies (4 female, 11 male[GR1], median age $12 \mathrm{y}$ ) were screened, 10 pts were enrolled for single center, phase I-II trial of safety and clinical efficacy of automatically produced cell therapy product, 5 were eligible for compassionate use of therapy.
Seven patients had relapsed B-ALL after HSCT, 6 pts had refractory relapse after chemotherapy, $1 \mathrm{pt}$ had refractory induction failure, $1 \mathrm{pt}$ had refractory primary mediastinal Bcell lymphoma (PMBCL). Eight patients received previous blinatumomab infusion.

Eight patients had high disease burden $>5 \%$ blast cells in BM, median 89\% (14-100), 6 pts had minimal residual disease (MRD) in BM.

The CliniMACS Prodigy T cell transduction (TCT) process was used to produce CD19 CAR-T cells from fresh patientderived leukapheresis product. Automatic production included CD4/CD8 selection, stimulation with MACS GMP T Cell TransAct, transduction with lentiviral (second generation CD19.4-1BB zeta) vector (Lentigen, Miltenyi Biotec company) and expansion over 10 days in the presence of TexMACS GMP Medium and MACS GMP IL-7/IL-15 combination. Final product was administered without cryopreservation after fludarabine/cyclophosphamide preconditioning. All patients received prophylactic tocilizumab at $8 \mathrm{mg} / \mathrm{kg}$.

Results: All production cycles were successful. Median transduction efficiency was 59\% (32-75). Median expansion of $\mathrm{T}$ cells was 36 fold (18-49). CD4/CD8 ratio in the final product was 0,6 . The cell products were administered at $1 * 10^{5} / \mathrm{kg}$ of CAR-T cells in $5 \mathrm{pts}, 5^{*} 10^{5} / \mathrm{kg}$ in $5 \mathrm{pts}, 1^{*} 10^{6} / \mathrm{kg}$ in $5 \mathrm{pts}$.

Cytokine release syndrome occurred in $8(53 \%)$ pts: grade I in 5 pts, grade II in 2 pts, grade IV in 1 pts. CAR-T cell related encephalopathy occurred in 4 (26\%) pts, including one fatal brain edema. Grade I neurotoxicity had 2 pts, grade II - $1 \mathrm{pt}$, grade V - 1 pt. Four patients were admitted to the intensive care unit (ICU). Three patients died until day 28 ( 2 due to sepsis, 1 due to fatal brain edema and sepsis, on autopsy in the brain vessels of this patient were found Klebsiella pneumoniae emboli)

Twelve patients were evaluable for response at day 28. Three pts had persistent leukemia, without evidence of CAR-T expansion. MRD-negative responses were achieved in 8 cases among 11 evaluable cases with bone marrow involvement. Patient with PMBCL had a decrease in metabolic activity on PET/CT scan. CD19(-) relapse after initial response was registered in 1 case at 109 day.

Conclusions: Production of CAR-T cells with the CliniMACS Prodigy TCT process is a robust option that provides a point-of-care manufacturing approach to enable rapid and flexible delivery of CAR-T cells to patients in need. Robustness and consistency of this approach provides a solid basis for multi-center academic trials in the field of adoptive cell therapy.

Disclosure: Nothing to declare 
Chronic leukaemia and other myeloproliferative disorders

\section{6}

Abstract already published.

\section{7}

Development and validation of a clinical-molecular transplant scoring system for myelofibrosis

Nico Gagelmann ${ }^{1}$, Markus Ditschkowski', Rashit Bogdanov $^{2}$, Swan Bredin', Marie Robin ${ }^{3}$, Bruno Cassinat ${ }^{3}$, Rabia Shahswar, Felicitas Thol', Michael Heuser, Gerard Socié $\hat{e}^{3}$, Dietrich Beelen', Anita Badbaran', Nicolaus Kröger ${ }^{1}$

${ }^{1}$ University Medical Center Hamburg-Eppendorf, Hamburg, Germany, ${ }^{2}$ West German Cancer Center, Essen, Germany, ${ }^{3}$ Hôpital Saint-Louis, Paris, France, ${ }^{4}$ Hannover Medical School, Hannover, Germany

Background: In the last decade, several prognostic scoring models such as the International Prognostic Scoring System (IPSS), the dynamic IPSS (DIPSS) or DIPSS-plus have been developed for diagnosed primary myelofibrosis (PMF) and are currently used for decision finding regarding allogeneic stem cell transplantation. Furthermore, the prognostic relevance of mutation profile resulted in a mutation-enhanced system (MIPSS70) in transplant-age PMF patients (70 years or younger). For secondary myelofibrosis, the MYSEC-PM was developed. While all scoring systems were developed in nontransplant populations and may be useful in decision finding regarding transplantation, uncertainty remains regarding usefulness of these systems to predict posttransplant outcome and thus counseling patients whether to proceed to transplant.

Methods: Bone marrow or peripheral blood samples were obtained before transplantation and mutations were detected using next-generation sequencing. The following myelofibrosis-associated genes were sequenced: $J A K 2$, CALR, MPL, ASXL1, IDH1/2, CBL, DNMT3A, TET2, SF3B1, SRSF2, U2AF1, EZH2, TP53, NRAS, KRAS, RUNX1, and FLT3. Cytogenetic analysis and reporting were performed according to the International System for Human Cytogenetic Nomenclature criteria using standardized techniques. We examined 361 myelofibrosis patients, of whom 260 had PMF and $101 \mathrm{SMF}$ at time of transplantation. A training cohort of 205 patients was used to create a clinical-molecular transplant scoring system (MTSS) predicting survival from Cox models, internally validated by use of bootstrap and cross-validation. Model discrimination was measured by the concordance index (C). The final MTSS was externally validated in a cohort of 156 patients and was furthermore applied to posttransplant non-relapse mortality as a secondary objective.
Results: Multivariable analysis on 5-year survival identified age $\geq 57$ years, Karnofsky performance status $<90 \%$, platelet count $<150 \times 10^{9} / \mathrm{L}$ and leukocyte count $>25 \times 10^{9} / \mathrm{L}$ at time of transplantation, HLA-mismatched unrelated donor, ASXL1 mutated and $C A L R$ - $/ M P L$-unmutated genotype being independent prognostic factors for outcome. The uncorrected concordance index for the final survival model was 0.723 (0.713-0.733), and bias-corrected indices were similar. A weighted score of 2 was assigned to transplantation from an HLA-mismatched unrelated donor and an CALR-IMPLunmutated genotype, whereas a score of 1 was assigned to older age, leukocytosis, thrombocytopenia, ASXL1 mutation, and poor performance status. The MTSS consisted of four distinct risk groups showing 5-year survival in the validation cohort of $83 \%$ (71-95\%) for low (score 0-2), 64\% (53-75\%) for intermediate (score 3-4), 37\% (17-57\%) for high (score 5), and $22 \%$ (4-39\%) for very high risk (score $>5$ ), respectively $(\mathrm{P}<0.001)$. Increasing score was predictive of non-relapse mortality $(\mathrm{P}<0.001)$ and remained applicable to $\mathrm{PMF}(\mathrm{C}=$ 0.718; 0.710-726) and SMF $(\mathrm{C}=0.701 ; 0.690-0.711)$ improving prognostic ability in comparison to current systems such as DIPSS $(\mathrm{C}=0.573 ; 0.564-0.582)$, DIPSS-plus $(\mathrm{C}=0.557$; 0.546-0.568), MIPSS70 ( $\mathrm{C}=0.587 ; 0.578-0.596)$, MIPSS70plus $(\mathrm{C}=0.547 ; 0.538-0.556)$, MIPSS70-plus version 2.0 $(\mathrm{C}=0.566 ; 0.558-0.574)$, GIPSS $(\mathrm{C}=0.544 ; 0.532-0.556)$ as well as MYSEC-PM $(\mathrm{C}=0.605 ; 0.593-0.617)$.

Conclusions: We show here that this internally and externally validated MTSS accurately discriminated different risk for death and may improve counseling patients with respect to transplant compared with currently existing systems, as well as facilitate design of clinical trials in the transplant setting.

Disclosure: Nothing to declare.

\section{O058}

Response to up-front azacitidine in juvenile myelomonocytic leukemia (JMML): Interim analysis of the prospective European multicenter study AZA-JMML-001

Charlotte M. Niemeyer ${ }^{\text {, }}$, Christian Flotho ${ }^{1}$, Daniel B. Lipka $^{2}$, Jan Starý, Claudia Rössig ${ }^{4}$, André Baruchel, Giuseppe Basso $^{6}$, Thomas Klingebiel ${ }^{7}$, Concetta Micalizzi $^{8}$, Gérard Michel ${ }^{9}$, Karsten Nysom ${ }^{10}$, Susana Rives ${ }^{11}$, Markus Schmugge Liner ${ }^{12}$, Marco Zecca ${ }^{13}$, Irith Baumann $^{14}$, Bouchra Benettaib ${ }^{15}$, Noha Biserna ${ }^{15}$, Jennifer Poon $^{15}$, Mathew Simcock ${ }^{16}$, Meera Patturajan ${ }^{15}$, Marry M. van den Heuvel-Eibrink ${ }^{17}$, Franco Locatelli ${ }^{18,19}$

${ }^{1}$ University of Freiburg, Freiburg, Germany, ${ }^{2}$ German Cancer Research Center (DKFZ), Heidelberg, Germany, ${ }^{3}$ Fakultní nemocnice v Motole, Prague, Czech Republic, ${ }^{4}$ University Children's Hospital Münster, Münster, Germany, ${ }^{5} \mathrm{CHU}$ Paris - Hôpital Universitaire Robert Debré (APHP), Paris, France, 
${ }^{6}$ Azienda Ospedaliera di Padova, Padua, Italy, ${ }^{7}$ Universitaetsklinikum Frankfurt, Frankfurt, Germany, ${ }^{8}$ Istituto Giannina Gaslini, Genoa, Italy, ${ }^{9}$ Hôpital de la Timone, Marseilles, France, ${ }^{10}$ Rigshospitalet, Copenhagen, Denmark, ${ }^{11}$ Hospital Sant Joan de Deu de Barcelona, Barcelona, Spain, ${ }^{12}$ Kinderspital Zürich, Zürich, Switzerland, ${ }^{13}$ Fondazione IRCCS Policlinico San Matteo, Pavia, Italy, ${ }^{14}$ Böblingen Hospital, Sindelfingen, Germany, ${ }^{15}$ Celgene Corporation, Summit, NJ, United States, ${ }^{16}$ Celgene Corporation, Uxbridge, United Kingdom, ${ }^{17}$ Prinses Máxima Center for Pediatric Oncology/ Hematology, Utrecht, Netherlands, ${ }^{18}$ IRCCS Ospedale Pediatrico Bambino Gesù, Rome, Italy, ${ }^{19}$ Sapienza, University of Rome, Rome, Italy

Background: JMML is a chemotherapy-resistant neoplasia of early childhood. Allogeneic hematopoietic stem cell transplantation (HSCT) is the only curative therapy, saving approximately $50 \%$ of these children. Relapse is the major cause of treatment failure, with chemotherapy prior to HSCT being notably unsuccessful. Novel therapies controlling the disorder prior to HSCT are urgently needed.

Methods: We conducted a phase 2, multicenter, open-label study to evaluate pharmacodynamics, safety, and antileukemic activity of azacitidine monotherapy prior to HSCT in patients with newly diagnosed JMML. Azacitidine was administered at $75 \mathrm{mg} / \mathrm{m}^{2} /$ day intravenously on days $1-7$ of a 28 -day cycle for 3 to 6 cycles. The primary endpoint was the number of patients with clinical complete remission or clinical partial remission (cPR) at Cycle 3 Day 28 (C3D28); secondary endpoints included overall survival following HSCT.

Results: Eighteen JMML patients (13 PTPN11-, 3 NRAS-, 1 $K R A S$-, 1 NF1-mutated) aged 0.2-7.0 years were enrolled. Median (range) WBC, platelet count and spleen size were: 19.7 $(4.3-59.0) \times 10^{9} / \mathrm{L}, 28(7-85) \times 10^{9} / \mathrm{L}$, and $4(2-14) \mathrm{cm}$ below the costal margin, respectively. DNA methylation class (Lipka et al. Nat Comm 2017; $\mathrm{n}=17$ ) was high, intermediate, or low in 10,5 , and 2 patients, respectively. Sixteen patients completed 3 cycles of therapy and 5 of them completed 6 cycles. Two patients discontinued treatment before completing 3 cycles due to disease progression. Six patients $(33 \%)$ experienced $\geq 1$ grade 3 or 4 manageable adverse event, consistent with the known azacitidine safety profile. Eleven patients (61\%) achieved cPR at C3D28 and 7 had progressive disease either at C3D28 or prior. Importantly, 8 of the 15 patients who needed platelet transfusions before or shortly after treatment initiation did not require transfusions at the time of HSCT. Seven of these 8 platelet responders had normalized their platelet count $\left(\geq 130 \times 10^{9} / \mathrm{L}\right)$. Palpable spleen size decreased in 11 responders by a median of $3.5 \mathrm{~cm}$ after 3 cycles and ranged from 0-2 cm below the costal margin after 6 cycles. Sixteen patients received allo-HSCT from a family or compatible unrelated donor following a busulfan- $(n=15)$ or treosulfan-based $(n=1)$ preparative regimen after a median of
57 days (36-112) from last azacitidine dose. Thirteen transplanted patients were leukemia-free at median follow-up of 15.7 months (0.1-31.7) after HSCT. Two children (both high methylation class) given HSCT relapsed after the allograft. Sixteen of the 18 patients were alive at a median follow-up of 19.8 months (2.6-37.3). One patient who discontinued treatment before cycle 3 died from disease progression, and 1 non-responder died from graft failure.

Conclusions: This study shows azacitidine monotherapy was well tolerated in children with newly diagnosed JMML. Although the long-term advantage of azacitidine therapy remains to be fully assessed, both the decrease in spleen size and significant platelet responses observed demonstrate that the drug was effective in JMML and provided clinical benefit to JMML patients in this study. This clinical trial has shown that azacitidine therapy prior to HSCT may be considered for patients with JMML.

\section{Clinical Trial Registry: NCT02447666}

Disclosure: Charlotte Niemeyer is a member of a board of directors or advisory committee and provides consultancy for Celgene.

Claudia Rössig is a member of a board of directors or advisory committee at Amgen, EUSAPharm, Roche, Celgene, Novartis, Pfizer, BMS; honoraria from Amgen, Roche, Pfizer.

André Baruchel provides consultancy (includes expert testimony) with Celgene, Novartis, Servier, JAZZ Pharma; research funding and honoraria from Shire; honoraria from Novartis, Jazz Pharma.

Susana Rives has received honoraria (for talks in Industria sponsored Satellite Symposia) from Novartis, JAZZ Pharma, Baxalta, Shire, Servier; Speaker's Bureau at Novartis, JAZZ Pharma, Baxalta, Shire, Servier, Amgen, Erytech Pharma; membership on an entity's board of directors or advisory committees at Novartis; received travel and accommodation expenses for medical congresses from Novartis, JAZZ Pharma, Baxalta, Shire, Servier, Amgen, Erytech Pharma.

Marco Zecca has received honoraria from CHIMERIX and JAZZ Pharma.

Marry M. van den Heuvel-Eibrink received honoraria from Celgene (consultation fee).

Bouchra Benettaib, Noha Biserna, Jennifer Poon, Mathew Simcock, Meera Patturajan are employees of and hold stock or other equity ownership in Celgene.

Christian Flotho, Daniel Lipka, Jan Starý, Karsten Nysom, Gérard Michel, Thomas Kilngebiel, Franco Locatelli, Giuseppe Basso, Concetta Micalizzi, Irith Baumann, Markus Schmugge Liner have nothing to disclose.

\section{9}

Ruxolitinib before allogeneic hematopoietic stem cell transplantation (HSCT) in patients with myelofibrosis: A preliminary descriptive report of the JAK ALLO phase II study 
Marie Robin ${ }^{1,2}$, Raphael Porcher ${ }^{3}$, Corentin Orvain ${ }^{4}$, Jacques Olivier Bay ${ }^{5}$, Hélène Labussière ${ }^{6}$, Anne Huynh ${ }^{7}$, Amandine Charbonnier, Edouard Forcade ${ }^{8}$, Sylvain Chantepie $^{10}$, Claude Bulabois ${ }^{11}$, Ibrahim Yakoub Agha ${ }^{12}$, Pascal Turlure ${ }^{13}$, Marie Dutrait ${ }^{14}$, Nicole Raus ${ }^{6}$, Françoise Boyer, Felipe Suarez ${ }^{15}$, Stephanie $N$ Guyen ${ }^{16}$, Jerome Cornillon ${ }^{17}$, Alban Villate ${ }^{18}$, Brigitte Dupriez ${ }^{19}$, Bruno Cassinat ${ }^{1}$, Valerie Rolland ${ }^{11}$, Marie Helene Schlageter $^{I}$, Gerard Socie ${ }^{I}$, Jean Jacques Kiladjian ${ }^{I}$

${ }^{1}$ Hopital Saint-Louis, APHP, Paris, France, ${ }^{2}$ University Paris 7, INSERM 1131, Paris, France, ${ }^{3}$ Hotel Dieu, APHP, Paris, France, ${ }^{4}$ Centre Hospitalier Universitaire (CHU) Angers, Angers, France, ${ }^{5} \mathrm{CHU}$ Clermont Ferrand, Clermont Ferrand, France, ${ }^{6} \mathrm{CHU}$ Lyon Sud, Lyon, France, ${ }^{7} \mathrm{CHU}$ Toulouse, Paris, France, ${ }^{8} \mathrm{CHU}$ Amiens Picardie, Amiens, France, ${ }^{9} \mathrm{CHU}$ Bordeaux, Bordeaux, France, ${ }^{10} \mathrm{CHU}$ Caen, Caen, France, ${ }^{11} \mathrm{CHU}$ Grenoble Alpes - Université Grenoble Alpes, Grenoble, France, ${ }^{12} \mathrm{CHRU}$ de Lille, Lille, France, ${ }^{13} \mathrm{CHU}$ Limoges, Limoges, France, ${ }^{14} \mathrm{CHU}$ Nancy, Nancy, France, ${ }^{15} \mathrm{CHU}$ Necker, APHP, Paris, France, ${ }^{16}$ Hôpita de la Pitie Saletrière, APHP, Paris, France, ${ }^{17} \mathrm{CHU}$ Saint Etienne, Saint Etienne, France, ${ }^{18} \mathrm{CHU}$ Tours, Tours, France, ${ }^{19} \mathrm{CH}$ Lens, Lens, France

Background: Allogeneic hematopoietic stem cell transplantation (HSCT) is the only curative treatment for patients with myelofibrosis; OS is from 30 to $60 \%$ depending on age, comorbidities, disease status and type of donors. JAK1/2 inhibitors have been reported to decrease constitutional symptoms and spleen size in one half of patients with a possible advantage of OS as compared to best current treatment. Retrospective studies show that ruxolitinib has been used in patients before HSCT with apparent good tolerance.

Methods: In 2012, we initiated a phase II prospective French collaborative (FILO and SFGM-TC) trial testing the role of ruxolitinib given before allogeneic hematopoietic transplantation in patients with myelofibrosis. Patients could be included if they were intermediate or high risk according to Lille score or IPSS. Patients had to start ruxolitinib after inclusion and were transplanted in case a donor has been identified within 4 months. Primary aim was progression-free survival at one year after HSCT.

Results: 76 patients could be included. 53 (70\%) had a primary myelofibrosis and $23(30 \%)$ had a myelofibrosis secondary to Essential Thrombocythemia $(n=10)$ or Polycythemia Vera $(n=13)$. At time of inclusion, $43(57 \%)$ patients had constitutional symptoms, $72(95 \%)$ had palpable splenomegaly, 48 (63\%) patients had hemoglobin $<10 \mathrm{gr} / \mathrm{dl}$, $23(30 \%)$ patients had peripheral blast cell count $>$ or $=$ at $1 \%$ and $16(21 \%)$ had thrombocytopenia $<100 \mathrm{G} / \mathrm{L}$. Median follow-up was 31 months. At 4 months, one patient has died, 11 patients had no donor, 18 had an HLA matched sibling donor, 31 had an HLA matched unrelated donor and 15 had a 9/10 HLA mismatched unrelated donor. 59 (78\%) patients with a donor at 4 months could be transplanted of whom 18 underwent a splenectomy before transplantation. Conditioning regimen was fludarabine $90 \mathrm{mg} / \mathrm{m} 2$ in combination with melphaln $140 \mathrm{mg} / \mathrm{m} 2$. GVHD prophylaxis was cyclosporine and mycophenolate mofetil with ATG in unrelated donor. Partial response under ruxolitinib was $26 \%$ while the whole complete response incidence was $46 \%$ at one year. OS and PFS at 2 years were 68\% (95\% CI: 59-80) and 53\% (95\% CI: 43-66). OS was significantly better in patients with HLA matched sibling donor as compared to patients without a donor or with an unrelated donor $(\mathrm{p}=0.008$, figure 1$)$. Cumulative incidence of acute GVHD on day 100 was $66 \%$ of whom $44 \%$ had grade 3-4 acute GVHD. Cardiogenic shock generally associated with severe sepsis or "sepsis like" syndrome occurred in 7 patients: 2 patients were not transplanted because of this complication while in the 5 other patients CS occurred within the 15 days after transplantation. Three renal failures secondary to tumor lysis syndrome were declared just after conditioning regimen initiation.

Figure 1. Overall survival in JAK ALLO patients.

Conclusions: OS was very good in patients with HLA matched related donor and significantly better than in patients with an unrelated donor. More analyses are currently ongoing to determinate the potential role of cytokine release in peri-transplantation time, as well as specific myelofibrosis and quality of life questionnaires assessing the impact of ruxolitinib in patients.

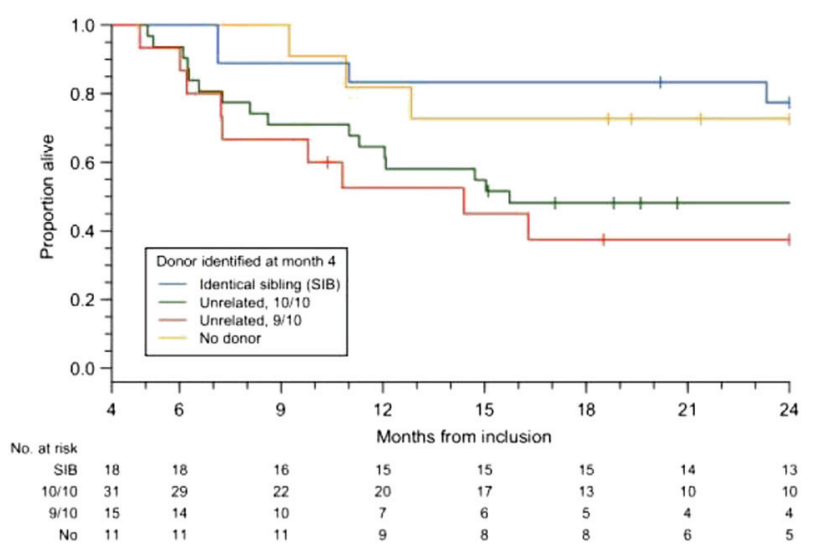

[[O059 Image] 1. Figure 1. Overall survival in JAK ALLO patients]
Clinical Trial Registry: NCT01795677
Disclosure: no

\section{0}

Impact of cytogenetics on prognosis in primary myelofibrosis after allogeneic hematopoietic stem cell transplantation 
Nico Gagelmann ${ }^{1}$, Markus Ditschkowski², Rashit Bogdanov $^{2}$, Swan Bredin ${ }^{3}$, Marie Robin ${ }^{3}$, Bruno Cassinat ${ }^{3}$, Rabia Shahswar, Felicitas Thol, Michael Heuser, Dietrich Beelen ${ }^{2}$, Anita Badbaran', Nicolaus Kröger ${ }^{1}$

${ }^{1}$ University Medical Center Hamburg-Eppendorf, Hamburg, Germany, ${ }^{2}$ West German Cancer Center, Essen, Germany, ${ }^{3}$ Hôpital Saint-Louis, Paris, France, ${ }^{4}$ Hannover Medical School, Hannover, Germany

Background: While cytogenetics may influence outcome in primary myelofibrosis (PMF) from diagnosis which lead to several prognostic systems incorporating different cytogenetic risk classifications, its definitive role specific after allogeneic stem cell transplantation is still unclear. Here, we aim to evaluate the role of currently existing cytogenetic risk classifications included in the Dynamic International Prognostic Scoring System (DIPSS)-plus and in the mutation-enhanced system (MIPSS70-plus version 2.0).

Methods: In this multicenter retrospective study, we examined 149 PMF patients undergoing allogeneic stem cell transplantation. Current cytogenetic risk stratifications used in DIPSS-plus and MIPSS70-plus version 2.0 were evaluated. According to DIPSS-plus, an unfavorable karyotype includes $+8,-7 / 7 \mathrm{q}, \mathrm{i}(17) \mathrm{q},-5 / 5 \mathrm{q}, 12 \mathrm{p}-$, inv(3), and $11 \mathrm{q} 23$ rearrangements while MIPSS70-plus version 2.0 consisted of very high risk (VHR: single/multiple abnormalities of $-7, \mathrm{i}(17 \mathrm{q})$, inv(3)/ $3 \mathrm{q} 21,12 \mathrm{p}-/ 12 \mathrm{p} 11.2,11 \mathrm{q}-/ 11 \mathrm{q} 23,+21$, or other autosomal trisomy, not including $+8 /+9$ ), favorable (normal karyotype or sole abnormalities of 13q-, $+9,20 \mathrm{q}-$, chromosome 1 translocation/duplication or sex chromosome abnormality including -Y), and unfavorable (all other abnormalities).

Results: The median follow-up period of all 149 PMF patients was 56 months and the median time from diagnosis to transplant was 26 months. After five years, 51 (34\%) patients had died. The median age at transplant was 57 years and $63 \%$ of patients were male. Most allografts were applied using peripheral blood (97\%) and were received from an HLA-matched unrelated donor (42\%), followed by mismatched unrelated (34\%), matched related $(23 \%)$, and mismatched related or haploidentical (1\%). Reduced intensity conditioning was applied to $75 \%$ of patients. Splenectomy before transplant was undergone in $15 \%$ and ruxolitinib was received by $27 \%$. Frequencies according to driver mutation genotype were: CALR (19\%), JAK2 (59\%), MPL (5\%), and triple negative (17\%). ASXL1 mutation was present in $38 \%$ while $64 \%$ had more than two mutations overall. 94 (63\%) patients had normal karyotype. Most frequent abnormalities were trisomy 8 in $11(7 \%)$, trisomy 9 in $5(3 \%)$, deletion in $20 \mathrm{q}$ in $17(11 \%)$, deletion in $13 \mathrm{q}$ in $11(7 \%)$, chromosome 7 in $5(3 \%)$, chromosome 5 in $5(3 \%)$, and chromosome 1 translocation/duplication in $2(1 \%)$. More than two abnormalities were present in $9(6 \%)$ patients. High cytogenetic risk category according to DIPSS-plus was present in $24(16 \%)$ while $12(8 \%)$ had VHR and $25(17 \%)$ had unfavorable risk according to MIPSS70-plus version 2.0. Regarding outcome, an unfavorable karyotype according to DIPSS-plus showed 5year OS of $62 \%(52-72 \%)$ vs $63 \%(57-70 \%)$, with causespecific hazard being $1.16(\mathrm{P}=0.69)$. 5-year relapse and nonrelapse mortality rates were not significantly different showing $9 \%$ and $33 \%$ for unfavorable karyotype vs $17 \%$ and $28 \%(\mathrm{P}=0.31$ and 0.52$)$. With respect to the three-tiered classification of MIPSS70-plus version 2.0, 5-year OS of VHR was $74 \%$ (62-86\%) and unfavorable was 52\% (42-62\%) vs favorable risk of $64 \%$ (58-70\%), with cause-specific hazard of $1.09(\mathrm{P}=0.74)$. Relapse and non-relapse mortality were $9 \%$ and $17 \%$ for VHR, $4 \%$ and $48 \%$ for unfavorable, and $19 \%$ and $26 \%$ for favorable risk $(\mathrm{P}=0.19$ and 0.48$)$.

Conclusions: Current cytogenetic risk stratifications do not predict outcome in PMF after allogeneic stem cell transplantation.

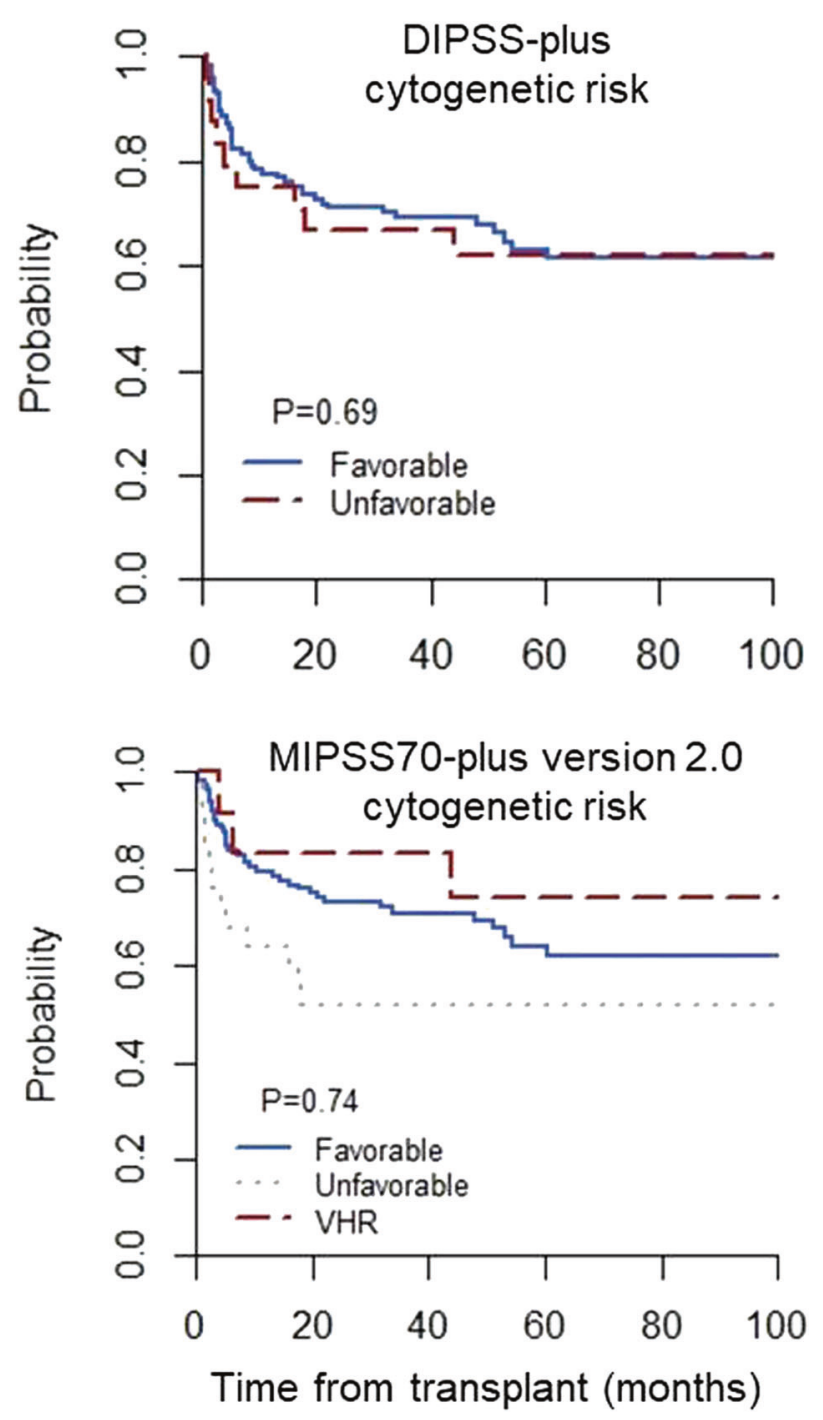


[[O060 Image] 1. Figure 1. Cytogenetic risk classification according to DIPSS-plus and MIPSS70-plus version 2.0]

Disclosure: Nothing to declare.

\section{1}

allogeneic transplants for myelofibrosis: Improved outcome in 2018

Stefania Bregante ${ }^{1}$, Anna Maria Raiola ${ }^{1}$, Alida Dominietto $^{I}$, Carmen di Grazia ${ }^{1}$, Maria Teresa van Lint ${ }^{l}$, Francesca Gualandi', Patrizia Chiusolo ${ }^{2}$, Sabrina Giammarco $^{2}$, Luca Laurenti ${ }^{2}$, Federica Sora ${ }^{\prime 2}$, Elena Rossi ${ }^{2}$, Valerio de Stefano ${ }^{2}$, Simona Sica ${ }^{2}$, Emanuele Angelucci ${ }^{1}$, Andrea Bacigalupo ${ }^{2}$

${ }^{1}$ AOU S.Martino IST IRCCS, Genova, Italy, ${ }^{2}$ Fondazione Policlinico Universitario Agostino Gemelli IRCCS, Roma, Italy

Background: The aim of this study was to assess the outcome of patients with Myelofibrosis allografted before and after 2010, in two transplant Centers (Genova San Martino and Rome Gemelli).

Methods: We have studied 147 patients, divided in two groups: 2001-2010 $(\mathrm{n}=59)$ and between 2011 and 2018 $(\mathrm{n}=88)$. The age was significantly older in the most recent group ( 53 vs 58 years, $\mathrm{p}<001$ ), and there was a greater use of alternative donors ( $38 \%$ vs $80 \%, \mathrm{p}<0.0001)$, and more patients with DIPSS-R high score ( $40 \%$ vs $47 \%, \mathrm{p}=0.1)$. The transplant score (based on transfusions $>20$ and splee size $>22 \mathrm{~cm}$ ) was intermediate-high risk in $82 \%$ and $58 \%$ $(\mathrm{p}<0.01)$ of patients respectively. The conditioning regimen was a combination of thiotepa, busulfan fludarabine (TBF) in $1 \%$ and $87 \%$ of patients grafted before and after 2010 ( $\mathrm{p}<$ 0.00001). The transplant risk score (TS) was based on spleen size $(>22 \mathrm{~cm})$ and pre-graft transfusion $(>20)$ as previously described (Bone Marrow Transplant. 2010 Mar;45(3):458-63)

Results: Outcome. The cumulative incidence (CI) of acute grade II-IV (25\%) and of chronic GvHD (18\%) was comparable in the two time periods. The 5 year CI of non relapse mortality (NRM) was reduced overall from $37 \%$ to $20 \%(\mathrm{p}=0.01)$ and the 5 year CI of relapse from $47 \%$ to $11 \%(\mathrm{p}=0.00006)$. As a consequence the overall 5 year actuarial survival has improved from $35 \%$ to $64 \%$ $(\mathrm{p}=0.003)$.

Predictive factors. The following factors predicted survival in multivariate Cox analysis: high risk transplant score (HR 2.5, $\mathrm{p}=0.01$ ), high risk DIPSS-R (HR 2.0, $\mathrm{p}=0.01$ ), the use of TBF (HR 0,50, $\mathrm{p}=0.02$ ), ALT donor (HT $1.8 \mathrm{~m} \mathrm{p}=0.04)$, donor age $>29$ years $(\mathrm{HR} 1.8, \mathrm{p}=0.06$ ) and $\mathrm{ABO}$ match (HR 0.64, $\mathrm{p}=0.08$ ).

DIPSS and Transplant score. When combining DIPSSR (high) and Transplant Score (int2-high) we could identify 3 groups : score 0 (DIPSS not high and TS not high) $(\mathrm{n}=40)$ score 1 (either high DIPSS or high TS) $(\mathrm{n}=51)$, score 2 (both DIPSS a TS high risk) $(\mathrm{n}=55)$. NRM was $9 \%, 27 \% 40 \%$ in these 3 group $(\mathrm{p}=0.0004)$, relapse was $17 \%, 29 \%, 25 \%(\mathrm{p}=0.1)$ and 5 year survival was $78 \%, 55 \%, 20 \%(\mathrm{p}<0.00001)$. In the current transplant era $(>2010)$ the 5 year disease free survival of these 3 groups is $78 \%, 70 \%, 35 \%(\mathrm{p}<0.00001)$. ABO matching further increases DFS for score 0 patients. NRM mortality for these 3 groups is currently $6 \%, 16 \%, 36 \%$ $(\mathrm{p}=0.01)$.

Conclusions: The 5 year survival of allografts in patients with Myelofibrosis has improved overall from $35 \%$ before 2010 , to $64 \%$ beyond 2010 , despite the current use of $80 \%$ ALT donors. Predictive factors are the Transplant Score (transfusions and spleen size), DIPSS-R and the use of 2 alkylating agents (TBF), the latter being the major change in the 2 transplant eras. Other factors include donor age $>30$ and $\mathrm{ABO}$ match . Combining DIPSS and TS identifies patients with a current 5 year disease free survival of $78 \%$, and a NRM of $6 \%$ : this may call for earlier grafts in transplant eligible patients.

Clinical Trial Registry: Not applicable

Disclosure: Nothing to disclose

\section{2}

Favorable outcome of allogeneic stem cell transplantation in myelofibrosis harboring MPL mutation

Daniele Mannina, Nico Gagelmann ${ }^{I}$, Markus Ditschkowski ${ }^{2}$, Rashit Bogdanov ${ }^{2}$, Swan Bredin ${ }^{3}$, Marie Robin ${ }^{3}$, Bruno Cassinat, Rabia Shahswar, Felicitas Thol, Michael Heuser, Dietrich Beelen', Anita Badbaran', Nicolaus Kröger ${ }^{1}$

${ }^{1}$ University Medical Center Hamburg-Eppendorf, Hamburg, Germany, ${ }^{2}$ West German Cancer Center, Essen, Germany, ${ }^{3}$ Hôpital Saint-Louis, Paris, France, ${ }^{4}$ Medical School, Hannover, Germany

Background: Primary and post-ET/PV myelofibrosis are myeloproliferative neoplasms harboring in most cases driving mutations in JAK2, CALR or MPL, and a variable number of additional mutations in other genes. Molecular analysis, today available for all the affected patients, allows 
the physicians to correlate mutational status with prognosis, potentially representing a powerful tool to guide the clinical management. Only about $10 \%$ of patients with myelofibrosis harbor alterations in MPL gene, consisting in MPL W515K or W515L point mutation. No data are available about the outcome of transplantation in the specific MPLmutated group.

Methods: We collected the data of 18 patients (male 8, female 10) transplanted in 4 EBMT centers (Hamburg Paris, Essen, Hannover) affected by MPL-mutated myelofibrosis (primary: 14; post-ET: 4), who underwent allo-SCT between 2005 and 2016. Median age was 59 years ( $\min 43, \max 67$ ). Prognostic risk assessed according to the international DIPSS system was high in 6 cases, intermediate- 2 in 7 cases, intermediate 1 in 5 cases. One patient was previously splenectomised, 8 patients received ruxolitinib before the transplantation, 7 were transfusion-dependent. All the patients underwent PBSC infusion, except one who received BMSC. In 7 cases the source was a sibling donor, in 9 a MUD, in 2 a HLA-mismatched UD (1 mismatch). Conditioning regimen was mostly based on the combination of singlealkylating agent and Fludarabine (12 Bu/Flu; $3 \mathrm{Mel} / \mathrm{Flu} /$ BCNU; $1 \mathrm{Mel} / \mathrm{Flu}$; $1 \mathrm{Treo} / \mathrm{Flu}$ ) with ATG infusion. Six patients received a fully myeloablative schema, 12 reduced-intensity conditioning. Only one patient received conditioning with TBI.

Results: Before the transplant, a panel of molecular analysis based on next-generation-sequencing revealed, in addition to the MPL mutation, alterations in ASXL1 (22\%), SRSF2 (11\%), SF3B1 (5.5\%), EZH2 (11\%), IDH1 (5.5\%), IDH2 (5.5\%), TET2 (33.5\%), TP53 (5.5\%). After the transplant, the incidence of acute GVHD was $72 \%$; only 3 patients (16.5\%) experienced acute GVHD grade 3-4. Chronic GVHD was registered in 50\% of cases (9 patients: 8 mild, 1 moderate, 0 severe). Relapse occurred in only 1 case. NRM incidence was $16.5 \%$ of cases, occurring in the first year after transplant. With a median follow up of 55 months, 5-year overall survival was $83.5 \%$, and 5-years PFS reported the same value, beeing the only relapse at $>10$ years after transplantation. The relapse occurred in the only patient who harbored mutations in both ASXL1 and EZH2 genes.

Conclusions: These retrospective data suggest that the particular group of MPL-mutated myelofibrosis may have a good outcome after stem cells transplantation. In particular, our data revealed very low rate of disease relapse (5.5\%) in comparison with the available historical controls regarding myelofibrosis in toto.
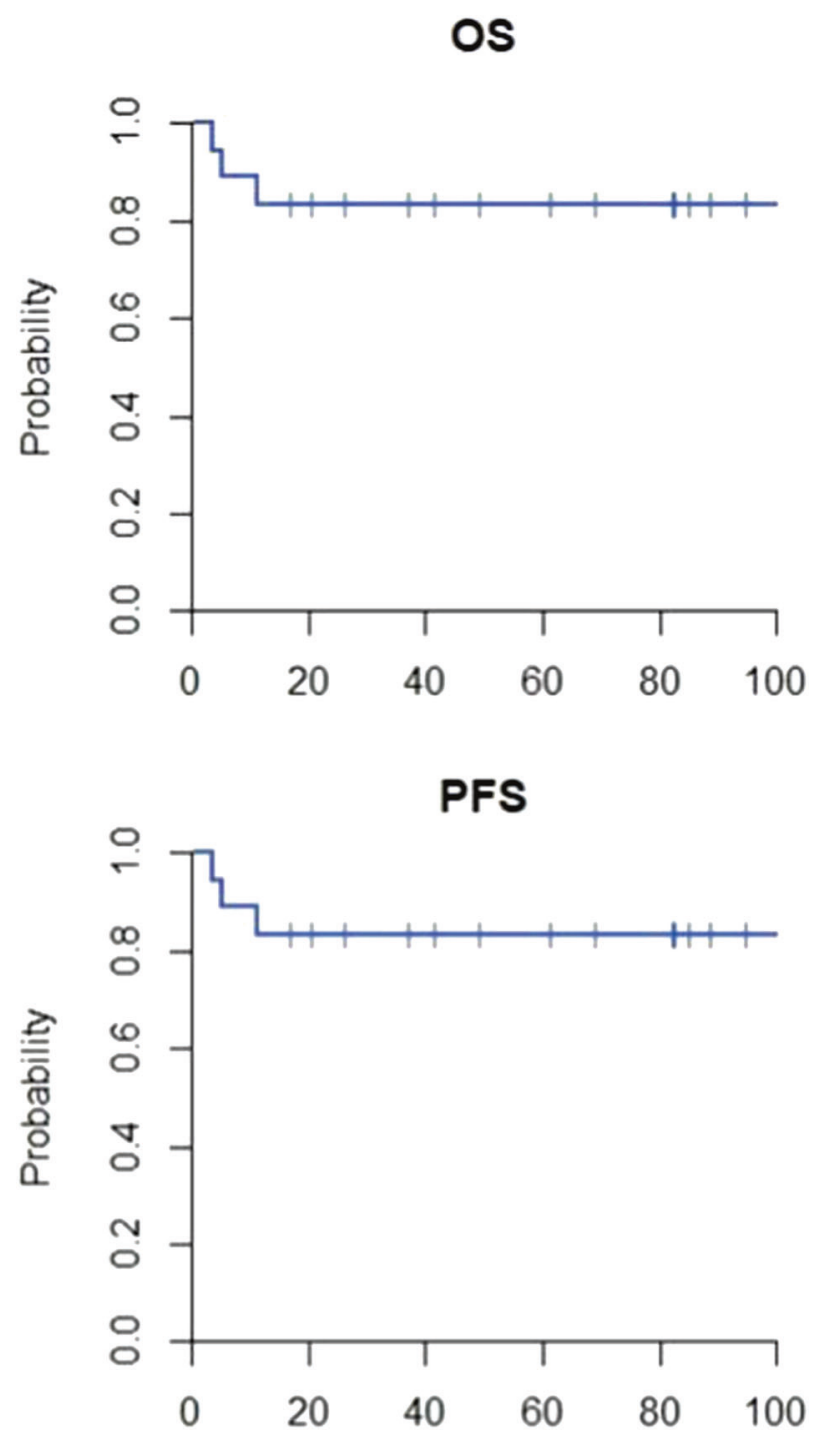

[[O062 Image] 1. OS and PFS of MPL patients after transplant]

Disclosure: Nothing to declare

\section{3}

TKI discontinuation after allogeneic stem cell transplantation in patients with chronic myeloid leukemia (CML): A retrospective study from the chronic malignances working party (CMWP)

Hugues de Lavallade, Dirk-Jan Eikema ${ }^{2}$, Jennifer Hoek $^{3}$, Marie Robin ${ }^{4}$, Maija Itälä-Remes ${ }^{5}$, Jakob Passweg $^{6}$, J. Cornelissen ${ }^{7}$, Arnold Ganser ${ }^{8}$, Charles Craddock $^{9}$, Mahmoud Aljurf ${ }^{10}$, Goda Choi ${ }^{11}$, Arnon Nagler ${ }^{12}$, Nicolaus Kröger ${ }^{13}$, Paul Browne ${ }^{14}$, Peter Dreger ${ }^{15}$, Christof Scheid $^{16}$, Mareike Verbeek ${ }^{17}$, Jürgen Finke ${ }^{18}$, Yves Chalandon ${ }^{19}$, Ibrahim Yakoub-Agha ${ }^{20}$ 
${ }^{1}$ King's College Hospital NHS Foundation Trust, London, United Kingdom, ${ }^{2}$ EBMT Statistical Unit, Leiden, Netherlands, ${ }^{3}$ EBMT Data Office, Leiden, Netherlands, ${ }^{4}$ Hospital St. Louis, Hematologie, Paris, France, ${ }^{5} \mathrm{HUCH}$ Comprehensive Cancer Center, Helsinki, Finland, ${ }^{6}$ University Hospital, Basel, Switzerland, ${ }^{7}$ Erasmus MC Cancer Institute, Rotterdam, Netherlands, ${ }^{8}$ Hannover Medical School, Hannover, Germany, ${ }^{9}$ Queen Elizabeth Hospital, Birmingham, United Kingdom, ${ }^{10}$ King Faisal Specialist Hospital \& Research Centre, Riyadh, Saudi Arabia, ${ }^{11}$ University Medical Center Groningen, Groningen, Netherlands, ${ }^{12}$ Chaim Sheba Medical Center, Tel-Hashomer, Israel, ${ }^{13}$ University Hospital Eppendorf, Hamburg, Germany, ${ }^{14}$ Hope Directorate St. James's Hospital, Dublin, Ireland, ${ }^{15}$ University of Heidelberg, Heidelberg, Germany, ${ }^{16}$ University of Cologne, Cologne, Germany, ${ }^{17}$ Klinikum Rechts der Isar, Munich, Germany, ${ }^{18}$ University of Freiburg, Freiburg, Germany, ${ }^{19}$ Hôpitaux Universitaire de Genève, University of Geneva, Geneva, Switzerland, ${ }^{20}$ Hopital Huriez, Lille, France

Background: A significant proportion of CML patients undergoing allogeneic stem cell transplantation (alloHSCT) restart TKI following transplant to prevent relapse. There is however no data to support if TKI can be discontinued following allo-HSCT and whether such patients can safely discontinue TKI remains controversial. Practices varies among transplant centres depending on countrywide practices, centre experience, duration of molecular remission, patients 'wish and analysis of retrospective data on the outcome of patients who discontinue TKI after transplant may provide further insight to help elaborating future guidelines. The present study objective is to investigate the outcome of patients with CML who discontinue TKI therapy after restarting TKI following allo-HSCT. This retrospective study may be helpful to support future guidelines.

Methods: Through the EBMT database of patients who received an allo-HSCT between March 2004 and September 2013, we identified $81 \mathrm{CML}$ patients who restarted TKI treatment post allo-HSCT and stopped it after at least 3 months of TKI therapy.

Results: Out of 81 patients who discontinued TKI, 21 were in first chronic phase (CP1) at the time of transplant, 31 in second or third chronic phase (CP1 and CP2) and 28 in accelerated or blastic phase $(\mathrm{AP} / \mathrm{BP})$ or primary refractory disease. One patient had missing data at the time to transplant.

Allo-HSCT conditioning was of reduced intensity (RIC) in 27 patients and myeloablative (MAC) in 54 patients, including TBI in 29 patients. TKI therapy was (re)started in all patients after a median time from transplant of 4.4 months (range, 0.4 to 57.6 months) for a duration of 9.7 (range, 3.1-62.3 months). After a median time from TKI discontinuation of 65.8 months (range 51.8-71.1), 5-years progression free survival (PFS) and Overall survival (OS) were $51 \%$ (95\% CI 39 to $62 \%$ ) and $62 \%$ (95\%CI 51 to $73 \%$ ) respectively. Patients in $\mathrm{CP} 1$ at the time of transplant had a significantly higher 5-years OS compared to those in CP2/ $\mathrm{CP} 3$ or $\mathrm{AP} / \mathrm{BP}, 100 \%$ vs $37 \%$ (95\%CI 17 to $58 \%$ ) and $59 \%$ (95\%CI 39 to $80 \%)$ respectively $(\mathrm{p}<0.001$, Figure 1$)$. Causes of death (COD) in the CP2/3 and AP/BP groups were relapse (12/31 and 5/28 respectively) and NRM (4/31 and 4/28 respectively, 1 missing COD in each group).

Conclusions: Post-transplant TKI discontinuation appears safe in patients who receive an allo-HSCT while still in first chronic phase. However, such approach in patients who transform to advanced phases before alloHSCT remains a matter of concerns given the high incidence of post allo-HSCT relapse. Further analysis to identify reason for restating TKI post-transplant and for subsequent discontinuation will be performed after further data is collected through the ongoing Data Quality Initiative (DQI) in CML.

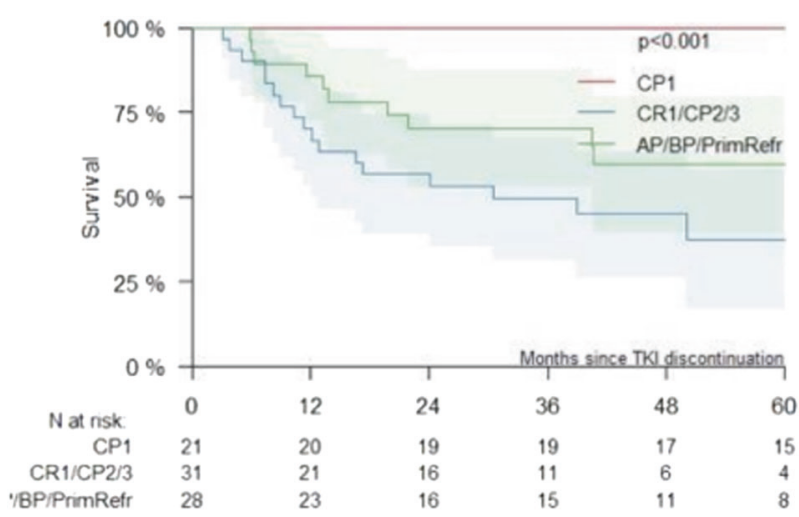

[[O063 Image] 1. OS according to pre-transplant status]

\begin{tabular}{ll}
\hline Demographics & Patients ( $\mathrm{n}=81)$ \\
\hline $\begin{array}{l}\text { Median age (range) } \\
\text { Gender (M/F, missing) }\end{array}$ & $\begin{array}{l}52 \text { years (18.5-67.3) } \\
52 / 28 \text { (one missing) }\end{array}$ \\
$\begin{array}{l}\text { Median interval diagnostic-transplant } \\
\text { (months, range) }\end{array}$ & $16.8(2.6-253.6)$ \\
$\begin{array}{l}\text { Pre-transplant response: } \\
\text { Haematological; Cytogenetic; }\end{array}$ & $48(16$ missing); $18(25$ \\
Molecular & missing); $8(20$ missing) \\
Conditioning: RIC/MAC & $27 / 54$ \\
Median TKI duration (months, range) & $9.7(3.1-62.3)$ \\
\hline
\end{tabular}

[[O063 Table] 1. Patient's characteristics]

Disclosure: HdL: research grants from Incyte and BMS travel grants from Incyte; honoraria from Incyte, BMS and NovartisYC: advisory board from Novartis, BMS, Pfizer and Incyte 
Travel grants from Novartis and BMS

\section{Conditioning regimen}

\section{4}

effect of the thiotepa dose in the TBF conditioning regimen in patients undergoing allogeneic stem-cell transplantation for AML in CR, report from the EBMT ALWP

Jean El Cheikh', Myriam Labopin', Ali Bazarbachi', Emanuele Angelucci $^{3}$, William Arcese ${ }^{4}$, Stella Santarone ${ }^{5}$, Francesca Bonifazi ${ }^{6}$, Angelo Michele Carella ${ }^{7}$, Luca Castagna $^{8}$, Bruno Benedetto', Anna Paola Iori ${ }^{10}$, Giorgio La Nasa ${ }^{11}$, Bipin Savani ${ }^{12}$, Arnon Nagler ${ }^{13}$, Mohamad Mohty $^{14}$

\footnotetext{
${ }^{1}$ American University of Beirut Medical Center, Beirut, Lebanon, ${ }^{2}$ EBMT Paris Study Office / CEREST-TC, Saint Antoine Hospital, Paris, France, ${ }^{3}$ Ospedale San Martino, Genova, Italy, ${ }^{4}$ Tor Vergata University of Rome, Rome, Italy, ${ }^{5}$ Medicina Trasfusionale e Biotecnologie, Pescara, Italy, ${ }^{6}$ Bologna University, S.Orsola-Malpighi Hospital, Bologna, Italy, ${ }^{7}$ IRCCS, Casa Sollievo della Sofferenza, Giovanni Rot, Italy, ${ }^{8}$ Istituto Clinico Humanitas, Milano, Italy, ${ }^{9}$ S.S.C.V.D Trapianto di Cellule Staminali, A.O.U Citta della Salute e della Scienza di Torino Presidio Molinette, Torino, Italy, ${ }^{10}$ Univ. La Sapienza, Rome, Italy, ${ }^{11}$ Centro Trapianti Unico Di CSE Adulti e Pediatrico A. O Brotzu, Cagliari, Italy, ${ }^{12}$ Vanderbilt University Medical Center, Nashville, TN, United States, ${ }^{13}$ Chaim Sheba Medical Center, Tel Hashomer, Israel, ${ }^{14}$ Hopital Saint Antoine, Paris, France
}

Background: Allogeneic stem-cell transplantation (alloSCT) is a well-established treatment modality for high-risk hematopoietic malignancies. However, the optimal intensity of myeloablation with a reduced-toxicity conditioning regimen to decrease relapse rate after allo-SCT without increasing TRM has not been well established. Thiotepa is an alkylating compound with antineoplastic activity and immunosuppressive properties, as well as the ability to penetrate the blood brain barrier. Thiotepa has become an integral part of the Thiotepa, Busulfan iv (Busilvex), and fludarabine (TBF) conditioning regimen, which is being used with increasing frequency, particularly for Haploidentical and cord-blood transplants. However, few studies have focused on analyzing the effect of Thiotepa dose in the TBF conditioning.

Methods: The aim of this study was to evaluate the optimal dose of Thiotepa, as part of the TBF conditioning for allo-SCT in adults with AML transplanted in complete remission $(\mathrm{CR})$, by comparing the transplantation outcomes of patients who received $5 \mathrm{mg} / \mathrm{kg}$ Thiotepa and 2 days of Busilvex $(6.4 \mathrm{mg} / \mathrm{kg})$ (T1B2F) versus those who received $10 \mathrm{mg} / \mathrm{kg}$ Thiotepa with 2 days of Busilvex (T2B2F) or 3 days of Busilvex (9.6 mg/kg) (T2B3F) using a large dataset from the EBMT registry.

Results: We identified 639 AML patients allotransplanted between January 2009 and June 2018 from matched related or unrelated donors or $\mathrm{T}$ replete haplo-identical donors. 127 patients $(20 \%)$ received (T1B2F); 113 patients $(18 \%)$ received (T2B2F); the remaining 399 patients (62\%) received (T2B3F). all the patients were in CR at transplant. Median follow-up was 20 months (IQR: 9-37). Outcomes are summarized in the table 1. Acute GVHD grade II-IV was $15 \%, 17 \%$ and $19 \%(\mathrm{p}=0.14)$ respectively. At 2 years the non-relapse mortality (NRM) was $22 \%, 25 \%$ and $21 \%$ respectively $(\mathrm{p}=0.62)$; the relapse incidence $(\mathrm{RI})$ was $18 \%, 19 \%$ and $17 \%(\mathrm{p}=0.37)$ respectively; the leukemia free survival (LFS) was $60 \%$ vs $56 \%$ vs $63 \%$ $(\mathrm{p}=0.21)$ respectively and the overall survival (OS) was $67 \%$ vs $62 \%$ vs $67 \%(\mathrm{p}=0.56)$ in the 3 groups, respectively. the $2-$ year GRFS was $50 \%, 43 \%$, and $55 \%$ respectively $(\mathrm{p}=0.02)$. In multivariate analysis, acute GVHD was higher for patients receiving $\mathrm{T} 2 \mathrm{~B} 2 \mathrm{~F}(\mathrm{p}=0.01$; HR 2.25) or $\mathrm{T} 2 \mathrm{~B} 3 \mathrm{~F}(\mathrm{p}=0.02 ; \mathrm{HR}$ 2.05) as well as for patients receiving transplant from Haploidentical donor or peripheral blood stem cells, whereas NRM was higher for older patients $(p=0.001$; HR 1.56), patients receiving $\mathrm{T} 2 \mathrm{~B} 3 \mathrm{~F}(\mathrm{p}=0.008$; HR 2.28) or Haploidentical transplant ( $\mathrm{p}=0.009$; HR 2.2). Importantly, OS was lower for older patients $(\mathrm{p}=0.001$; HR 1.4$)$ or for patients receiving T2B3F ( $\mathrm{p}=0.004$; HR 2.09). the multivariate analysis was adjusted to all the different factors between the 3 groups.

Conclusions: T2B2F is associated with higher incidence of acute GVHD compared to T1B1F whereas T2B3F associated with higher NRM, a higher incidence of acute GVHD and a lower OS compared to T1B1F. With the limitation of the retrospective nature of registry data, these results suggest that a lower dose-intensity of Thiotepa and Busilvex in the TBF regimen in general may yield better results in AML patients transplanted in complete remission.

\begin{tabular}{|c|c|c|c|c|c|c|c|c|c|}
\hline & RI & NRM & LFS & OS & GRFS & $\begin{array}{l}\text { Acute } \\
\text { GVHD } \\
\text { II-IV }\end{array}$ & $\begin{array}{l}\text { Acute } \\
\text { GVHD } \\
\text { III-IV }\end{array}$ & $\begin{array}{l}\text { chronic } \\
\text { GVHD }\end{array}$ & $\begin{array}{l}\text { extensive } \\
\text { chronic } \\
\text { GVHD }\end{array}$ \\
\hline $\begin{array}{l}\text { T1B2F } \\
(\mathrm{n}=127) \\
\text { T2B2F } \\
(\mathrm{n}=113) \\
\text { T2B3F } \\
(\mathrm{n}=399) \\
\mathrm{p} \text { value }\end{array}$ & $\begin{array}{l}18.1 \% \\
{[10.6-} \\
27.3] \\
18.7 \% \\
{[11.3-} \\
27.4] \\
16.7 \% \\
{[12.8-} \\
21] 0.37\end{array}$ & $\begin{array}{l}21.9 \% \\
{[14-30.9]} \\
25.1 \% \\
{[16.3-} \\
34.9] \\
20.7 \% \\
{[16.5-} \\
25.3] 0.62\end{array}$ & $\begin{array}{l}60.3 \% \\
{[49.8-} \\
70.9] \\
56.3 \% \\
{[45.5-} \\
67.1] \\
62.6 \% \\
{[57.2-} \\
68] 0.21\end{array}$ & $\begin{array}{l}67.2 \% \\
{[56.7-} \\
77.7] \\
62.2 \% \\
{[51-73.4]} \\
67.1 \% \\
{[61.8-} \\
72.4] 0.56\end{array}$ & $\begin{array}{l}50.4 \% \\
{[39.8-61]} \\
43.1 \% \\
{[32.6-} \\
53.6] \\
55.3 \% \\
{[49.9-} \\
60.8] 0.02\end{array}$ & $\begin{array}{l}15.1 \% \\
{[9.4-22.2]} \\
17 \% \\
{[10.6-} \\
24.8] \\
19.1 \% \\
{[15.3-} \\
23.2] 0.13\end{array}$ & $\begin{array}{l}4.2 \%[1.6- \\
9] 6.6 \% \\
{[2.9-12.4]} \\
8.1 \%[5.6- \\
11.1] 0.31\end{array}$ & $\begin{array}{l}31.4 \% \\
{[21.4-41.8] 34 \%} \\
{[23.4-44.8]} \\
28.4 \% \\
{[23.2-33.8] 0.14}\end{array}$ & $\begin{array}{l}15.5 \% \\
{[8.6-24.2]} \\
16.6 \% \\
{[9.1-26]} \\
9.5 \%[6.5- \\
13.2] 0.10\end{array}$ \\
\hline
\end{tabular}

[[0064 Table] 1. results at 2 years]

Clinical Trial Registry: na

Disclosure: no disclosure to decalre 


\section{5}

The role of anti-thymocyte globulin withthiotepaBusulfan-Fludarabine based conditioning in patients undergoing haploidentical stem cell transplant and posttransplant cyclophosphamide

Jean El Cheikh ${ }^{1}$, Raynier Devillier ${ }^{2}$, Remy Dulery, Radwan Massoud ${ }^{1}$, Nour Moukalled ${ }^{1}$, Nohra Ghaoui ${ }^{\text {, }}$ Thomas Pagliardini ${ }^{2}$, Fabrizio Marino ${ }^{4}$, Ali Bazarbachi', Lucas Castagna ${ }^{4}$, Mohammad Mohty ${ }^{3}$, Didier Blaise ${ }^{2}$

${ }^{1}$ American University of Beirut Medical Center, Beirut, Lebanon, ${ }^{2}$ Institut Paoli Calmettes, Marseille, France, ${ }^{3}$ Hôpital Saint-Antoine, AP-HP, Paris, France, ${ }^{4}$ Humanitas University, Milan, Italy

Background: TheThiotepa-Busulfan-Fludarabine (TBF) based conditioning regimen is widely used in T-cell repleted haploidentical transplantation (Haplo) with post-transplant cyclophosphamide. However, the use of Anti-thymocyte globulin (ATG) has not been well established. It decreases the incidence of graft versus host disease however some claim that it's at the cost of increased relapse. We conducted this multi centric study to compare the outcomes of patients who underwent Haplo with TBF conditioning regimen with ATG to those without.

Methods: This is a multicentric retrospective study. Data was collected from 4 centers, the American University of Beirut Medical Center, Hospital Saint Antoine Paris, institute Paoli Calmette Marseille, and Humanitas Research Hospital Milan. We included all consecutive adult patients who underwent Haplo with TBF conditioning. The conditioning consisted of thiotepa $5 \mathrm{mg} / \mathrm{kg}$ per day infused on days -7 and/or -6 , fludarabine $30 \mathrm{mg} / \mathrm{m} 2$ infused on day -5 to day -2 ; and busulfan $130 \mathrm{mg} / \mathrm{m} 2$ infused on day -5 to day -3. Graft versus host disease (GVHD) prophylaxis consisted of post transplantation cyclophosphamide $50 \mathrm{mg} /$ $\mathrm{kg}$ per day on day +3 and day +5 , cyclosporine on day +6 and readjusted according to level, and mycophenolate mofetil $500 \mathrm{mg}$ every 6 hours beginning on days +6 to +28 or +35 depending on the center. Patients who received ATG received a dose of $2.5 \mathrm{mg} / \mathrm{kg}$ per day.

Results: We included a total of 268 patients, 69 of whom (26\%) received ATG (group 2) as part of the conditioning chemotherapy. Patients who received ATG had a younger median age compared to the second group without ATG (group 1) (53 and 58 years respectively; p value 0.004). (63\% vs $61 \%)$ of each group had acute leukemia, and $(71 \%$ vs $70 \%$ ) were in complete remission at the time of transplant, while 47 patients (24\%) in the group 1 had progressive disease at transplant. 151 patients $(56.5 \%)$ had an intermediate disease risk index (DRI). In the ATG group, 59 patients (30\%) compared to $50(73 \%)$ in the other group received $5 \mathrm{mg} / \mathrm{kg}$ Thiotepa, while 140 (70) and 19 (27\%) received
$10 \mathrm{mg} / \mathrm{kg}$ respectively. Peripheral blood stem cells were the most common graft source in both groups (83\% and $88 \%$ respectively). At a median follow-up of 15.4 months, patients receiving ATG had a statistically significant decreased risk of acute graft versus host disease (aGVHD) (RR 0.47; p value $0.031)$, and non-relapse mortality (NRM) at 24 months (RR 0.5 ; $\mathrm{p}$ value 0.027 ). ATG also resulted in higher progression and overall survival at 24 months, which was not statistically significant $(66.2 \%$ and $59.8 \%$; $p$ value 0.168 , with $76.6 \%$ and $67.8 \%$; p value 0.056 respectively).

Conclusions: ATG as part of the pre-transplantation conditioning leads to significant reduction in aGVHD and NRM at 24 months without significant effects on PFS or OS.

Disclosure: Nothing to declare

\section{6}

Abstract already published.

\section{7}

Abstract already published.

\section{8}

Randomized, multicenter, phase II trial of clofarabine / ARA-C (CLARAC) or of flamsa treatment in high risk AML or advanced MDS scheduled for allogeneic SCT

Gernot Beutel ${ }^{1}$, Stefanie Buchholz, Marie von LilienfeldToal $^{2}$, Gerhard Behre ${ }^{3}$, Michael Stadler ${ }^{I}$, Inken Hilgendorf', Theodor Framke, Anika Großhennig', Elke Dammann ${ }^{1}$, Catherina Lück, Dietger Niederwieser ${ }^{3}$, Andreas Hochhaus', Armin Koch ${ }^{4}$, Matthias Eder', Arnold Ganser ${ }^{l}$

${ }^{1}$ Hannover Medical School, Hannover, Germany, ${ }^{2}$ Universitätsklinikum Jena, Jena, Germany, ${ }^{3}$ University of Leipzig, Leipzig, Germany, ${ }^{4}$ Hannover Medical School, Hannover, Germany

Background: High-risk leukemia is associated with poor prognosis and inferior outcome. In elderly or comorbid patients allogeneic SCT with myeloablativ conditioning regimen as the most effective treatment option is not available. Sequential regimen combining cytoreductive therapy with RIC has shown high antileukemic activity for high-risk patients with acceptable toxicity profile. This study is based on the observation that antileukemic effects have been described previously for the nucleoside analogue clofarabine.

Methods: The trial was designed as an investigatorinitiated prospective, multicenter, open-label, two-arm, parallel-group phase II study comparing ClAraC to FLAMSA regimen. FLAMSA regimen consists of Fludarabine $(30 \mathrm{mg} /$ $\mathrm{m} 2$, days -13 to -10$)$, Amsacrine $(100 \mathrm{mg} / \mathrm{m} 2$, days -13 to -10) and Cytarabine (2000 mg/m2, days -13 to -10$)$. ClAraC 
regimen consists of Clofarabine ( $30 \mathrm{mg} / \mathrm{m} 2$, days -16 to -12 ) and Cytarabine $(1000 \mathrm{mg} / \mathrm{m} 2$, days -16 to -12$)$. Both cytoreductive therapies were combined with $\mathrm{Bu} / \mathrm{Cy}$ (Busulfan, $4 \times 0.8 \mathrm{mg} / \mathrm{kg}$, days -6 to -5 and Cyclophosphamide 60 $\mathrm{mg} / \mathrm{kg}$, days -4 to -3 ). As GvHD-prophylaxis ATG, CsA and MMF were used. Patients with high risk AML or advanced MDS (IPSS $\geq$ int2) with contraindication for conventional conditioning or refractory to induction therapy were eligible. Primary objective was to demonstrate that event-free survival is improved by ClAraC instead of the FLAMSA. Secondary objectives were overall and relapse-free survival, mortality rate, safety profiles and cardiac toxicity.

Results: Between 2011 and 2017, 62 patients were recruited, 2 patients did not meet the in-/exclusion criteria. A total of 60 were randomized with 30 patients each in the ClAraC and FLAMSA group.

Mean time to event was $656.6 \pm 84.6$ days for FLAMSA and $565.6 \pm 49.2$ days for ClAraC, respectively $(\mathrm{p}=0.177$, figure 1). In total 38 of the adverse events were serious with fatal outcome of 3 patients in the ClAraC and 4 patients in the FLAMSA group. Cardiac toxicity was observed in 26 patients in the ClAraC treatment arm, whereas 27 patients were affected in the FLAMSA treatment arm $(\mathrm{p}=0.730)$.

Overall survival for ClAraC was numerically, but not statistically inferior to FLAMSA $(\mathrm{p}=0.134)$. A part of $16 / 30$ $(53.3 \%)$ patients died until the end of the study in the ClAraC treatment arm, whereas only 12/30 (40.0\%) died in the FLAMSA treatment arm $(\mathrm{p}=0.134)$.

Conclusions: This study did compare two different conditioning regimens for allogeneic stem cell recipients with high risk AML/MDS. 62 patients have been included and 60 were randomized. The treatment arms were wellbalanced at study baseline for relevant covariates. Superiority of the ClAraC treatment regimen over the FLAMSA regimen could not be demonstrated. Consistently hazard ratios for event free survival, overall survival and relapsefree survival were in favor of the control group with FLAMSA treatment. No differences were found regarding cardiac toxicity, rate of engraftment, or chimerism. Regarding general safety parameters, no relevant differences between the two treatment strategies were found.

Clinical Trial Registry: EudraCT-No. 2010-021944-17

Disclosure: Conflict of interest: Nothing to declare. Funding: Federal Ministry of Education and Research (BMBF).

\section{9}

Impact of molecular genetics on outcome after different conditioning intensity in myelofibrosis undergoing allogeneic stem cell transplantation

Nico Gagelmann ${ }^{1}$, Markus Ditschkowski ${ }^{2}$, Rashit Bogdanov $^{2}$, Swan Bredin ${ }^{3}$, Marie Robin ${ }^{3}$, Bruno Cassinat ${ }^{3}$,
Rabia Shahswar ${ }^{4}$, Felicitas Thol', Michael Heuser ${ }^{4}$, Dietrich Beelen ${ }^{2}$, Anita Badbaran ${ }^{1}$, Nicolaus Kröger ${ }^{1}$

${ }^{1}$ University Medical Center Hamburg-Eppendorf, Hamburg, Germany, ${ }^{2}$ West German Cancer Center, Essen, Germany, ${ }^{3}$ Hôpital Saint-Louis, Paris, France, ${ }^{4}$ Hannover Medical School, Hannover, Germany

Background: Currently, there is no direct evidence to recommend specific conditioning intensities in myelofibrosis undergoing allogeneic stem cell transplantation. Moreover, recent risk stratifications for diagnosed myelofibrosis (MF) included specific mutation profiles as prognostic factors. Using clinical-molecular data from four different centers from Germany and France, we sought to determine whether molecular genetics have an impact on outcome after reduced intensity (RIC) and myeloablative conditioning (MAC) stem cell transplantation in MF.

Methods: Previously published methods were used to sequence myelofibrosis-associated genes (i.a. CALR, JAK2, MPL, ASXL1, SRSF2, EZH2, IDH1/2, DNMT3A, TET2, TP53). Risk stratifications according to Dynamic International Prognostic Scoring System (DIPSS), mutation-enhanced system (MIPSS70), genetic inspired prognostic system (GIPSS), prognostic model for secondary myelofibrosis (MYSEC-PM), cytogenetics as well as other clinical and transplant-specific variables were included in analyses. Cox model with hazard ratios (HR) was used for survival (OS) and cumulative incidence for relapse and non-relapse mortality (NRM). Risk ratios (RR) were obtained for subgroup analysis.

Results: The study included $361 \mathrm{MF}$ patients (260 primary and 101 secondary MF) of whom 230 received RIC and 131 MAC. The median follow-up was 61 months in RIC and 73 months in MAC and the median age was 57 and 54 years. Patients receiving RIC were at higher risk according to DIPSS, MIPSS70, and MYSEC-PM, whereas frequencies of driver mutation genotype (CALR, JAK2, $M P L$, triple negative) as well as $A S X L 1$ mutation were similar. HLA-mismatched unrelated donors were more frequent in RIC. Most common conditioning regimens for RIC were Bu/Flu (83\%) and Flamsa (12\%), and Flu/Mel (37\%), Treo/Flu and TBI/Flu (21\%, respectively) for MAC.

No significant difference was found for RIC versus MAC with respect to OS (62\% vs $57 \%$; $\mathrm{p}=0.35)$, relapse ( $16 \%$ vs $13 \% ; \mathrm{p}=0.60)$ or NRM ( $28 \%$ vs $30 \% ; \mathrm{p}=0.68)$. Early relapse within five months was increased after RIC (7\% vs $3 \%$ ). Including molecular variables, RIC showed higher but not significantly different OS rates in patients with $<3$ mutations, in triple negative driver mutation genotype, ASXL1 mutation (Figure 1). When evaluating patients with an ASXL1 mutation, those 88 patients who had only one ASXL1 or one additional mutation seemed to benefit from RIC showing 5-year OS of $65 \%$ vs $20 \%$ for MAC 
$(\mathrm{p}=0.001)$, whereas no difference was identified when more than two additional mutations were present $(57 \%$ vs $65 \%$; $\mathrm{p}=0.27)$. Furthermore, in patients with an $A S X L 1$ mutation and one additional driver mutation (CALR, MPL, JAK2), 5year OS was significantly different showing $63 \%$ in RIC vs $18 \%$ in MAC $(\mathrm{p}=0.005)$. Regarding current risk stratifications, RIC showed significantly improved OS only in high risk DIPSS and MYSEC-PM, whereas no difference was found regarding the remaining systems such as DIPSS-plus, MIPSS70 and GIPSS (Figure 1).

Conclusions: The evaluation of different conditioning intensities in MF did not favor RIC or MAC regarding currently existing risk stratifications. With respect to molecular genetics, a proportion of patients specifically harboring up to two mutations including ASXL1 may benefit from RIC with respect to OS.

\begin{tabular}{|c|c|}
\hline Variable & $\mathbf{R R}$ \\
\hline \multicolumn{2}{|l|}{ Age } \\
\hline$<40$ & 2.50 \\
\hline $40-49$ & 0.43 \\
\hline $50-60$ & 0.71 \\
\hline$>60$ & 1.13 \\
\hline \multirow{2}{*}{\multicolumn{2}{|c|}{$\begin{array}{l}\text { ASXL1 mutation } \\
\text { No }\end{array}$}} \\
\hline & \\
\hline \\
\hline \multicolumn{2}{|l|}{$\begin{array}{l}\text { Yes } \\
\text { CMV match }\end{array}$} \\
\hline$n / n$ & 0.74 \\
\hline$n / p$ & 0.52 \\
\hline pin & 0.70 \\
\hline $\mathrm{p} / \mathrm{p}$ & 1.22 \\
\hline \multicolumn{2}{|l|}{ Diagnosis } \\
\hline PMF & 1.08 \\
\hline SMF & 0.62 \\
\hline \multicolumn{2}{|l|}{ DIPSS } \\
\hline Low & 7.27 \\
\hline Intermediate-1 & 0.69 \\
\hline Intermediate.2 & 0.86 \\
\hline High & 0.50 \\
\hline \multicolumn{2}{|l|}{ DIPSS-plus } \\
\hline Low & 3.00 \\
\hline Intermediate-1 & 4.41 \\
\hline Intermediate-2 & 0.86 \\
\hline High & 0.95 \\
\hline \multicolumn{2}{|l|}{ Driver mutation } \\
\hline CALR & 0.67 \\
\hline JAK2 & 1.03 \\
\hline MPL & 0.50 \\
\hline Triple negative & 0.73 \\
\hline \multicolumn{2}{|l|}{ GIPSS } \\
\hline Low & 0.60 \\
\hline Intermediate-1 & 1.90 \\
\hline Intermediate $\cdot 2$ & 2.01 \\
\hline High & 0.33 \\
\hline \multicolumn{2}{|l|}{ HLA-match } \\
\hline Matched/mismatched related 0 & 0.83 \\
\hline Matched unrelated & 0.78 \\
\hline Mismatched unrelated & 0.65 \\
\hline \multicolumn{2}{|l|}{ KPS } \\
\hline $90-100$ & 0.91 \\
\hline$<90$ & 0.84 \\
\hline \multicolumn{2}{|l|}{ MIPSS70 } \\
\hline Lowfintermediate & 1.22 \\
\hline High & 0.99 \\
\hline \multicolumn{2}{|l|}{ MIPSS70-plus 2.0} \\
\hline \multicolumn{2}{|l|}{ Very low } \\
\hline Low & 2.37 \\
\hline Intermediate & 1.00 \\
\hline High & 0.68 \\
\hline Very high & 1.20 \\
\hline \multicolumn{2}{|l|}{ MYSEC-PM } \\
\hline Low & 0.28 \\
\hline Intermediate-1 & 0.39 \\
\hline Intermediate-2 & 1.07 \\
\hline High & 0.58 \\
\hline \multicolumn{2}{|l|}{ Number of all mutations } \\
\hline $0-2$ & 0.74 \\
\hline$>2$ & 1.19 \\
\hline
\end{tabular}

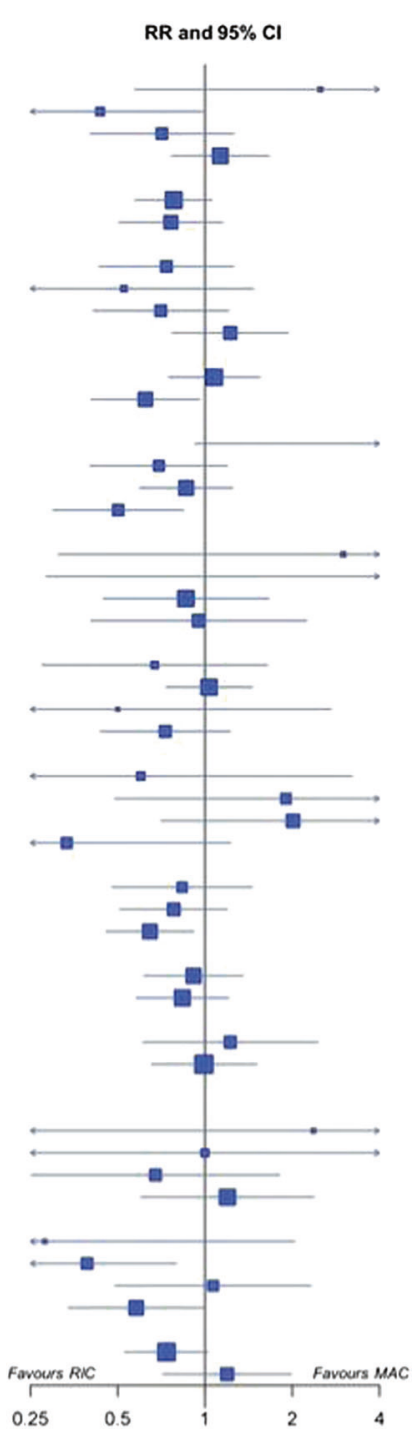

[[O069 Image] 1. Subgroup analysis on survival for RIC vs $M A C]$
Disclosure: Nothing to declare.

\section{O070}

Comparison of sequential conditioning regimens for allogeneic stem cell transplantation in relapsed/refractory AML: A study on behalf of the ALWP of EBMT

Thomas Heinicke ${ }^{1}$, Myriam Labopin ${ }^{2,3}$, Emmanuelle Polge $^{4}$, Johanna Tischer ${ }^{5}$, Arnold Ganser ${ }^{6}$, Nicolaus Kröger $^{7}$, Arne Brecht ${ }^{8}$, Lothar Kanz ${ }^{9}$, Christof Scheid ${ }^{10}$, Peter Dreger $^{11}$, Donald Bunjes ${ }^{12}$, Uwe Platzbecker ${ }^{13}$, Eva Wagner $^{14}$, Bipin N. Savani ${ }^{15}$, Arnon Nagler, ${ }^{4,16,17}$, Mohamad Mohty ${ }^{2,4}$

${ }^{1}$ Otto-von-Guericke University, Magdeburg, Germany, ${ }^{2}$ Hopital Saint-Antoine, Paris, France, ${ }^{3}$ Acute Leucemia Working Party of EBMT, Paris, France, ${ }^{4}$ Acute Leukemia Working Party of EBMT Office, Paris, France, ${ }^{5}$ University Hospital of Munich-Campus Grosshadern, Munich, Germany, ${ }^{6}$ Hannover Medical School, Hannover, Germany, ${ }^{7}$ University Hospital Eppendorf, Hamburg, Germany, ${ }^{8}$ DKD Helios-Klinikum, KMT- Abteilung, Wiesbaden, Germany, ${ }^{9}$ University Tübingen, Tübingen, Germany, ${ }^{10}$ University of Cologne, Cologne, Germany, ${ }^{11}$ University of Heidelberg, Heidelberg, Germany, ${ }^{12}$ University Hospital Basel Ulm, Ulm, Germany, ${ }^{13}$ University Hospital Leipzig, Leipzig, Germany, ${ }^{14}$ University Medical Center Mainz, Mainz, Germany, ${ }^{15}$ Vanderbilt University Medical Center, Brentwood, TN, United States, ${ }^{16}$ Tel-Hashomer and Tel Aviv University, Tel Hashomer, Israel, ${ }^{17}$ Tel Aviv University, Tel Aviv, Israel

Background: Sequential conditioning regimens (SR) have shown substantial activity in relapsed/refractory acute myeloid leukemia (r/rAML). The original prototype SR is the FLAMSA-CYTBI regimen. Various modifications of this protocol have been developed in recent years (see table 1). In the current study, we compared the outcomes of patients suffering from $\mathrm{r} / \mathrm{rAML}$ that had received their first allogeneic stem cell transplantation (alloSCT) after conditioning with a SR.

Methods: Adult patients with r/rAML who had received their first alloSCT following SR conditioning between 2000 and 2017 and were reported to the EBMT registry were analyzed. Patients were grouped according to the type of SR used as shown in table1. The FLAMSA-CYTBI group served as comparator for all others. The primary endpoint was leukemia-free survival (LFS). Secondary endpoints were overall survival (OS), relapse incidence (RI), nonrelapse mortality (NRM), refined graft-versus-host-diseasefree, relapse-free survival (GRFS), acute (a)GVHD and chronic (c)GVHD. Multivariate analysis was done using Cox regression. 
Results: A total of 2250 patients were eligible for the study of which 1240 (55\%) were male. Median follow-up was 41 months (IQR 14.4-78.1). Median age was 54.7 years (IQR: 44.2-62.2). Donors were matched siblings/syngeneic, unrelated and other relatives in 666(29.6\%), 1482(65.9\%) and $102(4.5 \%)$ of the cases, respectively. LFS, OS, RI, NRM, GRFS, aGVHD and cGVHD at 2 years for all patients were $29 \%(95 \%$ CI $26.9-31), 35.2 \%(95 \%$ CI: $33-$ $37.4)$, $46.4 \%(95 \%$ CI: $44.2-48.6), \quad 24.6 \%(95 \%$ CI: $22.8-$ 26.5), 22.2\%(95\%CI: 20.3-24.1), $28.2 \%(95 \%$ CI: 26.3 $30.1)$ and $31.5 \%(95 \%$ CI: 29.2 - 33.9), respectively. Univariate analysis revealed that LFS and OS were not significantly different between groups. However, lowest RI and highest NRM, aGVHD II-IV and aGVHD III-IV were seen in the MEL group with 34.3\%(26.7-42.1), 34.9\%(27.242.6), 37.6\%(30.2-45) and 26.1\%(19.6-33), respectively. Highest cGVHD was seen in the OTHERS group with 40.8\%(29.6-51.8). Highest GRFS was found in the TREO group with 30\%(18.5-41.4).

Multivariate analysis showed that compared to FLAMSA-CYTBI there was no significant difference in LFS, OS, GRFS, cGvHD or extensive cGVHDfor any of the other treatment groups. However, the BUCY and MEL groups were associated with reduced RI with a hazard ratio (HR) and $95 \% \mathrm{CI}$ of $0.79(0.64-0.98), \mathrm{p}=.03$, and $0.60(0.41-$ $0.87), \mathrm{p}=.007$, respectively. In addition, the CLOFA and MEL groups were associated with increased NRM with a HR of 1.49(1.03-2.15), $\mathrm{p}=.03$ and $1.60(1.06-2.42), \mathrm{p}=.025$, respectively. Acute GvHD II-IV and aGvHD III-IV were both higher in the MEL group with HR of 1.56 (1.06-2.29), $\mathrm{p}=.023$ and $2.12(1.36-3.3), \mathrm{p}<10 \mathrm{e}-3$, respectively.

\begin{tabular}{lll}
\hline Group & Regimens & Patients N \\
\hline CYTBI & ARAC+AMSA+FLUDA+Cy+TBI & 756 \\
BU & $\begin{array}{l}\text { ARAC+AMSA+FLUDA+Bu ARAC+Bu } \\
\text { +FLUDA+Idarub ARAC+Daunorub+Bu+Flu }\end{array}$ & 421 \\
BUCY & $\begin{array}{l}\text { ARAC+AMSA+FLUDA+Bu+Cy ARAC } \\
\text { +CCNU+Bu+Cy ARAC+FLUDA+Bu+Cy }\end{array}$ & 494 \\
CLOFA & ARAC+Clofa+Cy+TBI ARAC+Clofa+Bu & 191 \\
& + Cy ARAC+FLUDA+Bu+Clofa & \\
MEL & ARAC+AMSA+FLUDA+Mel ARAC & 171 \\
& +AMSA+FLUDA+Cy+Mel ARAC+FLUDA \\
& +Idarub+Mel & \\
TREO & ARAC+AMSA+FLUDA+Treo & 82 \\
OTHER & ARAC+AMSA+FLUDA+TBI ARAC+Cy & 135 \\
& +Daunorub+FLUDA ARAC+AMSA & \\
& + FLUDA+Cy ARAC+FLUDA+Idarub & \\
& / Other
\end{tabular}

[[O070 Table] 1. Table 1 Groups of Sequential Regimens]

Conclusions: Results of allogeneic transplantation in patients with r/rAML following the various sequential regimens are equivalent to those of the original FLAMSACYTBI with similar LFS, OS and GRFS.

Disclosure: Thomas Heinicke: Eurocept: travel grant; Gilead: travel grant; Jazz Pharmaceuticals. travel grant; all other authors: Nothing to declare.

\section{1}

Bendamustine-based versus BEAM conditioning regimen. A matched controlled study in diffuse large B-cell lymphoma from LYSA and LWP-EBMT

Baptiste Lapierre ${ }^{I}$, Ariane Boumendil ${ }^{2}$, Christian Creveuil $^{1}$, Sylvain Garciaz ${ }^{3}$, Frederic Peyrade ${ }^{4}$, Hervé Finel ${ }^{2}$, Irma Khvedelidze $e^{2}$, Sandra Malak ${ }^{5}$, Richard Lemal ${ }^{6}$, Mari Virgine Larcher ${ }^{7}$, Luc Matthieu Fornecker ${ }^{10}$, Nathalie Fegueux $^{11}$, Caroline Delette ${ }^{12}$, Ahmad Ibrahim ${ }^{13}$, Bertrand Joly $^{14}$, Loic Fouillard ${ }^{15}$, Steven Legouill ${ }^{16}$, Olivier Casasnovas $^{17}$, Mohammed Bekadja ${ }^{18}$, Adrien Chauchet ${ }^{19}$, Sylvain Chantepie ${ }^{1}$, Remy Gressin ${ }^{20}$, Ibrahim Yakoub Agha $^{21}$, Roch Houot ${ }^{22}$, Mohammad Mohty ${ }^{23}$, Edouard Durot $^{24}$, Sylvain Choquet ${ }^{25}$, Catherine Thieblemont ${ }^{26}$, Stephen Robinson ${ }^{27}$, Krimo Bouabdallah ${ }^{28}$, Sylvia Montoto $^{29}$, Gandhi Damaj ${ }^{1}$

${ }^{1}$ University Hospital, Caen, France, ${ }^{2}$ Saint-Antoine Hospital, European Society for Blood and Marrow Transplantation Lymphoma Working Party Paris Office, Paris, France, ${ }^{3}$ Institut Paoli Calmettes, Marseille, France, ${ }^{4}$ Centre Antoine Lacassagne, Nice, France, ${ }^{5}$ Institut Curie, Saint Cloud, France, ${ }^{6} \mathrm{CHU}$ Clermont Ferrand, Clermont Ferrand, France, ${ }^{7} \mathrm{CHU}$ Toulouse, Toulouse, France, ${ }^{10} \mathrm{CHU}$ Strasbourg, Strasbourg, France, ${ }^{11} \mathrm{CHU}$ Montpellier, Montpellier, France, ${ }^{12} \mathrm{CHU}$ Amiens Picardie, Amiens, France, ${ }^{13} \mathrm{Mak}-$ kassed General Hospital, Beirut, Lebanon, ${ }^{14} \mathrm{Centre}$ Hospitalier Sud Francilien, Corbeil-Essonnes, France, ${ }^{15}$ Centre Hospitalier Meaux, Meaux, France, ${ }^{16} \mathrm{CHU}$ Nantes, Nantes, France, ${ }^{17} \mathrm{CHU}$ Dijon, Dijon, France, ${ }^{18} \mathrm{EHU}$, Oran, Algeria, ${ }^{19} \mathrm{CHU}$ Besancon, Besancon, France, ${ }^{20} \mathrm{CHU}$ Grenoble Alpes - Université Grenoble Alpes, Grenoble, France, ${ }^{21} \mathrm{CHU}$ Lille, Lille, France, ${ }^{22} \mathrm{CHU}$ Rennes, Rennes, France, ${ }^{23}$ Hopital Saint-Antoine, Paris, France, ${ }^{24} \mathrm{CHU}$ Reims, Reims, France, ${ }^{25}$ Hopital Pitié-Salpétrière, Paris, France, ${ }^{26}$ Hopital Saint Louis, Paris, France, ${ }^{27}$ University Hospital Bristol NHS Foundation Trust, Bristol, United Kingdom, ${ }^{28} \mathrm{CHU}$ Bordeaux, Hôpital Haut-Leveque, Bordeaux, France, ${ }^{29} \mathrm{St}$ Bartholomew's Hospital, Barts Health NHS Trust, London, United Kingdom

Background: Bendamustine-based conditioning regimens have been extensively used prior to autologous stem cell transplantation (ASCT) for lymphoma. We recently reported on the toxicities in 474 lymphoma patients who received an ASCT after BeEAM (bendamustine, etoposide, 
cytarabine, melphalan) at LYSA centres. The aim of this study was to evaluate the efficacy of the BeEAM in comparison with BEAM regimen, in terms of overall survival (OS), lymphoma-free survival (LFS), relapse incidence (CIR) and non-relapse mortality (NRM), in patients with diffuse large B-cell lymphoma (DLBCL).

Methods: 131 patients with DLBCL from LYSA centers were matched to 230 patients from the EBMT database. Inclusion criteria were adult patients with DLBCL, who received $1^{\text {st }}$ ASCT between 2012 and 2016 after BeEAM or BEAM conditioning. Matched criteria were age at transplant, gender, stage at diagnosis, number of prior lines of chemotherapy, disease status at transplant and year of transplant. Hazard ratios for OS and LFS were estimated using Cox regression models stratified on the matched sets, to account for the matched-cohort design. Stratified Fine and Gray regression models were used for NRM and CIR

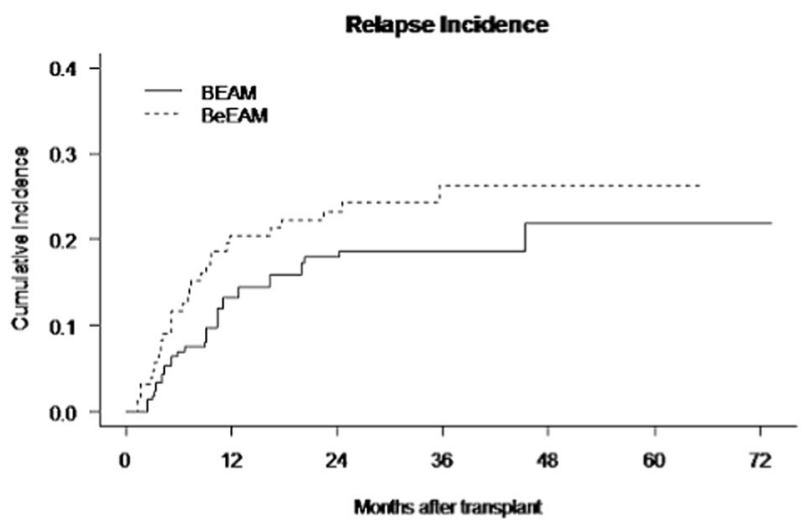

[[O071 Image] 1. figure 1: cumulative incidence of relapse]

\begin{tabular}{|c|c|c|c|}
\hline & BEAM $(n=230)$ & $\operatorname{BeEAM}(\mathrm{n}=131)$ & $P$ \\
\hline $\begin{array}{l}\text { Age at SCT, median (range) } \\
\text { (years) }\end{array}$ & $55,7(25,6-68,4)$ & $56,0(23,4-71,6)$ & 0.75 \\
\hline Gender, $\mathrm{n}(\%)$ male female & $\begin{array}{l}129(56,1 \%) 101 \\
(43,9 \%)\end{array}$ & $\begin{array}{l}74(56,5 \%) 57 \\
(43,5 \%)\end{array}$ & 1 \\
\hline AA Stage,n (\%) I-II III-IV & $\begin{array}{l}24(10,4 \%) 206 \\
(89,6 \%)\end{array}$ & $\begin{array}{l}17(13,0 \%) 114 \\
(87,0 \%)\end{array}$ & 1 \\
\hline $\begin{array}{l}\text { Disease status prior to AST, } n \\
(\%) \text { CR PR SD }\end{array}$ & $\begin{array}{l}207(90,0 \%) 22 \\
(9,6 \%) 1(0,4 \%)\end{array}$ & $\begin{array}{l}115(87,8 \%) 15 \\
(11,5 \%) 1(0,8 \%)\end{array}$ & 1 \\
\hline $\begin{array}{l}\text { Year of transplantation, n (\%) } \\
\text { 2012-2013 2014-2016 }\end{array}$ & $\begin{array}{l}194(84,3 \%) 36 \\
(15,7 \%)\end{array}$ & $\begin{array}{l}2(1,5 \%) 129 \\
(98,5 \%)\end{array}$ & $<0.001$ \\
\hline $\begin{array}{l}\text { Lymphome subtype, n (\%) } \\
\text { DLBCL t-DLBCL }\end{array}$ & $\begin{array}{l}173(82,4 \%) 37 \\
(17,6 \%)\end{array}$ & $\begin{array}{l}122(93,1 \%) \\
9(6,9 \%)\end{array}$ & 0.010 \\
\hline
\end{tabular}

Results: Patients' characteristics are detailed in Table 1. There were more patients with transformed NHL in the BEAM (18\%vs7\%). The median time to neutrophil count $>$ $0.5 \mathrm{G} / 1$ and platelet counts $>50 \mathrm{G} / 1$ were $10(4-24)$ and 10 (1- 124) days for BeEAM and 12 (1-34) and 12 (9-50) days for BEAM, respectively. Twenty-nine (22\%) patients in the
BeEAM and $35(15 \%)$ patients in the BEAM groups relapsed after a median time to relapse of 6 and 9 months, respectively. After a median follow-up from transplant of 33 months, 54 (15\%) patients died, 24 (18\%) in the BeEAM and $30(13 \%)$ in the BEAM groups, respectively. The main causes of death was lymphoma in 31 patients, (BeEAM:13, BEAM:18), infections in 16, (BeEAM:7, BEAM:9), secondary cancers in 2 patients (BeEAM).

There were no significant differences between BeEAM and BEAM regimens for OS: $\mathrm{HR}=1.02[(0.55-1.86)$, $\mathrm{p}=0.96]$, LFS: $\mathrm{HR}=1.27[(0.79-2.06), \mathrm{p}=0.32]$ or NRM: $H R=0.55[(0.21-1.43), p=0.22]$. However, relapse incidence (CIR) was higher in the BeEAM group $\mathrm{HR}=1.71$ [(1.01-2.86), $\mathrm{p}=0.045]$ (figure 1).

Conclusions: In this matched pair analysis, BeEAM and BEAM resulted in equivalent NRM, LFS and OS suggesting that both conditioning regimens may reasonably be employed in patients with DLBCL. The higher relapse rate following BeEAM requires further evaluation. A prospective randomized study will be required to confirm the equivalence of the two regimens.

[[O071 Table] 1. Table 1: Patient Characteristics]

Disclosure: No conflict of interest

Early complications / late effects \& quality of life / fertility

0072

Influence of inflammatory bowel disease on the outcome of allogeneic stem cell transplantation: A matched-pair analysis study from the transplant complications working party of EBMT

Zinaida Peric ${ }^{1}$, Christophe Peczynsky², Emmanuelle Polge $^{2}$, Nicolaus Kröger ${ }^{3}$, Henrik Sengeloev ${ }^{4}$, Peter Dreger $^{5}$, Grzegorz Helbig ${ }^{6}$, NH Russell, Donald Bunjes ${ }^{8}$, Gerard Socié $^{9}$, Dietger Niederwieser ${ }^{10}$, Victoria Potter ${ }^{11}$, Dietrich Beelen $^{12}$, Charles Crawley ${ }^{13}$, Adrian Bloor ${ }^{14}$, Jürgen Finke ${ }^{15}$, Christian Koenecke ${ }^{16}$, Grzegorz W Basak $^{17}$

${ }^{1}$ University Hospital Centre Zagreb, Zagreb, Croatia, ${ }^{2}$ EBMT Data Office Paris, Paris, France, ${ }^{3}$ University Hospital Eppendorf, Hamburg, Germany, ${ }^{4}$ National University Hospital Rigshospitalet, Copenhagen, Denmark, ${ }^{5}$ University of Heidelberg, Heidelberg, Germany, ${ }^{6}$ Silesian Medical Academy, Katowice, Poland, ${ }^{7}$ Nottingham University, Nottingham, United Kingdom, ${ }^{8}$ Universitätsklinikum Ulm, Ulm, Germany, ${ }^{9}$ Hopital St. Louis, Paris, France, ${ }^{10}$ University Hospital Leipzig, Leipzig, Germany, ${ }^{11}$ GKT School of Medicine, London, London, 
United Kingdom, ${ }^{12}$ University Hospital Essen, Essen, Germany, ${ }^{13}$ Addenbrookes Hospital, Cambridge, United Kingdom, ${ }^{14}$ Christie NHS Trust Hospital, Manchester, United Kingdom, ${ }^{15}$ University of Freiburg,, Freiburg, Germany, ${ }^{16}$ Institute of Immunology, Hannover, Germany, ${ }^{17}$ Medical University of Warsaw, Warsaw, Poland

Background: Inflammatory bowel diseases (IBD) are thought to increase the risk and severity of graft-versus-host disease (GVHD) and non-relapse mortality (NRM) after allogeneic hematopoietic stem cell transplantation (alloHSCT). Thus, IBD have been included in pre-transplant comorbidity risk scores, although formal analysis of alloHSCT outcomes in patients with IBD are lacking.

Methods: With this background, we designed an EBMT registry retrospective case-controlled analysis to assess outcomes of allo-HSCT in patients with IBD. The aim was to compare the incidence of GVHD, NRM and overall survival (OS) after allo-HSCT in the 2 groups of patients. Each patient with IBD was matched with 3 controls according to following factors: patient sex, patient age, disease, intensity of conditioning, donor type and HLA disparity, cell source and year of transplant. Group comparisons were done using logrank test or Gray test for competing risks outcomes.

Results: Between 2011 and 2015, 175 patients with IBD who underwent allo-HSCT for a hematologic malignancy were reported to EBMT. The cohort comprised 94 males and 81 females, with a median age of 53 years (range, 18 to 69 years) at the time of transplantation. The most frequent malignancies in the IBD group were acute leukemia (106 patients; $61 \%$ ) and myelodysplastic/myeloproliferative neoplasm (42 patients; 24\%). The donor was an identical sibling for 66 patients (38\%), and a matched unrelated donor for 56 patients (32\%). 93 patients (53\%) received a myeloablative conditioning regimen while 82 patients (47\%) received a reduced-intensity conditioning regimen. With a median follow-up of 37 months (range, 32-45) for the patients with IBD and 36 months (range, 33-39) for controls, the cumulative incidence of grade II-IV acute GVHD was $27 \%$ for the patients with IBD and $28 \%$ for controls (hazard ratio (HR) for IBD versus controls, 0.95; $95 \% \mathrm{CI}, 0.66$ to $1.36 ; \mathrm{p}=0.77)$. The cumulative incidence of extensive chronic GVHD at 36 months was $25 \%$ in patients with IBD and $17 \%$ in controls (HR, 1.54; $95 \%$ CI, 1.03 to 2.28; $\mathrm{p}=0.03$ ). NRM at 36 months was $27 \%$ for the patients with IBD and $25 \%$ for controls (HR, 1.07; 95\% CI, 0.76$1.52 ; \mathrm{p}=0.68$ ). The relapse incidence at 36 months was $32 \%$ in patients with IBD and $33 \%$ in patients without IBD (HR, 1.19 ; 95\% CI, 0.94-1.49; $\mathrm{p}=0.82$ ). OS at 36 months was $47 \%$ for the patients with IBD and $49 \%$ for matched controls (HR, 1.04; 95\% CI, 0.81-1.33; $\mathrm{p}=0.79$ ). Finally, GVHD-free relapse-free survival (GRFS) at 36 months was
$29 \%$ for the patients with IBD and $34 \%$ for matched controls (HR, 1.19; 95\% CI, 0.94-1.49; $\mathrm{p}=0.14$ ).

Conclusions: We report the largest matched-controlled study of allo-HSCT in patients with IBD conducted so far. Contrary to our expectations, we found no significant differences in acute GVHD, NRM or OS between the IBD group and controls. However, IBD patients had significantly more extensive chronic GVHD than the control group. Our results suggest that allo-HSCT should not be contraindicated if IBD alone is considered a comorbidity. However, IBD patients have a higher risk for developing severe forms of chronic GVHD, which could considerably impair their long-term quality of life.

Clinical Trial Registry: NA

Disclosure: NO disclosures

\section{3}

easix score predicts high-grade acute GVHD, nonrelapse mortality, and overall survival in adult patients undergoing unmodified or CD34-selected allogeneic HCT

Miriam Sanchez-Escamilla ${ }^{1,2}$, Patrick Hilden ${ }^{2}$, Molly Malloy $^{2}$, Josel D Ruiz ${ }^{2}$, Samira Fatmi ${ }^{2}$, Craig S Sauter ${ }^{2}$, Doris Ponce, ${ }^{2}$, Sergio A Giralt ${ }^{2}$, Michael Scordo ${ }^{2,3}$, Miguel-Angel Perales ${ }^{2,3}$

${ }^{1}$ IDIVAL (Research Institute of Marques de Valdecilla), Santander, Spain, ${ }^{2}$ Memorial Sloan Kettering Cancer Center, New York, NY, United States, ${ }^{3}$ Weill Cornell Medical College, Cornell University, Department of Medicine, New York, NY, United States

Background: The EASIX (Endothelial Activation and Stress Index) score is associated with non-relapse mortality (NRM) and overall survival (OS) after reduced intensity (RIC) AlloHCT (Luft et al, Lancet Haematol 2017). We aimed to validate the prognostic ability of EASIX in a cohort of both unmodified and CD34-selected alloHCT.

Methods: Between April 2008 and December 2016, 509 adult patients (pts) underwent unmodified RIC or non-myeloablative (NMA) alloHCT $(\mathrm{N}=149)$ with uniform GVHD prophylaxis of sirolimus/tacrolimus and low/ dose MTX or myeloablative conditioning (MAC) alloHCT using ex vivo CD34-selection $(\mathrm{N}=360)$ with the CliniMACS CD34 Reagent System (Miltenyi Biotech) as GVHD prophylaxis. The EASIX formula (LDH*Creatinine/Thrombocytes) was calculated at multiple timepoints (pre-alloHCT, day 30, day 100 and onset of acute GVHD). A $\log$ transformation using base $2(\log 2)$ was applied to all EASIX variables to reduce skew. A one unit increase in $\log 2$ EASIX is associated with a doubling 
(one-fold increase) of EASIX on the original scale. Relapse and death or relapse, were considered competing risks for NRM and acute GVHD, respectively.

Results: Median age was 55.6 years (19.6-78.7) and most pts were male (59\%). Most pts had myeloid malignancies $(72 \%)$ and received MAC $(70 \%)$. With a median follow up of 4.8 years $(0.7-10)$ among survivors, 1 and 3-year OS were $79.9 \%$ (95\% CI, 76.2-83.2) and 63.2\% (95\% CI, 5.7-67.3), respectively. The 1 and 3-year NRM were $13.2 \%$ (95\% CI, 10.4-16.3) and $22.2 \%(95 \%$ CI, 18.6-25.9), respectively. The 1-year cumulative incidence of grade 1-4, 2-4 and 3-4 acute GVHD was 44.4\% (95\% CI, 40.0-48.7), 33.0\% (95\% CI, 29.0-37.1) and $10.4 \%$ (95\% CI, 8.0-13.3), respectively. Causes of death in 211 pts at last follow up included relapse (34\%), GVHD (25\%) and infection (20\%).

The median [range] EASIX scores for unmodified vs. CD34-selected pts were 1.2 [0.4-27.8] vs. 0.9 [0.1-37.4], $\mathrm{p}=0.008$, pre-alloHCT, 2.1 [0.3-317.6] vs. 1.5 [0.2-284.7], $\mathrm{p}<0.001$, at day 30 , and 2.1 [0.3-97.9] vs. 1.3 [0.3-164.1], $\mathrm{p}<0.001$, at day 100, respectively. Higher pre-alloHCT EASIX score was associated with increased NRM $(\mathrm{HR}=1.17$ [95\% CI, 1.02-1.35], $\mathrm{p}=0.022)$ and grade 3-4 acute GVHD ( $\mathrm{HR}=1.23$ [95\% CI, 1.01-1.50], $\mathrm{p}=0.044)$, but not with OS or other acute GVHD grades. Higher EASIX score at d30, d100 and at onset of acute GVHD was significantly associated with increased risk of death and NRM (table 1 and figure 1).

Conclusions: Higher EASIX scores at day 30, day 100, and at onset of acute GVHD are associated with higher NRM and inferior OS with a more prominent association in calcineurin inhibitor-based unmodified alloHCT compared to CD34-selected alloHCT. Endothelial damage is an important contributor to poorer outcomes after alloHCT and EASIX formula provides an easy complimentary tool to predict outcomes in these patients.

\begin{tabular}{|c|c|c|c|c|c|c|c|}
\hline & \multicolumn{2}{|l|}{ OS } & \multicolumn{2}{|l|}{$N R M$} & \multicolumn{3}{|c|}{ Acute GVHD } \\
\hline & HR $(95 \% \mathrm{CI})$ & $\begin{array}{l}\mathrm{p}- \\
\text { value }\end{array}$ & HR $(95 \% \mathrm{CI})$ & p-value & Grade & $\begin{array}{l}\text { HR } \\
(95 \% \mathrm{CI})\end{array}$ & $\mathrm{p}$-value \\
\hline $\begin{array}{l}\text { Pre- } \\
\text { EASIX }\end{array}$ & $1.05(0.94-1.18)$ & 0.394 & $1.17(1.02-1.35)$ & 0.022 & $\begin{array}{l}\text { Grade } \\
1-4 \\
\text { Grade } \\
2-4 \\
\text { Grade } \\
3-4\end{array}$ & $\begin{array}{l}1.09 \\
(0.98- \\
1.21) \\
1.07 \\
(0.95- \\
1.21) \\
1.23 \\
(1.01- \\
1.50)\end{array}$ & $\begin{array}{l}0.106 \\
0.2570 .04\end{array}$ \\
\hline $\begin{array}{l}\text { EASIX } \\
\text { d30 }\end{array}$ & $1.33(1.20-1.47)$ & $<0.001$ & $1.54(1.37-1.74)$ & $<0.001$ & & & \\
\hline $\begin{array}{l}\text { EASIX } \\
\text { d100 }\end{array}$ & $1.36(1.23-1.51)$ & $<0.001$ & $1.52(1.34-1.72)$ & $\mathrm{p}<0.001$ & & & \\
\hline $\begin{array}{l}\text { EASIX- } \\
\text { aGVHD }\end{array}$ & $1.16(1.01-1.33)$ & 0.032 & $1.27(1.09-1.48)$ & 0.003 & & & \\
\hline
\end{tabular}

[[O073 Table] 1. Table 1. Univariate analysis]

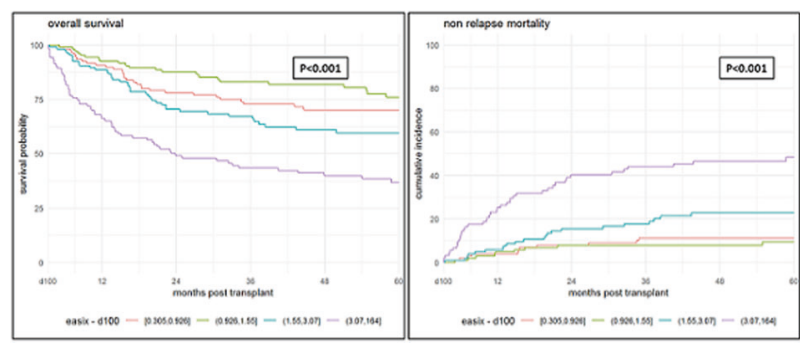

[[O073 Image] 1. Figure 1]

Disclosure: M.Sanchez-Escamilla: Was supported by Research Institute of Marques de Valdecilla (IDIVAL) Lopez-Albo Wenceslao Grant (WLA17/03).

CS Sauter: Juno Therapeutics: Consultancy, Research Funding; Sanofi-Genzyme: Consultancy, Research Funding; Spectrum Pharmaceuticals: Consultancy; Novartis: Consultancy; Precision Biosciences: Consultancy; Kite: Consultancy.

S Giralt: Consultancy: Amgen, Actinuum, Celgene, Johnson\&Johnson, JAZZ pharmaceutical, Milteny, Takeda, Novartis, Kite and Spectrum Pharma.

MA Perales: Consultancy: Merck; Ad Board: Incyte, Novartis, Abbvie and Nektar Therapeutics; Member and Scientific advisory board: MolMed and NexImmune; Personal fees and Clinical trial support; Merk: Personal fees; Takeda, Abbvie: and Novartis.

M Scordo: Consultancy: Angiocrine Bioscience and McKinsey \& Company.

P Hilden, M Maloy, SA Fatmi, JD Ruiz and D Ponce declare no conflict of interest.

\section{4}

subsequent malignant neoplasms after pediatric, adolescent and young adulthood stem-cell transplantation: a population-based cohort

Jason D Pole ${ }^{1}$, Fotios V Michelis ${ }^{2}$, Muhammad Ali ${ }^{3}$,Jeff Lipton $^{2}$, Lillian Sung, Kylie Lepic ${ }^{4}$, Joerg Krueger ${ }^{3}$, Mark Greenberg', David Hodgson ${ }^{2}$, KY Chiang ${ }^{3}$, Paul Nathan $^{3}$, Tal Schechcter ${ }^{3}$

${ }^{1}$ Pediatric Oncology Group of Ontario, Toronto, Canada, ${ }^{2}$ Princess Margaret Cancer Centre, University of Toronto, Toronto, Canada, ${ }^{3}$ The Hospital for Sick Children, Toronto, Canada, ${ }^{4}$ McMaster University, Hamilton, Canada

Background: Subsequent malignant neoplasms (SMNs) are one of the most important complications of hematopoietic stem cell transplantation (HSCT) and result in considerable morbidity and mortality. The reported rate of SMNs after HSCT in adults ranges between $1-11 \%$ at 10 -years. There is limited data on SMNs after pediatric, adolescent and young 
adult (AYA) HSCT, where the potential years of life gained is greater than among older adults. The objective of this study was to assess the incidence and types of SMNs in a cohort of survivors of childhood and AYA HSCTs that were performed for malignant indications.

Methods: All HSCT patients (age 0-30 years at time of transplant) who survived at least 2-years after HSCT in the province of Ontario, Canada between 1994 and 2017 were identified from 3 transplant centers. Clinical data were linked to provincial administrative databases and the Ontario Cancer Registry that identifies cancer cases based on pathology reports and electronic patient records.

Results: Four-hundred and forty-six 2-year allogeneic HCST survivors were included in this study. Of them, 36 (8\%) developed 45 SMNs at a median follow up of 13.1 years (range: $0.25-22.8$ years). The 10-year cumulative incidence of SMN was 4\% (2.1-6.3\%) and the 20-year cumulative incidence of SMN was 18\% (12.4-24.6\%). Several patients developed more than 1 SMN. The most common SMNs were: papillary carcinoma of the thyroid $(\mathrm{n}=11)$; secondary leukemia/lymphoma $(\mathrm{n}=10)$; squamous cell carcinoma of the skin/oral mucosa $(n=9)$; and adenocarcinoma of colon/lung $(n=5)$. Ten other types of SMNs were found including sarcoma, melanoma, nerve sheath tumor and breast cancer. Nine survivors died at a median of 7.7 months after SMN diagnosis. The 10-year cumulative incidence of SMN for 190 acute lymphoblastic leukemia survivors who received total-body irradiation was $5 \%(2.4 \%-10.6 \%)$.

Conclusions: Our findings corroborate the observation that children and AYA who undergo allogeneic HSCT are at a significant risk for developing SMN. Careful observation in the survivorship period is required for potential early detection.

Clinical Trial Registry: NA

Disclosure: Nothing to declare

\section{5}

Comorbidity profile of survivors at 20 years following allogeneic haematopoietic cell transplantation

\section{Ayesh Kumar Seneviratne ${ }^{I}$, Wilson Lam ${ }^{I}$, Jeffrey $H$. Lipton $^{1}$, Fotios V. Michelis ${ }^{1}$}

${ }^{1}$ Princess Margaret Cancer Centre, Toronto, Canada

Background: Allogeneic haematopoietic cell transplantation (HCT) is potentially curative for a variety of benign and malignant haematological disorders. Numerous longterm conditions and complications can arise as a result of the transplant that may decrease survival and quality of life. The purpose of the present study is to review the comorbidities of a single-centre cohort of allogeneic HCT recipients that survived twenty years post-transplant.

Methods: We retrospectively investigated 173 patients that underwent allogeneic HCT at the Princess Margaret Cancer Centre (Toronto, Canada) between 1979 and 1998 and who survived at least 20 years post-HCT while continuing follow-up at our centre. We documented performance status, comorbidities, number of medications and occurrence of secondary malignancies at 20 years, as well as survival following the 20 -year time-point.

Results: The median age of the cohort at 20 years postHCT was 56 years (range 37-77), 157 (91\%) of patients underwent transplant using a related donor. Eighty patients (46\%) underwent HCT for CML, 44 (25\%) for AML, 14 (8\%) for ALL, 12 (7\%) for aplastic anemia, $23(13 \%)$ for other indications. Bone marrow grafts were used in 163 (94\%) patients. Myeloablative conditioning was used in 163 (94\%) patients. Individual comorbidities were categorized into five major groups: endocrine $79(46 \%)$, cardiac 59 (34\%), secondary cancer $46(27 \%)$, psychosocial 39 (23\%), and other organ dysfunctions 94 (54\%). At the 20 year mark, median Karnofsky Performance Status was 100 (range 30-100). No comorbidities were seen in $24(14 \%)$ patients. The most frequent individual comorbidities were dyslipidemia $(\mathrm{n}=54,31 \%)$, hypertension $(\mathrm{n}=54,31 \%)$, osteoporosis $(\mathrm{n}=31,18 \%)$ and hypothyroidism $(\mathrm{n}=28$, $16 \%$ ). At the time of the 20 -year post-HCT follow-up, the median number of medications patients were taking was 2 (range 0-25).

Follow-up data after the 20-year mark was available for 146 (84\%) patients. Median follow-up of survivors after the 20-year mark was 59 months (range 4-219 months). Fiveyear overall survival of the 146 patients was $92 \%$ (95\% CI $85-96 \%$ ) and at 10 years was $89 \%$ (95\% CI 79-94\%). When grouped by age at the 20 -year mark, there was no significant difference in 5-year OS survival between ages 35-49 $(\mathrm{n}=48$, 5-year OS 91\%), 50-60 $(\mathrm{n}=58,5$-year OS 95\%) and 61-75 $(\mathrm{n}=40,5$-year OS $87 \%)(\mathrm{p}=0.90)$. When grouped by the number of concurrent comorbidities, there was a significant difference in OS between the groups with $0-1(n=54), 2-3$ $(\mathrm{n}=53)$ and $\geq 4$ comorbidities $(\mathrm{n}=39)$ (10-year OS 98\%, $87 \%$ and $67 \%$ respectively, $\mathrm{p}=0.0001$, Figure 1 ). When grouped by the number of medications patients were on at the 20-year mark, there was a borderline significant difference between the groups on $0-1 \quad(n=61), 2-4 \quad(n=46)$ and $\geq 5 \quad(n=39)$ different medications (10-year OS 93\%, $93 \%$ and $76 \%$ respectively, $\mathrm{p}=0.05$ ).

Conclusions: Long-term allogeneic HCT recipients may develop a number of long-term comorbidities that negatively influence survival even past the 20-year mark. These findings warrant the continuous long-term medical followup of allogeneic transplant patients, regardless of age or time that has lapsed post-HCT. 


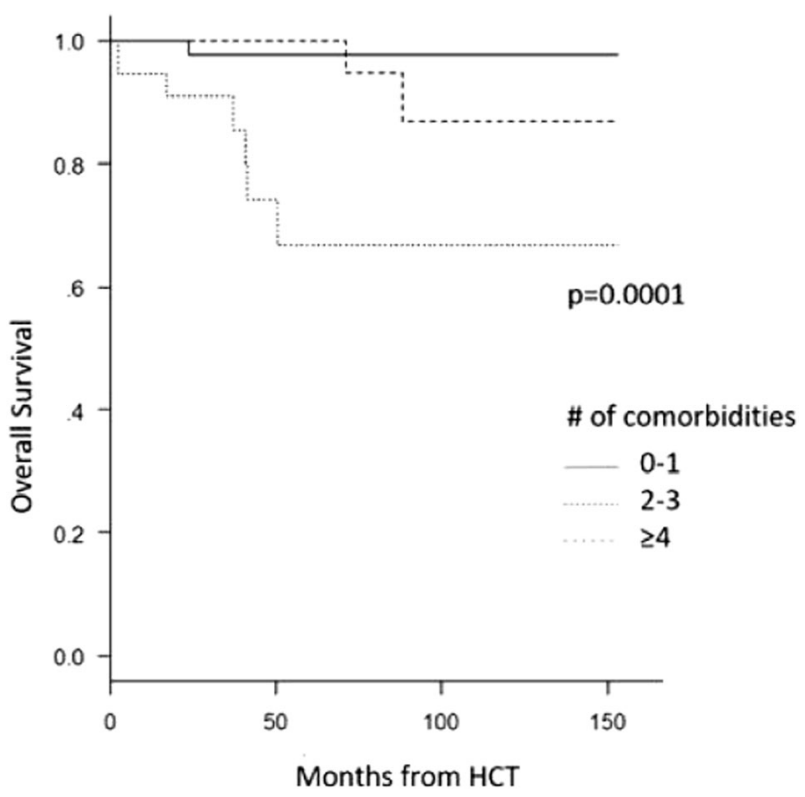

[[O075 Image] 1. Overal Survival of 20 year HCTsurvivors after 20 years]

Disclosure: Nothing to declare

\section{O076}

Predicting sinusoidal obstruction syndrome/venoocclusive disease (SOS/VOD) after ALLOSCT with the EASIX biomarker panel and the cibmtr clinical risk score

Olaf Penack, Sihe Jiang ${ }^{2}$, David Schult ${ }^{3}$, Joshua MajerLauterbach $^{3}$, Aleksandar Radujkovic ${ }^{3}$, Peter Dreger ${ }^{3}$, Thomas Luft ${ }^{3}$

${ }^{1}$ Charite Campus Virchow Klinikum, Berlin, Germany, ${ }^{2}$ Charité Univerisätsmedizin Berlin, Berlin, Germany, ${ }^{3}$ University Hospital Heidelberg, Heidelberg, Germany

Background: No standard procedure is in use to predict sinusoidal obstruction syndrome/venoocclusive disease (SOS/ VOD). Recently the SOS/VOD CIBMTR clinical risk score (age, Karnofsky, Sirolimus, Hepatitis B/C, conditioning regimen, disease type) has been established using the CIBMTR database (Biol Blood Marrow Transplant. 2018;24:2072). The Endothelial Activation and Stress Index (EASIX), based on the simple formula ' $\mathrm{LDH}(\mathrm{U} / \mathrm{l}) \times$ Creatinine $(\mathrm{mg} / \mathrm{dl}) /$ Thrombocytes $\left(10^{9} / 1\right)$ ', has been proven to predict mortality after GVHD (Lancet Haematol 2017;4:e414). The aim of the current study was to test prediction of SOS/VOD by EASIX compared with the CIBMTR score in two independent European cohorts.

Methods: SOS/VOD was defined according to the 2016 EBMT criteria. The capacity of EASIX and of the CIBMTR score for predicting SOS/VOD was tested retrospectively in 556 consecutive adult patients undergoing alloSCT at a single institution between 2001 and 2013 (training cohort). The primary endpoint was prediction of SOS/VOD by the CIBMTR score or by EASIX-d0 (EASIX measured at the day of alloSCT). Results were validated in an independent cohort of 446 adult alloSCT recipients from another single institution transplanted between 2013 and 2015. Incidence of SOS/VOD was assessed by uni- and multivariable Cox regression analyses using age, EASIX and the CIBMTR score as confounders.

Results: SOS/VOD was diagnosed in 35 patients $(6.3 \%$, median onset day +8 ) in the training cohort and in 45 patients $(10.1 \%$, median onset $d+9)$ in the validation cohort, respectively. In the training cohort, increasing EASIX-d0 was significantly associated with SOS/VOD incidence on multivariate analysis (HR per $\log 2$ increase 1.27, 95\%CI 1.03-1.57, $\mathrm{p}=0.023$ ). Similarly, EASIX-d0 predicted the incidence of SOS/VOD in the validation cohort (HR per $\log 2$ increase 1.35, 95\% CI 1.14-1.59, p< 0.001). Also, the CIBMTR score showed an association with SOS/VOD incidence which was however significant only in the training cohort (HR per $\log 2$ increase $1.55,95 \%$ CI $1.09-1.62, \mathrm{p}=0.016)$ but not in the validation cohort, (HR per $\log 2$ increase $1.43,95 \%$ CI $0.98-2.08, \mathrm{p}=0.060$ ). These results are visualized by comparing EASIX-d0 and CIBMTR-VOD scores in patients grouped according to later VOD development within the observation period (Figure 1, Kruskal-Wallis tests; 1A-B EASIX-d0 and 1C-D CIBMTR risk score). The association of EASIXd0 with VOD incidence was independent from CIBMTR score.

Conclusions: EASIX-d0 is very easy to test and predicted SOS/VOD in two separate cohorts of alloSCT recipients independent of the CIBMTR-VOD score. Patients with high EASIX-d0 scores might be candidates for clinical evaluation of intensified strategies to prevent SOS/VOD after alloSCT.
Training cohort
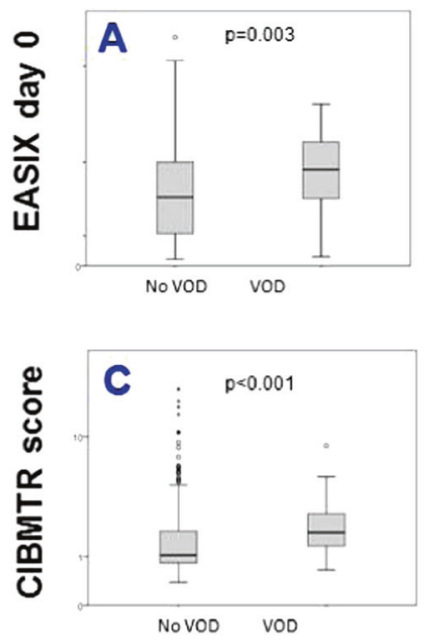

[[O076 Image] 1. Figure 1]
Validation cohort
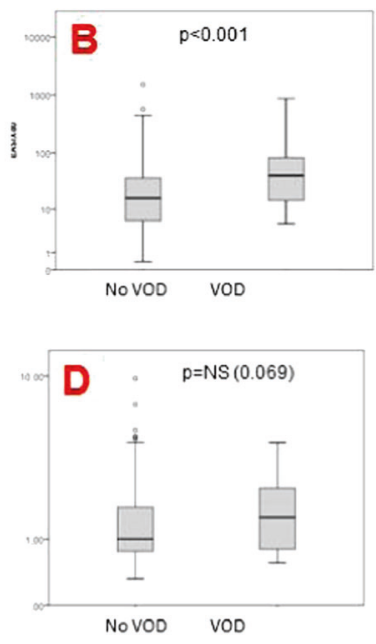
Disclosure: The authors thank Jazz Pharmaceuticals for providing financial support that was used for data collection. The company had no active part in this project, did not have access to data and was not involved in analyses or writing/editing of this abstract.

\section{O077}

Early hyperbilirubinaemia without SOS/VOD - A link between endothelial dysfunction and early mortality after allogeneic transplantation

Thomas Luft ${ }^{I}$, David Schult ${ }^{1}$, Joshua Majer-Lauterbach ${ }^{1}$, Sihe Jiang ${ }^{2}$, Aleksandar Radujkovic ${ }^{1}$, Peter Dreger ${ }^{1}$, Olaf Penack $^{2}$

${ }^{1}$ University Hospital Heidelberg, Heidelberg, Germany, ${ }^{2}$ Charité Universitätsmedizin Berlin, Berlin, Germany

Background: Endothelial dysfunction is a risk factor for early mortality after allogeneic stem cell transplantation (alloSCT) and is linked to transplant-associated thrombotic microangiopathy (TAM). Similar to TAM, diagnosis of sinusoidal obstruction syndrome / venoocclusive disease (SOS/VOD) is based on consensus criteria, and several scores have been proposed that include earlier or later stages of the diseases.

Early hyperbilirubinaemia occurs frequently after alloSCT. The pathophysiology is often elusive, and only in a subset of alloSCT recipients with hyperbilirubinaemia SOS/VOD is identified as the underlying mechanism. The aim of the current study was to explore clinical impact and pathophysiological context of early hyperbilirubinaemia without SOS/VOD.

Methods: In two independent cohorts of 864 and 446 patients, serum bilirubin levels before alloSCT and on days $0,3,5,7,14,21$ and 28 were retrospectively retrieved. SOS/VOD was defined according to the 2016 EBMT criteria. Patients with at least one bilirubin value of $>3 \mathrm{mg} / \mathrm{dl}$ between days 0-28 were grouped as "early hyperbilirubinaemia". We assessed overall survival (OS), non-relapse mortality (NRM) and time to relapse (TTR) with and without early hyperbilirubinaemia depending on coincident SOS/VOD, TAM, and refractory acute GVHD, and we investigated the impact of statin-based endothelial cell prophylaxis (pravastatin plus ursodeoxycholic acid). Serum bilirubin levels were correlated with the Endothelial Activation and Stress Index (EASIX, 'LDH(U/l) x Creatinine $(\mathrm{mg} / \mathrm{dl})$ / Thrombocytes $\left.\left(10^{9} / \mathrm{l}\right)^{\prime}\right)$, measured before alloSCT (EASIX-pre) or on the day of transplantation (EASIX-d0). Similarly, bilirubin levels were correlated with the SOS/VOD CIBMTR clinical risk score, and with serum markers of liver damage (alanine transaminase, ALT,
Gamma-glutamyltransferase, GGT) and endothelial cell distress markers (Angiopoietin-2).

Results: Early hyperbilirubinaemia was diagnosed in 156 $(18 \%)$ and $141(32 \%)$ patients of the training and validation cohort, and VOD diagnostic criteria were met by $23 \%$ and $30 \%$ of patients with early hyperbilirubinaemia, respectively. In all patients, early hyperbilirubinaemia was associated with increased NRM and OS (NRM, training: HR 2.03, 95\%CI 1.47-2.80, $\mathrm{p}<0.001$; NRM validation: HR $2.12,95 \% \mathrm{CI} 1.48-3.06, \mathrm{p}<0.001)$. Increased NRM in recipients with hyperbilirubinaemia without SOS/VOD was independent from TMA or refractory acute GVHD (Figure 1). EASIX-pre and EASIX-d0 correlated with day 0-28 bilirubin in both cohorts. EASIX-pre, EASIX-d0 and the CIBMTR-VOD score predicted risk of early hyperbilirubinaemia. However, only EASIX predicted risk of NRM in patients without SOS/VOD. Endothelial protection with statins and ursodeoxycholic acid was associated with reduced incidence of and reduced NRM after early hyperbilirubinaemia. Furthermore, pre-transplant Angiopoietin-2 correlated with early hyperbilirubinaemia, whereas ALT and GGT did not.

Conclusions: Early hyperbilirubinaemia represents a novel risk factor for NRM independent of TMA and refractory acute GVHD, even in patients not meeting the diagnostic criteria for VOD. The endothelial relationship of this condition is underlined by the observation that Angiopoietin-2, EASIX-pre and EASIX-d0 but not markers of liver damage associate with the incidence of early hyperbilirubinaemia. Therapeutic strategies aiming at normalization of endothelial dysfunction after alloSCT are attractive. As a first example, our data demonstrate reduced incidence of early hyperbilirubinaemia and reduced NRM thereafter in alloSCT recipients prophylactically treated with statins and ursodeoxycholic acid in the peri-transplant period.
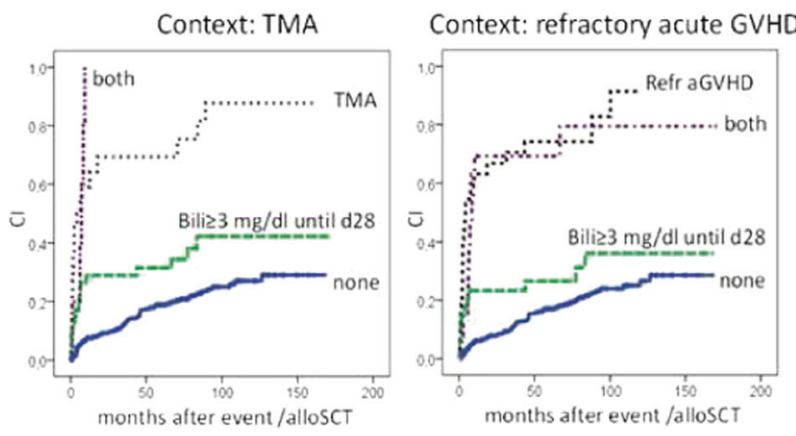

[[O077 Image] 1. Figure 1]

Clinical Trial Registry: not applicable

Disclosure: no conflicts of interest 


\section{8}

Crosstalk between complement and neutrophil activation in transplant-associated thrombotic microangiopathy

Eleni Gavriilaki, Ioanna Sakellari', Ioannis Batsis ${ }^{1}$, Despina Mallouri', Anna Vardi, Akrivi Chrysanthopoulou $^{2}$, Maria Georgoutsou-Spyridonos ${ }^{3}$, Zoi Bousiou, Michalis Iskas ${ }^{I}$, Tasoula Touloumenidou ${ }^{I}$, Apostolia Papalexandri $^{1}$, Dimitris Mastellos ${ }^{3}$, Konstantinos Ritis ${ }^{2}$, Ioannis Mitroulis $^{2,4}$, Achilles Anagnostopoulos ${ }^{1}$

${ }^{1}$ G. Papanicolaou Hospital, Thessaloniki, Greece, ${ }^{2}$ Democritus University of Thrace, Alexandroupolis, Greece, ${ }^{3}$ National Center for Scientific Research 'Demokritos', Athens, Greece, ${ }^{4}$ Technische Universität Dresden, Dresden, Germany

Background: Accumulating evidence has suggested complement activation in transplant-associated thrombotic microangiopathy (TA-TMA), mainly in the pediatric setting. To further understand its pathogenesis in adults, we hypothesized that both complement and neutrophils are activated in TA-TMA as previously observed in distinct thrombotic disorders.

Methods: We enrolled adult TA-TMA (International Working Group/IWG criteria), acute and/or chronic graftversus-host-disease (GVHD) and control hematopoietic cell transplantation (HCT) recipients in a 1:1:1 ratio (January 2015-June 2018). Complement activation was detected in patient sera and plasma with the modified Ham test (mHam), soluble C5b-9 and activated C3 fragments; while neutrophil activation with neutrophil extracellular traps (NETs) using extracellular DNA and myeloperoxidase/ MPO-DNA.

Results: We studied 10 TA-TMA, 10 GVHD and 10 control patients. TA-TMA patients suffered from severe acute and/or extensive chronic GVHD. TA-TMA presented at median +109 (9-930) day post-transplant. Full donor chimerism was evident in all patients. No significant difference in transplant characteristics was observed among groups, except for the significantly lower GVHD rate in the control group.

C5b-9 and mHam levels were significantly increased in TA-TMA compared to GVHD ( $p=0.004$ and $p=0.006$, Figure A) and control patients $(p=0.001$ and $p=0.005$, Figure B), while no significant difference was observed in activated plasma $\mathrm{C} 3$ levels in the plasma. In the multivariate analysis, C5b-9 levels were an independent predictor of TATMA diagnosis $(p=0.026)$. We next sought to determine the cutoff value of C5b-9 that distinguishes TA-TMA from other HCT recipients. In the receiver-operating characteristic curve, we found a significantly high area under the curve $(0.823, \mathrm{p}=003)$. Values higher than $321.5 \mathrm{ng} / \mathrm{ml}$ conferred a specificity of $70 \%$ with a very high sensitivity of $91.7 \%$ for TA-TMA diagnosis.

Regarding NETs, both extracellular and MPO-DNA were significantly increased in patients with TA-TMA compared to GVHD ( $<<0.001$ and $p=0.042$, Figure $C)$ and control patients $(p<0.001$ and $p=0.029$, Figure $D)$. Interestingly, increased complement activation markers (C5b-9 and mHam) were strongly associated with MPO-DNA $\left(r^{2}=0.461, p=0.001\right.$ and $r^{2}=0.403, p=0.018$, respectively $)$ and extracellular DNA $\left(\mathrm{r}^{2}=0.555, \mathrm{p}=0.001\right.$ and $\mathrm{r}^{2}=0.502$, $\mathrm{p}=0.002$, respectively).

Lastly, we studied changes of complement and neutrophil activation in 3 patients that received complement inhibition by eculizumab. Despite delayed initiation in the first two patients (28 and 18 days post TA-TMA diagnosis respectively), we observed laboratory response including evidence of reduced hemolysis, schistocytosis and transfusion needs. Both extracellular DNA and C5b-9 levels were significantly reduced post 2 doses of eculizumab $(\mathrm{p}<0.001)$. However, all patients succumbed to complications of endstage renal disease and infections after a median of $3(2-8)$ doses of eculizumab.

Conclusions: Our findings demonstrate for the first time a crosstalk between complement and neutrophils in adult TATMA. In addition, we were able to set a cut-off C5b-9 value for distinguishing complement activation in unselected patients diagnosed with the IWG criteria. Although complement inhibition by eculizumab seems to hinder this pathophysiological process, further studies are needed to clarify changes and identify optimal therapeutic targets in this complex setting.
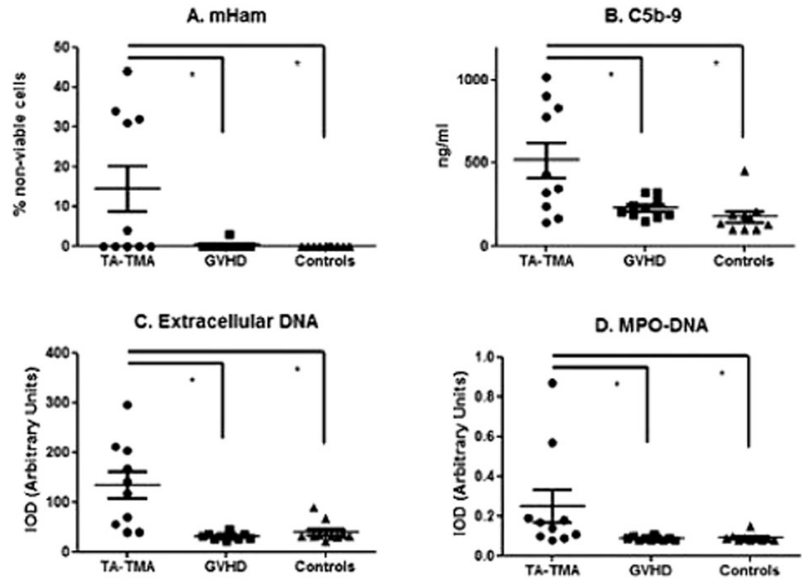

[[O078 Image] 1. Figure: Markers of complement $(A, B)$ and neutrophil $(C, D)$ activation]

Disclosure: E.G. was supported by the European Hematology Association Clinical Research Grant. The remaining authors declare no competing financial interest. 


\section{O079}

Pooled analysis of time to complete response after defibrotide treatment initiation in patients with hepatic veno-occlusive disease/sinusoidal obstruction syndrome after hematopoietic stem cell transplantation

\section{Paul Richardson ${ }^{1}$, Angela Smith ${ }^{2}$, Nancy Kernan ${ }^{3}$, Leslie Lehmann $^{4}$, Robert Ryan ${ }^{5}$, William Tappe ${ }^{5}$, Stephan Grupp $^{6}$}

${ }^{1}$ Jerome Lipper Multiple Myeloma Center, Boston, MA, United States, ${ }^{2}$ University of Minnesota, Minneapolis, MN, United States, ${ }^{3}$ Memorial Sloan Kettering Cancer Center, New York, NY, United States, ${ }^{4}$ Harvard Medical School, Boston, MA, United States, ${ }^{5} \mathrm{Jazz}$ Pharmaceuticals, Palo Alto, CA, United States, ${ }^{6}$ The Children's Hospital of Philadelphia, Philadelphia, PA, United States

Background: Hepatic VOD/SOS with multi-organ dysfunction (MOD; typically, renal or pulmonary) may be associated with $>80 \%$ mortality. Defibrotide is approved for treating severe hepatic VOD/SOS post-hematopoietic stem cell transplantation (HSCT) in patients aged $>1$ month in the European Union, and for hepatic VOD/SOS with renal or pulmonary dysfunction post-HSCT in the United States and Canada. Per prescribing guidelines, defibrotide $25 \mathrm{mg} / \mathrm{kg}$ /day (4 divided doses) is recommended for $\geq 21$ days. This pooled analysis examined time to complete response (CR) of VOD/ SOS and MOD symptoms relative to day of defibrotide initiation in patients who received defibrotide $25 \mathrm{mg} / \mathrm{kg} / \mathrm{day}$.

Methods: Time to CR and safety data were pooled from 2 studies that included patients with VOD/SOS and MOD post-HSCT who were treated with defibrotide: a phase 3 trial $(\mathrm{n}=102)$ and a phase 2 , randomized dose-finding trial $(\mathrm{n}=74$ receiving $25 \mathrm{mg} / \mathrm{kg} / \mathrm{day}$ ). Duration of therapy in patients who discontinued due to $\mathrm{CR}$ in an expanded-access program (TIND) in VOD/SOS patients with and without MOD postHSCT $(n=1000)$ was analyzed separately due to differences in the patient population and data monitoring protocol (eg, CR data by day were not collected). VOD/SOS diagnosis was defined by Baltimore criteria/biopsy for the phase 2 and 3 studies; in the T-IND, modified Seattle criteria also was permitted. Minimum recommended treatment duration was $\geq 14$ days (phase 2 ) or $\geq 21$ days (phase 3 , T-IND).

Results: The pooled phase 2 and 3 trials had 60 patients with $C R$ ( $n=34$ and $n=26$, respectively) and 116 patients without $C R$ ( $n=40$ and $n=76$, respectively).

Of 60 patients with CR (Figure) in the phase 2 and 3 trials, the median time to $\mathrm{CR}$ was 24.5 days (range: 7-123); of these, $53.3 \%$ achieved CR after 4 or more weeks of treatment. In the T-IND, 390 patients discontinued treatment due to $\mathrm{CR}$ (median time to discontinuation, 22 days after initiation of defibrotide; range, 2-64), with 57 patients $(14.6 \%)$ discontinuing on or after week 4 of treatment.

In the phase 2 and 3 studies ( $n=176), 58$ patients ( $33 \%)$ had treatment-related adverse events (TRAEs); most common were gastrointestinal (GI) hemorrhage (4.0\%), epistaxis $(4.5 \%)$, hypotension $(5.7 \%)$, and pulmonary alveolar hemorrhage (5.7\%). In the T-IND $(n=1000), 210$ patients $(21.0 \%)$ had $\geq 1$ TRAE; most common were pulmonary hemorrhage (4.6\%), GI hemorrhage (3.0\%), epistaxis (2.3\%), and hypotension $(2.0 \%)$.

Conclusions: Among patients with CR in the phase 2 and 3 studies, a significant number of patients achieve CR (40\%) after 4 weeks of persistent treatment, highlighting the importance of continued therapy as per label indication.

Support: Jazz Pharmaceuticals

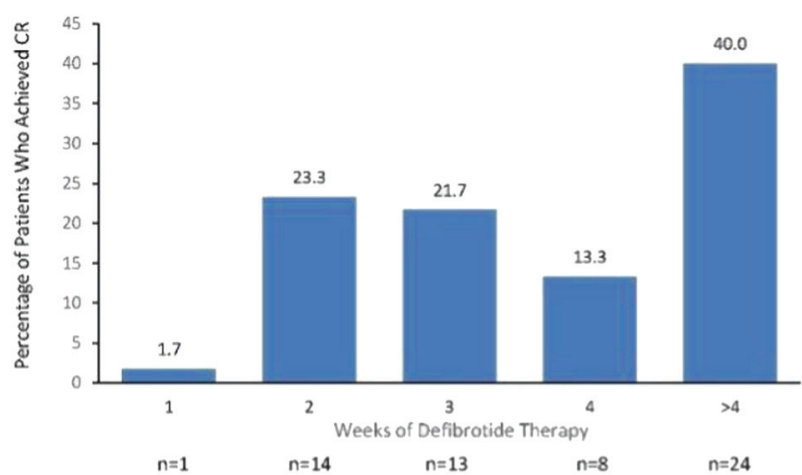

[[O079 Image] 1. Figure. Time to CR Among 60 Patients Achieving CR in Defibrotide Phase 2 and 3 Studies]

Clinical Trial Registry: ClinicalTrials.gov: NCT00003966, NCT00358501, and NCT00628498

Disclosure: Paul G. Richardson has served on advisory committees and as a consultant, and has received research funding from Jazz Pharmaceuticals.

Angela R. Smith and Leslie Lehmann have nothing to disclose.

Nancy A. Kernan received grants from Gentium during the conduct of the study, and her research was supported by The National Cancer Institute of the National Institutes of Health under award number P30 CA008748; the content is solely the responsibility of the author and does not necessarily represent the official views of the National Institutes of Health. She has a research grant from Jazz Pharmaceuticals.

Robert Ryan and William Tappe are employees of Jazz Pharmaceuticals and hold stock and/or stock options in Jazz Pharmaceuticals plc.

Stephan A. Grupp has served on a steering committee and as a consultant to Jazz Pharmaceuticals. 


\section{O080}

Sperm counts and prevalence of testosterone substitution at long-term follow-up after myeloablative allogeneic HSCT in childhood

Sidsel Mathiesen', Malene Mejdahl, Anu Suominen', Kaspar Sørensen ${ }^{1}$, Marianne Ifversen ${ }^{1}$, Päivi Lähteenmäki ${ }^{3}$, Hanne Frederiksen ${ }^{1}$, Anders Juul, Klaus Müller ${ }^{1}$, Kirsi Jahnukainen ${ }^{2,4}$

${ }^{1}$ Rigshospitalet, University of Copenhagen, Copenhagen, Denmark, ${ }^{2}$ Children's Hospital, University of Helsinki and Helsinki University Hospital, Helsinki, Finland, ${ }^{3}$ Turku University Hospital and Turku University, Turku, Finland, ${ }^{4}$ Karolinska Institutet and University Hospital, Stockholm, Sweden

Background: Little is known about the long-term effects of pediatric HSCT on the male reproductive axis. We investigated sperm counts and prevalence of testosterone substitution twenty years after pediatric HSCT and aimed at identifying risk factors for azoospermia and testosterone substitution.

Methods: This cross-sectional follow-up study included two national cohorts of adult males ( $\geq 18$ years) treated with myeloablative allogeneic pediatric HSCT before age 17 between 1980-2010 in Denmark and Finland. The study included medical history; physical examination including testicular volume and screening for chronic graft-versushost-disease (cGvHD); sex hormones and a semen sample. Cumulative (pre-HSCT and HSCT) gonadal irradiation including TBI and cumulative cyclophosphamide equivalent doses (CED) were estimated from patient files.

Results: 98/181 (54\%) of eligible patients (age 18-47 years) participated with a median (range) follow up time of 18 (8-35) years. $76 \%$ had malignant diagnoses and $74 \%$ were treated with TBI-conditioning. 91 delivered a semen sample. Results of semen and sex hormone analyses are listed in table 1. $30 / 98(31 \%)$ had detectable sperm counts (0.010-524.8 million), of these 15 were treated with chemotherapy only and 15 with TBI ( 2 with 2 Gy TBI and 13 with 10-12 Gy TBI including 5 with gonadal shielding).

In patients with detectable sperm in the ejaculate, increase in sperm counts was associated with time from HSCT ( $\beta=6.6$ million per year 95\%CI ((-0.18)-13.32), $\mathrm{p}=0.056$, time range 9-32 years) indicating late spermatogenic recovery. Testicular irradiation was a strong risk factor for azoospermia $(\mathrm{OR}=6.2$ 95\% CI $(2.0-20.7), \mathrm{p}<0.001)$ and testosterone substitution $\quad(\mathrm{OR}=5.0 \quad 95 \% \mathrm{CI} \quad(1.3-28.3)$, $\mathrm{p}=0.012$ ) and no patients with cumulative testicular irradiation doses $>12$ Gy had detectable sperms, figure 1 .

Pre-pubertal stage at HSCT was a risk factor for later testosterone substitution $\quad(\mathrm{OR}=10.4 \quad 95 \% \mathrm{CI} \quad(1.5-453)$, $\mathrm{p}=0.0060$ ). Risk of testosterone substitution was associated with time from transplantation (OR for +1 year $1.195 \% \mathrm{CI}$ (1.0-1.2), $\mathrm{p}=0.025)$. Cumulative CED adjusted for testicular irradiation was not a risk factor for azoospermia or testosterone substitution, nor was cGvHD.

\begin{tabular}{|c|c|c|c|}
\hline & $\begin{array}{l}\begin{array}{l}\text { Sperms } \\
\text { detected, } n=30\end{array}\end{array}$ & Azoospermia, $n=42$ & $\begin{array}{l}\text { Testosterone } \\
\text { substitution, } n=24\end{array}$ \\
\hline $\begin{array}{l}\text { Pre-pubertal/ } \\
\text { pubertal/post- } \\
\text { pubertal at HSCT (1 } \\
\text { missing), n }\end{array}$ & $23 / 5 / 2$ & $25 / 10 / 7$ & $22 / 1 / 01$ \\
\hline $\begin{array}{l}\text { Orchiectomy } \\
\text { unilateral/ } \\
\text { bilateral, } \mathrm{n}\end{array}$ & $1 / 0$ & $2 / 0$ & $2 / 4$ \\
\hline $\begin{array}{l}\text { Total testes volume } \\
(\mathrm{ml}) \text {, median } \\
\text { (range) }\end{array}$ & $40(7.5-27)$ & $24(4-52)$ & $6(0-30)$ \\
\hline $\begin{array}{l}\text { Chronic graft- } \\
\text { versus-host- } \\
\text { disease, } \mathrm{n}(\%)\end{array}$ & $7(23)$ & $12(29)$ & $8(33)$ \\
\hline $\begin{array}{l}\text { Follicle stimulating } \\
\text { hormone (U/l), } \\
\text { median (range) }\end{array}$ & $\begin{array}{l}5.38(1.40- \\
32.30)\end{array}$ & $17.05(4.29-64.40)$ & $0.47(0.01-52.50)$ \\
\hline $\begin{array}{l}\text { Luteinizing } \\
\text { hormone (U/l), } \\
\text { median (range) }\end{array}$ & $\begin{array}{l}4.31(0.06- \\
13.20)\end{array}$ & $8.07(2.53-22.2)$ & $0.11(0.01-31.00)$ \\
\hline $\begin{array}{l}\text { Inhibin B ( } \mathrm{pg} / \mathrm{ml}) \text {, } \\
\text { median (range) }\end{array}$ & $\begin{array}{l}109.5(10.0- \\
247.0)\end{array}$ & $19.00(1.00-134.00)$ & $4.00(1.00-30.00)$ \\
\hline $\begin{array}{l}\text { Sex hormone } \\
\text { binding globulin } \\
\text { (nmol/l), median } \\
\text { (range) }\end{array}$ & $\begin{array}{l}29.55(7.30- \\
61.40)\end{array}$ & $27.70(4.80-68.80)$ & $23.20(7.10-62.10)$ \\
\hline $\begin{array}{l}\text { Total testosterone } \\
\text { (nmol/l), median } \\
\text { (range) }\end{array}$ & $\begin{array}{l}18.40(8.06- \\
31.19)\end{array}$ & $13.66(1.65-26.20)$ & $18.55(3.99-53.82)$ \\
\hline
\end{tabular}

[[O080 Table] 1. Table 1: Results of semen sample, pubertal stage at HSCT, physical examination and sex hormones]

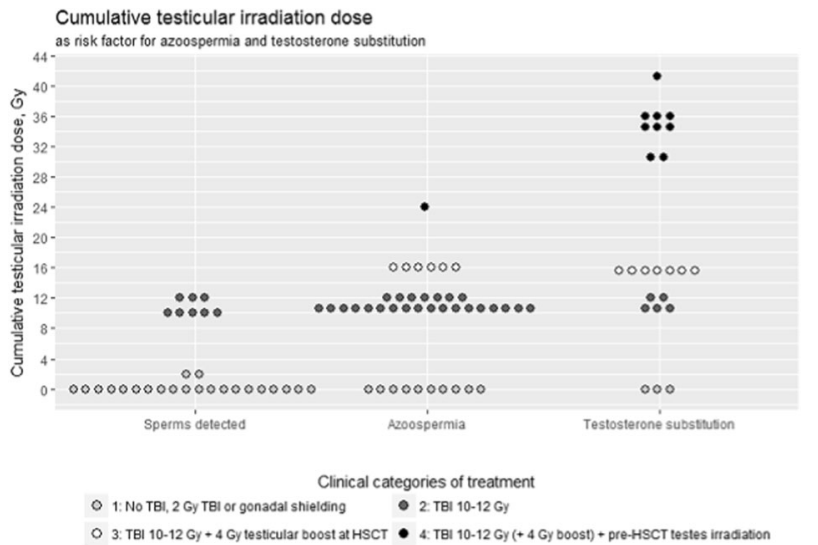

[[O080 Image] 1. Figure 1]

Conclusions: Late spermatogenic recovery is possible 10-30 years following myeloablative HSCT but depends on cumulative testicular irradiation dose; azoospermia was 
present in all patients treated with $>12$ Gy. Pre-pubertal stage at HSCT increases the risk for later testosterone substitution supporting the hypothesis that pre-pubertal Leydig cells are more sensitive to irradiation than more mature ones. Additionally, the risk of testosterone substitution increased with time from transplantation indicating a potential early androgen insufficiency in male HSCT recipients. Thus, close follow-up and focus on cumulative irradiation doses are needed.

Disclosure: Nothing to declare

\section{O081}

Late hospitalizations among survivors of childhood, adolescence and young adulthood hematopoietic stemcell transplantation performed for malignant indications

Jason D Pole ${ }^{1}$, Muhammad Ali', Fotios V Michelis ${ }^{3}$, Lillian Sung ${ }^{2}$, Jeff Lipton ${ }^{3}$, Kylie Lepic ${ }^{4}$, Joerg Krueger ${ }^{2}$, KY Chiang ${ }^{2}$, Mark Greenberg', Paul Nathan', Tal Schechcter $^{2}$

${ }^{1}$ Pediatric Oncology Group of Ontario, Toronto, Canada, ${ }^{2}$ The Hospital for Sick Children and University of Toronto, Toronto, Canada, ${ }^{3}$ Princess Margaret Cancer Centre and University of Toronto, Toronto, Canada, ${ }^{4} \mathrm{McMaster}$ University, Hamilton, Canada

Background: Hematopoietic stem cell transplantation (HSCT) has become a standard component of therapy for several malignant indications. As HSCT may improve survival for some cancers, the risk for late complications is of increasing concern. The frequency of hospitalizations can serve as a proxy measure of severe morbidity. However, knowledge regarding late hospitalizations is limited. The objectives of the study were to describe health care utilization as measured by hospitalizations beyond 2 years following transplant in survivors of pediatric/adolescent and young adult (AYA) HSCT for a malignant indication.

Methods: We linked data from 3 Ontario HSCT centers and provincial health care utilization data housed at Institute for Clinical Evaluative Sciences (ICES) to describe all hospitalizations and their indications. We also described intensive-care unit admissions. The study population included Ontario residents with cancer age 0-30 years who underwent HSCT between 1992 and 2015, who survived more than 2 years from transplant (index date). Hospitalizations were described from the index date until Dec 2017 or death.

Results: The cohort consisted of 446 survivors who were followed for a median of 11.4 years from index date (interquartile range (IQR) 2.6-18.5). Indications for transplant included: acute lymphoblastic leukemia $(\mathrm{n}=190$, 42.6\%); myeloid malignancy $(n=231,51.8 \%)$; and lymphoma $(n=25,5.6 \%)$. Of these, $150(33.6 \%)$ received a related-donor bone-marrow HSCT. At the time of HSCT, ages were: $0-9(n=132,29.6 \%) ; 10-18(n=137,30.7 \%)$; and $19-29(n=177,39.7 \%)$ years. There were 269 patients $(60 \%)$ with at least 1 hospitalization beyond 2 years from HSCT. There were a total of 1879 hospitalizations, resulting in a hospitalization rate of 0.43 per follow-up year. Average length of hospital stay was 6.1 days. A total of 97 intensivecare unit admissions were documented among 57 (12.8\%) patients. The most common indications for hospitalization were: graft-versus-host disease (GVHD) $(n=486,25.9 \%)$, relapse $(n=303,16.1 \%)$, infection $(n=186,9.8 \%)$, orthopedic procedures/fractures $(\mathrm{n}=90,5.4 \%)$, benign neoplasm $(\mathrm{n}=96,5.3 \%)$ and subsequent malignant neoplasm $(\mathrm{n}=83$, 4.7\%). Among those who did not relapse, 190/320 (59\%) were hospitalized. At 10 years following HSCT, the proportion of patients hospitalized was $10.5 \%$. An underlying diagnosis of acute myeloid leukemia (AML) $(\mathrm{p}=0.001)$ and chronic GVHD $(\mathrm{p}=0.014)$ were associated with increased rate of hospitalization. In the follow-up period, 73 (16.4\%) patients died.

Conclusions: We identified a high rate of late hospitalization in pediatric/AYA survivors who underwent HSCT for a malignant indication, even among those without relapse. A diagnosis of AML and chronic GVHD were associated with increased risk for hospitalization. Careful observation in the survivorship period is required for potential prevention of hospitalization.

Clinical Trial Registry: NA

Disclosure: Nothing to declare

\section{2}

Engraftment syndrome after haploidentical stem cell TRANSPLANTATION with post-transplant cyclophosphamide

Elena Landete ${ }^{1}$, Laura Solán ${ }^{1,2}$, Rebeca Bailén ${ }^{1,2}$, Nieves Dorado $^{1,2}$, Pascual Balsalobre ${ }^{1,2}$, Carolina MartínezLaperche $^{1,2}$, Javier Anguita ${ }^{1,2}$, José Luis Diez-Martín ${ }^{1,2}$, Mi Kwon ${ }^{1,2}$

${ }^{1}$ Gregorio Marañón General University Hospital, Madrid, Spain, ${ }^{2}$ Gregorio Marañón Health Research Institute (IiSGM), Madrid, Spain

Background: Engraftment syndrome (ES) is a clinical complication characterized by inflammatory signs and symptoms occurring during neutrophil recovery after stem cell transplantation (SCT). Its incidence varies depending on the clinical criteria used. The objective of this study was to analyze the incidence, clinical characteristics, risk factors and clinical outcomes of ES after haploidentical-SCT with post-transplant cyclophosphamide (HaploSCT) in a single center. 
Methods: 105 consecutive Haplo-SCT performed between 2010-2016 in our center were retrospectively reviewed. GVHD prophylaxis was performed with cyclophosphamide $50 \mathrm{mg} / \mathrm{kg} /$ day days +4 and +5 , MMF and CsA from day +5 . G-CSF was started in all cases from day +5 until engraftment. 11 cases were excluded from the analysis ( 9 due to death before engraftment and 2 due to primary graft failure). Maiolino and Spitzer's diagnostic criteria were used to define ES.

Results: Characteristics of the 94 transplant included are shown in Table 1. The ES incidence was $27.6 \%$, with median time to diagnosis of 17 days (IQR, 15-19). Median time to neutrophil engraftment in the ES cohort was 17 days (IQR, 14-20). Fever (100\%) and skin rash (77\%) were the most frequent clinical findings. There were other 5 cases of fever and skin rash during the peri-engraftment period with a final diagnosis of GVHD considering clinical outcome. 18 patients $(69 \%)$ received high doses of corticosteroids, with favorable response in $78 \%$ of cases. Of note, $28 \%$ cases also needed intensive supportive care. There were no deaths secondary to ES. Univariable analysis showed a higher risk of ES with use of brother/sister as cell donor (61\% cases of $E S ; p=0,003)$. No other risk factors were identified. No association was noted with acute or chronic GVHD. There was no significant difference in NRM, overall survival and progression-free survival between ES and non-ES patients.

\begin{tabular}{ll} 
SCT characteristics & \\
SCT, $\boldsymbol{n}$ & 94 \\
Age $($ years), median (range) & $46(33-58)$ \\
Sex, female $\boldsymbol{n}(\%) /$ male $\boldsymbol{n}(\%)$ & $28(30) / 66(70)$ \\
Diagnosis, $\boldsymbol{n}(\%)$ & \\
Acute myeloid leukaemia & $38(40)$ \\
MDS & $6(6)$ \\
HL & $21(22)$ \\
NHL & $11(12)$ \\
ALL & $11(12)$ \\
Others & $6(6)$ \\
Stem cell source PB/BM, $\boldsymbol{n}(\%)$ & $87(92) / 7(8)$ \\
Conditioning regimen, $\boldsymbol{n}$ (\%) & \\
Myeloablative & $39(41)$ \\
Non myeloablative & $55(59)$ \\
Engraftment (days), median (IQR) & $17(15-19)$ \\
Cellularity -TNC-, x10 ${ }^{8 / K g}$ median (IQR) & $8,66(5,81-10,71)$ \\
Median follow up, months (range) & $25(9,2-43,4)$ \\
2 years CI of relapse, \% & 26 \\
2 years Overall survival, \% & 61 \\
2 years event free survival, \% & 47 \\
\hline
\end{tabular}

[[O082 Table] 1. SCT characteristics]
Conclusions: In our experience, ES is a frequent complication of HaploSCT with post-transplant cyclophosphamide. To our knowledge, this is the largest study including only haploidentical SCT. Most cases of ES had a self-limited course or good response to corticosteroids, and there were no associated mortality. However ES can progress to multi-organ dysfunction with need of intensive supportive care. In our analysis, incidence of ES was higher with the use of sibling haplo-donors, and further studies are needed to confirm these results. We have not found relationship between ES and GVHD. Specific biomarkers may contribute to an early identification of this entity in order to install therapeutic measures.

Disclosure: Nothing to declare

\section{3}

Features and outcome of early cardiac toxicity associated with post-transplant cyclophosphamide in allogeneic stem cell transplantatioN

Rémy Duléry ${ }^{1}$, Razan Mohty ${ }^{2}$, Myriam Labopin ${ }^{1}$, Simona Sestili $^{2}$, Florent Malard, Eolia Brissot ${ }^{1}$, Giorgia Battipaglia $^{1}$, Clemence Mediavilla ${ }^{1}$, Anne Banet ${ }^{2}$, Zoé Van de Wyngaert $^{2}$, Annalisa Paviglianiti ${ }^{2}$, Ramdane Belhocine ${ }^{2}$, Françoise Isnard', Simona Lapusan', Rosa Adaeva ${ }^{2}$, Anne Vekhoff', Tounes Ledraa ${ }^{2}$, Ollivier Legrand ${ }^{1}$, Ariel Cohen $^{3}$, Agnès Bonnin', Stéphane Ederhy ${ }^{3}$, Mohamad Mohty $^{2}$

${ }^{1}$ Sorbonne University (UPMC), Paris, France, ${ }^{2}$ Saint Antoine Hospital, Paris, France, ${ }^{3}$ Saint Antoine Hospital, AP-HP, Sorbonne University (UPMC), Paris, France

Background: Data on risk factors and incidence of early cardiac events (ECE) after post-transplant Cyclophosphamide (PT-Cy) are scarce. Thus, we compared clinical outcomes between patients who received PT-Cy and patients who did not in a cohort study including all consecutive patients allografted in our center.

Methods: We analyzed all ECE occurring within 3 months after HSCT in 331 patients. Transthoracic echocardiography and EKG were performed before HSCT, at day +90 and in case of ECE. Prior to transplant, 276 patients $(83.4 \%)$ had at least one cardiovascular risk factor, $72(21.8 \%)$ cardiovascular disease history and $31(9.4 \%)$ left ventricular systolic dysfunction (LVSD) (defined by left ventricular ejection fraction $<53 \%$ ). Median age was 55 years (range, 15-76) and $60.4 \%$ of patients were males. Patients were transplanted for AML $(n=153,46 \%)$, ALL $(\mathrm{n}=49,15 \%)$, lymphoma $(\mathrm{n}=44,13 \%)$, multiple myeloma $(\mathrm{n}=8,2.4 \%)$, MDS $(\mathrm{n}=35,11 \%)$ and MPN $(\mathrm{n}=42,13 \%)$. Disease risk index was high or very-high in 93 patients (28\%). Conditioning regimen were MAC $(n=131,40 \%)$, 
RIC $(n=85,26 \%)$ or sequential (Flamsa-like) $(n=115$, $35 \%)$. Donors were matched related $(\mathrm{n}=89,27 \%)$, unrelated $(n=124,37 \%)$ or haploidentical $(n=118,36 \%)$. Stem cell source was peripheral blood $(n=310,94 \%)$ or bone marrow $(n=21,6 \%)$. GVHD prophylaxis included cyclosporine in all patients associated with mycophenolate mofetil $(n=267$, $81 \%)$, short courses of methotrexate $(n=23,7 \%)$ and/or antithymocyte globulin $(n=312,94 \%)$. In the PT-Cy group, 136 patients received PT-Cy at $50 \mathrm{mg} / \mathrm{kg} /$ day for at least 1 day and 100 patients for 2 days, including 13 patients with unrelated donor and 6 patients with matched related donor, either because of HLA-mismatch or renal insufficiency, or inclusion in a clinical trial.

Results: In univariate analysis, cumulative incidence of ECE was $21.3 \%$ in the PT-Cy group and $8.2 \%$ in the no PTCy group $(\mathrm{p}<0.001)$. The main complication was LVSD (15\% of patients in the PT-Cy group and $3 \%$ in the no PT$\mathrm{Cy}$ group, $\mathrm{p}<0.001)$. Other ECE included acute pulmonary edema $(n=13,4 \%)$, arrhythmia $(n=12,4 \%)$, pericarditis $(n=7,2 \%)$ and coronary artery syndrome $(n=3,1 \%)$ in the whole patient group. ECE resolved in 38 patients $(78 \%)$. Cardiovascular risk factors and the cumulative doses of anthracycline were not significantly associated with the incidence of ECE. In multivariate analysis, the main factors associated with ECE were the use of PT-Cy $[\mathrm{HR}=2.8,95 \%$ CI 1.5-5.1; $\mathrm{p}=0.001]$, older age $[\mathrm{HR}=1.3$, 95\% CI 1-1.6; $\mathrm{p}=0.04]$, sequential conditioning $[\mathrm{HR}=2.5$, 95\% CI 1.2-5; $\mathrm{p}=0.01]$. A trend was observed in case of a cardiovascular disease history $[\mathrm{HR}=1.8,95 \% \mathrm{CI}$ 0.9-3.4; $\mathrm{p}=0.07]$.

After a median follow-up of 36.5 months (IQR, 32-40), the 2-year cumulative incidences of NRM, relapse, OS and DFS were $28 \%$ vs. $22 \%$; $23 \%$ vs. $19 \%$; $56 \%$ vs. $63 \% ; 49 \%$ vs. 59\% in the PT-Cy and no PT-CY groups, respectively (p values non significant). At last-follow-up, 136 patients have died. The main causes of death were disease relapse $(n=51)$, GVHD/infection $(n=48)$ and ECE $(n=8)$.

Conclusions: Incidence of ECE is significantly higher in the PT-Cy group. In elderly patients or with a history of pretransplant cardiac event, an alternative to PT-Cy should be considered to prevent ECE.

Disclosure: Nothing to declare

\section{4}

Severe iron overload, measured by liver MRI at the preallo-HSCT, significantly impaired the long-term outcome of the procedure

Lourdes Aguirre', Emma Salvador ${ }^{2}$, Josune Zubicaray ${ }^{1}$, M. José Pizarro', Ane Ugarte', Anunciación Urquíal, Iratxe Urreta ${ }^{3}, J o s e ́$ Emparanza ${ }^{3}$, José M. Alustiza ${ }^{2}$, Carlos Vallejo ${ }^{1}$
${ }^{1}$ University Hospital Donostia, San Sebastián, Spain, ${ }^{2}$ University Hospital Donostia, OSATEK, San Sebastián, Spain, ${ }^{3}$ University Hospital Donostia, San Sebastián, Spain

Background: Pre-transplant iron overload (IO) has been suggested to have a detrimental effect on long-term survival of HSCT recipients. In this analysis, we wanted to check if IO affected adversely to the long-term outcome of our series de patients.

Methods: Once approved by the Clinical Trials and Ethics Committee, a liver MRI was systematically offered to patients who were admitted to undergo an allo-HSCT in our Center. Among the 131 pts consecutively transplanted between June 2015 and July 2018, 100 pts signed the informed consent and underwent a pre-HSCT MRI to assess the hepatic iron load. A Signal Intensity Ratio (SIR) method was employed for the measurement.

Results: 64 pts were male, and 36 were female. Median age was 54 years (range: 12-69). The baseline diseases were: 37 AML, 24 NHL, 16 ALL, 8 MDS, 8 cMPD, 6 MM, and 1 BMF. 67 underwent alternative donor transplants (56 unrelated, and 11 haplo-identical), and 33 HLA-id family donor transplants. Stem cell source was $\mathrm{PB}$ in 96 , and $\mathrm{BM}$ in 4 cases. Conditioning regimen was intensive in 51 pts, and RIC in 49; no non-myeloablative allo-HSCT were performed. Based on hepatic iron overload at pre-HSCT MRI, the patients were classified into the following groups: 23 pts $(23 \%)$ showed severe IO $(\mathrm{LIC}>80 \mathrm{micromol} / \mathrm{g}$ or $4.5 \mathrm{mg} / \mathrm{g}), 25 \mathrm{pts}(25 \%)$ moderate IO (LIC $36-80 \mathrm{micromol} / \mathrm{g}$ or $2.1-4.4 \mathrm{mg} / \mathrm{g}$ ) and 52 pts $(52 \%)$ no significant IO (LIC $<36$ micromol/g or $2.1 \mathrm{mg} / \mathrm{g}$ ). As shown in table 1 , majority of patients with severe iron overload had been heavily transfused, and had a high pre-HCST ferritin level. Surprisingly, pre-HSCT chelation had been employed only in 3 pts $(13 \%)$ of this group. Overall mortalities at days $+100,+180$ and +365 in the global series, and in severe versus non-severe IO group are reflexed in table 2 .

\section{Conclusions:}

1) our data shows that pre-allo-HSCT iron-overload correlates with previous PRBC transfusion load;

2) it also makes evident that IO is an important risk factors for post-transplant mortality.

3) our real-life study reflects that only a minority of heavily transfused pts had received chelation therapy previously to the allo-HSCT.

4) considering the relevance of pre-allo-HSCT iron overload, we strongly suggest to referring physicians to employ chelation therapy for patient candidates to transplant during the treatment of the underline disease. 


\begin{tabular}{|c|c|c|c|c|}
\hline & $\begin{array}{l}\text { Liver MRI } \\
\text { Pre-HSCT } \\
\text { (micromol/ } \\
\text { g) }\end{array}$ & $\begin{array}{l}\text { Liver } \\
\text { MRI Pre- } \\
\text { HSCT } \\
(\mathrm{mg} / \mathrm{g})\end{array}$ & $\begin{array}{l}\text { PRBC } \\
\text { transfused } \\
\text { pre-HSCT }\end{array}$ & $\begin{array}{l}\text { Ferritin } \\
\text { pre- } \\
\text { HSCT }\end{array}$ \\
\hline $\begin{array}{l}\text { Pts without severe } \\
\text { iron overload in pre- } \\
\text { HSCT liver MRI } \\
(N=77)\end{array}$ & $\begin{array}{l}1.4(0.3- \\
4.4)\end{array}$ & $\begin{array}{l}24.7(5.6- \\
79.6)\end{array}$ & $12(0-159)$ & $\begin{array}{l}919(18- \\
3382)\end{array}$ \\
\hline $\begin{array}{l}\text { Pts with severe iron } \\
\text { overload in pre-HSCT } \\
\text { liver } M R I(N=23)\end{array}$ & $\begin{array}{l}5.6(4.5- \\
13.9)\end{array}$ & $\begin{array}{l}99.5(80- \\
248)\end{array}$ & $28(5-120)$ & $\begin{array}{l}1880 \\
(454- \\
6195)\end{array}$ \\
\hline$p$ & $<0.05$ & $<0.05$ & $<0.05$ & $<0.05$ \\
\hline
\end{tabular}

[[O084 Table] 1. Table 1: Characteristics of pts based on pre-allo-HSCT iron overload (liver MRI)]

\begin{tabular}{llll}
\hline & $\begin{array}{l}\text { Day }+100 \\
\text { overall } \\
\text { mortality }\end{array}$ & $\begin{array}{l}\text { Day }+180 \\
\text { overall } \\
\text { mortality }\end{array}$ & $\begin{array}{l}\text { Day }+365 \\
\text { overal } \\
\text { mortality* }\end{array}$ \\
\hline Global $(N=100)$ & $6 / 100$ & $9 / 100$ & $18 / 68$ \\
$(6.0 \%)$ & $(9.0 \%)$ & $(26.5 \%)$ \\
$\begin{array}{l}\text { Pts without severe iron } \\
\text { overload in pre-HSCT liver }\end{array}$ & $3 / 77(3.9 \%)$ & $4 / 77(5.2 \%)$ & $8 / 48(16.7 \%)$ \\
$\begin{array}{l}\text { MRI }(N=77) \\
\text { Pts with severe iron } \\
\text { overload in pre-HSCT liver }\end{array}$ & $3 / 23$ & $5 / 23$ & $10 / 20$ \\
$\begin{array}{l}\text { MRI }(N=23) \\
p\end{array}$ & $<0.05$ & $(21.7 \%)$ & $(50.0 \%)$ \\
\hline
\end{tabular}

*Only pts with follow-up >1 year or death before

[[O084 Table] 2. Table 2:Overall short and long term mortality based on pre-allo-HSCT iron overload (liver MRI)]

Disclosure: Nothing to declare

\section{5}

Non-infectious neurologic complications after allogeneic hematopoietic stem cell transplantation (ALLO-HSCT)

Aitana Balaguer Rosello ${ }^{I}$, Luis Bataller ${ }^{I}$, Carlos Carretero $^{I}$, Carmen Freiria ${ }^{I}$, Ana Villalba ${ }^{I}$, Marta Santiago ${ }^{I}$, Maria Isabel Moreno', Juan Montoro', Jose Luis Piñana $^{I}$, Manuel Guerreiro ${ }^{I}$, Jose Ignacio Lorenzo ${ }^{I}$, Guillermo Sanz ${ }^{I}$, Miguel Ángel Sanz ${ }^{I}$, Jaime Sanz ${ }^{I}$

${ }^{1}$ Hospital Universitari i Politècnic la Fe, Valencia, Spain

Background: Neurologic complications (NCs) are associated with relevant morbidity and mortality after alloHSCT. The aim of this study was to analyze the incidence, characteristics, risk factors and outcomes of patients developing NCs after allo-HSCT.
Methods: We evaluated 971 consecutive adult patients (>17 years) who underwent allo-HSCT at our center between January 2000 and December 2016.

We collected data on neurological symptoms, diagnostic methods, time of onset and cause. NC was defined as any neurological event that occurred after starting the conditioning regimen and before relapse. NC due to central nervous system (CNS) infections o neoplastic infiltration were excluded. We considered both CNS and peripheral nervous system (PNS) complications.

Results: The current series comprised 467 allo-HSCT from matched sibling donor (MSD), 381 from umbilical cord blood (UCB), 49 matched unrelated donor (MUD) and 74 haploidentical donor (HAPLO). Median age was 41 years and most patients had acute leukemia (63\%). Median follow-up of surviving patients was 71 months (range, 11213). There were differences in median follow-up according to the donor source, being longer in UCB and MSD, 87 and 67 months respectively, and shorter in MUD and HAPLO, 23 and 24 months respectively $(P<0.0001)$.

Overall, NCs were documented in 149 cases, 64 after MSD transplants, 74 after UCB, 9 after MUD, and 5 after HAPLO. CNS complications (68\%) were more common than PNS events. The most frequent NC was encephalopathy $(31 \%)$ followed by myopathy $(13 \%)$ and neuropathy (13\%). $17 \%$ of the PNS complications were immunemediated. Median time to NC was 78 days (range, -5-3722)

\begin{tabular}{|c|c|c|c|c|c|}
\hline $\begin{array}{l}\text { Neurologic } \\
\text { complications } \\
\text { Events, n (\%) }\end{array}$ & $\begin{array}{l}\text { Days 0- } \\
30, \\
95(100)\end{array}$ & $\begin{array}{l}\text { Days } \\
31-100, \\
26(100)\end{array}$ & $\begin{array}{l}\text { Days } \\
101-365, \\
30(100)\end{array}$ & $\begin{array}{l}>1 \text { year, } \\
24(100)\end{array}$ & $\begin{array}{l}\text { Total, } \\
149(100)\end{array}$ \\
\hline Stroke & $6(9)$ & $2(8)$ & $4(13)$ & $3(13)$ & $15(10)$ \\
\hline $\begin{array}{l}\text { Posterior } \\
\text { reversible } \\
\text { encephalopathy } \\
\text { syndrome }\end{array}$ & $5(7)$ & 0 & 0 & $1(4)$ & $6(4)$ \\
\hline Encephalopathy & $25(36)$ & 7 (26) & $7(23)$ & $7(29)$ & $46(31)$ \\
\hline Isolated seizures & $4(6)$ & $1(4)$ & $3(10)$ & $1(4)$ & $9(6)$ \\
\hline Headache & $17(25)$ & 0 & $1(3)$ & $2(8)$ & $20(13)$ \\
\hline Myelopathy & $1(1)$ & $2(8)$ & 0 & $2(8)$ & $5(3)$ \\
\hline Neuropathy & $6(9)$ & $5(19)$ & $6(20)$ & $3(13)$ & $20(13)$ \\
\hline Myopathy & $5(7)$ & $7(27)$ & $6(20)$ & $2(8)$ & $20(13)$ \\
\hline $\begin{array}{l}\text { PNS immune- } \\
\text { mediated } \\
\text { disorders }\end{array}$ & 0 & $2(8)$ & $3(10)$ & $3(13)$ & $8(5)$ \\
\hline
\end{tabular}

[[O085 Table] 1. Neurologic complications according to post-transplant period]

1-year and 5-year cumulative incidence of $\mathrm{NCs}$ was $11 \%$ and $13 \%$, respectively. 5-year cumulative incidence was $11 \%$ after MSD, $17 \%$ after UCB, $16 \%$ after MUD, and $6 \%$ after HAPLO transplants $(P=0.014)$. 
Multivariable analyses identified alternative donors (hazard risk[HR],1.7; 95\%confidence interval[CI], 1.12$2.3 ; P=0.009)$ (Figure 1B), older age $(>40 \mathrm{y} ; \mathrm{HR} 1.6 ; 95 \% \mathrm{CI}$, $1.1-2.7 ; P=0.009$ ), acute GvHD grade II-IV (HR,3.3; $95 \%$ CI, 2.2-5; $P<0.00001)$ and extensive chronic GvHD (HR,3.2; 95\%CI, 1.7-5.9; $P=0.0002$ ) as risk factors for developing NCs.

The 5-year overall survival (OS) was $21 \%$ for patients who developed NCs and $41 \%$ for the rest $(P<0.0001$; Figure1C). Statistically significant differences in OS were seen in patients who developed CNS NCs $(\mathrm{P}=0.001)$, but not in patients who developed PNS complications $(\mathrm{P}=0.36)$.

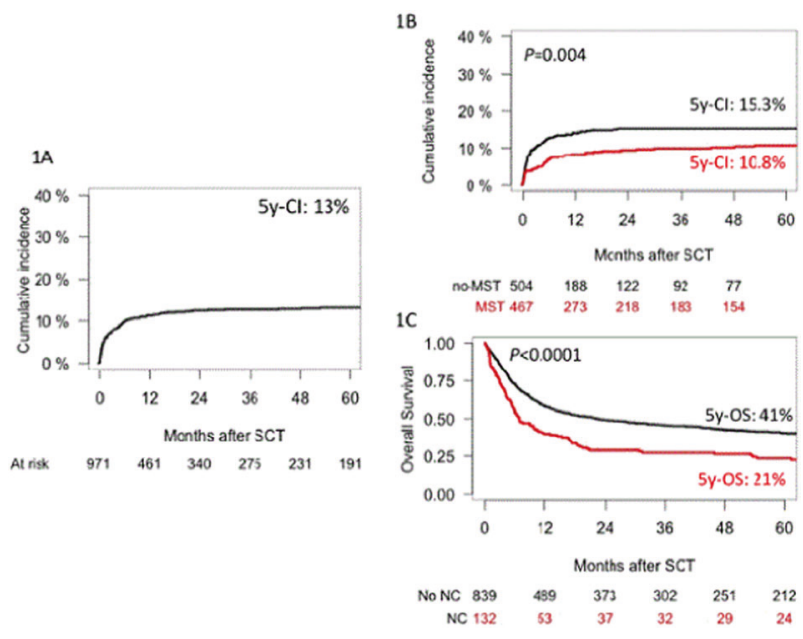

[[O085 Image] 1. Cumulative incidence of neurologic complications $(1 A, 1 B)$ and impact on $O S(1 C)]$

Conclusions: NCs are common and diverse after alloHSCT. NCs were more frequent in recipients allografted from alternative donors, recipients older than 40 years, and in those developing GvHD. CNS complications, but not PNS, are associated with poor OS.

Disclosure: Nothing to declare.

\section{6}

Abstract already published.

\section{7}

Using ciclosporin's area under the curve (AUC) to predict risk of acute kidney injury in non-myeloablative haematopoietic stem cell transplantation

VAIDIE Julien', Jean-Baptiste Woillard', Stéphane Girault $^{1}$, Pierre Marquet, Franck Saint-Marcoux ${ }^{1}$, Arnaud Jaccard ${ }^{1}$, Pascal Turlure

${ }^{1}$ CHU Limoges, Limoges, France
Background: Non myeloablative allogeneic stem cell transplantation (HSCT) by limiting toxicity, can be proposed to elderly patients. However, acute renal injuries related to anti-calcineurin, which are frequent in this population, can negatively impact the outcome. Currently, the exposure indexes to follow and the target to use are not consensually established. However, using the area under the curve (AUC) for therapeutic drug monitoring (TDM) is theoretically the best method to describe the patient's exposure. The primary objective of this study was to determine an AUC target for ciclosporin associated to the occurrence of acute kidney injury in HSCT patients.

Methods: We retrospectively studied all consecutive patients who received a non-myeloablative allogeneic stem cell transplantation at Limoges University Hospital from June 2009 to December 2015. Patients received Fludarabine $30 \mathrm{mg} / \mathrm{m} 2 /$ day between D-6 and D-2 before allograft and Busulfan $3.2 \mathrm{mg} / \mathrm{kg} /$ day at D-4 and D-3. GvH prophylaxis consisted in rabbit anti-lymphocyte serum at the dose of 2.5 $\mathrm{mg} / \mathrm{kg}$ at D-2 and D-1, and ciclosporin at the beginning dose of $3 \mathrm{mg} / \mathrm{kg}$ per os twice a day. Mycophenolate mofetil was added for patients with HLA-matched or mismatched unrelated donors. TDM of ciclosporin was done based on trough concentration $(\mathrm{C} 0)$ twice a week with concomitant renal evaluation using creatininemia. Dose adjustments were done in according to the SFGM-TC recommendations and renal tolerance. Ciclosporin's AUC was evaluated at day 1, day 14 and day 28 after allograft using bayesian estimation from a limited sampling strategy and a population pharmacokinetics model previously published. The association between ciclosporin AUC and acute kidney injury was investigated using a joint model. A ROC curve was then constructed to investigate an AUC threshold associated with the best sensibility/specificity ratio for acute kidney injury (AKI).

Results: We included 79 patients, with a median [minmax] age of 60 [33-70] years. 17\% of patients received an HLA-identical related donor, $70 \%$ an HLA-matched related donor and $13 \%$ an HLA-mismatched unrelated donor. The five years overall survival and survival without relapse or GVH probabilities were 73\% IC95 [63\%-84\%] and 60\% respectively IC 95 [56\%-64\%]. At M3, 39 patients (49\%) developed an AKI at a median time of 64 [7-100] days. The risk of developing AKI increased with ciclosporin AUC (per unit AUC increase, HR [95\%CI] = 1.10 [1.004-1.265]) $(\mathrm{p}=0.035)$. A threshold of $5,15 \mathrm{mg} * \mathrm{~h} / \mathrm{L}$ for ciclosporin AUC0-12h was associated with an AUC ROC of 0.64 IC95 $[0.57-0.71]$ (sensitivity $=70,27 \%$ IC95 [53\%-84.1\%] and specificity $=57.42 \%$ IC95 [49.2\%-65.3\%]). Interestingly, a very low correlation was observed between ciclosporin $\mathrm{C} 0$ and AUC ( $\mathrm{r}^{2}=0.55$ for the overall period). Additionally, an higher intra-individual variability was observed with $\mathrm{C} 0$ 
than AUC (coefficient of variation $=36 \%$ and $26 \%$ respectively).

Conclusions: We report in this study that a ciclosporin $\mathrm{AUC}=5.15 \mathrm{mg} * \mathrm{~h} / \mathrm{L}$ could be used as a high threshold for AKI. New evaluations of AUC in prospective studies are needed to better define the relevance of this marker in clinical practice.

Disclosure: No disclosure

\section{Experimental stem cell transplantation}

\section{8}

Abstract already published.

\section{O089}

Clonal Dynamics of single-donor derived human cord blood hematopoietic progenitor cells in murine xenografts revealed by cellular barcoding

Mirjam Belderbos ${ }^{1,2}$, Taco Koster ${ }^{2}$, Sabrina Jacobs ${ }^{2}$, Bertien Dethmers-Ausema ${ }^{2}$, Erik Zwart ${ }^{2}$, Gerald de Haan $^{2}$, Leonid Bystrykh ${ }^{2}$

${ }^{1}$ Princess Máxima Center for Pediatric Oncology, Utrecht, Netherlands, ${ }^{2}$ European Research Institute for the Biology of Ageing, Groningen, Netherlands

Background: Human cord blood (CB) provides an attractive source of hematopoietic stem cells for allogeneic transplantation of patients with a variety of diseases. A sufficient hematopoietic stem cell (HSC) dose, currently measured as $\mathrm{CD} 34+$ cells/kg recipient, is essential for successful engraftment. Nevertheless, the frequency of true HSC within the CD34+ population and the dynamics of their clonal offspring remain poorly understood and may differ between donors.

Methods: Here, we use cellular barcoding and multiplexed high-throughput sequencing to determine the frequency of repopulating cells among CD34+ cells from 20 individual human $\mathrm{CB}$ donors, and to quantify their contribution to each of the blood lineages over time in murine NOD/Scid/IL2Ry ${ }^{-/-}$(NSG) xenografts.

Results: In total, we detected a median of 14.3 (range 5168) clones in blood and 15.0 (range 1.7-85) clones in bone marrow, corresponding to HSPC frequencies of 1:100 to $1: 1000$. The number of retrieved clones correlated to barcoded CD34+ cell dose (Spearman $\mathrm{R}=0.85, \mathrm{p}=0.001$ ), yet could vary up to four-fold between mice transplanted with the same cell dose. Clonal patterns in blood early after transplant differed markedly from those at later time points, and became increasingly deterministic over time. The majority of clones displayed multilineage output, yet clones with bias towards lymphoid or myeloid lineages were also present. Similar to recent data on murine HSC clones, human $\mathrm{CB}$ clones were distributed asymmetrically across different bone marrow sites.

Conclusions: In conclusion, the frequency of NSGrepopulating cells among cord blood $\mathrm{CD} 34^{+}$cells is low, and highly variable between individual $\mathrm{CB}$ donors. Heterogeneity in HSC frequency, proliferation and lineage fate decisions may contribute to (non-)engraftment upon HSCT. Future research will be aimed at identifying the underlying mechanisms guiding HSC behavior upon transplantation and expanding our findings to human HSCT recipients.

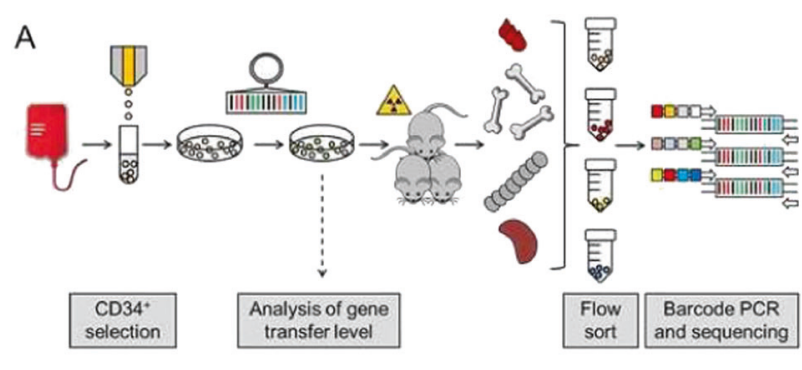

B

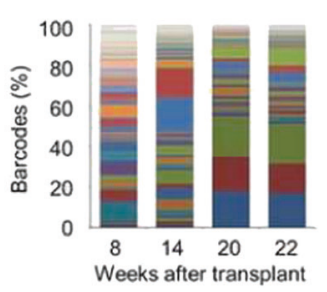

C

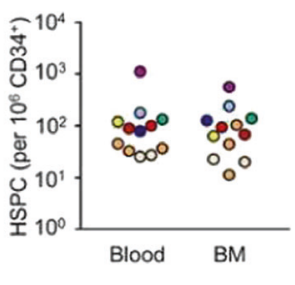

[[O089 Image] 1. Figure 1: Frequency and inter-donor variability in HSPC among cord blood CD34+ cells]

Clinical Trial Registry: n/a.

Disclosure: Nothing to declare.

O090

Abstract already published.

0091

Abstract already published.

\section{Gene Therapy}

0092

Effective lentiviral gene therapy for X-linked chronic granulomatous disease

Claire Booth', Donald B Kohn ${ }^{2}$, Elizabeth M Kang ${ }^{3}$, Sung-Yun Pai ${ }^{4}$, Kit L Shaw ${ }^{2}$, Giorgia Santilli', Myriam Armant $^{4}$, Karen Buckland ${ }^{5}$, Uimook Choi ${ }^{3}$, Suk See De 
Ravin $^{3}$, Morna Dorsey ${ }^{6}$, Caroline Kuo ${ }^{2}$, Diego LeonRico $^{5}$, Christine Rivat ${ }^{5}$, Katie Snell ${ }^{5}$, Jinhua Xu-Bayford ${ }^{5}$, Emma Morris ${ }^{5}$, Dayna Terrazas ${ }^{2}$, Leo D Wang ${ }^{7,4}$, Geraldine Honnet ${ }^{8}$, Peter Newburger ${ }^{9}$, Frederic D Bushman $^{10}$, Manuel Grez ${ }^{11}$, H Bobby Gaspar ${ }^{5,12}$, David A Williams $^{4}$, Harry L Malech ${ }^{3}$, Anne Galy, Adrian J Thrasher $^{5}$

${ }^{1}$ University College London/Great Ormond Street Hospital, London, United Kingdom, ${ }^{2}$ University of California, Los Angeles, CA, United States, ${ }^{3}$ National Institute of Allergy and Infectious Diseases, Bethesda, MD, United States, ${ }^{4}$ Boston Children's Hospital, Boston, MA, United States, ${ }^{5}$ Great Ormond Street Hospital, London, United Kingdom, ${ }^{6}$ University of California, San Fransisco, CA, United States, ${ }^{7}$ City of Hope, Beckman Research Institute, Duarte, CA, United States, ${ }^{8}$ Genethon, Evry, Paris, France, ${ }^{9}$ University of Massachusetts, Worcester, MA, United States, ${ }^{10}$ University of Pennsylvania, Philadelphia, PA, United States, ${ }^{11}$ Georg-Speyer Haus, Frankfurt, Germany, ${ }^{12}$ Orchard Therapeutics, London, United Kingdom

Background: Chronic Granulomatous Disease (CGD) is a rare genetic immune disorder that leaves patients susceptible to life-threatening infections, chronic inflammation and often long hospital stays. X-linked CGD (X-CGD) is caused by mutations in $C Y B B$ encoding the gp91phox subunit of the phagocyte NADPH-oxidase, which regulates cell $\mathrm{pH}$ and ionic content for efficient microbial killing. Allogeneic hematopoietic stem cell transplant (HSCT) has been a potentially curative approach for X-CGD patients, but is often complicated by lack of HLA-matched donors and risks of graft versus host disease, graft rejection, and procedure-related fatality. Previous attempts at autologous ex vivo gene therapy for X-CGD using gammaretroviral vectors have met with limited efficacy due to transient engraftment of gene corrected cells, gene silencing, and mutagenic activation leading to myelodysplasia. Here we report on 9 patients with a history of severe X-CGD-related complications, who received autologous ex vivo gene therapy (GT) using a novel self-inactivating lentiviral vector (G1XCGD LV) designed to limit the risk of mutagenesis through preferential expression of the missing g91phox subunit in mature myeloid cells.

Methods: Similar trials of GT with G1XCGD LV were initiated in the UK ( $n=3$, plus 1 compassionate use patient) and in the USA ( $\mathrm{n}=5,3$ sites). All patients had histories of severe, persistent infections, and inflammatory disease. GCSF and Plerixafor-mobilized CD34+ selected hematopoietic stem and progenitor cells were transduced ex vivo with G1XCGD LV. Subjects received near-myeloablative conditioning with single agent busulfan, targeted to net area-under-the-curve of $70,000 \mathrm{ng} / \mathrm{ml} * \mathrm{hr}$. Freshly prepared or cryopreserved quality-tested gene-modified cells, manufactured on-site, were administered intravenously. Primary endpoints were efficacy, as determined by percent of oxidase positive granulocytes by dihydrorhodamine [DHR] flow cytometry, and safety at 12 months.

Results: We report results for 7 patients (2-27 years) with 1-2.5 years of follow-up; 2 additional patients were treated but died within 3 months of GT from complications deemed related to pre-existing disease-related co-morbidities (severe pulmonary disease, anti-platelet antibodies). GT was welltolerated, only one serious adverse reaction (a systemic inflammatory process at engraftment of functional neutrophils) was reported as possibly related to GT. Patients experienced typical conditioning-related transient neutropenia, thrombocytopenia and mucositis. There has been no molecular evidence for clonal dysregulation or gene silencing through $\mathrm{CpG}$ dinucleotide methylation. Followup demonstrated sustained stable persistence of $12-46 \%$ oxidase $(+)$ neutrophils for $>12$ months in $6 / 7$ surviving patients (one, who remains clinically well, had a decline to $<5 \%$ after 3 months) [Figure]. These patients have maintained restoration of biochemical function and immunity (defined as $\geq 10 \%$ of oxidase $(+)$ by DHR) as of December 2018. Patients have been well, without new XCGD-related infections, and 4 are successfully weaned off prophylactic antibiotics.

Conclusions: These results are the first demonstration of effective autologous lentiviral GT at 12 months in severely affected X-CGD patients without evidence of genotoxicity. Corrected neutrophil function has been observed in 6 patients for $>12$ months and has been associated with significant clinical improvement, freedom from infections, and resolution of chronic inflammation. Results are supportive of extended clinical trials evaluating the safety and efficacy of G1XCGD LV-based gene therapy.

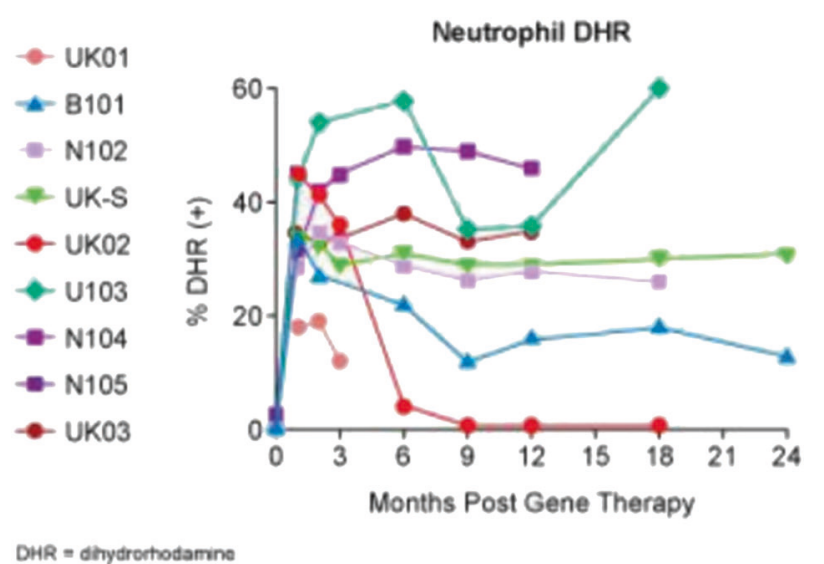

[[0092 Image] 1. Percent oxidase $(+)$ neutrophils over time] 
Clinical Trial Registry: USA: NCT02234934, Europe: NCT01855685

Disclosure: Donald Kohn: National Institutes of Health (grants), Orchard Therapeutics (grants, paid consultant), patent Lentiviral vector: gene therapy of ADA-SCID. Claire Booth: Honoraria from GSK: providing educational material on ADA-SCID. Sung-Yun Pai: Boston Children's Hospital (salary support), National Institutes of Health (grants). Kit Shaw: California Institute of Regenerative Medicine (grants); Orchard Therapeutics (personal fees, non-financial support) Caroline Kuo: California Institute of Regenerative Medicine, Gene Therapy Resource Program (grants). Dayna Terrazas: California Institute of Regenerative Medicine (grants). Leo Wang: NHLBI (grants), Genethon (non-financial support); Magenta Therapeutics (personal fees). H Bobby Gaspar: Orchard Therapeutics (employee, equity/stock holder), patent Lentiviral vector: gene therapy of ADA-SCID. David Williams: Orchard Therapeutics (co-founder, SAB member) Harry Malech: Orchard Therapeutics (unpaid member scientific advisory board) Adrian Thrasher: Orchard Therapeutics, Rocket Pharmaceuticals, Generation Bio (Board member, consultant); 4BIOCapital (board member). The following authors have nothing to declare: Elizabeth Kang, Giorgia Santilli, Myriam Armant, Karen Buckland, Uimook Choi, Suk See De Ravin, Morna Dorsey, Karen Buckland, Uimook Choi, Suk See De Ravin, Diego Leon-Rico, Christine Rivat, Katie Snell, Jinhua Xu-Bayford, Emma Morris, Geraldine Honnet, Peter Newburger, Frederic Bushman, Manuel Grez, Anne Galy.

\section{3}

Updated results from the ongoing northstar-2 (HGB207) trial of lentiglobin gene therapy in patients with transfusion-dependent $\beta$-thalassemia and non- $\boldsymbol{\beta}^{\mathbf{0}} / \boldsymbol{\beta}^{\mathbf{0}}$ genotypes

Franco Locatelli, Alexis Thompson ${ }^{2,3}$, Janet Kwiatkowski, ${ }^{4,5}$, Suradej Hongeng, ${ }^{6}$, John Porter ${ }^{7}$, Martin Sauer $^{8}$, Adrian Thrasher', Isabelle Thuret ${ }^{10}$, Heidi Elliot $^{11}$, Ge Tao ${ }^{11}$, Mohammed Asmal ${ }^{11}$, Mark Walters ${ }^{12}$

${ }^{1}$ IRCCS Bambino Gesù Children's Hospital, Rome, Italy, ${ }^{2}$ Ann and Robert H. Lurie Children's Hospital, Chicago, IL, United States, ${ }^{3}$ Northwestern University, Chicago, IL, United States, ${ }^{4}$ Children's Hospital of Philadelphia, Philadelphia, PA, United States, ${ }^{5}$ Perelman School of Medicine University of Pennsylvania, Philadelphia, PA, United States, ${ }^{6}$ Mahidol University and Ramathibodi Hospital, Bangkok, Thailand, ${ }^{7}$ University College London Hospitals, London, United Kingdom, ${ }^{8}$ Medizinische Hochschule Hannover, Hannover, Germany, ${ }^{9}$ UCL Great Ormond Street Institute of Child Health, London, United Kingdom,
${ }^{10}$ University Hospital of Marseille - Timone Hospital, Marseille, France, ${ }^{11}$ bluebird bio, Inc., Cambridge, MA, United States, ${ }^{12}$ UCSF Benioff Children's Hospital, Oakland, CA, United States

Background: Allogeneic hematopoietic stem cell (HSC) transplantation is potentially curative for patients with transfusion dependent $\beta$-thalassemia (TDT); however, it is associated with risks of morbidity and mortality and is limited by donor availability. Gene therapy has the potential to be an effective treatment option for patients with TDT, but without some of these limitations. LentiGlobin gene therapy contains autologous CD34+ HSCs transduced ex vivo with the $\mathrm{BB} 305$ lentiviral vector encoding $\beta$-globin with the T87Q amino-acid substitution. LentiGlobin is being studied in patients with TDT and non- $\beta^{0} / \beta^{0}$ genotypes in the ongoing, phase 3 Northstar-2 study (HGB-207; NCT02906202).

Methods: Northstar-2 is enrolling patients with TDT who had a history of $\geq 100 \mathrm{~mL} / \mathrm{kg} /$ year of red blood cells (RBCs) or $\geq 8 \mathrm{RBC}$ transfusions/year. To generate drug product (DP), autologous CD34+ cells were collected by apheresis after G-CSF and plerixafor mobilization and transduced with the BB305 lentiviral vector. Patients received myeloablative conditioning with single-agent busulfan before DP infusion. Primary endpoint was the proportion of patients achieving transfusion independence (TI, weighted average hemoglobin $[\mathrm{Hb}] \geq 9 \mathrm{~g} / \mathrm{dL}$ without RBC transfusions for $\geq 12$ months). Patients are followed for 2 years and offered participation in a long-term followup study. Statistics are presented as median (min-max).

Results: As of 14 September 2018, 16 patients (age 19 [8-34] years; 9 patients $\geq 18$ years) have been treated (follow-up 9.3 [0.7-20.4] months). Patients received a cell dose of $7.7 \times 10^{6}(5.0-19.4) \mathrm{CD} 34+$ cells $/ \mathrm{kg}$ with a DP vector copy number of 3.1 (2.1-5.6) vector copies/diploid genome and $82 \%(53-90 \%)$ of cells were transduced. Baseline liver iron content (LIC) was 6.4 (1.0-41.0) $\mathrm{mg} \mathrm{Fe} / \mathrm{g}$ $\mathrm{dw}$. Outcomes by age and baseline LIC will be reported. Times to neutrophil and platelet engraftment were 19 (1332 ) and 44.5 (20-84) days, respectively; 1 patient (1.0month follow-up) and 4 patients (1.0-2.1 months follow-up) had not achieved neutrophil and platelet engraftment, respectively, at time of analysis.

Of 11 patients with $\geq 3$ months of follow-up, $10(6 \geq 18$ years old) stopped chronic RBC transfusions and two have achieved TI. At last study visit, total $\mathrm{Hb}$ in these 10 patients was 11.1-13.3 g/dL consisting of 7.7-10.6 g/dL gene therapy-derived $\mathrm{Hb}, \mathrm{HbA}^{\mathrm{T} 87 \mathrm{Q}}$. One treated patient had no $\mathrm{RBC}$ transfusions for 11 months, then re-initiated transfusions due to low $\mathrm{Hb}$.

Non-hematologic grade $\geq 3$ adverse events post-infusion in $\geq 3$ patients included stomatitis, febrile neutropenia, 
epistaxis, pyrexia, and veno-occlusive liver disease (VOD). One grade 3 event of serious prolonged thrombocytopenia after platelet engraftment was considered possibly related to LentiGlobin. The three grade 4 serious VOD events were attributed to myeloablative conditioning (Table 1). The events resulted in extended hospitalization and resolved following defibrotide treatment. There were no deaths or graft failure and no evidence of vector-mediated replication of competent lentivirus or clonal dominance.

Conclusions: In Northstar-2, 10/11 patients with TDT and non $-\beta^{0} / \beta^{0}$ genotypes treated to date produced sufficient gene therapy-derived $\mathrm{Hb}, \mathrm{HbA}^{\mathrm{T} 87 \mathrm{Q}}$, to stop chronic transfusions following LentiGlobin gene therapy. Total $\mathrm{Hb}$ in patients off RBC transfusions remains stable at $>11$ $\mathrm{g} / \mathrm{dL}$. The safety profile of LentiGlobin remains generally consistent with myeloablative busulfan conditioning.

\begin{tabular}{|c|c|c|c|c|}
\hline Parameters & Patient 9 & $\begin{array}{l}\text { Patient } \\
10\end{array}$ & Patient 11 & $\begin{array}{l}\text { Patients who did } \\
\text { not have a VOD } \\
\text { event }(N=13)\end{array}$ \\
\hline $\begin{array}{l}\text { Age at consent, } \\
\text { years median (min- } \\
\text { max) }\end{array}$ & 12 & 12 & 34 & $20(10-24)$ \\
\hline \multirow[t]{2}{*}{ Gender } & \multirow[t]{2}{*}{ Male } & \multirow[t]{2}{*}{ Female } & \multirow[t]{2}{*}{ Male } & $\begin{array}{l}54 \% \text { female (7/ } \\
13)\end{array}$ \\
\hline & & & & $46 \%$ male $(6 / 13)$ \\
\hline VOD prophylaxis & No & No & $\begin{array}{l}\text { Ursodeoxycholic } \\
\text { acid Day-8 to } \\
+38\end{array}$ & $62 \%$ Yes $(8 / 13)$ \\
\hline \multicolumn{5}{|c|}{ Baseline, median (min-max) } \\
\hline $\begin{array}{l}\text { Imaging LIC mg } \\
\text { Fe/g dw }\end{array}$ & 1.0 & 5.6 & 8.5 & $7.2(1.4-41)$ \\
\hline$A S T, U / L$ & 23 & 14 & 40 & $21(17-67)$ \\
\hline$A L T, U / L$ & 32 & 7 & 43 & $24(9-164)$ \\
\hline $\begin{array}{l}\text { Total bilirubin, } \\
\mu \mathrm{mol} / \mathrm{L}\end{array}$ & 20.9 & 22.9 & 25.3 & $36.8(13.9-96.8)$ \\
\hline $\begin{array}{l}\text { Average busulfan } \\
\text { AUC, }{ }^{ \pm} \mu{ }^{*} \text { min } \\
\text { median (min-max) }\end{array}$ & 4595 & 4471 & 4545 & $\begin{array}{l}4428(3709- \\
8947)^{ \pm}\end{array}$ \\
\hline \multirow[t]{2}{*}{$\begin{array}{l}\text { Busulfan dosing } \\
\text { schedule }\end{array}$} & \multirow[t]{2}{*}{ Every $24 h r$} & \multirow[t]{2}{*}{$\begin{array}{l}\text { Every } \\
24 h r\end{array}$} & \multirow[t]{2}{*}{ Every $24 h r$} & $\begin{array}{l}58 \% \text { every } 24 \mathrm{hr} \\
(7 / 12)^{ \pm}\end{array}$ \\
\hline & & & & $\begin{array}{l}42 \% \text { every } 6 h r \\
(5 / 12)^{ \pm}\end{array}$ \\
\hline \multirow[t]{2}{*}{$\begin{array}{l}\text { Busulfan dose } \\
\text { adjustment during } \\
\text { conditioning }\end{array}$} & \multirow[t]{2}{*}{$\begin{array}{l}\text { One increase } \\
\text { and one } \\
\text { decrease in } \\
\text { dose }\end{array}$} & \multirow[t]{2}{*}{$\begin{array}{l}\text { One } \\
\text { increase } \\
\text { in dose }\end{array}$} & \multirow[t]{2}{*}{$\begin{array}{l}\text { One decrease in } \\
\text { dose }\end{array}$} & $\begin{array}{l}42 \% 1 \text { increase } \\
\text { and } \\
1 \text { decrease }(5 / 12) \\
\pm\end{array}$ \\
\hline & & & & $\begin{array}{l}25 \% \geq 1 \text { increase } \\
(3 / 12)^{ \pm} \\
25 \% \geq 1 \text { increase } \\
(3 / 12)^{ \pm} \\
8 \% \text { no dose } \\
\text { change }(1 / 12)^{ \pm}\end{array}$ \\
\hline \multicolumn{5}{|c|}{$\begin{array}{l}\text { ALT, alanine aminotransferase; AST, aspartate aminotransferase; AUC, are under } \\
\text { curve; hr, hours, VOD, veno-occlusive liver disease, }+ \text { Estimated average daily } \\
\text { busulfan exposure over } 4 \text { days. Target busulfan AUC of } 1100 \text { (min-max } 1000-1250) \\
\left.\mu M^{*} \text { min for every } 6 \text { hours dosing, or } 4500 \text { (min-max } 4000-5000\right) \mu M^{*} \text { min for once } \\
\text { daily dosing. After protocol amendment, target busulfan AUC was reduced to } 1050 \\
(\text { min-max } 950-1125) \mu M^{*} \text { min for every } 6 \text { hours dosing, or } 4200 \text { (min-max } 3800- \\
4500) \mu M^{*} \text { min for once daily dosing. }{ }^{ \pm} N=12 \text {, busulfan conditioning regimen was not } \\
\text { available for one patient at time of data snapshot. }\end{array}$} \\
\hline
\end{tabular}

[[O093 Table] 1. Table 1. Characteristics of patients who did or did not experience a VOD in the Northstar-2 study]

\section{Clinical Trial Registry: ClinicalTrials.gov NCT02906202}

Disclosure: Franco Locatelli: honoraria from and board member for Amgen; honoraria from Miltenyi; consultant and board member for Novartis and Bellicum; consultant for bluebird bio, Inc. Alexis Thompson: research funding from Amgen, Baxalta/Shire, bluebird bio, Inc., Celgene, Novartis, Biomarin, La Jolla Pharmaceutical; consultant for bluebird bio, Inc. Janet Kwiatkowski: consultant for bluebird bio, Inc. and Agios Pharmaceuticals; research funding from bluebird bio, Inc., Agios Pharmaceuticals, Novartis, Apopharma, and Terumo.John Porter: honoraria from Agios and Cerus; consultant for Novartis. Adrian Thrasher: consultant for and owns stock in Orchard Therapeutics; consultant and board member for Generation Bio and Rocket Pharmaceuticals. Isabelle Thuret: research funding from Addmedica, bluebird bio, Inc., and Novartis. Heidi Elliot, Ge Tao, Mohammed Asmal: employees of and hold stock in bluebird bio, Inc. Mark Walters: consultant for bluebird bio, Inc., bioverativ, Trucode, Editas, and Sangamo Therapeutics; medical director of ViaCord Processing Lab and AllCells, Inc. Suradej Hongeng and Martin Sauer: have nothing to declare.

\section{4}

Lentiviral hematopoietic stem cell gene therapy (HSCGT) for metachromatic leukodystrophy (MLD) provides sustained clinical benefit

Francesca Fumagalli, ${ }^{1,2}$, Valeria Calbi ${ }^{1,2}$, Maria Sessa ${ }^{1,2}$, Federica Cugnata ${ }^{3}$, Paola MV Rancoita ${ }^{3}$, Serena Acquati $^{1}$, Daniela Redaelli, Vanessa Attanasio ${ }^{I}$, Simona Miglietta $^{1}$, Sara Magni ${ }^{1}$, Salvatore Recupero ${ }^{2}$, Francesca Ferrua $^{1,2}$, Federica Barzaghi ${ }^{1,2}$, Maria Pia Cicalese ${ }^{1,2}$, Maddalena Migliavaccal, ${ }^{1,2}$ Francesca Tucci, ${ }^{1,2}$, Rossana Fiori $^{2}$, Maurizio Ferrari ${ }^{2,3}$, Ivana Spiga ${ }^{2}$, Paolo Silvani ${ }^{2}$, Marcella Facchini, Sara Locatelli ${ }^{I}$, Stefano Zancan ${ }^{1}$, Francesca Ciotti, Maddalena Fraschini ${ }^{2}$, Marina Sarzana $^{2}$, Alberto Zambon ${ }^{2}$, Andrea Calabria ${ }^{1}$, Eugenio Montini $^{I}$, Sabrina Canale ${ }^{1}$, Gigliola Antonioli, ${ }^{1,2}$, Michela Gabaldo $^{1}$, Giada Farinelli ${ }^{1}$, Francesco Morena ${ }^{4}$, Cristina Baldoli $^{2}$, Martino Sabata ${ }^{2}$, Clelia Di Serio ${ }^{3}$, Fabio Ciceri $^{2,3}$, Maria Grazia Natali Sora ${ }^{2}$, Maria Ester Bernardo $^{1,2}$, Luigi Naldini ${ }^{1,3}$, Alessandra Biffi, ${ }^{1,2,5}$, Alessandro Aiuti ${ }^{1,2,3}$

${ }^{1}$ San Raffaele Telethon Institute for Gene Therapy (SRTIGET), Milan, Italy, ${ }^{2}$ IRCCS San Raffaele Scientific Institute, Milan, Italy, ${ }^{3}$ Vita Salute San Raffaele University, Milan, Italy, ${ }^{4}$ University of Perugia, Perugia, Italy, ${ }^{5}$ University of Padova, Pediatric Onco-Hematology, Padova, Italy 
Background: Metachromatic leukodystrophy (MLD) is an ultra-rare and devastating demyelinating lysosomal storage disease caused by mutations in the Arylsulfatase A (ARSA) gene, currently with no approved treatment. We report an interim analysis of the safety and efficacy results of 20 early-onset MLD subjects treated with experimental autologous, ex-vivo, lentiviral-mediated hematopoietic stem cell gene therapy (HSC-GT) followed for up to 8 years posttreatment, as part of an ongoing, open-label, study. The study has completed the enrollment period; follow up visits are currently on-going.

Methods: GT consists of a formulation of autologous $\mathrm{CD}^{+}{ }^{+}$cells transduced ex vivo with a self-inactivating lentiviral vector encoding for the human ARSA gene and administered intravenously after Busulfan conditioning. Twenty early-onset MLD subjects (pre-symptomatic or early symptomatic) were enrolled and treated (9 Late Infantile [LI] and 11 Early Juvenile [EJ]). Co-primary efficacy endpoints were improvement in Gross Motor Function Measure (GMFM) score (10\%) and a significant increase in ARSA activity in peripheral blood mononuclear cells (PBMCs), evaluated 24 months after treatment. Safety endpoints include engraftment failure and long-term safety and tolerability of lentiviral-transduction.

Results: $18 / 20$ subjects are alive after a clinical follow-up of 3-8 years. Two EJ subjects, treated after onset of symptoms, died due to rapid disease progression 8- and 15months post-treatment. There was no treatment-related mortality, no evidence of abnormal clonal expansion, and no adverse events related to the Medicinal Product.

Durable and stable engraftment of gene-corrected cells were observed beginning 1-month post-treatment, with persistent vector copy number in $\mathrm{CD} 34^{+}$bone marrow cells and PBMCs throughout the follow-up for all 18 subjects. Reconstitution of ARSA activity in the hematopoietic system was observed in both populations (LI and EJ), stabilizing at normal to supranormal levels within three months. ARSA activity in CSF showed a similar pattern; normal levels were observed 9-12 months post-treatment, demonstrating effective enzymatic production in the central nervous system (CNS).

The majority of LI and EJ subjects treated before the onset of overt symptoms showed normal motor development, stabilization of motor dysfunction or a significant delay in disease progression, as measured by GMFM total score and gross motor function classification (GMFC)MLD. Cognitive function (measurements included performance and verbal IQ scores) was maintained within normal range for most subjects, independent of their symptomatic status at the time of treatment. Improvement or stabilization of central demyelination and peripheral nervous system (PNS) abnormalities were observed in most subjects treated.
Conclusions: This interim analysis demonstrates that HSC-GT continues to be a safe and well-tolerated treatment for all MLD subjects treated with a clinical follow-up $\leq 8$ years. All subjects achieved high levels of multi-linage engraftment, polyclonal hematological reconstitution and central and peripheral ARSA activity reconstitution within or above normal levels. Patients treated prior to symptom onset achieved a sustained clinical benefit in motor and cognitive function as well as on instrumental biomarkers of PNS and CNS demyelination, suggesting that autologous, ex-vivo HSC-GT is a highly promising therapeutic approach for LI and EJ MLD pre-symptomatic subjects. Further research is needed to support the benefit:risk profile in EJ patients.

Clinical Trial Registry: NCT01560182

https:/www.clinicaltrials.gov/ct2/show/NCT01560182? term $=$ NCT01560182\&rank $=1$

Disclosure: The San Raffaele Telethon Institute for Gene Therapy (SR-TIGET) is a joint venture between Telethon and Ospedale San Raffaele (OSR). ADA-SCID gene therapy (Strimvelis) was licensed to GlaxoSmithKline (GSK) in 2010 and received European marketing authorization in 2016. Alessandro Aiuti is the PI of the ADASCID long-term follow up clinical trial sponsored by GSK. Strimvelis was licensed to Orchard Therapeutics (OTL) in April 2018.

\section{5}

Lentiglobin gene therapy in patients with sickle cell disease: Updated interim results from HGB-206

Markus Y. Mapara', John F. Tisdale ${ }^{2}$, Julie Kanter ${ }^{3}$, Janet L. Kwiatkowski, ${ }^{4,5}$ Lakshmanan Krishnamurti, Manfred Schmidt ${ }^{7}$ Alexandra L. Miller ${ }^{8}$, Francis J. Pierciey Jr. ${ }^{8}$, Weiliang Shi ${ }^{8}$, Jean-Antoine Ribeil ${ }^{8}$, Mohammed Asmal ${ }^{8}$, Alexis A. Thompson ${ }^{9,10}$, Mark Walters $^{11}$

${ }^{1}$ Columbia University College of Physicians and Surgeons, New York, NY, United States, ${ }^{2}$ Sickle Cell Branch, NHLBI/NIDDK, National Institutes of Health, Bethesda, MD, United States, ${ }^{3}$ Medical University of South Carolina, Charleston, SC, United States, ${ }^{4}$ Children's Hospital of Philadelphia, Philadelphia, PA, United States, ${ }^{5}$ Perelman School of Medicine at the University of Pennsylvania, Philadelphia, PA, United States, ${ }^{6}$ Emory School of Medicine, Atlanta, GA, United States, ${ }^{7}$ GeneWerk GmbH, Heidelberg, Germany, ${ }^{8}$ bluebird bio, Inc., Cambridge, MA, United States, ${ }^{9}$ Ann and Robert H. Lurie Children's Hospital, Chicago, IL, United States, ${ }^{10}$ Northwestern University, Chicago, IL, United States, ${ }^{11}$ UCSF Benioff Children's Hospital Oakland, Oakland, CA, United States 
Background: $\beta$-globin gene transfer may reduce or eliminate complications in patients with sickle cell disease (SCD). LentiGlobin gene therapy (GT) comprises drug product (DP) made from autologous hematopoietic stem cells (HSCs) transduced with the BB305 lentiviral vector (LVV) encoding $\beta$-globin with an anti-sickling T87Q substitution $\left(\mathrm{HbA}^{\mathrm{T} 87 \mathrm{Q}}\right)$. The safety and efficacy of LentiGlobin GT in adults with SCD is being evaluated in a phase 1 study, HGB-206 (NCT02140554). Patients were initially treated with DP made from bone marrow harvested (BMH) HSCs (Group A, fully enrolled), then from DP made from $\mathrm{BMH}$ HSCs but using a refined manufacturing process (Group B, fully enrolled), and subsequently from plerixafor mobilized HSCs (Group C, currently enrolling).

Methods: Adults with severe SCD (history of recurrent vaso-occlusive crisis, acute chest syndrome, stroke, or tricuspid regurgitant jet velocity of $>2.5 \mathrm{~m} / \mathrm{s}$ ) were enrolled. Autologous CD34+ cells, collected by BMH or apheresis following mobilization with $240 \mu \mathrm{g} / \mathrm{kg}$ plerixafor, were transduced with BB305 LVV. After myeloablative busulfan conditioning (area under the curve goal of 5000 [range 4400 -5400] $\mu \mathrm{M}^{*}$ min daily), patients were infused with the transduced cells and monitored for safety and efficacy. Summary statistics are median (min-max).

Results: As of May 15, 2018, 22 patients had HSCs collected, 18 patients had DP manufactured and 15 patients were treated. Eleven patients (9 in Group A, 2 in Group B) underwent $\mathrm{BMH}$ and 12 patients (1 in Group B [who also had BMH], 11 in Group C) underwent mobilization/ apheresis. Median of $4.3(0.1-10.8) \times 10^{6}$ and 10.4 (3.8 -21.6) $\times 10^{6} \mathrm{CD} 34+$ cells $/ \mathrm{kg}$ were collected per $\mathrm{BMH}$ $(\mathrm{N}=26)$ and per mobilization cycle $(\mathrm{N}=17)$, respectively. Eighteen grade 3 adverse events (AEs) in 6 patients were attributed to $\mathrm{BMH}$ and 5 grade $3 \mathrm{AEs}$ in 3 patients to mobilization/apheresis. DP and treatment characteristics are shown in Table 1. DP characteristics were improved in Group B and Group C vs Group A. The safety profile postDP infusion was consistent with myeloablative conditioning and underlying SCD; most common non-hematologic grade $\geq 3$ AEs were stomatitis, febrile neutropenia, and vasoocclusive pain. No grade $\geq 3$ DP-related AEs, graft failure, veno-occlusive liver disease, replication competent lentivirus detection or clonal dominance were reported.

At last visit (Table 1), $\mathrm{HbA}^{\mathrm{T} 87 \mathrm{Q}}$ levels were higher in Group B (3.2-7.2 g/dL) vs Group A $(0.5-1.2 \mathrm{~g} / \mathrm{dL})$. In 4 Group $\mathrm{C}$ patients at the 3-month visit, $\mathrm{HbA}^{\mathrm{T} 87 \mathrm{Q}}$ (4.1 [3.26.0] $\mathrm{g} / \mathrm{dL}$ ) levels were equal to or exceeded HbS levels (3.3 [2.8-3.8] g/dL). In 1 Group $C$ patient at the 6-month visit, $\mathrm{HbA}^{\mathrm{T} 87 \mathrm{Q}}$ was $8.8 \mathrm{~g} / \mathrm{dL}$ and total $\mathrm{Hb}$ was $14.2 \mathrm{~g} / \mathrm{dL}$.

Conclusions: These data support the safety and feasibility of plerixafor-mediated HSC collection in patients with SCD. HGB-206 protocol changes have improved LentiGlobin DP characteristics yielding higher $\mathrm{HbA}^{\mathrm{T} 87 \mathrm{Q}}$ levels.
Additional data will determine the clinical effect of increased $\mathrm{HbA}^{\mathrm{T} 87 \mathrm{Q}} / \mathrm{HbS}$ ratios.

\begin{tabular}{llll}
\hline & Group & Group & Group \\
& A (N=7) & B (N=2) & C (N=6) \\
\hline Bone Marrow harvest (number) & $2(1-4)$ & $2.5(2-3)$ & NA \\
Mobilization cycles (number) & NA & $1 *$ & $1(1-2)$ \\
Cell dose (x 10 ${ }^{6}$ CD34+ cells/kg) & $2.1(1.6-$ & $2.7(2.2-$ & $7.1(3.0-$ \\
& $5.1)$ & $3-2)$ & $8.0)$ \\
DP vector copy number (copies/ & $0.6(0.3-$ & $3.1(1.4-$ & $4.0(2.8-$ \\
diploid genome) & $1-3)$ & $5.0)$ & $5.6)$ \\
Transduced cells (\%) & $25(8-42)$ & $87(46-$ & $81(78-$ \\
& & $95)$ & $88)$ \\
Neutrophil engraftment (days) & $22(17-$ & $26(23-$ & $19(18-$ \\
& $29)$ & $28)$ & $20)$ \\
Platelet engraftment (days) & $56(29-$ & $46(31-$ & $28(12-$ \\
& $63)$ & $61)$ & $64)^{ \pm}$ \\
Hsopitalization time from & $37(29-$ & $41(36-$ & $34(30-$ \\
conditioning to discharge (days) & $54)$ & $46)$ & $65)$ \\
Follow-up (months) & 24.2 & $11.4(8.5-$ & $3.0(1.2-$ \\
& $(22.8-$ & $14.3)$ & $6.0)$ \\
& $32.9)$ & & \\
\hline
\end{tabular}

Values are presented as median (min-max); *For research purposes; ${ }^{ \pm} \mathrm{n}=4$ Group $\mathrm{C}$ patients; platelet engraftment was pending in 2 Group $\mathrm{C}$ patients as of data cut; $\mathrm{NA}=$ not applicable

[[O095 Table] 1. Table 1. DP and Treatment Characteristics]

Clinical Trial Registry: ClinicalTrials.gov NCT02140554

Disclosure: Markus Mapara - consultant: Incyte.

Julie Kanter - Advisory Board member: NHLBI, ASH, AstraZeneca, bluebird bio, Inc. and Novartis; research funding: bluebird bio, Inc., Apopharma, Global Blood Therapeutics, Novartis, Pfizer, and Sancilio. Janet Kwiatkowski - consultant: bluebird bio, Inc., and Agios Pharmaceuticals; research funding: bluebird bio, Inc., Agios Pharmaceuticals, Novartis, Apopharma, and Terumo.

Manfred Schmidt - employee: GeneWerk GmbH and German Cancer Research Center; consultant - bluebird bio, Inc. Alexandra Miller, Francis Pierciey, Weiliang Shi, JeanAntoine Ribeil, and Mohammed Asmal - employees of and hold stock in bluebird bio, Inc. Alexis Thompson - research funding: Amgen, Baxalta/Shire, bluebird bio, Inc., Celgene, Novartis, Biomarin, and La Jolla Pharmaceutical; consultant: bluebird bio, Inc. Mark Walters - consultant: bluebird bio, Inc., Bioverativ, Trucode, Editas, and Sangamo Therapeutics; medical director: ViaCord Processing Lab and AllCells, Inc. John Tisdale and Lakshmanan Krishnamurti declare no conflicts of interest. 


\section{6}

A new promising, third-generation $\left(3^{\mathrm{RD}}\right)$ CAR-CD30 $T$ cells therapy for $\mathrm{CD30}+$ lymphoma patients

Biagio De Angelis ${ }^{1}$, Marika Guercio ${ }^{1}$, Domenico Orlando $^{I}$, Iolanda Boffa ${ }^{I}$, Simona Caruso ${ }^{I}$, Zeinab Abbaszadeh $^{I}$, Stefano Di Cecca ${ }^{l}$, Antonio Camera ${ }^{1}$, Matilde Sinibaldi ${ }^{1}$, Rita De Vito ${ }^{1}$, Luciana Vinti ${ }^{1}$, Mattia Algeri $^{1}$, Franco Locatelli ${ }^{1,2}$, Concetta Quintarelli, ${ }^{1,3}$

${ }^{1}$ Ospedale Pediatrico Bambino Gesú (OPBG), Roma, Italy, ${ }^{2}$ Sapienza, University of Rome, Rome, Italy, ${ }^{3}$ Università degli Studi di Napoli Federico II, Napoli, Italy

Background: The prognosis of most patients with chemotherapy-refractory or multiply-relapsed $\mathrm{CD} 30+$ (a cell membrane protein) Non-Hodgkin's Lymphoma (NHL) or Hodgkin lymphoma (HL) still remain poor. Targeting CD30 with monoclonal antibodies in HL and anaplastic large cell lymphoma (ALCL) was shown to induce remarkable clinical activity; however, occurrence of adverse events (mainly neuropathy) may result into treatment discontinuation in many patients. Immunotherapeutic approaches targeting CD30 by chimeric antigen receptor (CAR) has been demonstrated to be of value in two independent clinical trials (PMID: 27582488) (PMID:28805662), although clinical benefit was sub-optimal.

Methods: We designed two $3^{\text {rd }}$ generation, clinical-grade retroviral vectors carrying the cassette anti-CD30 singlechain variable fragment linked via CD8 hingetransmembrane domain, to the signaling domains of two costimulatory domains, namely either CD28/4-1BB or CD28/OX40 and CD3- $\zeta$.

The inducible Caspase-9 (iCasp9) safety switch was also included in the constructs with the goal of promptly controlling undue toxicity. As a selectable marker, we added, in frame with the CAR molecule, a peptide derived from CD34 antigen. The in vitro anti-tumor efficacy was evaluated by using Karpas299, L428 or HDML-2, in both short-term cytotoxic assay (represented by $\mathrm{Cr}^{51}$ release assays) and long-term co-cultures (7 days). Cytokine profile upon antigen stimulation was characterized, as well as Tcell exhaustion and memory marker profile. To assess the expansion, persistence, and antitumor effect of CAR.CD30 $\mathrm{T}$ cells in vivo, we used a NSG mouse model engrafted i.v. with human lymphoma cell lines (Karpas299 and L428) genetically modified with FF-luciferase, this allowing the monitoring of tumor growth by IVIS Imaging system. Persistence of CAR.CD30 $\mathrm{T}$ cells was evaluated every 15 days, together with a deep characterization of memory profile and policlonality of persisting $\mathrm{T}$ cells.

Results: Independently from the costimulatory domains $\mathrm{CD} 28 / \mathrm{OX} 40$ or $\mathrm{CD} 28 / 4-1 \mathrm{BB}$, the generated retroviral vectors showed similar transduction efficiency of $\mathrm{T}$ cells $(86.50 \pm 5.08 \%$ and $79.30 \pm 5.33 \%$, respectively). Nevertheless, CAR.CD30 incorporating CD28.OX40 costimulatory domains was associated with more stable expression of the CAR over time, during extensive in vitro culture (84.72 $\pm 5.30 \%$ vs $63.98 \pm 11.51 \% \mathrm{CAR}+\mathrm{T}$ cells at 30 days after transduction; $p=0.002$ ). This finding was also associated with the evidence that CAR.CD30-CD28.OX40 T cells showed a superior anti-lymphoma in vitro activity as compared to CAR.CD30-CD28.41BB $\mathrm{T}$ cells, when challenged at very high tumor/effector ratio (8:1).

Moreover, antigen-specific stimulation was associated to high levels of Th1 cytokine production, with CAR.CD30CD28.OX40 T cells secreting a significantly higher amount of IFN $\gamma(8306.03 \pm 3745.85 \mathrm{pg} / \mathrm{ml})$, IL2 $(13492.68 \pm 5837.77$ $\mathrm{pg} / \mathrm{ml})$ and TNF $\alpha(17661.00 \pm 11113.27 \mathrm{pg} / \mathrm{ml})$ as compared to CAR.CD30-CD28.41BB T cells $(6617.81 \pm 3025.67 \mathrm{pg} /$ $\mathrm{ml}, \mathrm{p}=0.040 ; 7616.67 \pm 4464.06 \mathrm{pg} / \mathrm{ml}, \mathrm{p}=0.008 ; 5824.63$ $\pm 1823.73 \mathrm{pg} / \mathrm{ml}, \mathrm{p}=0.02$; respectively). In NSG mouse lymphoma models, we proved that CAR.CD30-CD28. OX40 $\mathrm{T}$ cells had an extensive superior anti-tumor control than CAR.CD30-CD28.41BB T cells, leading to a significant reduction of bioluminescence at day $45\left(3.32 \times 10^{6}\right.$ vs $\left.2.29 \times 10^{10}, \mathrm{p}=0.04\right)$ and an increased overall survival of the treated mice ( $60 \%$ vs $10 \%$, at 180 days, $\mathrm{p}=0.0014$ ).

Conclusions: Overall, these data indicate that, in the context of CAR.CD30 T cells, the costimulatory machinery of CD28.OX40 is crucial for improving both persistence and ultimately the antitumor efficacy of the approach.

Disclosure: Nothing to declare

\section{Graft-versus-host disease - clinical}

\section{7}

Ruxolitinib in combination with corticosteroids for the treatment of steroid-refractory acute Graft-vs-host disease: 6-month follow-up data from the phase 2 REACH1 trial

Madan Jagasia ${ }^{1}$, Haris Ali ${ }^{2}$, Mark A. Schroeder ${ }^{3}$, Nirav N. Shah ${ }^{4}$, Yi-Bin Chen ${ }^{5}$, Fitzroy Dawkins ${ }^{6}$, Michael Arbushites $^{6}$, Chuan Tian ${ }^{6}$, Miguel-Angel Perales ${ }^{7}$

${ }^{1}$ Vanderbilt-Ingram Cancer Center, Nashville, TN, United States, ${ }^{2}$ City of Hope, Duarte, CA, United States, ${ }^{3}$ Washington University, St. Louis, MO, United States, ${ }^{4}$ Medical College of Wisconsin, Milwaukee, WI, United States, ${ }^{5}$ Massachusetts General Hospital, Boston, MA, United States, ${ }^{6}$ Incyte Corporation, Wilmington, DE, United States, ${ }^{7}$ Memorial Sloan Kettering Cancer Center, New York, NY, United States 
Background: Acute graft-vs-host disease (aGVHD) is a serious complication of allogeneic hematopoietic stem cell transplantation (allo-HSCT). Less than 50\% of patients (pts) achieve sustained responses with first-line corticosteroid (CS) treatment. Retrospective studies demonstrated clinical benefit with the Janus kinase (JAK)1/JAK2 inhibitor ruxolitinib (RUX) in pts with steroid-refractory (SR) aGVHD. Here we present 6-month follow-up data from patients enrolled in REACH1 (NCT02953678), a phase 2 trial evaluating RUX plus CS in SR aGVHD.

Methods: REACH1 was a single-arm, open-label, multicenter study. Eligible pts were aged $\geq 12$ years and developed grade II-IV SR aGVHD following allo-HSCT from any donor source for hematologic malignancies. SR aGVHD was defined as GVHD that progressed after 3 days or had not improved after 7 days of primary treatment with methylprednisolone $\geq 2 \mathrm{mg} / \mathrm{kg} / \mathrm{d}$ (or equivalent), development of GVHD in another organ after receiving $\geq 1 \mathrm{mg} / \mathrm{kg} / \mathrm{d}$ methylprednisolone for skin or skin plus upper gastrointestinal GVHD, or inability to tolerate CS taper. Pts received RUX $5 \mathrm{mg}$ twice daily (BID), with optional increase to $10 \mathrm{mg}$ BID in the absence of cytopenias. The primary endpoint was Day 28 overall response rate (ORR), and the key secondary endpoint was 6-month duration of response (DOR). ORR was defined as the proportion of patients demonstrating a complete response (CR), very good partial response, or partial response.

Results: The study enrolled 71 pts. Median age was 58 years, and $49.3 \%$ were male. Treatment was ongoing in 11 pts (15.5\%) at data cutoff (2 Jul 2018). At Day 28, ORR was $54.9 \%$ (CR, 26.8\%). Responses were observed irrespective of aGVHD grade and SR criteria. Best ORR at any time during treatment was $73.2 \%$ (CR, 56.3\%). Median (range) time to response was 7 (6-49) days. Median DOR with minimum 6 months follow-up was 345 days for both Day 28 responders (Figure 1) and for pts who had a best overall response at any time during treatment. Four pts (5.6\%) had malignancy relapse. Overall, nonrelapse mortality at 6 months was $44.4 \%$; results varied by Day 28 response (Day 28 responders, 21.2\%; other responders, 64.1\%; nonresponders, $78.9 \%$ ). Median overall survival had not been reached for Day 28 responders. The most frequently reported hematologic treatment-emergent adverse events (TEAEs) were anemia (64.8\%), thrombocytopenia $(62.0 \%)$, and neutropenia $(47.9 \%)$. Cytomegalovirus infection (12.7\%), sepsis (12.7\%), and bacteremia (9.9\%) were the most frequently reported infections. Fatal RUX-related TEAEs were sepsis and pulmonary hemorrhage (1 pt each) and were attributed to both RUX and CS.

Conclusions: In this first prospective trial of RUX in SR aGVHD, ORR was $54.9 \%$ by Day 28 and $73.2 \%$ at any time during treatment. Responses were rapid and durable. The AE profile was consistent with expectations for RUX and pts with SR aGVHD. RUX represents a promising therapeutic strategy.

Clinical Trial Registry: ClinicalTrials.gov identifier: NCT02953678

https://clinicaltrials.gov/ct2/show/NCT02953678?term= NCT02953678\&rank=1

Disclosure: Madan Jagasia and Haris Ali served on advisory boards for Incyte Corporation.

Mark A. Schroeder served as a consultant for Incyte Corporation, Amgen, Pfizer, Sanofi/Genzyme, and Flatiron and as a speaker for Merck and Takeda. Yi-Bin Chen served as a consultant for Incyte Corporation, Magenta, Takeda, and Kiadis.Fitzroy Dawkins, Michael Arbushites, and Chuan Tian are employees of Incyte Corporation.MiguelAngel Perales received honoraria from MolMed, NexImmune, Abbvie, Bellicum, Incyte Corporation, Nektar Therapeutics, Novartis, Merck, Medigene, Servier, and Takeda and received research funding from Incyte Corporation and Miltenyi. Nirav N. Shah has nothing to disclose

\section{8}

\section{Abstract already published.}

\section{9}

single cell immune profiling by mass cytometry of chronic graft-versus-host disease

Haowen Xiao ${ }^{1,2}$, Yang Gao ${ }^{1,2}$, Yi Luo ${ }^{2,3}$, Hongyu Shi ${ }^{4}$, Weiwei Yin ${ }^{5}$, Wei Chen ${ }^{5}$, Xun Zeng ${ }^{5}$, He Huang ${ }^{2,3}$

${ }^{1}$ Sir Run Run Shaw Hospital, Zhejiang University School of Medicine, Hangzhou, China, ${ }^{2}$ Zhejiang University, Hangzhou, China, ${ }^{3}$ The First Affiliated Hospital, Bone Marrow Transplantation Center, Hangzhou, China, ${ }^{4}$ Zhejiang PuLuoTing Health Tech Co. LTD, Hangzhou, China, ${ }^{5}$ Zhejiang University Hangzhou, China

Background: Chronic graft-versus-host disease (cGVHD) remains a major complication after allogeneic hematopoietic cell transplantation (allo-HSCT). Over the last decade, clinical success in patients with cGVHD has been hampered by the lack of insight into the complex pathobiological mechanisms of the disease and the paucity of specific therapeutic targets. Although, it is now evident that the clinical manifestations of cGVHD are the result of a highly complex immune pathology involving both donor B cells and $\mathrm{T}$ cells as well as other cells. Current work on immune cells involved in cGVHD pathobiology is limited by the number of parameters that conventional flow cytometry (FCM) can analyze because of cell autofluorescence and fluorescent dye spectral overlap. Mass cytometry time-offlight (CyTOF) substitutes rare earth elements for fluorophores to label antibodies, which allows simultaneous 
measurements of more than 40 parameters in single cells without correction for spectral overlap, and empowers us to understand cGVHD biology at the single-cell level.

Methods: We used mass cytometry with extensive antibody panels to perform in-depth immune profiling of peripheral blood samples from 34 patients following alloHSCT, in which 11 patients were without cGVHD, 7 patients experienced moderate cGVHD and 16 patients experienced severe cGVHD. The involved organs in patients with cGVHD are skin, liver and lung.

Results: We simultaneously stained cells with 42 antibody panels created for this study. The $\mathrm{T}$ cell panel was designed to identify different populations of naive, memory, effector, regulatory, and exhausted $\mathrm{T}$ cells. The panels also included markers for the identification of $\mathrm{B}$ cells, natural killer cells, NKT cells, DC cells, plasma cells, granulocytes, and myeloid cells. In 4 million measured cells, we identified 40 immune cell phenotypes, in which there were $22 \mathrm{~T}$ cell phenotypes, $6 \mathrm{~B}$ cell phenotypes, 6 monocyte phenotypes and 6 granulocyte phenotypes. To generate a comprehensive view of the immune ecosystem of cGVHD, we generated two-dimensional maps of the data using the dimensionality reduction algorithm t-SNE. This analysis showed a strong overlap between cGVHD of moderate and severe grades, but seperation from patients without cGVHD. Seven immune compositions were identified to be cGVHD-associated. Five distinct immune cells were correlated with specific cGVHD-involved organs (skin or lung), thereby presenting an in-depth human atlas of the immune cells in this disease.

Conclusions: This study revealed potential biomarkers and targets for immunotherapy of cGVHD and validated CyTOF as a valuable tool that can be used for immune profiling of cGVHD.

Disclosure: Nothing to declare

\section{0}

T cell costimulation blockade with abatacept for acute graft-versus-host disease prevention in matched and mismatched unrelated donor transplantation: Results of the first phase 2 trial

Muna Qayed $^{1,2}$, Benjamin Watkins ${ }^{1,2}$, Brandi Bratrude ${ }^{3}$, Kayla Betz, James Rhodes ${ }^{2}$, Shauna Sinclair ${ }^{4}$, Yvonne Suessmuth ${ }^{1}$, Alison Yu ${ }^{4}$, Carol Dean ${ }^{4}$, Kayla Cribbin ${ }^{4}$, Alexandria Narayan, ${ }^{4}$ Daniel Hunt ${ }^{3}$, Naomi Schwartz, Kyle Hebert, Marcelo Pasquini, ${ }^{5}$, Andre' Rogatko ${ }^{6}$, Mourad Tighiouart $^{6}$, Sungjin Kim ${ }^{6}$, Catherine Bresee ${ }^{6}$, Scott Gillespie ${ }^{1}$, Jeffrey Davis ${ }^{7}$, Sung Choi ${ }^{8}$, Christine Duncan $^{3}$, Roger Giller ${ }^{9}$, Michael Grimley ${ }^{10}$, Andrew Harris $^{11}$, David Jacobsohn ${ }^{12}$, Nahal Lalefar ${ }^{13}$, Maxim Norkin $^{14}$, Michael Pulsipher ${ }^{15}$, Shalini Shenoy ${ }^{16}$,
Aleksandra Petrovic ${ }^{4}$, Kirk Schultz, Bruce ${ }^{7}$ Blazar $^{17}$, Amelia Langston $^{1,18}$, John Horan ${ }^{1,2}$, Leslie Kean ${ }^{3,19}$

${ }^{1}$ Emory University, Atlanta, GA, United States, ${ }^{2}$ Aflac Cancer and Blood Disorders Center, Atlanta, GA, United States, ${ }^{3}$ Boston Children's Hospital, Boston, MA, United States, ${ }^{4}$ Seattle Children's Research Institute, Seattle, WA, United States, ${ }^{5}$ CIBMTR and Medical College of Wisconsin, Milwaukee, WI, United States, ${ }^{6}$ Cedars-Sinai Cancer Institute, Los Angeles, CA, United States, ${ }^{7}$ British Columbia Children's Hospital, Vancouver, Canada, ${ }^{8}$ University of Michigan Health System, Ann Arbor, MI, United States, ${ }^{9}$ Children's Hospital Colorado, Aurora, CO, United States, ${ }^{10}$ Cincinnati Children's Hospital Medical Center, Cincinnati, OH, United States, ${ }^{11}$ University of Utah, Salt Lake City, UT, United States, ${ }^{12}$ Children's National Medical Center, Washington DC, DC, United States, ${ }^{13} \mathrm{UCSF}$, Benioff Children's Hospital, Oakland, CA, United States, ${ }^{14}$ University of Florida Health Cancer Center, Gainesville, FL, United States, ${ }^{15}$ Children's Hospital Los Angeles, Los Angeles, CA, United States, ${ }^{16}$ Washington University in St. Louis, Saint Louis, MO, United States, ${ }^{17}$ University of Minnesota, Minneapolis, MN, United States, ${ }^{18}$ Winship Cancer Institute, Atlanta, GA, United States, ${ }^{19}$ Dana-Farber Cancer Institute, Boston, MA, United States

Background: We performed a Phase 2 trial in adults and children to test abatacept for AGVHD prevention ('ABA2'), based on our promising preclinical and pilot patient data.

Methods: ABA2 had 2 cohorts: A) HLA mismatched ('7/ $8^{\prime}, \mathrm{n}=43$ ), a single-arm study with pre-specified CIBMTR matched analysis (vs CNI+MTX or CNI+MTX + ATG). B) HLA-matched (' $8 / 8$ ', $\mathrm{n}=142)$, randomized double-blind, comparing $\mathrm{CNI}+\mathrm{MTX}+$ placebo vs $\mathrm{CNI}+\mathrm{MTX}+\mathrm{ABA}$ ('ABA'). Abatacept dosing was $10 \mathrm{mg} / \mathrm{kg}$ on $\mathrm{d}-1,+5$, $+14,+28$. ABA2 was designed as a screening Phase 2 trial, with relaxed Type 1 error (0.2) and standard Type 2 error (0.2). Power analysis assumed reduction of Gr 3-4 AGVHD from $30 \%-->10 \%$ in $7 / 8$ s and $20 \%-->10 \%$ in $8 / 8$ s. Median follow up $=708$ days $(7 / 8 \mathrm{~s})$ and 369 days $(8 / 8 \mathrm{~s})$.

Results: Reduced grade 3-4 AGVHD: ABA was associated with decreased d180 Gr 3-4 AGVHD. In 7/8s, Gr $3-4$ AGVHD $=2.5 \%(\mathrm{ABA})$ vs $31 \%(\mathrm{CNI}+\mathrm{MTX})$ and $22 \%$ (+ATG), (1-sided p $=0.001,0.005$ ). In 8/8s, Gr 3-4 AGVHD $=6.85 \%$ in ABA vs $14.6 \%$ in placebo, (1-sided $\mathrm{p}$ $=0.068$ ). Reduced grade 2-4 AGVHD in 8/8s: ABA was associated with decreased d $180 \mathrm{Gr} 2-4$ AGVHD. In 7/8s, Gr $2-4 \mathrm{AGVHD}=42 \%(\mathrm{ABA})$ vs $54 \%(\mathrm{CNI}+\mathrm{MTX})$ and $45 \%$ (+ATG, 1-sided $\mathrm{p}=0.098,0.25)$. In 8/8s, Gr 2-4 AGVHD $=44.5 \%$ in $\mathrm{ABA}$ vs $62.3 \%$ in placebo $(1$-sided $\mathrm{p}=0.004)$. Chronic GVHD: For 7/8s, 1 yr CGVHD $=38.8 \%$ (ABA) vs $43.5 \%$ (CNI+MTX) and $35.5 \%$ (+ATG, p $=0.4,0.99$ ). In $8 / 8$ s, data collection is ongoing. Safety indicators: There 
was no difference in neutrophil or platelet engraftment, $\mathrm{CMV}$ and EBV reactivation between $\mathrm{ABA}$ and controls. Cumulative incidence of relapse in $7 / 8 \mathrm{~s}$ at $2 \mathrm{yr} 9.4 \%$ (ABA) vs $20.6 \%(\mathrm{CNI}+\mathrm{MTX})$ and $23.4 \%(+\mathrm{ATG})(\mathrm{p}=0.115$, 0.085 ). In $8 / 8 \mathrm{~s}$, at $1 \mathrm{yr}$, it was $13.8 \%$ (ABA) vs $20.5 \%$ (Placebo, $\mathrm{p}=0.7$ ). Statistically significant survival advantage in 7/8s: For 7/8s, 1 yr non-relapse mortality $(\mathrm{NRM})=$ $10.5 \%(\mathrm{ABA})$ vs $32.7 \%(\mathrm{CNI}+\mathrm{MTX})$ and $26 \%(+\mathrm{ATG}$, $\mathrm{p}=0.024,0.365)$. For $8 / 8 \mathrm{~s}, \mathrm{NRM}=7.1 \%$ vs $14.6 \%$ at $1 \mathrm{yr}$ $(\mathrm{p}=0.5)$. Severe AGVHD free survival at 6 months for $7 /$ $8 \mathrm{~s}=97 \%(\mathrm{ABA})$ vs $55 \%(\mathrm{CNI}+\mathrm{MTX})$ and $59 \%(+\mathrm{ATG}$, $\mathrm{p}=0.001,0.006)$. For $8 / 8 \mathrm{~s}=89.0 \%(\mathrm{ABA})$ vs $76.8 \%$ (Placebo, $\mathrm{p}=0.049$ ). For $7 / 8 \mathrm{~s}$, relapse-free survival $(\mathrm{RFS})=73.7 \%(\mathrm{ABA})$ vs $38.7 \%$ in $\mathrm{CNI}+\mathrm{MTX}$ and $48.7 \%$ in + ATG $(\mathrm{p}=0.001,0.027)$. For $8 / 8 \mathrm{~s}, \mathrm{RFS}=79.1 \%$ for $\mathrm{ABA}$ vs $64.9 \%$ (placebo, $\mathrm{p}=0.38$ ). For $7 / 8 \mathrm{~s}$, overall survival $(\mathrm{OS})=71 \%(\mathrm{ABA})$ vs $47.5 \%(\mathrm{CNI}+\mathrm{MTX})$ and $58 \%$ ( + ATG, $\mathrm{p}=0.01,0.145$ ). For $8 / 8 \mathrm{~s}$, OS $=83.2 \%$ (ABA) vs 76.6 (placebo, $\mathrm{p}=0.32$ ).

Conclusions: Our results suggest that short-course ABA can safely prevent AGVHD without compromising relapse. Despite the modestly sized study, the comparative event size for $7 / 8$ s was high enough that the protective effect of ABA against Gr3-4 AGVHD was highly significant. For 8/ 8 s, there was a statistically significant improvement for $\mathrm{Gr}$ 2-4 GVHD and a trend toward an advantage in all parameters. For both cohorts, severe AGVHD free survival was statistically-significantly improved. These results are the first to demonstrate efficacy of in vivo $\mathrm{T}$ cell costimulation blockade in preventing AGVHD.

Clinical Trial Registry: Clinicaltrials.gov NCT01743131

Disclosure: Muna Qayed, Consultancy: Novartis, BristolMyers Squibb. Christine Duncan, Consultancy: Bluebird Bio, Magenta Therapeutics, Advanced Clinical, AbGenomics. Michael Pulsipher, Consultancy: Adaptive Biotech, CSL Behring, Novartis, Honoraria: Amgen, Novartis, Speakers Bureau: Novartis, Research funding: Adaptive Biotech. Shalini Shenoy, Consultancy: Novartis, Vertex, Bluebird Bio.

Bruce Blazar, Consultancy and Research funding: Kadmon Corporation. John Horan, Consultancy: BristolMyers Squibb, Leslie Kean honoraria and grant BristolMyers Squibb.Leslie Kean, Consultancy: Magenta Therapeutics, Kymab, Bioverativ, Equillium, EMDSerono, Research funding: Regeneron Pharmaceuticals, BristolMyers Squibb, Intellectual property: Bristol-Myers Squibb.

\section{1}

Biological differences between pediatric late acute graftversus-host disease (AGVHD) AND chronic GVHD (CGVHD) identified by day 100 biomarker analysis: Results of the PBMTC 1202/ABLE studies
Geoffrey Cuvelier ${ }^{1}$, Amina Kariminia ${ }^{2}$, Bernard $\mathrm{Ng}^{3}$, Sayeh Abdossamadi ${ }^{2}$, Peter Subrt ${ }^{2}$, Anat Halevy ${ }^{2}$, Sara Mostafavi ${ }^{3}$, Kirk R. Schultz ${ }^{2}$

${ }^{1}$ CancerCare Manitoba, Winnipeg, Canada, ${ }^{2}$ University of British Columbia, Vancouver, Canada, ${ }^{3}$ University of British Columbia, Vancouver, Canada

Background: In the NIH cGvHD diagnostic classification, patients with GvHD after 3 months are classified as either late aGvHD or cGvHD. To date, this is only a clinical classification, with no biological differences identified. Recently, the PBMTC 1202/ Applied Biomarker in Late Effects of Children and Adolescent (ABLE) study completed accrual of 302 pediatric allogeneic Hematopoietic Cell Transplantation (HCT) patients. We used day 100 biomarkers to identify biological differences between cGvHD and late aGvHD.

Methods: The PBMTC1202/ABLE study with 26 centers in Canada, US, and Europe prospectively collected peripheral blood samples at 3,6,12 month post HCT and at the onset of cGvHD in 302 children. A comprehensive analysis for previously identified cGvHD immune cell markers by flow and cytokines by ELISA on plasma and STRECK tubes shipped overnight and centrally evaluated at BC Children's Hospital. Clinical data was collected centrally with a thorough central clinical adjudication by the PBMTC study committee. Of those enrolled, 228 were evaluable at day 100 and classified as a) late aGvHD ( $\mathrm{N}=$ $58)$, b) cGvHD ( $\mathrm{N}=44)$, and c) controls that did not develop cGvHD $(\mathrm{N}=132)$. Univariate analysis was performed comparing late aGvHD, cGvHD, and no GvHD controls. Significant differences were defined as a biomarker with both a ROC AUC $\geq 0.60$ and $p$ value $\leq 0.05$ compared to controls.

Results: The profile of cGvHD included a cluster of abnormalities in memory and transitional B cells, conventional naïve and follicular helper $\mathrm{T}$ cells, and a loss of both recent thymic emigrant regulatory $\mathrm{T}$ cells and CD56 ${ }^{\text {bright }} \mathrm{NK}$ regulatory cells. Four inflammatory cytokines, ST2, Aminopeptidase N, CXCL9 and MMP3 (See Table 1) were increased. Patients clinically identified as late aGvHD had a more restricted biomarker pattern of limited B cell abnormalities and ST2.

Conclusions: Late acute GvHD is limited to restricted B cell and elevation of ST2. cGvHD is characterized by the identical B cell abnormalities but with the additional loss of regulatory function in CD56bright NKregs and RTE Treg cells. With the loss of regulatory function in cGvHD, there is an increase in $\mathrm{CD} 21^{\mathrm{lo}} \mathrm{B}$ cells, follicular T helper cells, and additional cytokines. These prognostic markers findings may suggest therapeutic targets that differ for late aGvHD compared to cGvHD. 


\begin{tabular}{|c|c|c|}
\hline Cell type & Late aGvHD & cGvHD \\
\hline B cells (total) & Decreased & Significantly decreased \\
\hline Transitional B cells & $\begin{array}{l}\text { Anergic Naïve B cells } \\
\text { and Naïve mature B } \\
\text { cells - Decreased }\end{array}$ & $\begin{array}{l}\text { Anergic Naïve B cells and } \\
\text { Naïve mature B cells - } \\
\text { Decreased }\end{array}$ \\
\hline CD21lo B cells & Normal & Increased \\
\hline Memory B cells & $\begin{array}{l}\text { Switched and } \\
\text { Unswitched Memory B } \\
\text { cells - Increased }\end{array}$ & $\begin{array}{l}\text { Switched and Unswitched } \\
\text { Memory B cells - Increased }\end{array}$ \\
\hline \multicolumn{3}{|l|}{ T cells (effector cells) } \\
\hline $\begin{array}{l}\text { Naïve helper T cells } \\
\text { and Follicular } \mathrm{T} \\
\text { helper cells }\end{array}$ & $\begin{array}{l}\text { Naïve helper T cells } \\
\text { and Follicular T helper } \\
\text { cells - Normal }\end{array}$ & $\begin{array}{l}\text { Naïve helper T cells and } \\
\text { Follicular T helper cells - } \\
\text { Increased }\end{array}$ \\
\hline \multicolumn{3}{|c|}{ Regulatory Populations } \\
\hline $\begin{array}{l}\text { NKreg cells and } \\
\text { Recent Thymus } \\
\text { emigrant (RTE) } \\
\text { Treg cells }\end{array}$ & $\begin{array}{l}\text { NKreg and RTE Treg } \\
\text { Cells - Normal }\end{array}$ & $\begin{array}{l}\text { NKreg and RTE Treg Cells - } \\
\text { Decreased }\end{array}$ \\
\hline Cytokines & ST2 - Increased & $\begin{array}{l}\text { Increased - ST2, } \\
\text { Aminopeptidase N, and } \\
\text { CXCL9. Significantly } \\
\text { increased - MMP3 }\end{array}$ \\
\hline
\end{tabular}

[[O101 Table] 1. Table 1 - Biomarker Differences Between Late aGvHD and $c G v H D$ Measured at Day 100: PBMTC1202/ABLE Study Results]

Clinical Trial Registry: https://clinicaltrials.gov/ct2/ show/NCT02067832

NCT02067832

Disclosure: None of the authors have any conflict of interest.

\section{2}

Treatment of steroid resistant acute graft versus host disease with an ANTI-CD26 Monoclonal antibody Begelomab

Elisabetta Metafuni ${ }^{1}$, Francesca Gualandi ${ }^{2}$, Anna Maria Raiola $^{2}$, Riccardo Varaldo ${ }^{2}$, Carmen Di Grazia ${ }^{2}$, Emanuele Angelucci ${ }^{2}$, Edoardo Benedetti ${ }^{3}$, Franco Locatelli, Antonio Risitano ${ }^{5}$, Maurizio Musso ${ }^{6}$, Francesco Zallio ${ }^{7}$, Fabio Ciceri ${ }^{8}$, Patrizia Chiusolo ${ }^{1}$, Alessandro Rambaldi ${ }^{9}$, Francesca Bonifazi ${ }^{10}$, Matteo Parma ${ }^{11}$, Massimo Martino $^{12}$, Francesco Onida ${ }^{13}$, Anna Paola Iori ${ }^{14}$, Carmine Selleri $^{15}$, Carlo Borghero ${ }^{16}$, Alice Bertaina ${ }^{4}$, Maria Teresa Van Lint ${ }^{17}$, Andrea Bacigalupo ${ }^{1}$

${ }^{1}$ Fondazione Policlinico Universitario Agostino Gemelli, Rome, Italy, ${ }^{2}$ IRCCS AOU San Martino IST, Genoa, Italy, ${ }^{3}$ Azienda Ospedaliero Universitaria Pisana, Hematology, Pisa, Italy, ${ }^{4}$ Ospedale Pediatrico Bambino Gesù, Rome, Italy, ${ }^{5}$ Università Federico II, Naples, Italy, ${ }^{6}$ Ospedale La Maddalena, Palermo, Italy, ${ }^{7}$ Ospedale San Biagio, Alexandria, Italy, ${ }^{8}$ Ospedale San Raffaele, Milan, Italy, ${ }^{9}$ Ospedale Papa Giovanni XXIII, Hematology, Bergamo, Italy, ${ }^{10}$ Policlinico S. Orsola, Institute Seragnoli, Bologna, Italy, ${ }^{11}$ Ospedale S. Gerardo, Monza, Italy, ${ }^{12}$ Ospedale Bianchi
Melacrino Morelli, Reggio Calabria, Italy, ${ }^{13}$ Policlinico Università di Milano, Milan, Italy, ${ }^{14}$ Policlinico Umberto I, Hematology, Rome, Italy, ${ }^{15}$ Ospedale S. Giovanni di Dio, Hematology, Salerno, Italy, ${ }^{16}$ Ospedale S. Bortolo, Vicenza, Italy, ${ }^{17}$ IRCCS AOU San Martino IST, Genoa, Italy

Background: We are reporting the outcome of 69 patients with steroid refractory acute graft versus host disease (SRGvHD), treated with an anti-CD26 monoclonal antibody $\left(\right.$ Begelomab $\left.^{\mathrm{R}}\right)$.

Methods: Twenty-eight patients were enrolled in two pilot studies EudraCT No. 2007-005809-21 and No. 2012001353-19, whereas 41 patient were treated on a multicenter follow up compassionate use of the antibody. The median age of the patients was respectively 42 and 44 years. At the time of anti-CD26 treatment, GvHD was overall recorded as grade II in 8 patients, grade III in 33 and grade IV in 28 patients. In the pilot sudies patients had failed 1 line of treatment, wheas in the follow up compassionate use, patients had failed one line $(n=18)$, two lines $(n=11)$, three lines $(n=11)$ or 4 lines of treatment $(n=1)$.

Results: There were no adverse events attributable to the antibody. Day 28 response was recorded in $75 \%$ and $63 \%$ in the pilot studies and follow up patients. Response in grade II GvHD was evaluable only in the pilot studies (57\%); response in grade III GvHD was recorded in $80 \%$ and $83 \%$ patients in the two groups; response in grade IV GvHD was recorded in $66 \%$ and $56 \%$ of patients in the two groups. Overall there were $60 \%$ responses for skin and liver stage 34 , and $70 \%$ responses for gut stage 3-4 GvHD. The cumulative incidence of non relapse mortality (NRM) at 6 months was $28 \%$ and $38 \%$. For day 28 responders, this figure was $19 \%$ and $22 \%$, for non responders it was $57 \%$ and $66 \%$ in the two groups. The overall survival at 1 year was $50 \%$ for the pilot studies and $33 \%$ for the follow up patients.

Conclusions: In conclusion, Begelomab induces a high remission rate on day +28 in patients with SR-GvHD, including a significant proportion of patients wih severe gut and liver GvHD.

Clinical Trial Registry: EudraCT No. 2007-005809-21

Eudtract No. 2012-001353-19

Disclosure: Nothing to declare

\section{3}

KD025-208: A phase 2A open-label trial of KD025-208 for steroid-dependent chronic graft-versus-host disease (CGVHD): Updated safety, efficacy, and pharmacodynamic results

Carlos R. Bachier', Behyar Zoghi', Amandeep Salhotra ${ }^{3}$, Daniel J. Weisdorf ${ }^{4}$, Aleksandr Lazaryan ${ }^{4}$, James Essell, Alexandra Zanin-Zhorov ${ }^{6}$, Jonathan M. Weiss ${ }^{6}$, Laurie $S$. 
Green $^{6}$, Olivier Schueller ${ }^{6}$, David Eiznhamer ${ }^{6}$, Zhongm- $^{2}$ ing Yang ${ }^{6}$, Sanjay K. Aggarwal ${ }^{6}$, Stephanie J. Lee ${ }^{7}$, Bruce R. Blazar, Madan Jagasia ${ }^{8}$

${ }^{1}$ Sarah Cannon Blood Cancer Network, Nashville, TN, United States, ${ }^{2}$ Texas Transplant Institute, Methodist Hospital, San Antonio, TX, United States, ${ }^{3}$ City of Hope National Medical Center, Duarte, CA, United States, ${ }^{4}$ University of Minnesota, Minneapolis, MN, United States, ${ }^{5}$ Oncology/Hematology Care, Cincinnati, OH, United States, ${ }^{6}$ Kadmon, New York, NY, United States, ${ }^{7}$ Fred Hutchinson Cancer Research Center, Seattle, WA, United States, ${ }^{8}$ Vanderbilt University Medical Center, Nashville, $\mathrm{TN}$, United States

Background: cGVHD is characterized by an imbalance between effector and regulatory arms of the immune system that results in overproduction of inflammatory cytokines including IL-17 and IL-21. Moreover, a persistent reduction in the number of regulatory $\mathrm{T}$ (Treg) cells limits the ability of the immune system to recalibrate this pro-inflammatory environment.

KD025 is an orally available Rho-associated coiled-coil kinase 2 (ROCK2) selective inhibitor. In vitro data suggest that KD025 modulates immune homeostasis by shifting the TH17/Treg balance towards a Treg phenotype.

Methods: KD025-208 is an open-label Phase 2a study in patients with steroid-dependent cGVHD after no more than 3 prior lines of treatment. Three cohorts $(\mathrm{C} 1: 200 \mathrm{mg}$ QD $(\mathrm{n}=17), \mathrm{C} 2$ : 200mg BID $(\mathrm{n}=16)$, and C3: 400mg QD $(\mathrm{n}=21))$ were enrolled. The primary endpoint is Overall Response Rate (ORR), defined per the 2014 NIH Consensus criteria.

Results: As of 13-September-2018, the median duration of treatment was 37, 33 and 27 weeks for $\mathrm{C} 1, \mathrm{C} 2$ and $\mathrm{C} 3$, respectively. The median age was 52 years (range 20-75) and median time from cGVHD diagnosis to KD025 treatment was 19 months. $67 \%$ of patients had received $\geq 2$ prior lines of therapy and $48 \%$ had $\geq 4$ organs involved at baseline. 20 patients remain on treatment with KD025, with median duration of treatment of 89 weeks $(n=6), 68$ weeks $(n=3)$ and 34 weeks $(n=11)$ for each cohort, respectively. The ORR was $65 \%$ in $\mathrm{C} 1,63 \%$ in $\mathrm{C} 2$ and $52 \%$ in $\mathrm{C} 3$. Responses were rapid, with $75 \%$ of responders achieving a response at the first assessment (8 weeks). Among responders, $82 \%, 50 \%$ and $36 \%$ have sustained responses for $\geq 20$ weeks in each cohort, respectively. Responses were observed across all affected organ systems, including CRs in upper GI, lower GI, esophagus, mouth, skin, joints/fascia, eyes, and liver. Two patients with lung cGVHD achieved PR. $69 \%$ of patients achieved reductions in corticosteroid dose and 7 patients discontinued corticosteroid treatment while receiving KD025. $72 \%$ of responders achieved a clinically meaningful improvement ( $\geq 7$-point reduction) in the Lee Symptom Scale (LSS) score. KD025 has been well tolerated. Commonly reported AEs ( $\geq 20 \%$ patients) were URTI, AST/ALT elevations, fatigue, nausea and diarrhea. Grade $\geq 3$ AEs occurring in $>3$ patients were GGT elevations $(n=6)$ and hyperglycemia $(n=4)$. No SAEs were considered related to study drug. Two patients discontinued treatment due to AEs considered possibly related to KD025 (headache, diarrhea). Three fatal events occurred (relapse of leukemia; lung infection; cardiac arrest); none were considered related to KD025. No increase in incidence of infection was observed. Consistent with the postulated KD025 mechanism of action, TH17 cells decreased and Treg cells increased in patients receiving KD025 treatment

Conclusions: Treatment with KD025 has demonstrated clinically meaningful responses in cGVHD patients. Responses were durable, led to reduction in steroid and/or immunosuppressant dose and reflected improvements in LSS score. KD025 treatment was well tolerated with no treatment-related SAEs and no increased risk of infection observed. Pharmacodynamic data are consistent with the postulated KD025 mechanism of action. A pivotal trial (KD025-213) of KD025 in cGVHD is currently enrolling.

Clinical Trial Registry: NCT02841995

https://clinicaltrials.gov/ct2/show/NCT02841995

Disclosure: Carlos Bachier: Speakers Bureau (Sanofi, Seattle Genetics) Daniel Weisdorf: Consultancy (Pharmacyclics, Seattle Genetics, SL Behring, FATE, Equillium) Alexandra Zanin-Zhorov: Employment (Kadmon Corporation, LLC) Jonathan Weiss: Employment (Kadmon Corporation, LLC) Laurie Green: Employment (Kadmon Corporation, LLC) Olivier Schueller: Employment (Kadmon Corporation, LLC) David Eiznhamer: Employment (Kadmon Corporation, LLC) Zhongming Yang: Employment (Kadmon Corporation, LLC) Sanjay Aggarwal: Employment (Kadmon Corporation, LLC)

Stephanie Lee: Consultancy (Kadmon Corporation LLC, Pfizer, Incyte, Amgen), Honoraria (Mallinckrodt) Bruce Blazar: Consulting and Research Funding (Kadmon Corporation) Madan Jagasia: Advisory Committee (Incyte)

\section{4}

Antithymocyte globulin inhibits chronic GVHD by suppressing naïve $\mathrm{CD} 4+T$ cells and enhancing CD56BRIGHT NK regulatory cells at day 100: Canadian BMT group 0801 study

Lena Naeije $e^{I}$, Amina Kariminia ${ }^{1}$, Sayeh Abdossamadi, Peter Subrt ${ }^{I}$, Boris Kuzeljevic ${ }^{2}$, Irwin Walker ${ }^{3}$, Kirk R. Schultz $^{1}$ 
${ }^{1}$ University of British Columbia, Vancouver, Canada, ${ }^{2} \mathrm{BC}$ Children's Hospital Research Institute, Vancouver, Canada,

${ }^{3}$ Juravinski Hospital and Cancer Centre, Hamilton, Canada

Background: Antithymocyte globulin (ATG) treatment significantly decreases later development of chronic graftversus-host disease (cGvHD). One phase 3 trial evaluating ATG was the Canadian BMT Group (CBMTG) 0801 study that found that ATG treatment resulted in significantly less cGvHD and dependence on immunosuppressive treatment at 1 year. The exact mechanism by which ATG decreases cGvHD is not known. We hypothesized that using known prognostic day 100 cGvHD biomarkers in the wellcontrolled CBMTG 0801 trial represented an optimal approach to understand ATG's biological impact on cGvHD in humans.

Methods: A separately developed CBMTG cGvHD biomarker study opened while CBMTG 0801 was ongoing and accrued 40 patients $(\mathrm{N}=25$; ATG treated and $\mathrm{N}=15$ controls) of the 203 CBMTG 0801 patients. Samples were collected at 3, 6, and 12 months and at the onset of cGvHD and evaluated at BC Children's' Hospital Research Institute, Vancouver, BC. Patients were evaluated for day 100 immune cellular markers previously associated with later cGvHD including: a) Naive helper $\mathrm{T}$ (Th) cells, b) recent thymic emigrant (RTE) Th cells; c) CD21 ${ }^{\text {low }}$ B cells; d) CD56 ${ }^{\text {bright }}$ NKreg cells; and e) Treg cells. Frequencies in the ATG treated and control group were evaluated to detect significant difference using non-parametric t-test MannWhitney test.

Results: Patients of this subpopulation (aged 16-70 years) were shown to be representative of the larger CBMTG 0801 study population. The ATG treated group had a significant decrease in total T cells, RTE Th cells, and naïve Th cells at day 100 compared to the control population $(\mathrm{p}<$ 0.0001 each population - See Table 1). ATG treatment had no impact on Tregcells, CD19+ B cells, and CD21lowB cells but there was a significant increase in CD56bright NKreg cells $(p<0.0001)$. We evaluated the ratio of Naïve Th effector cells to the regulatory NKregcell population and saw a $>100$ fold difference the ATG treated group (Naïve Th cell:NKregcell ratio $=0.06)$ compared to untreated patients (ratio of 9.2; $<<0.0001$ ). In this small population we found that the Naïve Th cell:NKregcell ratio was also high prognostic for later development of cGvHD (1.37 vs. $0.13 ; \mathrm{p}<0.0001)$.

Conclusions: These results suggest that ATG's major mechanism of action is related to its ability to simultaneously inhibit Naïve Th cells and enhance CD56 ${ }^{\text {bright }} \mathrm{NK}$ regcells after transplantation. While these results require confirmation, they support strategies that target the ratio of

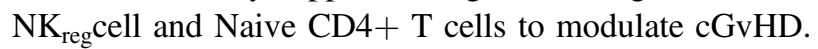

Clinical Trial Registry: NCT01217723
https://clinicaltrials.gov/ct2/show/NCT01217723?term= ATG $\&$ cntry $=$ CA\&city $=$ Hamilton\&rank $=2$

\begin{tabular}{|c|c|c|c|}
\hline $\begin{array}{l}\text { Immune Cell } \\
\text { Population }\end{array}$ & $\begin{array}{l}\text { ATG Treatment } \\
\text { group (Median } \\
\% \text { ) } \mathrm{N}=25\end{array}$ & $\begin{array}{l}\text { Control group (No } \\
\text { ATG treatment) } \\
\text { (Median\%) } \mathrm{N}=15\end{array}$ & $P$ value \\
\hline $\begin{array}{l}\text { CD56bright NKreg } \\
\text { cells (\% of total NK } \\
\text { population) }\end{array}$ & 0.8 & 0.85 & $<0.0001$ \\
\hline $\begin{array}{l}\text { Recent Thymus } \\
\text { Emigrant Th cells } \\
\text { (CD31+CD45RA } \\
+ \text { CD4 + T cells - \% } \\
\text { of CD4 + T cells) }\end{array}$ & 0.74 & 12.5 & $<0.0001$ \\
\hline $\begin{array}{l}\text { Total T cells }(\% \text { of } \\
\text { MNCs) }\end{array}$ & 0.80 & 14.5 & $<0.0001$ \\
\hline $\begin{array}{l}\text { Naive Helper T cells } \\
\text { (CD45RA+CD4 }+ \\
\text { T cells - \% of Th } \\
\text { Cells) }\end{array}$ & 0.94 & 11.7 & $<0.0001$ \\
\hline $\begin{array}{l}\text { Naive Helper } T \text { cell: } \\
\text { NKreg cell ratio }\end{array}$ & 0.06 & 9.2 & $<0.0001$ \\
\hline $\begin{array}{l}\text { CD21low B cells (\% } \\
\text { of } B \text { cells) }\end{array}$ & 17.1 & 18.9 & 0.68 \\
\hline \multirow{2}{*}{$\begin{array}{l}\text { Treg Cells (\% of CD4 } \\
+ \text { cells) }\end{array}$} & 6.68 & 4.09 & 0.13 \\
\hline & $\begin{array}{l}\text { cGvHD } \\
\text { Develops after } \\
100 \text { days } \\
\text { (Median \%) }\end{array}$ & $\begin{array}{l}\text { cGvHD Develops } \\
\text { after } 100 \text { days }\end{array}$ & $<0.0001$ \\
\hline $\begin{array}{l}\text { Naive Helper } T \text { cell: } \\
\text { NKreg cell ratio }\end{array}$ & 1.37 & 0.13 & $<0.0001$ \\
\hline
\end{tabular}

[[O104 Table] 1. Table 1 - Impact of ATG on Chronic GvHD Biomarkers Measured at Day 100 after Transplantation]

Disclosure: None of the Authors have any conflicts of interest to declare

\section{5}

Immune reconstitution - based score at diagnosis of CGVHD predicts GVHD severity and overall-survival: A novel prognostication tool for GVHD treatment tailoring

Maria Teresa Lupo-Stanghellini ${ }^{1}$, Fabio Serpenti ${ }^{2}$, Raffaella Milani ${ }^{1}$, Carlo Messina ${ }^{1}$, Raffaella Greco ${ }^{1}$, Stefania Girlanda $^{1}$, Carmine Liberatore ${ }^{I}$, Gabriele Casirati, Francesca Farina ${ }^{1}$, Sara Mastaglio ${ }^{1}$, Elena Guggiari, Francesca Lunghi ${ }^{1}$, Massimo Bernardi, Chiara Bonini $^{2,1}$, Andrea Assanelli ${ }^{1}$, Jacopo Peccatori', Consuelo Corti $^{1}$, Fabio Ciceri ${ }^{1,2}$, Francesca Lorentino ${ }^{I}$

${ }^{1}$ San Raffaele Scientific Institute, Milano, Italy, ${ }^{2}$ University Vita-Salute San Raffaele, Milano, Italy 
Background: Allogeneic stem cell transplantation (HSCT) survivors are at a relevant risk of developing chronic GvHD (cGvHD), which importantly affects quality of life and increases morbidity and mortality. Early identification of patients at risk of development of severe cGvHD related morbidity would be a relevant tool to tailor preventive strategies. We have previously demonstrated the role of immune reconstitution (IR) as predictive biomarker of occurrence of cGvHD. The aim of this study was to evaluate the prognostication power of IR at cGvHD onset through a new IR-based score.

Methods: We analyzed clinical data from 383 adult patients consecutively undergoing first allogeneic HSCT transplant between January 2011 and December 2016 at our Institution. A written consent was given for the use of medical records for research. Patients were divided into a test cohort (307 pts) and a validation cohort (76 pts). Median follow-up for surviving patients was 4 years.We built a Cox multivariate models for OS in patients with cGvHD of any severity. Variables included in the models were: patient age (according to median value), R-DRI score, type of donor (matched related donor vs matched unrelated vs haploidentical), main GvHD prophylaxis platform (ATG-based vs PtCy-based vs neither of the two), IR values (CD4, CD19, NK, IgA, IgM according to median values) at cGvHD diagnosis, history of prior aGvHD, Karnofsky PS, Plt $<100.000 / \mu \mathrm{L}$, ALC $<1000 / \mu L$, Eos $<$ $500 / \mu$ L.Once we identified the variables independently predicting OS by multivariate analysis, we derived a formula for a prognostic risk index by using the $\beta$ coefficients found in the model. Each patient was then assigned a score and we defined three groups of OS risk (low, intermediate and high) by dividing the score into three classes using the first and third quartiles. Finally, to evaluate predictive performance of the IR-score we calculated the receiver operating characteristics (ROC) curve via the area under the curve (AUC), to summarize the IR-score ability to correctly classify events and non-events.

Results: 115 patients (87 test-cohort, 28 validationcohort) were evaluated for cGvHD and outcome.

Our multivariate model defined the variables independently predicting OS at cGvHD onset: CD4+ count $>233$ / mcl, NK count $<115 / \mathrm{mcl}$, IgM $<0.45$ g/L, Karnofsky PS $<80 \%$.

Final score was calculated as follows: 2,4 (if CD4 $>233$ / $m c L)+2,1$ (if $N K<115 / m c L)+2,1$ (if IgM $<0,45)+4,3$ (if Karnofsky $<80$ ). Low risk patients were defined as having a score $\leq 2.4$, intermediate $>2,4$ and $\leq 4.5$, high risk $>4.5$. The $3 y-O S$ for low risk patients was $96 \%$, for intermediate $76 \%$ and for high risk $27 \%$ in the test-cohort and $100 \%, 66 \%$ and $35 \%$ in the validation-cohort (figure 1a-b). The ROC curve analysis supports the validity of the IR-score in our cohort of patients - AUC 85.5\%, with $95 \%$ confidence intervals higher than 50\%. Furthermore IR-score was able to stratify across NIH-severity classification (figure 1c).

Conclusions: Immune-Reconstitution score at diagnosis of cGvHD predicts GvHD severity and overall-survival. IRscore could be adopted to identify patients at high risk and modulate cGvHD treatments accordingly.

Disclosure: Chiara Bonini has research contract with Intellia Therapeutics. The other authors declare that they have no conflicts of interest.

\section{6}

Haploidentical transplantation with sirolimus-based GVHD prophylaxis and unmanipulated PBSC graft: Ten year experience in 354 adult patients

Raffaella Greco ${ }^{1}$, Serena Albanese ${ }^{2}$, Francesca Lorentino $^{I}$, Fabio Giglio ${ }^{I}$, Daniela Clerici ${ }^{I}$, Lorenzo Lazzari ${ }^{I}$, Maria Teresa Lupo Stanghellini ${ }^{I}$, Simona Piemontese ${ }^{I}$, Magda Marcatti ${ }^{1}$, Sara Mastaglio ${ }^{1}$, Andrea Assanelli, Luca Vago ${ }^{1}$, Chiara Bonini, Consuelo Corti ${ }^{1}$, Massimo Bernardi $^{1}$, Fabio Ciceri ${ }^{1}$, Jacopo Peccatori ${ }^{1}$

${ }^{1}$ IRCCS San Raffaele Scientific Institute, Italy, ${ }^{2}$ University Vita-Salute San Raffaele, Milano, Italy

Background: Haploidentical transplantation has emerged as a viable option for patients lacking a fully matched donor. We firstly explored the association of sirolimus and ATG, later followed by sirolimus with PT-Cy as GvHD strategy. Herein, we describe long-term outcomes of haploidentical HSCT using sirolimus-based GvHD prophylaxis.

Methods: Between October 2007 and March 2017, a total of 354 adult patients received an haploidentical HSCT for high-risk hematological malignancies. Disease risk index (DRI; Armand et al) was low-intermediate in 116, high in 148 and very high in 86 patients. Conditioning was myeloablative in 211 and reduced-intensity in 143 patients. Most patients $(\mathrm{n}=349)$ received unmanipulated PBSCs.

All patients received sirolimus and MMF as GvHD backbone prophylaxis plus ATG in 203 patients, and PT-Cy in 151. Conditioning regimen was based on treosulfanfludarabine; recipients of PT-Cy transplants were more likely to receive a regimen intensified by a $2^{\text {nd }}$ alkylating agent (melphalan or thiotepa). Median follow up was longer in ATG group (70 vs 26 monhs, $\mathrm{p}<0.01$ ). There were no differences in DRI.

Results: The majority of patients reached the neutrophil (89\% in ATG group vs $86 \%$ in PT-Cy group) and platelet (76\% vs $77 \%$ ) engraftment within 30 days after HSCT.

Immune-reconstitution was broad and fast, reaching more than $100 / \mathrm{ml} \mathrm{CD} 3+\mathrm{T}$ cells within a median of 35 vs 
33 days. The two groups were similar in terms of survival and main transplant outcomes.

In the ATG group, the cumulative incidence of grades IIIV and III-IV acute GvHD at 100 days was $26 \%$ and $20 \%$. Corresponding rates after PT-Cy were $35 \%$ and $20 \%$. The cumulative incidence of overall and severe chronic GvHD was $31 \%$ and $10 \%$ at 3 years in ATG group and $42 \%$ and $16 \%$ after PT-Cy .The cumulative incidences of relapse and NRM in ATG group were respectively $41 \%$ and $31 \%$ at 3 years. Corresponding rates after PT-Cy were $35 \%$ and $27 \%$. In ATG group, 3-year OS was $36 \%$, while GRFS was $24 \%$. The corresponding probabilities after PT-Cy were $44 \%$ and $24 \%$.

The only difference reported was a better PFS in favour of PT-Cy (38\% vs 29\%, $\mathrm{p}=0.04)$. Multivariate analysis confirmed the effect of DRI on transplant outcomes, with worse PFS in high $(\mathrm{p}=0.001$; HR 2.07; 95\%CI, 1.37-3.15) and very high $(\mathrm{p}<0.001$; HR 3.55; 95\% CI, 2.24-5.63) compared to low-intermediate DRI population.The protective role of PT-Cy compared to ATG-based platform on PFS was more relevant in patients with low-intermediate DRI $(\mathrm{p}=0.003)$, showing also lower relapse $(\mathrm{p}=0.009)$ and better OS (69\% vs $49 \%$ at 3-year); multivariate analysis confirmed this effect on PFS ( $\mathrm{p}=0.036$; HR $0.39 ; 95 \% \mathrm{CI}$, $0.16-0.94)$ and relapse $(\mathrm{p}=0.043$; HR 0.25 ; 95\%CI, 0.070.96).

Conclusions: Extended follow-up in 354 patients confirms Sirolimus-based GvHD prophylaxis as feasible and safe in haploidentical HSCT based on unmanipulated PBSC graft. Both ATG and PTCy association to sirolimus provide an effective prevention of GvHD and translate into a similar long-term overall survival. A significant advantage of SirPT-Cy on relapse rate warrants further investigation.

Clinical Trial Registry: NA

Disclosure: Nothing to declare

\section{7}

Human-derived $\alpha 1$-antirypsin is Effective in a cohort of high-risk patients with steroid-resistant gastrointestinal graft-versus-host disease

\section{Livia Giannoni ${ }^{1}$, Florence Morin ${ }^{1}$, Marie Robin ${ }^{1}$, Marine Peyneau $^{I}$, Marie Hélène Schlageter ${ }^{I}$, Simona Pagliuca ${ }^{I}$, Helene Moins-Teisserenc ${ }^{I}$, Flore Sicre de Fontbrune ${ }^{I}$, Regis Peffault de Latour ${ }^{I}$, Gerard Socié ${ }^{1}$, David Michonneau ${ }^{I}$}

${ }^{1}$ Hôpital Saint Louis, AP-HP, Paris, France

Background: Steroid-refractory graft-versus-host disease (SR-GVHD) is still responsible for high mortality in patients undergoing allogeneic stem cell transplantation; a number of agents is currently available in case of steroid- refractoriness, yet there is so far no consensus about a standard second-line treatment and overall survival (OS) remains poor. $\alpha 1$-antitripsyn $(\alpha \mathrm{AT})$ is a circulating $52-\mathrm{kDa}$ serine protease inhibitor found to enhance the production of anti-inflammatory cytokines and to favor the expansion of regulatory T-cells; it has therefore been tested in situations of altered tolerance and disproportionate inflammation, including GVHD. Two studies showed that treatment of SR acute GVHD with $\alpha$ AT is feasible and effective.

Methods: We retrospectively analyzed a series of patients who received exogenous $\alpha$ AT for SR acute gastrointestinal GVHD or overlap GVHD with acute gut features. SRGVHD was defined and graded according to standard criteria. $\alpha \mathrm{AT}$ was administered intravenously at a loading dose of $90 \mathrm{mg} / \mathrm{kg}$ at day 1 followed by $30 \mathrm{mg} / \mathrm{kg} /$ day every other day for a total of 8 doses. Response to treatment was defined according to published criteria; OS was estimated with the Kaplan-Meier method.A panel of 46 cytokines and immune cell subsets were measured before treatment and once weekly during treatment by a Luminex assay and by flow cytometry, respectively.

Results: Sixteen patients were treated for gut GVHD between September 2016 and March 2018. Median age was 50 years (range 18-56). $\alpha \mathrm{AT}$ was administered at a median time of 104 days from transplantation (range 38-215) and of 65 days from GVHD onset (range 12-198). Acute GVHD was scored as grade II in $28 \%$ of patients, grade III in $66 \%$, grade IV in $6 \%$. Sixty-seven percent of patients had already received one or more lines of treatment other than steroids, including ruxolitinib, etanercept, ATG; ORR was $44 \%$ with a CR rate of 27\%; median time to best response was 21 days (range 6-26), with a continued ORR at day 56 of $39 \%$. The overall rate of gastrointestinal responses was $61 \%$. Median follow-up of living patients was 440 days (range 84-602); median OS was 138 days and 1-year OS was $48 \%$ (95\% CI $26 \%$ - 74\%). The most common infectious event was CMV reactivation (29\%); 2 grade 3-4 infectious complications were recorded. There was no quantitative deficiency of blood AAT levels before treatment $(1.72 \mathrm{~g} / \mathrm{L} \pm 0.46)$; blood $\alpha \mathrm{AT}$ rose significantly during and after treatment. Baseline $\alpha A T$ level didn't differ between responding and nonresponding patients.

A cytokine profile was evaluable in 12 patients; no statistically significant increase or decrease in cytokine plasma concentration after $\alpha \mathrm{AT}$ infusion was observed. Surprisingly, a decrease in circulating $\mathrm{T}$ regs after exposure was found $(\mathrm{p}=0.002)$, regardless of patients' responding status.

Conclusions: Treatment with $\alpha \mathrm{AT}$ was safe and effective in a cohort of SR-aGVHD high risk, pre-treated patients and should be considered as a possible alternative. Changes in the cytokine milieu and T-cell subsets shown in murine models were not observed in a real-life setting. 
Disclosure: Nothing to declare

\section{8}

Post-transplant cyclophosphamide for GVHD prophylaxis in matched unrelated donor transplantation

Rebeca Bailén ${ }^{1,2}$, María Jesús Pascual-Cascón ${ }^{3}$, Pascual Balsalobre ${ }^{1,2}$, Jaime Sanz, Alberto Doblas-Marquez, Olga Benítez-Hidalgo ${ }^{3}$, José Luis Piñana ${ }^{4}$, Leyre Bento ${ }^{5}$, Antonia Sampol, Gillen Oarbeascoa ${ }^{1}$, Laura Solán ${ }^{1,2}$, Nieves Dorado ${ }^{1,2}$, Carolina Martínez-Laperche ${ }^{1,2}$, Javier Anguita $^{1,2,6}$, Ismael Buño ${ }^{1,2}$, José Luis Díez-Martín ${ }^{1,2,6}$, Mi Kwon ${ }^{1,2}$

${ }^{1}$ Hospital General Universitario Gregorio Marañón, Madrid, Spain, ${ }^{2}$ Instituto de Investigación Sanitaria Gregorio Marañón, Madrid, Spain, ${ }^{3}$ Hospital Regional de Málaga, Málaga, Spain, ${ }^{4}$ Hospital Universitari i Politècnic la Fe, Valencia, Spain, ${ }^{5}$ Hospital Universitario Son Espases, Mallorca, Spain, ${ }^{6}$ Universidad Complutense de Madrid, Madrid, Spain

Background: Post-transplant high dose cyclophosphamide (PT-CY) effectively prevents graft-versus-host disease (GVHD) after unmanipulated HLA-haploidentical hematopoietic stem cell transplantation (HSCT). The use of PT-CY in HLA-identical donor is less explored. In this study, we analyzed the results of PT-CY for GVHD prophylaxis in matched unrelated donor (MUD) HSCT and compared them with those obtained after prophylaxis with thymoglobulin (ATG), methotrexate (MTX) and cyclosporine (CsA).

Methods: 102 matched unrelated donor HSCT from 3 Spanish centers have been analyzed: 30 performed between 2010 and 2013 using ATG-MTX-CsA and 72 performed between 2014 and 2017 using PT-CY.

Results: Baseline characteristics and post-transplant complications are shown in Table 1. Peripheral blood was used as graft source in $87 \%$ of the patients in the ATG group and in $78 \%$ in the PT-CY group. GVHD prophylaxis consisted in ATG $2 \mathrm{mg} / \mathrm{m}^{2}$ days -4 to -2 , MTX days $+1,+3$, +6 and +11 , and CsA from day -1 in the ATG group. The PT-CY group received cyclophosphamide $50 \mathrm{mg} / \mathrm{kg} / \mathrm{d}$ on days +3 and +4 , followed by either CsA or tacrolimus and mycophenolate mofetil (MMF) from day +5 in 30 patients (42\%), cyclophosphamide on days +3 and +5 combined with CsA or tacrolimus from day 0 in 26 patients (36\%), or cyclophosphamide on days +3 and +5 combined with tacrolimus and sirolimus from day +5 in 16 patients (22\%). Cumulative incidence at 100 days of grade II-IV (67\% vs $46 \%, \mathrm{p}=0.02)$ and III-IV (41\% vs $3 \%, \mathrm{p}=0.002)$ acute GVHD, were significantly higher in the MTX-CsA group (Figure 1A). There were no differences in the 2-year cumulative incidence of chronic moderate to severe GVHD between the ATG and the PT-CY group (30\% vs $24 \%$, $\mathrm{p}=0.6$ ). After a median follow-up of 90 months for the ATG group and 26 months for the PT-CY group, 2-year overall survival (OS) was higher in the PT-CY group (45\% vs $60 \%)$ although not statistically significant $(\mathrm{p}=0.09)$ (Figure 1B). We found no differences between both cohorts in 2-year event-free survival (EFS) $(46 \%$ and $50 \%, \mathrm{p}=0.55)$ and the composite endpoint of GVHD-free and relapse-free survival (GFRS) (38\% vs $40 \%, \mathrm{p}=0.253$ ). The 2 -year cumulative incidence of relapse was significantly higher in the PT-CY group ( $26 \%$ vs $6.6 \%, \mathrm{p}=0.04$ ) and non-relapse mortality (NRM) at 2-years was higher in the ATG group ( $40 \%$ vs $22 \%$ ) but not statistically significant $(\mathrm{p}=0.06$ ).

Conclusions: In our experience, in spite of the limited number of patients, GVHD prophylaxis using PT-CY combined with additional immunosuppression after MUD HSCT, using mostly peripheral blood as graft source, reduced the cumulative incidence of aGVHD compared to standard prophylaxis with MTX-CsA. Prospective studies with longer follow-up are needed to confirm these observations.

Disclosure: Nothing to declare

\section{9}

Abstract already published.

\section{0}

Abstract already published.

\section{1}

Incorporating post-transplant cyclophosphamide-based prophylaxis as standard-of-care in HLA matched sibling or unrelated donor transplantation for patients with an increased risk OF GVHD

Rahinatu Awol, Irene García-Cadenas ${ }^{1}$, Rodrigo Martino $^{I}$, Albert Esquirol ${ }^{I}$, Silvana Saavedra ${ }^{1}$, Silvana Novelli, Ana Garrido ${ }^{I}$, Jordi Lopez, Miquel Granell, Carolina Moreno $^{I}$, Javier Briones ${ }^{I}$, Salut Brunet ${ }^{I}$, Jordi Sierra $^{I}$

${ }^{1}$ Hospital de la Santa Creu i Sant Pau, Sant Pau and Jose Carreras Leukemia Research Institutes, Autonomous University of Barcelona, Barcelona, Spain

Background: Post-transplant high dose cyclophosphamide (PT-CY) effectively prevents GVHD after haploidentical stem cell transplantation (SCT). However, the use of PT-CY in matched sibling and unrelated donor HSCT is less explored. We introduced PT-CY as a standard of care(SOC) for RIC and MAC allo-SCT, initially in recipients at high risk of severe GVHD. 
Methods: 44 consecutive patients who underwent alloSCT for hematological malignancies between January 2016 and August 2018 were included in this prospective singlecentre protocol. All patients had at least one baseline risk factor predicting development of severe GVHD (e.g. HLA mismatch, Fem-to-Male sex mismatch). PT-CY in combination with a second immunosuppressive drug was used as GVHD prophylaxis.

Results: Patients characteristics are summarized in Table 1. Median age was 56 (range, 19-67) years, with 6 male patients $(14 \%)$ receiving a graft from a female donor. More frequent allo-SCT indications were acute leukemia and MDS (54.5\%) followed by NHL (18\%). Eleven patients $(25 \%)$ were transplanted in advanced status. Donor was a sibling, matched unrelated or mismatched unrelated in $34 \%$, $25 \%$ and $41 \%$ respectively. Seven patients $(16 \%)$ received a myeloablative conditioning regimen including TBI ( 8 or $13.5 \mathrm{~Gy}$ ) while the remaining (84\%) received a RIC/RTC regimen based on Fludarabine, Busulfan or Melphalan +/Thiotepa $(3 \mathrm{mg} / \mathrm{kg})$. Median follow-up for survivors was 365 days (range:64-959).

Median time to neutrophil and platelet engrafment were +23 (12-36) and +22 (10-50) days, respectively (G-CSF not routinely used). Early toxicity was low, without cases of thrombotic microangiopathy, only 2 cases of drug-related renal failure $(4.5 \%)$ and 1 case of possible VOD. Before the introduction of mini-Thiotepa in the RIC protocols (FluBu/ FluMel) there was 1 case of primary graft failure (GF) and 5 cases of late graft failure $(6 / 28 ; 21 \%) ; 4$ cases were successfully regrafted with the mini-Thiotepa RIC. There have been no further cases of GF after its introduction $(n=8$ evaluable cases +4 second salvage alloRICs). Additional potential risk factors for GF were CD34+ $\leq 3 \times 10 \mathrm{e} 6 / \mathrm{kg}$ $(\mathrm{p}=0.07)$ and a high lymphocyte count at stem cell infusion $(\mathrm{p}=0.06)$.

The CI of grade 2-4 acute GVHD at day +120 was $28 \%$ (95\% CI: 14-48) with only 2 cases of refractoriness to steroids. Of the 37 evaluable patients, only 1 developed moderate chronic GVHD leading to a 1yr-CI of 3\%. NRM was $10.5 \%$ at $1 \mathrm{yr}$ and the CI of relapse was $31 \%(95 \% \mathrm{CI}$ : 21-40). All relapses occurred in patients with intermediate/ high rDRI. 1yr-OS was $76 \%$ and the estimated 1-year DFS was $63.6 \%$.

Conclusions: These outcomes confirmed the feasibility of both RIC and MAC allo-SCT using PT-Cy as SOC with a single immunosuppressive drug in patients at high risk of GVHD. An important observation was the high rate of GF with "classical" alloRIC platforms (FluBu/FluMel), which appears to be lower after introducing the mini-Thiotepa. Although these data need confirmation in larger cohorts, the current results suggests that PT-CY may pave the way to improving the quality of life of transplant survivors by markedly reducing severe GVHD.

\begin{tabular}{ll}
\hline Characteristics (N=44) & $\mathrm{N}(\%)$ \\
\hline Age, median (range) & $56(19-67)$ \\
Sex, male & $22(50 \%)$ \\
Baseline disease & \\
AL and MDS & $24(54 \%)$ \\
NHL/Hodgkin & $10(23 \%)$ \\
MPN/CML & $10(23 \%)$ \\
Others & $4(9 \%)$ \\
Advanced disease at transplant & $11(25 \%)$ \\
Donor type & \\
Identical sibling & $15(34 \%)$ \\
HLA (0 or 1 HLA mismatch) & $11 / 18(25 \% / 41 \%)$ \\
EBMT risk index: high (5-6) & $16(36 \%)$ \\
Conditioning & \\
Fludarabine-busulfan (RIC) & $16(36 \%)$ \\
Fludarabine-melphalan (RIC) & $13(30 \%)$ \\
MiniThiothepa modified RICs & $8(18 \%)$ \\
Fludarabine-TBI (8 or 13.5Gy) & $7(16 \%)$ \\
Stem cell source (PB) & $43(98 \%)$ \\
Second immunosuppressive agent: tarolimus/sirolimus & $40 / 4(91 \% / 9 \%)$ \\
CD34+ infused (x10E6), median (range) & $5,1(6,1-7,4)$ \\
\hline
\end{tabular}

Abbreviations: AL, acute leukaemia; CML, chronic myeloid leukaemia; MPN, myeloproliferative neoplasm; NHL, non-Hodgkin lymphoma; MDS, myeloproliferative syndrome; PB, peripheral blood; URD, unrelated donor; RIC, reduced intensity conditioning; TBI, total body irradiation

[[O111 Table] 1. Patients characteristics]

Disclosure: Nothing to declare

Graft-versus-host disease - preclinical and animal models

\section{2}

Protection of the endothelium during steroid-refractory GVHD

Steffen Cordes ${ }^{1}$, Zeinab Mokhtarias ${ }^{2}$, Maria Bartosova ${ }^{3}$, Katarina Riesner ${ }^{I}$, Sarah Mertlitz, Joerg Mengwasser ${ }^{I}$, Yu Shi, Martina Kalupa ${ }^{I}$, Aleixandria McGearey ${ }^{I}$, Jens Schrezenmeier ${ }^{1}$, Lars Bullinger ${ }^{I}$, Maribel Diaz-Ricart ${ }^{4}$, Marta Palomo ${ }^{5}$, Enric Carrreras, ${ }^{5}$, Gernot Beutel ${ }^{6}$, Claus Peter Schmitt ${ }^{3}$, Andreas Beilhack ${ }^{2}$, Olaf Penack ${ }^{1}$

${ }^{1}$ Charité Univerisätsmedizin Berlin, Berlin, Germany, ${ }^{2}$ University Würzburg, Würzburg, Germany, ${ }^{3}$ University Heidelberg, Heidelberg, Germany, ${ }^{4}$ University Barcelona, Barcelona, Spain, ${ }^{5}$ Carreras Research Institute, Barcelona, Spain, ${ }^{6}$ University Hannover, Hannover, Germany 
Background: Clinical data demonstrated that endothelium related factors predict mortality after the diagnosis of aGVHD, suggesting that the endothelium may be involved in the pathobiology of steroid-refractory aGVHD (SRaGVHD) (J Clin Oncol. 2018 Mar 10;36(8):789-800, Lancet Haematol. 2017 Sep;4(9):e414-e423, J Clin Oncol. 2014 Oct 20;32(30):3421-7, PNAS. 2011 Aug 11;118(6):168592). Along those lines, a $\mathrm{T}$ cell independent mechanism of steroid-refractory aGVHD has been suggested recently (Sci Rep. 2018 Aug 20;8(1):12475).

Methods: Intestinal biopsies from patients after alloSCT. Murine aGVHD models Balb/c $\rightarrow \mathrm{B} 6, \mathrm{~B} 6 \rightarrow \mathrm{BDF}$ and LP/ $\mathrm{J} \rightarrow \mathrm{B} 6$ with and without steroid treatment. Immunostaining, electron microscopy, light sheet fluorescence microscopy (LSFM), FACS. In vivo and in vitro assays for endothelial dysfunction. Treatment with phosphodiesterase type 5 inhibitor (PDE5) in SR-aGVHD models.

Results: We found a significant higher percentage of apoptotic vessels in duodenal and colonic mucosa biopsies of patients with grade III-IV aGVHD compared to no GVHD (Figure 1A). In murine experimental aGVHD, we detected severe microstructural endothelial damage and reduced endothelial pericyte coverage accompanied by reduced expression of endothelial tight junction proteins leading to increased endothelial leakage in aGVHD target organs. During intestinal aGVHD, colonic vasculature structurally changed, reflected by increased vessel branching and vessel diameter (Figure 1B). We analysed human biopsies and murine tissues from SR-GVHD vs. naive (untreated) aGVHD and found significantly lower lymphocyte infiltration in SRGVHD, demonstrating low inflammatory activity (Figure 1C). Our findings suggest that endothelium-related and T cell independent mechanisms play a previously unrecognized role during SR-aGVHD, providing the rationale for T-cell independent treatment strategies. As a first example for such an approach, we tested the endothelium-effective PDE5 inhibitor sildenafil and found reduced apoptosis as well as improved metabolic activity of endothelial cells in vitro. In accordance, sildenafil treatment resulted in improved survival and reduced target organ damage during experimental SRaGVHD (Figure 1D).

Conclusions: We show profound endothelial involvement after allo-HSCT and demonstrate that endothelialprotection with sildenafil ameliorates SR-aGVHD, providing a novel non-immunosuppressive treatment approach. These results can serve as rationale for translational development of endothelium-based therapies for SRaGVHD.

Disclosure: The authors declare no confilct of interest relevant to this study

\section{3}

Abstract already published.

\section{4}

The therapeutic effect of immune-modifying microparticles in an acute graft-versus-host disease model

\section{John Galvin ${ }^{1}$,Sara Beddow ${ }^{2}$,Stephen Miller ${ }^{2}$}

${ }^{1}$ University of Illinois Chicago, Chicago, IL, United States, ${ }^{2}$ Northwestern University, Chicago, IL, United States

Background: Inflammatory monocytes are recruited to target organs during acute Graft versus Host Disease (aGVHD). As seen in other autoimmune disorders, inflammatory monocytes play an important role in antigen presentation and cytokine production. These actions allow for a sustained activation and proliferation signal to T-cells. Previous studies have shown that IMP treatment in mouse models of colitis, encephalitis, myocardial infarction and peritonitis markedly reduced monocyte accumulation in the affected end-organs -- promoting tissue repair; reducing disease symptoms and increasing survival. Therefore, our objective was to test clinical outcomes after IMP treatment in a mouse model of aGVHD.

Methods: Murine aGVHD model: BALB/c mice were given 800 cGy total body irradiation, irradiated BALB/c mice were transplanted with $5 \times 10^{6} \mathrm{C} 57 \mathrm{BL} / 6$ bone marrow cells and $1 \times 10^{6} \mathrm{C} 57 \mathrm{BL} / 6$ spleen cells via tail vein.IMP treatment: IMPs were made with PLGA (Phosphorex Inc, Hopkinton MA) was administered to the recipient mice (1.4 $\mathrm{mg} / \mathrm{kg}$ body weight) by IV daily starting from day 5 to day 10 after bone marrow transplantation (BMT). PBS at the same volume was used as vehicle control.

In vivo bioluminescence imaging: Mice were given an intraperitoneal injection of luciferin $(150 \mathrm{mg} / \mathrm{kg}$ body weight) and then anesthetized and imaged using the IVIS Imaging system (Xenogen). Imaging data were analyzed and quantified with Living Image Software (Xenogen).

Results: IMP treated mice had significantly less severe acute GVHD symptoms (average score of 2.48) than the untreated $\mathrm{BM}+\mathrm{Sp}$ group (average score 3.96) starting at the time of IMP treatment (days 5-10) and remained with significantly reduced symptoms for the 30 day course (Figure 1). IMP treatment also rescued $\mathrm{BM}+\mathrm{Sp}$ mice from aGVHD associated mortality with a 30-day overall survival of $62 \%$ compared to $4 \%$ in the untreated $\mathrm{BM}+\mathrm{Sp}$ group (Figure 2). Intestinal tissue from the IMP treated mice compared to the $\mathrm{BM}+\mathrm{Sp}$ mice demonstrated less evidence of aGVHD (an average score of 1.25 and 2.75, respectively). Hepatic tissue from the IMP treated mice compared to the $\mathrm{BM}+\mathrm{Sp}$ mice demonstrated less evidence of aGVHD (an average score of 1.5 and 2.42, respectively). IMP treatment also significantly reduced INF- $\boldsymbol{\gamma}$ levels in the intestinal tissues of treated mice compared to untreated $\mathrm{BM}$ + Sp mice. In the mice infused with lymphoma cells (A20- 
luc), IMP treatment reduced aGVHD symptoms and death while preserving the GVL effect.

Conclusions: Our results demonstrate that IMPs significantly reduce symptoms and mortality in a murine model of aGVHD while preserving GVL. The reduction in inflammatory monocytes with IMPs leads to a reduction in inflammatory cytokines, hepatic lymphocyte infiltration and intestinal mucosal denudation. These findings highlight the potential of IMP therapy as a specific and potentially safe treatment in acute GVHD.

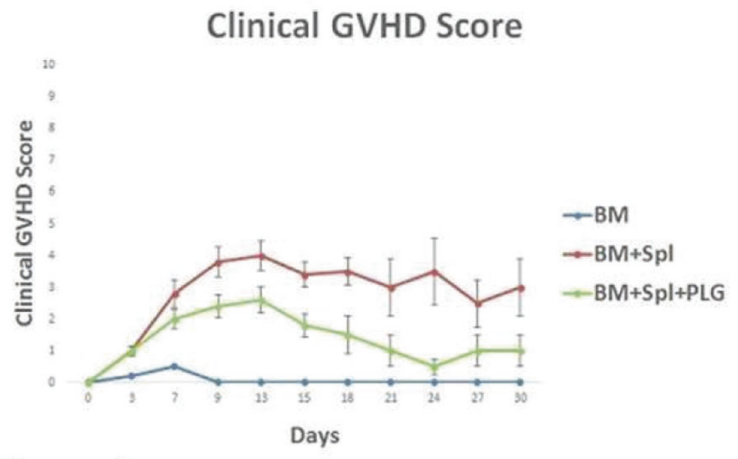

Figure 1.

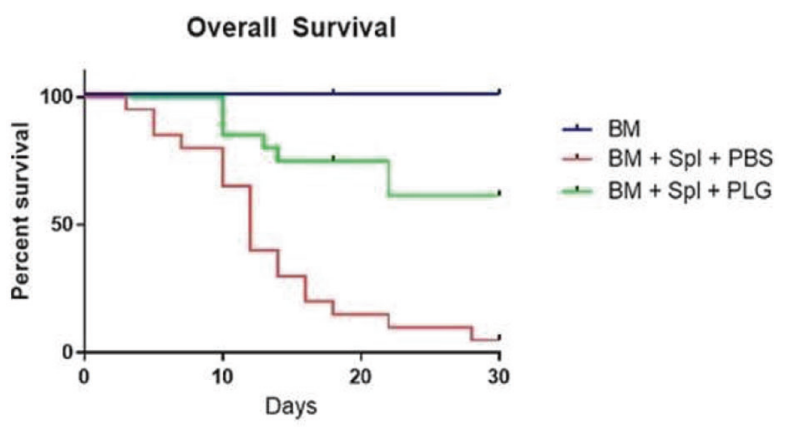

Figure 2.

[[O114 Image] 1. Figure 1 and 2]

Disclosure: Nothing to declare

Haemoglobinopathy and inborn errors of metabolism

\section{5}

Stem cell transplantation with haploidentical T-cell depleted peripheral blood stem cells yields comparable outcomes to matched sibling donor transplantation in advanced stage sickle cell disease

Juergen Foell, Johannes Schulte ${ }^{2}$, Anja Troeger ${ }^{1}$, Beatrix Pfirstinger ${ }^{I}$, Katharina Kleinschmidt, Petra Hofmann ${ }^{I}$, Ernst Holler', Wofgang Herr', Matthias Edinger $^{l}$, Charalampos Aslanidis ${ }^{1}$, Daniel Wolff ${ }^{1}$, Angelika Eggert ${ }^{2}$, Selim Corbacioglu ${ }^{1}$
${ }^{1}$ Universitätsklinikum Regensburg, Regensburg, Germany, ${ }^{2}$ Charité Universitätsmedizin Berlin, Berlin, Germany

Background: Despite significant improvements in the supportive Sickle cell disease (SCD) causes substantial morbidity and mortality. Allogeneic hematopoietic stem cell transplantation (HSCT) is currently the only curative option but is only offered if a matched sibling donor (MSD) is available. With a MSD availability of $<20 \% \mathrm{~T}$ cell depleted HSCT from a haploidentical donor (T-haplo-HSCT) is a potential alternative.

Methods: 29 patients (pts) with advanced stage SCD (asSCD) were transplanted with a $\mathrm{CD}^{+} / \mathrm{CD} 19^{+}$or $\alpha \beta / \mathrm{CD} 19$ ${ }^{+}$depleted T-haplo-HSCT (20 pts, median age 13 years, range 3-31 years) or with bone marrow (BM) from a MSD, 9 pts, median age 14, range 9-25 years). Indication for HSCT was asSCD with multiple SCD related complications. All pts underwent exchange transfusion before HSCT. In all pts the conditioning regimen consisted of treosulfan, thiotepa, fludarabine and ATG. Immunosuppression was carried out with cyclosporine A or tacrolimus and mycophenolate mofetil. The control group received a MSD BM allograft.

Results: In the T-haplo-SCT group the pts received a $\mathrm{CD}^{+}$ or $\alpha \beta^{+} \mathrm{T}$ - and $\mathrm{CD} 19^{+} \mathrm{B}$-cell depleted peripheral stem cell allograft with $13,1 \times 10^{6} \mathrm{CD} 34+$ cells $/ \mathrm{kg}$ body weight (range 8,1 to 77,9 ). All pts with a median follow up of 22 months (range 4-57 months) in the MSD group and 18 from 20 pts with a median follow up of 17 months (range 5-58 months) in the T-haplo-SCT group are alive. Engraftment was achieved in all pts with stable chimerism over $90 \%$, except for 4 pts with a stable MC in the T-haplo SCT group and 1 patient in the MSD group, but complete engraftment of red cell precursor in the BM. All pts are off immunosuppression with a stable almost complete chimerism. The conditioning regimen was well tolerated with no case of high-grade transplant related morbidity. The post-transplantation complications were comparable in both groups. One patient developed after severe rotavirus gastroenteritis a severe CMV pneumonitis and succumbed to an uncontrolled CMV pneumonitis. One patient in the T-haplo SCT group suffered from a late graft failure and developed a macrophage activation syndrome. He died in a septic event.None developed a Glucksberg Grade III-IV aGvHD and in the T-haplo SCT group 4 pts (20\%) and in the MSD group 2 pts (22\%) developed a steroid sensitive mild to moderate $\mathrm{cGvHD}$ with symptoms of fasciitis, oral as well as mild cutaneous GvHD. In both groups no severe or steroid refractory cGvHD was observed.

Conclusions: Our results demonstrate increasing evidence for the safety and efficacy of $\mathrm{CD}^{+} / \mathrm{CD} 19^{+}$or $\alpha \beta^{+} / \mathrm{CD} 19^{+}$depleted haploidentical HSCT in asSCD. The treosulfan based conditioning regimen was an excellent alternative to busulfan with a low incidence of transplant related morbidities and therefore 
most suitable for pts with SCD. These results open the option of a curative therapy for almost all SCD pts without a MSD.

Disclosure: Nothing to declare.

\section{6}

Abstract already published.

\section{Hematopoietic stem cells}

\section{7}

Abstract already published.

\section{8}

Comparison of outcomes post allogeneic hematopoietic cell transplantation using fresh versus cryopreserved peripheral blood stem cell grafts

Ahmad S. Alotaibi, ${ }^{1,2}$, Shruti Prem', Jeffrey H. Lipton', Dennis D. Kim ${ }^{1}$, Auro Viswabandya', Santhosh Thyagu', Rajat Kumar', Wilson Lam ${ }^{1}$, Arjun Law', Fotios V. Michelis $^{1}$

${ }^{1}$ Princess Margaret Cancer Centre, University Health Network, University of Toronto, Toronto, Canada, ${ }^{2}$ King Faisal Specialist Hospital \& Research Centre, Riyadh, Saudi Arabia

Background: Cryopreservation is routine practice with autografts, however in the allogeneic HCT setting the effects of cryopreservation have not been thoroughly investigated. We sought to compare allogeneic HCT outcomes using fresh versus cryopreserved grafts in a large single centre cohort.

Methods: Between 2003 and 2017, we retrospectively reviewed 951 consecutive adult patients who underwent allogenic peripheral blood HCT at our centre. Outcomes assessed included platelet $(\geq 20 \times 10 \mathrm{e} 9 / \mathrm{L})$ and neutrophil $(\geq 0.5 \times 10 \mathrm{e} 9 / \mathrm{L})$ engraftment, occurrence of acute graft-versushost disease (GvHD) in the first 100 days, overall survival (OS), cumulative incidence of relapse (CIR) and non-relapse mortality.

Results: Median follow up of survivors was 47 months (range 4-177 months). Fresh grafts were received by 525 patients, 426 received cryopreserved grafts, median age at HCT was 53 and 54 years respectively. Transplant indication was myeloid malignancy in 711 (75\%), lymphoid in $229(24 \%)$ patients. Myeloablative regimens were used in $506(53 \%)$ patients. The majority of fresh grafts were from unrelated donors $(82 \%)$ while most cryopreserved grafts were from matched related donors $(90 \%)$. In vivo T-cell depletion was performed in $65 \%$ of fresh and $18 \%$ of cryopreserved transplants. Median time to neutrophil engraftment for fresh versus cryopreserved grafts was 15 (8-52) and 15 (10-48) days respectively, while median time to platelet recovery was 16 (12-186) and 17 (8-171) days, respectively. For fresh versus cryopreserved grafts, grade II-IV acute GvHD was seen in $51 \%$ and $54 \%$, respectively $(\mathrm{p}=0.42)$ while grade III-IV acute GvHD was seen in $28 \%$ of patients in both groups $(\mathrm{p}=0.96)$.

On univariate analysis, OS for the entire cohort at 2 years was $50 \%(95 \% \mathrm{CI} 47-54 \%)$ and at 5 years was $40 \%(95 \% \mathrm{CI}$ $36-43 \%$ ). Two and 5 year OS was $47 \%$ (95\%CI 43-52\%) and $39 \%(95 \%$ CI 34-44\%) respectively for fresh grafts and $54 \%(95 \%$ CI $49-59 \%)$ and $41 \%(95 \%$ CI 36-46\%) respectively for cryopreserved grafts $(p=0.21)$. Cumulative incidence of relapse (CIR) of the entire cohort at 2 years was $18 \%(95 \%$ CI $16-21 \%)$ and at 5 years was $21 \%(95 \% \mathrm{CI}$ $19-24 \%)$. Two and 5 year CIR was $16 \%$ (95\%CI 13-20\%) and $19 \%(95 \%$ CI $15-22 \%)$ respectively for fresh grafts and $21 \%(95 \%$ CI $17-25 \%)$ and $25 \%$ (95\%CI 21-29\%) respectively for cryopreserved grafts $(\mathrm{p}=0.02$, Figure 1$)$.
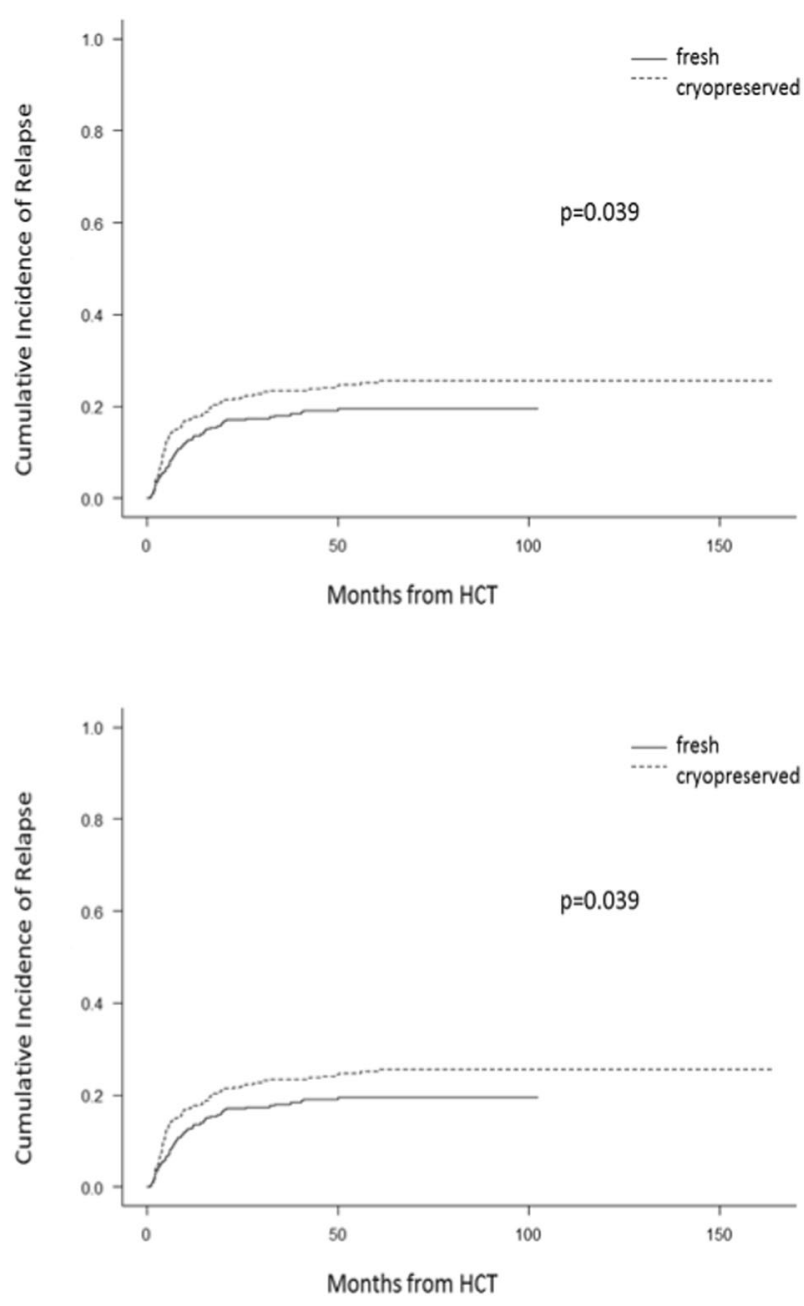

[[O118 Image] 1 . Figure 1] 
Multivariable analysis for OS verified no significant difference between fresh versus cryopreserved grafts $(\mathrm{p}=0.25)$. For CIR however, cryopreservation was the only independent predictor of relapse (HR 1.43 for cryopreserved, 95\%CI 1.07-1.91, $\mathrm{p}=0.02$ ), while for NRM cryopreservation was not an independent predictor of increased risk $(\mathrm{p}=0.06)$. When the multivariable analysis was repeated for related donor transplants only ( $n=474,385$ cryopreserved grafts, 89 fresh), this confirmed the independent increased relapse risk for cryopreserved grafts (HR 2.30, 95\%CI 1.21-4.38, $\mathrm{p}=0.01$ ).

Conclusions: We confirmed on univariate and multivariable analysis that there is no significant difference in OS between allogeneic transplants performed with fresh versus cryopreserved peripheral blood stem cell grafts, however there is a significant increase in relapse risk associated with cryopreservation.

Disclosure: Nothing to declare.

\section{9}

Post-transplantation cyclophosphamide versus low dose ATG FRESENIUS IN GVHD prophylaxiS: Similar graft versus host disease and relapse-free survival

Cristina Fernández Martínez ${ }^{1}$, Arancha Bermudez Rodríguez, ${ }^{1,2}$, Sara García Ávila', Almudena Cabero Martínez ${ }^{1}$, Lucrecia Yañez Sansegundo', Monica Lopez Duarte ${ }^{1}$, Joan Manuel Mora Barrios', Yolanda González Romero ${ }^{1}$, Sara Fernández Luis', David Gomez Lamas', Andrés Insunza Gaminde ${ }^{1}$, Iñigo Romón ${ }^{1}$, José Luis Arroyo ${ }^{3}$, Carlos Richard Espiga ${ }^{1}$, Enrique María Ocio San Miguel $^{1,2}$, Mercedes Colorado Araujo ${ }^{1}$

${ }^{1}$ Hospital Universitario Marqués de Valdecilla, Instituto de Formación e Investigación Marqués de Valdecilla (IDIVAL), Santander, Spain, ${ }^{2}$ Universidad de Cantabria, Santander, Spain, ${ }^{3}$ Banco de Sangre y Tejidos de Cantabria, Santander, Spain

Background: High dose post-transplantation Cyclophosphamide(PTCY) used in haploidentical transplantation (haplo-SCT) has demonstrated to be highly effective in acute and chronic GvHD prophylaxis; however it is associated with high relapse rates. Anti-T-Lymphocyte Globulin (ATG-Fresenius ${ }^{\circledR}$ ) is also effective as GVHD prophylaxis but its benefit in Overall Survival (OS) and Relapse Free Survival (RFS) is unclear. The aim of this study was to compare the effectiveness of two GVHD prophylaxis regimen employed in high risk transplantation: PTCY-haplo-SCT and low doses of ATG-F used in peripheral blood(PB) and mismatched transplantation. The primary endpoint was to evaluate the incidence and severity of aGVHD and cGVHD. As secondary endpoints we analysed the OS, RFS and GRFS (considering as events severe aGVHD, systemic therapy-requiring cGVHD, relapse or death). We also evaluated mortality related to transplantation(TRM) and post-transplant complications.

Methods: We retrospectively analysed 111 allo-SCT performed in our institution between 2012 and 2017. We analysed two cohorts: 49 haplo-SCT with PTCY $(50 \mathrm{mg} /$ $\mathrm{kg}$, days $+3,+4)$ followed by Tacrolimus and Mycophenolate (MMF); and $62 \mathrm{~PB}$ and/or mismatched transplants with low dose ATG-F (7 mg/Kg days -3,-2,-1) associated to calcineurin inhibitors starting on day -1 , with short course MTX (days $+1,+3,+6$ ) or MMF. In both cohorts, MMF was stopped on day +28 and calcineurin inhibitors were tappered on day +50 . Comparing both groups, we found differences in diagnosis (lymphoproliferative disorders 16 vs 4,p=0.003), high DRI-score (18 vs 11 , $\mathrm{p}=0.04)$, previous transplantation (12 vs $5, \mathrm{p}=0.02)$, reduced-intensity conditioning regimen (36 vs $20, \mathrm{p}<$ $0.001)$ and bone marrow as stem cells source (38 vs 9, $\mathrm{p}<0.001)$.

Results: Median time to neutrophil engraftment was similar in both groups: 17 vs 16 days. Conversely, median time to platelet recovery was longer in PTCY cohort (33vs18 days, $\mathrm{p}=0.016$ ).

There were no differences in aGVHD incidence (PTCY $30.6 \%$ vs ATG $36.4 \%$ ) or severe aGVHD (PTCY $4.1 \%$ vs ATG 9\%, $\mathrm{p}=0.852$ ). The global cGVHD incidence was PCTY $56.1 \%$ vs ATG 66\%. Mild, moderate and severe cGVHD incidence was $31.7 \%, 19.5 \%$ and $4.8 \%$, for PTCY vs $28 \%, 26 \%$ y $12 \%$ for $\operatorname{ATG}(\mathrm{p}=0.475)$. With a median follow-up of 27 months (28 months for PTCY and 22 months for ATG) the OS at 12 and 24 was: PCTY $67.8 \%$ and $61 \%$, and ATG $68.8 \%$ and $59.9 \%, \mathrm{p}=0.971)$. RFS (12 and 24 months) was: $61 \%$ and $57.9 \%$ for PTCY, and, $69 \%$ and $54 \%$ for $\operatorname{ATG}(\mathrm{p}=0.839)$.

GRFS at 12 and 24 months was $46.5 \%$ and $42.9 \%$, for PTCY patients and $40.7 \%$ and $33 \%$ for ATG patients $(\mathrm{p}=0.433)$. PTCY cohort seemed to develop more relevant non-infectious complications, but there were no differences among infectious complications. Image 1.

TRM was similar in both cohorts: PCTY $18.4 \%$ vs ATG $22.5 \%(\mathrm{p}=0.250)$. We neither found differences in early toxic mortality (<100 days): PCTY $16.3 \%$ vs ATG $14.5 \%$ for ATG $(\mathrm{p}=0,421)$.

Conclusions: Regardless the different transplant scenarios in which they were used, PTCY and low dose ATG seem to be equally effective in the prophylaxis of severe forms of acute and chronic GVHD, offering similar GRFS. Moreover,they show similar early toxicity and rate of infectious events.

Disclosure: We have nothing to disclose. 

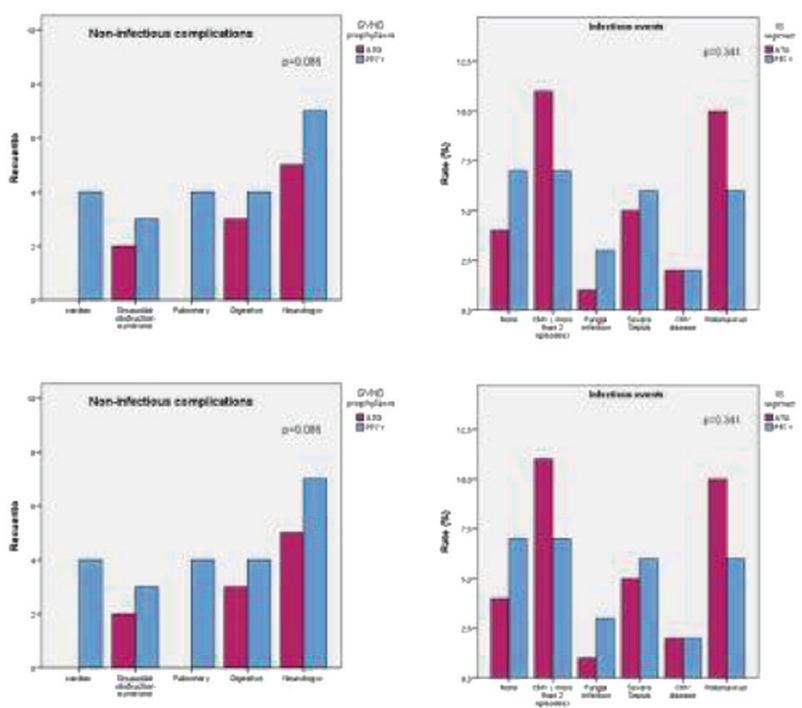

[[0119 Image] 1. Image1. Post transplantation complications.]

\section{Immunodeficiency diseases and macrophage}

\section{0}

Safety and efficacy of emapalumab in pediatric patients with primary hemophagocytic lymphohistiocytosis (PHLH)

Franco Locatelli, Michael B. Jordan', Carl E. Allen ${ }^{3}$, Simone Cesaro ${ }^{4}$, Julian Sevilla ${ }^{5}$, Anupama Rao ${ }^{6}$, Martina Ahlmann $^{7}$, Jose-Luis Dapena ${ }^{8}$, Barbara Degar ${ }^{9}$, Franca Fagioli $^{10}$, Timothy Garrington ${ }^{11}$, Michael Henry ${ }^{12}$, Maria Caterina Putti ${ }^{13}$, Carmelo Rizzari ${ }^{14}$, Fabrizio De Benedetti $^{15}$, Alexei A. Grom ${ }^{16}$, Walter Giovanni Ferlin ${ }^{17}$, Maria Ballabio $^{17}$, Cristina de Min $^{17}$

${ }^{1}$ Bambino Gesù Children's Hospital IRCCS, Rome, Italy, ${ }^{2}$ Cincinnati Children's Hospital Medical Center, Cincinnati, $\mathrm{OH}$, United States, ${ }^{3}$ Texas Children's Hospital, Texas Children's Cancer Center, Houston, TX, United States, ${ }^{4}$ University Hospital Verona, Verona, Italy, ${ }^{5}$ University Children Hospital Niño Jesús, Madrid, Spain, ${ }^{6}$ Great Ormond Street Hospital for Children, London, United Kingdom, ${ }^{7}$ University Children's Hospital Muenster, Muenster, Germany, ${ }^{8}$ University Hospital Vall d'Hebron, Barcelona, Spain, ${ }^{9}$ Dana-Farber Cancer Institute, Boston, MA, United States, ${ }^{10}$ Regina Margherita-S. Anna Hospital, Turin, Italy, ${ }^{11}$ Children's Hospital Colorado, Center for Cancer and Blood Disorders, Aurora, CO, United States, ${ }^{12}$ Phoenix Children's Hospital, Phoenix, AZ, United States,

${ }^{13}$ University of Padova, Padova, Italy, ${ }^{14}$ San Gerardo Hospital, University of Milano-Bicocca, Monza, Italy,
${ }^{15}$ Bambino Gesù Children's Hospital IRCCS, Rome, Italy, ${ }^{16}$ Cincinnati Children's Hospital Medical Center, Cincinnati, OH, United States, ${ }^{17}$ Novimmune SA, Geneva, Switzerland

Background: pHLH is a rare, genetic, hyper-inflammatory syndrome driven by high production of interferon (IFN)- $\gamma$. Emapalumab (NI-0501) is a monoclonal antibody that neutralizes IFN- $\gamma$ and is developed as a treatment for HLH.

Methods: This open-label phase 2/3 study (NCT01818492, data cut-off is July 20 2017) includes 34 patients $\leq 18$ years old with pHLH based on genetic confirmation, family history, or presence of $\geq 5 / 8 \mathrm{HLH}-$ 2004 diagnostic criteria. Patients were treatment-naïve (7) or had failed previous conventional HLH therapies (27). Emapalumab (1 mg/kg intravenously every 3-4 days, increased up to $10 \mathrm{mg} / \mathrm{kg}$ based on clinical and laboratory response parameters) was administered with dexamethasone $\left(5-10 \mathrm{mg} / \mathrm{m}^{2} /\right.$ day with tapering permitted). Treatment duration was 8 weeks with possible shortening to a minimum of 4 weeks or extension up to allogeneic hematopoietic stem cell transplantation (HSCT). The primary endpoint, Overall Response Rate (ORR), was objectively assessed based on normalization or $\geq 50 \%$ improvement in pre-defined criteria: fever, splenomegaly, cytopenias, hyperferritinemia, fibrinogen and/or D-Dimer levels, central nervous system (CNS) abnormalities, with no sustained worsening of sCD25 levels. An exact binomial test at one-sided 0.025 significance level was used to evaluate the null hypothesis that ORR be at most $40 \%$. Following completion of the study, patients entered an extension phase (NCT02069899).

Results: Patients (median age 1.0 yr, range 0.1-13 yr) entered the study with broad spectrum of pHLH clinical abnormalities, $>30 \%$ with CNS involvement. Mutations in pHLH-associated genes were present in $79 \%$ of patients. ORR was significantly higher than the pre-specified null hypothesis, thus meeting the primary efficacy endpoint (Table). Response rates based on Investigator's clinical judgement were $70.6 \%$ and $70.4 \%$ in the two groups.

Emapalumab was safe and well tolerated. Mild to moderate infusion-related reactions occurred in $27 \%$ of patients. Infection caused by pathogens potentially favored by IFN- $\gamma$ neutralization occurred in 1 patient (disseminated histoplasmosis, resolved with treatment). No off-target effects were observed. Most patients proceeded to HSCT $(50 \%$ of patients received myeloablative conditioning and $50 \%$ reduced intensity conditioning) with favorable outcome (engraftment in $86 \%$ and $89 \%$ of patients, respectively) and $90 \%$ of patients receiving HSCT were alive at 1 year post transplant. 
Conclusions: Emapalumab treatment promoted disease control, blunting the exacerbated immune response typical of the disease, with a favorable safety and tolerability profile. The results of the study also suggest that emapalumab may contribute to optimize post-transplant outcome of patients given HSCT. Based on these data, emapalumab received marketing authorization in the US from the Food and Drug Administration for the treatment of patients with primary HLH with refractory, recurrent or progressive disease or intolerance with conventional therapy.

\begin{tabular}{lll}
\hline & All patients (N=34) & $\begin{array}{l}\text { Failed conventional } \\
\text { therapy N=27 }\end{array}$ \\
\hline ORR, n/N (\%) & $22 / 34(64.7)^{*}$ & $17 / 27(63.0)^{* *}$ \\
Time to response, days (median [95\% CI] $)$ & $8.0[5.0-10.0]$ & $8.0[7.0-14.0]$ \\
$\begin{array}{l}\text { Cumulative duration of response, \% days } \\
\text { of treatment (median [Q1, Q3] }\end{array}$ & $75.7[33.3-91.2]$ & $75.4[30.8-91.2]$ \\
Patients reducing dexamethasone dose by & $16 / 34(47.1)$ & $12 / 27(44.4)$ \\
at least 50\%, n/N (\%) & $22 / 34(64.7)$ & $19 / 27(70.4)$ \\
Proceeded to HSCT, n/N (\%) & 52 & 55 \\
MUD, \% of patients w/HSCT & 29 & 28 \\
MMUD, \% of patients w/ HSCT & 14 & 11 \\
Haploidentical, \% of patients w/ HSCT & 6 \\
Matched sibling, \% of patients w/ HSCT & 5 & \\
Overall survival & $24 / 34(70.6)$ & $20 / 27(74.1)$ \\
Alive at last observation, n/N (\%) & $69[50-82]$ & $73[52-86]$ \\
12-month pOS estimate (\%[95\% CI]) & $27 / 34(79.4)$ & $22 / 27(81.5)$ \\
Survived to HSCT, n/N (\%)*** & $20 / 22(90.9)$ & $17 / 19(89.5)$ \\
Survived post HSCT, n/N (\%) & &
\end{tabular}

$* 95 \%$ CI: 46-80\%, $\mathrm{p}=0.0031 ; * * 95 \%$ CI: $42-81 \%$, $\mathrm{p}=0.0134$

***patients not receiving HSCT censored at day of last seen or day 180; CI, confidence interval; MUD, matched unrelated donor; MMUD, mismatched unrelated donor; $\mathrm{Q}$, quatile; pOS, probability of interval survival

[[O120 Table] 1. Table]

Disclosure: Franco Locatelli - I have relevant relationships to disclose: Novartis: Honoraria and Membership on an entity's Board of Directors or advisory committees, Amgen: Honoraria and Membership on an entity's Board of Directors or advisory committees, Miltenyi: Honoraria, MEDAC: Honoraria, Jazz Pharmaceuticals: Membership on an entity's board of directors. Michael Jordan - I have relevant financial relationship(s) to disclose: Novimmune: Consultancy and Membership on an entity's Board of Directors or advisory committees. Carl E. Allen - I have no relevant financial relationships to disclose

Simone Cesaro - I have no relevant financial relationships to disclose. I am employed at the Azienda Ospedaliera Universitaria Integrata Verona. Julian Sevilla - I have no relevant financial relationships to disclose. Anupama Rao - I have no relevant financial relationships to disclose. Martina Ahlmann - I have no relevant financial relationships to disclose. Jose-Luis Dapena - I have relevant financial relationships to disclose:Consultancy for: Amgen, Novartis,
Jazz Phamaceuticals, Shire, and Servier. Barbara Degar - I have no relevant financial relationships to disclose. Franca Fagioli - I have no relevant financial relationships to disclose. I am employed at the Regina Margherita-S. Anna Hospital, Turin, Italy. Timothy Garrington - I have no relevant financial relationships to disclose. Michael Henry I have no relevant financial relationships to disclose. I am employed at the Phoenix Children's Hospital. Maria Caterina Putti - I have no relevant financial relationships to disclose. Carmelo Rizzari - I have no relevant financial relationships to disclose. Fabrizio De Benedetti - I have relevant financial relationships to disclose: Novartis: Consultancy and Research Funding, SOBI: Consultancy and Research Funding, Roche: Consultancy and Research Funding, Sanofi: Consultancy and Research Funding, UCB: Consultancy, Eli-Lylli: Consultancy, Abbvie: Research Funding, Novimmune: Research Funding, Pfizer: Research Funding. Alexei A. Grom - I have relevant financial relationships to disclose:

Novartis: Consultancy, AB2Bio: Consultancy, Novimmune: Consultancy. Walter Giovanni Ferlin - I have relevant financial relationship to disclose: Novimmune: Employment, Equity Ownership and Patents \& Royalties. Maria Ballabio - I have relevant financial relationship to disclose: Novimmune: Employment and Equity Ownership. Cristina de Min - I have relevant financial relationship to disclose:

Novimmune: Employment and Equity Ownership

\section{1}

Haploidentical HSCT with post-transplant cyclophosphamide for primary immunodeficiencies and inherited disorders in children

Benedicte Neven ${ }^{1}$, Jean-Sebastien Diana ${ }^{1}$, Martin Castelle $^{1}$, Alessandra Magnani ${ }^{1}$, Jeremie Rosain ${ }^{1}$, Fabien Touzot $^{1}$, Baptiste Moreira ${ }^{1}$, Marie-Louise Fremond ${ }^{1}$, Coralie Briand ${ }^{I}$, Matthieu Bendavid, Romain Levy ${ }^{I}$, Guillaume Morelle ${ }^{1}$, Elsa Magrin ${ }^{1}$, Philippe Bourget ${ }^{\text {, }}$ Lucienne Chatenoud ${ }^{1}$, Capucine Picard ${ }^{1}$, Alain Fischer ${ }^{1}$, Despina Moshous ${ }^{1}$, Stephane Blanche ${ }^{1}$

\section{${ }^{1}$ Necker Children Hospital, Paris, France}

Background: Allogeneic hematopoietic stem cell transplantation (HSCT) is a potentially curative treatment for some inherited disorders, including selected primary immunodeficiencies (PIDs). In the absence of a wellmatched donor, HSCT from a haploidentical family donor (HIFD) may be considered. Various approaches are being developed to mitigate the risks of graft failure and graftversus-host disease (GVHD) and to speed-up immune reconstitution. Among those, high-dose, post-transplant 
cyclophosphamide (PTCY) is increasingly used in adult recipients. However, data on PTCY in children and those with inherited disorders in particular are scarce.

Methods: We reviewed the outcome of 27 children transplanted with HIFD and PTCY for PID $(n=22)$ or osteopetrosis $(n=5)$ in a single center. Median (range) age was 1.5 years (0.2 to 17)). Patients in our series had major risk factors for poor post-HSCT outcome such as active viral infections at the time of transplantation $(n=9)$, EBVrelated lymphoproliferation in partial remission $(n=3)$, previous kidney transplant $(\mathrm{n}=1)$. HSCT with PTCY was a primary $(n=21)$ or a rescue procedure after graft failure $(n=6)$. Conditioning regimen was myeloablative in most primary HSCTs and non-myeloablative in all rescue procedures.

Results: After a median follow-up of 17.3 months, 24 of the 27 patients engrafted. Twenty-one patients are alive and have been cured of the underlying disease. The two-year overall survival rate was $77.7 \%$. The cumulative incidences of acute GVHD grade $\geq$ II, chronic GVHD and autoimmune disease were $49 \%, 26.6 \%$, and $26.5 \%$, respectively. There were only two cases of grade III acute GVHD, all cases of cGVHD were limited and allowed to stop systemic immune-supression, autoimmunity consisted in 2 autoimmune hemolytic anemia in remission at last follow up, 1 vitiligo and 2 thyroiditis. The cumulative incidence of blood viral replication and life-threatening viral events were 58\% and $15.5 \%$, respectively. There was evidence of early $\mathrm{T}$ cell immune reconstitution including early anti-viral responses.

Conclusions: In the absence of an HLA-identical donor, HIFD HSCT with PTCY is a viable option for patients with life-threatening inherited disorders.

Disclosure: nothing to declare

\section{2}

Hematopoietic stem cell transplantation in children with GRISCELLI syndrome type 2: A single-center report on 35 patients

Ali Al-Ahmari, ${ }^{1,2}$,Maha Al-Mofareh ${ }^{1}$, Amal Al-Seraihy ${ }^{1}$, Abdullah Al-Jefri ${ }^{1}$, Suleimman Alsweedan', Ibrahim Ghemlas $^{I}$, Hawazen Alsaedi, Awatif Alanazi, Hassan Elsolh $^{1}$, Khawar Saddiqui ${ }^{I}$, Bander Al Saud ${ }^{1}$, Hasan Al Dhekri', Hamoud Al Musa', Saleh Al Muhsen ${ }^{1}$, Rand Arnaout ${ }^{1}$, Mouhab Ayas ${ }^{1}$

${ }^{1}$ King Faisal Specialist Hospital \& Research Centre, Riyadh, Saudi Arabia, ${ }^{2}$ Alfaisal University, College of Medicine, Riyadh, Saudi Arabia

Background: Griscelli Syndrome (GS) is a very rare autosomal recessive disease, characterized by skin hypopigmentation and silvery-gray hair. GS type 2 (GS2) patients suffer immunodeficiency and potentially fatal episodes of macrophage activation known as accelerated phases during early childhood. The only curative treatment modality for these patients is allogeneic Hematopoietic Stem Cell Transplantation (HSCT). We report the outcome of HSCT in 35 children with GS2. To date, this is the largest cohort of GS patients who have undergone transplantation at a single center.

Methods: We retrospectively reviewed 35 consecutive patients with GS2 who underwent HSCT at our institution between January 1993-December 2017. Median age at diagnosis and at transplant was $0.3(0-12)$ and $0.9(0.3-15.1)$ years respectively. Prior to HSCT, $28(80 \%)$ and $16(46 \%)$ patients had life-threatening accelerated phases (HLH) and CNS involvement respectively. All such patients were treated with chemotherapy and achieved remission at the time of HSCT. The source of grafts was matched related marrows in $19(54.3 \%)$ pts and partially mismatched unrelated cord in $14(40 \%)$ pts. Two patients received haploidentical and matched unrelated marrows respectively. Conditioning regimens were myloablative doses of busulfan/cyclophosphamide and busulfan/fludaribine in $27(77 \%)$ and $8(23 \%)$ patients respectively. All patients received GVHD prophylaxis. Growth factors were administered in $33(94.3 \%)$ and median CD34 dose used was 5.46 (2.1411.94) $10^{\wedge} 6$ and $5.45(1.8-13.5) 10^{\wedge} 5$ per $\mathrm{Kg}$ of body weight for marrow and cord respectively.

Results: Post infusion rate of engraftment was $88.6 \%$ (31 cases) with median time to neutrophil and platelets recovery were 15 (8-30) and 29 (14-61) days respectively. Cumulative incidence of acute GVHD was $28.6 \%$ (10), with an overall Grade of I, II and IV as 20\%, 60\% and $20 \%$ respectively. The post-transplant course was complicated by CMV infection, EBV viremia and veno-occlussive disease in 14(40\%), 7(20\%) and 7(20\%) patients respectively. Chimerism studies at the last contact were available for 24 patients. Full donor cell chimerism (100\%) was seen in 16 (67\%) of the transplanted patients. Post-transplant two patients experienced disease reactivation at a median time 45.5 (28-63) days. With a mortality rate of $37.1 \%$ (13) and a median follow-up time of 87.7 months, five-year cumulative probability of overall survival (OS) for our cohort of patients was $0.625 \pm 0.083$. Transplant related mortality counted as death within day 100 was $28.6 \%$ (10). Prominent causes of death were septic shock followed by ARDS. Cumulative probability of five years overall survival was significantly better in those who did not have HLH prior to SCT (1.0 \pm 0.0 vs. $0.529 \pm 0.096$, P-value: 0.037$)$. Of the 16 patients with neurological involvement before HSCT, 8 survived with residual sequelae in 3 patients. OS at five years was $0.500 \pm 0.125$ and $0.729 \pm 0.104(\mathrm{P}-$ Value: 0.328 ) in pts with and without CNS involvement at presentation respectively 
Conclusions: HSCT in patients with GS is potentially curative with long-term, disease-free survival. Early HSCT before the development of the accelerated phase showed better result.

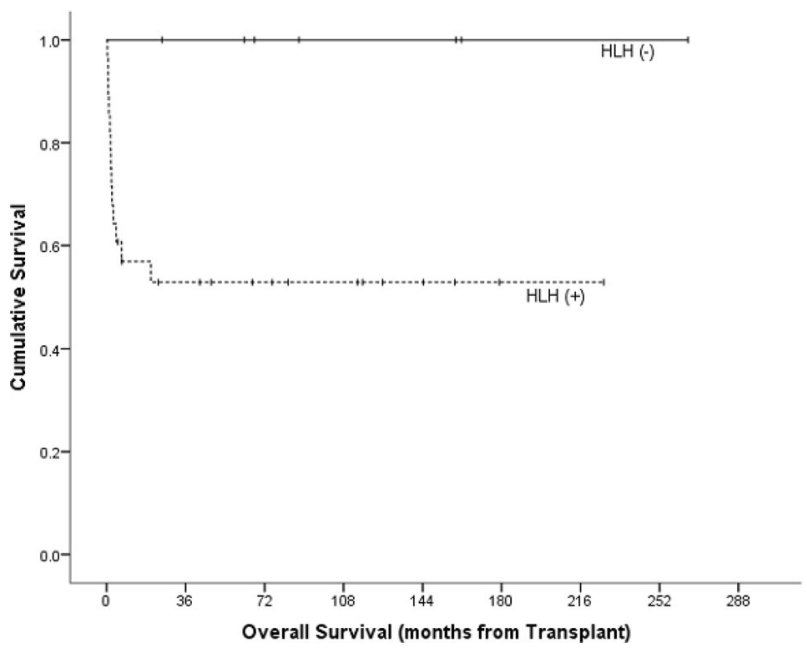

[[O122 Image] 1. Overall survival in pts with and without HLH at the diagnosis]

Disclosure: Nothing to declare

\section{3}

Hematopoietic stem cell transplantation for adult with primary immunodeficiency: A successful report from A single center experience

Ambroise Marçais ${ }^{1}$, Morgane Cheminant ${ }^{1}$, Nizar Mahlaoui $^{2}$, Fanny Lanternier ${ }^{I}$, Claire Rouzaud ${ }^{\prime}$, Romain Guery $^{I}$, Jeremie Rosain ${ }^{1}$, Émilie Catherinot ${ }^{3}$, Peter Van Endert $^{1}$, Louis-Jean Couderc ${ }^{3}$, Olivier Lortholary ${ }^{3}$, Capucine Picard ${ }^{1}$, Olivier Hermine ${ }^{1}$, Alain Fischer ${ }^{2}$, Felipe Suarez ${ }^{I}$

${ }^{1}$ Hopital Necker Enfants-Malades, Paris, France, ${ }^{2}$ Hopital Necker Enfants-Malades, Centre de Référence Déficits Immunitaires Héréditaires, Paris, France, ${ }^{3}$ Hopital Foch, Service de Pneumologie, Suresnes, France

Background: Primary immunodeficiencies (PIDs) are heterogeneous inborn disorders resulting in impaired cellular and/or humoral responses. PID present a wide range of clinical manifestations, ranging from infections to autoimmune, inflammatory and/or malignant complications. Allogeneic stem cell transplantation (alloSCT) is curative for pediatric PID with an excellent safety. In adults on the other hand, this therapeutic approach remains controversial.

Methods: This is a retrospective, monocentric study of 26 consecutive adult patients with PID who underwent an
alloSCT between 2011 and 2018. The objective was to assess the feasibility, effectiveness and safety of this procedure.

Results: Twenty-two (85\%) of 26 patients presented an inherited $\mathrm{T}$ or B cell deficiency and $4(15 \%)$ a phagocyte impairment. Twenty (77\%) patients had a genetic diagnosis. Besides infectious complications, $8(31 \%)$ patients had an history of lymphoma and $13(50 \%)$ an history of autoimmune/ inflammatory complication.

The median age at transplant was 24 years (range 17-37). Twelve (46\%) patients received a myeloablative conditioning (MAC), $12(46 \%)$ a reduced intensity conditioning (RIC) and 2 (8\%) had no conditioning (second transplant for severe combined deficiency). MAC included fludarabine (Flu)/Buslfan (Bu, dosage: $12.8 \mathrm{mg} / \mathrm{kg}$ ) based regimen $(\mathrm{n}=11)$ and $\mathrm{Bu}(12.8 \mathrm{mg} / \mathrm{kg}) /$ Cyclophosphamide $(\mathrm{Cy})$ based regimen $(\mathrm{n}=1)$. RIC included based $\mathrm{Flu} / \mathrm{Bu}$ (range dosage: $6.4-9.6 \mathrm{mg} / \mathrm{kg})$ regimen $(\mathrm{n}=9)$, Flu/Cy based regimen $(\mathrm{n}=1)$, Thiotepa/Flu/Bu regimen $(\mathrm{n}=1)$ and low dose TBI based regimen $(n=1)$. Among the 24 patients who received a conditioning, $4(17 \%)$ received Alemtuzumab and $11(46 \%)$ received antithymocyte globulin as part of the conditioning.

The stem cell source was bone marrow for $8(31 \%)$ and peripheral blood stem cells for $18(69 \%)$. The donor was matched related for $10(38 \%)$, matched unrelated for 12 $(46 \%)$, mismatch unrelated for $2(8 \%)$, and haplo-identical for $2(8 \%)$. All assessable patients had a successful engraftment. Eight $(31 \%)$ presented a grade II-IV acute GVHD (one grade III, 0 grade IV), and 5 (23\%) a chronic GVHD ( 2 limited and 3 extensive).

With a median follow-up of 29 months (3-90 months) post-transplant, the 3-years overall survival (OS) was $80.8 \%$. The transplant related mortality was $19.2 \%$ with 5 deaths. Among them, both patients transplanted with an haplo-identical donor died. All deaths occurred in the firstyear post-transplant. Thus, the use of an haploidentical donor was associated with an adverse outcome. Conversely, neither the conditioning nor the stem cell source was associated with a worse outcome. Except one patient with an history of aggressive B cell lymphoma who relapsed few months after alloSCT, no patient with an history of lymphoma relapsed. After a salvage treatment, the relapsing patient remained in complete remission 5 years later. At last follow-up, all surviving patients had a stable, mixed donorrecipient or full donor chimerism without any sign of active infection.

Conclusions: AlloSCT in the PID setting is an effective therapeutic with an acceptable toxicity and should be considered in case of severe infectious, inflammatory and/or malignant complications in young adult patients with PID when an appropriate donor is available.

Disclosure: No relevant conflicts of interest to declare 


\section{4}

The comparison of different approaches to immunosuppression after hematopoietic stem cell transplantation with TCR $\alpha \beta / C D 19$ graft depletion in a big cohort of patients with primary immunodeficiencies

Alexandra Laberko ${ }^{1}$, Elena Gutovskaya ${ }^{2}$, Irina Shipitsina $^{2}$, Svetlana Radigina ${ }^{2}$, Svetlana Kozlovskaya ${ }^{2}$, Elvira Sultanova $^{2}$, Larisa Shelikhova ${ }^{2}$, Michael Maschan ${ }^{2}$, Anna Shcherbina $^{1}$, Alexei Maschan ${ }^{2}$, Dmitry Balashov ${ }^{2}$

${ }^{1}$ Dmitry Rogachev National Medical Research Center of Pediatric Hematology, Immunology, Moscow, Russian Federation, ${ }^{2}$ Dmitry Rogachev National Medical Research Center of Pediatric Hematology, Moscow, Russian Federation

Background: According to several studies hematopoietic stem cell transplantation (HSCT) with TCR $\alpha \beta / \mathrm{CD} 19$ graft depletion demonstrates low incidence of graft versus host disease (GVHD) in patients with primary immunodeficiencies (PID).

Methods: We analyzed the results of allogenic HSCT with TCR $\alpha \beta / C D 19$ graft depletion in 148 patients with various PID (excluding classic SCID) who received HSCT from May 2012 to September 2018 in our center. The median age at HSCT was 3,5 years (range 0,43-17,63). 112 patients received HSCT from matched unrelated, 31 haploidentical donors, 5 - siblings. The conditioning regimens included: Fludarabin (Flu) $150 \mathrm{mg} / \mathrm{m} 2$ with 1 alkylator (Treosulfan (Treo) $36-42 \mathrm{~g} / \mathrm{m} 2$ ) in 33 patients, with 2 alkylators (Treo $36-42 \mathrm{~g} / \mathrm{m} 2$ with Melphalan $140 \mathrm{mg} / \mathrm{m} 2$ or Thyotepa $10 \mathrm{mg} / \mathrm{kg}$ ) in 74 . Twenty-five patients received 2 alkylators with addition of G-CSF $30 \mathrm{mg} / \mathrm{kg}$ and Plerixafor $24 \mathrm{mg} / \mathrm{kg}$. Seventeen patients with Nijmegen breakage syndrome (NBS) received reduced intensity conditioning with Busulfan $4 \mathrm{mg} / \mathrm{kg}$ or Treo $30 \mathrm{mg} / \mathrm{m} 2$, Cyclophosphamide $40 \mathrm{mg} / \mathrm{kg}$ and Flu $150 \mathrm{mg} / \mathrm{m} 2$. In all but 2 patients serotherapy was used: 10 patients - horse ATG $-90 \mathrm{mg} / \mathrm{kg}$, 133 - rabbit ATG (Thymoglobulin) $5 \mathrm{mg} / \mathrm{kg}, 3-1 \mathrm{mg} / \mathrm{kg}$ anti CD52 monoclonal antibodies. In 55 patients two or more immunosuppressive drugs were used after transplantation (combination of calcineurin inhibitor (CNI) with short course of methotrexate, or mycophenolate mofetil, or abatacept), 66 patients received one immunosupressive agent (CNI). From November 2017 no posttransplant immunosuppression was used (27 patients).

Primary end points were: incidence of acute GVHD, graft failure (GF) and transplant related mortality (TRM). Because of the supposed influence of conditioning regimen on GF and TRM incidence, to the analysis of GF and TRM were included only 74 patients with similar conditioning regimen with 2 alkylators (excluding patients with NBS, with 1 alkylator and addition of G-CSF and plerixafor in conditioning regimen). Median follow up after HSCT was 1,95 years (range 0,04-6,3 years).

Results: Overall survival (OS) in 148 patients was 0,85 (95\% CI 0,79-0,91). Cumulative incidence (CI) of acute GVHD in patients with two and more immunosupressants was 0,164 (95\% CI 0,09 - 0,3), with one - 0,17 (95\% CI 0,1 - 0,29), with no immunosupression - 0,11 (95\% CI 0,04 $0,32), p=0,843$. CI of graft failure was: in patients with two and more immunosuppressive agents $(\mathrm{n}=25)-0,24(95 \%$ CI 0,12 - 0,48), with one $(\mathrm{n}=32)-0,17$ (95\% CI 0,1 - 0,29), without immunosuppression $(n=17)-0,06(95 \%$ CI 0,01 $0,44), p=0,172$. CI of TRM in patients with two and more immunosuppressants was $0,22(95 \%$ CI $0,1-0,47)$, with one - $0,1(95 \%$ CI $0,02-0,28)$, without immunosuppression- 0,26 (95\% CI $0,09-0,73), \mathrm{p}=0,42$.

Conclusions: We conclude that in our group of PID patients presence or absence of immunosuppression after HSCT with TCR $\alpha \beta / C D 19$ graft depletion and its extent made no significant difference on the incidence of acute GVHD, graft failure and TRM. Considering these results HSCT with TCR $\alpha \beta / C D 19$ graft depletion without posttransplant immunosupression could be recommended for patients with PID.

Disclosure: No conflict of interest to disclose

\section{5}

Treatment forms and clinical course of LPS-Responsive beige-like anchor protein deficiency and introduction of the immune deficiency and -dysregulation activity (=IDDA) score

Victoria Katharina Tesch ${ }^{1}$, Hassan Abolhassani ${ }^{2}$, Bodo Grimbacher $^{3}$, Arjan Lankaster ${ }^{4}$, Andrew Gennery, Bella Shadur $^{6}$, Polina Stepensky ${ }^{6}$, Yuliya Mareika ${ }^{7}$, Svetlana Sharapova $^{7}$, Elif Karakoc-Aydiner ${ }^{8}$, Jaques G. Rivière', Marina Garcia-Prat ${ }^{9}$, Nicolette Moes ${ }^{10}$, Filomeen Haerynck $^{10}$, Luis I. Gonzales- Granado ${ }^{11}$, Juan Luis Santos Pérez $^{12}$, Anna Mukhina ${ }^{13}$, Asghar Aghamohammadi ${ }^{14}$, Lennart Hammarström ${ }^{15}$, Figen Dogu ${ }^{16}$, Aydan I İkincio$\breve{g u l l a r t}^{16}$, Sevgi Bal ${ }^{16}$, Safa Baris ${ }^{17}$, Sara Sebnem Kilic ${ }^{18}$, Neslihan Edeer Karaca ${ }^{19}$, Necil Kutukculer ${ }^{19}$, Hermann Girschick $^{20}$, Antonios Kolios ${ }^{21}$, Sevgi Keles ${ }^{22}$, Markus G Seidel $^{23}$

${ }^{1}$ Medical University of Graz, Graz, Austria, ${ }^{2}$ Karolinska Institutet, Stockholm, Sweden, ${ }^{3}$ Medical Center - University of Freiburg, University of Freiburg, Center for Chronic Immunodeficiency at Center for Translational Cell Research, Freiburg, Germany, ${ }^{4}$ Leiden University Hospital, Leiden, Netherlands, ${ }^{5}$ Newcastle University, Newcastle, United Kingdom, ${ }^{6}$ Hadassah Hebrew University Medical Center, Jerusalem, Israel, ${ }^{7}$ Belarusian State Medical 
University, Minsk, Belarus, ${ }^{8}$ Marmara University, Isstanbul, Turkey, ${ }^{9}$ Universitat Autonoma de Barcelona, Barcelona, Spain, ${ }^{10}$ Ghent University Hospital, Ghent, Belgium, ${ }^{11}$ Hospital 12 de Octubre, Madrid, Spain, ${ }^{12}$ Hospital Virgen de las Nieves, Granada, Spain, ${ }^{13}$ Dmitry Rogachev Federal Research Centre of Pediatric Hematology, Oncology and Immunology, Moscow, Russian Federation, ${ }^{14}$ Tehran University of Medical Sciences, Tehran, Iran, Islamic Republic of, ${ }^{15}$ Karolinska Institutet, Stockholm, Sweden, ${ }^{16}$ Ankara University, Ankara, Turkey, ${ }^{17}$ Marmara University, Istanbul, Turkey, ${ }^{18}$ Uludag University, Bursa, Turkey, ${ }^{19}$ Ege University Hematology Department, Izmir, Turkey, ${ }^{20}$ University of Würzburg Medical Center, Würzburg, Germany, ${ }^{21}$ Universitätsspital Zürich, Zürich, Switzerland, ${ }^{22}$ Necmettin Erbakan Üniversitesi, Konya, Turkey, ${ }^{23}$ Medical University of Graz, Graz, Austria

Background: LPS-responsive beige-like anchor protein (LRBA) deficiency is a severe primary immunodeficiency with a variable clinical phenotype, including features overlapping with common variable immunodeficiency, autoimmune lymphoproliferative syndrome, and immune dysregulation polyendocrinopathy enteropathy X-linked syndrome and an association with lymphoma. Recent findings strongly support HSCT in patients with severe presentation of LRBA deficiency. However, there are no up-to-date follow-up and survival data of patients not undergoing transplantation beyond previous publications of large cohort studies. This study was conducted to increase the knowledge on transplant experience and to elucidate the clinical course of both transplanted and untransplanted patients with LRBA deficiency under various targeted treatment modalities.

Methods: We performed an international Inborn errors working party- and European Society for Immunodeficiencies-wide survey in 2018 to collect information about the HSCT experience and the clinical course of both transplanted and untransplanted patients with LRBA deficiency. We included an assessment of the disease activity and treatment responses with a specially developed immune deficiency and dysregulation activity (IDDA) score, which weighs the sum of organ involvement (scored by $0-4$ ) by days of hospitalization and performance scores, taking into account the type of therapy and response per treatment phase, which we herewith introduce.

Data were obtained in accordance with the Declaration of Helsinki and an institutional review board review by means of retrospective chart review by local physicians.

Results: We analyzed the data of 68 LRBA deficient patients from 23 centers, including 35 unpublished patients. 20 patients of our cohort underwent HSCT between 2005 and 2018. Overall survival of patients undergoing transplantation (median FU 30 months) was 70\% (14/20 patients); all deaths were due to transplant-related mortality and occurred within 3 months of HSCT. 83.3\% (40/48) of untransplanted patients (2-68 years) are currently alive. Out of the surviving transplanted patients $(n=14) 6$ are in complete remission, 4 in good partial remission (no treatment) and 4 in partial remission (receiving therapy), implying that 10 out of 14 transplanted patients $(71.4 \%)$ are currently without treatment. Only 5 out of 40 living untransplanted patients $(12.5 \%)$ do currently not receive therapy. Most commonly used drugs in LRBA deficiency were Steroids (27.7\%), Sirolimus (18.8\%) and Abatacept $(18.8 \%)$. Disease activity, measured by IDDA score, was significantly lower in patients treated with Abatacept $(\mathrm{p}<$ 0.0001). No immunosuppression-associated malignancy was detected in our cohort. Analyzation of the IDDA score of all living patients revealed significantly lower disease activity in patients transplanted and alive, than in those who did not undergo HSCT $(\mathrm{p}=0.01)$.

Conclusions: In conclusion our findings do, concordant with previous results, support HSCT, especially in patients with a severe phenotype of LRBA deficiency. However, the question of HSCT indication and optimal time point cannot yet be generally resolved. In terms of conventional treatment, Abatacept is clearly favorable in untransplanted patients. Nevertheless, it is not available in many countries and the long-term dependency on this treatment with its associated potential risks remains. Finally, our newly introduced IDDA score for assessment of disease activity may also prove useful for other syndromes with immune dysregulation.

Disclosure: Nothing to declare

\section{Infectious complications}

\section{6}

Multi-antigen vaccine (TRIPLEX) based on attenuated poxvirus prevents cytomegalovirus viremia in a multicenter placebo-controlled, double blinded, randomized phase 2 clinical trial in allogeneic HCT recipients

Ibrahim Aldoss' ${ }^{\text {, Lindsey Baden }}$, Ella Ariza-Heredia ${ }^{3}$, Corinna La Rosa ${ }^{4}$, Jeffrey Longmate ${ }^{5}$, Cindy Slape ${ }^{1}$, Lupe Duarte ${ }^{1}$, Andy Dagis ${ }^{4}$, Len Farol ${ }^{5}$, Auayporn Nademanee ${ }^{1}$, Monzr Al Malki, Nicolas C. Issa ${ }^{2}$, Alisha Pandit ${ }^{2}$, Ester Haddad Arbona ${ }^{2}$, John Zaia ${ }^{4}$, Stephen Forman', Ryotaro Nakamura ${ }^{1}$, Don Diamond ${ }^{4}$

${ }^{1}$ City of Hope National Medical Center, Duarte, CA, United States, ${ }^{2}$ Brigham and Women's Hospital/Dana Farber Cancer Institute, Boston, MA, United States, ${ }^{3}$ University of Texas MD Anderson Cancer Center, Houston, TX, United States, ${ }^{4}$ City of Hope, Beckman Research Institute, 
Duarte, CA, United States, ${ }^{5}$ Southern California Kaiser Permanente/City of Hope, Duarte, CA, United States

Background: Cytomegalovirus (CMV), is a major complication of allogeneic hematopoietic stem cell transplant (HCT) recipients. To overcome morbidity associated with CMV infection, antivirals are given, and while they successfully suppress viremia, they do so in the absence of immune reconstitution. A different approach is to use a vaccine to bolster immunity to CMV early post-HSCT and sustain it at least until the patient is immune reconstituted. We selected a highly attenuated, non-proliferating viral vector referred to as modified vaccinia Ankara (MVA) to insert 3 immunodominant CMV antigens pp65, IE1-exon4, and IE2-exon5. This novel vaccine (Triplex) demonstrated excellent tolerability and immunogenicity in a clinical study of 24 healthy adults (La Rosa, et al. Blood 2017). These findings prompted us to conduct a test of vaccine protective efficacy in at-risk HCT recipients in a multi-center, randomized and placebo-controlled Phase 2 clinical trial (NCT02506933).

Methods: Eligible patients were CMV-seropositive and undergoing HCT for hematologic malignancies from matched related/unrelated donors without the use of exvivo or in-vivo $\mathrm{T}$ cell depletion. The randomization (1:1) was stratified by donor serostatus and HCT center. Patients were enrolled pre-HCT, and on $\mathrm{d} 28$ were requalified for eligibility based on lack of prior CMV reactivation, $\geq$ Grade 3 GVHD, high-dose steroids, or other significant transplantrelated morbidity. After randomization patients received Triplex or placebo injections on $\mathrm{d} 28$ and $\mathrm{d} 56$ post-HCT and followed for one year. The primary endpoint is defined as any CMV reactivation $[\geq 500 \mathrm{CMV}$ genome copies $(\mathrm{gc}) /$ $\mathrm{mL}$, low-level reactivation prompting antiviral therapy, or CMV disease prior to d100 post-HCT. Based on the objective of reducing reactivation from $30 \%$ to $10 \%$ or from $40 \%$ to $15 \%$ on the vaccine arm, a sample size of 102 was determined to provide at least $90 \%$ power at a one-sided 0.10 level of significance by log-rank test.

Results: The patient clinical/HCT characteristics were balanced between the vaccine arm $(n=51)$ and placebo arm $(\mathrm{n}=51)$. The vaccine was well tolerated with no significant difference in grade 3-4 AEs or SAEs in both arms. Triplex displayed potent immunogenicity, as many vaccine recipients with either CMV-positive or -negative donors showed strong reconstitution of both CD4 and CD8 CMV-specific immunity that initiated soon after the first injection and was elevated for at least 100d post-HCT. Most notably there was a reduction of primary endpoint-defining reactivation through $\mathrm{d} 100$ in the vaccine arm (5 events, 9.8\%) compared to the placebo arm (10 events, 19.6\%, $\mathrm{p}=0.08$ ). Greater detail regarding protocol-specified secondary endpoints including time to viremia, its duration, frequency and duration of antiviral drug treatment, late CMV viremia, time to engraftment and incidence of acute and chronic GVHD, relapse and non-relapse mortality will be presented.

Conclusions: In summary, Triplex was well tolerated in seropositive HCT recipients, and was significantly better than placebo in preventing viremia cases accompanied by improved CMV-specific T cell reconstitution.

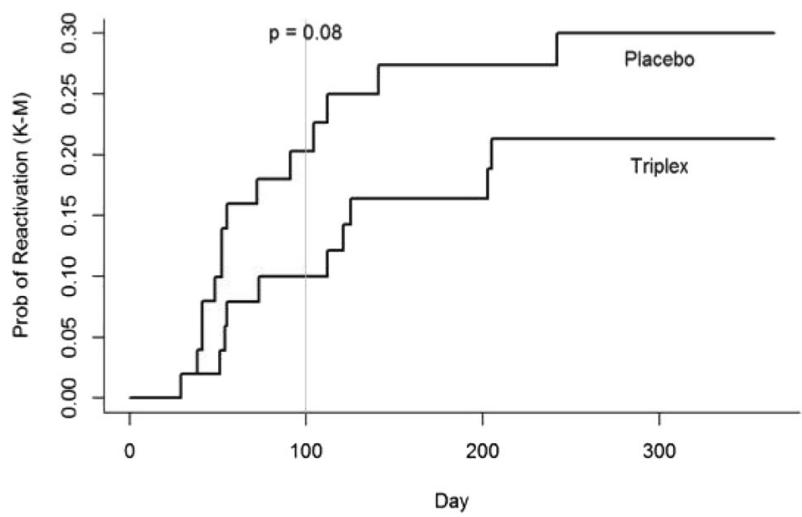

[[O126 Image] 1. Figure]

Clinical Trial Registry: NCT02506933

Disclosure: Ryotaro Nakamura, MD: Merck Advisory Board meeting attendence, Jazz Pharmaceuticals, collaborating investigator, Celgene; support for and invited lecture. Ella J Ariza-Heredia, MD: Imunotec Research: Research Funding. Stephen J. Forman, MD: Mustang Biotech, Consultant, Intellectual Property Rights and Royalty. Don J. Diamond, PhD: Helocyte, Intellectual property right, Advisory Board, Research Funding and Royalty, Pfizer, pharmaceutical manufacturer provided adjuvant PF03512676 at no cost

\section{7}

Long-term antipneumococcal antibodies after different schedules of vaccination in allogeneic hematopoietic cell transplant recipients

Christine Robin ${ }^{1}$, Mathilde Bahuaud ${ }^{2}$, Rabah Redjoul, Mohamed Jeljeli $^{4}$, Mathieu Leclerc ${ }^{1}$, Ludovic Cabanne ${ }^{3}$, Florence Beckerich, Sebastien Maury, Catherine Cordonnier ${ }^{1}$

${ }^{1}$ APHP Henri Mondor Hospital and UPEC, Creteil, France, ${ }^{2}$ APHP Hopital Cochin and University Paris Descartes, Paris, France, ${ }^{3}$ APHP Henri Mondor Hospital, Hematology, Creteil, France, ${ }^{4}$ APHP Hopital Cochin and University Paris Descartes, Paris, France 
Background: Due to the risk of invasive pneumococcal disease (IPD) after allogeneic transplant, the ECIL7 guidelines recommend 3 doses of pneumococcal conjugate vaccine (PCV) starting 3 months after transplant, followed by either one polysaccharide 23-valent vaccine (PSV23) dose or a 4th PCV in case of chronic GvHD. However, no data strongly support recommendations from the $2^{\text {nd }}$ year. The aim of this study was to assess the anti-pneumococcal antibody $(\mathrm{Ab})$ concentrations, years after different schedules of vaccination.

Methods: Patients who received allogeneic HCT more than 18 months ago and were seen in consultation between Jan-Nov 2018 were screened for IgG Ab concentrations for the 7 pneumococcal serotypes shared by PCV7, PCV13, and PSV23 (4, 6B, 9V, 14, 18C, 19F, 23F), using modified enzyme linked immunosorbent assay (Wernette et al.2003). Patients were considered protected if their $\mathrm{Ab}$ concentrations were $\geq 0.35 \mu \mathrm{g} / \mathrm{mL}$ for the 7 serotypes tested. From 2001, all patients received 3 PCVs during the $1^{\text {st }}$ year of transplant, then either one dose of PSV23 or (from 2009) a $4^{\text {th }}$ PCV. For patients transplanted for more than 12 months when PCV became available, we locally recommended $\geq 2$ PCV and one PSV23.

Results: Sixty-one patients were assessed at a median of 8.3 years (range: 1.7-39.1) after transplant. The mean age was 53 years (range: $21-79$ ), M/F sex ratio 0,85 . Most patients had acute leukemia $(32,52.5 \%)$ or lymphoproliferative diseases $(13,21.5 \%)$. The donor was HLA-identical in $42(69 \%)$ cases. Conditioning regimen was myeloablative in $37(61 \%)$, reduced-intensity or non-MA in $12(19.5 \%)$ each. None patient had experienced IPD since transplant nor had received immunoglobulins for at least 6 months or rituximab for at least 23 months at time of assessment.

Fifty-four patients (88.5\%) received 3 consecutive PCV: $46(75.5 \%)$ during the first 12 months and 8 (13\%) thereafter. Five (8\%) patients received $2 \mathrm{PCV}$ and only one patient $(1.5 \%)$ received a unique dose of PCV. The $1^{\text {st }} \mathrm{PCV}$ was administered at a median of 3.5 (range: 3-201) months after HCT. Fifty-seven (93.5\%) patients received one PSV23 at a median of 13 months (range: 0.9-385) and 33 (54\%) of them received a 2nd PSV23 thereafter. Finally, 39/ $61(64 \%)$ patients received the recommended program.

The overall protection rate was $29 / 61(47.5 \%)$ at a median time of 8.3 years after HCT. No differences were observed between patients who received the recommended program $(18 / 39,46 \%)$ and the others $(11 / 22,50 \%)$. However, this latter group was often vaccinated $>10$ years after HCT and therefore not protected for years, but may have developed a better response to only one or $2 \mathrm{PCV}$. In all groups, the $\mathrm{Ab}$ concentrations were heterogeneous from one serotype to an other. Recipient age, donor age, underlying disease, conditioning, donor type, previous GvHD, lymphocyte count or gammaglobulin serum levels were not associated with seroprotection.

Conclusions: Although the early antipneumococcal vaccination program is now well established, half of the patients vaccinated according to the guidelines were not protected anymore years later. Prospective studies are needed to establish an optimal long-term programme.

Disclosure: Nothing to declare

\section{8}

Risk factors associated with the development of clinically significant cytomegalovirus (CMV) infection in the allogeneic hematopoietic-cell transplant (HCT) letermovir prophylaxis trial

Francisco M Marty ${ }^{1,2}$, Misoo C Ellison ${ }^{3}$, Werner Rabitsch $^{4}$, Maija Itälä-Remes ${ }^{5}$, Alida Dominietto ${ }^{6}$, Karl Peggs $^{7}$, Peter Browett ${ }^{8}$, Mariela Moreno ${ }^{9}$, Randi Y Leavitt ${ }^{3}$, Amita Joshi ${ }^{3}$, Cyrus Badshah ${ }^{3}$, Wendy W Yeh $^{3}$

${ }^{1}$ Dana-Farber Cancer Institute, Boston, MA, United States, ${ }^{2}$ Brigham and Women's Hospital, Boston, MA, United States, ${ }^{3}$ Merck \& Co., Inc., Kenilworth, NJ, United States, ${ }^{4}$ Medizinische Universität Wien, Vienna, Austria, ${ }^{5}$ Turun Yliopistollinen Keskussairaala, Turku, Finland, ${ }^{6}$ Ospedale San Martino, Genoa, Italy, ${ }^{7}$ University College London Cancer Institute, London, United Kingdom, ${ }^{8}$ Aukland City Hospital, Aukland, New Zealand, ${ }^{9}$ Hospital Nacional Edgardo Rebagliati Martins, Lima, Peru

Background: In a phase 3 trial, letermovir prophylaxis in CMV-seropositive HCT recipients through Week 14 postHCT resulted in lower incidence of clinically-significant CMV infection (cs-CMVi) compared to those who received placebo through Week 24 post-HCT (57/325 [18\%] vs. 71/ 170 [42\%], letermovir vs. placebo) and was well tolerated overall. We evaluated risk factors associated with development of cs-CMVi to further inform the clinical use of letermovir prophylaxis and future trial designs.

Methods: The 495 participants without detectable CMV DNA at randomization (primary efficacy population) were analyzed. CS-CMVi was defined as CMV viremia requiring antiviral preemptive therapy (PET) or CMV diseasepatients without cs-CMVi who died or withdrew from the trial on or before Week 24 were censored at the time of those events for this analysis. Potential risk factors for csCMVi by Week 24 post HCT were examined using univariate and multivariate Cox proportional hazards models. Candidate covariates were included in the multivariate Cox model if they were associated with cs-CMVi in the univariate analysis or had been previously identified in the literature as significant risk factors. To avoid collinearity, the trial high-risk for reactivation of CMV stratification 
covariate was replaced with an updated covariate that only included patients who underwent HCT with a mismatched donor, cord blood, ex-vivo T-cell depletion, or received alemtuzumab. The haploidentical HCT and matched unrelated donor type were considered separately from the trial high-risk CMV categories. Graft-versus-host disease (GVHD) and systemic glucocorticoid exposure were modeled as time-dependent covariates. Race was dichotomized into Asian and non-Asian. The effect of study treatment was not included in the model due to nonproportional hazards. Instead, letermovir treatment was used as a stratification variable. Hazard ratios and $95 \%$ confidence intervals $(\mathrm{CI})$ were calculated.

Results: There were 128 cs-CMVi events (25.9\%) among 495 patients by Week 24 post-HCT for an incidence rate of $0.19 / 100$ patient-days (95\% CI, 0.16-0.22). Multivariate Cox modeling demonstrated that haploidentical HCT (HR 2.77, 95\% CI 1.51-5.07, $\mathrm{p}=0.001$ ), Asian race (HR 2.19, 95\% CI 1.26-3.79, $\mathrm{p}=0.005$ ), time-dependent GVHD (HR 2.13, 95\% CI 1.45-3.14, $\mathrm{p}<0.001$ ), time-dependent glucocorticoid use (HR 1.97, 95\% CI 1.35-2.87, p< 0.001 ), antithymocyte globulin (ATG) use (HR 1.80, 95\% CI 1.23-2.64, $\mathrm{p}=0.003$ ) and donor CMV seronegativity (HR $1.69,95 \%$ CI 1.18-2.43, $\mathrm{p}=0.004$ ), were significantly associated with increased risk of developing cs-CMVi.

Conclusions: Donor CMV seronegativity, haploidentical HCT, GVHD, glucocorticoid use, ATG use, and Asian race conferred significant risk of cs-CMVi through Week 24 post-HCT in a phase 3 study of letermovir prophylaxis. These results identify HCT patient groups that would benefit the most from letermovir prophylaxis.

Clinical Trial Registry: ClinicalTrials.gov, NCT02137772; EudraCT, 2013-003831-31

Disclosure: Francisco Marty has received research grant support from Chimerix, Merck, Shire; consulting honoraria from Chimerix, Fate, Merck, Roche Molecular Diagnostics, Shire.

Werner Rabitsch,Maija Itälä-Remes, Alida Dominietto, Karl Peggs, Peter Browett, and Mariela Moreno received research grant support from Merck for the conduct of this trial.

Misoo Ellison, Randi Leavitt, Amita Joshi, Cyrus Badshah, and Wendy Yeh are employees of Merck \& Co., Inc., and may own stock in the company.

\section{9}

Cytomegalovirus MIR-US4-1/UL-148D reduce the relapse in patients with acute myeloid leukemia after haploidentical hematopoietic stem cell transplantation by strengthening NKG2C $+\mathrm{NK}$ reconstitution via PLZF/ KLRC1
Meng Lv ${ }^{1}$, Xiang-Yu Zhao ${ }^{1}$, Xiao-Su Zhao ${ }^{\text {, }}$, Yan Hong ${ }^{1}$, Zheng-Li Xu ${ }^{I}$, Ying-Jun Chang ${ }^{1}, Y u$ Wang $^{I}$, Xiao-Hui Zhang $^{I}$, Lan-Ping X ${ }^{I}$, Kai-Yan Liu ${ }^{I}$, Xiao-Jun Huang ${ }^{I}$

${ }^{1}$ Peking University People's Hospital, Beijing, China

Background: Conflicting results have been reported regarding whether cytomegalovirus(CMV) infection influence the graft-versus-leukemia (GVL) effect post hematopoietic stem cell transplantation (HSCT). In the present prospective trial (ChiCTR-OCH-10000940), we investigated the impact of CMV reactivation and CMV derived microRNA on relapse for acute myeloid leukemia (AML) patients following T-cell replete human leukocyte antigen (HLA) haploidentical HSCT (Haplo-HSCT).

Methods: 769 adult patients (age 18-60) with de novo AML in complete remission (CR) perusing T-cell replete haplo-HSCT with ATG+G-CSF protocol were consecutively enrolled at Peking University People's Hospital between 2010 and 2016. All patients were evaluated for donor-patient CMV serostatus before HSCT. CMV DNA emia were positive for $>1000$ copies/mL CMV by PCR tests on peripheral blood (PB). Refractory CMV infection was defined as CMV DNAemia lasting for $>2$ weeks in spite of treatment. PB samples of 8 patients with refractory CMV infection (4 relapse and 4 without AML relapse) were sequencing for CMV derived small RNA. CMV-miR-US41/UL-148D were then chosen as target miRNAs and tested by stem-loop Taqman qPCR in consecutive patients $(n=157)$. Cumulative incidence of relapse (CIR) and treatment related mortality (TRM) were calculated using competing risks, Cox model was tested with patient age, sex, WBC count at diagnosis, CR status, courses to achieving $\mathrm{CR}$, cytogenetic risk group, minimal residual diseases before HSCT, CMV infection, CMV-miR-US4-1/ UL-148D expression. US4-1/UL-148D were further explored for the association with $\mathrm{T}$ cells and natural killer (NK) cells reconstitution and target mRNA validation.

Results: In the total cohort of 769 patients, the 3-year overall survival (OS) and Leukemia-free survival (LFS) were $75.3 \%$ and $78.4 \%$, CIR and TRM were $15.9 \%$ and $8.8 \%$. CMV infection occurred in $71.5 \%$ recipients at a median time of 4 weeks after haplo-HSCT, and the duration was 2 weeks. $49.7 \%$ recipients experienced refractory CMV infection. CMV infection (yes vs. no, refractory vs no) did not affect CIR in univariate analysis, while in multivariate analysis, cytogenetic risk group was identified as the only independent prognostic factor affecting CIR (HR 1.72, 95\% CI, 1.004-2.947; $p=0.032$ ). In the cohort testing for CMV microRNA, $35.0 \%$ patients were identified expression of US4-1, 40.1\% patients for UL-148D, while $29.3 \%$ patients had co-expression. The relapse incidence was significantly lower in patients with vs. without co-expression of miR- 
US4-1/UL-148D. Co-expression of miR-US4-1/UL-148D was the only independent risk factor for reducing CIR (HR $0.414,95 \%$ CI, 0.165-1.036; $p=0.019$ ). US4-1/UL-148D as also found to promote reconstitution of $\mathrm{NKG} 2 \mathrm{C}+\mathrm{NKs}$. Luciferase assay identify PLZF as target of US4-1 while KLRC1 targeted by miR-UL-148D, which suggest potential role of shifting $\mathrm{NKG} 2 \mathrm{C} / \mathrm{NKG} 2 \mathrm{~A}$ balance to $\mathrm{NKG} 2 \mathrm{C}$ domination.

Conclusions: The present study is the first prospective trial to evaluate CMV infection on relapse for AML patients following T-cell replete haplo-HSCT with ATG + G-CSF protocol. Our results suggested CMV-miR-US4-1/UL148D co-expression rather than CMV infection reduces the relapse incidence, the stronger GVL effect might be associated with strengthening NKG2C $+\mathrm{NK}$ cells reconstitution and alloreactivity via PLZF/KLRC1 pathway.

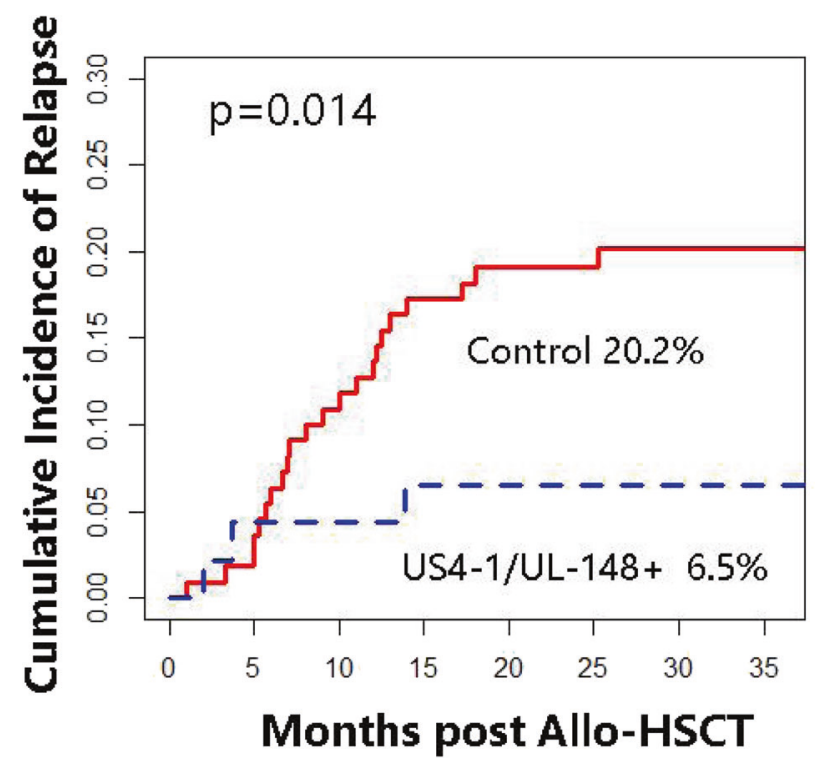

[[O129 Image] 1. miR-US4-1/UL-148D reduces relapse in AML after haplo-HSCT]

Clinical Trial Registry: ChiCTR-OCH-10000940

Disclosure: Nothing to declare

\section{0}

A randomised, placebo-controlled phase 3 study to evaluate the efficacy and safety of ASP0113, a first-inclass, DNA-based vaccine in $\mathrm{CMV}$-seropositive allogeneic haematopoietic cell transplant recipients

Johan Maertens' ${ }^{1}$, Arancha Bermúdez, Aaron Logan ${ }^{3}$, Mohamed Kharfan-Dabaja ${ }^{4}$, Patrice Chevallier ${ }^{5}$, Rodrigo Martino $^{6}$, Gerald Wulf ${ }^{7}$, Dominik Selleslag ${ }^{8}$, Kazuhiko Kakihana $^{9}$, Amelia Langston ${ }^{10}$, Dong-Gun Lee ${ }^{11}$, Carlos Solano $^{12}$, Shinichiro Okamoto ${ }^{13}$, Larry R. Smith ${ }^{14}$,
Michael Boeckh ${ }^{15}$, John Wingard $^{16}$, Beth Cywin ${ }^{17}$, Christine Fredericks $^{17}$, Christopher Lademacher ${ }^{17}$, Xuegong Wang $^{17}$, James Young ${ }^{17}$, Per Ljungman ${ }^{18}$

${ }^{1} \mathrm{KU}$ Leuven and UZ Leuven, Leuven, Belgium, ${ }^{2}$ Hospital Universitario Marqués de Valdecilla, Santander, Spain, ${ }^{3}$ University of California, San Francisco, CA, United States, ${ }^{4} \mathrm{H}$. Lee Moffitt Cancer Center and Research Institute and University of South Florida, Tampa, FL, United States, ${ }^{5} \mathrm{CHU}$ de Nantes, Nantes, France, ${ }^{6}$ Hospital de la Santa Creu I Sant Pau, Barcelona, Spain, ${ }^{7}$ University Medical Center Göttingen, Göttingen, Germany, ${ }^{8} \mathrm{AZ}$ Sint-Jan Brugge-Oostende, Brugge, Belgium, ${ }^{9}$ Komagome Hospital, Tokyo, Japan, ${ }^{10}$ Emory University, Atlanta, GA, United States, ${ }^{11}$ Catholic Hematology Hospital and The Catholic University of Korea, Seoul, Korea, Republic of, ${ }^{12}$ Institute of Research INCLIVA and University of Valencia, Valencia, Spain, ${ }^{13}$ Keio University School of Medicine, Tokyo, Japan, ${ }^{14}$ Vical Inc., San Diego, CA, United States, ${ }^{15}$ Fred Hutchinson Cancer Research Center and University of Washington, Seattle, WA, United States, ${ }^{16}$ University of Florida, Gainesville, FL, United States, ${ }^{17}$ Astellas Pharma Global Development, Inc., Northbrook, IL, United States, ${ }^{18}$ Karolinska University Hospital and Karolinska Institutet, Stockholm, Sweden

Background: ASP0113 is a first-in-class, DNA-based vaccine designed for the prevention of cytomegalovirus (CMV) infection; it contains two plasmids encoding glycoprotein B (gB) and phosphoprotein 65 (pp65). This study aimed to investigate the efficacy, safety and immunogenicity of ASP0113 compared with placebo in allogeneic haematopoietic cell transplant (allo-HCT) recipients.

Methods: This was a randomised, double-blind, placebocontrolled phase 3 study. CMV-seropositive allo-HCT recipients received five intramuscular $1 \mathrm{ml}$ doses of $5 \mathrm{mg} /$ $\mathrm{ml}$ ASP0113 or placebo (phosphate buffered saline) in a 1:1 ratio on Days -14 to $-3,14$ to $40,60 \pm 5,90 \pm 10$ and 180 \pm 10 , relative to the day of transplant (Day 0). Plasma CMV viral load was determined through 1 year and analysed at the central laboratory. Treatment-emergent adverse events (TEAEs) were recorded through 30 days after the last randomized treatment injection. Immunogenicity was measured by $\mathrm{T}$-cell responses to pp65 and gB-specific antibody levels through 1 year after the first randomized treatment injection. The primary endpoint was the proportion of patients with a composite of all-cause mortality and adjudicated CMV end-organ disease (EOD) through 1 year post transplant. Secondary endpoints were CMV viraemia rate, adjudicated CMV-specific antiviral therapy (AVT) rate, a composite of protocol-defined CMV viraemia and adjudicated CMV-specific AVT use, first occurrence of 
adjudicated CMV-specific AVT or adjudicated CMV EOD and mortality through 1 year post transplant.

Results: Overall, 514 patients were randomized, of whom 501 received $\geq 1$ dose of randomized treatment (ASP0113 $n=246$; placebo $n=255$ ). There was no statistically significant difference in the proportion of patients who achieved the primary endpoint between the ASP0113 $(n=87$ [35.4\%] $)$ and placebo $(n=77$ [30.2\%]) groups, respectively (odds ratio 1.27; 95\% confidence interval [CI] $0.87-1.85 ; \mathrm{p}=0.205)$. There were no statistically significant differences between groups for any secondary endpoints (Table). The incidence of TEAEs was similar between groups, except for a greater incidence of drugrelated TEAEs in the ASP0113 group $(n=194$ [78.9\%]) compared with placebo $(\mathrm{n}=74$ [29.0\%]) attributed to injection site-related TEAEs. Mean T-cell response to pp65 increased over time in both groups and was significantly greater with placebo compared with ASP0113 $(p=0.027)$. There was no statistically significant difference between groups for mean $\mathrm{gB}$-specific antibody response.

Conclusions: ASP0113 did not demonstrate efficacy in the reduction of overall mortality and CMV EOD through 1 year post transplant. ASP0113 demonstrated a similar safety profile to placebo, with the exception of injection siterelated TEAEs that were more frequent in the ASP0113 group. Participants in this study will be followed up to 5.5 years post transplant for long-term safety assessments.

\begin{tabular}{|c|c|c|c|}
\hline & $\begin{array}{l}\text { ASP0113 } \\
(\mathrm{n}=246)\end{array}$ & $\begin{array}{l}\text { Placebo } \\
(\mathrm{n}=255)\end{array}$ & $\begin{array}{l}\text { Hazard ratio }(\mathrm{HR}) / 1 \text {-hazard } \\
\text { ratio }(1-\mathrm{HR}) / \text { odds ratio }(\mathrm{OR} \\
(95 \% \mathrm{CI}) ; \mathrm{p} \text { value }\end{array}$ \\
\hline $\begin{array}{l}\text { CMV viraemia rate, } \\
\%(95 \% \mathrm{CI})\end{array}$ & $56.7(50.1-62.8)$ & $58.6(52.0-64.6)$ & $\begin{array}{l}\text { HR: } 0.96(0.76-1.22) \\
p=0.748\end{array}$ \\
\hline $\begin{array}{l}\text { Adjudicated CMV-specific } \\
\text { AVT rate, } \%(95 \% \text { CI) }\end{array}$ & $54.6(48.1-60.6)$ & $53.2(46.8-59.1)$ & $\begin{array}{l}\text { HR: } 1.02(0.80-1.29) \\
p=0.888\end{array}$ \\
\hline $\begin{array}{l}\text { A composite of protocol- } \\
\text { defined CMV viraemia and } \\
\text { adjudicated CMV-specific } \\
\text { AVT use, n (\%) }\end{array}$ & $150(61.0)$ & $155(60.8)$ & $\begin{array}{l}\text { OR: } 1.05(0.73-1.51) \\
\mathrm{p}=0.802\end{array}$ \\
\hline $\begin{array}{l}\text { First occurrence of } \\
\text { adjudicated CMV-specific } \\
\text { AVT or adjudicated CMV } \\
\text { EOD rate, } \%(95 \% \mathrm{CI})\end{array}$ & $55.4(48.9-61.5)$ & $54.4(48.0-60.3)$ & $\begin{array}{l}\text { 1-HR: }-0.01 \quad(-0.28-0.20) \\
\mathrm{p}=0.928\end{array}$ \\
\hline Mortality, n (\%) & $78(31.7)$ & $72(28.2)$ & $\begin{array}{l}\text { OR: } 1.18(0.81-1.73) \\
p=0.393\end{array}$ \\
\hline
\end{tabular}

[[O130 Table] 1. Secondary endpoints]

\section{Clinical Trial Registry: ClinicalTrials.gov: NCT01877655}

Disclosure: JM: Personal fees and non-financial support from Astellas, Basilea, Cidara, F2G, Gilead, Merck and Pfizer; research support fees from Gilead and Merck. AB: Research support fees from Astellas. ALogan: Research support fees from Astellas and Novartis. MK-D: Speaker bureau participation fees from Alexion, Incyte and Seattle Genetics. RM: Research support fees from Astellas. GW:
Personal fees from Astellas. KK: Research support fees from Astellas; personal fees from Bristol-Myers Squibb, Chugai, Dainippon Sumitomo, Kyowa Hakko Kirin, and Takeda. D-GL: Research support fees from Astellas and GlaxoSmithKline; consultancy fees from Gilead, MSD, Pfizer and SL VaxiGen. SO: Research support fees from Astellas; personal fees from Clinigen and Mitsubishi Tanabe Pharma.LRS: Personal fees from Astellas. MB: Research support and consultancy fees from Astellas, Chimerix, Merck, Shire and Vir Bio; research support fees from Lophius Biosciences; consultancy fees from Artemis Therapeutics, Helocyte, Moderna and Oxford Immunotec. JW: Consultancy fees from Astellas, Celgene, Merck, Pluristem and Shire. BC, CF, CL, XW and JY: Employees of Astellas. PL: Research support fees from Astellas, Merck and Shire; consultancy fees from AiCuris, Astellas and Vical; non-financial support from Astellas. PC, DS, ALangston and CS: No conflicts of interest.

\section{1}

Abstract already published.

\section{2}

Invasive fungal diseases caused by rare pathogens in large cohort of pediatric and adult patients after hematopoietic stem cell transplantation (HSCT) \& chemotherapy

Marina Popova, Yuliya Rogacheva, Alisa Volkova, Anastasya Frolova ${ }^{1}$, Inna Markova ${ }^{1}$, Alexandr Shvetcov ${ }^{I}$, Ilya Nikolaev ${ }^{I}$, Svetlana Ignatieva ${ }^{2}$, Tatyana Bogomolova ${ }^{2}$, Asmik Gevorgian ${ }^{1}$, Olesya Paina, Tatyana Bykova $^{I}$, Elena Darskaya ${ }^{I}$, Oleg Goloshchapov ${ }^{I}$, Elena Morozova $^{I}$, Mariya Vladovskaya ${ }^{I}$, Sergey Bondarenko ${ }^{1}$, Ivan Moiseev ${ }^{1}$, Ludmila Zubarovskaya ${ }^{I}$, Nikolay Klimko ${ }^{2}$, Boris Afanasyev ${ }^{I}$

${ }^{1}$ R.M. Gorbacheva Institute of Pediatric Oncology Hematology and Transplantation, I.P.Pavlov First St. Petersburg State Medical University, Saint Petersburg, Russian Federation, ${ }^{2}$ I.I. Mechnikov North-Western State Medical University, Ministry of Health of Russia, Saint Petersburg, Russian Federation

Background: The number of publications on the invasive fungal diseases caused by rare pathogens (rare IFD) after HSCT and chemotherapy is limited.

Methods: The retrospective analysis of rare IFD cases in large cohort of hematological patients after HSCT and chemotherapy for a 10-year period. From 2008 to 2017 in R. Gorbacheva Memorial Institute of Children Oncology, Hematology and Transplantation (CIC725) have been performed 2738 HSCT including 1845 allogeneic (allo- 
HSCT) and 893 autologous HSCT (auto-HSCT). During the observation period 40 probable and proven rare IFD (EORTC/MSG 2008 criteria) cases were diagnosed in children and adults with hematological malignances and non-malignant hematological diseases after allo-HSCT $(n=30)$, auto-HSCT $(n=2)$, and chemotherapy $(n=8)$. The median age was 23 (2-59) y.o., males - 60\% $(n=24)$. The median follow up time for rare IFD cases was 3 months; for survivors - 30 months.

Results: Incidence of rare IFD in HSCT recipients was $1,5 \%$, it was higher after allo-HSCT $(1,6 \%)$ than autoHSCT $(0,2 \%)(\mathrm{p}<0,01)$. In eight patients, this complication developed after CT and four of them proceed to allo-HSCT. The most frequent underlying diseases were acute lymphoblastic leukemia (33\%) and acute myeloid leukemia (30\%). The median time of onset of rare IFD after allo-HSCT was 104 (21-1057) days, auto-HSCT - 138 (60-216), after start of CT - 161 (79-189). Etiology of rare IFD was identified by culture in $65 \%$ cases: Rhizopus spp. - 27\%, Paecilomyces spp. - 7\%, Fuzarium spp. - 5\%, Malassezia furfur - 5\%, Trichosporon asahii - 3\%, Scedosporium apiosperium $2 \%$, Scopulariopsis gracilis - 2\%, Rhizomucor pusillus $2 \%$, Lichtheimia corymbifera - 2\%, mix rare IFD with Rhizopus spp. + Paecilomyces spp. - 5\%, Paecilomyces spp. + Fuzarium spp. - 5\%. 35\% cases (mucormycosis) were diagnosed by microscopy. In $45 \%$ cases rare IFD developed after or in combination with invasive aspergillosis, and 2 patients had both preexisting invasive aspergillosis and co-infection with mucormycosis. The main site of infection were lungs $(82 \%)$, the main clinical symptom - febrile fever (95\%). Antifungal therapy was used in all patients: lipid amphotericin B - 30\%, lipid amphotericin B + caspofungin - 22,5\%, voriconazole $17,5 \%$, posaconazole - $12,5 \%$, lipid amphotericin $\mathrm{B}+$ posaconazole - $10 \%$, and echinocandins - 7,5\%. Surgery was used in $10 \%$ patients. Overall survival at 12 weeks from the diagnosis of rare IFD was 50\%. The 12-weeks overall survival was better in patients after CT and autoHSCT $(80 \%)$ than allo-HSCT $(42 \%), \mathrm{p}=0,048$.

Conclusions: The incidence of rare IFD in HSCT recipients was $1,5 \%$ and depends on type of transplantation. Rare IFD is a late complication after chemotherapy and HSCT and usually develops after or in combination with invasive aspergillosis. Higher incidence and worst prognosis rare IFD is observed in allo-HSCT recipients.

Disclosure: Nothing to declare

\section{3}

Incidence and outcome of kaposi sarcoma after hsct: A retrospective analysis on behalf of IDWP

Simone Cesaro ${ }^{1}$, Federica Minniti', Steffie van der Werf ${ }^{2}$, Gloria Tridello ${ }^{1}$, Peter Bader ${ }^{3}$, Gerard Socie ${ }^{4}$, Per
Ljungman $^{5}$, Grant McQuaker6, Stefano Giardino ${ }^{7}$, Duygu Uckan-Cetinkaya ${ }^{8}$, Achilles Anagnostopoulos', Hakan Ozdogu $^{10}$, Rik Schots ${ }^{11}$, Pavel Jindra ${ }^{12}$, Marco Ladetto ${ }^{13}$, Wilfried Schroyens ${ }^{14}$, Malgorzata Mikulska ${ }^{15}$, Jan Styczynski ${ }^{16}$

${ }^{1}$ Azienda Ospedaliera Universitaria Integrata Verona, Verona, Italy, ${ }^{2}$ EBMT Data Office, Leiden, Netherlands, ${ }^{3}$ Universitaetsklinikum Frankfurt Goethe-Universitaet, Frankfurt, Germany, ${ }^{4}$ Hopital St. Louis, Paris, France, ${ }^{5}$ Karolinska University Hospital, Stockholm, Sweden, 6 Beatson West of Scotland Cancer Centre, Glasgow, United Kingdom, ${ }^{7}$ Istituto Giannina Gaslini, Genova, Italy, ${ }^{8}$ Hacettepe University Children's Hospital, Ankara, Turkey, ${ }^{9}$ George Papanicolaou General Hospital, Thessaloniki, Greece, ${ }^{10}$ Baskent University Hospital, Adana, Turkey, ${ }^{11}$ Universitair Ziekenhuis Brussel, Brussels, Belgium, ${ }^{12}$ Charles University Hospital, Pilsen, Czech Republic, ${ }^{13} \mathrm{H}$ SS. Antonio e Biagio, Alessandria, Italy, ${ }^{14}$ Antwerp University Hospital (UZA), Antwerp, Belgium, ${ }^{15}$ Ospedale San Martino, Genova, Italy, ${ }^{16}$ University Hospital, Bydgoszcz, Poland

Background: Kaposi's sarcoma (KS) is an angioprolipherative disease which occurs in immunosuppressed patients, often associated with infection by Human Herpes Virus 8 (HHV-8). In solid organ transplantation (SOT) KS is relatively common, the risk being 60-500 folds higher than that of general population and representing around $23 \%$ of secondary cancers. Also hematopoietic stem cell transplantation (HSCT) is a risk factor for the development of KS but until now only few case reports were published. We assessed retrospectively the incidence, the clinical characteristics, and the outcome of $\mathrm{KS}$ in the EBMT database.

Methods: The cases of KS were identified by EBMT registry (ProMISe) and by inviting all 569 EBMT centers to notify KS cases. The clinical features, type of therapy, survival rate and causes of death were retrieved from of ProMise or, if lacking, by specific case report form sent to participating centers. The center response rate was 74/ 569 (13\%).

Results: Fourteen centers reported 17 patients with KS, all KS were diagnosed from 2004 to 2017, but one case that was diagnosed in 1987 . The analysis was limited to $13 / 17$ patients because KS was diagnosed before HSCT in 4 patients: they were 13 patients, 3 females and 10 males who developed KS after allo HSCT (10) and auto HSCT (3); moreover, KS occurred after a second HSCT in 2 patients and after a third HSCT in 1 patient. The search of HHV8 in tumour tissue was done in 9 cases and resulted positive in $8 /$ 9. The underlying disease were: $46 \%$ leukemia, $23 \%$ lymphoma, $15 \%$ myeloma, $8 \%$ myelodysplastic syndrome 
(MDS), $8 \%$ bone marrow failure. The source of stem cells was bone marrow in 4 patients (31\%) and peripheral blood in 9 patients $(69 \%)$. The median age at KS diagnosis was 49.7 years (range 6.3-61.4). Considering the number of HSCT performed in the participating center from 2004 to 2017, the incidence rate was $0.17 \%$ in allogeneic transplantations (9/5345), $0.05 \%$ in autologous transplantations $(3 / 5857)$ and $0.11 \%(12 / 11202)$ in the whole group. The interval of time between HSCT and the development of KS was 7 months (range -0.3-61.2). The organ involvement was: $62 \%$ skin (8 patients), $31 \%$ lymph nodes (4), $8 \%$ gingival (1). Apart from withdrawal of immunosuppression, 2 patients received chemotherapy, 3 patients received radiotherapy, and 1 patient received radio and chemotherapy; moreover 5 patients received antiviral treatment with ganciclovir or foscarnet.

Eight patients $(62 \%)$ are alive whereas 5 patients $(38 \%)$ died at a median time of 8.1 months, range $0.5-12.3$. The causes of death were infection in 2 cases, secondary malignancy/PTLD in 2 and relapse/progression in 1 whereas no case of death directly associated to KS.

Conclusions: KS is a rare complication of the immunosuppressive status related to HSCT that generally occurs within the first year after HSCT. The low prevalence and the rarity of this complication do not justify the adoption of screening program for HHV-8. On the other hand, the role of the virus in febrile status in immunosuppressed patients and the risk factors for the development of $\mathrm{KS}$ are not well known.

Disclosure: The authors have no conflict of interest

\section{Lymphoma}

\section{4}

Tandem autologous-reduced intensity allogeneic stem cell transplantation in relapsed hodgkin lymphoma: A retrospective study of the LWP-EBMT

Leyre Bento ${ }^{1}$, Ariane Boumendil ${ }^{2}$, Hervé Finel ${ }^{2}$, Irma Khvedelidze $^{2}$, Didier Blaise ${ }^{3}$, Nathalie Fegueux ${ }^{4}$, Luca Castagna $^{5}$, Noël Milpied ${ }^{6}$, Patrice Chevallier ${ }^{7}$, Nicola Mordini $^{8}$, Pauline Brice, ${ }^{9}$ Eric Deconinck ${ }^{10}$, Martin Gramatzki $^{11}$, Paolo Corradini ${ }^{12}$, Mathilde Hunault ${ }^{13}$, Maurizio Musso ${ }^{14}$, Anna Tsoulkani ${ }^{15}$, Dolores Caballero $^{16}$, Sandro Nati $^{17}$, Silvia Montoto ${ }^{18}$, Anna Sureda ${ }^{19}$

${ }^{1}$ Son Espases University Hospital, IdISBa, Palma de Mallorca, Spain, ${ }^{2}$ Lymphoma Working Party EBMT, Paris, France, ${ }^{3}$ Institut Paoli Calmettes, Marseille, France, ${ }^{4} \mathrm{CHU}$ Lapeyronie, Montpellier, France, ${ }^{5}$ Istituto Clinico Humanitas, Milano, Italy, ${ }^{6} \mathrm{CHU}$ Bordeaux, Bordeaux, France, ${ }^{7} \mathrm{CHU}$ Nantes, Nantes, France, ${ }^{8}$ Az. Ospedaliera S. Croce e
Carle, Cuneo, Italy, ${ }^{9}$ Hôpital St. Louis, Paris, France, ${ }^{10}$ Hopital Jean Minjoz, Besancon, France, ${ }^{11}$ University Medical Center Schleswig-Holstein, Kiel, Germany, ${ }^{12}$ Istituto Nazionale dei Tumori, Milano, Italy, ${ }^{13} \mathrm{CHRU}$, Angers, Angers, France, ${ }^{14}$ Ospedale La Maddalena, Palermo, Italy, ${ }^{15}$ Nottingham University, Nottingham, United Kingdom,

${ }^{16}$ Hospital Clínico Salamanca, Salamanca, Spain, ${ }^{17}$ Azienda Ospedaliera Universitaria San Martino, Genova, Italy, ${ }^{18}$ Queen Mary, University of London. Centre for Haemato-Oncology, London, United Kingdom, ${ }^{19}$ Institut Català d'Oncologia, Hospital Duran i Reynals, Barcelona, Spain

Background: Autologous stem cell transplantation (autoSCT) is considered the standard treatment for patients with relapsed or refractory (R/R) Hodgkin lymphoma (HL). For those with high-risk disease, an alternative consolidation strategy with allogeneic SCT (allo-SCT) could be a potential option to improve the outcome. However, allo-SCT with a reduced-intensity conditioning (RIC) needs around 3 months for the graft-versus-lymphoma effect (GVL) to develop, thus in patients with an aggressive HL the disease might progress before this happens. In this setting, a tandem auto-RIC-SCT approach has the potential of combining cytoreduction to keep the lymphoma under control and the potential benefit of a GVL effect. To better understand the safety and efficacy of a tandem auto-RIC-SCT approach we conducted a retrospective analysis of patients treated with this strategy between January 2004 and December 2015 and reported to the EBMT registry.

Methods: Patients were included if they had received an auto-SCT followed by a planned RIC-SCT in $<6$ months with no disease relapse between the procedures. The primary endpoint was progression-free survival (PFS) after the tandem procedure. Secondary endpoints were overall survival (OS), cumulative incidence of non-relapse mortality (NRM), incidence of relapse (IR) and graft versus host disease (GVHD).

Results: One-hundred and thirty patients [58\% male, median age at auto-SCT, 30 years (range: 18-65)] fulfilled the inclusion criteria. The median time between diagnosis and auto-SCT was 16 months (range: 2-174) and the median number of lines prior to auto-SCT 2 (2-4). Disease status at auto-SCT was complete response in $32 \%$, partial response in $27 \%$ and the remaining $41 \%$ were transplanted with active disease. The median time from auto to allo-SCT was 3 months (1-6). Forty percent underwent an identical sibling allo-SCT, $39 \%$ unrelated and $21 \%$ haplo. TBI was used in $35 \%$ of the patients as a part of RIC. GVHD prophylaxis was cyclosporine-methotrexate in $36 \%$ of the patients, cyclosporine-micofenolate mofetil in $16 \%$ and post-transplant cyclophosphamide in the remaining $17 \%$. $91 \%$ of the patients engrafted after RIC-SCT. After a median follow-up of 44 months (6-130), 33\% of the patients 
have relapsed and $34 \%$ died. The main causes of death were SCT-related in 53\% and disease in 35\%. 36-months PFS, OS, IR and NRM were 53\% (44-63), 72\% (64-80), 34\% (25-43) and 13\% (8-20), respectively. Cumulative incidence of grade 3-4 acute GVHD at 100 days after RIC-SCT was $10 \%$ (5-16) and chronic GVHD at 36 months 48\% (38-58). PFS and IR were influenced by patient sex $(\mathrm{p}=0.025$ and $0.04)$ and disease status at allo-SCT ( $\mathrm{p}=0.024$ and 0.001$)$; engraftment by TBI before allo-SCT $(\mathrm{p}=0.039)$ and stem cell source $(\mathrm{p}=0.04)$; acute grade 3-4 GVHD by donor type $(\mathrm{p}=0.037)$ and chronic GVHD by allo-SCT conditioning $(\mathrm{p}=0.016)$ and donor sex $(\mathrm{p}=0.01)$.

Conclusions: This is the largest series analysing the efficacy and safety of a tandem auto-RIC-SCT approach in R/R HL. The low NRM and IR with promising PFS and OS suggest that this might be an effective approach in this highrisk population.

Disclosure: Nothing to declare.

\section{5}

Post-transplant cyclophosphamide-based GVHD prophylaxis compared to standard prophylaxis in patients with lymphoma receiving HLA identical transplantation: A retrospective study from the LWP of EBMT

Stefania Bramanti', Ariane Boumendil ${ }^{2}$, Hervè Finel ${ }^{3}$, Irma Khvedelidze ${ }^{4}$, Boris Afanasyev ${ }^{5}$, Norbert Schmitz, Didier Blaise ${ }^{7}$, Fabio Ciceri, ${ }^{8}$ Edouard Forcade ${ }^{9}$, Jan Cornelissen $^{10}$, Urpu Salmenniemi ${ }^{11}$, Christof Scheid ${ }^{12}$, Yener Koc ${ }^{13}$, Renato Fanin ${ }^{14}$, Tobias Gedde-Dahl ${ }^{15}$, Jacques-Olivier Bay ${ }^{16}$, Benedetto Bruno ${ }^{17}$, Luca Castagna $^{18}$, Paolo Corradini ${ }^{19}$, Bulabois Claude-Eric ${ }^{20}$, Choi Goda $^{21}$, Mathieu Leclerc ${ }^{22}$, Ellen Meijer ${ }^{23}$, Giuseppe Milone $^{24}$, Ghulam Mufti ${ }^{25}$, Johanna Tischer ${ }^{26}$, Stephen Robinson $^{27}$, Silvia Montoto ${ }^{28}$

${ }^{1}$ Istituto Clinico Humanitas, Rozzano, Italy, ${ }^{2}$ EBMT Lymphoma Worky Party, Paris, France, ${ }^{3}$ EBMT Lymphoma Worky Party, LWP Study Coordinator, Paris, France, ${ }^{4}$ EBMT ALWP and LWP, Paris, France, ${ }^{5}$ First State Pavlov Medical University of St. Petersburg, St. Petersburg, Russian Federation, ${ }^{6}$ Asklepios Klinik St. Georg, Hamburg, Germany, ${ }^{7}$ Institut Paoli Calmettes, Marseille, France, ${ }^{8}$ Ospedale San Raffaele s.r.l., Milano, Italy, ${ }^{9} \mathrm{CHU}$, Bordeaux, France, ${ }^{10}$ Erasmus MC Cancer Institute, Rotterdam, Netherlands, ${ }^{11}$ University Hospital, Turku, Finland, ${ }^{12}$ University of Cologne, Cologne, Germany, ${ }^{13}$ Medical Park Hospitals, Antalya, Turkey, ${ }^{14}$ Azienda Ospedaliera Universitaria, Udine, Italy, ${ }^{15}$ Rikshospitalet, Oslo, Norway, ${ }^{16} \mathrm{CHU}$, Clermont Ferrand, France, ${ }^{17}$ A.O.U. Città della Salute e della Scienza di Torino, Torino, Italy, ${ }^{18}$ Istituto Clinico Humanitas, Milano,
Italy, ${ }^{19}$ Istituto Nazionale dei Tumori, Milano, Italy, ${ }^{20} \mathrm{CHU}$ de Grenoble, Grenoble, France, ${ }^{21}$ University Medical Center, Groningen, Netherlands, ${ }^{22}$ Hôpital Henri Mondor, Créteil, France, ${ }^{23}$ University Medical Center, Amsterdam, Netherlands, ${ }^{24}$ Ospedale Ferrarotto, Catania, Italy, ${ }^{25}$ GKT School of Medicine, London, United Kingdom, ${ }^{26}$ Klinikum Grosshadern, Munich, Germany, ${ }^{27}$ University Hospital Bristol NHS Foundation Trust, Bristol, United Kingdom, ${ }^{28}$ Queen Mary University of London, Centre for HaematoOncology, London, United Kingdom

Background: Allogeneic stem cell transplantation (allo$\mathrm{SCT}$ ) is a valid option in patients with refractory/relapsed lymphoma but GVHD remains the major cause of mortality and morbidity. Calcineurin-inhibitors (CNI) combined with methotrexate or ATG is the conventional strategy for preventing GVHD, resulting in an incidence of cGVHD of 41$60 \%$. Post-transplant cyclophosphamide (PT-Cy) reduces the risk of severe cGVHD and improves survival in acute leukemia patients receiving allo-SCT from matched sibling (MSD) or unrelated donors (UD). The aim of this retrospective registry-based study was to compare PT-Cy-based GVHD prophylaxis to standard prophylaxis in the setting of MSD or UD for patients with lymphoma.

Methods: Three thousand eight hundred sixty-four lymphoma patients undergoing an HLA -id SCT(HLA identical stem cell transplantation) registered in Promise were included in the study (Table 1). Outcomes between PT-Cy vs no-PT-Cy were compared a) with a multivariate cause-specific cox model adjusted on RIC/MAC, donor type, source of stem cell, age of the patient, donor gender, patient gender, diagnosis and, disease status at SCT; and b) by matching one PT-Cy patient (118) with two no-PT-Cy patients (217) using the same covariates.

Results: In univariate analysis, comparing PT-Cy and noPT-Cy, the 100-day CI of grade 2-4 aGVHD was $26 \%$ and $27 \%(\mathrm{p}=0.7)$, the 1 -year CI of non-relapse mortality (NRM) was $12 \%$ and $16 \%(\mathrm{p}=0.6)$ and the 3-year relapse incidence (RI) was $38 \%$ and $33 \%(\mathrm{p}=0.2)$, respectively. The 1 -year CI of cGVHD was $32 \%$ vs $42 \%$ ( $\mathrm{p}=0.1$ ), 3-year PFS for PT$\mathrm{Cy}$ and no-PT-Cy was $43 \%$ and $46 \%(\mathrm{p}=0.5)$ and 3 -year OS, $54 \%$ and $57 \%(\mathrm{p}=0.9)$, respectively. In multivariate analysis, prophylaxis with PT-Cy was not associated with a reduced risk of aGVHD, overall or extensive cGVHD, NRM, RI, nor with an improved PFS or OS. Likewise, in the matched-pair analysis PT-Cy did not impact on any of these outcomes.

Conclusions: This study demonstrates that in lymphoma patients who underwent an HLA-id SCT, GVHD prohphylaxis strategies employing PT-Cy- based achieve equivalent transplant outcomes to those seen with CNI- based strategies. 


\begin{tabular}{llll}
\hline N. & PTCY 142 & NO PTCY 3722 & P \\
\hline Median follow-up (range) & 21 months (<1-75) & 33 months (<1-95) & $<0.0001$ \\
Conditioning regimen RIC & $86(61 \%)$ & $2495(68 \%)$ & 0.0973 \\
$\begin{array}{l}\text { Donor type Unrelated } \\
\text { Stem cell source PBSC BM }\end{array}$ & $68(52 \%)$ & $1662(45.2 \%)$ & 0.6 \\
$\begin{array}{l}\text { Disease Hodgkin DLBCL Mantle } 60(42 \%) 36(25 \%) 23 \\
\text { cell Follicular Peripheral T-cell }\end{array}$ & $(16 \%) 12(9 \%) 11(7 \%)$ & $(15 \%) 772(21 \%) 338(9 \%)$ & 0.0012 \\
$\begin{array}{l}\text { Peripheral T-cell lymphoma } \\
\text { Disease status at allo CR PR }\end{array}$ & $72(51 \%) 31(22 \%)$ & $1812(49 \%) 873(23 \%)$ & 0.9 \\
$\begin{array}{l}\text { Refractory } \\
\text { TCD No ATG/Campath Ex-vivo }\end{array}$ & $94(69 \%)$ & $1037(28 \%)$ & \\
& $(32 \%) 2(2 \%)$ & $1714(46 \%) 1921(52 \%)$ & $<0.0001$ \\
D/R gender F/F F/M M/F M/M & $21(15 \%) 36(25 \%) 31$ & $519(14 \%) 829(23 \%) 711$ & 0.6 \\
& $(22 \%) 54(38 \%)$ & $(19 \%) 1583(43 \%)$ & \\
\hline
\end{tabular}

[[O135 Table] 1. Table 1.]

Disclosure: Nothing to declare

\section{6}

Myeloablative conditioning may contribute to disease control after stem cell transplantation in blastic plasmacytoid dentric cell neoplasia

Sascha Dietrich ${ }^{1}$, Herve Finel, Jian-Jian Lu ${ }^{2}$, Ariane Boumendil $^{2}$, Henrik Sengeloev ${ }^{3}$, Gérard Socié $e^{4}$, Helene Labussiere-wallet ${ }^{5}$, Sebastien Maury ${ }^{6}$, Jan Cornelissen ${ }^{7}$, Patrice Chevallier ${ }^{8}$, Metthew Colin ${ }^{9}$, Hildegard Greinix ${ }^{10}$, Thomas Heinicke ${ }^{11}$, Lothar Kanz ${ }^{12}$, Dietrich Beelen ${ }^{13}$, Ellen Meijer ${ }^{14}$, Christoph Schmid ${ }^{15}$, Hans Martin ${ }^{16}$, Gerard Michel $^{17}$, Luca Castagna ${ }^{18}$, Christoph Schmeid ${ }^{19}$, Kerstin Schäfer ${ }^{20}$, Anna Tsoulkani ${ }^{21}$, Joerg Bittenbring ${ }^{22}$, Jürgen Finke ${ }^{23}$, Yener Koc ${ }^{24}$, Arnon Nagler ${ }^{25}$, Mohamad Mohty $^{26}$, Stephen Robinson ${ }^{27}$, Peter Dreger ${ }^{28}$, Silvia Montoto $^{29}$

${ }^{1}$ Universität Heidelberg, Heidelberg, Germany, ${ }^{2}$ Lymphoma Working Party EBMT, Paris, France, ${ }^{3}$ Rigshospitalet, Copenhagen, Denmark, ${ }^{4}$ Hopital St. Louis, Paris, France, ${ }^{5}$ Centre Hospitalier Lyon Sud, Lyon, France, ${ }^{6}$ Hôpital Henri Mondor, Créteil, France, ${ }^{7}$ Erasmus MC Cancer Institute, Rotterdam, Netherlands, ${ }^{8}$ Nantes University Hospital, Nante, France, ${ }^{9}$ Freeman Hospital, New Castel, United Kingdom, ${ }^{10}$ University Hospital Graz, Graz, Austria, ${ }^{11}$ Universitaetsklinium Magdeburg, Magdeburg, Germany, ${ }^{12}$ Uniklinik Tübingen, Tübingen, Germany, ${ }^{13}$ University Hospital Essen, West-German Cancer Center, Essen, Germany, ${ }^{14}$ VU University Medical Center Amsterdam, Amsterdam, Netherlands, ${ }^{15}$ Klinikum Augsburg, Augsburg, Germany, ${ }^{16}$ University Frankfurt, Frankfurt, Germany, ${ }^{17}$ Hôpital d'Enfants de la Timone, Marseille, France, ${ }^{18}$ Istituto Clinico Humanitas, Milano, Italy, ${ }^{19}$ University of Cologne, Cologne, Germany, ${ }^{20}$ Klinikum Nürnberg, Nürnberg, Germany, ${ }^{21}$ Nottingham University, Nottingham, United Kingdom, ${ }^{22}$ University of Saarland, Homburg, Germany, ${ }^{23}$ University of Freiburg, Freiburg, Germany, ${ }^{24}$ Medical Park Hospitals, Antalya, Germany,
${ }^{25}$ Chaim Sheba Medical Center, Tel-Hashomer, Israel, ${ }^{26}$ Hôpital Saint Antoine, Paris, France, ${ }^{27}$ University of Bristol, Bristol, United Kingdom, ${ }^{28}$ University of Heidelberg, Heidelberg, Germany, ${ }^{29}$ Queen Mary, University of London. Centre for Haemato-Oncology, London, United Kingdom

Background: Blastic plasmacytoid dentric cell neoplasia (BPDCN) is a rare and clinically aggressive hematopoietic malignancy, which preferentially involves the skin, the bone marrow, and, occasionally, the lymph nodes. It mainly affects elderly patients and has a poor prognosis with conventional chemotherapy. The treatment for this condition is heterogeneous, some patients treated with lymphoma-type regimens and others receiving intensive acute leukemiatype chemotherapy. Although preliminary case series suggest that hematopoietic stem cell transplantation (SCT) could provide sustained disease control in patients with BPDCN, the role of SCT and potential graft-versus-tumor effects (GVT) in this condition is yet undefined.

Methods: Between 2009 and 201726 patients were included in an EBMT prospective non-interventional study (NIS) on the value of SCT in BPDCN. These were compared with 133 patients with BPDCN registered with the EBMT during the same time period outside the NIS. No differences with regard to overall survival (OS), line of treatment or year of transplant was observed between patients included in the NIS and the remainder from the EBMT database, and they were therefore analyzed together.

Results: One hundred and forty-two patients were treated with an allogeneic SCT (alloSCT) and 17 patients with autologous or syngeneic SCT (AUTOSYN). The median follow-up was 17 months with no differences between the alloSCT and AUTOSYN groups. Disease status at SCT was complete remission (CR) in $88 \%$, and $90 \%$ patients received the SCT as part of first-line treatment. Two-year OS after AUTOSYN was 70\% (95\% CI 46-99\%), whereas it was $65 \%$ (95\% CI 53-83\%) following alloSCT. Of those patients who received an alloSCT, $71 \%$ were transplanted from an unrelated donor (UD), and 29\% from an identical sibling donor (SIB). Reduced intensity conditioning (RIC) was used in $47 \%$ and myeloablative conditioning (MAC) in $53 \%$ of the patients. In allotransplanted patients, multivariate competitive risk analysis of NRM and relapse incidence considering age, conditioning intensity, line of treatment, remission status prior to alloSCT and donor type revealed a statistically significant reduction of relapse incidence $(p=0.02$, HR 0.2 CI 0.1-0.7) without increased NRM for patients who received MAC compared to RIC. Multivariate cox regression analysis for OS considering the same co-variates confirmed that MAC was associated with a significantly reduced mortality risk $(\mathrm{p}=0.05$, CI $0.4, \mathrm{HR}$ 
0.1-0.99) along with being in CR at alloSCT ( $\mathrm{p}<0.001$, HR

9.1, CI 2.8-29.0).

Conclusions: This study confirms on a large data set that SCT is an effective and potentially curative treatment for patients with BPDCN. The superiority of MAC and the efficacy of AUTOSYN suggest that apart from GVT, highdose therapy might be an important contributor to long-term disease control in this condition.

Clinical Trial Registry: NA

Disclosure: NA

\section{7}

Allogeneic hematopoietic cell transplantation in elderly non-hodgkin lymphoma patients: A time trends analysis from the center for international blood and marrow transplant research (CIBMTR)

Mehdi Hamadani, Kwang Woo Ahn ${ }^{1}$, Carlos Litovich', Timothy Fenske ${ }^{1}$, Anna Sureda ${ }^{2}$, Mohamed KharfanDabaja $^{3}$, Nirav Shah ${ }^{4}$

${ }^{1}$ CIBMTR (Center for International Blood and Marrow Transplant Research), Milwaukee, WI, United States, ${ }^{2}$ Institut Català d'Oncologia - Hospitalet, Barcelona, Spain, ${ }^{3}$ Mayo Clinic, Miami, FL, United States, ${ }^{4}$ Medical College of Wisconsin, Milwaukee, WI, United States

Background: Allogeneic hematopoetic cell transplantation (allo-HCT) is a curative therapy for patients with relapsed/ refractory and high-risk non-Hodgkin lymphoma (NHL). However, no large studies have evaluated the trends in the utilization of allo-HCT in elderly NHL patients ( $\geq 65$ years). Using the CIBMTR registry we report here a time trends analysis of allo-HCT use in elderly NHL subjects

Methods: We identified 727 NHL patients ( $\geq 65$ years of age) undergoing a first alloHCT during 2000-2015 in the United States (U.S.). Study cohort was divided into the following time-periods for analysis; 2000-2005 vs. 2006-2010 vs. 2011-2015. Primary outcome was overall survival (OS). Secondary outcomes included progression-free survival (PFS), relapse/progression (R/P) and non-relapse mortality (NRM).

Results: Baseline patient characteristics are shown in Table-1. During the three study time-periods median patient age (67-68-year), use of reduced-intensity conditioning regimens and proportion of patients with chemosensitive disease remained stable. In the most recent era (2011-2015) a higher proportion of patients had T-cell NHL, history of prior autografts, good performance status (KPS 90-100) and high HCT-CI, while fewer subjects received a HLAmatched sibling HCT.

The cumulative incidence of day 100 grade 2-4 acute graft-versus-host disease (GVHD) for 2000-2005 vs. 20062010 vs. $2011-2015$ cohorts was $25 \%$ vs. $35 \%$ vs. $31 \%$ respectively ( $\mathrm{p}=0.47)$. The cumulative incidence of chronic GVHD at 1 year was $21 \%$ vs. $34 \%$ vs. $31 \%$, in similar order $(\mathrm{p}=0.07)$. The 4 -year probabilities of NRM and $\mathrm{R} / \mathrm{P}$ of 2000-2005 vs. $2006-2010$ vs. 2011-2015 time-periods were $34 \%$ vs. $29 \%$ vs. $30 \%(\mathrm{p}=0.69)$ and $48 \%$ vs. $40 \%$ vs. $40 \%$ $(\mathrm{p}=0.39)$, respectively (Figure). The 4-year probabilities of PFS and OS (2000-2005 vs. 2006-2010 vs. 2011-2015) showed significantly improved outcomes in the most recent time-periods as following: $17 \%$ vs. $31 \%$ vs. $30 \%(\mathrm{p}=0.02)$ and $21 \%$ vs. $42 \%$ vs. $44 \%$ ( $<<0.001$ ), respectively (Figure). On multivariate analysis, compared to the 2000-2005 cohort, the 2011-2015 cohort showed a $28 \%$ reduction in the risk of mortality $(\mathrm{RR}=0.72,95 \% \mathrm{CI}=0.52-1.008$, $\mathrm{p}=0.056$ ). The most common cause of death was relapse of primary disease in all time-periods.

Conclusions: Utilization of allo-HCT has steadily increased in elderly NHL patients in the U.S. since 2000. In the recent years despite decline in the use of HLAmatched sibling donors and transplanting elderly patients with higher HCT-CI and more heavily pretreated disease, survival outcomes have improved. Age alone should not be a determinant for allo-HCT eligibility in NHL.

\begin{tabular}{|c|c|c|c|}
\hline & $\begin{array}{l}2000- \\
2005(\mathrm{~N}=76)\end{array}$ & $\begin{array}{l}2006-2010 \\
(\mathrm{~N}=238)\end{array}$ & $\begin{array}{l}2011-2015 \\
(\mathrm{~N}=413)\end{array}$ \\
\hline Median age, Years (range) & $67(65-75)$ & $67(65-76)$ & $68(65-77)$ \\
\hline KPS $\geq 90 @ H C T(\%)$ & $16(21)$ & $125(53)$ & $245(59)$ \\
\hline HCT-CI $\geq 3$ (\%) & Not available & $61(26)$ & $182(44)$ \\
\hline B-cell NHL/ T-cell NHL (\%) & $64(84) / 12(16)$ & $208(87) / 30(13)$ & 315 (76) / 98 (24) \\
\hline Chemosensitive @HCT (\%) & $56(74)$ & $192(81)$ & $361(87)$ \\
\hline Prior auto-HCT (\%) & $17(22)$ & $68(29)$ & $138(33)$ \\
\hline MAC / RIC (\%) & $6(8) / 70(92)$ & $13(5) / 225(95)$ & $30(7) / 383(93)$ \\
\hline Matched sibling HCT (\%) & $46(61)$ & $87(37)$ & $120(29)$ \\
\hline $\begin{array}{l}\text { Median follow-up of survivors, } \\
\text { months (range) }\end{array}$ & $128(3-168)$ & $73(13-120)$ & $36(6-65)$ \\
\hline
\end{tabular}

[[O137 Table] 1. Table 1]
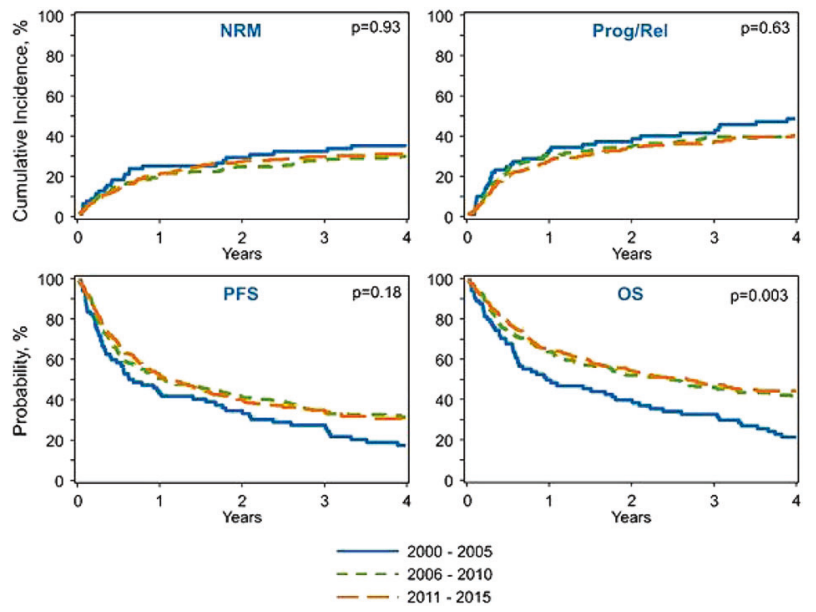

[[O137 Image] 1. Figure 1] 
Clinical Trial Registry: NA

Disclosure: No conflicts of interest to disclose

\section{8}

Graft source and pre-transplant disease status are the main variables affecting the outcome of $T$ cell-replete haploidentical transplantation with post-transplant cyclophosphamide for hodgkin lymphoma

Jacopo Mariotti ${ }^{1}$, Raynier Devillier ${ }^{2}$, Stefania Bramanti ${ }^{1}$, Laura Giordano, Barbara Sarina', Sabine Furst', Angela Granata ${ }^{2}$, Valerio Maisano ${ }^{2}$, Thomas Pagliardini $^{2}$, Chiara De Philippis ${ }^{1}$, Maria Kogan ${ }^{1}$, Catherine Faucher $^{2}$, Samia Harbi ${ }^{2}$, Christian Chabannon ${ }^{2}$, Carmelo Carlo-Stella ${ }^{1}$, Reda Bouabdalla ${ }^{2}$, Armando Santoro ${ }^{1}$, Didier Blaise $e^{2}$, Luca Castagna ${ }^{1}$

${ }^{1}$ Humanitas Cancer Center, Rozzano, Italy, ${ }^{2}$ Institut Paoli Calmettes, Marseille, France, ${ }^{3}$ Humanitas Clinical and Research Center, Rozzano, Italy

Background: Haploidentical stem cell transplantation (Haplo-SCT) with post-transplant cyclophosphamide (PT$\mathrm{Cy}$ ) represents a potential curative strategy for patients with Hodgkin lymphoma (HL) when a matched related or unrelated donor is not available ${ }^{1}$. While bone marrow (BM) was originally the preferred stem cell source, more recently peripheral blood stem cell (PBSC) is more often used. Some retrospective studies suggest that the risk to develop acute and chronic graft-versus-host-disease (GVHD) is higher with PBSC than BM, while PBSC may reduce the risk of relapse $^{2}$. Here we analyzed the effect of stem cell source in $91 \mathrm{HL}$ patients receiving Haplo-SCT with PT-Cy, with the aim to evaluate if the final outcome is modified by the use of PBSC or BM.

Methods: From April 2009 to January 2017, 91 consecutive patients with poor prognosis HL received a Haplo-SCT with PT-Cy either from a PBSC $(n=38)$ or BM $(n=53)$. The two cohorts were similar for most characteristics, but the PBSC group had more patients with an unfavorable hematopoietic stem cell transplant comorbidity index (HCT-CI) score $\geq 3(\mathrm{p}=0.002)$ and had received a non myeloablative conditioning (NMAC; $\mathrm{p}=0.001$ ).

Results: Cumulative incidence of neutrophil $>500 / \mathrm{ul}$ at day +30 and of platelet $>20000 / \mathrm{ul}$ at day +60 were $96 \%$ (95\% CI: 89-98) and 96\% (95\% CI: 88-99), respectively, with no significant differences between the PBSC and BM cohorts. With a median follow-up of 22.8 months, there was no difference between PBSC and BM graft in terms of cumulative incidence of grade 2-4 acute GVHD (29\% vs $21 \%, \mathrm{p}=0.3$ ), grade $3-4$ acute GVHD ( $3 \%$ vs $4 \%, \mathrm{p}=0.7$ ) and moderate-severe chronic GVHD (9\% vs $7 \%, \mathrm{p}=0.7$ ). This was also confirmed by multivariate analysis. In the whole population, the 2-year overall survival (OS), 2-year progression-free survival (PFS) and 1-year GVHD/relapse free survival (GRFS) rates were $67 \%, 58 \%$ and $58 \%$, respectively. We observed a trend for improved OS (74\% vs $62 \%, p=0.07)$ and PFS $(62 \%$ vs $56 \%, p=0.1)$ for recipients of PBSC relative to BM cells, but pre-transplant disease status was the only significant variable by univariate analysis (Table I). By multivariate analysis, pre-transplant active disease status, transplant from a BM and HCT-CI $\geq 3$ remained the only independent predictors of adverse outcome in terms of OS; PFS and GRFS (Table I). Nonrelapse mortality was not affected by graft source both by univariate and multivariate analysis, while pre-transplant disease status was the only variable affecting the chance of disease relapse.

Conclusions: Overall these data suggest that PBSC is associated with better outcome, in terms of OS, PFS and GRFS, relative to BM cells as graft source for patients undergoing Haplo-SCT with PT-Cy. In addition, the risk of acute and chronic GVHD is not increased after PBSC relative to $\mathrm{BM}$ graft.

\section{References:}

1. Mariotti J, Devillier R, Bramanti S, et al. Biol Blood Marrow Transplant. 2018;24:627-632

2. Martínez C, Gayoso J, Canals C, et al. J Clin Oncol. 2017;35:3425-3432

Disclosure: The authors have no conflict of interests to disclose

\section{9}

Consensus CRS and neurotoxicity regrading of "Juliet": Phase II prospective study of tisagenlecleucel therapy in patients with relapsed/ refractory large B cell lymphoma

Richard Maziarz ${ }^{1}$, Frederick Locke ${ }^{2}$, Solveig Ericson ${ }^{3}$, Elisha Rusch ${ }^{3}$, Vadim Romanov ${ }^{3}$, James Signorovitch ${ }^{4}$, David Maloney, Stephen Schuster ${ }^{6}$

${ }^{1}$ Oregon Health \& Science University, Portland, OR, United States, ${ }^{2}$ Moffitt Cancer Center, Tampa, FL, United States, ${ }^{3}$ Novartis, Inc, Morristown, NJ, United States, ${ }^{4}$ Analysis Group, Inc, Boston, MA, United States, ${ }^{5}$ Fred Hutchinson Cancer Research Center, Seattle, WA, United States, ${ }^{6}$ University of Pennsylvania Perelman School of Medicine, Philadelphia, PA, United States

Background: Patients with relapsed or refractory diffuse large B cell lymphoma have improved outcomes with CD19 directed immune effector cell therapy. Tisagenlecleucel has been shown to eradicate CD19-expressing B cells and showed efficacy against B-cell lymphomas with 54\% overall response, and of those responders, the median 
duration of response had not been achieved by 19 months. Cytokine release syndrome (CRS) was determined by Penn scale (Porter, Sci Trans Med, 2015) and neurotoxicity (NT) by NCI CTC, recorded at $57 \%$ and $20 \%$ overall with $23 \%$ \& $11 \%$ grade III/IV respectively.

Methods: Four medical experts who had managed patients with DLBCL using different CAR T-cell therapy protocols and products independently reviewed the extracted adverse event data from the case report forms and re-graded CRS using the more commonly used Lee scale (Lee, Blood, 2014) and the NT grading for encephalopathy (modified Car $\mathrm{T}$ related encephalopathy syndrome (mCRES) (Neelapu, Nature Reviews in Clinical Oncology, 2018) while blinded to the original trial grading and other experts' grading. Re-grading assessments and disagreements concerning the assigned Lee and NCI CTC grades were later discussed and reconciled among reviewers during a live meeting. As per the investigational charter, in cases that could not be reconciled, the most conservative final assessment of any reviewer determined the final grading for any individual case.

Results: CRS: 64 of 111 (58\%) patients were originally recorded as CRS by Penn Scale, and 63 were regraded as CRS by Lee criteria. 18 of 64 subjects received anticytokine therapy overall. With this blinded reassessment, more patients were categorized as grade 1 (Lee vs Penn: 26 vs 17), fewer patients as grades 2 and 3 (18 vs 23 and 10 vs 15 , respectively) and the same number of patients as grade 4 (9 vs 9).

NT: Results were compared with data on NT determined by NCI CTC criteria of tisagenlecleucel, in which NT was broadly defined as the occurrence of any nervous system or psychiatric $\mathrm{AE}$ (eg, anxiety, dizziness, headache, peripheral neuropathy, and sleep disorder). 68 of 111 subjects were identified as having NT by CTC criteria: 34 (30.6\%) patients were identified as having grade 1/2 NT, $11(9.9 \%)$ patients as having grade $3 \mathrm{NT}, \& 5(4.5 \%)$ patients as having grade 4 NT. Evalution by mCRES grading system revealed low rates of encephalopathy/delirium: 5 (4.5\%) patients had grade 1/2 NT, $6(5.4 \%)$ patients had grade $3 \mathrm{NT}$, and 8 (7.2\%) patients had grade 4 NT. No grade 5 events were seen and the presence of CRS was associated with higher likelihood of concomitant NT.

Conclusions: These results demonstrate difficulties in identifying cross protocol toxicity assessments. Harmonized grading scales being developed for future studies will facilitate comparison of the safety profiles of different CAR T-cell and other immune effector cell products. Further analyses are ongoing of these data with the new ASBMT consensus CRS guidelines.

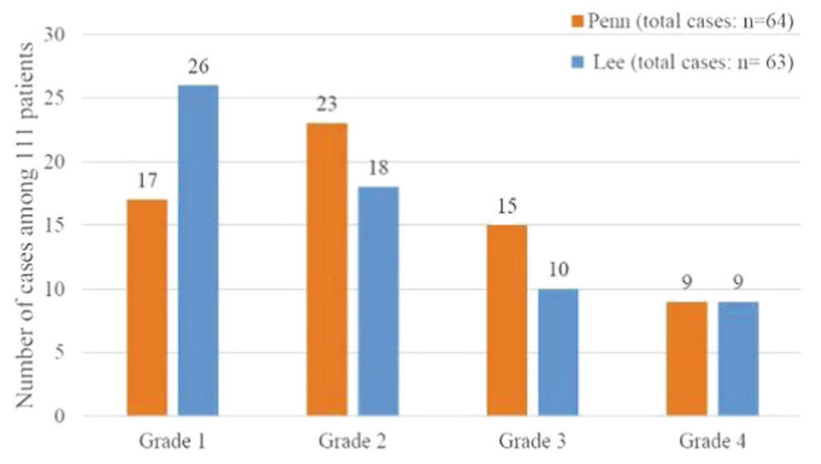

[[O139 Image] 1. CRS: Penn vs Lee scale grading of Juliet trial subjects]

Clinical Trial Registry: NCT02445248

Disclosure: Richard T. Maziarz: Honoraria, Membership of Advisory Committee and Research Funding: Novartis; Consultancy and Honoraria: Incyte, Juno Therapeutics; Honoraria: Kite Therapeutics; Patents \& Royalties: Athersys, Inc.;Employment: Oregon Health \& Science University (OHSU); The potential conflict of interest re: consultant services to and payment from Novartis has been reviewed and managed by OHSU. Stephen J. Schuster: Honoraria, Membership on an entity's Board of Directors or advisory committees and Research Funding: Celgene; Consultancy and Honoraria: Dava Oncology; Honoraria and Research Funding: Genentech; Membership on an entity's Board of Directors or advisory committees: Gilead; Consultancy, Honoraria, and Research Funding: Merck; Honoraria, Membership on an entity's Board of Directors or advisory committees and Research Funding: Novartis; Consultancy, Honoraria and Membership on an entity's Board of Directors or advisory committees: Nordic Nanovector. Vadim V. Romanoff: Employment: Novartis. Elisa S. Rusch: Employment: Novartis. James Signorovitch: Employment: Analysis Group, which

received research funding from Novartis. Solveig G. Erickson: Employment: Novartis. David G. Maloney: Research Funding: Juno Therapeutics, GlaxoSmithKline; Honoraria: Janssen Scientific Affairs, Seattle Genetics, Roche/Genentech. Frederick L. Locke: Other: Scientific Advisor: Kite Pharma, Novartis; Consultancy: Cellular BioMedicine Group Inc.

\section{0}

Abstract already published.

\section{1}

\section{Abstract already published.}


Minimal residual disease, tolerance, chimerism and immune reconstitution

\section{2}

TREG/TCON immunotherapy promotes HSC-derived B lymphopoiesis and boosts $B$ cell immunity after haploidentical transplantation

Sara Piccinelli, Antonella Mancusi ${ }^{1}$, Loredana Ruggeri ${ }^{1}$, Alessandra Carotti ${ }^{1}$, Adelmo Terenzi ${ }^{1}$, Samanta Bonato ${ }^{\prime}$, Matteo Paradiso ${ }^{1}$, Mauro Di Ianni ${ }^{2}$, Massimo Fabrizio Martelli $^{1}$, Andrea Velardi, Antonio Pierini ${ }^{1}$

${ }^{1}$ University of Perugia, Institute of Hematology-Centro di Ricerche Emato-Oncologiche (CREO), Perugia, Italy, ${ }^{2}$ University of Chieti-Pescara, Pescara, Italy

Background: Adoptive transfer of $\mathrm{CD} 4^{+} \mathrm{CD} 25^{+} \mathrm{FOXP} 3^{+}$ regulatory $\mathrm{T}$ cells (Tregs) effectively prevents conventional $\mathrm{T}$ cell (Tcons) mediated graft-versus-host disease while it does not impair graft-versus-leukemia effect in haploidentical hematopoietic cell transplantation (haplo-HCT). Recent studies show that mouse bone marrow Tregs localize in the hematopoietic stem cell (HSC) niche, where they contribute to HSCs maintenance and promote donor engraftment and B cell lymphopoiesis. We are investigating if human Tregs promote B cell reconstitution and immunity in preclinical models and in haplo-HCT patients.

Methods: B cell reconstitution was analysed monthly by FACS in bone marrow (BM) and peripheral blood (PB) samples from 51 patients who underwent either Treg/Tcon haplo-HCT (33 patients), or T-cell depleted haplo-HCT (8 patients) or haplo-HCT with high dose post-transplant $\mathrm{Cy}$ (PTCy, 10 patients). Diagnosis was acute leukemia in 39 patients, lymphoma in 9 and multiple myeloma in 3. PB total immunoglobulin (Ig) and anti-Cytomegalovirus (CMV) IgM were also monitored together with CMV viremia. For the mouse model, donor derived human Treg and $\mathrm{CD}^{+} 4^{+}$hematopoietic stem cells (HSCs) were coinfused in sublethally irradiated (2 Gy) immune-deficient NSG mice and donor engraftment and $\mathrm{B}$ cell reconstitution were analysed in mouse PB twice a month by FACS.

Results: B cell reconstitution was faster after Treg/Tcon haplo-HCT when compared to other haplo-HCT protocols. B cell counts were higher in PB of patients that received Treg/ Tcon haplo-HCT $(\mathrm{p}=.02)$ and were comparable to those of healthy subjects by 4 months after transplant $(131 \pm 121$ cells/ $\mathrm{mm}^{3}$, Fig.1A). We could detect early frequencies of CD34 ${ }^{+} \mathrm{CD} 38^{+} \mathrm{CD} 10^{+} \mathrm{CD} 127^{+}$common lymphoid progenitors, $\mathrm{CD}_{4}{ }^{+} \mathrm{CD} 10^{+} \mathrm{CD} 38^{+} \mathrm{CD} 19^{-} \quad$ Pre/Pro-B, $\quad \mathrm{CD} 45^{+} \mathrm{CD} 10$ ${ }^{+} \mathrm{CD} 38^{+} \mathrm{CD} 19^{+}$Pre-B, and Pro-B cells in the BM of these patients, that resulted in an increased production of CD38
${ }^{+} \mathrm{CD}_{19}{ }^{+} \mathrm{CD}^{-} \mathrm{IgM}^{+}$immature $\mathrm{B}$ cells, CD38 ${ }^{+} \mathrm{CD} 19^{+} \mathrm{CD} 5$ ${ }^{+} \mathrm{IgM}^{+}$transitional B cells and $\mathrm{CD} 19^{+} \mathrm{CD} 20^{+}$mature B cells.

We used a mouse model of xenotransplantation to understand whether donor B cell reconstitution in Treg/ Tcon haplo-HCT is boosted by a Treg-mediated effect on donor human HSCs. We found that infusion of human Tregs facilitated donor HSC engraftment. HSC-derived mature B cells were rapidly abundant and easily detectable 60 days after HSC infusion in PB of Treg-treated animals.

To evaluate donor $\mathrm{B}$ cell function we analysed Ig production in response to $\mathrm{CMV}$ reactivation in transplanted patients. Post-transplant hypogammaglobulinemia was rapidly corrected in Treg/Tcon haplo-HCT patients. Total IgM were higher compared to other haplo-HCT protocols and reached normal levels by 3 months after transplant (152 $\pm 194 \mathrm{mg} / \mathrm{dL}$,Fig.1B). CMV reactivation rate was similar among haplo-HCT protocols $(\sim 73 \%)$, but it occurred later after Treg/Tcon immunotherapy $(45+/-17$ days vs 33 $+/-11$ days). New production of anti-CMV specific IgM was documented in $60 \%$ of CMV seropositive patients 76 + -/33 days after Treg/Tcon haplo-HCT, while anti-CMV specific IgM were undetectable after other haplo-HCT protocols within the first 6 months after transplant.
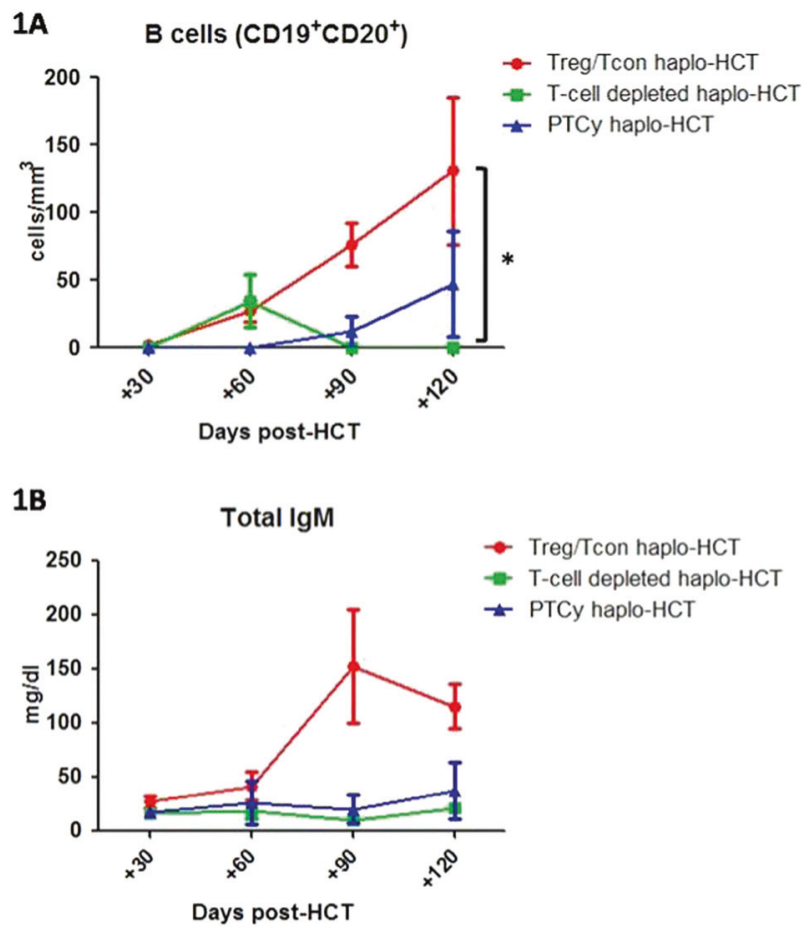

[[O142 Image] 1. Peripheral blood B cell reconstitution (1A) and total IgM levels (1B) after haplo-HCT]

Conclusions: Adoptive transfer of human Tregs boosts donor HSC engraftment and facilitates the reconstitution of functional HSC-derived donor B cells. Such results suggests 
that Treg/Tcon haplo-HCT patients could be early vaccinated after transplant. Treg/Tcon immunotherapy promotes control of infections and B cell immunity in patients undergoing haplo-HCT.

Disclosure: Nothing to declare

\section{3}

Integral analysis of minimal residual disease and hematopoietic chimerism for relapse prediction using digital droplet PCR

Miguel Waterhouse, Dietmar Pfeifer ${ }^{2}$, Jesus DuqueAfonso $^{2}$, Justus Duyster ${ }^{2}$, Hartmut Bertz ${ }^{2}$, Jürgen Finke ${ }^{2}$

${ }^{1}$ Freiburg University Medical Center, Freiburg, Germany, ${ }^{2}$ Freiburg University Medical Center, Freiburg, Germany

Background: Allogeneic cell transplantation (HSCT) success prediction is partly based on minimal residual disease (MRD) and hematopoietic chimerism testing. We developed a droplet digital PCR platform (dPCR) for the simultaneous detection of MRD and hematopoietic chimerism.

Methods: A panel of 12 deletion/insertion polymorphic markers and frequent molecular targets used for MRD testing: NPM1, RUNX1-RUNXIT1 (t 8;21), DNMT3A, MLL-PTD, CBFß-MYH11 (Inv 16), KRAS, MLL-AF10, IDH 1/2, cKIT, BCR-ABL (t9; 22), EVII and WTI expression were included in a single dPCR platform for MRD and hematopoietic chimerism analysis. A total of 225 patients were evaluated with a mean follow-up of 653 days (range: 30-5895 days).

Results: Hematopoietic chimerism analysis revealed mixed chimerism (MC) in 93 patients (41\% of all patients). $\mathrm{MC}$ was detected more frequently in patients with reduced intensity conditioning (68\%) when compared with full conditioning (47\%). The mean percentage of host derived DNA in peripheral blood was 13\% (range: $0.1-90 \%$ ). Three different patterns of MC were observed: increasing, decreasing or stable MC. In those patients with stable or decreasing MC $(n=46)$, as well as patients with complete donor chimerism $(n=132)$ the molecular targets used for MRD monitoring were not detectable. In 47 out of 93 patients, increasing $\mathrm{MC}$ was detected. This group of patient showed in addition either a positive MRD marker, increased WTl expression or both. In 37 patients with increasing MC, a positive MRD marker and increased WT1 expression hematologic relapse of the underlying disease was observed. In patients with increasing MC and a positive MRD marker, we analysed whether MC or the molecular target for MRD was first detected. In $40 \%$ of the cases MC was detected before the molecular marker used for MRD assessment, while in $13 \%$ of the patients MRD positivity was detected before MC. In the remaining patients ( $47 \%$ of the patients) MC and MRD positivity were detected simultaneously. The mean time between either $\mathrm{MC}$ or MRD detection in peripheral blood and relapse was 116 days (range: 15-385 days). Patients with increasing MC and MRD positivity, whether or not they responded to treatment, showed a similar kinetic pattern for the chimerism and MRD markers. In those patients that responded to molecular relapse treatment $(n=10)$ the mean time to achieve complete donor chimerism or MRD PCR negativity was 305 days and 294 days respectively.

Conclusions: The combination of MRD and chimerism markers in a dCPR platform represents a sensitive and accurate diagnostic tool for the comprehensive assessment of the molecular remission status after HSCT. In addition, by using the developed APCR platform costs and turnaround times can be reduced.

Disclosure: Nothing to declare

\section{4}

Preemptive interferoN- $\alpha$ treatment in acute leukemia patients with minimal residual disease after allogeneic hematopoietic stem cell transplantation: Long-term results from two prospective studies

Xiao-Dong Mo ${ }^{1}$, Xiao-Hui Zhang ${ }^{1}$, Lan-Ping $X u^{1}, Y u$ Wang $^{1}$, Chen-Hua Yan', Huan Chen ${ }^{1}$, Yu-Hong Chen ${ }^{1}$, Ya-Zhen Qin ${ }^{I}$, Kai-Yan Liu ${ }^{I}$, Xiao-Jun Huang ${ }^{I}$

${ }^{1}$ Peking University People's Hospital, Beijing, China

Background: Minimal residual disease (MRD) monitoring can help to indicate impending relapse of acute leukemia after allogeneic hematopoietic stem cell transplantation (allo-HSCT). Because impending relapse can be altered with early detection of low-volume disease and timely therapies, preemptive intervention is a reasonable option for patients with MRD which can spare those in remission from further therapy. Chemotherapy plus donor leukocyte infusion (Chemo-DLI) is the most important preemptive intervention, but it may lead to several complications, such as severe graft-versus-host disease (GVHD) and pancytopenia. Hypomethylating agents (HMAs) represent another potential preemptive intervention, but it only delayed the time to hematologic relapse and the long-term efficacy may be unsatisfactory. Thus far, few studies had identified the longterm efficacy of preemptive intervention with drugs in patients with MRD after allo-HSCT.Two prospective studies (NCT02027064 and NCT02185261) reported that preemptive interferon- $\alpha$ (IFN- $\alpha$ ) treatment can help clear minimal residual disease (MRD) and prevent relapse after allogeneic hematopoietic stem cell transplantation (alloHSCT). In this extension study, we aimed to identify the 
long-term clinical outcomes of preemptive IFN- $\alpha$ treatment in acute leukemia patients who were MRD positive after allo-HSCT $(n=118)$.

Methods: MRD was monitored by multiparameter flow cytometry (MFC) and TaqMan-based reverse transcriptionreal time polymerase chain reaction (PCR). A patient was considered to be MRD-positive when a single bone marrow sample tested positive by PCR or MFC. Recombinant human IFN- $\alpha-2 b$ injections were administered subcutaneously for 6 cycles (twice or thrice weekly in every 4-week cycle).

Results: The 4-year cumulative incidence of total chronic graft-versus-host disease (cGVHD) and severe cGVHD after IFN- $\alpha$ treatment was $58.5 \%$ (95\% CI, 49.5-67.5\%) and $10.2 \%$ (95\% CI, 4.7-15.7\%), respectively. The 4-year cumulative incidence of relapse and non-relapse mortality (NRM) after IFN- $\alpha$ treatment was $16.1 \%$ (95\% CI, 9.4$22.8 \%$ ) and $5.6 \%$ (95\% CI, 1.1-10.1\%), respectively. The 4year probabilities of disease-free survival and overall survival (OS) after IFN- $\alpha$ treatment were $78.3 \%$ (95\% CI, 70.6-86.0\%) and $84.0 \%$ (95\% CI, 77.1-90.9\%). In multivariate analysis, severe acute GVHD was associated with a higher risk of NRM and poorer OS, and mild to moderate cGVHD was associated with a lower risk of relapse and better survival.

Conclusions: These data confirmed that preemptive IFN$\alpha$ treatment showed long-term efficacy in patients who were MRD-positive after allo-HSCT.

Clinical Trial Registry: The study was registered at http://clinicaltrials.gov as \#NCT02185261 and \#NCT02027064.

Disclosure: The authors declare no competing financial interests.

\section{5}

A phase II clinical trial of leuprolide for enhancement of immune reconstitution after ex vivo CD34+ cell-selected allogeneic HCT with TBI-based conditioning

Christina Cho ${ }^{1,2}$, Sean M. Devlin ${ }^{1}$, Scott T. Avecilla ${ }^{1}$, Hugo Castro-Malaspina ${ }^{1,2}$, Sergio A. Giralt ${ }^{1,2}$, Ann A. Jakubowski, ${ }^{1,2}$,Molly Maloy ${ }^{1}$, Peter Maslak ${ }^{2,1}$, Richard J. O'Reilly $^{1,1}$, Esperanza B. Papadopoulos ${ }^{1,2}$, Katherine Smith $^{1}$, Roni Tamari ${ }^{1,2}$, Marcel R.M. van den Brink ${ }^{1,2}$, Miguel A. Perales ${ }^{1,2}$

${ }^{1}$ Memorial Sloan Kettering Cancer Center, New York, NY, United States, ${ }^{2}$ Weill Cornell Medical College, Cornell University, New York, NY, United States

Background: T-cell depletion using ex vivo CD34+ cell selection reduces GvHD risk after allogeneic HCT, but delayed immune reconstitution, particularly T-cell reconstitution, has limited improvement in survival. Sex steroids negatively impact lymphopoiesis, likely by thymic atrophy, and our preclinical models have shown that androgen blockade with the GnRH agonist leuprolide enhances thymopoiesis (Goldberg, JI 2009; Velardi, JEM 2014). We hypothesized that peri-HCT leuprolide could improve immune reconstitution among recipients of CD34selected HCT.

Methods: This was a phase II clinical trial of leuprolide in CD34-selected myeloablative allogeneic PBSCT for hematologic malignancies in patients aged 18-60 (NCT01746849). All participants received conditioning with TBI $1375 \mathrm{cGy}$, thiotepa $10 \mathrm{mg} / \mathrm{kg}$, and cyclophosphamide $120 \mathrm{mg} / \mathrm{kg}$; antirejection prophylaxis with rabbit ATG $5-7.5 \mathrm{mg} / \mathrm{kg}$; and palifermin $60 \mathrm{mg} / \mathrm{kg} / \mathrm{d}$ on days -13 to -11 and 0 to +2 . Patients received a 3 -month depot of leuprolide $11.25 \mathrm{mg}$ 2-6 weeks before conditioning and a second depot 3 months later. Primary endpoint was an absolute CD4+ count $>=200$ by 6 months post-HCT. Patients who died, relapsed, or otherwise did not have flow data available at day +180 were excluded from primary endpoint analysis but included in outcome analyses. We excluded flow data after secondary cell infusions (DLI, CTLs, second HCT, CD34+ cell boost) but followed recipients of these interventions for survival analysis. Descriptive statistics summarized absolute levels of lymphocyte subset counts at select time intervals. A KruskalWallis rank sum test compared counts among patients who received leuprolide/palifermin, historical controls who received palifermin alone, and historical controls who received neither. Kaplan-Meier functions estimated OS/ RFS. Cumulative incidence functions estimated GvHD/ NRM.

Results: Thirty-two patients received at least one dose of leuprolide. Median age was 39 years (range 21-57). Twenty-six(82\%) had acute leukemia, 3(9\%) MDS/MPN, and 3(9\%) CML. All but one had an 8/8-matched donor. At median follow-up of 24 months among survivors (range 761 ), estimated 2 Y OS was 74\%(95\%CI 60-91\%) and RFS $62 \%(95 \% \mathrm{CI} 46-83 \%)$. CI of TRM at $2 \mathrm{Y}$ was $10 \%(95 \% \mathrm{CI}$ $2-24 \%)$. CI of grade III-IV acute GvHD was $3 \%(95 \% \mathrm{CI}$ $0.2-14 \%$ ). Of 27 patients with evaluable flow data, 30\% achieved a CD4 + count $>=200$ by $180+/-30$ days, not significantly different from historical controls. Median lymphocyte counts at $180+/-30$ days did not differ significantly among groups (Table; Figure).

Conclusions: This phase II study did not demonstrate significant quantitative improvement in immune reconstitution after leuprolide with palifermin in recipients of TBIbased CD34-selected HCT. TCR sequencing to identify possible improvement in T-cell diversity in this cohort is forthcoming. One potential explanation for these results lies in the initial surge in sex steroid levels immediately after 
GnRH agonist use, which has been associated with thymic cellular degeneration in mouse (Velardi, JEM 2014). As direct GnRH antagonism might circumvent this effect, a follow-up phase II trial evaluating peri-transplant degarelix for enhancement of immune reconstitution is in progress.

\begin{tabular}{|c|c|c|c|c|}
\hline $\begin{array}{l}\text { CELLS/ } \\
\text { MCL, } \\
\text { MEDIAN } \\
\text { (IQR) }\end{array}$ & $\begin{array}{l}\text { NO } \\
\text { PALIFERMIN } \\
\text { OR } \\
\text { LEUPROLIDE } \\
(n=21)\end{array}$ & $\begin{array}{l}\text { PALIFERMIN } \\
(\mathrm{n}=82)\end{array}$ & $\begin{array}{l}\text { PALIFERMIN } \\
\text { AND } \\
\text { LEUPROLIDE } \\
(\mathrm{n}=27)\end{array}$ & $p$ \\
\hline $\mathrm{CD} 3+$ & $273(142-482)$ & $227(136.25-675.7$ & 5) $275(138.5-659.5$ & 5) 0.90 \\
\hline $\mathrm{CD} 4+$ & $130(92-151)$ & $118(76.5-191.5)$ & $131(58-200.5)$ & 0.98 \\
\hline $\mathrm{CD} 8+$ & $87(25-193)$ & $76.5(34-402.75)$ & $118(53.5-333)$ & 0.57 \\
\hline CD19+ & $142.5(119-392)$ & $247.5(119-392)$ & $219(152.5-391)$ & 0.15 \\
\hline
\end{tabular}

[[O145 Table] 1. Absolute Lymphocyte Subset Counts 180+/-30 Days Post-HCT]
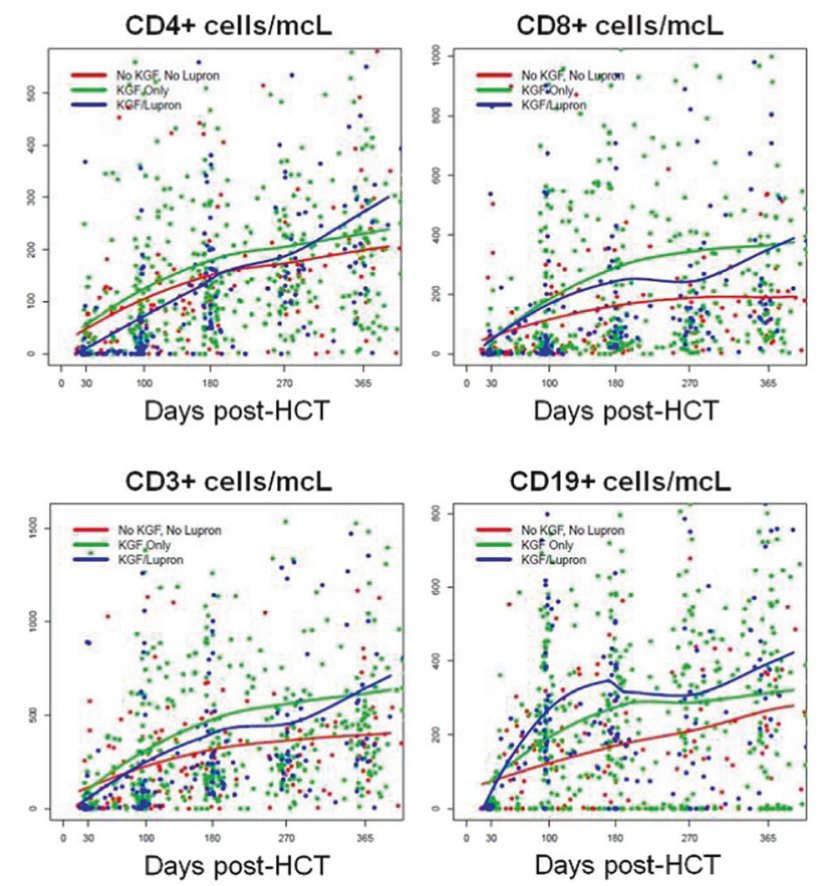

[[O145 Image] 1. Loess Smoothed Curves Estimating Overall Trends in Absolute Lymphocyte Subset Counts Post-HCT]

Clinical Trial Registry: NCT01746849 (https://clinica ltrials.gov/ct2/show/NCT01746849).

Disclosure: O'Reilly, Richard: Atara Biotherapeutics (consultancy, patents \& royalties, research funding). Perales, Miguel-Angel: Takeda (personal fees); Novartis (personal fees); Incyte (membership on an entity's Board of Directors or advisory committees, personal fees, clinical trial support); Merck (personal fees); Abbvie (personal fees).

\section{6}

Abstract already published.

\section{7}

CMV-specific $T$ cells restricted by donor, host and shared HLA molecules reconstitute with different kinetics and differentially impact the incidence of CMV reactivations after allogeneic-HSCT

Elena Tassi ${ }^{1}$, Maddalena Noviello ${ }^{I}$, Pantaleo De Simone ${ }^{1}$, Francesca Serio ${ }^{I}$, Maria Teresa Lupo-Stanghellini, Giacomo Oliveira', Sara Racca ${ }^{1}$, Roee Dvir ${ }^{1}$, Lorenzo Lazzari $^{1}$, Daniela Clerici, Fabio Giglio ${ }^{1}$, Francesca Lorentino $^{I}$, Consuelo Corti ${ }^{l}$, Massimo Bernardi ${ }^{l}$, Liselotte Brix $^{2}$ Fabio Ciceri' ${ }^{2}$,Jacopo Peccatori ${ }^{1}$, Raffaella Greco ${ }^{1}$, Chiara Bonini $^{1}$

${ }^{1}$ San Raffaele Scientific Institute, Milano, Italy, ${ }^{4}$ Immudex ApS, Copenhagen, Denmark, ${ }^{5}$ Università Vita-Salute San Raffaele, Milano, Italy

Background: In allogeneic hematopoietic stem cell transplantation (allo-HSCT) recipients, Cytomegalovirus (CMV) reactivation and disease are frequent causes of morbidity and mortality, that may be evaded by CMV-specific $\mathrm{T}$ cell reconstitution.

Methods: We designed a prospective, single-center observational study to assess if the kinetic and quality of CMV specific T-cell reconstitution impact the incidence and severity of CMV reactivations. We report data on the first 54 consecutive patients affected by hematological malignancies receiving allo-HSCT followed by Cyclophosphamide and Rapamycin between December 2017 and August 2018. Patients received allo-HSCT from family (siblings $=9$; HLA haploidentical $=21$ ), unrelated HLA matched $(n=23)$ donors or cord blood $(n=1)$. The CMV serostatus of host $(\mathrm{H})$ and donor $(\mathrm{D})$ pairs was: $\mathrm{H}^{+} / \mathrm{D}^{+}(\mathrm{n}=36,67 \%)$, $\mathrm{H}^{+} / \mathrm{D}^{-}(\mathrm{n}=17,31 \%)$ and $\mathrm{H}^{-} / \mathrm{D}^{+}(\mathrm{n}=1,2 \%) ; \mathrm{H}^{-} / \mathrm{D}^{-}$were excluded. CMV DNAemia was assessed weekly in whole blood (WB).

Absolute numbers of polyclonal and CMV-specific $\mathrm{T}$ cells were quantified by flow cytometry using Troucount $^{\mathrm{TM}}$ Tubes (BD) and Dextramer ${ }^{\circledR}$ CMV-Kit (IMMUDEX), respectively, in the graft and fresh WB at days -7 , $+30,+45,+60,+90,+120,+150$ and +180 . Dextramers permit the identification of CMV-specific lymphocytes restricted for several HLA class I molecules: $A^{* 01: 01 / ~}$ $* 02: 01 / * 03: 01 / * 24: 02$ and $B^{*} 07: 02 / * 08: 01 / * 35: 01$. These alleles allowed the longitudinal evaluation of $48(89 \%)$ patients. 
Results: At a median follow-up of 150 days post-HSCT, $28(58 \%)$ patients experienced a CMV-related clinically relevant event (CRE, median +50 days), including 7 patients $(15 \%)$ with CMV disease. For each time-point, we compared the absolute number of CMV-specific lymphocytes in patients experiencing or not a subsequent CRE. At +45 days, we observed lower CMV-specific CD8

${ }^{+} \mathrm{T}$ cells in patients prone to reactivate CMV than in not reactivating patients (median $\mathrm{CMV}$-specific $\mathrm{CD}^{+}$cells/ $\mathrm{mL}=0.125$ vs $1.69, \mathrm{p}=0.026$ ). Furthermore, patients with any Dextramer positivity at +45 days displayed a lower incidence of CRE compared with subjects who were negative (CRE probability: 0.55 vs $0.83, \mathrm{p}=0.038$ ). Patient stratification based on different thresholds of Dextramerpositive cells confirms the inverse association between CRE and CMV-specific immunity (CRE incidence in patients with: 0 cells $/ \mathrm{mL}=0.83,<1$ cells $/ \mathrm{mL}=0.66, \geq 1$ cells $/ \mathrm{mL}=$ $0.36 ; \mathrm{p}=0.046)$. We observed a higher CRE incidence in CMV $\mathrm{H}^{+} / \mathrm{D}^{-}$pairs than in $\mathrm{H}^{+} / \mathrm{D}^{+}(0.89$ vs $0.44, \mathrm{p}=0.03$ ). Taking advantage of the HLA mismatched-HSCT setting, we then dissected CMV-specific T-cell response according to HLA restriction elements $(H / D=$ shared $n=40, D$ restricted $n=12$, H-restricted $n=19$ ). In $\mathrm{H}^{+} / \mathrm{D}^{+}$pairs, we observed a fast and similar kinetic of reconstitution of CMV-specific lymphocytes restricted by H/D and D HLAs. Conversely, in $\mathrm{H}^{+} / \mathrm{D}^{-}$pairs, we detected only $\mathrm{CMV}$-specific $\mathrm{CD}^{+}$lymphocytes restricted for H/D haplotypes. Hostrestricted cells remained undetectable for the first 150 days after HSCT.

Conclusions: When the donor is CMV seropositive, a rapid and effective reconstitution of CMV-specific D-and H/D-restricted memory T cells occurs. If the donor is CMV seronegative, only H/D-restricted lymphocytes are observed early after allo-HSCT in $\mathrm{H}^{+} / \mathrm{D}^{-}$pairs. These findings indicate that CMV reactivation can prime H/D-restricted $\mathrm{T}$ cells presumably educated in the donor thymus; conversely, D- and H-restricted donor-derived lymphocytes have not yet undergone neither cross-priming nor thymic education, which might be required for full protection from CMV.

Disclosure: C.B. received research support from Molmed s.p.a. and Intellia Therapeutics. L.B. is employed by Immudex ApS. Non of the other authors has any relevant conflict of interest to disclose.

\section{8}

5-Azactidine is safe and effective therapy for prevention of disease relapse in high-risk patients with acute myeloid leukemia and myelodysplastic syndrome following allogeneic stem-cell transplantation

Ivetta Danylesko ${ }^{1}$, Noga Shem-Tov ${ }^{1}$, Adriana Del-Giglio ${ }^{1}$, Ronit Yerushalmi $^{1}$, Arnon Nagler ${ }^{1}$, Avichai Shimoni ${ }^{I}$
${ }^{1}$ Chaim Sheba Medical Center, Tel Hashomer, Ramat Gan, Israel

Background: Allogeneic stem-cell transplantation (SCT) is curative approach in patients with AML or MDS. However, disease recurrence is the major cause of treatment failure. 5azacitidine has been used in standard treatment of MDS and also in AML patients not eligible for standard chemotherapy. There is limited data on the safety and efficacy of azacitidine after SCT. We explored the use of low-dose azacitidine for prevention and treatment of relapse of AML/ MDS after SCT.

Methods: Patients in CR after SCT who were considered to be at high-risk for relapse were given azacitidine at $32 \mathrm{mg} / \mathrm{m}^{2}$ for 5 days every 28 days, planned for 2 years. Patients with overt relapse after SCT were given $32-75 \mathrm{mg} /$ $\mathrm{m}^{2}$ for 5 days until progression.

Results: Ninety-four patients, median age 64 years (2477) were given azacitidine after SCT from HLA-matched sibling $(n=29)$, matched-unrelated $(n=64)$ or haploidentical $(n=1)$ donors. Diagnosis was AML $(n=66)$ or MDS $(n=28)$. The conditioning regimen was myeloablative $(n=56)$ or reduced-intensity $(n=38) .22$ patients were given prophylactic azacitidine; 15 were in CR after SCT but at high-risk for relapse due to active disease $(n=7)$ or positive minimal-residual disease (MRD) $(\mathrm{n}=3)$ prior to $\mathrm{SCT}$, or poor-risk cytogenetics $(n=5)$. Seven patients were given azacitidine as secondary prevention after achieving CR with chemotherapy for post-transplant relapse. 19 patients were given azacitidine pre-emptively for mixed-chimerism, positive MRD after SCT or early relapse $(<10 \%$ marrow blasts). Patients in the combined prophylactic/ preemptive group $(\mathrm{n}=41)$ started azacitidine in a median of 2.4 months (1.2-14.9) after SCT and received a median of 8 courses (150). 4 patients were also given donor-lymphocyte infusions (DLI) concomitantly with azacitidine. 23 are still on therapy, 12 died or progressed, 2 stopped after long remission, 3 stopped due to patient request and only 1 discontinued due to toxicity. 17 of 22 patients given prophylactic azacitidine remained in CR. 16 of 19 patients given azacitidine preemptively achieved $\mathrm{CR}$ and 10 remained in CR. With median follow-up of 14 months (3$39), 30$ of the 41 patients in the prophylactic/ pre-emptive group are alive and 11 died. The estimated 3-year OS and PFS are 58\% (95\%CI, 29-86) and 50\% (95\%CI, 27-74), respectively. The expected PFS in this group of high-risk patients is less than $20 \% .53$ patients were given azacitidine for overt relapse after SCT. Patients were given a median of 3 courses (2-36) and 16 were also given DLI. 10 patients achieved CR, 10 stable disease and 33 progressive disease. 5 patients are still on therapy, 44 died or progressed and 4 had to discontinue due to hematological toxicity. With median follow-up of 15 months (3-43), 12 are alive and 5 
are progression-free. The 3-year OS and PFS are 17\% (95\% CI, 5-29) and 7\% (95\%CI, 0-13), respectively.

Conclusions: Azacitidine is safe and effective therapy when used prophylactically in high-risk AML/MDS patients to prevent relapse or preemptively to treat MRD or early relapse after SCT. Azacitidine maintenance may improve outcome in this high-risk patient group and should be further explored. Results of azacitidine treatment in overt post-transplant relapse are limited.

Disclosure: Nothing to declare

\section{9}

Novel mass cytometry analysis identifies reciprocal changes in NKREG and $\mathrm{CD4}_{\mathrm{EM}}$ as the dominant early immune reconstitution signature associated with subsequent acute GVHD after RIC-AHST

James Aries ${ }^{1}$, Jennifer Ball, Sarah Charrot ${ }^{1}$, Matthew Mee $^{I}$, Sofie Van Gassen ${ }^{2}$, Sameena Iqbal, John Gribben $^{I}$, Jeff Davies ${ }^{I}$

${ }^{1}$ Barts Cancer Institute, Queen Mary University of London, Centre for Haemato-Oncology, London, United Kingdom, ${ }^{2}$ Ghent University Hospital, VIB Inflammation Research Centre, Ghent, Belgium

Background: Treatment failure after allogeneic haematopoietic stem-cell transplantation (AHST) using reducedintensity conditioning (RIC) results from either too much alloreactivity and harmful acute Graft-versus-Host Disease (aGvHD) or not enough (reducing Graft-versus-Tumour effects). Studies have identified individual reconstituting immune cell subsets associated with development of clinical alloreactivity but the functionally dominant parameters remain unknown. We therefore used mass cytometry (MS) technology to determine multiple parameters simultaneously to identify dominant cellular immune reconstitution signatures associated with development of clinical alloreactivity after AHST.

Methods: Phenotypic markers identifying $>30 \mathrm{~T}, \mathrm{~B}$ and NK cell subsets known to influence alloreactivity were combined in a single MS panel. Peripheral blood from 52 patients with haematological cancer was analysed at D+30 after T-replete HLA-matched RIC-AHST using uniform conditioning. Test samples were spiked with CD45barcoded healthy control cells. Three complementary high-dimensional analytic tools were used to identify immune signatures in $\mathrm{CD}_{4} 5^{+}$lineage ${ }^{+}$cells across the whole cohort and identify differences between patients grouped by subsequent occurrence of aGvHD.

Results: Significant batch effects were effectively reduced with a novel R-based algorithm normalising data to control cells. Unsupervised clustering analysis using
Phenograph and FlowSOM algorithms identified 24 and 40 phenotypically distinct clusters respectively. Diversity analysis demonstrated lower cluster diversity $(\mathrm{P}=0.06)$ in the 20 patients who subsequently developed aGvHD consistent with perturbation of phenotypic clusters in these patients. Comparison of individual cluster abundance identified 2 cluster groups significantly different between patients who subsequently developed aGvHD and those who did not. A cluster containing cells with a $\mathrm{CD} 56^{\text {bright }} \mathrm{CD} 16^{\text {neg }} \mathrm{CD} 27^{+/-}$regulatory/tolerant $\mathrm{NK}$ cell (NKreg) phenotype was significantly reduced in patients who subsequently developed aGvHD using both Phenograph and FlowSOM algorithms $(p<0.001)$. A differentiating cell population with this phenotype was also identified in forward analysis using the CITRUS algorithm. Notably, this reduction in NKreg was accompanied by a significant increase in abundance of a cluster of $\mathrm{CCR}^{+} \mathrm{CD}^{+} 5 \mathrm{RA}^{-}$ CCR7 ${ }^{-}$CD4 effector memory T-cells (Tem) in patients who subsequently developed aGvHD. There was a significant negative correlation $(\mathrm{p}=0.01)$ between NKreg and Tem. In contrast there was no inverse correlation between CD4 regulatory $\mathrm{T}$-cells and $\mathrm{CD} 4 \mathrm{em}$. These changes were independent of clinically significant CMV reactivation. Finally, we determined the impact of time to aGvHD on this novel immune signature. Reciprocal changes in NKreg and CD4 Tem abundance were more significant in patients who developed aGvHD before D60, consistent with a dynamically evolving immune signature.

Conclusions: We show proof-of-concept that a novel pipeline can be applied to MS data to measure multiple immune reconstitution parameters after AHST. Importantly, this pipeline identified concomitant loss of NKreg and increase of CD4 Tem in patients who subsequently developed aGvHD. This is consistent with loss of NK cell-mediated control of alloreactive CD4 Tem cells as the dominant immune process preceding the development of aGvHD after RIC-AHST. Our data provide mechanistic insight into evolution of alloreactivity and support the development of strategies to maintain or expand NKreg numbers early post-transplant to reduce harmful aGvHD.

Disclosure: This study was funded by Barts and The London Charity, Greg Wolf Foundation \& Campbell-Savours Fund. Aries: Funding (as above). Ball: Bloodwise Funding. Mee: Cancer Research UK Funding. Gribben: Cancer Research UK: Research Funding; Unum: Equity Ownership; Abbvie: Honoraria; Kite: Honoraria; Acerta Pharma: Honoraria, Research Funding; Medical Research Council:Research Funding; Janssen: Honoraria, Research Funding; NIH: Research Funding; TG Therapeutics: Honoraria; Celgene: Consultancy, Honoraria, Research Funding; Roche: Honoraria; Pharmacyclics: Honoraria; Wellcome Trust:Research Funding; Novartis: Honoraria. Davies Cancer Research UK: Research Funding, Bloodwise Funding 


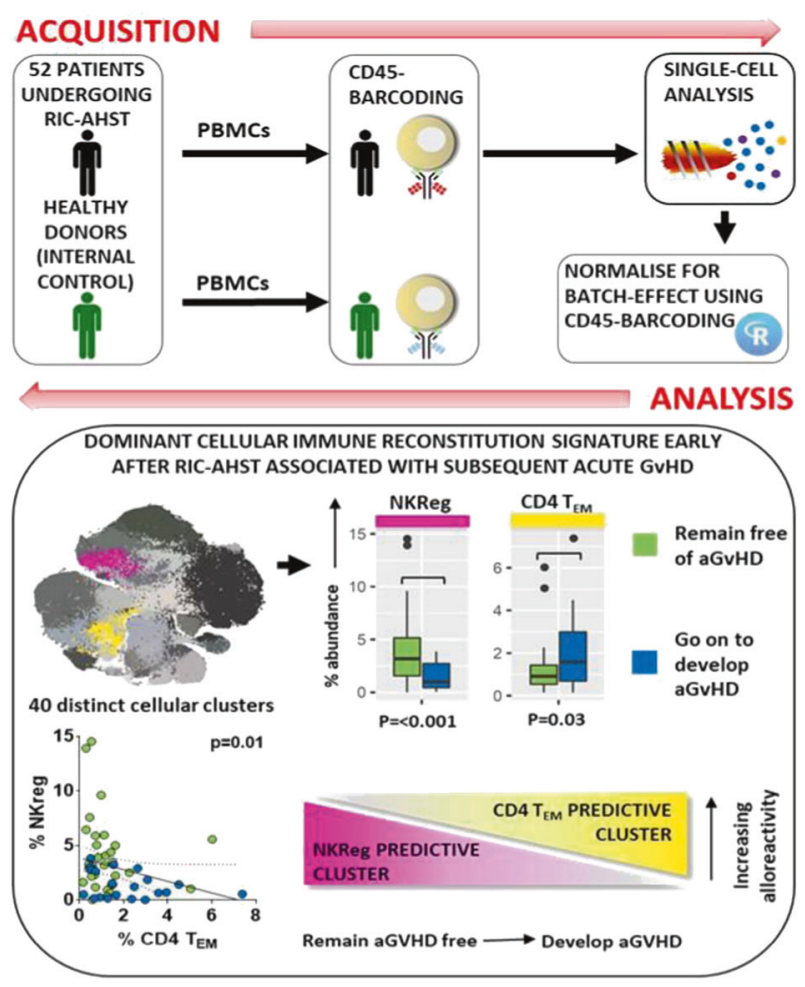

[[O149 Image] 1. Dominant immune reconstitution signature early after RIC-AHST associated with subsequent acute $G v H D$ ]

\section{Multiple myeloma}

\section{0}

Abstract already published.

\section{1}

Impact of high-risk cytogenetics in newly diagnosed multiple myeloma undergoing upfront stem cell transplantation: A study from the EBMT chronic malignancies working party

Nico Gagelmann ${ }^{1}$, Dirk-Jan Eikema ${ }^{2}$, Simona Iacobelli, Johan Maertens ${ }^{3}$, Linda Koster ${ }^{4}$, Denis Caillot ${ }^{5}$, Didier Blaise $^{6}$, Péter Remémyi ${ }^{7}$ Claude-Eric Bulabois ${ }^{8}, J a k o b$ Passweg $^{9}$, Xavier Leleu ${ }^{10}$, Samo Zver ${ }^{11}$, Guido Kobbe ${ }^{12}$, Alessandro Rambaldi ${ }^{13}$, Hareth Nahi ${ }^{14}$, Patrice Chevallier $^{15}$, Mark Ringhoffer ${ }^{16}$, Alexander M Martin ${ }^{17}$, Urpu Salmenniemi $^{18}$, Xavier Poiré ${ }^{19}$, Stig Lenhoff $f^{20}$, Pietro Pioltelli $^{21}$, Nicola Mordini ${ }^{22}$, Stefan Schönland ${ }^{23}$, Laurent Garderet $^{24}$, Ibrahim Yakoub-Agha ${ }^{25}$, Nicolaus Kröger ${ }^{1}$

${ }^{1}$ University Medical Center Hamburg-Eppendorf, Hamburg, Germany, ${ }^{2}$ EBMT Statistical Unit, Leiden, Netherlands,
${ }^{3}$ University Hospital Gasthuisberg, Leuven, Belgium, ${ }^{4}$ EBMT Data Office, Leiden, Netherlands, ${ }^{5}$ Hôpital d`Enfants Dijon, Dijon, France, ${ }^{6}$ Institut Paoli-Calmettes, Marseille, France, ${ }^{7}$ Dél-pesti Centrumkórház - Országos Hematológiai és Infektológiai Intézet, Budapest, Hungary, ${ }^{8}$ Service d'Hématologie Pôle Cancer et Maladies du Sang CHU Grenoble Alpes Université Grenoble Alpes, Grenoble, France, ${ }^{9}$ University Hospital Basel, Basel, Switzerland, ${ }^{10}$ Hopital La Miletrie, Poitiers, France, ${ }^{11}$ University Med. Center Ljubljana, Ljubljana, Slovenia, ${ }^{12}$ Klinik für Hämatologie, Onkologie und klinische Immunologie Heinrich Heine Universität, Düsseldorf, Germany, ${ }^{13}$ University of Milan and Ospedale Papa Giovanni XXIII, Bergamo, Italy, ${ }^{14}$ Karolinska Institutet and University Hospital, Stockholm, Sweden, ${ }^{15} \mathrm{CHU}$ Nantes, Nantes, France, ${ }^{16}$ Klinikum Karlsruhe gGmbH, Karlsruhe, Germany, ${ }^{17}$ Leicester Royal Infirmary, Leicester, United Kingdom, ${ }^{18}$ Turku University Hospital, Turku, Finland, ${ }^{19}$ Cliniques Universitaires St. Luc, Brussels, Belgium, ${ }^{20}$ Skanes University Hospital, Lund, Sweden, ${ }^{21}$ Ospedale San Gerardo, Clinica Ematologica dell Universita Milano-Biocca, Monza, Italy, ${ }^{22} \mathrm{Az}$. Ospedaliera S. Croce e Carle, Cuneo, Italy, ${ }^{23}$ University of Heidelberg, Heidelberg, Germany, ${ }^{24}$ Hospital Saint Antoine, Paris, France, ${ }^{25} \mathrm{CHU}$ de Lille, LIRIC, INSERM U 995, Université de Lille, Lille, France

Background: Current consensus identifies $\mathrm{t}(4 ; 14), \mathrm{t}(14 ; 16)$, $\mathrm{t}(14 ; 20)$, gain and/or deletion in chromosome 1 , and del(17/ $17 p)$ as high-risk cytogenetics in newly diagnosed multiple myeloma (NDMM). However, evidence on outcome of specific abnormalities after transplantation as first-line treatment is limited. We analyzed high-risk NDMM patients reported to the European Society for Blood and Marrow Transplantation (EBMT) registry undergoing upfront stem cell transplantation.

Methods: Upfront transplantation was defined as first autologous transplant within 12 months from MM diagnosis. Survival and cumulative incidence were calculated from date of first transplant (95\% confidence interval). End points were progression-free survival (PFS), overall survival (OS), relapse and non-relapse mortality (NRM). Cox model with hazard ratios (HR) was used for multivariable OS analyses and cumulative incidence method for relapse incidence and NRM.

Results: Within the EBMT registry, 623 high-risk NDMM patients according to cytogenetics underwent single autologous $(n=446)$, tandem autologous $(n=105)$, autologous-allogeneic $(n=72)$ stem cell transplantation between 2000 and 2015. The median follow-up of all patients was 58 months (95\% CI, 52-63 months), the median age was 59 years (range, 25-76 years) and the median time between diagnosis and transplantation was 5.6 months (range, 2.2-11.7 months). Frequencies 
according to cytogenetic were: $\operatorname{del}(17)(\mathrm{n}=333,54 \%), \mathrm{t}$ $(4 ; 14)(n=344,55 \%)$, gain or deletion in chromosome 1 $(\mathrm{n}=85,14 \%), \mathrm{t}(14 ; 16) \quad(\mathrm{n}=19,3 \%)$. Two or more cytogenetic abnormalities were documented in 143 patients (23\%). $56 \%$ of patients were male and most patients had $\operatorname{IgG}(53 \%)$ or $\operatorname{IgA}(26 \%)$ paraproteins. A Karnofsky performance status $<90 \%$ had $29 \%$ of patients while frequencies according to International Staging System (ISS) I/II/III were $21 \% / 57 \% / 22 \%$. Complete remission (CR) at time of transplantation was achieved by $18 \%$. In univariable analysis, presence vs absence of $\operatorname{del}(17)$ or $\mathrm{t}(4 ; 14)$ did not show different 5 -year OS being $53 \%$ vs $44 \%(\mathrm{p}=0.48)$ and $45 \%$ vs $53 \%(\mathrm{p}=0.26)$. Furthermore, no difference in outcome was found with respect to 5-year PFS ( $\mathrm{p}=0.82$ and 0.25 ), relapse ( $\mathrm{p}=0.54$ and 0.22 ), and NRM ( $\mathrm{p}=0.42$ and 0.82 ). Two or more abnormalities showed significantly worse 5-year OS and PFS of $44 \%$ and $17 \%$ vs one abnormality showing $51 \%$ and $21 \%(\mathrm{p}=0.04$ and 0.02 ). Relapse incidence was significantly higher in patients with two or more abnormalities resulting in $80 \%$ vs $76 \%$ $(\mathrm{p}=0.02)$ while NRM showed no difference ( $4 \%$ vs $3 \%$, $\mathrm{p}=0.78$ ). In multivariable analysis including cytogenetics and clinical variables, two or more high-risk abnormalities (HR, 1.49, 1.04-2.14; $\mathrm{p}=0.03$ ), older age (HR, 1.03, 1.01$1.04 ; \mathrm{p}=0.004)$ and stage III vs I (HR, 1.72, 1.13-2.61; $\mathrm{p}=0.01$ ) were independent predictors of worse OS while Karnofsky performance status $90-100 \%$ showed improved OS (HR, 0.72, 0.53-0.97; $\mathrm{p}=0.03$ ). Presence of either del (17) or $\mathrm{t}(4 ; 14)$ showed no difference in OS (HRs, 0.98 and $1.12 ; \mathrm{p}=0.93$ and 0.65$)$. Regarding PFS, only $<\mathrm{CR}$ at time of transplantation (HR, 1.64, 1.20-2.23; $\mathrm{p}=0.002)$ and stage III (HR, 1.59, 1.16-2.18; $\mathrm{p}=0.004)$ were associated with worse outcome.

Conclusions: In NDMM patients with at least one highrisk cytogenetic abnormality undergoing upfront transplantation, outcome was similar between $\operatorname{del}(17)$ and $t(4 ; 14)$ while the presence of two or more high-risk cytogenetic abnormalities showed significantly worse OS compared with only one high-risk abnormality.

Disclosure: Nothing to declare.

\section{2}

The role of renal impairment at diagnosis in multiple myeloma undergoing autologous transplantation. A retrospective analysis of the CMWP

Christof Scheid ${ }^{1}$, Jennifer Hoek ${ }^{2}$, Nigel H Russell ${ }^{3}$, Didier Blaise $^{4}$, Jiri Mayer ${ }^{5}$, Per Ljungman $^{6}$, Stig Lenhoff ${ }^{7}$, Guido Kobbe $^{8}$, Simona Iacobelli ${ }^{2}$, Cecilia Isaksson', Nicolaas Schaap $^{10}$, Grzegorz Basak ${ }^{11}$, Patrick Hayden ${ }^{12}$, Soledad González Muñiz ${ }^{13}$, Jane Apperley ${ }^{14}$, Alessandro Rambaldi $^{15}$, Keith M.O. Wilson ${ }^{16}$, Rocio Parody Porras ${ }^{17}$, Esa Jantunen $^{18}$, Achilles Anagnostopoulos ${ }^{19}$, Stefan
Schönland ${ }^{20}$, Ibrahim Yakoub-Agha ${ }^{21}$, Nicolaus Kröger ${ }^{22}$, Curly Morris $^{23}$, Laurent Garderet ${ }^{24}$

${ }^{1}$ Uniklinik Köln, Klinik I für Innere Medizin, Köln, Germany, ${ }^{2}$ EBMT Statistical Unit, Leiden, Netherlands, ${ }^{3}$ Nottingham University, Nottingham, United Kingdom, ${ }^{4}$ Institut Paoli Calmettes, Marseille, France, ${ }^{5}$ University Hospital Brno, Brno, Czech Republic, ${ }^{6}$ Karolinska University Hospital, Stockholm, Sweden, ${ }^{7}$ Skanes University Hospital, Lund, Sweden, Lund, Sweden, ${ }^{8}$ Heinrich Heine Universitaet, Düsseldorf, Germany, ${ }^{9}$ Umea University Hospital, Umea, Sweden, ${ }^{10}$ Radboud University - Nijmegen Medical Centre, Nijmegen, Netherlands, ${ }^{11}$ The Medical University of Warsaw, Warsaw, Poland, ${ }^{12}$ St. James`s Hospital, Dublin, Ireland, ${ }^{13}$ Hospital Universitario Central de Asturias, Oviedo Asturias, Spain, ${ }^{14}$ Hammersmith Hospital, London, United Kingdom, ${ }^{15}$ ASST Papa Giovanni XXIII, Bergamo, Italy, ${ }^{16}$ University Hospital of Wales, Cardiff, Wales, United Kingdom, ${ }^{17}$ ICO - Hospital Duran i Reynals, Barcelona, Spain, ${ }^{18}$ Kuopio University Hospital, Kuopio, Finland, ${ }^{19}$ George Papanicolaou General Hospital, Thessaloniki, Greece, ${ }^{20}$ University of Heidelberg, Heidelberg, Germany, ${ }^{21} \mathrm{CHU}$ de Lille, LIRIC, INSERM U 995, Université de Lille, Lille, France, ${ }^{22}$ University Hospital Eppendorf, Hamburg, Germany, ${ }^{23}$ Queens University of Belfast, Belfast, Ireland, ${ }^{24}$ Hospital Saint Antoine, Paris, France

Background: Renal impairment (RI) is frequent in newly diagnosed myeloma patients and is considered to be a risk factor for worse overall survival. With active myeloma therapy renal function often improves or even normalises. However, it is unclear whether renal impairment at diagnosis is a persisting biological risk factor or rather a potentially reversible organ complication.

Methods: From the EBMT CALM study database all myeloma patients having received a first autologous transplant between 2008 and 2012 with information on renal function both at diagnosis and at transplant were extracted. Renal function was classified according to the calculated glomerular filtration rate (GFR) rate as normal ("normal", GFR > $50 \mathrm{ml} / \mathrm{min}$ ), moderately impaired ("moderate", GFR 30-50 $\mathrm{ml} / \mathrm{min}$ ) or severely impaired ("severe", GFR < $30 \mathrm{ml} / \mathrm{min}$ ). Categorial variables were tested by Chi-Square test. OS was determined from transplantation and calculated by Kaplan Meier with logrank testing.

Results: 1905 patients fulfilled the selection criteria and were included. At diagnosis, 1447 patients had normal, 184 moderate and 274 severe RI. Median age at diagnosis was 58, 60 and 59 years in the RI subgroups. Genetic information was available in only a subgroup of patients. $\mathrm{t}(4 ; 14)$ was present in $14 \%, 22 \%$ and $13 \%$ respectively and 
del17 was found in 7\%, $8 \%$ and $4 \%$. Bortezomib-based induction therapy was given in $53 \%, 58 \%$ and $64 \%$ of cases ( $\mathrm{p}=0.018$ ). OS differed significantly between the RI groups with a median of 84,72 and 62 months, respectively $(\mathrm{p}<$ 0.001 , Fig 1). In contrast renal function at transplant had no impact on OS with a median of 78 months (no RI at transplant), 79 months (moderate RI at transplant) and not reached (severe RI at transplant). Most of the 274 patients with severe RI at diagnosis had improved their renal function by the time of transplantation. However, this did not positively impact on OS: Patients with no RI at transplant had a median OS of 37 months $(n=132)$, while it was 65 months for moderate RI $(n=71)$ and not reached in patients transplanted with persisting severe RI $(n=71, p<$ 0.001).

Conclusions: From this large analysis including almost 2000 myeloma patients renal impairment at diagnosis has been found to be a risk factor for OS, while renal function at transplant did not impact on post transplant survival. These findings support the safety and efficacy of autologous transplantation in patients with severe RI at transplant. On the other hand improving renal function between diagnosis and transplant does not seem to improve prognosis.. Our analysis supports the notion that RI at diagnosis appears to be a surrogate parameter for a more aggressive disease course.

Disclosure: Nothing to declare

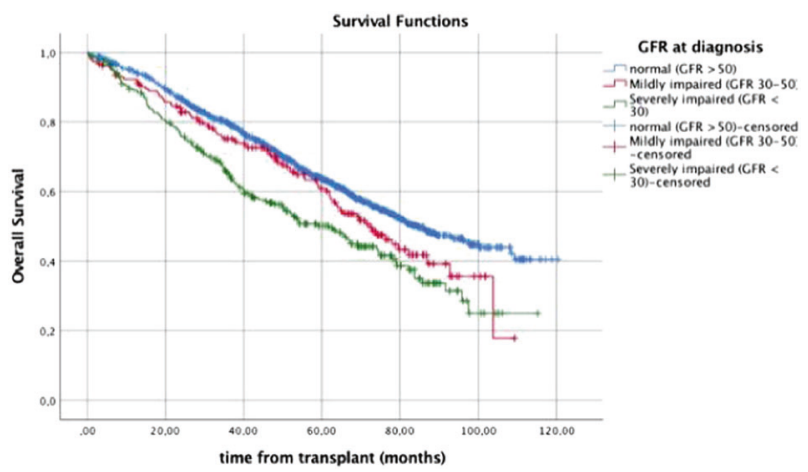

[[O152 Image] 1. Fig.1 Oerall survival according to RI at diagnosis]

\section{3}

Analysis of outcomes in patients with myeloma who had a second allohct EITHER for disease relapse or graft failure: AN EBMT CMWP study

Patrick Hayden ${ }^{1}$, Dirk-Jan Eikema ${ }^{2}$, Liesbeth de Wreede ${ }^{3}$, Linda Koster ${ }^{4}$, Nicolaus Kroger ${ }^{5}$, Herman Einsele ${ }^{6}$, Eefke Petersen $^{7}$, Emanuele Angelucci ${ }^{8}$, Michael Potter ${ }^{9}$, Jakob
Passweg ${ }^{10}$ Carlos Richard Espiga ${ }^{11}$, Veronique Leblond $^{12}$, Uwe Platzbecker ${ }^{13}$, Johanna Tischer ${ }^{14}$, Fabio Ciceri $^{15}$, Joan Hendrik Veelken ${ }^{16}$, Per Ljungman ${ }^{17}$, Nicolaas Schaap ${ }^{18}$, Noel Milpied ${ }^{19}$, Angelo Michele Carella $^{20}$, Virginie Gandemer ${ }^{21}$, William Arcese ${ }^{22}$, Adrian Bloor $^{23}$, Attilio Olivieri ${ }^{24}$, Laure Vincent ${ }^{25}$, Meral Beksac $^{26}$, Stefan Schonland ${ }^{27}$, Ibrahim Yakoub-Agha ${ }^{28}$

${ }^{1}$ Trinity College Dublin, St. James's Hospital, Dublin, Ireland, ${ }^{2}$ EBMT Statistical Unit, Leiden, Netherlands, ${ }^{3}$ LUMC, Leiden, Netherlands, ${ }^{4}$ EBMT Data Office, Leiden, Netherlands, ${ }^{5}$ University Hospital Eppendorf, Hamburg, Germany, ${ }^{6}$ Universitatsklinikum Wurzburg, Wurzburg, Germany, ${ }^{7}$ University Medical Centre Utrecht, Utrecht, Netherlands, ${ }^{8}$ Ospedale San Martino, Genova, Italy, ${ }^{9}$ Royal Marsden NHS Trust, London, United Kingdom, ${ }^{10}$ University Hospital Basel, Basel, Switzerland, ${ }^{11}$ Hospital Universitario Marqués de Valdecilla, Santander, Spain, ${ }^{12}$ Hopital la Pitié-Salpétrière, Paris, France, ${ }^{13}$ Medical Clinic and Policlinic 1, Leipzig, Leipzig, Germany, ${ }^{14} \mathrm{Kli}-$ nikum Grosshadern, Munich, Germany, ${ }^{15}$ Ospedale San Raffaele, Milano, Italy, ${ }^{16}$ Leiden University Hospital, Leiden, Netherlands, ${ }^{17}$ Karolinksa University Hospital, Stockholm, Sweden, ${ }^{18}$ Radboud University - Nijmegen Medical Centre, Nijmegen, Netherlands, ${ }^{19} \mathrm{CHU}$ Bordeaux, Bordeaux, France, ${ }^{20}$ Casa Sollievo della Sofferenza, San Giovianni, Italy, ${ }^{21}$ Centre Hospitalier Universitaire de Rennes Sud, Rennes, France, ${ }^{22}$ Rome Transplant Network - Policlinico Tor Vergata, Rome, Italy, ${ }^{23}$ Christie Hospital NHS Foundation Trust, Manchester, United Kingdom, ${ }^{24}$ Azienda Ospedali Riuniti di Ancona, Ancona, Italy, ${ }^{25}$ CHU Lapeyronie, Montpellier, France, ${ }^{26}$ Ankara University Faculty of Medicine, Ankara, Turkey, ${ }^{27}$ University of Heidelberg, Heidelberg, Germany, ${ }^{28} \mathrm{CHU}$ de Lille, LIRIC, INSERM U 995, Universite de Lille, Lille, France

Background: The options for patients with myeloma (MM) who relapse or develop graft failure after an alloSCT are limited. A second alloHCT is occasionally feasible though is high-risk. We performed a retrospective analysis to assess outcomes in this cohort.

Methods: Data on patients with MM who underwent a second alloHCT at EBMT centres between 1994 and 2017 were obtained from the EBMT registry.

Results: A total of 273 patients (165 M,108 F) with MM (51\% IgG, 22\% IgA, 23\% LC) underwent a second alloSCT. The median (range) age at the first alloHCT was 48.5 (20.1-67.9) years and 78.1\% were $>/=P R$. When comparing the indications for the $2^{\text {nd }}$ alloHCT - relapsed MM (74\%) or graft failure (26\%) - patients with graft failure were significantly more likely to have received a mismatched (related/unrelated) or unrelated donor for the $1^{\text {st }}$ alloSCT $(58 \%$ vs. $31 \%)(\mathrm{p}=0.001)$, were more commonly 
female (57\% vs. 38\%) $(\mathrm{p}=0.018)$ and were more likely to have had a RIC as opposed to a MAC alloSCT (79\% vs. $59 \%)(\mathrm{p}=0.015)$. The median (range) interval between the $1^{\text {st }}$ and $2^{\text {nd }}$ alloHCTs was 3.5 (7.7-66.6) months in cases of graft failure and 40.5 (68.6-170.4) months for those who had relapsed. At a median $(95 \% \mathrm{CI})$ follow-up of 107.2 (84.9 - 141.7) months following the second alloHCT, Overall Survival (OS) was $41 \%(35-47 \%)$ at two years and $26 \%(20-32 \%)$ at five years. There was no difference in OS at five years based on the indication for alloHCT: $25 \%$ (17$32 \%$ ) for relapse and $32 \%(19-45 \%)$ for graft failure $(\mathrm{p}=0.595)$ (Figure 1). Neutrophil engraftment following the $2^{\text {nd }}$ alloHCT was achieved by Day +28 in $94 \%(90-98 \%)$ and $80 \%(69-92 \%)$ of the relapse and graft failure patients, respectively. The cumulative incidence of aGvHD (II-IV) and cGVHD (at five years) following the $2^{\text {nd }}$ alloHCT was $27 \%(21-33 \%)$ and $39 \%(32-47 \%)$, respectively.RelapseFree Survival was $12 \%(7-17 \%)$ at 5 years, $7 \%(2-13 \%)$ in those transplanted for disease relapse and 22\% (10-35\%) in those transplanted for graft failure $(\mathrm{p}=0.061)$. The Cumulative Incidence of Relapse and NRM at five years was $64 \%$ $(57-71 \%)$ and $24 \%(18-30 \%)$, respectively. The five-year OS following the second alloSCT was 14\% (1-28\%) for those relapsing between 12 and 24 months after the first alloHCT and $31 \%(21-40 \%)$ for those relapsing later than 24 months $(\mathrm{p}=0.07)$.On univariate analysis, OS at five years was superior in patients who had had HLA identical sibling donors as opposed to other donor sources: $32 \%$ (24$40 \%)$ vs. $17 \% \quad(9-25 \%) \quad(\mathrm{p}<0.001)$. On multi-variate analysis, donor source (HLA identical sibling vs. other) remained a predictive factor for OS $(\mathrm{p}=0.014)$.

Conclusions: In this high-risk MM cohort, one quarter of patients remained alive five years after the $2^{\text {nd }}$ alloHCT with similar outcomes seen following disease relapse and graft failure. However, the relapse-free survival rate was low in those transplanted for relapsed MM. Later relapses after the first alloHCT appear to fare better and the best outcomes are seen using matched sibling donors. A $2^{\text {nd }}$ alloHCT therefore remains an option to be considered for selected MM patients.

Disclosure: Nothing to declare

\section{4}

Minimal residual disease (MRD) ratio before and after autologous stem cells transplantation (ASCT) in multiple myeloma (MM)

\section{Riccardo Boncompagni, Michela Staderini ${ }^{1}$, Chiara Nozzoli $^{1}$, Elisabetta Antonioli ${ }^{1}$, Barbara Accogli, Ric- cardo Saccardi ${ }^{1}$}

${ }^{1}$ Careggi University Hospital, Florence, Italy,
Background: In the last ten years, Multiparametric Flow Cytometry (MFC) has been standardized and routinely applied for the detection of MRD as a prognostic factor in MM patients across different lines of therapy. We assessed the MRD carried out before and after ASCT in a series of consecutive MM patients in order to investigate whether the ratio of the two determinations might increase the prognostic potential.

Methods: We collected bone marrow samples for MRD assessment at the end of induction therapy and 3 months after ASCT from 61 MM patients treated between 2013 and 2017 achieving at least a Very Good Partial Remission (VGPR) with a bortezomib-based induction therapy, according to the most recent International Myeloma Working Group (IMWG) criteria (Kumar S et al, Lancet Oncol 2016). MFC-determined MRD was evaluated according to EuroFlow recommendations (Kalina $\mathrm{T}$ et al, Leukemia 2012). All patients were examined with 18fluorodeoxyglucose positron emission tomography/computed tomography (FDG-PET/CT) scan before and after the ASCT.

Results: Post-induction therapy MRD was found predictive of post-ASCT MRD status. Indeed, patients transplanted in a MRD positive status had a significantly increased risk to maintain a MRD positivity status after transplantation (Odds Ratio - OR - 15,053, p = 0,002). Detection of post-ASCT MRD had a negative impact on median PFS (28 months vs not reached respectively, $\mathrm{p}=$ 0,001). In Cox-Regression analysis, a complete remission status (CR) with an undetectable MRD after the ASCT resulted to be the major protective factor from relapse (Hazard Ratio - HR - 0,012, p =0,005), while patients with a detectable MRD before and after the ASCT had the worse PFS (22 months, HR 2,958; $\mathrm{p}=0,029)$. Risk analysis showed 3 different PFS risk groups: "high" for the patients with MRD detectable before and after the ASCT, "intermediate" for patients with MRD positivity before the ASCT who achieve a negativity after, and "low" in the case of MRD undetectable before and after. In our study, response evaluated by FDG-PET/CT showed no correlation with PFS.

Conclusions: Multiparametric flow cytometry is a relatively recent method to assay $\mathrm{MM} M R D$, and its role in $\mathrm{MM}$ therapeutic path is still under investigation. According to our data, a detectable MRD after the ASCT is a major relapse risk. Interestingly we found that it can be early predicted by the post-induction MRD status and its negativization after ASCT has a modest impact on this. Therefore, we support the concept of treatment escalation when a CR is not reached after the induction treatment, in order to undergo to the ASCT in the best possible response. However, double MRD determination before and after 
ASCT may increase the prediction potency of currently validated.

Disclosure: Nothing to declare

\section{5}

Multiple myeloma treatment in real-world clinical practice: A focus on induction regimens prior to autologous stem cell transplantation from the prospective, multinational, non-interventional emmos study

Mohamad Mohty ${ }^{1}$, Evangelos Terpos ${ }^{2}$, Maria-Victoria Mateos $^{3}$, Michele Cavo ${ }^{4}$, Sandra Lejniece ${ }^{5}$, Meral Beksac $^{6}$, Wojciech Legiec ${ }^{7}$, Mohamed Amin Bekadja ${ }^{8}$, Meletios Dimopoulos ${ }^{2}$, Svetlana Stankovic ${ }^{9}$, Maria Soledad Durán ${ }^{10}$, Valerio De Stefano ${ }^{11}$, Alessandro Corso ${ }^{12}$, Yulia Kochkareva ${ }^{13}$, Edward Laane ${ }^{14}$, Christian Berthou $^{15}$, Hans Salwender ${ }^{16}$, Zvenyslava Masliak ${ }^{17}$, Valdas Pečeliūnas ${ }^{18}$, Wolfgang Willenbacher ${ }^{19}$, João Silva $^{20}$, Vernon Louw ${ }^{21}$, Damir Nemet ${ }^{22}$, Zita Borbényi ${ }^{23}$, Uri Abadi ${ }^{24}$, Robert Schou Pedersen ${ }^{25}$, Peter Černelč ${ }^{26}$, Anna Potamianou ${ }^{27}$, Catherine Couturier ${ }^{28}$, Caroline Feys $^{29}$, Florence Thoret-Bauchet ${ }^{30}$, Mario Boccadoro ${ }^{4}$

${ }^{1}$ Hôpital Saint-Antoine, Paris, France, ${ }^{2}$ National and Kapodistrian University of Athens, Alexandra General Hospital, Athens, Greece, ${ }^{3}$ Hospital Universitario de Salamanca, CIC, IBMCC (USAL-CSIC), Salamanca, Spain, ${ }^{4}$ Seràgnoli Institute of Hematology, University of Bologna, Bologna, Italy, ${ }^{5}$ Riga East Clinical University Hospital, Riga, Latvia, ${ }^{6}$ Ankara University, Ankara, Turkey, ${ }^{7}$ Medical University of Lublin, Lublin, Poland, ${ }^{8}$ Etablissement Hospitalier Universitaire, Oran, Algeria, ${ }^{9}$ University Clinic of Hematology, Skopje, Macedonia, the Former Yugoslav Republic of, ${ }^{10}$ Complejo Hospitalario De, Jaén, Spain, ${ }^{11}$ Catholic University, Rome, Italy, ${ }^{12}$ Fondazione IRCCS Policlinico San Matteo, University of Pavia, Pavia, Italy, ${ }^{13}$ State Budget Healthcare Institution of Moscow. City Outpatient Clinic 68 of Healthcare, Moscow, Russian Federation, ${ }^{14}$ North Estonia Medical Centre Foundation, Tallinn, Estonia, ${ }^{15}$ Université de Bretagne Occidentale (UBO), Brest, France, ${ }^{16}$ Asklepios Klinik Altona, Hamburg, Germany, ${ }^{17}$ Institute of Blood Pathology and Transfusion Medicine, Lviv, Ukraine, ${ }^{18}$ Vilnius University Hospital Santariskiu Klinikos Hematology, Oncology and Transfusion Medicine Center, Vilnius, Lithuania, ${ }^{19}$ Universitätsklinik Innsbruck Innere Medizin $\mathrm{V}$, Innsbruck, Austria, ${ }^{20}$ University Health Network (UHN), Toronto, Canada, ${ }^{21}$ University of the Free State, Bloemfontein, South Africa, ${ }^{22}$ Clinical Hospital Centre Zagreb, Zagreb, Croatia, ${ }^{23}$ Szegedi Tudomanyegyetem, Szeged, Hungary, ${ }^{24}$ Meir Medical Center, Kfar Saba, Israel, ${ }^{25}$ Regionshospitalet i Holstebro, Medicinsk Afdeling, Holstebro, Denmark, ${ }^{26}$ University Medical Centre Ljubljana,
Ljubljana, Slovenia, ${ }^{27}$ Janssen-Cilag Pharmaceuticals SACl, Athens, Greece, ${ }^{28}$ Janssen-Cilag, Issy-les-Moulineaux, France, ${ }^{29}$ Janssen Research \& Development, Beerse, Belgium, ${ }^{30}$ Janssen-Cilag, Paris, France

Background: The prospective, multinational, noninterventional EMMOS study aimed to document, and describe real-world treatment regimens and disease progression in patients with $\mathrm{MM}$ at different stages of the disease.

Methods: Adult patients initiating any new MM therapy between 2010 and 2012 were eligible. A multi-staged patient/site recruitment model was applied to minimize selection bias, and enrolment was stratified by country, region, and practice type. Patients' medical/disease features, treatment history and remission status were recorded at baseline, and prospective data on treatment, efficacy and safety were collected electronically every 3 months until 2 years after last enrolment. Responses were investigatorassessed. Overall findings from EMMOS were previously reported. Here, we are presenting additional analyses focusing on the induction regimens used in the subgroup of patients who proceeded to auto-SCT frontline.

Results: A total of 2358 patients (775 with stem-cell transplant [SCT] and 1583 without) were enrolled. Patient demographics/baseline characteristics were as expected. Of 380 recipients of SCT after enrolment, 299 (79\%) underwent auto-SCT frontline. $90 \%$ of the auto-SCT patients were aged $\leq 65$ years. Among these 299 frontline auto-SCT patients, the majority had a single transplant $(87 \%)$. The most frequent induction regimen was bortezomibthalidomide-dexamethasone (VTD; $\mathrm{n}=95 ; \quad 32 \%)$, bortezomib-dexamethasone (VD; $\mathrm{n}=56 ; \quad 19 \%)$, bortezomib-cyclophosphamide-dexamethasone (VCD; $\mathrm{n}=49 ; \quad 16 \%$ ), doxorubicin-bortezomib-dexamethasone (PAD; $\mathrm{n}=26 ; \quad 9 \%$ ), and cyclophosphamidedexamethasone-thalidomide (CTD; $\mathrm{n}=26 ; 9 \%$ ). Only $1 \%$ of patients received a bortezomib-lenalidomidedexamethasone (VRD) induction regimen, while lenalidomide was shown to be the most frequently used agent in lines 2 and 3 at time of relapse. In the VTD subgroup, most patients received $100 \mathrm{mg}$ thalidomide dose during induction. The majority of administration schedule was based on 21 days cycles, while a few other schedules were seen corresponding to 35 days cycles or to delay due to adverse events or other specific reasons. The most prevalent number of VTD cycles was 3 and 4 (42\% and 38\%, respectively). Lower or higher number of cycles was only marginal $(6 \%$ and $14 \%$, respectively). Out of the 95 patients with VTD induction, $39 \%$ achieved $\mathrm{CR}$ as best response, $28 \% \mathrm{nCR}$ or VGPR and $16 \%$ PR. After auto-SCT, the best overall response rates (ORR) at any time during frontline therapy were $>85 \%$ for those patients whose treatment included 
VTD, with $\geq 60 \%$ of patients achieving a best response of $\geq$ VGPR. The ORR in patients receiving 'other' induction therapies was $71 \%$ and the $\geq$ VGPR rate was $29 \%$. Finally, following auto-SCT, the ORRs for patients receiving VTD induction were around $80 \%, 60 \%$, and $33 \%$ in lines $2-3,4$, and $5+$, respectively.

Conclusions: This analysis provides prospective, realworld data on therapy of patients with MM receiving autoSCT. VTD is the most widely used induction regimen prior to auto-SCT. Moreover, the response rates are in line with reported rates in phase III clinical trials. While other induction regimens are being developed, VTD is likely to remain a standard of care, because access to novel agents will continue to vary greatly from country to country due to factors such as affordability, local guidelines/restrictions, and regulatory decisions. (ClinicalTrials.gov NCT01241396; Funding: Janssen Global Services, LLC.)

Clinical Trial Registry: ClinicalTrials.gov
NCT01241396

Disclosure: Study funded by Janssen Global Services, LLC

\section{6}

Abstract already published.

\section{7}

Abstract already published.

New drug- and cell-based immune therapies

\section{8}

Clinical results of a first-in-human phase 1 study Bispecific anti-CD19, ANTI-CD20 chimeric antigen receptor modified ( $\mathrm{LV20.19CAR}) \mathrm{T}$ cells for relapsed, refractory, non-Hodgkin lymphoma

Nirav Shah ${ }^{1}$, Fenlu Zhu ${ }^{1}$, Carolyn Keever-taylor ${ }^{1}$, Dina Schneider $^{2}$, Winfried Kruger ${ }^{2}$, Andrew Worden', Mehdi Hamadani, Timothy Fenske ${ }^{1}$, Bryon Johnson ${ }^{1}$, Boro Dropulic $^{2}$, Rimas Orentas ${ }^{3}$, Parameswaran Hari ${ }^{1}$

${ }^{1}$ Medical College of Wisconsin, Milwaukee, WI, United States, ${ }^{2}$ Lentigen, A Miltenyi Biotec Company, Gaithersburg, MD, United States, ${ }^{3}$ Seattle Children's Research Institute, Seattle, WA, United States

Background: CAR-T cell therapy against the CD19 antigen is a breakthrough treatment for patients with relapsed/ refractory (R/R) B-cell non-Hodgkin lymphoma (NHL). Despite impressive outcomes, non-response and relapse with CD19 negative disease remain challenges. Through dual B-cell antigen targeting of CD20 and CD19, with a first-in-human bispecific lentiviral CAR-T cell (LV20.19CAR), we attempt to improve response rates while limiting relapses due to CD19 antigen loss. Production was optimized with point of care automated manufacturing using the CliniMACS Prodigy, a compact GMP compliant tabletop device in an ISO7 clean room.

Methods: Patients were treated on our Phase 1 dose escalation + expansion trial (NCT03019055) to demonstrate feasibility of point of care manufacturing and safety of a bispecific 41BB/CD3z LV20.19CAR T cell for adults with R/R B-cell NHL. Safety was assessed by incidence of dose limiting toxicities (DLTs) within 28 days postinfusion. Dose was escalated in incremental $3+3$ fashion with a starting dose of $2.5 \times 10^{5}$ cells $/ \mathrm{kg}$ and a target cell dose of $2.5 \times 10^{6}$ cells $/ \mathrm{kg}$. Lymphodepletion was with fludarabine $30 \mathrm{mg} / \mathrm{m}^{2}$ x 3 days and cyclophosphamide 500 $\mathrm{mg} / \mathrm{m}^{2}$ x 1 day. Patients received either fresh uncryopreserved CAR-T cell infusions $(\mathrm{N}=7)$ or cells thawed $(\mathrm{N}=3)$ after cryopreservation.

Results: 10 patients have completed treatment: 9 patients in dose escalation and 1 patient in dose expansion. Median age was 55 years (46-67) and histology included DLBCL in 4 patients, MCL in 4 patients, and CLL in 2 patients. In dose escalation, 3 patients were treated at $2.5 \times 10^{5}$ cells $/ \mathrm{kg}$, 3 patients at $7.5 \times 10^{5}$ cells $/ \mathrm{kg}$, and 3 patients at $2.5 \times 10^{6}$ cells/kg with no DLTs to report. No patient experienced grade 3-4 cytokine release syndrome (CRS) or grade 3-4 neurotoxicity (NTX) allowing start of a dose expansion cohort at the $2.5 \times 10^{6}$ cells $/ \mathrm{kg}$ level. In total, 6 patients had Grade 1-2 CRS and 3 patients had Grade 1-2 NTX. Mean time to CRS was Day +9 post-infusion and no patient required ICU level care. 4 patients required 1-2 doses of tocilizumab.

The day 28 overall response rate for all patients was $80 \%$ with 5/10 achieving a complete response (CR) and 3/10 achieving a partial response (PR). All patients in CR remain in remission, the longest 15 months from treatment. CAR-T persistence is demonstrated in Figure 1. Two patients had progressive disease (PD) at Day 28 and 2 patients with PR, eventually progressed. All progressing patients underwent repeat biopsy, and all retained either CD19 or CD20 positivity. Target dose LV20.19 CAR T cells were produced in all patients indicating $100 \%$ feasibility of our manufacturing process.

Conclusions: Phase 1 results from the first-in-human bispecific LV20.19 CAR T clinical trial demonstrate that near patient manufacturing and infusion of $2.5 \times 10^{6}$ cells/ $\mathrm{kg}$ is safe for further investigation with no DLTs among treated patients. Point of care production logistics aided the administration of fresh CAR-T cells in the majority. With limited toxicity and $60 \%$ sustained response in this relapsed refractory population, this approach to CAR-T production and dual B-cell targeting merits further investigation. 
Clinical Trial Registry: NCT03019055

https://clinicaltrials.gov/ct2/show/NCT03019055

Disclosure: Nirav Shah, Fenlu Zhu, Bryon Johnson, and Parameswaran Hari report research funding from Lentigen Technology

Dina Schneider, Winfried Kruger, Andrew Worden, Boro Dropulic are employees of Lentigen Technology.

\section{9}

CAR-T cell therapy bridging to allogeneic hematopoietic cell transplantation for patients with refractory and relapsed acute lymphoblastic leukemia

Jia Chen ${ }^{1,2}$, Yi $\mathrm{Fan}^{1,2}$, Yang $\mathrm{Xu}^{1,2}$, Suning Chen ${ }^{1,2}$, Huiying Qiu ${ }^{1,2}$, Xiaowen Tang ${ }^{1,2}$, Yue Han ${ }^{1,2}$, Chengcheng $F u^{1,2}$, Depei $W^{1,2}$

${ }^{1}$ First Affiliated Hospital of Soochow University, Hematology, Suzhou, China, ${ }^{2}$ Jiangsu Institute of Hematology, Suzhou, China

Background: Refractory and relapsed (R/R) acute lymphoblastic leukemia (ALL) always leads to a dismal outcome. Allogeneic hematopoietic cell transplantation (HCT) is the only potentially curative modality for R/R ALL, but the long-term survival post-HCT remains unsatisfying. CAR-T cell therapy targeting to CD19 produces promising response for R/R ALL patients, but the recurrence is the major concern. We investigated the effectiveness of a tandem protocol using CAR-T cell therapy followed by allogeneic HCT.

Methods: We conducted a prospective study to enroll the patients with R/R ALL. Major inclusion and exclusion criteria are: 1) definitely diagnosed as ALL; 2) primary refractory (failed to achieve CR after induction) patients or relapsed patients with no response for salvage therapy; 3) with an available donor for allogeneic HCT; 4) without severe organ dysfunction or uncontrolled infection. Patients enrolled received CAR-T cell therapy targeting to CD19 with a total dose of $5 \sim 10 \times 10^{6} / \mathrm{kg}$ of recipient weight, and the preparative regimen before CAR-T cell infusion consisted of fludarabin and cyclophosphamide. After the evaluation at 30 days post CAR-T cell infusion, patients started the allogeneic HCT procedure using a myeloablative conditioning (modified $\mathrm{Bu} / \mathrm{Cy}$ ). The control group consisted of patients with $\mathrm{R} / \mathrm{R}$ ALL and underwent allogeneic HCT without CAR-T therapy in the same time frame.

Results: Totally 24 patients were enrolled in this study from December, 2016 through April, 2018, including 17 primary refractory patients and 7 relapsed patients (details in Table 1). Twenty patients (83.3\%) achieved CR after CAR-T cell therapy, and 6 patients (25\%) developed Grade
2 or higher CRS. No irreversible toxicities emerged and all the patients moved to the HCT procedure. When comparing with the control group (Figure 1), the 1-year cumulative incidence of relapse was $14.8 \pm 8.0 \%$ for the trial group versus $46.4 \pm 12.4 \%$ for the control group $(P=0.090)$, and 1 -year overall survival was $71.4 \pm 17.1 \%$ versus $54.0 \pm$ $11.4 \%(\mathrm{P}=0.067)$.

Conclusions: We concluded that CAR-T cell therapy bridging to allogeneic HCT is a promising approach for $\mathrm{R} / \mathrm{R}$ ALL patients, which leads to both high response and low risk of relapse. Besides, CAR-T cell therapy is a safe modality as salvage treatment for R/R ALL patients, which had little negative impact for following HCT.

\begin{tabular}{|c|c|c|c|}
\hline & & $\begin{array}{l}\text { Trial } \\
\text { Group } \\
(n=24)\end{array}$ & $\begin{array}{l}\text { Control } \\
\text { Group } \\
(n=20)\end{array}$ \\
\hline Age (median, range) & & $\begin{array}{l}27(13- \\
48)\end{array}$ & $31(16-47)$ \\
\hline \multirow[t]{2}{*}{ Sex (cases) } & Male & 15 & 11 \\
\hline & Female & 9 & 9 \\
\hline $\begin{array}{l}\text { High risk patients } \\
(\mathrm{NCCN}) \text { (cases) }\end{array}$ & & 11 & 10 \\
\hline $\begin{array}{l}\text { No. of previous } \\
\text { chemotherapies } \\
\text { (median, range) }\end{array}$ & & $4(2-9)$ & $5(3-8)$ \\
\hline \multirow[t]{3}{*}{ Donor (cases) } & $\begin{array}{l}\text { Haplo- } \\
\text { idientical donor }\end{array}$ & 16 & 15 \\
\hline & $\begin{array}{l}\text { Matched } \\
\text { sibling donor }\end{array}$ & 6 & 2 \\
\hline & $\begin{array}{l}\text { Matched } \\
\text { unrelated donor }\end{array}$ & 2 & 2 \\
\hline
\end{tabular}

[[O159 Table] 1. Characteristics of patients]
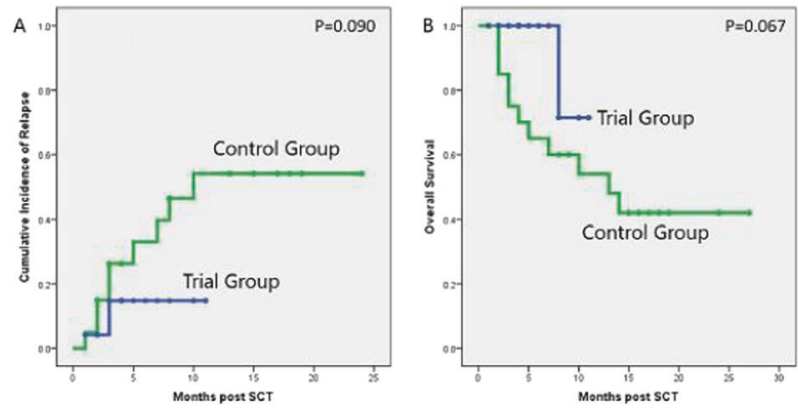

[[O159 Image] 1. Comparison of CIR and OS]

Disclosure: Nothing to declare

0160

Abstract already published. 


\section{1}

Abstract already published.

\section{Paediatric issues}

\section{2}

The role of anti-GD2 $\mathrm{CH14.18/CHO}$ monoclonal antibody based immunotherapy within the HR-NBL1/ SIOPEN trial. comparison of two treatment ERAS

Ruth Ladenstein ${ }^{1}$, Ulrike Poetschger ${ }^{2}$, Dominique Valteau-Couanet ${ }^{3}$, Roberto Luksch, Victoria Castel ${ }^{5}$, Shifra Ash ${ }^{6}$, Genevieve Laureys ${ }^{7}$, Penelope Brock ${ }^{8}$, Jean Marie Michon ${ }^{9}$, Cormac Owens ${ }^{10}$, Toby Trahair ${ }^{11}$, Godfrey Chi Fung Chan ${ }^{12}$, Ellen Ruud ${ }^{13}$, Henrik Schroeder ${ }^{14}$, Maja Beck Popovic ${ }^{15}$, Guenter Schreier ${ }^{16}$, Hans Loibner $^{17}$, Peter Ambros ${ }^{18}$, Keith Holmes ${ }^{19}$, Maria Rita Castellani $^{20}$, Mark N Gaze (1) Alberto Garaventa $^{22}$, Andrew DJ Pearson ${ }^{23}$, Holger N Lode LA $^{24}$

${ }^{1}$ St. Anna Children's Hospital and Children's Cancer Research Institute (CCRI), Medical University, Vienna, Austria, ${ }^{2}$ Children's Cancer Research Institute (CCRI), St. Anna Kinderkrebsforschung, Studies \& Statistics for Integrated Research and Projects, Vienna, Austria, ${ }^{3}$ Gustave Roussy, Institut de Cancérologie, Paris-Sud University, Paris, France, ${ }^{4}$ Fondazione IRCCS Istituto Nazionale dei Tumori, Milan, Italy, ${ }^{5}$ Hospital Universitario y Politecnico $\mathrm{La} \mathrm{Fe}$, Valencia, Spain, ${ }^{6}$ Schneider Children's Medical Center of Israel, Sackler Faculty of Medicine Tel Aviv University, Petach Tikvah, Israel, ${ }^{7}$ University Hospital Ghent, Ghent, Belgium, ${ }^{8}$ Great Ormond Street Hospital, London (retired), London, United Kingdom, ${ }^{9}$ Institut Curie, Paris, France, ${ }^{10}$ Our Lady's Children's Hospital, Crumlin, Dublin, Ireland, ${ }^{11}$ Sydney Children's Hospital, Randwick, Australia, ${ }^{12}$ Queen Mary Hospital, Hong Kong, Hong Kong, ${ }^{13}$ Rikshospitalet, Oslo, Norway, ${ }^{14}$ University Hospital of Aarhus, Aarhus, Denmark, ${ }^{15}$ University Hospital Lausanne, Lausanne, Switzerland, ${ }^{16}$ AIT Austrian Institute of Technology $\mathrm{GmbH}$, Center for Health \& Bioresources, Graz, Austria, ${ }^{17}$ Apeiron Biologics AG, CEO (retired), Vienna, Austria, ${ }^{18}$ Children's Cancer Research Institute (CCRI), Tumour Biology, Vienna, Austria, ${ }^{19}$ St George's Hospital (retired), London, United Kingdom, ${ }^{20}$ Fondazione IRCCS Istituto Nazionale dei Tumori, Nuclear Medicine, Milan, Italy, ${ }^{21}$ National Institute for Health Research University College London Hospitals Biomedical Research Centre, University College London Hospitals NHS Foundation Trust, London, United Kingdom, ${ }^{22}$ Istituto Giannina Gaslini, Genova, Italy, ${ }^{23}$ Institute of Cancer Research, Royal Marsden Hospital (retired), Sutton, United Kingdom, ${ }^{24}$ University Medicine Greifswald, Greifswald, Germany
Background: We explored the effect of dinutuximab beta (ch14.18/CHO) on outcome within the HR-NBL1/SIOPEN trial population by comparing an era prior immunotherapy availability.

Methods: The analysis cohort consists of the immunotherapy population (IP) (2009-2013) and a matched control population (CP) (2002-2009). All patients had rapid COJEC induction, up to two TVD courses and high-dose chemotherapy (BuMel or CEM) followed by autologous stem cell reinfusion (HDC/ASCR) within 9 months since diagnosis; local control included surgery and local radiotherapy (21Gy) followed by maintenance with six cycles of isotretinoin. IP patients had additional five cycles of dinutuximab beta short-term infusions with or without subcutaneous interleukin-2. CP patients had to be part of the HDC randomization. The median time between ASCR and initiation of immunotherapy was 109 days in the IP. Only patients without progressive disease at this landmark time point were included in the CP. Median follow-up was 5.8 years (IQR: 4.2 to 8.2 years) for 844 eligible patients.

Results: The 5y-EFS of the IP (378 patients) was 57\% $\pm 3 \%$ compared to $42 \% \pm 2 \%$ for the CP (466 patients; $\mathrm{p}<$ 0.001). Both populations were balanced for sex, age, stage 4, MYCN amplification and response prior HDC. Multivariate analysis showed an independent higher risk for the CP $(\mathrm{p}=0.0002$, HR 1.573), for CEM ( $\mathrm{p}=0.0029$; HR 1.431 ), a response $<\mathrm{CR}$ prior to maintenance therapy $(p=0.0043$, HR 1.494) and for $>1$ metastatic compartment at diagnosis $(\mathrm{p}<0.001$, HR 2.665). After adjustment for risk factors, the benefit of immunotherapy was confirmed in BuMel- $(\mathrm{p}=0.0066$; HR 1.439) and in CEM-treated patients $(\mathrm{p}=0.0107$; HR 2.334).

Conclusions: Results suggest a patient benefit from dinutuximab beta based immunotherapy with or without IL2 within the HR-NBL1 trial.

Clinical Trial Registry: The trial was registered with ClinicalTrials.gov (number NCT01704716) and EudraCT (number 2006-001489-17). https://www.siopen-r-net.org

Disclosure: The academic data supported APEIRON to obtain the dinutuximab beta product licensure in May 2017 in the European Union (EMA). SIOPEN and CCRI had an agreement in place with APEIRON regarding the provision of academic data. Ruth Ladenstein and Holger Lode acted as consultants for APEIRON on behalf of SIOPEN for the ch14.18/CHO development.

The other authors declared no conflicts of interest.

\section{3}

Brincidofovir is an effective treatment for adenoviremia in immunocompromised patients

Reem Elfeky ${ }^{1}$, Juliana M Furtado-Silva ${ }^{2}$, Arina Lazar$e v a^{2}$, Oana Ciocarlie ${ }^{2}$, Robert Chiesa ${ }^{2}$, Persis Amrolia ${ }^{1,2}$, 
Austen Worth', Claire Booth', Giovanna Lucchini', Bilyana Doncheva ${ }^{2}$, Waseem Qasim ${ }^{1,2}$, Paul Veys ${ }^{1,2}$, Kanchan Rao $^{2}$

${ }^{1}$ UCL Great Ormond Street Institute of Child Health, London, United Kingdom, ${ }^{2}$ Great Ormond Street Hospital, London, United Kingdom

Background: Immunocompromised patients especially post stem cell transplant are at risk of life threatening adenoviremia. Brincidofovir (BCV) is a novel oral lipid conjugate of Cidofovir with potent activity against adenoviruses (AdV) but clinical data in paediatrics are sparse.

Methods: Between August 2015 and July 2018, 24 immunocompromised patients ( 20 post-HSCT, 1 after gene therapy for ADA SCID, 2 primary immune deficiency patients and one patient with high risk neuroblastoma) received $\mathrm{BCV}$ at Great Ormond Street Hospital for management of uncontrolled viraemia \pm viral induced disease; adenoviraemia $(n=22)$, Ganciclovir resistant CMV retinitis $(n=1)$ and BK haemorrhagic cystitis $(n=1)$. 19 out of 24 (79\%) received serotherapy with Alemtuzumab $(n=12)$ or ATG $(n=6)$ before viral reactivation. Where possible immunosuppression was withdrawn in combination to BCV therapy. Viral load was detected in blood by PCR as copies/ml. Data on response to BCV was divided into complete response $(\mathrm{CR})$ with undetectable virus in blood and resolution of symptoms, partial response (PR) with at least $1 \log$ drop in viral load after BCV, no response (NR) with no change in the viral load and stationary disease and progressive disease (PD) with evidence of at least a log rise in viral load by PCR or organ disease progression.

Results: The median viral load at the start of BCV was 5.4 million copies/ml (range: $4142-85$ million copies $/ \mathrm{ml}$ ). $\mathrm{BCV}$ was used as a first line treatment in 8 cases and as second line in 16 cases after failure of first line therapy $(n=8)$, toxicity $(n=5)$ or $\operatorname{both}(n=3) .13 / 24(54 \%)$ patients had evidence of viral induced organ disease at time of BCV administration; 11 AdV disease (encephalitis, pneumonitis, hepatitis and colitis), 1 Ganciclovir resistant CMV retinitis and 1 BK haemorrhagic cystitis. 19/24 (79\%) patients achieved either a CR $(n=15)$ or PR $(n=4)$ and $8 / 13(61 \%)$ patients with organ disease achieved a CR.

Two patients with AdV disease and PR received donor derived cytotoxic $\mathrm{T}$ lymphocytes to achieve $\mathrm{CR}$. At a median follow-up of 20 months (range: 7-53.8), patients who were in CR or PR did not show any evidence of viral reactivation after $\mathrm{BCV}$ discontinuation despite no evidence of immune reconstitution (IR). Four patients had evidence of disease progression with significant rise in viral load while on BCV therapy and all 4 died. The patient with Ganciclovir resistant CMV attained CR of CMV retinitis.
Among 22 patients with AdV viraemia \pm disease; 18 (81\%) achieved either CR $(n=14)$ or PR $(n=4)$. Nine cases had concomitant $\mathrm{BK} \pm$ cystitis at the time of $\mathrm{BCV}$ therapy and all had NR. Toxicity was observed in $4 / 24$ cases; renal impairment $(n=1)$, transaminitis $(n=1)$ and diarrhoea $(n=2)$. Median CD3, CD4 and CD8 were persistently low both pre-BCV and at the end of treatment; 100 cells/ul vs 290 cells/ul, 40 cells/ul vs 70 cells/ul and 20 cells/ul vs 120 cells/ul; $p=0.2, p=0.34, p=0.5$ respectively (Figure 1). At last follow-up, $15 / 24(63 \%)$ were alive. Of these, viral infection related mortality was $4 / 24(17 \%)$.

Conclusions: $\mathrm{BCV}$ is an effective and well tolerated treatment in immune compromised patients with $\mathrm{AdV}$ infection with a response rate of $80 \%$.

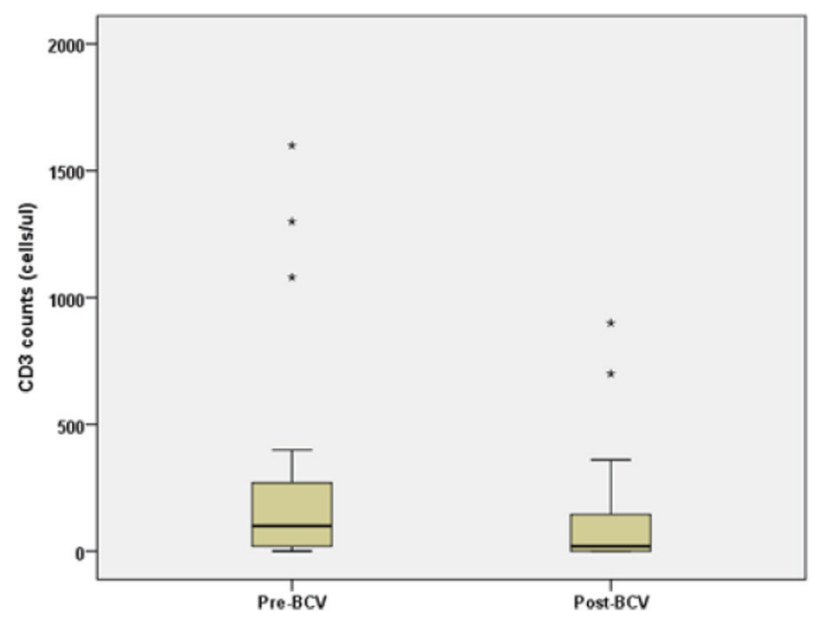

[[O163 Image] 1. Figure 1: CD3 counts during BCV therapy]

Clinical Trial Registry: Not applicable

Disclosure: Nothing to declare

\section{4}

Superior graft-versus-leukemia effect associated with transplantation of haplo-identical compared with HLAmatched sibling donor grafts for high-risk pediatric AML: A multi-centre study

Yu Wang ${ }^{1}$, Feng-Mei Zheng ${ }^{1}, X i$ Zhang $^{2}$, Chun-Fu $\mathrm{Li}^{3}$, Lan-Ping Xu ${ }^{1}$, Xiao-Hui Zhang ${ }^{1}$, Yi-Fei Cheng ${ }^{1}, \mathrm{Li} \mathrm{Gao}^{2}$, Yue-Lin $\mathrm{He}^{3}$, Xiao-Jun Huang ${ }^{1}$

${ }^{1}$ Peking University People's Hospital, Beijing, China, ${ }^{2}$ Xinqiao Hospital,Army Military Medical University, Chongqing, China, ${ }^{3}$ Nanfang Hospital, Southern Medical University, Guangzhou, China

Background: More than $50 \%$ of children referred for hematopoietic cell transplantation (HCT) lack a suitable 
matched donor. A haploidentical donor is readily available to almost all children. We sought to better define the role of haploidentical HCT for high-risk pediatric acute myeloid leukemia (AML).

\begin{tabular}{llll}
\hline Characteristics & MSD-HCT(n=23) & HID-HCT(n=69) & P value \\
\hline Age of recipient & $11(4-18)$ & $12(1-16)$ & 0.62 \\
Cytogenetic risk & & & 0.28 \\
intermediate & $11(48 \%)$ & $43(62 \%)$ & \\
poor & $8(35 \%)$ & $13(19 \%)$ & \\
unknown & $4(17 \%)$ & $13(19 \%)$ & \\
Diseases status & & & 0.36 \\
CR1 & $17(74 \%)$ & $55(80 \%)$ & \\
CR2 & $6(26 \%)$ & $11(16 \%)$ & \\
Beyond CR2 & 0 & $3(4 \%)$ & \\
\hline
\end{tabular}

[[O164 Table] 1. Patient, disease and HCT characteristics]

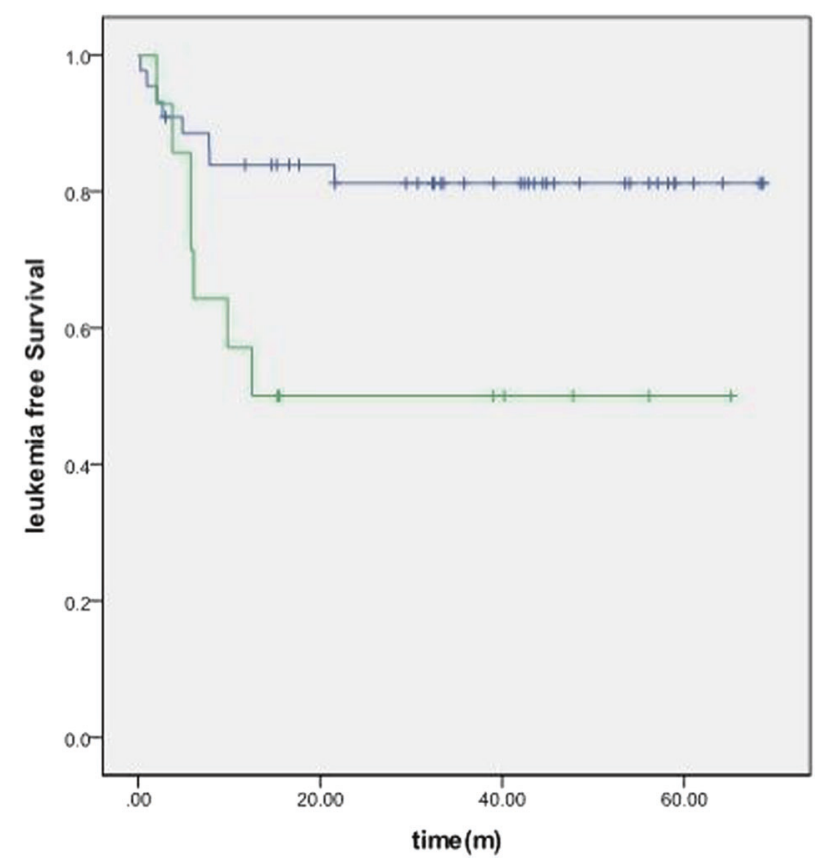

[[O164 Image] 1. Leukemia-free-survival between groups among CRI patients. Green line: MSD-HCT; blue line: HID-HCT]

Methods: To test the hypothesis that haploidentical HCT would be a valid option for high-risk pediatric AML patients lacking a matched donor, we designed a diseasespecific, multi-centre study. We retrospectively analyzed 179 consecutive patients under 18 years with high-risk AML underwent matched sibling donor (MSD) $(n=23)$ or haploidentical donor (HID) HCT $(\mathrm{n}=156)$ between July, 2013 and Dec, 2017. A 1:3 ratio matched pair analysis was implemented with the following matching factors: cytogenetic risk, disease status (CR1/CR2/>CR2), age and sex of patients, sex of donor, and graft type.

Results: All patients achieved myeloid recovery with a median time of $15 \mathrm{~d}$ and $13 \mathrm{~d}$ for MSD cohort and HID group $(\mathrm{p}=0.002)$. The cumulative incidence of grade II-IV acute graft-versus-host-disease (GVHD) in MSD cohort (13\%) was significantly lower than in HID group ( $35 \%, \mathrm{P}=0.048)$; the incidence of chronic GVHD was comparable between the two groups. The cumulative incidence of relapse in MSD cohort (39\%) was significantly higher than in HID group ( $16 \%, \mathrm{P}=0.037)$; the incidence of $\mathrm{NRM}$ was 0 and $10 \%(\mathrm{p}=0.12)$, respectively. The 3 -year overall survival $(65 \%$ versus $75 \%, \mathrm{P}=0.68)$ and leukemia free survival (61\% versus $73 \%, \mathrm{P}=0.29$ ) were comparable in MSD-HCT compared with HID-HCT. In a multivariate analysis, HIDHCT remained a significant factor for reduced relapse rate (HR 0.259(0.092-0.731), $\mathrm{P}=0.011$ ) in comparison with MSD-HCT. In subgroup analysis for patients with known cytogenetics and transplanted in the first complete remission $(\mathrm{n}=58)$, the cumulative incidence of relapse in MSD cohort $(50 \%, \mathrm{n}=14)$ was significantly higher than in HID group $(9 \%, \mathrm{n}=44, \mathrm{P}=0.001)$; and leukemia free survival $(50 \%$ versus $81 \%, \mathrm{P}=0.021$ ) were significantly lower in MSDHCT compared with HID-HCT. In a multivariate analysis among these subgroup of patients, HID-HCT remained a significant factor for increased LFS (HR 0.304(0.1020.905), $\mathrm{P}=0.032$ ) in comparison with MSD-HCT.

Conclusions: In conclusion, unmanipulated haploidentical-HCT achieves outcomes comparable to those of ISD-HCT for high-risk pediatric AML patients and even exerts greater GVL effect in some circumstances. Such transplantation was proved to be a valid alternative treatment for high-risk pediatric AML patients lacking a matched donor. Larger prospective studies are needed to confirm these findings.

Disclosure: Nothing to declare.

\section{5}

Unmanipulated haploidentical transplantation using PT-CY IN pediatric patients with acute leukemia, a survey from the PDWP OF EBMT

Annalisa Ruggeri ${ }^{1}$, Jacques-Emmanuel Galimard ${ }^{2}$, Boris Afanasyev ${ }^{3}$, Frabca Fagioli ${ }^{4}$, Akif Yesilipek ${ }^{5}$, Abdelghani Tbakhi $^{6}$, José Maria Fernandez Navarro ${ }^{7}$, Edoardo Lanino $^{8}$, Arnaud Dalissier ${ }^{9}$, Vanderson Rocha $^{10}$, Franco Locatelli $^{1}$, Selim Corbacioglu ${ }^{11}$

${ }^{1}$ Bambino Gesù Children's Hospital IRCCS, Roma, Italy, ${ }^{2}$ Paris Study Office / CEREST-TC, Saint Antoine Hospital, 
INSERM UMR 938 and Université Pierre et Marie Curie, Paris, France, ${ }^{3}$ BMT Unit, St Petersburg, Russian Federation, ${ }^{4}$ BMT Unit, Torino, Italy, ${ }^{5}$ Medical Park Hospitals, Antalya, Turkey, ${ }^{6}$ King Hussein Cancer Center, Amman, Jordan, ${ }^{7}$ BMT Unit, Valencia, Spain, ${ }^{8}$ Ospedale Gaslini, Genova, Italy, ${ }^{9} \mathrm{EBMT}$ Data Office, Paris, France, ${ }^{10} \mathrm{BMT}$ Unit, Sao Paolo, Brazil, ${ }^{11}$ BMT Unit, Regensburg, Germany

Background: Use of unmanipulated HLA-haploidentical donor transplants (haplo-HSCT) is constantly increasing in the last years. Few cases of Haplo-HSCT using posttransplant-cyclophosphamide (PT-Cy) for pediatric patients were reported by single center and registry studies, with an incidence of grade II-IV aGVHD ranging from $30 \%$ to $40 \%$ and cGVHD approaching up to $40 \%$, although with low incidence of extensive disease.

Methods: We investigated the outcomes of children $(<$ $=18 \mathrm{y}$ ) undergoing haplo-HSCT using PT-CY as GVHD prophylaxis transplanted between 2010-2017 and registered to the PDWP-EBMT.

Results: 210 patients were analyzed (ALL, $\mathrm{n}=122,58 \%$; AML, $\mathrm{n}=88,42 \%$ ); median age at haplo-HSCT was 10 years, while median follow-up was 16 months (IQR 9-24). Disease status at haplo-HSCT was CR1 for 31\%, CR2 43\% and advanced for $26 \%$. Poor-risk cytogenetics was reported in $36 \%$ of AML, and $11 \%$ had Ph+ ALL. Conditioning regimen was myeloablative (MAC) in $70 \%(\mathrm{n}=146)$ and reduced-intensity (RIC) in the remaining 30\% $(n=64)$. Thiotepa/Busulfan/Fludarabine (TBF) was the most common MAC (22\%), while RIC were mainly TBI-based (28\%). All patients received PT-Cy, in association with Tacrolimus/MMF in $33 \%$ or CSA/MMF in $28 \%$ or Tacrolimus/Sirolimus in $12 \%$. ATG was used in 15 cases. Bone-marrow (BM) was the stem cell source in 141 patients (67\%) and peripheral blood stem cells (PBSC) in the remaining 69 (33\%). Engraftment rate was $89 \%$ with 22 patients experiencing graft failure. Cumulative incidence (CI) of day-100 acute GVHD grade II-IV and grade III-IV were $33 \%$ and $14 \%$ respectively, and CI of 2-y chronic GVHD was $20 \%$ (9\% extensive disease). 2-y CI of NRM was $19 \%$ and relapse $41 \%$. Disease recurrence and infections were the most common causes of death. $2 \mathrm{y}-\mathrm{OS}$ and LFS were $49 \%$ and $40 \%$. $2 \mathrm{y}-\mathrm{OS}$ was $53 \%$ and $47 \%$ $(\mathrm{p}=0.91)$ for AML and ALL; it was $80 \%, 47 \%$ and $22 \%$ ( $\mathrm{p}<$ 0.001 ) for patients transplanted in CR1, CR2 and advanced disease. For $2 \mathrm{y}$-LFS, no significant difference was found according to the type of conditioning regimen (39\% MACchemotherapy-based, 43\% MAC-TBI based and 38\% for RIC, $p=0.97$ ). The use of PBSC was associated with higher CI of grade II-IV aGVHD (45\% versus $26 \%$ for BM recipients, $\mathrm{p}<0.001$ ).
In multivariable analysis, disease status and Karnofskiperformance-status (KPS) were factors independently associated with the main outcomes (OS, LFS, GRFS, RI). OS: CR2 vs CR1, HR 2.06 (95\%CI0.99-4.2, $\mathrm{p}=0.053$ ); advanced disease status, HR $5.2(95 \% \mathrm{CI} 2.5-11, \mathrm{p}<0.01)$; KPS $>90 \%$, HR 0.51 (95\%CI 0.3-0.8, $\mathrm{p}=0.01)$.

Disease status (CR2 vs CR1, HR 0.49 95\%CI 0.25-0.96, $\mathrm{p}=0.04$ ) and use of PBSC (HR 2.2, 95\%CI 1.27-3.82, $\mathrm{p}<$ 0.01 ) were independent factors associated with the risk of grade II-IV aGVHD. There was a trend for lower risk of cGVHD for patients transplanted for ALL (HR 0.38, 95\% CI $0.14-1, \mathrm{p}=0.05$ ).

Conclusions: PT-Cy is effective in preventing severe GVHD in children with leukemia receiving an unmanipulated haploidentical-donor transplant. Disease status remain the most important factor for outcomes. The use of PBSC as stem cell source increases the risk of grade II-IV aGVHD. The effect of long-term complications, and morbidity related to GVHD, should be considered cautiously especially in the setting of a pediatric population.

Disclosure: No disclosure

\section{6}

Hematopoetic stem cell transplantation in patients with thalassemia major: Better in younger

Gülsün Karasu', Vedat Uygun', Volkan Hazar', Suar Çakı Kılıç ${ }^{1}$, Seda Irmak Öztürkmen ${ }^{2}$, Hayriye Daloglu ${ }^{2}$, Suna Celen ${ }^{1}$, Akif Yeşilipek ${ }^{2}$

${ }^{1}$ MedicalPark Göztepe Hospital, İstanbul, Turkey, ${ }^{2}$ MedicalPark Antalya Hospital, Antalya, Turkey

Background: Allogeneic hematopoietic stem cell transplantation (HSCT) is the only curative option for patients with beta thalassemia major. Although limited study in the literature has evaluated the impact of age on success of transplantation, more data are needed. In this study, we aimed to evaluate the effect of age of the patients on transplant outcome in cases who underwent HSCT with the diagnosis of thalassemia major.

Methods: All cases who underwent stem cell transplantation with thalassemia major were included. All thalassemia major patients with a median age of 7 years (range 7 month,17.7 years) underwent allogeneic HSCT using myeloablative conditioning regimen. Cyclosporine and methotrexate were used as GVHD prophylaxis. In total, 169 patients underwent HSCT at age younger than 7 and 159 patients underwent at age older than 7 years and all patients were assigned to two different groups according to transplantation age. The distribution of donor type and stem cell sources by age groups is shown in Table 1. No statistical difference was found between the two age groups 
in terms of donor type and stem cell source. Patients in two different age groups were compared with cox regression analysis in terms of overall survival, thalassemia free survival and thalassemia- GVHD free survival.

Results: A total of 299 patients; 162 patients under 7 years of age, 137 patients aged 7 and over, were engrafted and remained transfusion independent with full or mixed chimerism. Four patients, two from each age group, did not engrafted and had primary rejection. Four patients under seven years of age developed secondary rejection, whereas in the group of patients older than 7 years of age, 17 patients experienced secondary rejection $(\mathrm{p}<0.05)$. A total of 42 patients developed acute GVHD (12.8\%)and their rates were similar in both age groups $(10.6 \%$ vs $15 \%)$. Chronic GVHD development rates in two group was also similar (9\% vs 7\%). The median follow-up time was 34 months (range 0.5-93 months). The 3 -year overall survival rates (OS), thalasemia- free survival rates (TFS), thalassemiaGVHD free survival rates (DFS) were shown in the table 2. Both thalassemia-free survival and thalassemia-GVHD free survival were higher in patients who underwent transplantation under seven years of age. There was no difference in overall survival.

\begin{tabular}{llll}
\hline $\mathrm{n}$ & & $\begin{array}{l}\text { Patients }<7 \\
\text { years } \mathrm{n}=169\end{array}$ & $\begin{array}{l}\text { Patients } \geq 7 \\
\text { years } \mathrm{n}=159\end{array}$ \\
\hline Donor type & MSD & 98 & 97 \\
& MRD & 34 & 42 \\
& MUD & 37 & 20 \\
Stem & PBSC & 28 & 38 \\
cell source & BM & 118 & 105 \\
& BM & 23 & 16 \\
& + CB & & \\
\hline
\end{tabular}

[[O166 Table] 1. Donor type and stem cell source distribution in two different age groups]

\begin{tabular}{llll}
\hline & $\begin{array}{l}\text { Overall } \\
\text { survival } \\
\text { (3 years) }\end{array}$ & $\begin{array}{l}\text { Thalassemia-free } \\
\text { survival (3 years) }\end{array}$ & $\begin{array}{l}\text { Thalassemia GVHD free } \\
\text { survival (3 years) }\end{array}$ \\
\hline $\begin{array}{l}\text { Patients } \\
<7 \text { years }\end{array}$ & $94.0 \% \pm 2.0$ & $91.8 \% 2.2$ & $88.3 \% \pm 2.5$ \\
$\begin{array}{l}\text { Patients } \\
\mathbf{7} \text { years }\end{array}$ & $92.9 \% \pm 2.2$ & $81.3 \% 3.2$ & $76.9 \% \pm 3.5$ \\
$\boldsymbol{p}$ & 0.86 & 0.012 & 0.011 \\
\hline
\end{tabular}

[[O166 Table] 2. The 3 -year survival comparison of two different age groups]

Conclusions: The results of our study show that the rates of rejection are high, thalassemia free and thalassemia / gvhd free survival are low in patients who underwent stem cell transplantation over seven years of age. In the light of successful transplantation results from unrelated donors, the delay in age of transplantation in thalassemia patients should also be avoided.

Disclosure: Nothing to declare

\section{7}

HLA-haploidentical Transplantation with regulatory and conventional $T$-cell adoptive immunotherapy in pediatric patients with high-risk acute leukemia

Maria Speranza Massei ${ }^{I}$, Ilaria Capolsini ${ }^{I}$, Katia Perruccio $^{I}$, Elena Mastrodicasa ${ }^{I}$, Francesco Arcioni ${ }^{I}$, Grazia Gurdo $^{I}$, Carla Cerri ${ }^{1}$, Loredana Ruggeri ${ }^{2}$, Alessandra Carotti $^{2}$, Franca Falzetti ${ }^{2}$, Tiziana Zei ${ }^{2}$, Roberta Iacucci Ostini $^{2}$, Cynthia Aristei ${ }^{2}$, Moira Panizza ${ }^{2}$, Mauro March$e^{e s}{ }^{1}$, Olivia Minelli ${ }^{1}$, Massimo Fabrizio Martelli ${ }^{2}$, Andrea Velardi $^{2}$, Maurizio Caniglia ${ }^{1}$

${ }^{1}$ Santa Maria della Misericordia Hospital, Perugia, Italy, ${ }^{2}$ Institute of Hematology and Bone Marrow Transplant, University of Perugia and Santa Maria della Misericordia Hospital, Perugia, Italy

Background: Post-transplant relapse is still a major cause of treatment failure in high-risk acute leukemia (AL) patients. In order to separate the GvL effect from GVHD, we investigated the role of a thymic-derived $\mathrm{CD} 4^{+} \mathrm{CD} 25^{+}$ FoxP $^{+}$regulatory $\mathrm{T}$ cells (Tregs). The Perugia center reported results from 69 adult high-risk AL patients who received an HLA haploidentical T-cell-depleted hematopoietic transplant and adoptive immunotherapy with donor Tregs and conventional $\mathrm{T}$ cells (Tcons) (and no posttransplant pharmacologic immunosuppressive GvHD prophylaxis) (Di Ianni et al., Blood 2011, Martelli et al. Blood 2014). Adoptive immunotherapy with Tregs and Tcons prevented post-transplant leukemia relapse and largely protected patients from GvHD. In this report we present a pediatric cohort of high risk leukemia patients who received a haploidentical Treg/Tcon-based hematopoietic transplant.

Methods: Twelve pediatric patients, median age of nine years (range, 5-19) with high-risk acute leukemia underwent HLA-haploidentical stem cell transplantation with regulatory and conventional T-cell adoptive immunotherapy between September 2016 and December 2017. Eleven had ALL (three $\mathrm{Ph}+$ ), one secondary AML. Seven patients were transplanted in CR1 $(3 \mathrm{Ph}+\mathrm{ALL}, 1$ ALL in CR after second-line induction, 1 ALL with extramedullary leukemia, 1 ALL with $\mathrm{t}(19 ; 11), 1$ secondary AML after medulloblastoma), two patients in CR2, three in CR3. Median time from diagnosis to transplantation was 18.5 months (range, 5-48), median time from relapse to 
transplantation for CR2 and CR3 patients was 3 months (range, 3-15). Conditioning regimen included hyperfractionated TBI, $13.5 \mathrm{~Gy}$, thiotepa $10 \mathrm{mg} / \mathrm{Kg}$, fludarabine 200 $\mathrm{mg} / \mathrm{m}^{2}$, cyclophosphamide $30 \mathrm{mg} / \mathrm{Kg}$. One patient did not receive TBI and was treated with thiotepa $8 \mathrm{mg} / \mathrm{Kg}$, fludarabine $160 \mathrm{mg} / \mathrm{m}^{2}$, treosulfan $14 \mathrm{~g} / \mathrm{m}^{2}$ and thymoglobulin $6 \mathrm{mg} / \mathrm{Kg}$. All patients received positively selected CD34+ cells (mean $11.3 \times 10^{6} / \mathrm{Kg}$, range $7.2-17.7$ ), $2 \times 10^{6}$ / $\mathrm{Kg}$ T-regs on day $-4,0.5 \times 10^{6} / \mathrm{Kg} \mathrm{T}$-cons on day -1 (4 patients received $1 \times 10^{6} / \mathrm{Kg}$ Tcons). Three patients were transplanted from NK alloreactive donors (Ruggeri et al., Science 2002).

Results: All patients achieved primary, sustained fulldonor engraftment (median neutrophils engraftment 14 days, range 9-19; median platelets 15 days, 9-19). Five patients (42\%) developed $\geq$ grade 2 aGvHD (2/5 had concomitant HCV hepatitis and developed liver aGvHD), none developed cGvHD. The immune recovery was good in all patients despite immune suppressive therapy in patients with aGvHD. Median values of T-cells at 30 days were:

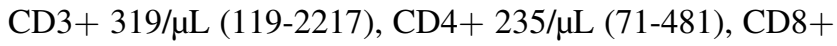
$405 / \mu \mathrm{L}(18-1465)$, at 100 days $927 / \mu \mathrm{L}$ (136-1656), 244/ $\mu \mathrm{L}$

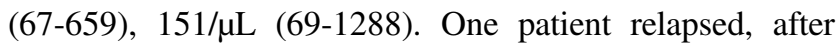
achieving CR3, he is currently receiving a second transplant from a different haploidentical familial donor. The incidence of NRM was $25 \%$ (3/12 patients). Causes of NRM were: 1 aGvHD, 1 invasive aspergillosis, 1 thrombotic microangiopathy. Nine of the 12 patients are alive at a median follow-up of 19 months (2-22 months), cGvHD/ leukemia-free survival is $75 \%$.

Conclusions: These preliminary data in 12 very high risk pediatric patients showed that HLA-haploidentical transplantation with regulatory and conventional T-cell adoptive immunotherapy prevented leukemia relapse (relapse: 1/12 patients) and was associated with an encouraging DFS $(75 \%)$.

Disclosure: Nothing to declare

\section{8}

Results of HSCT with TCR $\alpha \beta$ depletion from haploidentical donors in pediatric acute leukemia patients in complete remission

Anna Bogoyavlenskaya ${ }^{1}$, L Shelikhova ${ }^{I}$, M Ilushina $^{1}, Z$ Shekhovtsova $^{I}$, D Balashov ${ }^{I}$, I Shipitsina ${ }^{I}$, D Shasheleva ${ }^{I}$, R Khismatullina ${ }^{I}$, S Blagov ${ }^{1}$, A Livshits ${ }^{1}$, A Mitrakov ${ }^{1}, S$ Kozlovskaya $^{1}$, S Kovrygin ${ }^{1}$, Y Skvortsova ${ }^{1}$, E Kurnikova ${ }^{1}$, Y Muzalevsky', A Kazachenok ${ }^{I}$, M Fadeeva $^{I}$, A Nechesnyuk $^{I}$, Y Olshanskaya ${ }^{I}, I$ Kalinina ${ }^{I}, N$ Miakova ${ }^{I}, D$ Litvinov $^{1}$, G Novichkova $^{1}$, Maschan $^{1}$, M Maschan $^{1}$

${ }^{1}$ Dmitry Rogachev National Medical Research Center of Pediatric Hematology, Moscow, Russian Federation
Background: Relapse, GvHD and associated non-relapse mortality are the main obstacles to successful HSCT in children with leukemia. $\alpha \beta$ T cell depletion was developed to prevent GvHD and improve immune reconstitution in recipients of mismatched grafts.

Methods: A total of 89 children with AL in CR (38 AML, 51 ALL, 36 female, 53 male, median age 8,7 y) underwent allo HSCT from haploidentical donor between 06.2012 and 07.2017. 64 pts received treosulfan-based conditioning, 25 - TBI-based (ALL). Either melphalan $(n=41)$ or thiophosphamide $(n=31)$ or etoposide $(n=16)$ were added, fludarabine was used in all pts. Two types of GvHD prophylaxis were used: Type $1(\mathrm{n}=18)$ : hATG 50 $\mathrm{mg} / \mathrm{kg}$ and post-HSCT tacro/mtx, type $2(\mathrm{n}=71)$ : thymoglobulin(rATG) $5 \mathrm{mg} / \mathrm{kg}$, rituximab $200 \mathrm{mg} / \mathrm{m}^{2}$ with either bortezomib on days $+2,+5(n=62)$ or tacro $(n=6)$ or without $(n=3)$; a $\mathrm{T}$ cell depletion with CliniMACS was used in all cases. The median dose of CD34+ cells was 8 $\mathrm{x} 10^{6} / \mathrm{kg}$, a $\mathrm{T}$ cells $-16 \times 10^{3} / \mathrm{kg}$.

Results: Five patients $(5,6 \%)$ died before engraftment due to septic event. Primary engraftment was achieved in all evaluable pts (100\%) with full donor chimerism. Among the whole cohort the CI of GvHD grades II - IV and III - IV was 17,9\% (95\% CI: $12-28)$ and 3,4\% (95\% CI: $1-10)$ respectively. The CI of cGvHD was $19 \%$ (95\% CI: $12,5-$ 29). CI of aGvHD was significantly lower in a group with type 2 of GvHD prophylaxis: $11,2 \%$ (95\% CI:6-22) vs $44 \%$ (95\% CI:26-74), $\mathbf{p}=\mathbf{0 , 0 0 2}$. rATG was also effective in prevention of cGvHD: CI at 2 year after HSCT was $15 \%$ vs $33 \%, \mathbf{p}=\mathbf{0 , 0 8}$. 3-year pTRM was $12,3 \%$ (95\%CI: 7-21) and CI of relapse was 19,8\% (95\%CI: 13-31) without different between rATG and hATG. 3y pEFS was 68\% (95\%CI: 5978 ), in AML pts $83 \%$ (95\%CI: 69-96), versus $57 \%$ (95\%CI: 43-70) in ALL, $\mathbf{p}=\mathbf{0 , 0 0 7}$, without difference between rATG and hATG. Among ALL pts, who received TBI-containing regimen, pEFS was $68 \%$ (95\% CI: 50-86), as compared to 46\% (95\%CI: 27-65) without TBI, $\mathrm{p}=\mathbf{0 , 0 4 4}$ and $\mathrm{pOS}$ was $74 \%$ (95\%CI: 62-86). In the group with available immune reconstitution data $(n=68) \alpha \beta$ T cell recovery at day +30 was associated with a trend to decreased incidence of relapse, CI of relapse was $29 \%$ (95\% CI:15 - 54) in those with $\alpha \beta$-T cell count < median vs $15 \%$ (95\% CI: 6-38) in those with $\alpha \beta$-cell count $>$ median, $\mathbf{p}=\mathbf{0 , 2 5}$ and decreased TRM: $16 \%$ (95\% CI: 6,5-39) among $\alpha \beta$ T"low[ПMO1] “vs pTRM $0 \%$ among $\alpha \beta$ T"high" $\mathbf{p}=\mathbf{0 , 0 3 6}$. pEFS among $\alpha \beta$ T" high" was $85 \%(+/-10)$ vs $55 \%(+/-14)$ among $\alpha \beta$ T"low", p=0,019, pOS $89 \%(+/-10)$ vs $59 \%(+/-20)$ respectively. Recovery of the NK and gd-T cell was not associated with improved survival.

Conclusions: We confirm that the depletion of $\alpha \beta$ T cells from haplo graft in combination with intensive conditioning regimen provides a high chance of long-term survival in a cohort of children with high-risk AL in remission, 
especially for AML pts. Our analysis suggests that early $\alpha \beta$ $\mathrm{T}$ cell recovery is associated with a relatively low nonrelapse mortality and relapse rate.

Disclosure: Nothing to declare

\section{Stem cell donor}

\section{9}

Prognostic value of functional HLA-DPB1 mismatching after allogeneic stem cell transplantation from 8/8 HLAmatched unrelated donor

Francesca Lorentino $^{1}$, Sacchi Nicoletta ${ }^{2}$, Elena Oldani ${ }^{3}$, Valeria Miotti, Alessandra Picardi ${ }^{5}$, Anna Maria Gallina $^{2}$, Paolo Bernasconi ${ }^{6}$, Riccardo Saccardi ${ }^{7}$, Lucia Farina $^{8}$, Fabio Benedetti ${ }^{9}$, Michela Cerno ${ }^{4}$, Benedetto Bruno $^{10}$, Alessandro Rambaldi, Francesca Patriarca ${ }^{4}$, Fabio Ciceri', Katharina Fleischhauer ${ }^{11}$, Luca Vago ${ }^{1}$, Francesca Bonifazi ${ }^{2}$

${ }^{1}$ San Raffaele Scientific Institute, Milan, Italy, ${ }^{2}$ Italian Bone Marrow Donor Registry, Genova, Italy, ${ }^{3}$ Ospedale Papa Giovanni XXIII, Bergamo, Italy, ${ }^{4}$ Azienda Ospedaliera Universitaria - Udine, Udine, Italy, ${ }^{5}$ Tor Vergata University Hospital, Rome, Italy, ${ }^{6}$ Fondazione IRCCS Policlinico San Matteo, Pavia, Italy, ${ }^{7}$ AOUC Azienda OspedalieroUniversitaria Careggi, Firenze, Italy, ${ }^{8}$ Istituto Nazionale dei Tumori, Milan, Italy, ${ }^{9}$ University of Verona, Verona, Italy, ${ }^{10}$ A.O.U Citta della Salute e della Scienza di Torino, Torino, Italy, ${ }^{11}$ Essen University Hospital, Essen, Germany, ${ }^{12}$ Bologna University, S.Orsola-Malpighi Hospital, Institute of Hematology \& Medical, Oncology L \& A Seràgnoli, Bologna, Italy

Background: Hematopoietic stem cell transplantation (HSCT) from HLA-A, -B, -C and -DRB1-matched unrelated donors (8/8 UD) is performed across HLA-DPB1 barrier in more than $85 \%$ of cases. Clinically tolerable (permissive) mismatches (mm) at HLA-DPB1 locus have been classified by different immunogenetic models. Here we compare the prognostic value of these models.

Methods: 1033 adult patients received an 8/8 UD-HSCT in a GITMO center in 2012-2015. Only 422 donor-recipient pairs were typed for HLA-DPB1, $382(90 \%)$ having at least one $\mathrm{mm}$. Included diseases were acute leukemia (55\%), lymphoma and multiple myeloma (29\%), myelodysplastic and myeloproliferative syndromes (16\%). Disease status was early (45\%), intermediate (26\%) or advanced (29\%). Stem cell source was peripheral blood in $81 \%$ of cases. Graft-versus-host disease (GvHD) prophylaxis was ATGbased in $91 \%$ of patients. We adopted four models to classify permissive HLA-DPB1 $\mathrm{mm}$ : I) differential immunogenicity of alleles belonging to 3 groups of T-cell epitopes (Crivello, BBMT 2015); II) a similar model subdividing alleles in 4 TCE groups (TCE4, Crocchiolo, Blood 2009); III) differences in "delta functional distance" scores of 12 polymorphic aminoacids in HLA-DPB1 peptide-binding groove (Crivello, Blood 2016); IV) unidirectional mismatches in the $\mathrm{GvH}$ direction stratified according to the rs9277534 SNP in the HLA-DPB1 $3^{\prime}$ UTR region, associated to HLA-DPB1 expression (Petersdorf, NEJM 2015). While the first three models were applicable to all 382 HLA-DPB1 $\mathrm{mm}$ patients, the latter was restricted to 229 of them. The TCE4 model appeared the most restrictive one, with only $36 \%$ of mm considered to be permissive.

Median follow-up was $3.2 \mathrm{y}$.

Results: HLA-DPB1 permissive (P) mm pairs defined by TCE4 model $(\mathrm{N}=135)$ had superior 3-y overall survival (OS) and GvHD-free \& Relapse-Free Survival (GRFS) compared to non-permissive (NP) $\mathrm{mm}(\mathrm{N}=247)(60 \pm 8 \%$ vs $49 \pm 7 \%$, p .05; and $36 \pm 8 \%$ vs $29 \pm 5 \%$, p .04, respectively). A higher transplant-related mortality (TRM) and a higher 3y CI of extensive cGvHD were experienced by NP compared to $\mathrm{P}$ mm $(30 \pm 6 \%$ versus $21 \pm 6 \%$, p .09; $12 \pm 4 \%$ versus $4 \pm 2 \%, \mathrm{p} .01$, respectively). No effect was found for aGvHD or relapse. In Cox multivariate analysis (including age, donor/host gender and CMV, disease status, HCT-CI, conditioning intensity, stem cell source, ATG use, 5-loci HLA-matching, center effect), TCE4 functional DPB1 matching was prognostic for OS (HR 1.6 for NP compared to P, p .01), GRFS (HR 1.4, p .02), TRM (HR 1.9, p .01), cGvHD (HR 1.6, p .03) and extensive cGvHD (HR 3.6, p< $.01)$. The predicted HLA-DPB1 mismatched allele expression in the recipient was associated with 100-d CI of grade $\geq 2$ aGvHD: $32 \pm 10 \%$ in high expression $(\mathrm{N}=76)$ versus $16 \pm 6 \%$ in low expression $(\mathrm{N}=153), \mathrm{p}<.01$. This was confirmed in multivariate analysis for grade $\geq 2$ aGvHD (HR 2.2, p<.01), however, without higher hazards for TRM and overall mortality. The overlap among the four models and their adjusted HR for OS is shown in Figure 1.

Conclusions: functional HLA-DPB1 matching is of prognostic value in 8/8 UD-HSCT outcomes. In our cohort, TCE4 appears superior to other models in predicting survival and stratifying risks of TRM and $\mathrm{cGvHD}$, while aGvHD is best predicted by HLA-DPB1 expression model.

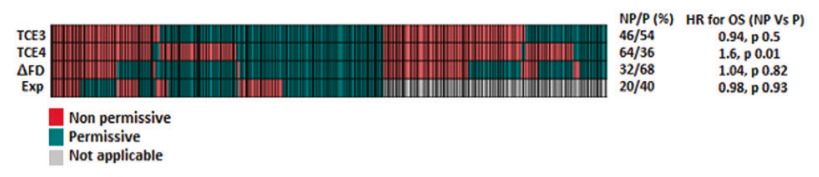

[[O169 Image] 1. Figure 1. Overlap among HLA-DPB1 functional matching models] 
Disclosure: Nothing to declare

\section{0}

Abstract already published.

\section{1}

Haploidentical transplant with post-transplant cyclophosphamide versus matched unrelated donor for all: Combined analysis from EBMT acute leukemia working group and the haploidentical transplant research consortium

Monzr M. Al Malki', Dongyun Yang ${ }^{1}$, Asad Bashey ${ }^{2}$, Amado Karduss-Urueta ${ }^{3}$, Nelli Bejanyan ${ }^{4}$, Francesca Ferraro $^{5}$, Sally Mokhtari, Armin Ghobadi, Ryotaro Nakamura ${ }^{1}$, Stephen J. Forman ${ }^{1}$, Richard Champlin ${ }^{6}$, Mohamad Mohty ${ }^{7}$, Stefan O. Ciurea ${ }^{6}$, Arnon Nagler ${ }^{8}$

${ }^{1}$ City of Hope National Medical Center, Duarte, CA, United States, ${ }^{2}$ BMT and Acute Leukemia Program at Northside Hospital, Blood and Marrow Transplant Group of Georgia, Atlanta, GA, United States, ${ }^{3}$ Instituto de CancerologiaClinica Las Americas, Bone Marrow Transplant Program, Medellin, Colombia, ${ }^{4}$ Moffitt Cancer Center, Tampa, FL, United States, ${ }^{5}$ Washington University School of Medicine, St. Louis, MO, United States, ${ }^{6}$ University of Texas MD Anderson Cancer Center, Houston, TX, United States, ${ }^{7}$ Hôpital Saint-Antoine, Université Pierre \& Marie Curie and INSERM UMRs U 938, Service d'Hématologie Clinique et Thérapie Cellulaire, Paris, France, ${ }^{8}$ Chaim Sheba Medical Center, Tel Hashomer, Israel

Background: Allogeneic hematopoietic cell transplantation (HCT) is an effective therapy for patients with high-risk acute lymphoblastic leukemia (ALL). Multiple studies comparing haploidentical HCT (HaploHCT) with matched donor transplants in acute myeloid leukemia and lymphoma showed similar outcomes. Recently, 2 retrospective studies evaluating outcomes of HaploHCT using PTCy-based GVHD prophylaxis for patients with ALL showed promising results. However, it remains unclear how outcomes of patients with ALL treated with a haploidentical donor (HAPLO) compare with HLA matched unrelated donor (MUD) transplants.

Methods: We, therefore retrospectively compared outcomes of 506 patients with ALL who underwent haploHCT with PTCy, reported from the participating centers (HIT-RC and EBMT) from 01/2005 to 6/2018, with a matched cohort of 1012 patients (1:2) who underwent MUD-HCT and were reported to EBMT. Patients were matched for sex, age at transplant $(\leq 40$ or $>40)$, disease type (B-ALL Vs. T-ALL), disease stage (CR1 Vs. CR2 Vs. other), disease risk (high Vs. others), Philadelphia chromosome status (positive Vs negative), and conditioning regimen (MAC Vs. RIC/NMA). Other significant differences in patient/transplant characteristics were adjusted for. Median age of 33 years (range $=18-73$ ) for HAPLO and 36 years (range $=18-76$ ) for MUD; $\mathrm{p}=0.0009$. Median time from diagnosis to transplant was significantly longer for haploHCT recipients (at 6 months: 24\% Vs 29\%; p< 0.0001). More patients in the HAPLO group received a femaleto-male transplantation and bone marrow graft compared with MUD (28\% Vs. $15 \% ; \mathrm{p}<0.0001$ and $48 \%$ Vs $17 \%$; $<<0.0001$, respectively). Calcineurin/methotrexate-based regime was the most commonly used prophylaxis in MUD-HCT, with ATG being added in $65 \%$ of cases.

Results: With a median follow up of 2.0 and 3.0 years in HAPLO and MUD, respectively, there was no statistical differences in the overall survival (OS) between HAPLO and MUD-HCT recipients after adjusting for all covariates (HR $=0.96,95 \%$ CI: 0.77-1.20; $p=0.73$ ). Probability of OS in the HAPLO Vs. MUD group were $61.2 \%$ and $60.4 \%$ at 1 -year and $44.7 \%$ and $48.7 \%(\mathrm{p}=0.24)$ at 3 -years. Similar to that, there was no statistical difference in progression free survival (PFS) $(\mathrm{HR}=0.94,95 \% \mathrm{CI}: 0.77-1.15 ; \mathrm{p}=0.55)$, relapse rate $(\mathrm{HR}=1.05 ; 95 \% \mathrm{CI}: 0.81-1.35 ; \mathrm{p}=0.72)$, and non-relapse mortality (NRM) $(\mathrm{HR}=0.85 ; 95 \% \mathrm{CI}: 0.62$ 1.16; $\mathrm{p}=0.3$ ); Figure 1 .

We then analyzed the data in subgroups based on the conditioning intensity. In the MAC group, day +28 neutrophil recovery was similar between HAPLO (86.6\%) and MUD $(87.3 \%),(\mathrm{p}=0.21)$. Corresponding rates after RIC were $83.5 \%$ and $88.3 \%(\mathrm{p}=0.14)$. In multivariable analysis, OS, PFS, NRM, and relapse rate were not statistically different between patients receiving HCT from HAPLO or MUD, regardless of the intensity of the conditioning regimen; (Table 1).

Conclusions: In conclusion, in this large retrospective analysis, outcomes of patients with ALL undergoing transplant from a haploidentical donor with post-transplant cyclophosphamide is comparable with those undergoing $8 / 8$ MUD transplantations.

Disclosure: No relevant conflict of interest

\begin{tabular}{lllll}
\hline & $\begin{array}{l}\text { MAC } \\
(95 \% \text { CI })\end{array}$ & P value & $\begin{array}{l}\text { RIC/NMA } \\
(95 \% \text { CI })\end{array}$ & P value \\
\hline OS & $0.91(0.70-1.18)$ & 0.47 & $0.96(0.62-1.50)$ & 0.87 \\
PFS & $0.93(0.73-1.19)$ & 0.56 & $0.90(0.60-1.34)$ & 0.60 \\
NRM & $0.73(0.5-1.05)$ & 0.09 & $1.40(0.75-2.61)$ & 0.29 \\
$\begin{array}{l}\text { Relapse } \\
\text { Rate }\end{array}$ & $1.23(0.89-1.69)$ & 0.20 & $0.65(0.41-1.03)$ & 0.06 \\
\hline
\end{tabular}

[[O171 Table] 1. Table 1. OS, EFS, NRM and relapse between HAPLO and MUD groups for MAC and RIC/NMA conditioning] 


\section{2}

NON-FIRST DEGREE RELATIVE DONOR AND THE OUTCOME OF PATIENTS RECEIVING T CELL-REPLETE HAPLOIDENTICAL TRANSPLANTATION WITH POST-TRANSPLANT CYCLOPHOSPHAMIDE

Jacopo Mariotti', Stefania Bramanti, Lucia Brunello ${ }^{2}$, Anna Maria Raiola ${ }^{3}$, Francesca Patriarca4, Massimo Martino $^{5}$, Antonio Risitano ${ }^{6}$, Angelo Michele Carella ${ }^{7}$, Alessandro Busca ${ }^{2}$, Serena Marotta ${ }^{6}$, Emanuela Merla ${ }^{7}$, Andrea Bacigalupo ${ }^{8}$, Emanuele Angelucci ${ }^{3}$, Benedetto Bruno $^{2}$, Luca Castagna ${ }^{1}$

${ }^{1}$ Humanitas Cancer Center, Rozzano, Italy, ${ }^{2}$ Ospedale Universitario Molinette San Giovanni Battista, Turin, Italy, ${ }^{3}$ Ospedale Policlinico San Martino, Genoa, Italy, ${ }^{4}$ Azienda Sanitaria Universitaria Integrata di Udine, Clinica Ematologica e Unità di Terapie Cellulari 'Carlo Melzi', Udine, Italy, ${ }^{5}$ Azienda Ospedaliera Bianchi-Melacrino-Morello, Reggio Calabria, Italy, ${ }^{6}$ Federico II University of Naples, Bone Marrow Transplant Center, Naples, Italy, ${ }^{7}$ Fondazione Casa Sollievo della Sofferenza, San Giovanni Rotondo, Italy, ${ }^{8}$ Policlinico Gemelli, Rome, Italy

Background: The safety and efficacy of haploidentical transplantation (Haplo-SCT) with post-transplant cyclophosphamide (PT-Cy) after first-degree related donors has been widely demonstrated. However, some patients lack an eligible first-degree related haploidentical donor. A prospective clinical trial of non-first-degree relative Haplo-SCT with PT-Cy was conducted at the Johns Hopkins Hospital, showing equivalent rates of graft-versus-host-disease (GVHD) and non-relapse-mortality (NRM). On the contrary, in the so called GIAC platform, the authors from the Beijing group found an increased risk of extensive chronic GVHD using collateral related (CRD) relative to immediate related donors (IRD) ${ }^{2}$. Here we report a multicentric retrospective study with the aim to evaluate the outcome HaploSCT with PT-Cy from a CRD.

Methods: From August 2010 to November 2017, 680 consecutive patients were treated with Haplo-SCT with PTCy at 6 different Italian Bone Marrow Transplant centers.

Results: 25 patients received a transplant from a CRD ( $n=17$ cousin, $n=8$ nephew) and 665 from an IRD (either sibling, parent or child). We performed a matched pair analysis (R-software) between $24 \mathrm{CRD}$ and 24 IRD transplants. Cases were matched for: gender $(p=1)$, disease type (both cohorts with: 9 AML, 7 MDS, 2 ALL, 2 NHL, 1 PMF and $1 \mathrm{MM} ; \mathrm{p}=1$ ), pre-transplant disease status (IRD: 8 CR, 3 PR, 13 SD/PD; CRD: 8 CR, 1 PR, 15 SD/PD, $\mathrm{p}=0.74)$, disease-risk index (DRI, $\mathrm{p}=1)$, HCT-CI $(\mathrm{p}=1)$, conditioning regimen (IRD: 15 RIC, 9 MAC; CRD: 12 RIC
12 MAC; $p=0.56)$. Median follow-up was 14,6 months and was similar in both cohorts $(p=0.78)$. 2-year overall survival (OS) was $41.5 \%$ for the all patients and did not differ between transplants from a IRD or a CRD $(36 \%$ vs $47 \%, \mathrm{p}=0.33$; Table I). 2-year progression-free survival (PFS) and GVHD/relapse-free survival (GRFS) was 39\% and $37 \%$, respectively. 1-year non-relapse mortality (NRM) was $22 \%$ and was similar between IRD and CRD $(\mathrm{p}=0.62$, Table I). 6-months cumulative incidence of grade 2-4 acute GVHD, grade 3-4 acute GVHD and 1-year moderate-severe chronic GVHD was 23\%, $8 \%$ and $19 \%$, respectively. Again, there was no difference between CRD and IRD transplants in terms of grade 2-4 acute GVHD and moderate-severe chronic GVHD (Table I). By univariate analysis the main variables associated with a worse outcome were: RIC relative to MAC regimen for OS ( $p=0.04$, Table I), HCT$\mathrm{CI} \geq 3$ relative to $\mathrm{HCT}-\mathrm{CI}<3$ both for grade $2-4$ acute GVHD and moderate-severe chronic GVHD (Table I). Moreover, we found that acute GVHD was more frequent with increasing donor age (hazard ratio (HR): 1.06, $\mathrm{p}=0.006$ ).

Conclusions: Our results confirm previous findings that a CRD haploidentical transplant is a viable option for HaploSCT when a first-degree donor is available. Main long-term outcome are not different in this matched pair analysis. Extending our analysis to a larger cohort of patients receiving a CRD is warranted to confirm our preliminary results.

\section{References:}

1. Elmariah H, Kasamon YL, Zahurak M, et al. Biol Blood Marrow Transplant 2018; 24: 1088-1102

2. Zhang YY, Liu DH, Liu KY. Bone Marrow Transplant 2014; 49: 496-501

Disclosure: The authors have no conflict of interest to disclose

Stem cell mobilization, collection and engineering

\section{3}

TAT-MYC, a recombinant chimeric protein, safely enhances graft performance following hematopoietic stem cell transplantation

Jerry Stein ${ }^{1,2}$, Greg Bird ${ }^{3}$, Brian Turner $^{3}$, Thomas Payne $^{3}$, Yosef Refaeli ${ }^{3,4}$

${ }^{1}$ Schneider Children's Medical Centerl, Petach Tikva, Israel, ${ }^{2}$ Tel Aviv University, Pediatrics, Tel Aviv, Israel, ${ }^{3}$ Taiga Biotechnologies, Inc., Aurora, CO, United States, ${ }^{4}$ University of Colorado, Aurora, CO, United States 
Background: Enhancing stem cell performance can improve the results of hematopoietic stem cell transplant (HSCT) for diseases in which engraftment is unpredictable, and in patients receiving pre-HSCT conditioning regimens of progressively decreasing intensity. In a series of preclinical studies, we explored the use of Tat-Myc, a chimeric recombinant protein, to improve the performance of hematopoietic stem cell grafts.

Methods: Tat-Myc recombinant protein encompasses 9 amino acids from the $\mathrm{N}$-terminal nuclear localization domain of HIV-Tat, coupled to the entire coding sequence of c-Myc, with an appended histidine tag to aid in protein purification. The construct was expressed in bacteria and purified to pharmacological grade purity under GLP conditions.

Results: Brief (1 hour) culture of fibroblasts or hematopoietic cells in medium containing 10 microgram/ $\mathrm{ml}$ Tat-Myc results in rapid nuclear localization of the recombinant protein, whence it disappears within 48 hours as measured by Western blot. Flow cytometric assays have been validated to measure the uptake of Tat-Myc recombinant protein into nucleated marrow cells. Marrow homing of Tat-Myc recombinant protein-treated murine marrow increased 5-fold as compared to that of control cells. Incubation of activated murine $\mathrm{T}$ cells with Tat-Myc recombinant protein conferred resistance to Granzyme B cytotoxicity, but did not protect cells from effects of cyclophosphamide. Tat-Myc recombinant protein-treated marrow could be serially transplanted in mice for three generations. Murine bone marrow harvested from 5fluorouracil treated mice and briefly incubated with TatMyc recombinant protein outcompeted control marrow when transplanted in sub-lethally irradiated immunedeficient mice even at ratios of 1:9 treated:control cells. $\mathrm{T}$ - and B-cell reconstitution following transplantation in immune deficient mice was superior following Tat-Myc vs control incubation of murine marrow. Engraftment of human umbilical cord blood (UCB) cells in sub-lethally irradiated immune deficient mice was markedly improved following Tat-Myc incubation as compared to that of control UCB cells.

The transforming potential Tat-Myc protein was extensively explored. Tat-Myc culture hematopoietic cells did not display aneuploidy in cytogenetic or spectral karyotypic analyses. Intramuscular injection of 10 micrograms of TatMyc protein in $\mathrm{p} 53^{+/-}$mice for 12 consecutive weeks did not result in tumor formation. To exaggerate potentially transformative effects of Tat-Myc protein, murine marrow was co-incubated with both Tat-Myc and Tat-Bcl2 recombinant proteins prior to transplantation into irradiated immune deficient mice. Mice were followed for 24 weeks and none developed malignancies. Serially transplanted marrow incubated with both Tat-Myc and Bcl-2 proteins did not result in malignancies in recipient mice. Of $>500$ mice that have been exposed to Tat-Myc recombinant protein in our experiments, none has developed a tumor.

Conclusions: In preclinical studies, brief incubation with Tat-Myc recombinant protein enhances homing, engraftment and immune reconstitution of murine and human cells in recipient mice following transplantation. Brief exposure to the recombinant protein (as opposed to transduction of the MYC gene) does not cause malignant transformation of cells. We are currently developing clinical trials using Tat-Myc protein to enhance engraftment following HSCT.

Disclosure: Greg Bird, Brian Turner, Thomas Payne, Yosef Refaeli are employees and or shareholders in Taiga Biotechnologies. Jerry Stein has received laboratory support from Taiga Biotechnologies

\section{4}

Graft $\gamma \delta$ T-cell receptor sequencing identifies public clonotypes associated to HSCT efficacy in AML patients and unravels CMV impact on repertoire distribution

\section{Lucas CM Arruda ${ }^{1}$, Ahmed Gaballa ${ }^{1}$, Michael Uhlin ${ }^{1,2,3}$}

${ }^{1}$ Karolinska Institutet, CLINTEC, Stockholm, Sweden, ${ }^{2}$ Royal Institute of Technology, Science for Life Laboratory, Stockholm, Sweden, ${ }^{3}$ Karolinska University Hospital, Stockholm, Sweden

Background: Although the impact of donor graft composition on clinical outcome after hematopoietic stem cell transplantation (HSCT) has been studied, little is known about the role of intra-graft $\gamma \delta$ T-cell receptor (TCR) repertoire on clinical outcome following HSCT. Using high-throughput sequencing we sought to analyze the TCR $\gamma$-chain (TRG) repertoire of $\gamma \delta$ T-cells within donor stem cell grafts and address its potential impact on clinical response in the corresponding patients.

Methods: We analyzed twenty peripheral blood stem cell grafts from matched unrelated donors and classified as CMV-positive/negative. The respective acute myeloid leukemia recipients were followed for disease relapse and acute graft- $v s$-host disease (aGvHD) development postHSCT. $\gamma \delta$ T-cells were isolated using magnetic beads and the gDNA extracted for next-generation sequencing (ImmunoSEQ, Adaptive Biotechnologies). TRG characteristics were assessed using VDJTools, VDJviz, tcR and ImmunoSEQ Analyzer platforms.

Results: Deep sequencing showed similar median total/ unique reads in all grafts as well as similarly low unique CDR3 TRG ratios. Grafts presented multiple clonal overrepresentations, with no differences on TCR richness between patient groups. Grafts received by the non- 
relapse patient group had an increased proportion of long CDR3 sequences (54-57 nucleotides) compared to relapse patients $[0.41 \% \mathrm{vs} 0.27 \%(\mathrm{P}=0.02)$ and $0.11 \% \mathrm{vs} 0.04 \%$ $(\mathrm{P}=0.04)$ ]. Grafts from CMV-positive donors presented significantly reduced diversity (inverse Simpson's DI: $30.70 \mathrm{vs} 81.21, \quad \mathrm{P}=0.02)$, decreased proportion of CDR3 sequences having 24, 27, 42 and 51 nucleotides $[0.27 \%$ vs $0.61 \% \quad(\mathrm{P}=0.01), \quad 0.57 \% \mathrm{vs} 1.12 \% \quad(\mathrm{P}=0.02)$, $5.11 \%$ vs $7.54 \%(\mathrm{P}=0.007)$ and $0.61 \% \mathrm{vs} 2.1 \%(\mathrm{P}=0.04)]$, and an increase of sequences with 30-39 nucleotides (5.32\%vs $4.13 \%, \mathrm{P}=0.03)$. Hyperexpanded clones took up 2.5 times more space in the CMV positive grafts $(49.33 \%$ vs $19.38 \%, \mathrm{P}=0.007)$, who presented a skewed nonGaussian distribution. For all samples, the segments TRGV9 and TRGJP were the most frequent. Nonrelapsing group received grafts with lower usage of TRGV4, TRGV5 and TRGJP2 segments and higher usage of TRGJP1 compared to relapse patients [4.13\%vs7.46\% $(\mathrm{P}=0.04), \quad 1.12 \% \mathrm{vs} 4.77 \% \quad(\mathrm{P}=0.02), \quad 3.02 \% \mathrm{vs} 5.52 \%$ $(\mathrm{P}=0.02)$ and $6.62 \% \mathrm{vs3} .23 \%(\mathrm{P}=0.02)] . \mathrm{CMV}$-positive donor grafts presented a lower TRGV2 and TRGJP expression (6.29\%vs8.99\%, $\mathrm{P}=0.04, \quad$ and $30.99 \%$ vs60.63\%, $\mathrm{P}=0.01$ ) as well as a higher TRGJP1 gene usage (11.04\%vs3.90\%, $\mathrm{P}=0.04)$. The V9-JP combination was the most frequent pairing in all samples. Non-relapse patients received grafts with lower usage of the pairing V4-J2, V5-J2, V8-JP2 [3.12\%vs6.57\% ( $\mathrm{P}=0.04), 1.06 \%$ vs4.38\% $(\mathrm{P}=0.02)$ and $1.33 \%$ vs $1.91 \%(\mathrm{P}=0.03)]$ and higher usage of V2-JP1 pairing $(1.28 \% \mathrm{vs} 0.36 \%, \mathrm{P}=0.03)$ than relapse patients. The TCR usage of the sequence pairs V2-J2, V2-JP2, V4-JP2, V9-JP, and V9-JP2 was lower in CMV-positive grafts $[4.62 \% \mathrm{vs} 6.94 \%(\mathrm{P}=0.04)$, $0.59 \%$ vs $1.33 \% \quad(\mathrm{P}=0.04), \quad 0.31 \%$ vs $0.09 \% \quad(\mathrm{P}=0.02)$, $30.75 \%$ vs $60.30 \% \quad(\mathrm{P}=0.02) \quad$ and $\quad 0.07 \% \mathrm{vs} 0.29 \%$ $(\mathrm{P}=0.01)$ ]. We identified 12 public clones shared exclusively between the grafts received by non-relapsing patients in addition to four private over-represented sequences exclusively present in grafts given to nonrelapse patients, taking from $2.00 \%$ to $6.23 \%$ of the TRG repertoire and longer than 45 nucleotides. We also identified five private over-represented and one public CDR3 sequence associated to CMV infection. Additionally, CMV-positive grafts presented the highest percentage or repertoire taken by private over-represented clones, ranging from $13.72 \%$ to $41.61 \%$.

Conclusions: Our findings show that the TRG composition is not associated to aGvHD incidence, CMV infection reshapes the TRG repertoire and several public sequences are associated to clinical remission.

Disclosure: The authors have nothing to disclose.
Stem cell source

\section{5}

\section{Abstract already published.}

\section{6}

Outcomes of allogenic hematopoietic stem cell transplantation for mature $T$ - and NK-cell lymphoma in kyoto stem cell transplantation group (KSCTG)

Mizuki Watanabe ${ }^{I}$ Junya Kanda ${ }^{1}$, Yasuyuki Arai, Takayuki Ishikawa ${ }^{2}$, Kazunori Imada ${ }^{3}$, Yasunori Ueda ${ }^{4}$, Takashi Akasaka ${ }^{5}$, Akihito Yonezawa ${ }^{6}$, Masaharu Nohgawa $^{7}$, Nobuyoshi Arima ${ }^{8}$, Mitsuru Ito ${ }^{9}$, Tomoharu Takeoka $^{10}$, Toshinori Moriguchi', Kazuhiro Yago ${ }^{12}$, Hiroko Tsunemine ${ }^{13}$, Naoyuki Anzai ${ }^{14}$, Mitsumasa Watanabe $^{15}$, Tadakazu Kondo ${ }^{1}$, Akifumi Takaori-Kondo ${ }^{1}$

${ }^{1}$ Kyoto University, Kyoto, Japan, ${ }^{2}$ Kobe City Medical Center General Hospital, Kobe, Japan, ${ }^{3}$ Osaka Red Cross Hospital, Osaka, Japan, ${ }^{4}$ Kurashiki Central Hospital, Kurshiki, Japan, ${ }^{5}$ Tenri Hospital, Tenri, Japan, ${ }^{6}$ Kokura Memorial Hospital, Fukuoka, Japan, ${ }^{7}$ Japanese Red Cross Wakayama Medical Center, Wakayama, Japan, ${ }^{8}$ Medical Research Institute Kitano Hospital, Osaka, Japan, ${ }^{9}$ Kyoto City Hospital, Kyoto, Japan, ${ }^{10}$ Ohtsu Red Cross Hospital, Ohtsu, Japan, ${ }^{11}$ Kyoto-Katsura Hospital, Katsura, Japan, ${ }^{12}$ Shizuoka General Hospital, Shizuoka, Japan, ${ }^{13}$ Shinko Hospital, Kobe, Japan, ${ }^{14}$ Takatsuki Red Cross Hospital, Takatsuki, Japan, ${ }^{15}$ Hyogo Prefectural Amagasaki General Medical Center, Amagasaki, Japan

Background: Prognosis of mature T- and NK-cell lymphomas remains poor despite development of novel therapeutic agents. Accordingly, these lymphomas are still good candidates of allogenic hematopoietic stem cell transplantation (allo-HSCT) to achieve long-lasting remission of the diseases. However, the analysis of transplantations for these lymphomas is scarce, mainly due to the rarity of these lymphomas. Hence, we analysed the data of these transplantations operated in 15 different Japanese institutions as a multi-institutional joint research and examined factors that affected the outcomes in aims of figuring out better transplant strategies against these lymphomas.

Methods: A total of 116 patients who received alloHSCTs for mature T- and NK-cell lymphomas (PTCLNOS, $n=38 ;$ NK/T cell lymphoma nasal type, $n=22 ; A I T L$, $\mathrm{n}=20 ;$ ALCL, $\mathrm{n}=12$; EATL, $\mathrm{n}=8$; Other lymphomas, $\mathrm{n}=16$ ) from 1998 to 2016 in 15 institutions were examined. Median age at transplantation was 50 (range, 18-69). Forty patients received transplantation from related bone marrow transplantation (BMT) / peripheral blood stem cell 
transplantation (PBSCT), 36 received unrelated BMT/ PBSCT, and 40 received unrelated single cord blood transplantation (CBT). Lymphoma statuses at transplantation were CR/CR-unconfirmed in 35 patients, $\mathrm{PR}$ in 18, and others (SD, PD, relapse, and induction failure) in 62 patients. Median follow-up time for survivors was 27 months.

Results: Overall survival (OS) and relapse-free survival (RFS) rates at 2 years after transplantation were 49\% 37\%, respectively. Cumulative incidences of non-relapse mortality (NRM) and relapse at 2 years were $21 \%$ and $43 \%$, respectively. In multivariable analysis, performance status (PS) over 1 (PS $>1$ vs PS $<=1$, HR 2.49, $\mathrm{P}=0.005$ ) and lymphoma progression at transplantation $(\mathrm{CR} / \mathrm{CR}-\mathrm{u} / \mathrm{PR}$ vs others, HR 1.95, $\mathrm{P}=0.043$ ) showed significant negative impacts on OS. CBT was strongly associated with better OS compared to unrelated BMT/PBSCT (HR 0.34, $\mathrm{P}=0.003$ ) and comparable to related BMT/PBSCT. Lymphoma control status at transplantation was significantly associated with relapse (relapse/induction failure vs CR/CR-u/PR, HR 2.61, $\mathrm{P}=0.017$ ). Poor PS over 1 at transplantation ( $\mathrm{PS}>1$ vs $\mathrm{PS}<=1$, HR $3.35, \mathrm{P}=0.023)$ and reduced-intensity conditioning (RIC) regimen (RIC vs myeloablative, HR $3.54, \mathrm{P}=0.025)$ were associated with higher risk of NRM.

Conclusions: Allo-HSCT could improve overall survival of patient with mature $\mathrm{T}$ - or NK-cell lymphomas, if performed at appropriate timing with good disease control of partial remission (PR) or better. Cord blood unit could be a favorable alternative donor source when related donors are not available. On the other hands, RIC regimen did not decrease the risk of NRM in allo-HSCT for mature T- and NK-cell lymphoma patients in our setting. Out study also suggested that major problem of allo-HSCT is still a high frequency of relapse after transplantation. Better disease control is mandatory to improve the outcomes of allo-HSCT for mature T- or NK-cell lymphomas.

Disclosure: Nothing to declare

\section{Late-breaking abstracts}

\section{7}

Comparable outcome after 10/10 and 9/10 HLAmatched unrelated donor stem cell transplantation (HSCT) using post-transplant cyclophosphamide (PTCY) for GVHD prophylaxis, on behalf of the ALWP-EBMT

Francesca Lorentino ${ }^{1}$, Myriam Labopin ${ }^{2,3,4}$, Fabio Ciceri $^{1}$, Luca Vago ${ }^{1}$, Katharina Fleischhauer ${ }^{5}$, Boris Afanasyev $^{6}$, Nicolaus Kröger ${ }^{7}$, Jan J. Cornelissen ${ }^{8}$, Montserrat Lovira, Ellen Meijer ${ }^{10}$, Antonin Vitek ${ }^{11}$,
Ahmet Elmaagacli $^{12}$, Didier Blaise ${ }^{13}$, Arnon Nagler ${ }^{2,14}$, Mohamad Mohty ${ }^{2,3,4}$

${ }^{1}$ San Raffaele Scientific Institute, Milan, Italy, ${ }^{2}$ Université Pierre and Marie Curie, Paris, France, ${ }^{3}$ Unite' $^{\prime}$ de Recherche Mixte en Sante' (UMR_S) 938, INSERM, Paris, France, ${ }^{4}$ Hôpital Saint-Antoine, AP-HP, Paris, France, ${ }^{5}$ Essen University Hospital, Institute for Experimental Cellular Therapy, Essen, Germany, ${ }^{6}$ Raisa Gorbacheva Memorial Research Institute, The First Pavlov State Medical University of St-Petersburg, St Petersburg, Russian Federation, ${ }^{7}$ University Hospital Eppendorf, Hamburg, Germany, ${ }^{8}$ Erasmus MC Cancer Institute University Medical Center Rotterdam, Rotterdam, Netherlands, ${ }^{9}$ Hospital Clinic, Institute of Hematology \& Oncology, Barcelona, Barcelona, Spain, ${ }^{10}$ University Medical Center, Amsterdam, Netherlands, ${ }^{11}$ Institute of Hematology and Blood Transfusion, Prague, Prague, Czech Republic, ${ }^{12}$ Asklepios Clinic St. Georg, Hamburg, Germany, ${ }^{13}$ Institut Paoli Calmettes, Marseille, France, ${ }^{14}$ Chaim Sheba Medical Center, TelHashomer, Israel

Background: PTCy is largely adopted as GvHDprophylaxis backbone in haploidentical transplantation. The encouraging results prompted investigations to assess PTCy also in unrelated donor (UD) setting. High-resolution HLA-matching contributes to UD-HSCT success; however, due to the selective in-vivo deletion of alloreactive T-cells, PTCy could modulate HLA-matching impact on UDHSCT.

Methods: We compared the outcomes of acute leukemia patients receiving 10/10 $(n=431)$ and 9/10 $(n=234)$ HLAallele matched UD-HSCT with PTCy GvHD-prophylaxis, reported to the ALWP-EBMT in 2010-2017. Primary endpoint was GvHD-free \& Relapse-free survival (GRFS). Secondary endpoints were leukemia-free survival (LFS), overall survival (OS), acute and chronic GvHD, relapse and transplant-related mortality (TRM). Table 1 illustrates patients' characteristics. The power to detect a 2-years $10 \%$ difference GRFS between the 2 groups was $83 \%$ (alpha 2-sided $=5 \%$ ).

Results: Outcome endpoints at 2 years were not different between 10/10 and 9/10 UD-HSCT (GRFS: $42 \pm 5 \%$ and 43 $\pm 7 \%, \mathrm{p}=0.5$; LFS: $56 \pm 5 \%$ and $55 \pm 7 \%, \mathrm{p}=0.7$; OS: $63 \pm 5 \%$ and $60 \pm 8 \%, \mathrm{p}=0.9$, respectively). The 100-day CI of grade $\geq 2$ and grade $\geq 3$ aGvHD were comparable for $10 / 10$ and $9 / 10 \mathrm{UD}(31 \pm 5 \%$ and $28 \pm 6 \%, \mathrm{p}=0.4$ and $10 \pm 3 \%$ and 9 $\pm 3 \%, p=0.5$, respectively). Likewise, the 2 -y CI of cGvHD and extensive cGvHD were similar between $10 / 10$ and $9 / 10$ UD $(32 \pm 5 \%$ and $38 \pm 7 \%, \mathrm{p}=0.2$ and $17 \pm 4 \%$ and $15 \pm 5 \%$, $\mathrm{p}=0.4$, respectively). The 2 -y CI of TRM was $19 \pm 4 \%$ after $10 / 10$ and $17 \pm 5 \%$ after $9 / 10$ UD-HSCT $(\mathrm{p}=0.4)$. Relapse 
incidence at 2 -y was $25 \pm 5 \%$ for $10 / 10$ and $28 \pm 6 \%$ for $9 / 10$ UD-HSCT $(\mathrm{p}=0.6)$.

After adjustment for diagnosis, patient age, disease status, Karnofsky PS, donor/patient gender and CMV, conditioning intensity, additional use of ATG, the multivariable model showed no effect of donor HLA-matching on outcomes. Compared to 10/10 UD, the hazards for GRFS was 0.9 for $9 / 10 \mathrm{UD}(\mathrm{p}=0.2)$, the HR for LFS was 0.9, $\mathrm{p}=0.3$ and the HR for $\mathrm{OS}$ was $1, \mathrm{p}=0.8$. Moreover, compared to 10/10 UD, 9/10 UD-HSCT yelded similar hazards for grade $\geq 2$ aGvHD (HR: $0.8, \mathrm{p}=0.2$ ), cGvHD (HR: 1.2, $\mathrm{p}=0.3$ ), TRM (HR: 0.7, $\mathrm{p}=0.2$ ) and relapse (HR: $1, p=0.9)$. No interaction was found between donor type and additional ATG use. Variables associated with GRFS were active disease (HR 2.1 compared to $1^{\text {st }} \mathrm{CR}, \mathrm{p}<10^{-5}$ ) and Karnofsky PS $\geq 90 \%$ (HR 0.6, $\mathrm{p}<10^{-5}$ ).

Conclusions: In the present series of acute leukemia patients transplanted with PTCy, we report comparable survival with $9 / 10$ and 10/10 HLA-matched UD-HSCT, across all disease stages, suggesting that this platform could alleviate the detrimental effect of single HLA-allele mismatching. These results warrant prospective comparative trials of PTCy versus standard use of ATG-based GVHD prophylaxis in matched and mismatched UDHSCT.

\begin{tabular}{llll}
\hline & $\begin{array}{l}10 / 10-\mathrm{UD} \\
(\mathrm{n}=431)\end{array}$ & $9 / 10-\mathrm{UD}(\mathrm{n}=234)$ & $p$ \\
\hline $\begin{array}{l}\text { Median follow- } \\
\text { up, months }\end{array}$ & $18(5-35)$ & $20(11-31)$ & 0.5 \\
$\begin{array}{l}\text { Patient median age } \\
\text { Diagnosis: AML; ALL }\end{array}$ & $43(18-76)$ & $48(19-73)$ & 0.1 \\
& $118(27 \%)$ & $170(73 \%) ;$ & 0.9 \\
Disease status: CR1; & $278(64 \%) ; 82$ & $134(57 \%) ; 57$ & 0.2 \\
CR>1; Advanced & $(19 \%) ; 71(17 \%)$ & $(24 \%) ; 43(19 \%)$ & \\
Karnofsky PS $\geq 90 \%$ & $314(75 \%)$ & $162(72 \%)$ & 0.3 \\
Cell source: PB; BM & $386(90 \%) ;$ & $205(88 \%) ;$ & 0.4 \\
& $45(10 \%)$ & $29(12 \%)$ & \\
Conditioning: & $193(45 \%) ;$ & $99(42 \%) ;$ & 0.5 \\
RIC; MAC & $238(55 \%)$ & $135(58 \%)$ & \\
Additional ATG use & $126(29 \%)$ & $75(32 \%)$ & 0.4 \\
\hline
\end{tabular}

[[O177 Table] 1. Table 1. Population description] Disclosure: Nothing to declare

\section{8}

Myeloablative conditioning for first allogeneic HSCT in pediatric all: FTBI or chemotherapy? - An update of the retrospective multicenter EBMT-PDWP study

Andre Manfred Willasch ${ }^{1}$, Christina Peters ${ }^{2}$, Petr Sedlacek $^{3}$, Jean-Hugues Dalle ${ }^{4}$, Vassiliki Kitra-Roussou ${ }^{5}$, Akif
Yesilipek $^{6}$, Jacek Wachowiak ${ }^{7}$, Arjan Lankester ${ }^{8}$, Arcangelo Prete ${ }^{9}$, Amir Ali Hamidieh ${ }^{10}$, Marianne Ifversen ${ }^{11}$, Jochen Buechner ${ }^{12}$, Gergely Krivan ${ }^{13}$, Rose-Marie Hamladji $^{14}$, Cristina Diaz de Heredia ${ }^{15}$, Elena Skorobogatova $^{16}$, Gerard Michel ${ }^{17}$, Franco Locatelli ${ }^{18}$, Alice Bertaina $^{19}$, Paul Veys ${ }^{20}$, Sophie Dupont ${ }^{21}$, Reuven Or ${ }^{22}$, Tayfun Güngör ${ }^{23}$, Olga Aleinikova ${ }^{24}$, Sabina Sufliarska ${ }^{25}$, Mikael Sundin ${ }^{26}$, Jelena Rascon ${ }^{27}$, Ain Kaare ${ }^{28}$, Damir Nemet $^{29}$, Franca Fagioli ${ }^{30}$, Thomas Erich Klingebiel, Jan Styczynski, ${ }^{31}$ Marc Bierings ${ }^{32}$, Kalman Nagy ${ }^{33}$, Manuel Abecasis ${ }^{34}$, Boris Afanasyev ${ }^{35}$, Marc Ansari ${ }^{36}$, Kim Venntenranta ${ }^{37}$, Amal Alseraihy ${ }^{38}$, Alicja Chybicka ${ }^{39}$, Stephen Robinson ${ }^{40}$, Yves Bertrand ${ }^{41}$, Alphan Kupesiz ${ }^{42}$, Ardeshir Ghavamzadeh ${ }^{43}$, Antonio Campos ${ }^{44}$, Arnaud Dalissier $^{45}$, Myriam Labopin ${ }^{46}$, Selim Corbacioglu ${ }^{47}$, Jaques Emmanuel Galimard ${ }^{45}$, Peter Bader ${ }^{\text {I }}$

${ }^{1}$ Goethe University Frankfurt, Frankfurt am Main, Germany, ${ }^{2}$ St Anna Children's Hospital, Vienna, Austria, ${ }^{3}$ University Hospital Motol, Prague, Prague, Czech Republic, ${ }^{4}$ Hôpital Robert Debré and Paris-Diderot University, Paris, France, ${ }^{5}$ Aghia Sophia Children's Hospital, Thivon and Papadiamantopoulou, Athens, Greece, ${ }^{6}$ Bahcesehir University, Istanbul, Turkey, ${ }^{7}$ University of Medical Sciences, Poznan, Poland, ${ }^{8}$ Leiden University Medical Center, Leiden, Netherlands, ${ }^{9}$ University of Bologna, Bologna, Italy, ${ }^{10}$ Tehran University of Medical Sciences, Hematology-Oncology and Stem Cell Transplantation Research Center, Teheran, Iran, Islamic Republic of, ${ }^{11}$ Copenhagen University Hospital, Rigshospitalet, Copenhagen, Denmark, ${ }^{12}$ Oslo University Hospital Rikshospitalet, Oslo, Norway, ${ }^{13}$ United St. Istvan and St. László Hospital, Budapest, Hungary, ${ }^{14}$ Service Hématologie Greffe de Moelle, Centre Pierre et Marie Curie, Alger, Algeria, ${ }^{15}$ Hospital Universitario Vall d'Hebron, Barcelona, Spain, ${ }^{16}$ Russian Children's Hospital, Moscow, Russian Federation, ${ }^{17}$ Timone Enfants Hospital, AP-HM and Aix-Marseille University, Marseille, France, ${ }^{18}$ IRCCS Ospedale Pediatrico Bambino Gesù, Università di Pavia, Rome, Italy, ${ }^{19}$ Stanford University Medical Center, Stanford, CA, United States, ${ }^{20}$ NHS Foundation Trust, Great Ormond Street Hospital for Children, London, United Kingdom, ${ }^{21}$ Cliniques Universitaires Saint-Luc, Brussels, Belgium, ${ }^{22}$ Hadassah-Hebrew University Medical Center, Jerusalem, Israel, ${ }^{23}$ University Children's Hospital, Children`s Research Center (CRC), Zurich, Switzerland, ${ }^{24}$ Republic Clinical Research Centre, Minsk, Belarus, ${ }^{25}$ Comenius University Medical School, Limbová, Bratislava, Slovakia, ${ }^{26}$ Astrid Lindgren Children's Hospital, Karolinska University Hospital, Pediatric Blood Disorders, Immunodeficiency and Stem Cell Transplantation, Stockholm, Sweden, ${ }^{27}$ Affiliate of Vilnius University Hospital Santariskiu Klinikos, Center of Pediatric Oncology and Hematology, Children's Hospital, Vilnius, Lithuania, 
${ }^{28}$ Tartu University Hospital, Tartu, Estonia, ${ }^{29}$ University Hospital Centre, Internal Clinic, Zagreb, Croatia, ${ }^{30}$ Regina Margherita Children's Hospital, City of Science and Health of Turin, Turino, Italy, ${ }^{31}$ Nicolaus Copernicus University Torun, Collegium Medicum, Bydgoszcz, Poland, ${ }^{32}$ University Medical Centre Utrecht Pediatrics, Urecht, Netherlands, ${ }^{33}$ Child Welfare Center, Borsod County Teaching Hospital, Miskolc, Hungary, ${ }^{34}$ Instituto Portugues Oncologia, Lisboa, Portugal, ${ }^{35}$ Saint Petersburg State Medical I.P. Pavlov University, Raisa Gorbacheva Memorial Research Institute for Pediatric Oncology, Haematology and Transplantation, Saint Petersburg, Russian Federation, ${ }^{36}$ Geneva University Hospital, Geneva, Switzerland, ${ }^{37}$ University of Helsinki, Hospital for Children and Adolescents, Helsinki, Finland,

${ }^{38}$ King Faisal Specialist Hospital \& Research Center, Riyadh, Saudi Arabia, ${ }^{39}$ Wroclaw Medical University, Cape of Hope Medical Center, Wroclaw, Poland, ${ }^{40}$ NHS Foundation Trust, University Hospitals Bristol, Bristol, United Kingdom, ${ }^{41}$ IHOP and Claude Bernard University, Lyon, France, ${ }^{42}$ Akdeniz University, School of Medicine, Antalya, Turkey, ${ }^{43}$ Shariati Hospital, HematologyOncology and BMT Research, Teheran, Iran, Islamic Republic of, ${ }^{44}$ Portuguese Institute of Oncology, Porto, Portugal, ${ }^{45}$ EBMT Paediatric Diseases Working Party, Paris, France, ${ }^{46}$ EBMT Paris Study Office, Paris, France, ${ }^{47}$ University of Regensburg, Regensburg, Germany

Background: It is still a matter-of-debate if chemotherapy (CHT) can replace fractionated total-body-irradiation (fTBI) in myeloablative conditioning (MAC) for allo-HSCT in pediatric ALL. We hereby present the latest update of this international multicenter EBMT-PDWP-study (Blood 2017, 130(Suppl. 1):911).

Methods: This update was done to extend the time of follow-up (FU, date of analysis: 01/OCT/2018). To compare outcomes of fTBI vs CHT-conditioning, we performed a retrospective EBMT-registry study. Children aged 2-18 years (y) after MAC for first allo-HSCT of BM/ PBSC from MSD/UD in CR1/CR2 between 2000-2012 were included. Propensity-score-weighting was used to control pretreatment imbalances of observed variables. This statistical method ensured that analyzed groups differed only in the parameter under investigation (here: conditioning).

Results: In total 3054 pts (CR1: 1498 (49\%), CR2: 1556 (51\%)) were included. CR1: 1041 pts (69\%) received BM and 457 pts (31\%) PBSC from MSD (756 (50\%)) or UD (742 (50\%)). CR2: 1064 pts $(68 \%)$ received BM and 492 pts $(32 \%)$ PBSC from MSD (672 (43\%)) or UD (884 $(57 \%))$. Overall, conditioning was fTBI- in $2630(86 \%)$ and CHT-based in 424 pts (14\%). Busulfan/Cyclophosphamide/ Etoposide $(\mathrm{Bu} / \mathrm{Cy} /$ Eto) was the most frequently applied CHT-regimen in CR1 (66 (31\%)) and Bu/Cy in CR2 (68
$(32 \%)$ ). The remaining conditionings included 5 combinations (other-chemo).

1498 pts in CR1 were conditioned with fTBI (1285), Bu/ Cy/Eto (66) or other-chemo (147) with median-FU of 6.9, 6.2 and $4.5 \mathrm{y}$. In weighted univariate analysis, 5-y-OS was $68.8 \%$ after fTBI, $75.6 \%$ after other-chemo and $81.4 \%$ after $\mathrm{Bu} / \mathrm{Cy} /$ Eto. In weighted Cox-model, taking into account the center-effect, pts having received $\mathrm{Bu} / \mathrm{Cy} /$ Eto had a lower risk to experience an event compared to fTBI $(\mathrm{HR}=0.53$, $\mathrm{P}=.029$ ). 5-y-LFS (range: $58.4 \%$ (other-chemo) to $72.6 \%$ (Bu/Cy/Eto)), 5-y-RI (range: $17.1 \%$ (Bu/Cy/Eto) to $31.8 \%$ (other-chemo)) and 5-y-NRM (range: 9.8\% (other-chemo) to $13.8 \%$ (fTBI)) did not show significant differences in weighted Cox-model.

1556 pts in CR2 were conditioned with fTBI (1345), Bu/ Cy (68) or other-chemo (143) with median-FU of 6.2, 5.2 and $5.8 \mathrm{y}$. In weighted univariate analysis, 5-y-OS was $31.1 \%$ after other-chemo, $43.5 \%$ after $\mathrm{Bu} / \mathrm{Cy}$ and $58.8 \%$ after fTBI. In weighted Cox-model, pts having received other-chemo had a higher risk to experience an event compared to fTBI $(\mathrm{HR}=2.00, \mathrm{P}=<.0001) .5$-y-LFS was $25.2 \%$ after other-chemo, $32.4 \%$ after $\mathrm{Bu} / \mathrm{Cy}$ and $53.7 \%$ after fTBI. In weighted Cox-model, children having received $\mathrm{Bu} / \mathrm{Cy}$ and other-chemo had a higher risk to experience an event compared to fTBI $(\mathrm{HR}=1.78, \mathrm{P}=.005$; $\mathrm{HR}=1.92, \mathrm{P}<.0001) .5-\mathrm{y}-\mathrm{RI}$ was $30.6 \%$ after fTBI, $49.3 \%$ after BuCy and $53.7 \%$ after other-chemo. In weighted Coxmodel, pts having received $\mathrm{Bu} / \mathrm{Cy}$ and other-chemo had a higher risk to experience an event compared to fTBI $(\mathrm{HR}=2.06, \mathrm{P}=.006 ; \mathrm{HR}=2.13, \quad \mathrm{P}<.0001) . \quad 5$-y-NRM (range: $18.3 \%$ (BuCy) to $21.1 \%$ (other-chemo)) did not show significant differences in weighted Cox-model.

Conclusions: This recent study-update ensured a substantial FU. We confirmed the clear superiority of fTBIconditioning compared to CHT with regard of LFS and RI for ALL-pts undergoing allo-HSCT in CR2. For pts in CR1 we could not find significant differences between fTBI and CHT-conditioning. These retrospective findings are currently re-evaluated in the prospective, randomized, international trial (ALL-SCTped-2012-FORUM).

Disclosure: Nothing to declare.

\section{9}

Abstract already published.

0180

Effective cancer immunotherapy with ERBB2-CARengineered cytokine-induced killer (CIK) cells in highrisk soft tissue sarcomas

Michael Merker ${ }^{I}$, Juliane Wagner ${ }^{1}$, Vida Meyer ${ }^{1}$, Evelyn Ullrich $^{1}$, Thomas Klingebiel ${ }^{1}$, Winfried S. Wels ${ }^{2}$, Peter Bader $^{1}$, Eva Rettinger ${ }^{I}$ 
${ }^{1}$ University Hospital Frankfurt, Frankfurt, Germany, ${ }^{2}$ Institute for Tumor Biology and Experimental Therapy, GeorgSpeyer-Haus, Frankfurt, Germany

Background: Pediatric patients with high-risk alveolar rhabdomyosarcoma (aRMS) above the age of 10 years cannot be cured by conventional therapies. Immune cells targeting ErbB2 with a chimeric antigen receptor (CAR) were recently considered for these patients. Cytokineinduced killer (CIK) cells already capable of natural killer (NK)-like anti-tumor capacity additionally redirected with an ErbB2 CAR may provide overall disease control in these high-risk tumors.

Methods: ErbB2-CAR modified CIK cells were generated from conventional CIK cells (WT-CIK) by lentiviral gene transduction on day 4 of culture. The codon-optimized CAR sequence consists of an IgG heavy-chain signal peptide, an ErbB2-specific antibody fragment scFv (FRP5) and a modified $\mathrm{CD} 8 \alpha$ hinge region, as well as CD28 transmembrane and intracellular domains and a $\mathrm{CD} 3 \zeta$ intracellular domain. $1 \times 10^{5}$ luciferase gene-transduced RH30 (aRMS) cells were engrafted in immunodeficient $\left.\mathrm{NOD} / \mathrm{SCID} / \gamma^{-} \mathrm{c}^{-} \mathrm{NSG}\right)$ mice. Mice were randomly selected into 5 different treatment groups (DBPS on day $+1,2.5 \times 10^{6}$ WT-CIK or ErbB2 CAR-CIK cells on days +1 and +36 , $2.5 \times 10^{6}$ WT-CIK or ErbB2 CAR-CIK cells on days +22 and +57$)$. Mice were monitored by bioluminescence imaging (BLI) until day +100 . Tumor engraftment and immune cell homing at tumor sites were analyzed by FACS, chimerism and immunohistochemistry analyses.

Results: Human RMS xenografts were established in all mice treated with DBPS only. Control-mice showed a median survival of 62 days. Human RMS was identified in all analyzed organs, with the highest tumor burden seen in livers of DBPS-treated mice.

Mice injected with WT or ErbB2-CAR CIK cells on days +1 and +36 showed a significant improved $(\mathrm{p}<0.014$ and $\mathrm{p}<0.01)$ disease-free survival, respectively. Furthermore, no signs of tumor engraftment were shown by BLI in ErbB2 CAR-CIK cell treated mice while some of the mice treated with WT-CIK cells developed positive tumor signals between weeks 7 and 10. In 4 out of 6 (64\%) WT- and in all $(8$ of $8,100 \%)$ CAR-CIK cells treated mice no residual tumor cells were identified by PCR-based analysis. In contrast, tumor cells were detectable in all mice with delayed anti-tumor treatment applied on day +22 and +57 . However, tumor growth was lower in these groups. Correspondingly, BLI showed delayed tumor engraftment in mice with WT- and even more with CAR-CIK cell treatment given on day 22 . Treatment on day 22 resulted in a significantly improved survival of ErbB2-CAR CIK cell treated mice $(\mathrm{p}<0.01)$, while survival was not improved after WT-CIK cell infusion ( $p>0.07$ ). Within all treatment groups, immune cells were detected by chimerism and FACS analyses. FACS analyses showed a significant increase of NK-like T cells $(\mathrm{p}<0.01$ and $<0.05$, WTand ErbB2-CAR CIK cells). Additionally, a higher, but not significant, amount of effector memory and stem cell memory $\mathrm{T}$ cells were detected.

Conclusions: These pre-clinical in vivo results indicate that ErbB2- CAR redirection of CIK cells improves both homing and NK-like cytotoxicity of CIK cells in the presence of ErbB2-positive tumors, implying that this therapy may represent a step forward in the treatment of patients with resistant, relapsed and advanced RMS.

Disclosure: Michael Merker, Juliane Wagner, Vida Meyer, Thomas Klingebiel, Winfried S. Wels and Eva Rettinger have nothing to declare. Peter Bader declares the following potential conflicts of interest: Novartis (consultancy: included expert testimony, speaker bureau, Honoraria), Medac (Research Funding, Patents and Royalties), Riemser (Research Funding), Neovii (Research Funding), Amgen (Honoraria).

\section{1}

Genesis - a phase III randomized double-blind, placebocontrolled trial, evaluating safety and efficacy of BL8040 and G-CSF in mobilization of HCS'S for autologous transplantation-multiple myeloma

\section{Hemda Chen', Zachary D. Crees ${ }^{2}$, Keith Stockerl- Goldstein $K^{2}$, Abi Vainstein ${ }^{1}$, Ella Sorani, Osnat Bohana-Kashtan ${ }^{1}$, John F Dipersio ${ }^{2}$}

${ }^{1}$ BiolineRx, Tel Aviv, Israel, ${ }^{2}$ Washington University in St. Louis, St. Louis, WA, United States

Background: CXCR4 mediates retention of hematopoietic stem cells (HSCs) in the bone marrow (BM) niche. BL8040, a novel, high affinity CXCR4 antagonist is a potent mobilizer of HSCs to the peripheral blood with numerous potential clinical applications, including mobilization of CD34+ cells for autologous HSC transplantation (autoHSCT) in Multiple Myeloma (MM). This study aims to evaluate the efficacy of single dose BL-8040 plus G-CSF in mobilization of $\geq 6.0 \times 10^{6} \mathrm{CD} 34+$ cells $/ \mathrm{kg}$ in up to 2 apheresis sessions for auto-HSCT in MM.

Methods: A Phase III study composed of an open-label, single-arm lead-in Part1 followed by a randomized, doubleblinded, placebo-controlled Part2. Eligible MM patients age $18-78$ will receive G-CSF (10 $\mu \mathrm{g} / \mathrm{kg}$; SC) daily for up to 8 days and one dose of BL-8040 $(1.25 \mathrm{mg} / \mathrm{kg}$; SC) or placebo on day 4 followed by up to 2 apheresis sessions; and if needed a second dose of BL-8040 or placebo on day 6 followed by up to 2 apheresis sessions. 
Part1 included up to 3 cohorts ( 10 patients/cohort), with Data Monitoring Committee (DMC) review after each cohort. Part2 will include 177 patients randomized 2:1.

Results: Part1 enrolled 11 patients, median age 61 (5770). $9 / 11$ patients $(82 \%)$ reached the primary endpoint of $\geq 6 \times 10^{6} \mathrm{CD} 34+$ cells $/ \mathrm{kg}$ with one dose of BL-8040 and up to 2 apheresis sessions. $7 / 11$ patients (64\%) collected $\geq 6 \times 10^{6} \mathrm{CD} 34+$ cells $/ \mathrm{kg}$ in one apheresis session. Administration of BL-8040 resulted in a 7.86-fold average increase in circulating peripheral CD34+ cells (range 1.62-15.75, median 7.5, $\mathrm{n}=9$ ). Additional CD34+ immunophenotyping/subset analyses are currently underway.

BL-8040 plus G-CSF was found to be safe and well tolerated. Following these promising results, DMC recommended early continuation to Part2 of the Phase III trial.

Conclusions: The GENESIS lead-in results demonstrate BL-8040 is a potent mobilizer of HSCs, with potential to improve mobilization rates while minimizing mobilizationrelated healthcare costs.

\section{Clinical Trial Registry: NCT03246529}

Disclosure: Hemda Chen -Medical Director, BiolineRx

Abi Vainstin - VP of medical affairs, BiolineRx

Ella Sorani- VP of R\&D, BiolineRx

Osnat Bohana-Kashtan- project manager, BiolineRx

John F. DiPersio - consultant for BiolineRx

\section{2}

Real life experience in the treatment of pediatric, adolescent and young adult all patients using commercially available CAR-T-cells

Peter Bader', Jan Soerensen', Eva Rettinger', Andre Willasch $^{1}$, Anne Sonntagbauer ${ }^{1}$, Sümmeyye Elgaz', Franziska Kalensee ${ }^{1}$, Hermann Kreyenberg ${ }^{1}$, Marc Ansari $^{2}$, Christine Wolschke ${ }^{3}$, Richard Noppeney, Gunnar Cario ${ }^{5}$, Guido Kobbe ${ }^{6}$, Tobias Feuchtinger ${ }^{7}$, Marion Subklewe ${ }^{8}$, Thomas Klingebiel, Martin Hutter ${ }^{1}$, Evelyn Ullrich $^{1}$, Halvard Bönig', Sabine Huenecke ${ }^{1}$, Andrea Jarisch $^{1}$

${ }^{1}$ University Hospital Frankfurt, Frankfurt, Germany, ${ }^{2}$ Geneva University Hospital, Children and Adolescents, Geneva, Switzerland, ${ }^{3}$ Universitsklinik Hamburg Eppendorf, Hamburg, Germany, ${ }^{4}$ University Hospital Essen, Essen, Germany, ${ }^{5}$ University Medical Center Schleswig-Holstein, Kiel, Germany, ${ }^{6}$ University Medical Center Düsseldorf, Düsseldorf, Germany, ${ }^{7}$ LMU, von Hauner Kinderspital, Munich, Germany, ${ }^{8}$ University Hospital Munich, Munich, Germany, ${ }^{9}$ German Red Cross Blood Center Frankfurt and Institute of Transfusion Medicine and Immunohematology, Frankfurt, Germany,
Background: Children, adolescents and young adult patients with ALL with second relapse, relapse after allogeneic SCT or patients with primary refractory disease have a poor prognosis with conventional treatment concepts. In this patient group several studies using second generation CD19 chimeric antigen receptor T- cells (CAR-T cells) demonstrated high efficacy with two year survival rates of up to $65 \%$. Recently, two different CAR-T cell products were approved by the FDA and in 2018 also by the EMA in Europe: Tisagenlecleucel $\left(\mathrm{Kymriah}^{\circledR}\right)$ for the treatment of patients with B-precursor ALL who are i) refractory, ii) in second relapse or iii) who relapsed after allogeneic SCT (relapsed/refractory ALL; r/r ALL) as well as for diffuse large cell lymphoma (DLBCL) and Axicabtagen Ciloleucel $\left(\right.$ Yescarta $^{\circledR}$ ) for the treatment of B-cell lymphoma. Here we report our first results using commercially available CART-cell product Tisagenlecleucel $\left(\mathrm{Kymriah}^{\circledR}\right)$ in patients with ALL which were treated by the University Hospital for Children and Adolescents Frankfurt am Main $(n=9)$, the Department of Medicine III, University Hospital LMU Munich $(\mathrm{n}=1)$, and the von Hauner Kinderspital, LMU Munich, Germany $(\mathrm{n}=1)$.

Methods: Between October and December 2018 eleven patients received apheresis for CAR-T cell generation. Nine patients suffered from $\mathrm{r} / \mathrm{r}$ c-ALL, and two from $\mathrm{r} / \mathrm{r} \mathrm{B}-$ precursor ALL. Eight patients had relapsed after allogeneic HSCT, one patient each suffered from first r/relapse, second r/relapse or from primary r/ALL. In 9/11 (82\%) patients CAR-T cell production was successful after one and in 2 patients $(18 \%)$ after a second apheresis.

Median patients' age was 16.7 years (1.1-25.4). Between apheresis and start of lymphodepleting chemotherapy (LDC), 10/11 patients received low dose chemotherapy according to the FRAPOSTALL protocol (Willasch et al. 2016) and one patient was treated with inotuzumab. Production slots were immediately available, resulting in turn-around-time from apheresis to product delivery of 3-4 weeks. Disease status at start of LDC was CR w/o MRD $(n=4), C R$ MRD pos. $(n=2), C R i(n=1)$, persistence of blasts $(n=2)$, and disease progression $(80-100 \%$ blasts, $\mathrm{n}=2$ ). LDC consisting of FLU-CYC was given to $9 / 10$ patients, one patient did not receive LDC.

Results: CAR-T cells could be transfused to $10 / 11$ patients at a median dose of $1.5 \mathrm{Mio} / \mathrm{kgBW}$ (0.145 Mio 8.5). In one patient, in whom a second viral transduction procedure was necessary; neither LDC nor CAR-T cell transfusion could be given because of diseases progression and deterioration of the patient's general condition. Cytokine release syndrome (CRS) grade I was observed in one patient; $8 / 10$ patients did not develop CRS. Cytokine related encephalopathy syndrome (CRES) grade II was observed in $1 / 10$ patients. At day $+288 / 10$ patients $(80 \%)$ achieved MRD negative CR. The two patients with 
progressive disease at time of LDC did not respond although CAR-T cells could be seen morphologically under the microscope. This might be explained by multidrug related phenomenon protecting refractory leukemia from CAR-T cell attack.

Conclusions: Commercial available CAR-T cell product Tisagenlecleucel $\left(\mathrm{Kymriah}^{\circledR}\right)$ showed high efficacy in $\mathrm{r} / \mathrm{r}$ ALL patients to re-induce CR.
Clinical Trial Registry: Commercial available CAR-T cell product Tisagenlecleucel $\left(\mathrm{Kymriah}^{\circledR}\right)$ showed high efficacy in r/r-ALL patients to re-induce CR.

Disclosure: PB: Novartis (consultancy: included expert testimony, speaker bureau, Honoraria); Medac (Research Funding, Patents and Royalties); Riemser (Research Funding); Neovii (Research Funding); Amgen (Honoraria). AJ: Novartis and Bluebird: (Consultancy). All other author declare no COI. 